\title{
Assinaturas Parciais e o Teorema de Yoshida-Nicolaescu
}

José Carlos CorrêA Eidam

\author{
TESE APRESENTADA \\ AO \\ Instituto de Matemática e Estatística \\ DA \\ Universidade de São Paulo \\ PARA \\ OBTENÇÃO DO GRAU DE DOUTOR \\ EM \\ Matemática
}

Área de Concentração: Geometria Diferencial

Orientador: Prof. Dr. Paolo Piccione

- SÃo PAUlo, ABRIL de 2005 -

DURANTE A REALIZAÇÃo DESTE TRABALHO,

O AUTOR RECEBEU O APOIO FINANCEIRO DA FAPESP, PROCESSO 01/00046-3. 

Este exemplar corresponde à versão final da tese defendida por José Carlos Corrêa Eidam em 01/04/2005.

BANCA EXAMINADORA

Prof. Dr. Paolo Piccione (Presidente) - IME/USP

Prof. Dr. Daniel Victor Tausk - IME/USP

Prof. Dr. Levi Lopes de Lima - UFC

Prof. Dr. Francesco Mercuri - IMECC/Unicamp

Prof. Dr. Orlando Lopes - IMECC/Unicamp 

"O senhor entende, o que conto assim é resumo; pois no estado do viver, as coisas vão enqueridas com muita astúcia: um dia é todo para a esperança, o seguinte para a desconsolação.

João Guimarães Rosa, Grande Sertão: Veredas 


\section{Introdução}

Em 1960, I. Gel'fand propôs em seu artigo [43] o problema geral de determinar a relação entre invariantes analíticos e topologicos de operadores elípticos em variedades. Ele sugere que seja possível expressar o índice de Fredholm de um operador elíptico somente em termos topológicos. Este tipo de relação já havia aparecido em trabalhos anteriores de F. Hirzebruch, R. Seeley, M. Agranovic, A. Dynin, A. Vol'pert entre outros. F. Hirzebruch já havia provado nos anos 50 a famosa fórmula da assinatura, a qual expressa a assinatura de uma variedade riemanniana compacta orientada $X$ de dimensão $4 k$ como a integral sobre $X$ de uma forma diferencial invariantemente definida sobre $X$. F. Hirzebruch e K. Kodaira também provaram por métodos analíticos e topológicos, o famoso teorema de Riemann-Roch para variedades algébricas, conforme [51], [57]. Respostas parciais para o problema foram dadas pelos referidos autores em [4], [5], [35], [36], [89], [90], [101], mas o problema geral ainda não havia sido resolvido com sucesso até que em 1963, M. Atiyah e I. Singer em [10] publicaram a primeira prova do teorema que posteriormente passou a chamar-se Teorema de Atiyah-Singer. Outras provas podem ser encontradas em [11], [12], [18], [46], [60]. Este teorema afirma que que se $E$ é um fibrado complexo sobre a variedade compacta $X$ e $P$ é um operador elíptico agindo em seções de $E$, então

$$
\text { ind } P=\int_{X} \pi_{!} \operatorname{ch}(\sigma) \wedge \hat{\mathrm{A}}(X)^{2},
$$

onde ind $P$ denota o índice de Fredholm de $P, \pi_{!}$é o isomorfismo de Thom, ch denota o isomorfismo de Chern entre K-teoria e cohomologia, $\sigma$ é uma certa classe na K-teoria de TX que depende exclusivamente de $P$ e $E$ e $\hat{A}(X)$ é uma classe de cohomologia de $X$ invariantemente definida.

O problema análogo para variedades com bordo é ainda mais delicado que o primeiro. Neste caso, eram conhecidas na época apenas algumas situações nas quais era possível calcular o índice de operadores elípticos em variedades com bordo em termos topológicos, como o teorema de Hellwig-Vekua, conforme [17]. As primeiras respostas para a questão vêm no início dos anos 70 com o trabalho de M. Atiyah, P. Gilkey, V. Patodi e I. Singer; o ponto crucial nesta situação é entender a assimetria espectral de um operador elíptico auto-adjunto $B$ em uma variedade compacta, i.e., quão simétrico é o espectro de $B$ com relação a origem. Para isso, é introduzido o $\eta$-invariante de um operador elíptico auto-adjunto, conforme [14], [15]. A filosofia de trabalho nesta situação consiste em utilizar o núcleo do calor de uma variedade compacta $Y$ para relacionar a topologia de $Y$ com a análise de um operador elíptico definido em $Y$, pois o núcleo do calor de $Y$ é um objeto que depende explicitamente da topologia de $Y$ e o índice de $P$ pode ser escrito em termos de um certo traço do núcleo do calor, conforme [45], [46], [63], [74]. Outra dificuldade no tratamento desta situação é a escolha de condições de fronteira adequadas para o operador $P$, i.e., condições de fronteira sob as quais $P$ define um operador Fredholm. Tais condições de fronteira são as chamadas condições de Atiyah-Patodi- 
Singer e generalizam as conhecidas condições de Lopatinsky-Shapiro, conforme [98]. Sob tais condições de fronteira, é válido o Teorema de Atiyah-Patodi-Singer, o qual afirma que se $P$ é um operador elíptico em uma variedade compacta orientada $X$ com bordo $Y$ e admite a forma cilíndrica em um colar de $Y$, i.e., $P$ admite a forma

$$
P=G\left(\frac{\partial}{\partial t}+B\right)
$$

onde $t$ denota a coordenada transversal ao bordo de $X, G$ é um isomorfismo de $E$ e $B$ é um operador elíptico auto-adjunto independente de $t$ definido em $Y$, então vale a fórmula

$$
\text { ind } P=\int_{X}\left(\pi ! \operatorname{ch}(\sigma) \wedge \tilde{\mathrm{A}}(X)^{2}\right)-\frac{\operatorname{dim}(\operatorname{ker} B)+\eta_{B}(0)}{2},
$$

onde $\sigma$ é uma classe na K-teoria de TX como antes, $\tilde{\mathrm{A}}(X)$ é uma classe de cohomologia de $X$ relacionada com $\hat{\mathrm{A}}(X)$ e $\eta_{B}$ é o $\eta$-invariante de $B$.

A partir daí, a teoria sofreu diversas modificações e serviu para provar vários resultados importantes em topologia e geometria. Vamos agora descrever a linha que seguirá o nosso trabalho.

Iniciamos descrevendo uma idéia muito interessante de A. Calderón e R. Seeley (conforme [91]) que reduz o estudo de problemas de fronteira em variedades com bordo a um tipo especial de problema de Cauchy. Consideremos o problema de fronteira

$$
\left\{\begin{array}{l}
P u=0 \\
\left.u\right|_{Y}=g
\end{array}\right.
$$

onde $P$ é um operador elíptico de primeira ordem ${ }^{1}$ agindo sobre seções de um fibrado $E$ sobre uma variedade compacta $X$ com bordo $Y$. Podemos estender $P$ a um operador elíptico $\tilde{P}$ agindo em seções de um fibrado $\tilde{E}$ (contendo $E$ como sub-fibrado) sobre uma variedade compacta $\tilde{X}$ contendo $X$. Dessa maneira, estudar o problema 0.2 é equivalente a estudar o problema

$$
\left\{\begin{array}{l}
\tilde{P} u=0 \mathrm{em} \mathrm{X} \\
\left.u\right|_{Y}=g
\end{array}\right.
$$

Isso nos induz a considerar a seguinte situação abstrata: dados uma variedade compacta orientada sem bordo $X, Y \subset X$ uma hipersuperfície fechada qualquer tal que $X=X_{+} \cup X$. com $X_{ \pm}$variedades com bordo $Y$ e $X_{+} \cap X_{-}=Y$ e $P$ um operador elíptico de primeira ordem agindo em seções de um fibrado $E$ sobre $X$, ao invés de estudar o problema 0.2, passamos a estudar o problema de Cauchy com respeito à subvariedade $Y$

$$
\left\{\begin{array}{l}
P u=0, u \in \Gamma\left(\left.E\right|_{X_{ \pm}}\right) \\
\left.u\right|_{Y}=g
\end{array},\right.
$$

onde $\Gamma\left(\left.E\right|_{X_{ \pm}}\right)$denota o espaço de seções de $E$ definidas em $X_{ \pm}$. Como é de praxe na teoria, vamos supor que $P$ admita a forma cilíndrica em um bi-colar de $Y$ e que as soluções da equação $P u=0$ em $X_{+}$e $X_{-}$sejam inteiramente determinadas pela sua restrição (ou traço) a $Y$.

\footnotetext{
${ }^{1}$ Supomos que $P$ é de primeira ordem apenas por conveniência. É possível fazer uma construção semelhante no caso de um operador de ordem qualquer, conforme [91].
} 
Neste ponto, surge uma questão interessante. Dada uma variedade $\tilde{X}$ particionada por uma subvariedade $Y$ como no parágrafo anterior, podemos nos perguntar qual a relação do operador $P$ com os problemas de fronteira 0.3. Consideremos os espaços de dados de Cauchy $\mathrm{H}_{+}(P)$ e $\mathrm{H}_{-}(P)$ de $P$ em $X_{+}$e $X_{-}$definidos como o fecho em $\mathrm{L}^{2}\left(\left.E\right|_{\gamma}\right)$ do conjunto das $g \in \mathrm{C}^{\infty}(E)$ tais que existe $u \in C^{\infty}\left(\left.E\right|_{X_{ \pm}}\right)$com $\left.u\right|_{Y}=g$. Pela elipticidade de $P$, é fácil ver que $\operatorname{ker} P$ é isomorfo à interseç̧ão $\mathrm{H}_{+}(P) \cap \mathrm{H}_{-}(P)$. Portanto, é de se esperar que tais subespaços tenham íntima relação com o operador $P$. O primeiro resultado que explicita esta relação é a chamada conjectura de Bojarski (veja [19], [25]), a qual afirma que

$$
\text { ind } P=\text { ind }\left(\mathrm{H}_{+}(P), \mathrm{H}_{-}(P)\right) \text {, }
$$

onde ind $\left(\mathrm{H}_{+}(P), \mathrm{H}_{-}(P)\right)$ é o índice de Fredholm do par Fredholm $\left(\mathrm{H}_{+}(P), \mathrm{H}_{-}(P)\right)$ de subespaços de $L^{2}(E)$.

Os espaços de dados de Cauchy descritos no parágrafo anterior também podem ser utilizados para conhecermos propriedades analíticas de operadores elípticos. Um exemplo simples desta situação é o caso do operador de Cauchy-Riemann $P=\frac{\partial}{\partial \ddot{z}}$ agindo em funções complexas (ou pares de funções reais) no plano complexo estendido $\mathbb{C}^{*}$ particionado pelo círculo unitário $Y \doteq S^{1}$. Escrevendo $P$ em coordenadas polares, vemos que $P$ admite a forma cilíndrica em uma vizinhança de $S^{1}$. Os espaços de dados de Cauchy de $P$ coincidem com os espaços de funções em $S^{1}$ cuja série de Fourier tem somente termos de ordem positiva (negativa) e portanto, têm intersecção trivial. Essa propriedade simplesmente reflete o conhecido fato

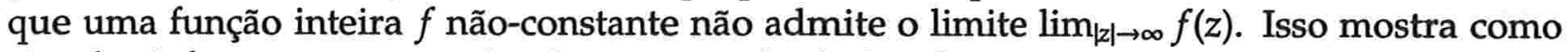
simples informações a respeito dos espaços de dados de Cauchy de um operador elíptico $P$ podem fornecer informações não-triviais a respeito de soluções da equação $P u=0$. Estes espaços são inspirados nos espaços de Hardy da teoria de funções analíticas (conforme [86]).

Outro invariante útil para a descrição da topologia de variedades é o fluxo espectral de uma família a um parâmetro de operadores de Fredholm auto-adjuntos (conforme [75]). Este invariante foi definido por M. Atiyah, V. Patodi e I. Singer nos anos 70. Eles conjecturaram que se $P$ é um operador elíptico em uma variedade compacta $X$ com bordo $Y$, admite a forma cilíndrica $P=G\left(\frac{\partial}{\partial t}+B(t)\right)$ em um colar de $Y$ e existem $\lim _{t \rightarrow \pm \infty} B(t)$ e não possuem autovalor nulo, então ind $P$ coincide com ofluxo espectral da família $B(t)$, conforme [84].

Uma classe particularmente importante de operadores satisfazendo as hipóteses usuais que estamos assumindo é a dos operadores de Dirac. Estes são operadores de primeira ordem definidos para seções de um fibrado de Clifford em termos de uma conexão qualquer. A importância destes operadores é justificada pela sua íntima relação com a geometria de $X$. No estudo de variedades de dimensão 3, é possível descrever o invariante de Casson de uma variedade tridimensional munida de uma decomposição de Heegard ao longo de uma superfície separante $\Sigma$ em termos do fluxo espectral de uma família de operadores de Dirac $\{P(s)\}_{s \in[0,1]}$, conforme [29]. A grosso modo, este invariante mede quantos autovalores de $P(0)$ deixam de ser negativos quando $s$ varia entre 0 e 1 .

As situações descritas anteriormente relacionando o fluxo espectral com invariantes topológicos e analíticos de variedades justificam a importância do estudo do fluxo espectral de famílias a um parâmetro de operadores elípticos de primeira ordem, em especial, de operadores de Dirac.

Há ainda uma outra situação em que o fluxo espectral de famílias a um parâmetro de operadores elípticos é utilizado, a qual descrevemos agora. Em dimensão ímpar, a fórmula (0.4) se trivializa, pois o índice de um operador elíptico em uma variedade de dimensão ímpar 
é nulo (conforme [60]). Neste caso, a variante adequada da conjectura de Bojarski é o teorema de Yoshida-Nicolaescu. Aqui, consideramos uma família a um parâmetro $\{P(s)\}_{s \in[0,1]}$ de operadores de Dirac sobre $M$ tais que $P(0)$ e $P(1)$ sejam bijetores; neste caso, a variante adequada da fórmula 0.4 é

$$
\operatorname{sf}(\{P(s)\})=\mu\left(\left\{\mathrm{H}_{+}(s)\right\},\left\{\mathrm{H}_{-}(s)\right\}\right),
$$

onde sf denota o fluxo espectral e $\mu$ denota o índice de Maslov de um par Fredholm de curvas de subespaços lagrangianos do espaço $\mathrm{L}^{2}\left(\left.E\right|_{\gamma}\right)$ munido da estrutura simplética dada pelo endomorfismo $G$, conforme [69], [103].

O objetivo deste trabalho é provar a fórmula 0.5 utilizando a teoria de assinaturas parciais, sem a hipótese de bijetividade nos pontos extremos assumida por L. Nicolaescu em [69]. $O$ método aqui utilizado também difere radicalmente do método utilizado no referido artigo. Naquela situação, obtém-se primeiramente uma maneira de calcular o índice de Maslov de um par Fredholm de curvas de lagrangianos $\gamma_{1}(s), \gamma_{2}(s), s \in[0,1]$, tais que $\gamma_{1}(0), \gamma_{2}(0)$ e $\gamma_{1}(1), \gamma_{2}(1)$ são transversais e cujas intersecções são isoladas de dimensão 1 . Usando um argumento bastante delicado, ele prova que é possível obter, genericamente, perturbações de um operador de Dirac que possuem somente um número finito de degenerescências e a partir daí, obtém a fórmula geral. Um argumento perturbativo como este não funcionaria para provar a fórmula no caso de degenerescências nos extremos, já que tais degenerescências não são estáveis por perturbações. Um ponto essencial é saber exatamente como devemos contar as degenerescências nos pontos extremos tanto no cálculo do fluxo espectral como do índice de Maslov. Em [34], M. Daniel provou uma fórmula semelhante a 0.5 no caso degenerado, utilizando uma convenção para o cálculo do fluxo espectral e do índice de Maslov que consiste em perturbar um pouco a curva de modo a eliminar as degenerescências nos extremos. A convenção que utilizaremos para contar a contribuição dos pontos extremos na contagem do fluxo espectral e do índice de Maslov é mais simples no sentido que não depende de nenhum tipo especial de perturbação da curva. A teoria de assinaturas parciais é baseada no trabalho de M. Farber e J. Levine [39].

No primeiro capítulo, descrevemos os fundamentos da teoria de operadores ilimitados em espaços de Hilbert, incluindo a teoria de convergência gap e o conceito fundamental de famílias holomorfas de tipo (A) de operadores, introduzido por T. Kato em [55]. Também é introduzida a noção de convergência de subespaços na métrica gap, com vistas à sua utilização na construção da topologia adequada em variedades grassmannianas de dimensão infinita. Estudamos a teoria de Fredholm para operadores limitados e ilimitados e a teoria correspondente para subespaços. Chamamos a atenção para o fato que o caminho aqui seguido na obtenção dos resultados é original: primeiramente, provamos alguns resultados elementares sobre operadores de Fredholm limitados, depois passamos ao caso de pares Fredholm de subespaços e finalmente, a teoria geral para operadores ilimitados é obtida como conseqüência simples das construções anteriores. Achamos razoável incluir uma subseção referente à perturbações compactas de operadores e subespaços por ser este um tópico geralmente negligenciado pelos analistas funcionais. Grande parte das perturbações que aparecem na teoria usual de operadores Fredholm (em geral, para operadores diferenciais e espaços de dados de Cauchy) são perturbações compactas e o desconhecimento das propriedades elementares deste tipo de perturbação tem produzido alguns inconvenientes na teoria.

Descrevemos também no primeiro capítulo, de maneira original, a construção do fluxo espectral para curvas de operadores Fredholm auto-adjuntos não necessariamente limitados em um espaço de Hilbert. É empregada uma idéia abstrata de P. Piccione [44] que permite 
estender uma família de homomorfismos definidos apenas para pequenas curvas a todo o grupóide fundamental de um espaço topológico, desde que uma certa condição de compatibilidade seja satisfeita. Em [13], M. Atiyah e I. Singer provam que o fluxo espectral é um isomorfismo entre o grupo fundamental da componente não-contrátil do espaço de operadores Fredholm auto-adjuntos e $\mathbb{Z}$. Já no caso ilimitado, essa situação não ocorre; pelo contrário, B. Booss-Bavnbek, M. Lesch e J. Phillips provaram em [23] que o espaço de operadores Fredholm auto-adjuntos ilimitados em um espaço complexo é conexo por caminhos! Isso mostra que as topologias dos espaços de operadores Fredholm auto-adjuntos limitados e ilimitados são radicalmente diferentes. A prova deste resultado dada na subseção 1.4.3 é original e funciona tanto no caso real quanto no caso complexo, uma vez que não fazemos uso da transformada de Cayley. No final da seção 1.4, apresentamos a teoria de assinaturas parciais para curvas de operadores Fredholm auto-adjuntos ilimitados. Esta teoria foi introduzida por R. Giambó, P. Piccione e A. Portaluri em [44] no caso limitado, baseada no trabalho de M. Farber e J. Levine [39]. Em nosso trabalho, fazemos a extensão adequada para o caso ilimitado, com as devidas modificações. Esta é uma das justificativas para a introdução, no início do capítulo, do conceito de famílias holomorfas de tipo (A), uma vez que, para este tipo de família, a teoria de assinaturas parciais fornece resultados bastante simples e interessantes.

Introduzimos também a teoria básica de espaços simpléticos e subespaços lagrangianos de dimensão infinita, fixando os conceitos básicos de modo a obter o máximo possível de informação a respeito do comportamento dos subespaços lagrangianos. Durante a redação deste trabalho, P. Piccione e D. Tausk provaram em [77] que dada qualquer família enumerável $\mathcal{L}$ de lagrangianos em um espaço simplético de dimensão infinita, existe um lagrangiano complementar a qualquer $L \in \mathcal{L}$. Este fato é muito mais geral que as proposições 1.5.31, 1.6.50 e o corolário 1.6.51, apesar de que, estes são suficientes para nosso trabalho. Antes de estudarmos as variedades grassmannianas em dimensão infinita, descrevemos na subseção 1.6.1 um conjunto de resultados imprescindíveis para a boa compreensão do assunto, como o teorema de Kuiper. Chamamos a atenção para o teorema 1.6.13, que generaliza fatos bem conhecidos em dimensão finita para dimensão infinita e, até onde o autor sabe, é original. Depois de construirmos uma estrutura diferenciável nas variedades grassmannianas usuais, estudamos a sua topologia, i.e., seu tipo de homotopia. Construímos um difeomorfismo entre a grassmanniana de perturbações compactas de um lagrangiano fixado e o quociente $\mathrm{U}(\infty) / \mathrm{O}(\infty)$, determinando assim o tipo de homotopia do primeiro espaço. O teorema 1.6.46 é original e prova que os espaços de perturbações Fredholm e perturbações compactas de um dado lagrangiano têm mesmo tipo de homotopia; e em particular, o espaço de perturbações Fredholm de um lagrangiano fixado tem o tipo de homotopia do quociente $U(\infty) / O(\infty)$. Este resultado foi provado incorretamente em [69]. Sua versão corrigida foi submetida à publicação em [38].

Finalizamos o primeiro capítulo com uma construção do índice de Maslov para uma curva de lagrangianos com respeito a um lagrangiano fixado e para pares de curvas de lagrangianos. O método empregado faz uso da mesma idéia de P. Piccione utilizada para definir o fluxo espectral. Chamamos a atenção para o teorema 1.7.5, o qual ainda não havia sido observado em dimensão infinita e é análogo ao caso do fluxo espectral agindo no grupo fundamental do espaço de operadores Fredholm auto-adjuntos limitados. O índice de Maslov para pares de curvas é definido recorrendo à definição do índice de Maslov para uma curva com respeito a um lagrangiano fixado. A seção encerra mostrando como a teoria de assinaturas parciais pode ser usada para o cálculo do índice de Maslov. 
O segundo capítulo inicia fixando os conceitos analíticos básicos para o nosso trabalho. Definimos operadores (pseudo) diferenciais, símbolos e espaços de Sobolev no contexto de variedades diferenciáveis. Na subseção 2.1.3, fornecemos uma prova da fórmula de GreenStokes baseada num argumento simples dado por R. Palais e R. Seeley em [71]. A subseção 2.1.4 contém os fundamentos básicos necessários para o estudo de problemas de fronteira para operadores elípticos e segue de perto o belíssimo artigo [91]. Os resultados mais utilizados sobre projetores de Calderón e operadores de Poisson encontram-se na subseção 2.1.5.

A seção 2.2 foi escrita para dar o exemplo mais comum de operador elíptico auto-adjunto de primeira ordem que verifica as hipóteses do teorema central deste trabalho. São construídas as álgebras de Clifford, os grupos $\operatorname{Pin}(n)$ e $\operatorname{Spin}(n)$ e determinadas as representações das álgebras de Clifford tanto no caso real quanto no caso complexo. Descrevemos estruturas spin em variedades com o intuito de fornecer um ambiente bastante natural para os operadores de Dirac. De fato, os teoremas do índice de Atiyah-Singer produzem resultados muito interessantes em variedades spin, conforme [60].

Os operadores de Dirac são introduzidos na seção 2.3. Tais operadores são definidos para seções de um fibrado especial $S \rightarrow X$, que é um módulo esquerda sobre a álgebra de Clifford de $X$, e dependem única e exclusivamente de uma conexão em $S$. Na referida subseção, mostramos como os operadores de Dirac podem ser utilizados para reescrever resultados geométricos e analíticos conhecidos, como o teorema de Hodge. Além disso, fornecemos exemplos de como escrever invariantes topológicos de variedades em termos de índices de operadores de Dirac, através da técnica de quebra de simetria para operadores de Dirac compatíveis. Na subseção 2.3.2, apresentamos resultados sobre propriedades de continuação única para operadores diferenciais e situamos o caso de operadores de Dirac na teoria.

Nosso trabalho encerra com o teorema de Yoshida-Nicolaescu na subseção 2.4. O problema geral é situado na teoria de problemas de fronteira desenvolvida anteriormente; como conseqüência, propriedades importantes dos espaços de dados de Cauchy são obtidas como, por exemplo, a conjectura de Bojarski. Além disso, mostramos que os espaços de dados de Cauchy formam um par Fredholm de lagrangianos, no caso de operadores elípticos auto-adjuntos verificando uma certa propriedade de continuação única. Os resultados principais do nosso trabalho são os teoremas 2.4 .17 e 2.4.19. Estes resultados foram submetidos à publicação em [37]. 


\section{Agradecimento}

"Como são preciosos para mim os teus pensamentos, ó Deus! Como é grande a soma deles!"

Livro dos Salmos, Cap. 139, Vers. 37

"O sofrimento e a dor são sempre obrigatórios para uma consciência ampla e um coração profundo."

F. Dostoiévski, Crime e Castigo

"Descobri que não há nada melhor para o homem do que ser feliz e praticar o bem enquanto vive. Descobri também que poder comer, beber e ser recompensado pelo seu trabalho é um presente de Deus. Sei que tudo o que Deus faz permanecerá para sempre; a isso nada se pode acrescentar e disso nada se pode tirar. Deus assim faz para que os homens o temam."

Livro de Eclesiastes,

Cap. 3, Vers. 12-14

Agradeço primeiramente a Deus por ter me dado a maravilhosa oportunidade de conhecêlo um pouco mais durante o tempo em que me dediquei a este trabalho. Sem tê-Lo como guia, nenhuma linha deste trabalho teria sido escrita.

Sou eternamente grato à minha mulher, Raphaela, por seu amor e pelo constante estímulo e suporte nos dias em que estar sozinho seria um problema a mais; aos meus pais, Zé e Vic, pelos tantos momentos de carinho e compreensão e às minhas queridas Carla e Patrícia, por nossos bons tempos em família.

Aos meus amigos Peterson (Véio), Marcone e Antônio agradeço pelas experiências, auxílio e companhia nos muitos dias nebulosos. Também aos meus colegas do IME deixo registrado o meu reconhecimento.

Sou grato ao Prof. Paolo Piccione por me ensinar coisas que eu jamais aprenderia sozinho. Expresso meus sinceros agradecimentos também ao Prof. Paulo D. Cordaro pela ajuda sempre pronta e irrestrita.

Finalmente, agradeço à Fapesp pelo auxílio financeiro durante a realização deste trabalho. 
Dedico este trabalho aos meus pais. 


\section{Sumário}

1 Operadores Lineares e Subespaços $\quad 1$

1.1 Preliminares . . . . . . . . . . . . . . . . . . . . 1

1.2 Convergência de operadores e subespaços . . . . . . . . . . . . . 3

1.2.1 Abertura entre subespaços . . . . . . . . . . . . . . 3

1.2.2 Distância entre operadores fechados . . . . . . . . . . . . . . . . 9

1.2 .3 Espectro e convergência . . . . . . . . . . . . . . . . . 16

1.2.4 Famílias holomorfas de operadores . . . . . . . . . . . . . . 24

1.3 Teoria de Fredholm . . . . . . . . . . . . . . . . . . . . 30

1.3.1 Operadores Fredholm . . . . . . . . . . . . . . . . . 30

1.3.2 Pares Fredholm de subespaços . . . . . . . . . . . . . . . . . 34

1.3.3 Operadores Fredholm ilimitados . . . . . . . . . . . . . . . . . 39

1.3.4 Pares comensuráveis de subespaços . . . . . . . . . . . . . . 46

1.4 O Fluxo Espectral . . . . . . . . . . . . . . . . . . . . . . . . . 50

1.4.1 Decomposições do Espectro . . . . . . . . . . . . . . . . 50

1.4.2 Construção do Fluxo Espectral . . . . . . . . . . . . . . . . . . . . . . 54

1.4.3 A Topologia dos Espaços de Operadores Auto-adjuntos . . . . . . . . . 57

1.4.4 Assinaturas Parciais e Fluxo Espectral . . . . . . . . . . . . . . . . . 62

1.5 Estruturas Simpléticas . . . . . . . . . . . . . . . . . . 68

1.5 .1 Preliminares . . . . . . . . . . . . . . . . . 68

1.5.2 Subespaços Lagrangianos . . . . . . . . . . . . . . . . . 69

1.6 Variedades Grassmannianas . . . . . . . . . . . . . . . . . . . . 78

1.6.1 Grupos de Operadores e Ações . . . . . . . . . . . . . . . . . . . . 78

1.6.2 Estruturas Diferenciáveis . . . . . . . . . . . . . . . . . 87

1.6 .3 Topologia . . . . . . . . . . . . . . . . . . . 93

1.7 Índice de Maslov e Assinaturas Parciais . . . . . . . . . . . . . . . . . 98

2 Problemas de Fronteira e Operadores de Dirac 109

2.1 Preliminares . . . . . . . . . . . . . . . . . . . . . . . . 109

2.1.1 Operadores Pseudo-Diferenciais . . . . . . . . . . . . . . . 110

2.1 .2 Espaços de Sobolev . . . . . . . . . . . . . . . . . . . . . 113

2.1.3 A Fórmula de Green-Stokes . . . . . . . . . . . . . . . . . . . . . 117

2.1.4 Operadores Elípticos Bijetores . . . . . . . . . . . . . . . . . . . . . 119

2.1 .5 O Projetor de Calderón . . . . . . . . . . . . . . . . . . . . . . . 124

2.2 Estruturas de Clifford . . . . . . . . . . . . . . . . . . . . . . . . . 127

2.2.1 Álgebras de Clifford e suas Representações . . . . . . . . . . . . . . . . . 127

2.2 .2 Estruturas $\mathrm{Spin} \ldots \ldots \ldots \ldots \ldots \ldots \ldots$ 
2.2.3 Fibrados de Clifford, fibrados spinor e conexões . . . . . . . . . . . . 136

2.3 Operadores de Dirac . . . . . . . . . . . . . . . . . . . . . . . . 141

2.3.1 Definição e Exemplos . . . . . . . . . . . . . . . . . . . 141

2.3.2 Propriedades de Continuação Única . . . . . . . . . . . . . . . . . . . . . . . . . . . . . . . . . . . . . . . . . .

2.4 O Teorema de Yoshida-Nicolaescu . . . . . . . . . . . . . . . . 150

Índice Remissivo . . . . . . . . . . . . . . . . . . . . . . . 167

Índice de Símbolos e Notações . . . . . . . . . . . . . . . . . . . . . . . . 171

xviii 
"Não co'os manjares novos e esquisitos, Não co'os passeios moles e ociosos, Não co'os vários deleites e infinitos Que afeminam os peitos generosos, Não co'os nunca vencidos apetitos, Que a fortuna tem sempre tão mimosos, Que não sofre a nenhum que o passo mude Para alguma obra heróica de virtude:

Mas com buscar, co'o seu forçoso braço, As honras que ele chame proprias suas; Vigiando e vestindo o forjado aço, Sofrendo tempestades e ondas cruas, Vencendo os torpes frios no regaço Do sul, e regiões de abrigo nuas; Engolindo o corrupto mantimento Temperado co'um árduo sofrimento;

$E$ com forçar o rosto, que se enfia, A parecer seguro, ledo, inteiro, Para o pelouro ardente que assovia E leva a perna ou braço ao companheiro. Destarte o peito um calo honroso cria, Desprezador das honras e dinheiro, Das honras e dinheiro que a ventura Forjou, e não virtude justa e dura.

Destarte se esclarece o entendimento, Que experiências fazem repousado, $E$ fica vendo, como de alto assento, $O$ baixo trato humano embaraçado. Este, onde tiver força o regimento Direito e não de afeitos ocupado, Subirá (como deve) a ilustre mando, Contra vontade sua, e não rogando."

Luís de Camões, Os Lusíadas, Canto Sexto, 96-99 




\section{Capítulo 1}

\section{Operadores Lineares e Subespaços}

Neste capítulo, trataremos de alguns fatos básicos a respeito de operadores lineares, os quais são úteis para o estudo de problemas de fronteira para operadores diferenciais.

Iniciamos introduzindo os conceitos de abertura máxima e mínima para subespaços, tendo em vista a sua grande utilidade, em especial na construção de uma teoria adequada de convergência para operadores ilimitados em geral. Construída esta teoria, estudaremos como se comporta o espectro de um operador ilimitado com respeito à perturbações pequenas em termos da abertura máxima. Vários resultados obtidos são estendidos e melhorados sob a hipótese de que tais perturbações são analíticas, num sentido que tornaremos preciso oportunamente.

Serão tratados pares (semi) Fredholm de subespaços, tendo em vista, como antes, a utilidade destes conceitos no estudo de operadores (semi) Fredholm. Verificaremos também como se comportam os operadores (semi) Fredholm quando sujeitos à pequenas perturbações.

Estudaremos ofluxo espectral de uma curva de operadores Fredholm auto-adjuntos (i)limitados e também um pouco da topologia dos espaços de operadores de Fredholm autoadjuntos. Neste capítulo é introduzida a técnica das assinaturas parciais para o cálculo do fluxo espectral de uma curva analítica de operadores auto-adjuntos.

Em seguida, trataremos de estruturas simpléticas em espaços de Hilbert, e de alguns espaços associados à estas estruturas, como a grassmanniana de lagrangianos $\Lambda$. Descreveremos explicitamente uma estrutura de variedade de Banach analítica real nas variedades grassmannianas. Encerramos o capítulo com a definição do índice de Maslov para (pares de) curvas de lagrangianos Fredholm e mostraremos como a teoria de assinaturas parciais pode ser utilizada para calculá-lo.

\subsection{Preliminares}

Sejam $\mathrm{H}, \mathrm{H}^{\prime}$ espaços de Hilbert, ambos reais ou ambos complexos. Um operador linear entre $\mathrm{H}$ e $\mathrm{H}^{\prime}$ é simplesmente uma transformação linear definida em algum subespaço $\mathrm{D}(T) \subset \mathrm{H}$, chamado domínio de $T$, com valores em $\mathrm{H}^{\prime}$. O núcleo, a imagem e o gráfico de $T$ são definidos por $\operatorname{ker} T \doteq\{u \in \mathrm{D}(T): T u=0\}, \operatorname{Im} T \doteq\{T u: u \in \mathrm{D}(T)\}$ e $\mathrm{G}(T) \doteq\{(u, T u): u \in \mathrm{D}(T)\} \subset \mathrm{H}^{\oplus} \mathrm{H}^{\prime}$, respectivamente. ${ }^{1}$ Definimos a nulidade e a deficiência de $T$ por nul $T \doteq \operatorname{dim} \operatorname{ker} T$ e $\operatorname{def} T \doteq$

\footnotetext{
${ }^{1} \mathrm{O}$ espaço $\mathrm{H} \oplus \mathrm{H}^{\prime}$ será sempre munido do produto interno $\left\langle\left(u, u^{\prime}\right),\left(v, v^{\prime}\right)\right\rangle=\langle u, v\rangle+\left\langle u^{\prime}, v^{\prime}\right\rangle$, a menos de menção explícita.
} 
codim $\operatorname{Im} T$, respectivamente. ${ }^{2}$ Lembramos que um operador $T: D(T) \subset H \rightarrow H^{\prime}$ é dito fechado se $G(T)$ for fechado. O conjunto dos operadores fechados densamente definidos ${ }^{3}$ é denotado por $\mathscr{C}\left(\mathrm{H}, \mathrm{H}^{\prime}\right)$. Quando $\mathrm{H}=\mathrm{H}^{\prime}$, abreviamos $\mathscr{C}(\mathrm{H}, \mathrm{H}) \doteq \mathscr{C}(\mathrm{H})$. Obviamente, se $T \in \mathscr{C}\left(\mathrm{H}_{1} \mathrm{H}^{\prime}\right)$ então $\operatorname{ker} T$ é fechado. O subconjunto dos operadores auto-adjuntos em $\mathrm{H}$ é denotado por $\mathscr{C}^{\text {sa }}(\mathrm{H})$, ou simplesmente $\mathscr{C}^{\text {sa }}$ quando não houver perigo de confusão. Dados operadores lineares $S, T$, escreveremos $S \subset T$ para indicar que $G(S) \subset \mathrm{G}(T)$, i.e., que $T$ é uma extensão de $S$.

Denotaremos por $\mathscr{L}\left(\mathrm{H}, \mathrm{H}^{\prime}\right)$ o espaço de Banach dos operadores lineares limitados cujo domínio é $\mathrm{H}$ com valores em $\mathrm{H}^{\prime}$. Obviamente, $\mathscr{L}\left(\mathrm{H}, \mathrm{H}^{\prime}\right) \subset \mathscr{C}\left(\mathrm{H}, \mathrm{H}^{\prime}\right)$. Escrevemos $\mathscr{L}(\mathrm{H}, \mathrm{H})=$ $\mathscr{L}(\mathrm{H})$. O subespaço dos operadores limitados auto-adjuntos em $\mathrm{H}$ será denotado por $\mathscr{L}^{\text {sa }}(\mathrm{H})$.

Um operador $\mathrm{K}: \mathrm{H} \rightarrow \mathrm{H}$ é dito de posto finito se $\operatorname{Im} K$ tem dimensão finita, e é dito compacto se a imagem por $\mathrm{K}$ da bola unitária de $\mathrm{H}$ é um subconjunto relativamente compacto. Claramente, todo operador de posto finito é compacto. Não é difícil ver que tanto o conjunto dos operadores de posto finito quanto o conjunto dos operadores compactos são ideais de $\mathscr{L}(\mathrm{H})$, e que um operador é compacto se e só se é limite na norma de operadores de uma seqüência de operadores de posto finito. O conjunto dos operadores compactos em $\mathrm{H}$ é denotado por $\mathscr{L}_{c}(\mathrm{H})$.

O espectro de um operador fechado $T \in \mathscr{C}(\mathrm{H})$ é definido como o conjunto

$$
\Sigma(T) \doteq\{\lambda \in \mathbb{K}: T-\lambda \text { não tem inverso em } \mathscr{L}(\mathrm{H})\},
$$

com $\mathbb{K}=\mathbb{R}$ ou $\mathbb{C}$, conforme $H$ seja real ou complexo. É fácil ver que $\Sigma(T)$ é fechado; o complementar de $\Sigma(T)$ é chamado de resolvente de $T$ e denotado por $\mathrm{P}(T)$. A função holomorfa $\mathrm{R}(\zeta ; T) \doteq(T-\zeta)^{-1}$ definida em $\mathrm{P}(T)$ é chamada de resolvente de $T$ e satisfaz a identidade do resolvente

$$
\mathrm{R}(\zeta ; T)-\mathrm{R}\left(\zeta^{\prime} ; T\right)=\left(\zeta-\zeta^{\prime}\right) \mathrm{R}(\zeta ; T) \mathrm{R}\left(\zeta^{\prime} ; T\right),
$$

para $\zeta, \zeta^{\prime} \in \mathrm{P}(T)$. O raio espectral de $T \in \mathscr{L}(\mathrm{H})$ é definido como $\operatorname{spr}(T) \doteq \sup _{\lambda \in \Sigma(T)}|\lambda|$.

Um operador $T \in \mathscr{C}\left(\mathrm{H}, \mathrm{H}^{\prime}\right)$ com imagem fechada é dito semi-Fredholm à esquerda (direita) se nul $T<\infty(\operatorname{def} T<\infty)$. $T$ é dito semi-Fredholm se for semi-Fredholm à esquerda ou à direita. Dizemos que $T$ é Fredholm se for semi-Fredholm à esquerda $e$ à direita. Definimos o índice de Fredholm de $T$ por

$$
\text { ind } T \doteq \operatorname{nul} T-\operatorname{def} T .
$$

Denotamos por $\mathscr{C} \mathscr{F}\left(\mathrm{H}, \mathrm{H}^{\prime}\right)$ o conjunto dos operadores Fredholm fechados densamente definidos em $\mathrm{H}$ com valores em $\mathrm{H}^{\prime}{ }^{4} \mathrm{O}$ conjunto dos operadores Fredholm auto-adjuntos em $\mathrm{H}$ é denotado por $\mathscr{C} \mathscr{F}^{\text {sa }}(\mathrm{H})$, ou $\mathscr{C} \mathscr{F}^{\text {sa }}$ quando não houver perigo de confusão. O conjunto dos operadores Fredholm limitados definidos em $\mathrm{H}$ com valores em $\mathrm{H}^{\prime}$ é denotado por $\mathscr{F}\left(\mathrm{H}, \mathrm{H}^{\prime}\right)$. De maneira análoga, definimos $\mathscr{F}^{\mathrm{sa}}(\mathrm{H})$.

Finalmente, denotaremos por $\mathrm{GL}(\mathrm{H})$ o grupo linear de $\mathrm{H}$, i.e., o grupo formado pelos operadores lineares inversíveis em $\mathscr{L}(\mathrm{H})$. O conjunto $\left\{T \in \mathrm{GL}(\mathrm{H}): T^{*} T=T T^{*}=I\right\}$ é chamado de grupo unitário de $\mathrm{H}$ e denotado por $\mathrm{U}(\mathrm{H})$ se $\mathrm{H}$ for complexo e de grupo ortogonal de $\mathrm{H}$ se $\mathrm{H}$ for real, denotado neste caso por $\mathrm{O}(\mathrm{H})$. Quando não estivermos fazendo diferença entre o caso real e o caso complexo, vamos denotar o grupo unitário (ortogonal) de $\mathrm{H}$ simplesmente por $U(H)$. Denotamos por $\mathrm{GL}_{c}(\mathrm{H}), \mathrm{U}_{c}(\mathrm{H})$ e $\mathrm{O}_{c}(\mathrm{H})$ os subgrupos de $\mathrm{GL}(\mathrm{H}), \mathrm{U}(\mathrm{H})$ e $\mathrm{O}(\mathrm{H})$, respectivamente, formados pelos operadores da forma $I+K$, com $K$ compacto.

\footnotetext{
${ }^{2} \mathrm{~A}$ codimensão de um subespaço $\mathrm{M}$ de $\mathrm{H}$ é definida como $\operatorname{codim}(\mathrm{M})=\operatorname{dim}(\mathrm{H} / \mathrm{M})$. Quando quisermos frisar que a codimensão é relativa a $H$, usaremos a notação $\operatorname{codim}_{H}(M)$.

${ }^{3} \mathrm{Ou}$ seja, $\mathrm{D}(T)$ é denso em $\mathrm{H}$.

${ }^{4}$ Não se faz nenhuma hipótese de limitação na definição de um operador (semi) Fredholm.
} 
Dados $\mathrm{M}, \mathrm{N}$ subespaços fechados, denotamos por $P_{\mathrm{M}}$ a projeção ortogonal sobre $\mathrm{M}$ e por $P_{\mathrm{N}}^{\mathrm{M}}$ a restrição de $P_{\mathrm{N}}$ a $\mathrm{M}$. As letras $\mathrm{M}, \mathrm{N}, \ldots$ serão sempre usadas para denotar subespaços de $\mathrm{H}$. Dados $\mathrm{M}, \mathrm{N}$ subespaços de $\mathrm{H}$, definimos a nulidade e a deficiencia $\operatorname{por} \operatorname{nul}(\mathrm{M}, \mathrm{N}) \doteq \operatorname{dim}(\mathrm{M} \cap \mathrm{N}) \mathrm{e}$ $\operatorname{def}(M, N) \doteq \operatorname{codim}(M+N)$. Dizemos que dois subespaços fechados $M, N$ são complementares se $\mathrm{M} \cap \mathrm{N}=0$ e $\mathrm{M}+\mathrm{N}=\mathrm{H}$. Neste caso, a projeção sobre $\mathrm{N}$ paralelamente a $\mathrm{M}$ é denotada por $P_{\mathrm{M}, \mathrm{N}}$. A notação $M \oplus N$ será reservada para indicar o subespaço $\{(u, v): u \in M, v \in N\} \subset \mathrm{H} \oplus \mathrm{H}$.

Vamos usar o símbolo $\doteq$ para indicar igual por definição, i.e., o membro esquerdo é igual por definição ao membro direito.

\subsection{Convergência de operadores e subespaços}

Nesta seção, estabeleceremos alguns fatos importantes sobre um conceito de convergência bastante geral, que engloba o conceito de convergência em norma para operadores limitados. Nosso ambiente de trabalho natural é o espaço $\mathscr{C}\left(\mathrm{H}, \mathrm{H}^{\prime}\right)$ dos operadores fechados entre $\mathrm{H}$ e $\mathrm{H}^{\prime}$. Definiremos uma métrica neste espaço que, restrita ao conjunto de operadores limitados, é equivalente àquela definida pela norma. Em seguida, estudaremos o comportamento do espectro de um operador fechado mediante perturbações e provaremos alguns resultados gerais sobre perturbações analíticas.

\subsubsection{Abertura entre subespaços}

A noção adequada de convergência para operadores fechados é dada em termos da convergência de seus gráficos. Para isso, é necessário estabelecer uma teoria abstrata de convergência para subespaços fechados; este será o objetivo desta subseção.

Definição 1.2.1 Define-se a abertura entre $\mathrm{M}$ e $\mathrm{N}$ como

$$
\delta(\mathrm{M}, \mathrm{N}) \doteq \sup _{\substack{u \in \mathrm{M} \\\|u\|=1}} \operatorname{dist}(u, \mathrm{~N})=\left\|P_{\mathrm{N}^{1}}^{\mathrm{M}}\right\|=\left\|P_{\mathrm{N}^{+}} P_{\mathrm{M}}\right\|
$$

A abertura máxima entre $\mathrm{M}$ e $\mathrm{N}$ é definida como

$$
\hat{\delta}(\mathrm{M}, \mathrm{N})=\max (\delta(\mathrm{M}, \mathrm{N}), \delta(\mathrm{N}, \mathrm{M})) \text {. }
$$

Não é difícil provar que $\delta$ verifica todas as propriedades de uma métrica, exceto a simetria. Isto justifica a definição de $\hat{\delta}$, que é de fato uma métrica. A próxima proposição, além de provar este fato, fornece uma fórmula explícita para calcular $\hat{\delta}$. Esta métrica foi introduzida por Cordes e Labrousse em [33].

Proposição 1.2.2 Se $\mathrm{M}, \mathrm{N}$ são subespaços fechados de $\mathrm{H}$, então $\hat{\delta}(\mathrm{M}, \mathrm{N})=\left\|P_{\mathrm{M}}-P_{\mathrm{N}}\right\|$. Em particular, $\hat{\delta}$ define uma métrica limitada no conjunto dos subespaços fechados de $\mathrm{H}$. Além disso, o conjunto dos subespaços fechados munido desta métrica é completo.

Prova. Temos que $P_{\mathrm{M}}-P_{\mathrm{N}}=\left(P_{\mathrm{N}^{\perp}}+P_{\mathrm{N}}\right) P_{\mathrm{M}}-P_{\mathrm{N}}\left(P_{\mathrm{M}^{\perp}}+P_{\mathrm{M}}\right)=P_{\mathrm{N}^{\perp}} P_{\mathrm{M}}-P_{\mathrm{N}^{-1}} P_{\mathrm{M}^{\perp}}$. Se $u \in \mathrm{M}$ então

$$
\begin{aligned}
\left\|P_{\mathrm{N}^{\perp}} u\right\|^{2} & =\left\|P_{\mathrm{N}^{\perp}} P_{\mathrm{M}} u\right\|^{2} \\
& \leq\left\|P_{\mathrm{N}^{\perp}} P_{\mathrm{M}} u\right\|^{2}+\left\|P_{\mathrm{N}} P_{\mathrm{M}^{\perp}} u\right\|^{2} \\
& =\left\|P_{\mathrm{N}^{\perp}} P_{\mathrm{M}} u-P_{\mathrm{N}^{\prime}} P_{\mathrm{M}^{\perp}} u\right\|^{2} \\
& =\left\|\left(P_{\mathrm{M}}-P_{\mathrm{N}}\right) u\right\|^{2},
\end{aligned}
$$


$\operatorname{logo} \delta(\mathrm{M}, \mathrm{N}) \leq\left\|P_{\mathrm{M}}-P_{\mathrm{N}}\right\|$. De maneira análoga, $\delta(\mathrm{N}, \mathrm{M}) \leq\left\|P_{\mathrm{M}}-P_{\mathrm{N}}\right\|$, e portanto, $\hat{\delta}(\mathrm{M}, \mathrm{N}) \leq$ $\left\|P_{\mathrm{M}}-P_{\mathrm{N}}\right\|$. Para a desigualdade reversa, observamos que $\left\|P_{\mathrm{N}^{+}} P_{\mathrm{M}} u\right\| \leq \delta(\mathrm{M}, \mathrm{N})\left\|P_{\mathrm{M}} u\right\|$. Além disso,

$$
\begin{aligned}
\left\|P_{\mathrm{N}} P_{\mathrm{M}^{\perp}} u\right\|^{2} & =\left\langle P_{\mathrm{N}} P_{\mathrm{M}^{\perp}} u, P_{\mathrm{M}^{\perp}} u\right\rangle \\
& =\left\langle P_{\mathrm{M}^{\perp}} P_{\mathrm{N}} P_{\mathrm{M}^{\perp}} u, P_{\mathrm{M}^{\perp}} u\right\rangle \\
& \leq \delta(\mathrm{N}, \mathrm{M})\left\|P_{\mathrm{N}} P_{\mathrm{M}^{\perp}} u\right\|\left\|P_{\mathrm{M}^{\perp}} u\right\|,
\end{aligned}
$$

de onde concluímos que $\left\|P_{\mathrm{N}} P_{\mathrm{M}^{\perp}} u\right\| \leq \delta(\mathrm{N}, \mathrm{M})\left\|P_{\mathrm{M}^{\perp}} u\right\| \leq \hat{\delta}(\mathrm{M}, \mathrm{N})\left\|P_{\mathrm{M}^{\perp}} u\right\|$. Portanto,

$$
\begin{aligned}
\left\|\left(P_{\mathrm{M}}-P_{\mathrm{N}}\right) u\right\|^{2} & =\left\|P_{\mathrm{N}^{\perp}} P_{\mathrm{M}} u\right\|^{2}+\left\|P_{\mathrm{N}} P_{\mathrm{M}^{\perp}} u\right\|^{2} \\
& \leq \hat{\delta}(\mathrm{M}, \mathrm{N})^{2}\left(\left\|P_{\mathrm{M}} u\right\|^{2}+\left\|P_{\mathrm{M}^{\perp}} u\right\|^{2}\right) \\
& =\hat{\delta}(\mathrm{M}, \mathrm{N})^{2}\|u\|^{2},
\end{aligned}
$$

provando que $\hat{\delta}(\mathrm{M}, \mathrm{N})=\left\|P_{\mathrm{M}}-P_{\mathrm{N}}\right\|$.

Para ver que $\hat{\delta}$ é completa, basta observar que o conjunto das projeções ortogonais é fechado em $\mathscr{L}(\mathrm{H})$ que é completo. Diretamente da definição, também decorre que $\delta$ e $\hat{\delta}$ são $\leq 1$.

Na próxima proposição, estabeleceremos alguns fatos simples a respeito de $\hat{\delta}$ e $\delta$.

Proposição 1.2.3 São verdadeiras as afirmações seguintes a respeito de dois subespaços fechados $\mathrm{M}, \mathrm{N}$ de $\mathrm{H}$ :

1. $S e U \in \mathrm{U}(\mathrm{H})$ então $\delta(\mathrm{M}, \mathrm{N})=\delta(U(\mathrm{M}), U(\mathrm{~N}))$;

2. $\delta(\mathrm{M}, \mathrm{N})=\delta\left(\mathrm{N}^{\perp}, \mathrm{M}^{\perp}\right) e \hat{\delta}(\mathrm{M}, \mathrm{N})=\hat{\delta}\left(\mathrm{M}^{\perp}, \mathrm{N}^{\perp}\right)$;

3. Se $\hat{\delta}(\mathrm{M}, \mathrm{N})<1$ então existe $U \in \mathrm{U}(\mathrm{H})$ tal que $U(\mathrm{M})=\mathrm{N}$. Em particular, $\mathrm{M}$ e $\mathrm{N}$ têm a mesma dimensão. Dito de outra forma, se $P, Q$ são projeções ortogonais e $\|P-Q\|<1$, então existe $U \in \mathrm{U}(\mathrm{H})$ tal que $U P U^{*}=Q$.

Prova. A primeira afirmação é óbvia, levando em consideração a identidade $P_{U(\mathrm{M})}=U P_{\mathrm{M}} U^{*}$ e a proposição 1.2.2. A segunda afirmação decorre do fato geral que se $P, Q$ são projeções ortogonais, então $\|P Q\|=\left\|(P Q)^{*}\right\|=\left\|Q^{*} P^{*}\right\|=\|Q P\|$. Provemos agora o terceiro ítem. Para simplificar a notação, escrevamos $P_{\mathrm{M}}=P, P_{\mathrm{M}^{\perp}}=P_{\perp}, P_{\mathrm{N}}=Q$ e $P_{\mathrm{N}^{\perp}}=Q_{\perp}$. Definimos $U^{\prime}=Q P+Q_{\perp} P_{\perp}, V^{\prime}=P Q+P_{\perp} Q_{\perp}$. Temos que $U^{\prime}(\mathrm{M}) \subset \mathrm{N}, U^{\prime}\left(\mathrm{M}^{\perp}\right) \subset \mathrm{N}^{\perp}, V^{\prime}(\mathrm{N}) \subset \mathrm{M}$ e $V^{\prime}\left(\mathrm{N}^{\perp}\right) \subset M^{\perp}$. Temos também que $U^{\prime} V^{\prime}=V^{\prime} U^{\prime}=I-R$, onde $R=(P-Q)^{2}$. Como $\|P-Q\|<1$ por hipótese, então $(1-R)^{-\frac{1}{2}}$ é bem definido pela fórmula clássica

$$
(1-R)^{-\frac{1}{2}}=\sum_{n=0}^{\infty}\left(\begin{array}{c}
-1 / 2 \\
n
\end{array}\right)(-R)^{n} .
$$

Pondo $U=(1-R)^{-\frac{1}{2}} U^{\prime}$ e $V=(1-R)^{-\frac{1}{2}} V^{\prime}$, temos que $U V=V U=I$. Como $R$ comuta com $P$ e $Q$, segue que $U(\mathrm{M}) \subset \mathrm{N}$ e $U\left(\mathrm{M}^{\perp}\right) \subset \mathrm{N}^{\perp}$. Como $R$ é auto-adjunto, temos que $U$ é unitário ${ }^{5}, \operatorname{logo}$ $U(\mathrm{M})=\mathrm{N}$.

\footnotetext{
${ }^{5}$ Em geral, o resultado vale sob a hipótese mais fraca de que $\operatorname{spr}(P-Q)<1$, conforme [55], Pag.33.
} 
OBSERVAÇÃo 1.2.4 Na prova do terceiro ítem da proposição 1.2.3, vemos que o operador $U$ depende continuamente de $\mathrm{M}$ e $\mathrm{N}$.

Passaremos agora a descrever o conceito de abertura mínima entre dois subespaços. Este conceito é de certa forma dual ao conceito de abertura máxima no sentido de que, enquanto a abertura máxima serve para medir o distanciamento entre subespaços, a abertura mínima serve para medir a aproximação entre os mesmos.

Definição 1.2.5 Dados subespaços fechados $\mathrm{M}, \mathrm{N}$ de $\mathrm{H}$, definimos a abertura mínima entre $\mathrm{M}$ e $\mathrm{N}$ por

$$
\gamma(\mathrm{M}, \mathrm{N}) \doteq \inf _{u \in \mathrm{M} \backslash \mathrm{N}} \frac{\operatorname{dist}(u, \mathrm{~N})}{\operatorname{dist}(u, \mathrm{M} \cap \mathrm{N})}=\inf _{u \in \mathrm{M} \backslash \mathrm{N}} \frac{\left\|P_{\mathrm{N}^{\perp}} u\right\|}{\left\|P_{(\mathrm{MNN})^{\perp}} u\right\|},
$$

caso $M \not \subset N$. Se $M \subset N$, definimos $\gamma(M, N)=1$.

A principal utilidade da abertura mínima é estabelecida no próximo lema.

LEMA 1.2.6 M + Néfechado se e só se $\gamma(\mathrm{M}, \mathrm{N})>0$.

Prova. Suponhamos o resultado válido para subespaços $M, N$ com intersecção trivial. Provemos que o mesmo é válido para quaisquer subespaços. Dados $M, N$ quaisquer, seja $L=M \cap N$. Considerando o espaço quociente $\hat{H}=H / L$ e $\hat{M}, \hat{N}$ as imagens de $M, N$ pela projeção canônica, é fácil ver que $M+N$ é fechado se e só se $\hat{M}+\hat{N}$ é fechado e vale a fórmula

$$
\gamma(\mathrm{M}, \mathrm{N})=\inf _{u \in M \backslash N} \frac{\operatorname{dist}(u, \mathrm{~N})}{\operatorname{dist}(u, \mathrm{~L})}=\inf _{u \in M \backslash N} \frac{\operatorname{dist}(u+L, \hat{\mathrm{N}})}{\|u+\mathrm{L}\|}=\gamma(\hat{\mathrm{M}}, \hat{\mathrm{N}}) .
$$

Como $\hat{\mathrm{M}} \cap \hat{\mathrm{N}}=0$, segue o desejado.

Provemos agora o lema no caso $M \cap N=0$. Suponhamos que $Z=M+N$ é fechado. Pelo teorema do gráfico fechado, a projeção $P: Z \rightarrow M$ ao longo de $N$ é contínua, logo

$$
\gamma(\mathrm{M}, \mathrm{N})=\inf _{\substack{u \in \mathbb{M} \\ u \neq 0}} \frac{\operatorname{dist}(u, \mathrm{~N})}{\|u\|}=\left(\sup _{\substack{u \in \mathbb{M} \\ v \in \mathrm{N}}} \frac{\|u\|}{\|u+v\|}\right)^{-1}=\|P\|^{-1}>0 .
$$

Reciprocamente, se $\gamma(\mathrm{M}, \mathrm{N})>0$, então dados $u_{n} \in \mathrm{M}$ e $v_{n} \in \mathrm{N}$ tais que $u_{n}+v_{n} \rightarrow w$, temos

$$
\left\|u_{n}-u_{m}\right\| \leq \frac{\operatorname{dist}\left(u_{n}-u_{m}, \mathrm{~N}\right)}{\gamma(\mathrm{M}, \mathrm{N})} \leq \frac{\left\|\left(u_{n}-u_{m}\right)+\left(v_{n}-v_{m}\right)\right\|}{\gamma(\mathrm{M}, \mathrm{N})} \rightarrow 0, m, n \rightarrow \infty,
$$

logo, existe $u \in \mathrm{M}$ tal que $u_{n} \rightarrow u$. Disso, $v_{n} \rightarrow w-u \in \mathrm{N}$, portanto, $w \in \mathrm{M}+\mathrm{N}$, terminando a demonstração.

ObSERVAÇÃo 1.2.7 No caso em que $\mathrm{M} \cap \mathrm{N}=0$ e $\mathrm{M}+\mathrm{N}=\mathrm{H}$, a prova do lema 1.2.6 mostra que $\gamma(\mathrm{M}, \mathrm{N})=\left\|P_{\mathrm{N}, \mathrm{M}}\right\|^{-1}$, onde $P_{\mathrm{N}, \mathrm{M}}: \mathrm{H} \rightarrow \mathrm{M}$ denota a projeção sobre $\mathrm{M}$ ao longo de $\mathrm{N}$.

O lema seguinte nos dá algumas propriedades de $\gamma$.

Lema 1.2.8 Para quaisquer subespaços fechados $\mathrm{M}, \mathrm{N}$ de $\mathrm{H}$, temos que $\gamma(\mathrm{M}, \mathrm{N}) \leq \delta(\mathrm{M}, \mathrm{N})$, se $\mathrm{M} \not \subset \mathrm{N} e$ $\gamma(\mathrm{M}, \mathrm{N})=\gamma(\mathrm{N}, \mathrm{M})$. 
Prova. A primeira desigualdade é óbvia. Para provar que $\gamma$ é simétrica com relação a $\mathrm{M}$ e $\mathrm{N}$, suponhamos primeiramente que $\gamma(\mathrm{M}, \mathrm{N})>0 \mathrm{e} L=\mathrm{M} \cap \mathrm{N}=0$. Pela observação 1.2.7 vemos que $\gamma(\mathrm{M}, \mathrm{N})=\left\|P_{\mathrm{N}, \mathrm{M}}\right\|^{-1}$. De maneira análoga, $\gamma(\mathrm{N}, \mathrm{M})=\left\|P_{\mathrm{M}, \mathrm{N}}\right\|^{-1}=\left\|I-P_{\mathrm{N}, \mathrm{M}}\right\|^{-1}$, e portanto, é suficiente provar que $\|P\|=\|I-P\|$ para qualquer projeção $P$ definida em um espaço de Hilbert. Isso decorre do lema 1.2.9 a seguir. Caso $\gamma(\mathrm{M}, \mathrm{N})=0$, pelo lema 1.2.6 temos que $\mathrm{M}+\mathrm{N}$ não é fechado, e portanto, $\gamma(\mathrm{N}, \mathrm{M})=0=\gamma(\mathrm{M}, \mathrm{N})$. Caso $\mathrm{M} \cap \mathrm{N} \neq 0$, definindo $\hat{\mathrm{H}}, \hat{\mathrm{M}}$ e $\hat{\mathrm{N}}$ com no lema 1.2.6, temos que $\hat{\mathrm{M}} \cap \hat{\mathrm{N}}=0$ e $\gamma(\mathrm{M}, \mathrm{N})=\gamma(\hat{\mathrm{M}}, \hat{\mathrm{N}})=\gamma(\hat{\mathrm{N}}, \hat{\mathrm{M}})=\gamma(\mathrm{N}, \mathrm{M})$.

Lema 1.2.9 Seja $0 \neq P \neq$ I uma projeção (não necessariamente ortogonal) em $\mathrm{H}$. Então:

1. $\|P\| \geq 1$ e $\|P\|=1$ se e só se $P$ é ortogonal;

2. $\|P\|=\|I-P\|$.

Prova. Se $P$ é qualquer projeção em $\mathrm{H}$, então $\|P\|=\left\|P^{2}\right\| \leq\|P\|^{2}$, logo $\|P\| \geq 1$. Se $P$ é ortogonal, obviamente $\|P\|=1$, pois $\|u\|^{2}=\|P u\|^{2}+\|(I-P) u\|^{2} \geq\|P u\|^{2}$, para qualquer $u \in \mathrm{H}$. Reciprocamente, se $\|P\|=1$, então tomando $N=\operatorname{ker} P$ e $M=\operatorname{Im} P$, temos que $N^{\perp} \subset M$. De fato, se $u \in \mathrm{N}^{\perp}$, então $\langle u,(I-P) u\rangle=0$, portanto,

$$
\|P u\|^{2}=\|u\|^{2}+\|(I-P) u\|^{2} \geq\|u\|^{2},
$$

logo, como $\|P\|=1$, temos $(I-P) u=0$, portanto, $u \in \mathrm{M}$. Como $P^{*}$ também é projeção com $\left\|P^{*}\right\|=\|P\|=1$, então temos que $\mathrm{M}=\operatorname{Im} P=\left(\operatorname{ker} P^{*}\right)^{\perp} \subset \operatorname{Im} P^{*}=(\operatorname{ker} P)^{\perp}=\mathrm{N}^{\perp}$ e disso, $\mathrm{M}=\mathrm{N}^{\perp}$, provando que $P$ é uma projeção ortogonal. Isso prova a primeira afirmação do enunciado.

Provemos agora que $\|P\|=\|I-P\|$. Obviamente, podemos excluir o caso $\|P\|=1$, pois neste caso $P$ e, portanto, $I-P$ é ortogonal, e a igualdade é decorrência da primeira afirmação. Sejam $1<a<\|P\|$ e $u \in \mathrm{H}$ tal que $\|P u\| \geq a\|u\|$. Definindo $v \doteq u-\frac{\langle u, P u\rangle}{\|P u\|^{2}} P u$, temos que $\|v\|^{2}=\|u\|^{2}-\frac{\left.\langle u, P u\rangle\right|^{2}}{\|P u\|^{2}}$. Além disso, $(I-P) v=(I-P) u \neq 0$, pois $\|P u\|>\|u\|$. Temos que

$$
\frac{\|(I-P) v\|^{2}}{\|v\|^{2}}=\frac{\|(I-P) u\|^{2}\|\| P u \|^{2}}{\|u\|^{2}\|P u\|^{2}-|\langle u, P u\rangle|^{2}} \geq \frac{\|P u\|^{2}}{\|u\|^{2}} \geq a^{2} ，
$$

sendo que a penúltima desigualdade segue de

$$
\begin{aligned}
\|u\|^{2}\|(I-P) u\|^{2}-\|u\|^{2}\|P u\|^{2}+|\langle u, P u\rangle|^{2}= & \left(\|u\|^{2}-\mid\langle u, P u\rangle\right)^{2}+ \\
& +2\|u\|^{2}(|\langle u, P u\rangle|-\operatorname{Re}\langle u, P u\rangle) \geq 0 .
\end{aligned}
$$

Vamos dar um exemplo de dois subespaços fechados de um espaço de Hilbert cuja soma não é fechada.

ExEMPLo 1.2.10 Seja $\mathrm{H}$ de dimensão infinita e $\mathrm{T}: \mathrm{H} \rightarrow \mathrm{H}$ um operador compacto auto-adjunto injetor. Temos que $(\operatorname{Im} T)^{\perp}=\operatorname{ker} T=0$, portanto $\operatorname{Im} T$ é densa. Se $\operatorname{Im} T$ é fechada, então $T: \mathrm{H} \rightarrow \mathrm{H}$ é um isomorfismo compacto, contrariando a hipótese sobre a dimensão de $\mathrm{H}$. Portanto, $\operatorname{Im} T$ não é fechada. Os subespaços $\mathrm{M}=\mathrm{H} \oplus 0$ e $\mathrm{N}=\mathrm{G}(T)$ de $\mathrm{H} \oplus \mathrm{H}$ são tais que $\mathrm{M} \cap \mathrm{N}=0$ e $\mathrm{M}+\mathrm{N}=\mathrm{H} \oplus \operatorname{Im} T$, que claramente não é fechado. 
Vamos agora tratar de um conceito semelhante à abertura mínima, definido para um operador fechado. Assim como é possível determinar se a soma $\mathrm{M}+\mathrm{N}$ é fechada simplesmente calculando o número $\gamma(\mathrm{M}, \mathrm{N})$, vamos mostrar que é possível fazer algo semelhante para provar que a imagem de um operador fechado é fechada. O próximo lema é o primeiro passo nesta direção.

LemA 1.2.11 Seja $T: \mathrm{D}(T) \subset \mathrm{H} \rightarrow \mathrm{H}^{\prime}$ um operador fechado tal que $\operatorname{ker} T=0$. Então $\operatorname{Im} T$ é fechada se e só se existe $\alpha>0$ tal que $\|T u\| \geq \alpha\|u\|$, para todo $u \in \mathrm{D}(T)$.

Prova. De fato, se existe tal $\alpha>0$, então dada $v_{n}=T u_{n} \rightarrow v$, temos que $\left\{u_{n}\right\}$ é de Cauchy, portanto, existe $u=\lim _{n \rightarrow \infty} u_{n} \in \mathrm{H}$. Como $T$ é fechado, $u \in \mathrm{D}(T)$ e $v=T u \in \operatorname{Im} T, \log$, $\operatorname{Im} T$ é fechada.

Reciprocamente, se $\operatorname{Im} T$ é fechada então $T^{-1}: \operatorname{Im}(T) \rightarrow D(T)$ é um operador fechado cujo domínio é um espaço de Banach. Pelo teorema do gráfico fechado, segue que $T^{-1}$ é limitado, e portanto, tomando $\alpha=\left\|T^{-1}\right\|^{-1}$, o resultado segue.

Se $T: \mathrm{D}(T) \subset \mathrm{H} \rightarrow \mathrm{H}$ é um operador fechado, considerando $\tilde{T}=\left.T\right|_{\mathrm{D}(T) \cap(\operatorname{ker} T)^{\perp}}$, temos que $\tilde{T}$ é fechado injetor. Pelo lema 1.2.11, segue que $\operatorname{Im} T=\operatorname{Im} \tilde{T}$ é fechada se e só se existe $\alpha>0$ tal que

$$
\|T u\|=\|\tilde{T} u\| \geq \alpha\|u\|,
$$

para todo $u \in \mathrm{D}(T) \cap(\operatorname{ker} T)^{\perp}$. Isto justifica a seguinte definição.

DefinIÇão 1.2.12 Dado $T: D(T) \subset \mathrm{H} \rightarrow \mathrm{H}$ um operador fechado, definimos o módulo mínimo reduzido de $T$ por

$$
\gamma(T) \doteq \inf _{T u \neq 0} \frac{\|T u\|}{\operatorname{dist}(u, \operatorname{ker} T)}=\inf _{u \in \mathrm{D}(T) \cap(\operatorname{ker} T)^{\perp}} \frac{\|T u\|}{\|u\|} .
$$

Caso $T$ seja injetor, $\gamma(T)$ é também chamado de módulo mínimo de $T$.

OвSERVAÇÃo 1.2.13 Vemos imediatamente que $\gamma(T)=\left\|\tilde{T}^{-1}\right\|^{-1}$, com $\tilde{T}$ definido no comentário que antecede a definição 1.2.12.

A próxima proposição decorre diretamente da discussão que antecede a definição 1.2.12.

Proposição 1.2.14 Um operador fechado $T: \mathrm{D}(T) \subset \mathrm{H} \rightarrow \mathrm{H}$ tem imagem fechada se e só se $\gamma(T)>0$.

Podemos também reformular a noção de abertura mínima entre subespaços em termos do módulo mínimo reduzido.

Lema 1.2.15 Dados $\mathrm{M}, \mathrm{N}$ subespaços fechados de $\mathrm{H}$, tem-se que $\gamma(\mathrm{M}, \mathrm{N})=\gamma\left(P_{\mathrm{N}^{\perp}}^{\mathrm{M}}\right)$.

Prova. Vemos que $\operatorname{ker}\left(P_{\mathrm{N}^{\perp}}^{\mathrm{M}}\right)=\mathrm{M} \cap \mathrm{N}$, portanto

$$
\gamma(\mathrm{M}, \mathrm{N})=\inf _{u \in \mathrm{M} \backslash \mathrm{N}} \frac{\operatorname{dist}(u, \mathrm{~N})}{\operatorname{dist}(u, \mathrm{M} \cap \mathrm{N})}=\inf _{u \notin \operatorname{ker}\left(P_{\mathrm{N}^{\perp}}^{\mathrm{M}}\right)} \frac{\left\|P_{\mathrm{N}^{\perp}}^{\mathrm{M}} u\right\|}{\operatorname{dist}\left(u, \operatorname{ker}\left(P_{\mathrm{N}^{\perp}}^{\mathrm{M}}\right)\right)}=\gamma\left(P_{\mathrm{N}^{\perp}}^{\mathrm{M}}\right) .
$$

Os lemas 1.2.6, 1.2.15 e a proposição 1.2.14 nos permitem concluir o seguinte corolário.

Corolário 1.2.16 $\mathrm{M}+\mathrm{N}$ é fechado se e só se $\operatorname{Im}\left(P_{\mathrm{N}^{\perp}}^{\mathrm{M}}\right)$ é fechada. 
A próxima proposição mostra que $\gamma$ é invariante por adjunção.

Proposição 1.2.17 Se $T \in \mathscr{C}\left(\mathrm{H}, \mathrm{H}^{\prime}\right)$ é densamente definido, então $\gamma(T)=\gamma\left(T^{*}\right)$. Em particular, $\operatorname{Im} T$ é fechada se e só se $\operatorname{Im} T^{*}$ é féchada.

Prova. Considerando $\tilde{T}:(\operatorname{ker} T)^{\perp} \rightarrow \overline{\operatorname{Im} T}$ e $\widetilde{T^{*}}:\left(\operatorname{ker} T^{*}\right)^{\perp} \rightarrow \overline{\operatorname{Im} T^{*}}$ definidos como nos comentários que antecedem a definição 1.2 .12 , temos que $\tilde{T}$ e $\widetilde{T}^{*}$ são adjuntos. Isto significa que $\widetilde{\left(T^{*}\right)}=\tilde{T}^{*}$, e portanto, pela observação 1.2.13,

$$
\gamma(T)=\left\|\tilde{T}^{-1}\right\|^{-1}=\left\|\left(\tilde{T}^{-1}\right)^{*}\right\|^{-1}=\left\|\left(\tilde{T}^{*}\right)^{-1}\right\|^{-1}=\left\|\left(\widetilde{T^{*}}\right)^{-1}\right\|^{-1}=\gamma\left(T^{*}\right) .
$$

A proposição 1.2.17 tem um corolário interessante para o estudo de pares de subespaços.

CoRolário 1.2.18 Se $\mathrm{M}, \mathrm{N}$ são subespaços fechados então $\gamma(\mathrm{M}, \mathrm{N})=\gamma\left(\mathrm{N}^{\perp}, \mathrm{M}^{\perp}\right)$.

PROva. Como $\left(P_{\mathrm{N}^{\perp}}^{\mathrm{M}}\right)^{*}=P_{\mathrm{M}}^{\mathrm{N}^{\perp}}$, o corolário decorre diretamente da proposição 1.2.17 e do lema 1.2.15.

Decorre do corolário 1.2.18 e do lema 1.2.6 que $\mathrm{M}+\mathrm{N}$ é fechado se e só se $\mathrm{M}^{\perp}+\mathrm{N}^{\perp}$ é fechado. Em geral, é verdade que $(M+N)^{\perp}=M^{\perp} \cap N^{\perp}$ para quaisquer $M, N$. A relação dual $(M \cap N)^{\perp}=M^{\perp}+N^{\perp}$ não é sempre verdadeira, pois $(M \cap N)^{\perp}$ é sempre fechado, mas $M^{\perp}+N^{\perp}$ nem sempre o é. Geralmente, apenas a inclusão $(M \cap N)^{\perp} \supset M^{\perp}+N^{\perp}$ é verdadeira. Se soubermos de antemão que $M^{\perp}+N^{\perp}$ é fechado, então $M^{\perp}+N^{\perp}=\left(M^{\perp}+N^{\perp}\right)^{\perp \perp}=(M \cap N)^{\perp}$. Estas observações provam a seguinte proposição.

Proposição 1.2.19 Seja $\mathrm{M}, \mathrm{N}$ um par de subespaços fechados de $\mathrm{H}$. Então $(\mathrm{M}+\mathrm{N})^{\perp}=\mathrm{M}^{\perp} \cap \mathrm{N}^{\perp} e$ $(\mathrm{M} \cap \mathrm{N})^{\perp} \supset \mathrm{M}^{\perp}+\mathrm{N}^{\perp}$. Além disso, $\mathrm{M}+\mathrm{N}$ é fechado se e só se $\mathrm{M}^{\perp}+\mathrm{N}^{\perp}$ óe, e sob estas condições,

$$
\begin{gathered}
(M \cap N)^{\perp}=M^{\perp}+N^{\perp}, \\
\operatorname{nul}\left(M^{\perp}, N^{\perp}\right)=\operatorname{def}(M, N), \operatorname{def}\left(M^{\perp}, N^{\perp}\right)=\operatorname{nul}(M, N),
\end{gathered}
$$

Observação 1.2.20 Se $T \in \mathscr{C}\left(\mathrm{H}, \mathrm{H}^{\prime}\right)$ e $M$ é um subespaço fechado tal que $M \oplus \operatorname{ker} T=\mathrm{H}$, então o operador $U=P_{(\operatorname{ker} T)^{\perp}}^{\mathrm{M}}: \mathrm{M} \rightarrow(\operatorname{ker} T)^{\perp}$ é um isomorfismo. De fato, $U^{-1}$ é a restrição a $(\operatorname{ker} T)^{\perp}$ da projeção $P_{\text {ker } T, M}$ sobre $\mathrm{M}$ relativa à decomposição $\mathrm{H}=\operatorname{ker} T \oplus \mathrm{M}$, a qual denotamos por $P_{\operatorname{ker} T)^{\perp}}^{(\operatorname{M}}$. Pela definição de $\delta$, temos que $\|U\|=\delta(\mathrm{M}, \operatorname{ker} T)$. Dado qualquer $u \in \mathrm{M}$ não-nulo, pondo $v=U u \in(\operatorname{ker} T)^{\perp}$, temos que

$$
\frac{\|T v\|}{\left\|U^{-1}\right\|\|v\|} \leq \frac{\|T u\|}{\|u\|} \leq \frac{\|T v\|}{\|U\|^{-1}\|v\|},
$$

portanto

$$
\left\|P_{\operatorname{ker} T, M}^{(\operatorname{ker} T)^{\perp}}\right\|^{-1} \gamma(T) \leq \inf _{u \in M \backslash 0} \frac{\|T u\|}{\|u\|} \leq \delta(\mathrm{M}, \operatorname{ker} T) \gamma(T) .
$$

Isso mostra que o papel de $(\operatorname{ker} T)^{\perp}$ na definição 1.2.12 é de certa forma irrelevante: podemos escolher qualquer subespaço fechado $M$ tal que $M \oplus \operatorname{ker} T=H$.

É possível relacionar $\gamma(\mathrm{M}, \mathrm{N})$ e $\delta\left(\mathrm{M}, \mathrm{N}^{\perp}\right)$. 
LemA 1.2.21 Se $\mathrm{M} \neq \mathrm{N}$ são subespaços de $\mathrm{H}$, então $1-\delta\left(\mathrm{M}, \mathrm{N}^{\perp}\right)^{2} \geq \gamma(\mathrm{M}, \mathrm{N})^{2}$.

Prova. De fato, $\delta\left(\mathrm{M}, \mathrm{N}^{\perp}\right)^{2}=\sup _{\|u\|=1}\left\|P_{\mathrm{N}}^{\mathrm{M}} u\right\|^{2} \geq 1-\inf _{\|u \in\|=1}\left\|P_{\mathrm{N}^{\perp}}^{\mathrm{M}} u\right\|^{2}=1-\gamma\left(P_{\mathrm{N}^{\perp}}^{\mathrm{M}}\right)^{2}$.

Vamos ainda citar mais alguns fatos interessantes e eventualmente úteis sobre o módulo mínimo (reduzido) de um operador. Mais resultados podem ser encontrados em [47].

Proposição 1.2.22 Sejam $T \in \mathscr{C}\left(\mathrm{H}, \mathrm{H}^{\prime}\right)$ e $\mathrm{M}$ um subespaço fechado de $\mathrm{H}$.

1. Se $\mathrm{M} \supset \operatorname{ker} T$ então $\gamma\left(\left.T\right|_{\mathrm{MnD}(T)}\right) \geq \gamma(T)$.

2. Se $\mathrm{M}+\operatorname{ker} T$ é fechado e $\gamma(T)>0$, então $T(\mathrm{M})$ é fechado.

3. Se nul $T<\infty$ e $\gamma(T)>0$ então $T(M)$ é fechado.

4. Se Té Fredholm, então $T(\mathrm{M})$ é fechado.

Prova. O ítem 1 decorre diretamente da definição de $\gamma$. Para provar o ítem 2, basta observar que se $S$ é a restrição de $T$ a $D(T) \cap(M+\operatorname{ker} T)$, então a hipótese sobre $M+\operatorname{ker} T$ implica que $S$ é fechado. Pelo primeiro ítem, segue que $\gamma(S) \geq \gamma(T)>0$, portanto, $T(M)=\operatorname{Im} S$ é fechado. Os ítens 3 e 4 decorrem diretamente dos ítens 2 e 3 .

\subsubsection{Distância entre operadores fechados}

Uma das principais utilidades do conceito de abertura máxima entre subespaços é que o mesmo nos permite definir uma noção muito útil de distância entre operadores fechados.

Definição 1.2.23 Dados $S, T \in \mathscr{C}\left(\mathrm{H}, \mathrm{H}^{\prime}\right)$, definimos $\delta(S, T) \doteq \delta(\mathrm{G}(S), \mathrm{G}(T))$ e

$$
\hat{\delta}(S, T) \doteq \max (\delta(S, T), \delta(T, S)) .
$$

OBservação 1.2.24 Vemos que $\delta$ pode ser escrita como

$$
\delta(S, T)=\sup _{\substack{u \in D(S) \\ u \neq 0}} \frac{\operatorname{dist}((u, S u), \mathrm{G}(T))}{\left(\|u\|^{2}+\|S u\|^{2}\right)^{1 / 2}} .
$$

Observação 1.2.25 Se $S, T$ são fechados e $\mathrm{D}(S) \subset \mathrm{D}(T)$, então decorre imediatamente da observação 1.2.24 que

$$
\delta(S, T) \leq \sup _{\substack{u \in D(S) \\ u \neq 0}} \frac{\|(S-T) u\|}{\left(\|u\|^{2}+\|S u\|^{2}\right)^{1 / 2}}=\left\|S-\left.T\right|_{D(S)}\right\|_{G(S)},
$$

onde $\|\cdot\|_{G(S)}$ denota a norma do gráfico com respeito ao operador $S$.

Decorre da proposição 1.2.2 que $\hat{\delta}$ define uma métrica em $\mathscr{C}\left(\mathrm{H}, \mathrm{H}^{\prime}\right)$. Esta métrica foi introduzida por $\mathrm{H}$. Cordes e J. Labrousse em [33], e faz sentido no caso mais geral de operadores entre espaços de Banach. Porém, no caso de espaços de Hilbert, usando a proposição 1.2.2, é possível obter uma definição mais explícita de $\delta$. Para isso, notemos alguns fatos elementares. 
LEMA 1.2.26 Se $S, T \in \mathscr{C}\left(\mathrm{H}, \mathrm{H}^{\prime}\right)$ então $\delta\left(S^{*}, T^{*}\right)=\delta(T, S)$ e $\hat{\delta}\left(S^{*}, T^{*}\right)=\hat{\delta}(S, T)$.

Prova. Lembramos que para qualquer $T \in \mathscr{C}\left(\mathrm{H}, \mathrm{H}^{\prime}\right)$ temos

$$
\mathrm{G}(T)^{\perp}=J\left(\mathrm{G}\left(T^{*}\right)\right),
$$

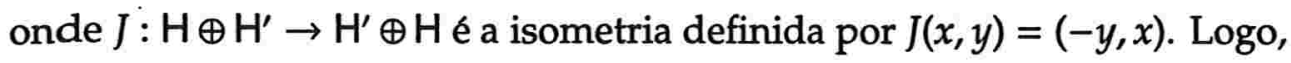

$$
\delta\left(S^{*}, T^{*}\right)=\delta\left(\mathrm{G}\left(S^{*}\right), \mathrm{G}\left(T^{*}\right)\right)=\delta\left(J\left(\mathrm{G}(S)^{\perp}\right), J\left(\mathrm{G}(T)^{\perp}\right)\right)=\delta(\mathrm{G}(T), \mathrm{G}(S))=\delta(T, S),
$$

pelo primeiro ítem da proposição 1.2.3.

Lema 1.2.27 Se $T: \mathrm{D}(T) \subset \mathrm{H} \rightarrow \mathrm{H}^{\prime}$ é fechado densamente definido, então $R(T) \doteq\left(I+T^{*} T\right)^{-1}$ é um operador auto-adjunto limitado com domínio $\mathrm{H}$ tal que $\|R(T)\| \leq 1 e\|T R(T)\| \leq 1$. Temos também que $R\left(T^{*}\right) T \subset T R(T) e(T R(T))^{*}=T^{*} R\left(T^{*}\right)$.

Prova. A equação 1.2.3 diz que cada par $\left(u, u^{\prime}\right) \in \mathrm{H} \oplus \mathrm{H}^{\prime}$ pode ser escrito como soma $(v, T v)+\left(-T^{*} v^{\prime}, v^{\prime}\right)$, com $v \in \mathrm{D}(T)$ e $v^{\prime} \in \mathrm{D}\left(T^{*}\right)$. Em particular, pondo $u^{\prime}=0$, segue que cada $u \in \mathrm{H}$ pode ser escrito como $u=v-T^{*} v^{\prime}$ e $v^{\prime}=-T v$, portanto, $u=\left(I+T^{*} T\right) v$. Logo, $S=I+T^{*} T$ é sobrejetor. Como $\langle S u, u\rangle=\langle u, u\rangle+\langle T u, T u\rangle$, temos que $S$ é injetor, e portanto $S^{-1} \doteq R(T)$ é bem definido e simétrico. Além disso, se $R(T) u=v$, então $u=v+T^{*} T v$, $\mathrm{e}$

$$
\|R(T) u\|^{2}=\|v\|^{2}=\langle u, v\rangle-\langle T v, T v\rangle \leq\langle u, v\rangle \leq\|u\|\|v\|=\|u\|\|R(T) u\|,
$$

portanto, $\|R(T)\| \leq 1 \mathrm{e}$

$$
\|T R(T) u\|^{2}=\|T v\|^{2}=\langle u, v\rangle-\langle v, v\rangle \leq\langle u, v\rangle \leq\|u\|\|v\|=\|u\|\|R(T) u\| \leq\|u\|^{2},
$$

$\log \|T R(T)\| \leq 1$.

Lema 1.2.28 Dado $T \in \mathscr{C}\left(\mathrm{H}, \mathrm{H}^{\prime}\right)$, a projeção ortogonal $P_{T}: \mathrm{H} \oplus \mathrm{H}^{\prime} \rightarrow \mathrm{G}(T) \subset \mathrm{H} \oplus \mathrm{H}^{\prime}$ sobre o gráfico de $T$ tem a forma

$$
\left(\begin{array}{cc}
R(T) & T^{*} R\left(T^{*}\right) \\
T R(T) & I-R\left(T^{*}\right)
\end{array}\right)
$$

Prova. Como $\mathrm{G}(T)^{\perp}=J \mathrm{G}\left(T^{*}\right)$, dados $u \in \mathrm{H}, v \in \mathrm{H}^{\prime}$, o sistema de equações

$$
\left\{\begin{aligned}
x-T^{*} y & =u \\
T x+y & =v
\end{aligned}\right.
$$

tem solução única $(x, y) \in \mathrm{H} \oplus \mathrm{H}^{\prime}$. Usando o lema 1.2.27,

$$
x=R(T) u+R(T) T^{*} v,
$$

assim,

$$
\begin{aligned}
P_{T}(u, v)=(x, T x) & =\left(R(T) u+R(T) T^{*} v, T\left(R(T) u+R(T) T^{*} v\right)\right) \\
& =\left(R(T) u+T^{*} R\left(T^{*}\right) v, T R(T) u+\left(I-R\left(T^{*}\right)\right) v\right)
\end{aligned}
$$

provando que $P_{T}$ tem a forma 1.2.4.

Vamos agora verificar que a métrica $\hat{\delta}$ é equivalente a uma métrica dada de maneira mais explícita. 
Proposição 1.2.29 Se $S, T \in \mathscr{C}\left(\mathrm{H}, \mathrm{H}^{\prime}\right)$, definimos

$$
\mu(S, T)=\left(\|R(S)-R(T)\|^{2}+\left\|R\left(S^{*}\right)-R\left(T^{*}\right)\right\|^{2}+2\|S R(S)-T R(T)\|^{2}\right)^{1 / 2},
$$

com $R(\cdot)$ definido no lema 1.2.27. Temos que

$$
2^{-1 / 2} \hat{\delta}(S, T) \leq \mu(S, T) \leq 2^{1 / 2} \hat{\delta}(S, T) ;
$$

em particular, $\mu$ também define uma métrica sobre $\mathscr{C}\left(\mathrm{H}, \mathrm{H}^{\prime}\right)$ equivalente a $\hat{\delta}$.

Prova. Pela proposição 1.2.2 e pelos lemas 1.2.27 e 1.2.28, temos que

$$
\hat{\delta}(S, T)=\left\|\left(\begin{array}{cc}
R(S)-R(T) & (S R(S)-T R(T))^{*} \\
S R(S)-T R(T) & R\left(T^{*}\right)-R\left(S^{*}\right)
\end{array}\right)\right\| .
$$

Portanto, $\hat{\delta}(S, T)^{2} \leq 2\left(\|R(S)-R(T)\|^{2}+\left\|R\left(S^{*}\right)-R\left(T^{*}\right)\right\|^{2}+2\|S R(S)-T R(T)\|^{2}\right)=2 \mu(S, T)^{2}$. Por outro lado, a desigualdade $1.2 .5 \operatorname{diz}$ que $\hat{\delta}(S, T)^{2} \geq\|R(S)-R(T)\|^{2}+\|S R(S)-T R(T)\|^{2}$ e $\hat{\delta}(S, T)^{2} \geq$ $\left\|R\left(S^{*}\right)-R\left(T^{*}\right)\right\|^{2}+\|S R(S)-T R(T)\|^{2}, \log , 2 \hat{\delta}(S, T)^{2} \geq \mu(S, T)^{2}$. $\hat{\delta}$.

Vejamos que alguns subconjuntos familiares de $\mathscr{C}\left(\mathrm{H}, \mathrm{H}^{\prime}\right)$ são abertos com respeito à métrica

Proposição 1.2.30 Se $T \in \mathscr{L}\left(\mathrm{H}, \mathrm{H}^{\prime}\right), S \in \mathscr{C}\left(\mathrm{H}, \mathrm{H}^{\prime}\right)$ e $\hat{\delta}(S, T)<\left(1+\|T\|^{2}\right)^{-1 / 2}$ então $S \in \mathscr{L}\left(\mathrm{H}_{1} \mathrm{H}^{\prime}\right)$ e

$$
\|S-T\| \leq \frac{\delta(S, T)\left(1+\|T\|^{2}\right)}{1-\delta(S, T)\left(1+\|T\|^{2}\right)^{1 / 2}} .
$$

Prova. Fixado $u \in \mathrm{D}(S) \operatorname{com}\|u\|^{2}+\|S u\|^{2}=1$ e dado $\hat{\delta}(S, T)<\delta<\left(1+\|T\|^{2}\right)^{-1 / 2}$, lembrando da definição de $\delta(S, T)$, segue que existe $v \in \mathrm{D}(T)$ tal que $\|u-v\|^{2}+\|S u-T v\|^{2}<\delta^{2}$. Temos que

$$
\|(S-T) u\|^{2} \leq(\|S u-T v\|+\|T\|\|u-v\|)^{2} \leq \delta^{2}\left(1+\|T\|^{2}\right)
$$

$\mathrm{e}$

$$
1=\|u\|^{2}+\|S u\|^{2} \leq\left(1+\|T\|^{2}\right)\|u\|^{2}+\|(S-T) u\|^{2}+2\|T\|\|u\|\|\mid(S-T) u\| .
$$

Multiplicando 1.2.7 por 1.2.8, segue que

$$
\|(S-T) u\|^{2} \leq \delta^{2}\left(1+\|T\|^{2}\right)\left(\left(1+\|T\|^{2}\right)\|u\|^{2}+\|(S-T) u\|^{2}+2\|T \mid\| u\|\|(S-T) u \|\right),
$$

ou seja,

$$
\left(1+\|T\|^{2}\right)\|u\|^{2}+2\|T\|\|u\|\|(S-T) u\|+\left(\frac{1-\delta^{2}\left(1+\|T\|^{2}\right)}{\delta^{2}\left(1+\|T\|^{2}\right)}\right)\|(S-T) u\|^{2} \geq 0 .
$$

Logo,

$$
\|(S-T) u\| \leq \frac{\delta\left(1+\|T\|^{2}\right)\left(\left(1-\delta^{2}\right)^{1 / 2}+\delta\|T\|\right)}{1-\delta^{2}\left(1+\|T\|^{2}\right)}\|u\| \leq \frac{\delta\left(1+\|T\|^{2}\right)}{1-\delta\left(1+\|T\|^{2}\right)^{1 / 2}}\|u\| .
$$

Como esta desigualdade é homogênea em $u$, então é válida para todo $u \in \mathrm{D}(S)$, e isso implica que $S-T$ é limitado, e portanto, $S$ é limitado. Em particular, vale 1.2.6. Como $\mathrm{D}(S)$ é denso, temos que $\mathrm{D}(\mathrm{S})=\mathrm{H}$, portanto $S \in \mathscr{L}\left(\mathrm{H}, \mathrm{H}^{\prime}\right)$.

No próximo lema, vamos deduzir uma desigualdade importante relacionando a distância $\hat{\delta}$ e a norma usual de operadores limitados. 
Lema 1.2.31 Se $T \in \mathscr{C}\left(\mathrm{H}, \mathrm{H}^{\prime}\right)$ e $A \in \mathscr{L}\left(\mathrm{H}, \mathrm{H}^{\prime}\right)$, então

$$
\hat{\delta}(T, T+A) \leq\|A\| .
$$

Prova. Dado $u \in \mathrm{D}(T)$ com $\|u\|^{2}+\|T u\|^{2}=1$, temos que

$$
\delta(T, T+A) \leq \operatorname{dist}((u, T u), \mathrm{G}(T+A)) \leq\|(u, T u)-(u,(T+A) u)\|=\|A u\| \leq\|A\| .
$$

Corolário 1.2.32 $\mathscr{L}\left(\mathrm{H}, \mathrm{H}^{\prime}\right) \subset \mathscr{C}\left(\mathrm{H}, \mathrm{H}^{\prime}\right)$ é aberto e a restrição da métrica $\hat{\delta}$ a $\mathscr{L}\left(\mathrm{H}, \mathrm{H}^{\prime}\right)$ é equivalente à métrica definida pela norma.

Prova. Decorre diretamente da proposição 1.2 .30 e do lema 1.2.31.

OBSERVAÇão 1.2.33 No caso de operadores limitados, é bem conhecida a série de Neumann

$$
(I-T)^{-1}=\sum_{n=0}^{\infty} T^{n},
$$

válida para $T \in \mathscr{L}(\mathrm{H})$ com $\|T\|<1$. Esta fórmula implica que se $T$ é limitado e inversível, então $T+A=\left(I+A T^{-1}\right) T$ é inversível se $\left\|A T^{-1}\right\|<1$, (em particular, se $\|A\|<\left\|T^{-1}\right\|^{-1}$ ) e $\left\|(I-T)^{-1}\right\| \leq 1 /(1-\|T\|)$. Como corolário, concluímos que o conjunto dos operadores inversíveis é aberto em $\mathscr{L}(\mathrm{H})$.

Dizemos que um operador $T \in \mathscr{C}\left(\mathrm{H}, \mathrm{H}^{\prime}\right)$ é inversível se o operador $T^{-1}$ existe e pertence a $\mathscr{L}\left(\mathrm{H}^{\prime}, \mathrm{H}\right)$. Vejamos que, como no caso de operadores limitados, o conjunto dos operadores inversíveis é aberto em $\mathscr{C}\left(\mathrm{H}, \mathrm{H}^{\prime}\right)$.

Proposição 1.2.34 Sejam $T, S \in \mathscr{C}\left(\mathrm{H}, \mathrm{H}^{\prime}\right)$ com $T$ inversível. Se e $\hat{\delta}(S, T)<\left(1+\left\|T^{-1}\right\|^{2}\right)^{-1 / 2}$ então $S$ é inversivel e

$$
\left\|S^{-1}-T^{-1}\right\| \leq \frac{\hat{\delta}(S, T)\left(1+\left\|T^{-1}\right\|^{2}\right)}{1-\hat{\delta}(S, T)\left(1+\left\|T^{-1}\right\|^{2}\right)^{1 / 2}} .
$$

Prova. Vejamos que para $S$ nas condições acima, $\operatorname{ker} S=0$. De fato, se $u \in \mathrm{D}(S),\|u\|=1$, e $S u=0$, dado $0<\delta<\left(1+\left\|T^{-1}\right\|^{2}\right)^{-1 / 2}$, então existe $v \in \mathrm{D}(T)$ tal que $\|u-v\|^{2}+\|T v\|^{2}<\delta^{2}$, logo,

$$
\begin{aligned}
1=\|u\|^{2} \leq(\|u-v\|+\|v\|)^{2} & \leq\left(\|u-v\|+\left\|T^{-1}\right\|\|T v\|\right)^{2} \\
& \leq\left(\|u-v\|^{2}+\|T v\|^{2}\right)\left(1+\left\|T^{-1}\right\|^{2}\right) \\
& <\delta^{2}\left(1+\left\|T^{-1}\right\|^{2}\right),
\end{aligned}
$$

uma contradição. Assim, existe $S^{-1}: \operatorname{Im} S \rightarrow \mathrm{H}$. Como $\hat{\delta}\left(S^{*}, T^{*}\right)<\left(1+\left\|\left(T^{*}\right)^{-1}\right\|^{2}\right)^{-1 / 2}$, trocando no argumento anterior $T, S$ por $T^{*}, S^{*}$, respectivamente, temos que $0=\operatorname{ker} S^{*}=(\operatorname{Im} S)^{\perp}, \log$ o Im $S$ é densa. Portanto, $S^{-1} \in \mathscr{C}\left(\mathrm{H}^{\prime}, \mathrm{H}\right)$ e $\hat{\delta}\left(S^{-1}, T^{-1}\right)=\hat{\delta}(S, T)<\left(1+\left\|T^{-1}\right\|^{2}\right)^{-1 / 2}$. Pela proposição 1.2.30, $S^{-1} \in \mathscr{L}\left(\mathrm{H}^{\prime}, \mathrm{H}\right)$ e a estimativa 1.2.11 decorre imediatamente da desigualdade 1.2.6.

Em geral, é bastante delicado estimar a distância entre operadores fechados. Entretanto, em alguns casos particulares, é possível obter uma estimativa razoável para $\delta$. 
Proposição 1.2.35 Se $T \in \mathscr{C}\left(\mathrm{H}, \mathrm{H}^{\prime}\right)$ e $A \in \mathscr{L}\left(\mathrm{H}, \mathrm{H}^{\prime}\right)$, então

$$
\delta(S+A, T+A) \leq \max \left(2,1+\|A\|^{2}\right) \delta(S, T) .
$$

Prova. Basta observar que para quaisquer $u \in \mathrm{D}(S)$ e $v \in \mathrm{D}(T)$,

$$
\|u-v\|^{2}+\|(S+A) u-(T+A) v\|^{2} \leq \max \left(2,1+\|A\|^{2}\right)\left(\|u-v\|^{2}+\|S u-T v\|^{2}\right)
$$

e

$$
\|u\|^{2}+\|S u\|^{2} \leq \max \left(2,1+\|A\|^{2}\right)\left(\|u\|^{2}+\|(S+A) u\|^{2}\right)
$$

e usar a observação 1.2.24.

ObSERVAÇão 1.2.36 Um argumento semelhante ao da proposição 1.2.35 mostra que se $A$ é $T$-limitado (conforme definição 1.2.39) com $T$-norma $b<1$, então

$$
\delta(S+A, T+A) \leq(1-b)^{-1}\left(a^{2}+b^{2}\right)^{1 / 2},
$$

com $a$ como na equação 1.2.14.

No caso em que $H=H^{\prime}$, temos um critério bastante útil para provar convergência em termos da distância $\hat{\delta}$.

Teorema 1.2.37 Sejam $T, T_{n} \in \mathscr{C}(\mathrm{H}), n \geq 1$, e suponhamos que $\mathrm{P}(T) \neq \emptyset$. Então $\hat{\delta}\left(T_{n}, T\right) \rightarrow 0$ se existem $\zeta \in \mathrm{P}(T), N=N(\zeta, T)$ tais que

1. $\zeta \in \mathrm{P}\left(T_{n}\right)$ para $n \geq N$;

2. $\left\|\mathrm{R}\left(\zeta ; T_{n}\right)-\mathrm{R}(\zeta ; T)\right\| \rightarrow 0$ se $n \rightarrow \infty$.

Reciprocamente, se $\hat{\delta}\left(T_{n}, T\right) \rightarrow 0$, então (1) e (2) são verificadas para todo $\zeta \in \mathrm{P}(T)$.

Prova. Suponhamos que $\hat{\delta}\left(T_{n}, T\right) \rightarrow 0$ se $n \rightarrow \infty$. Pela proposição 1.2.35, dado $\zeta$ qualquer, temos

$$
\hat{\delta}\left(T_{n}-\zeta, T-\zeta\right) \leq\left(2\left(1+|\zeta|^{2}\right)\right)^{1 / 2} \hat{\delta}\left(T_{n}, T\right) .
$$

Em particular, $\hat{\delta}\left(T_{n}-\zeta, T-\zeta\right) \rightarrow 0$ se $n \rightarrow \infty$. Pela proposição 1.2 .34 , se $\zeta \in \mathrm{P}(T)$, temos que existe $N \in \mathbb{N}$ tal que $\zeta \in \mathrm{P}\left(T_{n}\right)$ para todo $n \geq N$. Usando ${ }^{6}$ a desigualdade 1.2 .6 e o fato que $\hat{\delta}\left(R^{-1}, S^{-1}\right)=\hat{\delta}(R, S)$ para quaisquer $R, S$ fechados, segue que $\left\|\mathrm{R}\left(\zeta ; T_{n}\right)-\mathrm{R}(\zeta ; T)\right\| \rightarrow 0$ se $n \rightarrow \infty$, para todo $\zeta \in \mathrm{P}(T)$.

Reciprocamente, se as condições 1 e 2 valem para algum $\zeta \in P(T)$, então

$$
\begin{aligned}
\hat{\delta}\left(T_{n}, T\right) & \leq\left(2\left(1+|\zeta|^{2}\right)\right)^{1 / 2} \hat{\delta}\left(T_{n}-\zeta, T-\zeta\right) \\
& \leq\left(2\left(1+|\zeta|^{2}\right)\right)^{1 / 2} \hat{\delta}\left(\mathrm{R}\left(\zeta ; T_{n}\right), \mathrm{R}(\zeta ; T)\right) \\
& \leq\left(2\left(1+|\zeta|^{2}\right)\right)^{1 / 2}\left\|\mathrm{R}\left(\zeta ; T_{n}\right)-\mathrm{R}(\zeta ; T)\right\| \rightarrow 0,
\end{aligned}
$$

se $n \rightarrow \infty$, pelo lema 1.2.31.

Podemos melhorar consideravelmente a conclusão do teorema 1.2.37.

\footnotetext{
${ }^{6} \mathrm{~N}$ pode ser tomado uniforme em subconjuntos limitados de $\mathrm{P}(T)$.
} 
Proposição 1.2.38 Se $T \in \mathscr{C}(\mathrm{H})$ e $K \subset \mathrm{P}(T)$ é compacto, então existe $\delta>0$ tal que $K \subset \mathrm{P}(S)$ e

$$
\|\mathrm{R}(\zeta ; S)-\mathrm{R}(\zeta ; T)\| \leq \frac{\mathrm{C}\left(1+\|\mathrm{R}(\zeta ; T)\|^{2}\right) \hat{\delta}(S, T)}{1-C\left(1+\|\mathrm{R}(\zeta ; T)\|^{2}\right)^{1 / 2} \hat{\delta}(S, T)},
$$

para qualquer $S \in \mathscr{C}(\mathrm{H}) \operatorname{com} \hat{\delta}(S, T)<\delta$, onde $C \doteq\left\{\sup _{\zeta \in K}\left(2\left(1+|\zeta|^{2}\right)\right)^{1 / 2}\right\}^{-1}$.

Prova. Seja $\zeta \in K \subset \mathrm{P}(T)$. Pela desigualdade 1.2.12 e pela proposição 1.2 .34 , se $\hat{\delta}(S, T)<$ $\left.\operatorname{Cinf}_{\zeta \in K}\left(1+\|\mathrm{R}(\zeta ; T)\|^{2}\right)^{-1 / 2}\right\} \doteq \delta$ então $\zeta \in \mathrm{P}(S)$. A estimativa 1.2.13 decorre da desigualdade 1.2.11.

Estudemos agora a estabilidade de algumas propriedades por outro tipo importante de perturbação.

Definição 1.2.39 Dados operadores $T \in \mathscr{C}\left(\mathrm{H}, \mathrm{H}^{\prime}\right)$ e $A$ qualquer, dizemos que $A$ é $T$-limitado se $\mathrm{D}(A) \supset \mathrm{D}(T)$ e existem $a, b \geq 0$ tais que

$$
\|A u\| \leq a\|u\|+b\|T u\|,
$$

para $u \in \mathrm{D}(T)$. O ínfimo dos $b \geq 0$ que verificam a desigualdade 1.2.14 para algum $a \geq 0$ é chamado de $T$-norma de $A$. O operador $A$ é dito $T$-compacto se $\mathrm{D}(A) \supset \mathrm{D}(T)$ e cada seqüência $\left\{u_{n}\right\}$ em $\mathrm{D}(T)$ tal que $\sup _{n}\left\|u_{n}\right\|+\left\|T u_{n}\right\|<\infty$ admite uma subseqüência $\left\{u_{n_{j}}\right\}$ tal que $\left\{A u_{n_{j}}\right\}$ é convergente.

Observação 1.2.40 É imediato que se $A$ é T-compacto, então $A$ é $T$-limitado. Também é óbvio que se $A \in \mathscr{L}\left(\mathrm{H}, \mathrm{H}^{\prime}\right)$ então $A$ é $T$-limitado para qualquer $T$, com $T$-norma zero.

Vejamos que o fato de um operador $T$ ser fechado ou possuir inverso limitado é estável por perturbações $T$-limitadas com $T$-norma $<1$ e por perturbações $T$-compactas.

Proposição 1.2.41 Sejam $T \in \mathscr{C}\left(\mathrm{H}, \mathrm{H}^{\prime}\right)$ e A um operador qualquer entre $\mathrm{H}$ e $\mathrm{H}^{\prime}$.

1. Se Aé T-limitado com T-norma $<1$, então $T+A$ é fechado.

2. Se Aé T-compacto então $T+A$ é fechado.

3. $\operatorname{Se} T^{-1} \in \mathscr{L}\left(\mathrm{H}^{\prime}, \mathrm{H}\right)$ e A é $T$-limitado com $a\left\|T^{-1}\right\|+b<1$, então $T+A$ possui inverso em $\mathscr{L}\left(\mathrm{H}^{\prime}, \mathrm{H}\right)$ com norma $\leq\left\|T^{-1}\right\| /\left(1-b-a\left\|T^{-1}\right\|\right)$.

Prova. Se $A$ é T-limitado, temos que

$$
(1-b)\|T u\| \leq\|(T+A) u\|+a\|u\|,
$$

portanto, se $b<1$, então dada $\left\{u_{n}\right\}$ tal que $u_{n} \rightarrow u$ e $(T+A) u_{n} \rightarrow v$, pondo $u=u_{n}-u_{m}$ em 1.2.15 concluímos que $\left\{T u_{n}\right\}$ é Cauchy, portanto, como $T$ é fechado, $u \in \mathrm{D}(T)=\mathrm{D}(T+A)$ e $v=(T+A) u$. Logo, $T+A$ é fechado.

Se $A$ é $T$-compacto, então dada $\left\{u_{n}\right\}$ em $\mathrm{D}(T)$ tal que $u_{n} \rightarrow u$ e $(T+A) u_{n} \rightarrow v$, temos que $\sup _{n}\left\|T u_{n}\right\|<\infty$. Senão, tomando $v_{n}=u_{n} /\left\|T u_{n}\right\|$, temos que $v_{n} \rightarrow 0,(T+A) v_{n} \rightarrow 0$ e $\left\{T v_{n}\right\}$ é limitada. Como $A$ é $T$-compacto, existe $\left\{v_{n_{j}}\right\}$ tal que $A v_{n_{j}} \rightarrow w$. Disso, $T v_{n} \rightarrow-w$. Como $v_{n} \rightarrow 0$ e $T$ é fechado, temos $w=0$. Isto é uma contradição, pois $\left\|T v_{n}\right\|=1$. Portanto, $\sup _{n}\left\|T u_{n}\right\|<\infty$. 
Logo, existe subseqüência $\left\{u_{n_{i}}\right\}$ tal que $\left\{A u_{n_{i}}\right\}$ converge; em particular, $\left\{T u_{n_{j}}\right\}$ converge. Como $T$ é fechado, então $u \in \mathrm{D}(T)=\mathrm{D}(T+A)$ e $(T+A) u=v$.

Finalmente, seja $A$ satisfazendo as hipóteses do terceiro ítem do enunciado. Escrevendo $T+A=\left(I+A T^{-1}\right) T$, como

$$
\left\|A T^{-1} u\right\| \leq a\left\|T^{-1} u\right\|+b\|u\| \leq\left(a\left\|T^{-1}\right\|+b\right)\|u\|,
$$

segue que $\left(I+A T^{-1}\right)^{-1} \in \mathscr{L}\left(\mathrm{H}^{\prime}, \mathrm{H}\right)$ e $(T+A)^{-1}=T^{-1}\left(I+A T^{-1}\right)^{-1}$, usando a série de Neumann, conforme a observação 1.5.1.

ObSERVAÇão 1.2.42 Se $T \in \mathscr{C}\left(\mathrm{H}, \mathrm{H}^{\prime}\right)$, então o produto interno $\langle u, v\rangle_{T}=\langle u, v\rangle+\langle T u, T v\rangle$ torna $\mathrm{D}(T)$ um espaço de Hilbert, o qual denotaremos por $\mathrm{H}_{T}$. A norma $\|\cdot\|_{T}$ induzida por $\langle\cdot, \cdot\rangle_{T}$ é chamada de norma do gráfico de $T$. Observamos que $A$ é $T$-limitado se e só se $\hat{A}: \mathrm{H}_{T} \rightarrow \mathrm{H}^{\prime}$ dado por $\hat{A} u=A u$ é limitado. Também é imediato verificar que $A$ é T-compacto se e só se $\hat{A}$ é compacto.

Finalizamos esta subseção estudando algumas propriedades dos espaços de operadores fechados. O espaço $\mathscr{C}\left(\mathrm{H}, \mathrm{H}^{\prime}\right)$ não é um espaço vetorial, pois não temos uma operação de soma bem definida para operadores fechados em geral. Apesar disso, temos uma operação de multiplicação escalar bem definida, pois multiplicar um operador fechado por uma constante não altera o seu domínio. A seguir, vamos verificar alguns fatos a respeito da continuidade da multiplicação escalar.

Lema 1.2.43 Para $\lambda \neq 0$ fixado, e $S, T \in \mathscr{C}\left(\mathrm{H}, \mathrm{H}^{\prime}\right)$ quaisquer, temos

$$
\hat{\delta}(\lambda T, \lambda S) \leq \frac{\left(1+|\lambda|^{2}\right)^{1 / 2}}{\min (1,|\lambda|)} \hat{\delta}(T, S) .
$$

Em particular, $\mathscr{C}\left(\mathrm{H}, \mathrm{H}^{\prime}\right) \ni T \mapsto \lambda T \in \mathscr{C}\left(\mathrm{H}, \mathrm{H}^{\prime}\right)$ é contínua.

Prova. Se $u \in \mathrm{D}(T), u \neq 0$ e $v \in \mathrm{D}(S)$ então

$$
\begin{aligned}
\frac{\|(u, \lambda T u)-(v, \lambda S v)\|^{2}}{\|u\|^{2}+\|\lambda T u\|^{2}} & \leq\left(1+|\lambda|^{2}\right) \frac{\|u-v\|^{2}+\|T u-S v\|^{2}}{\|u\|^{2}+|\lambda|^{2}\|T u\|^{2}} \\
& \leq \frac{\left(1+|\lambda|^{2}\right)}{\min \left(1,|\lambda|^{2}\right)} \frac{\|u-v\|^{2}+\|T u-S v\|^{2}}{\|u\|^{2}+\|T u\|^{2}}
\end{aligned}
$$

provando 1.2.16.

Lema 1.2.44 Para $T \in \mathscr{C}\left(\mathrm{H}, \mathrm{H}^{\prime}\right)$ fixo e $\lambda, \mu \in \mathbb{C} \backslash\{0\}$, temos

$$
\hat{\delta}(\lambda T, \mu T) \leq \frac{|\lambda-\mu|}{\min (|\lambda|,|\mu|)} .
$$

Em particular, $\mathbb{C} \backslash\{0\} \ni \lambda \mapsto \lambda T \in \mathscr{C}\left(\mathrm{H}, \mathrm{H}^{\prime}\right)$ é contínua. 
Prova. Se $\|u\|^{2}+\|\lambda T u\|^{2}=1$ então

$$
\|(u, \lambda T u)-(u, \mu T u)\|=\frac{|\lambda-\mu|}{|\lambda|}\|\lambda T u\| \leq \frac{|\lambda-\mu|}{|\lambda|}\left(\|u\|^{2}+\|\lambda T u\|^{2}\right)^{1 / 2}=\frac{|\lambda-\mu|}{|\lambda|},
$$

portanto,

$$
\delta(\lambda T, \mu T) \leq \frac{|\lambda-\mu|}{|\lambda|} .
$$

Trocando os papéis de $\lambda$ e $\mu$, concluímos 1.2.17.

Usando os lemas 1.2.43 e 1.2.44 vamos provar um resultado de continuidade.

Proposição 1.2.45 A aplicação

$$
\begin{aligned}
\mathbb{C} \backslash\{0\} \times \mathscr{C}\left(\mathrm{H}, \mathrm{H}^{\prime}\right) & \rightarrow \mathscr{C}\left(\mathrm{H}, \mathrm{H}^{\prime}\right) \\
(\lambda, T) & \mapsto \lambda T
\end{aligned}
$$

é contínua. Em particular, dada qualquer $f: X \rightarrow \mathbb{C} \backslash\{0\}$ contínua, a aplicação $X \times \mathscr{C}\left(\mathrm{H}, \mathrm{H}^{\prime}\right) \ni$ $(x, T) \mapsto f(x) T \in \mathscr{C}\left(\mathrm{H}, \mathrm{H}^{\prime}\right)$ é continua.

Prova. Fixados $\lambda \in \mathbb{C} \backslash\{0\}$ e $T \in \mathscr{C}\left(\mathrm{H}, \mathrm{H}^{\prime}\right)$, os lemas 1.2.43 e 1.2.44 mostram que para qualquer $\mu \neq 0$ temos

$$
\begin{aligned}
\hat{\delta}(\lambda T, \mu S) & \leq \hat{\delta}(\lambda T, \lambda S)+\hat{\delta}(\lambda S, \mu S) \\
& \leq \frac{\left(1+|\lambda|^{2}\right)^{1 / 2}}{\min \left(1,|\lambda|^{2}\right)} \hat{\delta}(T, S)+\frac{|\lambda-\mu|}{\min (|\lambda|,|\mu|)},
\end{aligned}
$$

provando a continuidade da aplicação 1.2.18.

Corolário 1.2.46 A aplicação

$$
\begin{aligned}
\mathbb{R} \backslash\{0\} \times \mathscr{C}^{\mathrm{sa}}(\mathrm{H}) & \rightarrow \mathscr{C}^{\mathrm{sa}}(\mathrm{H}) \\
(\lambda, T) & \mapsto \lambda T
\end{aligned}
$$

é contínua. Em particular, dada qualquer $f: X \rightarrow \mathbb{R} \backslash\{0\}$ contínua, a aplicação $X \times \mathscr{C}^{\text {sa }}(\mathrm{H}) \ni(x, T) \mapsto$ $f(x) T \in \mathscr{C}^{\text {sa }}(\mathrm{H})$ é contínua. O mesmo vale com $\mathscr{C}_{F}{ }^{\text {sa }}(\mathrm{H})$ em lugar de $\mathscr{C}^{\text {sa }}(\mathrm{H})$.

Observação 1.2.47 Os lemas 1.2.43, 1.2.44 e a proposição 1.2.45 valem também no caso real com as adaptações óbvias.

\subsubsection{Espectro e convergência}

Consideremos um operador fechado $T \in \mathscr{C}(\mathrm{H})$, com $\mathrm{H}$ complexo, e suponhamos que exista uma curva fechada simples $\Gamma$ que separa $\Sigma(T)$ em duas partes $\Sigma^{\prime} e \Sigma^{\prime \prime}$. Isto significa que $\Gamma \subset \mathrm{P}(T)$ e se $\Omega$ é a região aberta determinada por $\Gamma$, então $\Sigma^{\prime} \subset \Omega, \Sigma^{\prime \prime} \subset \mathbb{C} \backslash \Omega$ e $\Sigma^{\prime} \cup \Sigma^{\prime \prime}=\Sigma(T)$. 


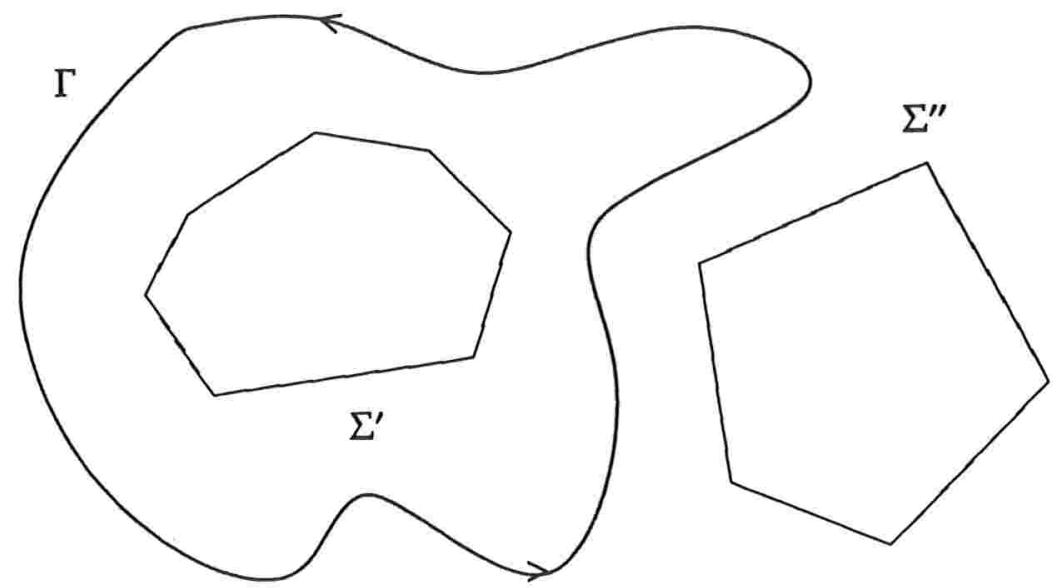

Definimos o operador

$$
P \doteq-\frac{1}{2 \pi i} \int_{\Gamma} \mathrm{R}(\zeta ; T) d \zeta
$$

Um cálculo direto usando o teorema de Cauchy prova que $P^{2}=P$, i.e., $P$ é uma projeção. Definimos $M^{\prime}=\operatorname{Im} P$ e $M^{\prime \prime}=\operatorname{ker} P$. Como $T$ comuta com $R(\zeta ; T)$, para $\zeta \in P(T)$, temos que $M^{\prime}, M^{\prime \prime}$ são invariantes por $T$. Denotemos por $T_{M^{\prime}}, T_{M^{\prime \prime}}$ as restrições de $T$ a $M^{\prime}, M^{\prime \prime}$, respectivamente.

Proposição 1.2.48 Temos que $\Sigma\left(T_{\mathrm{M}^{\prime}}\right)=\Sigma^{\prime}$ e $\Sigma\left(T_{\mathrm{M}^{\prime \prime}}\right)=\Sigma^{\prime \prime}$. Em particular, $T_{\mathrm{M}^{\prime}}$ é limitado.

Prova. É imediato que $\mathrm{P}(T) \subset \mathrm{P}\left(T_{\mathrm{M}^{\prime}}\right)$ e $\mathrm{P}(T) \subset \mathrm{P}\left(T_{\mathrm{M}^{\prime \prime}}\right)$. Vejamos que $\Sigma^{\prime \prime} \subset \mathrm{P}\left(T_{\mathrm{M}^{\prime}}\right)$. De fato, temos que, se $\mathrm{R}_{\mathrm{M}^{\prime}}(\zeta)$ denota a restrição de $\mathrm{R}(\zeta ; T)$ a $\mathrm{M}^{\prime}$, então $\mathrm{R}_{\mathrm{M}^{\prime}}(\zeta) u=\mathrm{R}(\zeta ; T) u=\mathrm{R}(\zeta ; T) P u$ para $u \in M^{\prime}$. Se $\zeta \in P(T) \backslash \Gamma$, então pela identidade do resolvente 1.1.1,

$$
\mathrm{R}(\zeta ; T) P=-\frac{1}{2 \pi i} \int_{\Gamma} \frac{\mathrm{R}(\zeta ; T)-\mathrm{R}\left(\zeta^{\prime} ; T\right)}{\zeta-\zeta^{\prime}} d \zeta^{\prime}
$$

Para $\zeta \notin \Omega$, temos

$$
\mathrm{R}(\zeta ; T) P=-\frac{1}{2 \pi i} \int_{\Gamma} \frac{\mathrm{R}\left(\zeta^{\prime} ; T\right)}{\zeta-\zeta^{\prime}} d \zeta^{\prime},
$$

$\log$, $R_{M^{\prime}}(\zeta)$, admite continuação analítica fora de $\Gamma$. Por analiticidade de $R_{M^{\prime}}(\zeta)$, segue que o membro direito de 1.2.21 é de fato igual a $R_{M^{\prime}}(\zeta)$ para $\zeta \notin \Omega$. Isso mostra que $\mathbb{C} \backslash \Omega \subset P\left(T_{M^{\prime}}\right)$, em particular, $\Sigma^{\prime \prime} \subset \mathrm{P}\left(T_{M^{\prime}}\right)$ e portanto, $\Sigma\left(T_{M^{\prime}}\right) \subset \Sigma^{\prime}$.

Se $\zeta \in \Omega$, a equação 1.2.20 nos dá que

$$
\mathrm{R}(\zeta ; T)(I-P)=\frac{1}{2 \pi i} \int_{\Gamma} \frac{\mathrm{R}\left(\zeta^{\prime} ; T\right)}{\zeta-\zeta^{\prime}} d \zeta^{\prime},
$$

portanto $\mathrm{R}(\zeta ; T)(I-P)$ admite continuação analítica a $\Omega$, e, como antes, segue que $\Sigma\left(T_{\mathrm{M}^{\prime \prime}}\right) \subset \Sigma^{\prime \prime}$. Como $\zeta \in \Sigma^{\prime}$ não pertence a $\mathrm{P}\left(T_{\mathrm{M}^{\prime}}\right) \cap \mathrm{P}\left(T_{\mathrm{M}^{\prime}}\right)$ (senão, teríamos que $\zeta \in \mathrm{P}(T)$, pois $\mathrm{R}(\zeta ; T)=$ $\left.\mathrm{R}_{\mathrm{M}^{\prime}}(\zeta) P+\mathrm{R}_{\mathrm{M}^{\prime \prime}}(\zeta)(I-P)\right)$, temos que $\Sigma\left(T_{\mathrm{M}^{\prime}}\right)=\Sigma^{\prime}$ e $\Sigma\left(T_{\mathrm{M}^{\prime \prime}}\right)=\Sigma^{\prime \prime}$.

Como $T$ e $P$ comutam, $T$ é fechado e $T R(\zeta ; T)=I+\zeta \mathrm{R}(\zeta ; T)$, temos que

$$
P T \subset T P=-\frac{1}{2 \pi i} \int_{\Gamma} T R(\zeta ; T) d \zeta=-\frac{1}{2 \pi i} \int_{\Gamma} \zeta \mathrm{R}(\zeta ; T) d \zeta,
$$


de onde concluímos que $T_{M^{\prime}}$ é limitado.

Um caso de particular interesse é o seguinte. Suponhamos que $\lambda \in \Sigma(T)$ seja um ponto isolado e consideremos $\Gamma$ uma circunferência centrada em $\lambda$ que não contenha em seu interior nenhum outro ponto de $\Sigma(T)$. Neste caso, $\Sigma^{\prime}=\{\lambda\}$ e $\Sigma^{\prime \prime}=\Sigma(T) \backslash\{\lambda\}$. Consideremos a expansão de Laurent de $\mathrm{R}(\zeta ; T)$ em torno de $\zeta=\lambda$

$$
\mathrm{R}(\zeta)=\sum_{n=-\infty}^{\infty}(\zeta-\lambda)^{n} A_{n}
$$

com

$$
A_{n}=\frac{1}{2 \pi i} \int_{\Gamma}(\zeta-\lambda)^{-n-1} \mathrm{R}(\zeta ; T) d \zeta,
$$

para $n \in \mathbb{Z}$. Um cálculo semelhante àquele feito para provar que $P$ definido em 1.2.19 é uma projeção mostra que $A_{n} A_{m}=\left(\eta_{n}+\eta_{m}-1\right) A_{n+m+1}$, onde $\eta_{n}=0$ para $n<0$ e $\eta_{n}=1$ para $n \geq 0$. Em particular, vemos que $P=-A_{-1}$. Pondo $D=-A_{-2}=(T-\lambda) P$ e $S=A_{0}$, temos $A_{-k}=-D^{k-1}$ para todo $k \geq 2$ e $A_{n}=S^{n+1}$ para todo $k \geq 0$. Logo, 1.2.22 é equivalente a

$$
\mathrm{R}(\zeta ; T)=-\frac{P}{\zeta-\lambda}-\sum_{n=1}^{\infty} \frac{D^{n}}{(\zeta-\lambda)^{n+1}}+\sum_{n=0}^{\infty}(\zeta-\lambda)^{n} S^{n+1},
$$

para $\zeta \neq \lambda$. Temos que $D P=P D=D, S P=P S=0$ e $(T-\lambda) S=I-P$. Além disso, $\Sigma(D)=\{0\}$.

A equação 1.2.23 é particularmente interessante quando $M^{\prime}=\operatorname{Im} P$ tem dimensão finita. Neste caso, $\left.D_{\mathrm{M}^{\prime}} \doteq D\right|_{\mathrm{M}^{\prime}}$ é nilpotente ${ }^{7}$, e portanto $D$ é nilpotente. Isso significa que 1.2.23 contém somente um número finito de potências negativas de $\zeta-\lambda$. Além disso, $\lambda$ é um autovalor de $T_{M^{\prime}}$, em particular, $\lambda$ é um autovalor de $T$. O número dim $\mathrm{M}^{\prime}$ é chamado de multiplicidade (algébrica) de $\lambda$ e denotado por $\operatorname{mul}(\lambda)$. Os operadores $P$ e $D$ são chamados de projetor e nilpotente correspondentes a $\lambda$, respectivamente.

Podemos desenvolver um raciocínio análogo para o caso de $\lambda_{1}, \ldots, \lambda_{m} \in \Sigma(T)$ pontos isolados. Neste caso, obtemos

$$
\mathrm{R}(\zeta ; T)=-\sum_{j=1}^{m}\left\{\frac{P_{j}}{\zeta-\lambda_{j}}+\sum_{n=1}^{\infty} \frac{D_{j}^{n}}{\left(\zeta-\lambda_{j}\right)^{n+1}}\right\}+\mathrm{R}_{0}(\zeta ; T),
$$

onde $\mathrm{R}_{0}(\zeta ; T)=\sum_{j=1}^{m} \sum_{n=0}^{\infty}\left(\zeta-\lambda_{j}\right)^{n} S^{n+1}$ e $P_{j}, D_{j}$ denotam o projetor e o nilpotente associados a $\lambda_{j}, j=1, \ldots, m$. Temos que $P_{j} P_{k}=\delta_{j k} P_{j}, P_{j} D_{j}=D_{j} P_{j}=D_{j}$ e $\left(T-\lambda_{j}\right) P_{j}=D_{j}$, para $j, k=1, \ldots, m$. Temos que $\mathrm{R}_{0}(\zeta ; T)$ é holomorfa em todo o plano complexo e $\mathrm{R}_{0}(\zeta ; T) P_{0}=P_{0} \mathrm{R}_{0}(\zeta ; T)=\mathrm{R}_{0}(\zeta ; T)$, se $P_{0}=1-\left(P_{1}+\ldots+P_{m}\right)$.

Se $\operatorname{mul}\left(\lambda_{j}\right)<\infty$ para $j=1, \ldots, m$, então cada $\lambda_{j}$ é autovalor de $T$ e é válida a seguinte decomposição para $\left.T\right|_{\operatorname{Im} P}$ :

$$
T P=\sum_{j=1}^{m}\left(\lambda_{j} P_{j}+D_{j}\right),
$$

onde $P=P_{1}+\ldots+P_{m}$. Isto significa que no subespaço $\operatorname{Im} P$, que tem dimensão finita, $T$ admite uma decomposição análoga à forma canônica de Jordan para operadores em espaços de dimensão finita.

\footnotetext{
${ }^{7}$ Isso porque um operador $A$ em um espaço de dimensão finita tal que $\operatorname{spr}(A)=\{0\}$ é necessariamente nilpotente.
} 
Definição 1.2.49 Dado um operador $T \in \mathscr{C}(\mathrm{H})$ dizemos que $\left\{\lambda_{1}, \ldots, \lambda_{m}\right\}$ é um sistema finito de autovalores de $T$ se cada $\lambda_{j}$ é um ponto isolado de $\Sigma(T)$ e $\operatorname{mul}\left(\lambda_{j}\right)<\infty$, para $j=1, \ldots, m$. A multiplicidade total do sistema é definida por $\sum_{j=1}^{m} \operatorname{mul}\left(\lambda_{j}\right)$.

Os comentários que antecedem a definição 1.2.49 provam a seguinte proposição.

Proposição 1.2.50 Se $\left\{\lambda_{1}, \ldots, \lambda_{m}\right\}$ é um sistema finito de autovalores de $T \in \mathscr{C}(\mathrm{H})$, então vale 1.2.24, onde $P_{j}, D_{j}$ denotam os projetores $e$ os nilpotentes correspondentes $a \lambda_{j}$, respectivamente, $e$ $P=P_{1}+\ldots+P_{m}$.

Estudaremos agora o comportamento de um sistema finito de autovalores de $T \in \mathscr{C}(\mathrm{H})$, quando sujeitamos $T$ a uma pequena perturbação em termos da distância $\hat{\delta}$. Seja $\Gamma$ uma curva fechada simples que separa $\Sigma(T)$ em duas partes $\Sigma^{\prime}(T)$ e $\Sigma^{\prime \prime}(T)$, como no começo desta subseção. O próximo teorema mostra que a decomposição $\Sigma(T)=\Sigma^{\prime}(T) \cup \Sigma^{\prime \prime}(T)$ varia continuamente com $T$.

Teorema 1.2.51 Existe $\delta>0$ dependendo de $T$ e $\Gamma$ tal que se $\hat{\delta}(S, T)<\delta$, então $\Sigma(S)$ é separado por $\Gamma$ em duas partes $\Sigma^{\prime}(S)$ e $\Sigma^{\prime \prime}(S)$. Nas decomposições $H=M^{\prime}(T) \oplus \mathrm{M}^{\prime \prime}(T)=\mathrm{M}^{\prime}(S) \oplus \mathrm{M}^{\prime \prime}(S)$ correspondentes, temos que $\mathrm{M}^{\prime}(T)$ e $\mathrm{M}^{\prime}(S)$ são isomorfos e o mesmo é válido para $\mathrm{M}^{\prime \prime}(T)$ e $\mathrm{M}^{\prime \prime}(S)$. Além disso, se $P(S), P(T)$ denotam as projeções correspondentes a $\Sigma^{\prime}(S)$ e $\Sigma^{\prime}(T)$, respectivamente, então $\|P(S)-P(T)\| \rightarrow 0$ se $\hat{\delta}(S, T) \rightarrow 0$.

Prova. A existência de $\delta>0$ é garantida pela proposição 1.2.38, onde tomamos $K=\Gamma \subset \mathrm{P}(T)$. Isto implica que $\Gamma \subset \mathrm{P}(S)$, e portanto, $\Sigma(S)$ é separado em duas partes $\Sigma^{\prime}(S)$ e $\Sigma^{\prime \prime}(S)$, com $\Sigma^{\prime}(S)$ contida no interior da região determinada por $\Gamma$. A projeção $P(S)$ correspondente a $\Sigma^{\prime}(S)$ é dada por

$$
P(S)=-\frac{1}{2 \pi i} \int_{\Gamma} \mathrm{R}(\zeta ; S) d \zeta,
$$

portanto, o teorema segue da proposição 1.2.38 e do ítem 3 da proposição 1.2.3.

O teorema 1.2.51 é particularmente interessante quando $\Sigma^{\prime}(T)$ é um sistema finito de autovalores.

Trorema 1.2.52 Seja $\left\{\lambda_{1}, \ldots, \lambda_{n}\right\}$ um sistema finito de autovalores de $T \in \mathscr{C}(\mathrm{H})$ com multiplicidade total $m$ e $\Gamma$ uma curva fechada simples contendo $\left\{\lambda_{1}, \ldots, \lambda_{n}\right\}$ em seu interior. Então existe $\delta>0$ tal que se $\hat{\delta}(S, T)<\delta$ então a parte de $\Sigma(S)$ contida no interior de $\Gamma$ é um sistema finito de autovalores de $S$ com multiplicidade total $m$.

Podemos interpretar o teorema 1.2.52 como continuidade de um sistema finito de autovalores. Na próxima subseção estudaremos esta questão mais detalhadamente.

Para finalizar esta subseção, vamos estudar a continuidade do cálculo funcional para operadores auto-adjuntos, tanto no caso limitado quanto ilimitado.

Proposição 1.2.53 O cálculo funcional

$$
\begin{aligned}
\mathrm{C}(\mathbb{R}) \times \mathscr{L}^{\mathrm{sa}}(\mathrm{H}) & \rightarrow \mathscr{L}^{\mathrm{sa}}(\mathrm{H}) \\
(f, T) & \mapsto f(T)
\end{aligned}
$$

define uma aplicação contínua. ${ }^{8}$

\footnotetext{
${ }^{8} \mathrm{C}(\mathbb{R})$ denota o espaço das funções reais contínuas em $\mathbb{R}$, munido da estrutura de espaço de Frechét fornecida pelas seminormas $\|f\|_{n} \doteq \sup _{|\lambda| \leq n}|f(\lambda)|, n \in \mathbb{N}$.
} 
Prova. Dados $S, T \in \mathscr{L}^{\text {sa }}(\mathrm{H})$, com $\|S-T\| \leq 1, f, g \in \mathrm{C}(\mathbb{R})$ e $p$ um polinômio qualquer em $\mathbb{R}$, temos

$$
\begin{aligned}
\|g(S)-f(T)\| & \leq\|g(S)-f(S)\|+\|f(S)-p(S)\|+\|p(S)-p(T)\|+\|p(T)-f(T)\| \\
& \leq \sup _{|\lambda| \leq\|T\|+1}|g(\lambda)-f(\lambda)|+2 \sup _{|\lambda| \leq\|T\|+1}|f(\lambda)-p(\lambda)|+\|p(S)-p(T)\| .
\end{aligned}
$$

A continuidade de 1.2.26 segue do teorema de Weierstrass.

OвSERVAçÃo 1.2.54 Podemos usar o mesmo argumento da prova da proposição 1.2.53 para provar que o cálculo funcional é contínuo sobre o espaço dos operadores normais ${ }^{9}$. De fato, cada operador normal $T \in \mathscr{L}(\mathrm{H})$ admite uma família espectral, i.e., uma medida $E_{T}$ definida sobre os borelianos de $\mathbb{C}$, com suporte em $\Sigma(T)$, a valores no espaço de projeções ortogonais tal que $T=\int \lambda d E_{T}$. O cálculo funcional para $T$ é definido como $f(T) \doteq \int f(\lambda) d E_{T}$, para qualquer $f: \Sigma(T) \rightarrow \mathbb{C}$ boreliana limitada. Propriedades inteiramente análogas às do cálculo funcional para operadores auto-adjuntos limitados são verificadas nesta situação. Também é verdade que a aplicação

$$
\begin{aligned}
\mathrm{C}(\mathbb{C}) \times \mathscr{L}_{\text {nor }}(\mathrm{H}) & \rightarrow \mathscr{L}_{\text {nor }}(\mathrm{H}) \\
(f, T) & \mapsto f(T),
\end{aligned}
$$

onde $\mathscr{L}_{\text {nor }}(\mathrm{H})$ denota o subconjunto dos operadores normais, é contínua. A prova é semelhante à prova da proposição 1.2.53; ao invés de tomar um polinômio em $\lambda$ aproximando $f$, devemos tomar um polinômio em $\lambda$ e $\bar{\lambda}$, o qual existe pelo teorema de Weierstrass-Stone. Mais detalhes sobre operadores normais podem ser encontrados em [87], Cap.12.

No caso ilimitado, a continuidade do cálculo funcional é uma questão bem mais delicada. A questão crucial é saber comparar os domínios de $T$ e $f(T)$, para $T \in \mathscr{C}^{\text {sa }}(\mathrm{H})$, com hipóteses adequadas sobre a função $f$. Primeiramente, estabeleceremos um resultado local simples.

Proposição 1.2.55 Seja $T \in \mathscr{C}^{\text {sa }}(\mathrm{H})$ e suponhamos que $a, b \in \mathrm{P}(T) \cap \mathbb{R}$. Então existe um vizinhança aberta $\mathscr{U}$ de $\mathrm{T}$ em $\mathscr{C}^{\mathrm{sa}}(\mathrm{H})$ tal que a aplicação

$$
\begin{aligned}
\mathrm{C}_{c}([a, b]) \times \mathscr{U} & \rightarrow \mathscr{L}^{\mathrm{sa}}(\mathrm{H}) \\
(f, S) & \mapsto f(S)
\end{aligned}
$$

é contínua. ${ }^{10}$

Prova. Seja $\delta>0$ fornecido pela proposição 1.2.38, $\operatorname{com} K=\{a, b\}$. Pondo $\mathscr{U}=\left\{S \in \mathscr{C}^{\mathrm{sa}}(\mathrm{H})\right.$ : $\delta(S, T)<\delta\}$, o teorema 1.2.51 implica que a aplicação $\mathscr{U} \ni S \mapsto \chi_{[a, b]}(S) \in \mathscr{L}^{\text {sa }}(\mathrm{H})$ é contínua. ${ }^{11}$ Como $f(S)=f\left(\chi_{[a, b]}(S)\right)$ para toda $f \in C_{c}([a, b])$, o resultado segue da proposição 1.2.53 e da continuidade da inclusão $\mathrm{C}_{c}([a, b]) \subset \mathrm{C}(\mathbb{R})$.

As proposições 1.2.38 e 1.2.55 implicam o seguinte corolário.

\footnotetext{
${ }^{9} T \in \mathscr{L}(\mathrm{H})$ é normal se $T T^{*}=T^{*} T$.

${ }^{10} \mathrm{C}_{c}(Y)$ denota o espaço das funções reais contínuas com suporte compacto contido em $Y$ munido da norma usual $\|f\|=\sup _{\lambda \in Y}|f(\lambda)|$.

${ }^{11}$ De fato, basta tomar a curva $\Gamma$ como uma circunferência com um diâmetro ligando $a$ e $b$.
} 
CoROLÁrio 1.2.56 Fixada $f \in \mathrm{C}_{c}(\mathbb{R})$ e pondo

$$
\mathscr{V} \doteq\left\{T \in \mathscr{C}^{\text {sa }}: \text { Existem } a, b \in \mathbb{R} \cap \mathrm{P}(T) \text { com supp } f \subset(a, b)\right\},
$$

temos que $\mathscr{V}$ é aberto em $\mathscr{C}^{\mathrm{sa}}$ e $\mathscr{V} \ni T \mapsto f(T) \in \mathscr{L}^{\mathrm{sa}}(\mathrm{H})$ é contínua.

Podemos estender consideravelmente a classe de funções e operadores para os quais a proposição 1.2.55 é válida. Para isso, definimos o espaço

$$
\begin{aligned}
\mathscr{C}_{\Sigma}^{\text {sa }} & \doteq\left\{T \in \mathscr{C}^{\text {sa }}: \inf \mathrm{P}(T) \cap \mathbb{R}=-\infty \text { e } \sup \mathrm{P}(T) \cap \mathbb{R}=\infty\right\} \\
& =\bigcap_{n \in \mathbb{N}}\left\{T \in \mathscr{C}^{\text {sa }}: \mathrm{P}(T) \cap(-\infty, n) \neq \emptyset, \mathrm{P}(T) \cap(n, \infty) \neq \emptyset\right\} .
\end{aligned}
$$

Pela proposição 1.2 .38 , temos que $\mathscr{C}_{\Sigma}^{\text {sa }}$ é um $\mathrm{G}_{\delta}$ de $\mathscr{C}^{\text {sa }}$. Denotamos ${ }^{12}$ por $\mathrm{C}_{0}(\mathbb{R})$ o espaço das funções reais contínuas tais que $\lim _{|\lambda| \rightarrow \infty} f(\lambda)=0$ munido da norma do sup usual.

Proposição 1.2.57 A aplicação

$$
\begin{aligned}
\mathrm{C}_{0}(\mathbb{R}) \times \mathscr{C}_{\Sigma}^{\mathrm{sa}} & \rightarrow \mathscr{L}^{\mathrm{sa}}(\mathrm{H}) \\
(f, T) & \mapsto f(T)
\end{aligned}
$$

é contínua.

Prova. Dados $f, g \in \mathrm{C}_{0}(\mathbb{R}), S, T \in \mathscr{C}_{\Sigma}^{\text {sa }}$, e $\chi \in \mathrm{C}_{c}(\mathbb{R})$, temos que

$$
\begin{aligned}
\|g(S)-f(T)\| & \leq\|g(S)-f(S)\|+\|f(S)-\chi(S)\|+\|\chi(S)-\chi(T)\|+\|\chi(T)-f(T)\| \\
& \leq \sup _{\lambda \in \mathbb{R}}|g(\lambda)-f(\lambda)|+2 \sup _{\lambda \in \mathbb{R}}|\chi(\lambda)-f(\lambda)|+\|\chi(S)-\chi(T)\| .
\end{aligned}
$$

O resultado decorre da densidade $\operatorname{de~}_{c}(\mathbb{R})$ em $C_{0}(\mathbb{R})$ e do corolário 1.2.56.

O próximo teorema nos fornece uma versão da proposição 1.2.53 no caso ilimitado. Para isso, introduzimos o espaço

$X=\left\{f \in \mathrm{C}(\mathbb{R}): \theta_{f} \in \mathrm{C}_{0}(\mathbb{R})\right.$ e existe $\alpha>0$ tal que $|f(\lambda)| \geq \alpha|\lambda|$ para todo $\left.\lambda \in \mathbb{R}\right\} \subset \mathrm{C}(\mathbb{R})$, onde $\theta_{f}(\lambda) \doteq f(\lambda)-\lambda, \lambda \in \mathbb{R}$. Sobre $X$, a topologia de $C(\mathbb{R})$ coincide com a topologia dada pela distância $d(f, g) \doteq \sup _{\lambda \in \mathbb{R}}|f(\lambda)-g(\lambda)|=\sup _{\lambda \in \mathbb{R}}\left|\theta_{f}(\lambda)-\theta_{g}(\lambda)\right|$.

Teorema 1.2.58 A aplicação

$$
\begin{aligned}
X \times \mathscr{C}_{\Sigma}^{\mathrm{sa}} & \rightarrow \mathscr{C}^{\mathrm{sa}} \\
(f, S) & \mapsto f(S)
\end{aligned}
$$

é contínua.

Prova. Dada $f \in X$, como $\theta_{f} \in \mathrm{C}_{0}(\mathbb{R})$, temos que $\mathrm{D}(f(S))=\mathrm{D}(S)$. Além disso, dado $S \in \mathscr{C}_{\Sigma}^{\text {sa }}$, denotando por $\left\{E_{\lambda}\right\}_{\lambda \in \mathbb{R}}$ a família espectral de $S$, temos

$$
\alpha^{2}\|S u\|^{2}=\alpha^{2} \int_{\mathbb{R}}|\lambda|^{2} d\left\|E_{\lambda}(u)\right\|^{2} \leq \int_{\mathbb{R}}|f(\lambda)|^{2} d\left\|E_{\lambda}(u)\right\|^{2}=\|f(S) u\|^{2} .
$$

\footnotetext{
${ }^{12}$ Um subconjunto de um espaço topológico é dito $\mathrm{G}_{\delta}$ se for uma intersecção enumerável de abertos.
} 
Temos que para quaisquer $S, S^{\prime} \in \mathscr{C}_{\Sigma}^{\mathrm{sa}}, u \in \mathrm{D}\left(S^{\prime}\right), v \in \mathrm{D}(S)$,

$$
\begin{aligned}
& \frac{\|u-v\|^{2}+\left\|f\left(S^{\prime}\right) u-f(S) v\right\|^{2}}{\|u\|^{2}+\left\|f\left(S^{\prime}\right) u\right\|^{2}} \leq \frac{\|u-v\|^{2}+2\left\|S^{\prime} u-S v\right\|^{2}+2\left\|\theta_{f}\left(S^{\prime}\right) u-\theta_{f}(S) v\right\|^{2}}{\|u\|^{2}+\left\|f\left(S^{\prime}\right) u\right\|^{2}} \\
& \leq \frac{2\left(1+4\left\|\theta_{f}(S)\right\|^{2}\right)}{\min (1, \alpha)^{2}} \frac{\|u-v\|^{2}+\left\|S^{\prime} u-S v\right\|^{2}}{\|u\|^{2}+\left\|S^{\prime} u\right\|^{2}}+ \\
&+4\left\|\theta_{f}\left(S^{\prime}\right)-\theta_{f}(S)\right\|^{2},
\end{aligned}
$$

pela desigualdade 1.2.27. Logo, pela observação 1.2.24

$$
\delta\left(f\left(S^{\prime}\right), f(S)\right) \leq \frac{\left(2\left(1+4\left\|\theta_{f}(S)\right\|^{2}\right)\right)^{1 / 2}}{\min (1, \alpha)} \delta\left(S^{\prime}, S\right)+2\left\|\theta_{f}\left(S^{\prime}\right)-\theta_{f}(S)\right\|
$$

Dada $g \in X$, pela observação 1.2.25 e pelo fato que $g\left(S^{\prime}\right)-f\left(S^{\prime}\right)$ é limitado, temos

$$
\delta\left(g\left(S^{\prime}\right), f\left(S^{\prime}\right)\right) \leq \sup _{\substack{u \in \mathrm{D}\left(S^{\prime}\right) \\ u \neq 0}} \frac{\left\|g\left(S^{\prime}\right) u-f\left(S^{\prime}\right) u\right\|}{\left(\|u\|^{2}+\left\|g\left(S^{\prime}\right) u\right\|^{2}\right)^{1 / 2}} \leq \sup _{\lambda \in \mathbb{R}}|g(\lambda)-f(\lambda)|,
$$

Como $\delta\left(g\left(S^{\prime}\right), f(S)\right) \leq \delta\left(g\left(S^{\prime}\right), f\left(S^{\prime}\right)\right)+\delta\left(f\left(S^{\prime}\right), f(S)\right)$, a proposição segue das desigualdades 1.2.28 e 1.2.29 e da proposição 1.2.57.

No caso em que $\mathrm{H}$ é complexo, uma importante ferramenta disponível para o estudo da topologia do espaço dos operadores auto-adjuntos é a transformada de Cayley, definida como

$$
\mathrm{k}(T) \doteq(T-i)(T+i)^{-1}
$$

se $T \in \mathscr{C}^{\text {sa }} \cdot{ }^{13}$ Vamos usar a notação $\mathrm{k}(\lambda) \doteq(\lambda-i) /(\lambda+i)$, para $\lambda \in \mathbb{C}$.

Lema 1.2.59 A aplicação $\tilde{\delta}(S, T) \doteq\|\mathrm{k}(S)-\mathrm{k}(T)\|$ define em $\mathscr{C}^{\text {sa }}$ uma métrica equivalente à métrica $\delta$.

Prova. Usando a notação do lema 1.2 .27 e da proposição 1.2 .29 , temos que $R(T)=\left(I+T^{2}\right)^{-1}=$ $(T+i)^{-1}(T-i)^{-1}, \operatorname{logo},(T \pm i)^{-1}=T R(T) \mp i R(T)$, se $T \in \mathscr{C}^{\text {sa }}$ e portanto $R(T)=\left((T-i)^{-1}-(T+i)^{-1}\right) / 2 i$ e $T R(T)=\left((T-i)^{-1}+(T+i)^{-1}\right) / 2$. Como $\left((T \pm i)^{-1}\right)^{*}=(T \mp i)^{-1}$ e $\mathrm{k}(T)=I-2 i(T+i)^{-1}$, o resultado segue pela proposição 1.2.29.

OBSERVAÇÃo 1.2.60 Já havíamos observamos que convergência na métrica $\delta$ implica em convergência dos resolventes, conforme o teorema 1.2.37. O lema 1.2.59 dá informações bem mais precisas a respeito deste fato no caso auto-adjunto.

Proposição 1.2.61 A imagem de ké

$$
\mathrm{U}_{\mathrm{inj}}(\mathrm{H}) \doteq\{U \in \mathrm{U}(\mathrm{H}): \operatorname{ker}(I-U)=0\} .
$$

\footnotetext{
${ }^{13}$ A idéia é transformar o operador $T$, geralmente ilimitado, em um operador unitário tendo controle sobre a mudança que ocorre no espectro de $T$ durante a transformação. Como a função $z \mapsto(z-i) /(z+i)$ leva $\mathbb{R}$ analiticamente sobre $S^{1} \backslash\{1\}$, isso nos mostra que a definição de $k(T)$ em 1.2.30 é razoável.
} 
Prova. A equação $\mathrm{k}(T)=I-2 i(T+i)^{-1}$ mostra que se $(I-\mathrm{k}(T)) u=0$ então $(T+i)^{-1} u=0$, logo, $u=0$. Agora vamos provar que qualquer $U \in \mathrm{U}_{\text {inj }}(\mathrm{H})$ é a transformada de Cayley de algum operador auto-adjunto. De fato, a hipótese sobre $U$ nos permite definir $T: D(T)=$ $\operatorname{Im}(I-U) \rightarrow \mathrm{H}$ como $T \doteq i(I+U)(I-U)^{-1}$. Como $\operatorname{ker}\left(I-U^{*}\right)=\operatorname{ker}(I-U)=0$ (pois $U$ é normal), temos que $T$ é densamente definido. Vejamos que $T$ é simétrico. Dados $u, w \in \mathrm{D}(T)$, sejam $v, z \in \mathrm{H}$ tais que $u=v-U v$ e $w=z-U z$. Logo, como $U$ é uma isometria,

$$
\langle T u, w\rangle=i\langle v+U v, z-U z\rangle=\langle v-U v, i(z+U z)\rangle=\langle u, T w\rangle .
$$

Isso implica que $\|T u+i u\|^{2}=\|T u\|^{2}+\|u\|^{2}, u \in \mathrm{D}(T)$, logo a correspondência

$$
\mathrm{G}(T) \ni(u, T u) \mapsto T u+i u \in \operatorname{Im}(T+i)
$$

é uma isometria. Decorre diretamente da definição de $T$ que $T+i=2 i(I-U)^{-1}$, portanto, $\operatorname{Im}(T+i)=\mathrm{H}, \operatorname{logo}, T$ é fechado. Não é difícil provar que, de fato, $T=i(I-U)^{-1}(I+U)$, i.e., $I+U$ e $(I-U)^{-1}$ comutam. Como $T$ é simétrico, $T \subset T^{*}=-i\left(I-U^{*}\right)^{-1}\left(I+U^{*}\right)=-i\left(I+U^{*}\right)\left(I-U^{*}\right)^{-1}$, pelo mesmo argumento. Usando o mesmo cálculo que fizemos para provar que $T$ é simétrico, com $U^{*}$ em lugar de $U$, concluímos que $T^{*}$ é simétrico, portanto, $T \subset T^{*} \subset T^{* *}=T$, pois $T$ é fechado. Logo, $T$ é auto-adjunto.

Finalmente, vejamos quek $(T)=U$. De fato, pela definição de $T$, temos que $T+i=2 i(I-U)^{-1}$ e $T-i=2 i U(I-U)^{-1}$. Logo, $\mathrm{k}(T)=(T-i)(T+i)^{-1}=U$.

A próxima proposição nos dá informação sobre o espectro da transformada de Cayley de um operador auto-adjunto.

Proposição 1.2.62 Se T é auto-adjunto e $\lambda \in \mathbb{C} \backslash\{-i\}$ então

$$
\mathrm{k}(T)-\mathrm{k}(\lambda)=\frac{2 i}{\lambda+i}(T-\lambda)(T+i)^{-1}=\frac{1}{\lambda+i}(T-\lambda)(I-\mathrm{k}(T)) .
$$

Em particular, $\Sigma(\mathrm{k}(T))=\mathrm{k}(\Sigma(T)), \Sigma_{\mathrm{ess}}(\mathrm{k}(T))=\mathrm{k}\left(\Sigma_{\mathrm{ess}}(T)\right) e \Sigma_{\text {disc }}(\mathrm{k}(T))=\mathrm{k}\left(\Sigma_{\text {disc }}(T)\right)$, conforme $a$ definição 1.4.4.

Prova. A equação 1.2.31 decorre diretamente da definição de k.

A proposição 1.2.62 implica o seguinte corolário.

Corolário 1.2.63 A imagem de $\mathscr{C} \mathscr{F}$ sa por ké

$$
\mathrm{U}_{\text {inj }}^{\text {Fred }}(\mathrm{H}) \doteq\{U \in \mathrm{U}(\mathrm{H}): \operatorname{ker}(U-I)=0 \text { e U+I é Fredholm }\} .
$$

Em particular, ké um homeomorfismo de $\mathscr{C} \mathscr{F}$ sa sobre $\mathrm{U}_{\text {inj }}^{\text {Fred }}(\mathrm{H})$.

Prova. Temos que $T \in \mathscr{C} \mathscr{F}$ sa se e só se $0 \notin \Sigma_{\text {ess }}(T)$. Pela proposição 1.2.62, concluímos que $T \in \mathscr{C} \mathscr{F}$ sa se e só se $\mathrm{k}(0)=-1 \notin \Sigma_{\text {ess }}(\mathrm{k}(T))$; pela proposição 1.4 .5 , isso ocorre se e só se $\mathrm{k}(T)+I$ é Fredholm. O resultado decorre da proposição 1.2.61.

Podemos utilizar a transformada de Cayley para mostrar que o cálculo funcional é contínuo também no caso ilimitado, sob hipóteses adequadas. Para isso, introduzimos o espaço

$$
y \doteq\left\{f \in C(\mathbb{R}): \lim _{\lambda \rightarrow \pm \infty} f(\lambda)= \pm \infty \text { e existe } A>0 \text { tal que }|f(\lambda)| \leq A|\lambda|, \lambda \in \mathbb{R}\right\} \subset \mathrm{C}(\mathbb{R})
$$


munido da topologia herdada de $C(\mathbb{R})$. Um cálculo simples usando a definição da transformada de Cayley mostra que a aplicação $f \mapsto \mathrm{kfk}^{-1}$ é um homeomorfismo entre y e o espaço

$$
z \doteq\left\{\varphi \in \mathrm{C}\left(S^{1}, S^{1}\right): \text { existe } \alpha>0 \text { tal que }|\varphi(z)-1| \geq \alpha|z-1|, z \in S^{1}\right\}
$$

munido ${ }^{14}$ da topologia herdada de $\mathrm{C}\left(S^{1}, S^{1}\right)$.

TeORema 1.2.64 A aplicação

$$
\begin{aligned}
Z \times \mathrm{U}_{\mathrm{inj}}(\mathrm{H}) & \rightarrow \mathrm{U}_{\mathrm{inj}}(\mathrm{H}) \\
(\varphi, U) & \mapsto \varphi(U)
\end{aligned}
$$

é contínua. Em particular,

$$
\begin{aligned}
y \times \mathscr{C}^{\text {sa }} & \rightarrow \mathscr{C}^{\text {sa }} \\
(f, T) & \mapsto f(T)
\end{aligned}
$$

é contínua.

Prova. Se $\varphi \in Z$ e $U \in U_{\text {inj }}(H)$ então $\varphi(U) \in U_{\text {inj }}(H)$, pois

$$
\|(\varphi(U)-I) u\|^{2}=\int_{S^{1}}|\varphi(z)-1|^{2} d\left\|E_{U}(z) u\right\|^{2} \geq \alpha^{2} \int_{S^{1}}|z-1|^{2} d\left\|E_{U}(z) u\right\|^{2}=\|(U-I) u\|^{2},
$$

para todo $u \in \mathrm{H}$, conforme a notação da observação 1.2.54. A continuidade de 1.2.32 segue também da referida observação. A continuidade de 1.2.33 segue imediatamente dos comentários que sucedem o corolário 1.2.63.

Corolário 1.2.65 Pondo $y_{0} \doteq\{f \in y: f(0)=0\}$, temos que

$$
\begin{aligned}
y_{0} \times \mathscr{C} \mathscr{F}^{\mathrm{sa}} & \rightarrow \mathscr{C} \mathscr{F}^{\mathrm{sa}} \\
(f, T) & \mapsto f(T)
\end{aligned}
$$

é contínua.

Observação 1.2.66 Caso $\mathrm{H}$ seja real, todos os resultados de continuidade obtidos nesta subseção são válidos com as adaptações óbvias, desde que nos restrinjamos a operadores autoadjuntos em $\mathrm{H}$. De fato, se $T$ é um operador auto-adjunto em $\mathrm{H} \mathrm{e} \mathrm{H}^{\mathbf{C}}$ denota o complexificado de $\mathrm{H}$, definimos $T^{\mathbb{C}}$ em $\mathrm{H}^{\mathbb{C}}$ por $T^{\mathbb{C}}(u+i v)=T u+i T v$. Vemos que $T^{\mathbb{C}}$ é auto-adjunto e tem espectro $\Sigma(T)+i \Sigma(T) \doteq\{\lambda+i \mu: \lambda, \mu \in \Sigma(T)\}$.

\subsubsection{Famílias holomorfas de operadores}

Dado um operador linear $T: V \rightarrow V$, com $\operatorname{dim} V<\infty$, o espectro de $T$ reduz-se a um conjunto finito de números complexos. Estes números são raízes da equação polinomial

$$
\operatorname{det}(T-\lambda)=0 \text {. }
$$

\footnotetext{
${ }^{14} \mathrm{C}\left(S^{1}, S^{1}\right)$ denota o espaço das funções contínuas $S^{1} \rightarrow S^{1}$ munido da topologia compacto aberta.
} 
Podemos nos perguntar como é a dependência entre as raízes $\lambda$ da equação 1.2.34 e o operador $T$. Em geral, tal dependência pode ser bastante irregular, mas existe um caso no qual a situação é bastante regular, o qual passamos a descrever.

Se $T(z): V \rightarrow V$ é uma família de operadores holomorfa com respeito ao parâmetro $z \in \Omega \subset \mathbb{C}$, então os autovalores de $T(z)$ são raízes da equação $1.2 .34 \mathrm{com} T(z)$ em lugar de $T$. Esta é uma equação polinomial em $\lambda$ cujos coeficientes são funções holomorfas em $z$. $O$ próximo teorema é fundamental no que diz respeito ao comportamento dos autovalores de $T(z)$ com respeito ao parâmetro $z$.

Teorema 1.2.67 Se $T(z)$ é uma família holomorfa de operadores em $V$ e $\lambda_{0}$ é autovalor de $T\left(z_{0}\right)$ com multiplicidade algébrica $m$, então para $z$ próximo de $z_{0}, T(z)$ possui $m$ autovalores (contando a multiplicidade) próximos de $\lambda_{0}$. Estes autovalores são ramos de uma função holomorfa multivalorada numa vizinhança de $z_{0}$ e podem ser expressos como séries de Puiseux

$$
\lambda_{0}+\sum_{k=1}^{\infty} a_{k}\left(z-z_{0}\right)^{k / m},
$$

considerando as $m$ definições de $z^{1 / m}$. Como na subseção 1.2.3, é possível definir o projetor $P_{k}(z) e$ o nilpotente $D_{k}(z)$ associados ao autovalor $\lambda_{k}(z)$ de $T(z)$ e as fórmulas correspondentes, em especial 1.2.24, são válidas também nesta situação. $P_{k}(z), D_{k}(z)$ também são ramos de uma função multivalorada holomorfa numa vizinhança de $z_{0}$ e podem ser expressos como séries de Puiseux correspondentes.

A prova do teorema 1.2.67 pode ser encontrada em [55], Cap.II. Apesar do teorema 1.2.67 fornecer informação detalhada a respeito dos autovalores de uma família holomorfa de operadores, a situação ainda é bastante complicada se houver pontos de ramificação para os autovalores. Vamos nos restringir a um caso no qual isto não ocorre.

Seja $V$ um espaço com produto interno e $T(z), z \in \Omega$, uma família holomorfa de operadores em $V$. Suponhamos que $\Omega$ intersecta o eixo real, é simétrico com relação ao mesmo e além disso, $T(z)^{*}=T(\bar{z})$, se $z \in \Omega$. Em particular, $T(z)^{*}=T(z)$ para $z \in \Omega \cap \mathbb{R}$. Uma família com estas propriedades é dita simétrica. Para estas famílias, é válido o seguinte teorema, cuja prova também encontra-se em [55], Cap.II.

Teorema 1.2.68 Se T(z) é uma família holomorfa simétrica, então os autovalores $\lambda_{k}(z)$ e os projetores $P_{k}(z)$ são holomorfos no eixo real (i.e., não possuem ponto de ramificação no eixo real) $e$ os nilpotentes $D_{k}(z)$ são nulos. Em particular, $T(z)$ é diagonalizável.

OBservação 1.2.69 Se $T(z), z \in I \subset \mathbb{R}$, é analítica real e $T(z)$ é auto-adjunto, então estendendo $T$ a uma vizinhança complexa de $I$, concluímos que as funções $\lambda_{k}(z)$ e $P_{k}(z), z \in I \subset \mathbb{R}$, correspondentes são analíticas reais.

Nesta seção, procuraremos estender as construções anteriores e o teorema 1.2.68, tanto quanto possível, para o caso de operadores fechados. Primeiramente, é necessário estender o conceito de família holomofa de operadores.

DeFinição 1.2.70 Uma família holomorfa de operadores fechados é uma aplicação $T$ definida em um aberto $\Omega \subset \mathbb{C}$ a valores em $\mathscr{C}(\mathrm{H})$ tal que para cada $z_{0} \in \Omega$ e $\zeta_{0} \in \mathrm{P}\left(T\left(z_{0}\right)\right)$, o resolvente $\mathrm{R}(\zeta, z) \doteq \mathrm{R}(\zeta ; T(z))=(T(z)-\zeta)^{-1}$ é uma função holomorfa numa vizinhança de $\left(\zeta_{0}, z_{0}\right)$. 
OBSERvaçÃo 1.2.71 Se $T(z)$ é holomorfa então $T: \Omega \rightarrow \mathscr{C}(\mathrm{H})$ é contínua, pelo teorema 1.2.37.

Proposição 1.2.72 T(z) é uma família holomorfa se e só se existe um espaço de Hilbert $\mathrm{H}^{\prime}$ e famílias holomorfas (no sentido usual) de operadores limitados $U(z), V(z) \in \mathscr{L}\left(\mathrm{H}^{\prime}, \mathrm{H}\right)$ tais que $U(z)$ é bijeção entre $\mathrm{H}^{\prime}$ e $\mathrm{D}(T(z))$ e $T(z) U(z)=V(z)$, para $z \in \Omega$.<smiles>[14CH3][14CH2][14CH3]</smiles>

Prova. Se $T(z)$ é holomorfa no sentido da definição 1.2.70, basta tomar $\mathrm{H}^{\prime}=\mathrm{H}, U(z)=\mathrm{R}(z, \zeta)$ e $V(z)=I+\zeta U(z)$. Reciprocamente, se existem $\mathrm{H}^{\prime}, U(z)$ e $V(z)$ como no enunciado, então temos que $(T(z)-\zeta) U(z)=V(z)-\zeta U(z)$ e $V\left(z_{0}\right)-\zeta U\left(z_{0}\right)$ é bijeção contínua entre $\mathrm{H}^{\prime}$ e $\mathrm{H}$, se $\zeta \in$ $\mathrm{P}\left(T\left(z_{0}\right)\right)$. Pelo teorema do gráfico fechado, $\left(V\left(z_{0}\right)-\zeta U\left(z_{0}\right)\right)^{-1} \in \mathscr{L}\left(\mathrm{H}, \mathrm{H}^{\prime}\right), \operatorname{logo},(V(z)-\zeta U(z))^{-1}$ é holomorfa numa vizinhança de $z_{0} \in \Omega$. Disso, $\mathrm{R}(\zeta, z)=U(z)(V(z)-\zeta U(z))^{-1}$ é holomorfa numa vizinhança de $z_{0}$.

OBSERVAÇÃo 1.2.73 Se $T(z)$ é limitado e $T(z)$ é holomorfa no sentido usual, então decorre trivialmente da proposição 1.2.72 que $T(z)$ é holomorfa no sentido dado pela definição 1.2.70.

A caracterização equivalente fornecida pela proposição 1.2.72 será útil quando estudarmos famílias holomorfas de tipo (A).

Usando a proposição 1.2.38, a observação 1.2.71 e o fato que o conjunto dos operadores limitados inversíveis é aberto em $\mathscr{L}(\mathrm{H})$, concluímos diretamente o seguinte fato.

Proposição 1.2.74 Se $T(z), z \in \Omega$, é uma família holomorfa de operadores fechados, então o conjunto

$$
U=\{(\zeta, z) \in \mathbb{C} \times \Omega: \zeta \in \mathrm{P}(T(z))\}
$$

é aberto e $\mathrm{R}(\zeta, z) \doteq(T(z)-\zeta)^{-1}$ é holomorfa em $U$.

Se $T(z)$ é uma família holomorfa de operadores fechados, $z_{0} \in \Omega$ e $\Gamma$ é uma curva fechada que separa o espectro de $T\left(z_{0}\right)$ em duas partes $\Sigma^{\prime}\left(z_{0}\right)$ (interior a $\Gamma$ ) e $\Sigma^{\prime \prime}\left(z_{0}\right)$, como na subseção 1.2.3, definimos como antes o projetor

$$
P(z)=-\frac{1}{2 \pi i} \int_{\Gamma} R(\zeta ; T(z)) d \zeta
$$

para $z$ próximo de $z_{0}$. Como $T$ é contínua, pela proposição 1.2.38, segue que para $z$ suficientemente próximo de $z_{0}, \Gamma \subset \mathrm{P}(T(z))$, e portanto, $\Sigma(T(z))$ também é separado por $\Gamma$ em duas partes $\Sigma^{\prime}(z)$ interior a $\Gamma$ e $\Sigma^{\prime \prime}(z)$. A estas decomposições de $\Sigma(T(z))$ correspondem decomposições $\mathrm{H}=\mathrm{M}^{\prime}(z) \oplus \mathrm{M}^{\prime \prime}(z)$ de $\mathrm{H}$, onde $\mathrm{M}^{\prime}(z)=\operatorname{Im} P(z)$ e $\mathrm{M}^{\prime \prime}(z)=\operatorname{ker} P(z)$. Como $T(z)$ é holomorfa, segue que $P(z)$ é holomorfa, para $z$ próximo de $z_{0}$. Neste caso, (cf. [55], Pag. 99) é possível obter isomorfismos $U(z)$ tais que $U(z)\left(\mathrm{M}^{\prime}\left(z_{0}\right)\right)=\mathrm{M}^{\prime}(z)$, i.e., $P(z)=U(z) P\left(z_{0}\right) U(z)^{-1}$. Como $T(z)$ comuta com $P(z)$, segue que $T^{\grave{T}}(z) \doteq U(z)^{-1} T(z) U(z)$ comuta com $P\left(z_{0}\right)$. Em particular, $M^{\prime}\left(z_{0}\right)$ e $M^{\prime \prime}\left(z_{0}\right)$ são subespaços invariantes para $\check{T}(z)$, para $z$ próximo de $z_{0}$. No caso em que $\Sigma^{\prime}\left(z_{0}\right)$ é um sistema finito de autovalores de $T\left(z_{0}\right)$, o próximo teorema nos dá algo mais preciso a respeito da variação de $M^{\prime}\left(z_{0}\right)$. 
Teorema 1.2.75 Se $\Sigma^{\prime}\left(z_{0}\right)$ é um sistema finito de autovalores de $z_{0}$, então $\Sigma^{\prime}(z)$ é um sistema finito de autovalores de $T(z)$ consistindo de ramos de funções holomorfas com singularidades algébricas próximo de $z_{0} .^{15}$

Prova. Pelo teorema 1.2.52 e pela observação 1.2.71, segue que para $z$ suficientemente próximo de $z_{0}, \Sigma^{\prime}(z)$ é um sistema finito de autovalores de $T(z)$.

Temos que $\mathrm{M}^{\prime}\left(z_{0}\right)$ tem dimensão finita e é invariante por $\dddot{T}(z)$. Logo, pelo teorema 1.2.67, existem funções $\lambda_{k}(z)$, projetores $\breve{P}_{k}(z)$ e nilpotentes $\breve{D}_{k}(z)$, tais que

$$
\check{T}(z) \check{P}_{k}(z)=\lambda_{k}(z) \check{P}_{k}(z)+\check{D}_{k}(z), k=1, \ldots, n,
$$

e as funções $\lambda_{k}(z), \breve{P}_{k}(z), \breve{D}_{k}(z)$ são ramos de funções holomorfas multivaloradas com, no máximo, uma singularidade algébrica em $z=z_{0} . \breve{P}_{k}(z)$ e $\check{D}_{k}(z)$ são operadores agindo em $\mathrm{M}^{\prime}\left(z_{0}\right)$, portanto, $\breve{P}_{k}(z)=P\left(z_{0}\right) \breve{P}_{k}(z)=\breve{P}_{k}(z) P\left(z_{0}\right)$ e $\breve{D}_{k}(z)=P\left(z_{0}\right) \breve{D}_{k}(z)=\breve{D}_{k}(z) P\left(z_{0}\right)$, para $k=1, \ldots, n$. Definindo $P_{k}(z)=U(z) \breve{P}(z) U(z)^{-1}$ e $D_{k}(z)=U(z) \breve{D}(z) U(z)^{-1}$, temos

$$
T(z) P_{k}(z)=\lambda_{k}(z) P_{k}(z)+D_{k}(z), k=1, \ldots, n,
$$

como queríamos.

Com respeito à uma família holomorfa de operadores fechados arbitrários e um sistema finito de autovalores de $T\left(z_{0}\right)$, o teorema 1.2.75 é o melhor que se pode obter. Como no caso de dimensão finita, passemos agora ao caso de famílias holomorfas de operadores auto-adjuntos.

DeFinição 1.2.76 Uma família holomorfa de operadores auto-adjuntos é uma família holomorfa de operadores $T(z)$ definida em um domínio $\Omega \subset \mathbb{C}$ simétrico com respeito ao eixo real tal que $T(z)^{*}=T(\bar{z})$, para todo $z \in \Omega$. Em particular, $T(z)$ é auto-adjunto para $z \in \Omega \cap \mathbb{R}$.

Sejam $T(z)$ uma família holomorfa de operadores auto-adjuntos e $\left\{\lambda_{1}, \ldots, \lambda_{m}\right\}=\Sigma^{\prime}\left(z_{0}\right)$ um sistema finito de autovalores de $T\left(z_{0}\right)$. Suponhamos $z_{0}$ real (isso implica que $\Sigma^{\prime}\left(z_{0}\right) \subset$ $\mathbb{R})$, e tomemos $\Gamma$ uma curva fechada simples simétrica com respeito ao eixo real contendo $\left\{\lambda_{1}, \ldots, \lambda_{m}\right\}$ em seu interior. Então o teorema 1.2.75 implica que os autovalores $\lambda_{k}(z)$ de $T(z)$ contidos no interior de $\Gamma$, para $z$ próximo de $z_{0}$, são ramos de funções holomorfas multivaloradas com no máximo singularidades algébricas em $z_{0}$. A família correspondente de projetores definida em 1.2.35 verifica que $P(z)^{*}=P(\bar{z}), z \in \Omega$. De fato, esta relação é verdadeira para $z$ real, e por continuação analítica, segue que a mesma é válida para todo $z \in \Omega$. Os operadores $U(z)$ correspondentes (veja os comentários que antecedem o teorema 1.2.75) verificam $U(z)^{*}=U(\bar{z})$, em particular, $U(z)$ é unitário se $z$ é real, cf. [55], Cap.II-6.2. Isto implica que $\breve{T}(z)=U(z)^{-1} T(z) U(z)$ é um operador auto-adjunto em $\mathrm{H}$, para $z$ real. Já vimos que $\breve{T}(z)$ deixa $\mathrm{M}^{\prime}\left(z_{0}\right)$ invariante, e portanto, pelo teorema 1.2.68 aplicado à restrição de $\breve{T}(z)$ à $\mathrm{M}^{\prime}\left(z_{0}\right)$, concluímos o seguinte teorema.

Teorema 1.2.77 Se T(z) é uma família holomorfa de operadores auto-adjuntos e $\Sigma^{\prime}\left(z_{0}\right)$ é um sistema finito de autovalores de $T\left(z_{0}\right)$, com $z_{0}$ real, então os autovalores de $T(z)$ pertencentes a $\Sigma^{\prime}(z)$ dependem analiticamente de $z$ se $z \in \mathbb{R}$ é suficientemente próximo de $z_{0}$. O mesmo é verdadeiro para os projetores $P_{k}(z)$ e os nilpotentes $D_{k}(z)$ são nulos.

\footnotetext{
${ }^{15}$ Os projetores e os nilpotentes correspondentes também são representados por ramos de funções multivaloradas holomorfas numa vizinhança de $z_{0}$, possuindo no máximo uma singularidade algébrica em $z_{0}$.
} 
Segue do teorema 1.2.77 que os autovalores $\lambda_{k}(z)$ de $T(z)$ podem ser estendidos analiticamente a um intervalo $I_{0}$ contendo $z_{0}$. Este intervalo, em geral, pode ser menor do que a componente conexa de $\Omega \cap \mathbb{R}$ que contém $z_{0}$. Em [55], Pag.387-390, são exibidos exemplos deste fenômeno. Porém, para uma classe ampla de famílias holomorfas, este fenômeno não ocorre, e é sobre estas famílias que falaremos daqui por diante.

Definição 1.2.78 Uma família $T: \Omega \rightarrow \mathscr{C}(\mathrm{H})$ é holomorfa de tipo $(A)$ se $\mathrm{D}=\mathrm{D}(T(z))$ é constante para $z \in \Omega$ e para cada $u \in \mathrm{H}$, a aplicação $z \in \Omega \mapsto T(z) u \in \mathrm{H}$ é holomorfa. A definição faz sentido também se $\Omega \subset \mathbb{R}$; neste caso, entendemos holomorfa como analítica real.

Lema 1.2.79 Toda família holomorfa de tipo (A) é holomorfa no sentido da definição 1.2.70.

Prova. Fixemos $z_{0} \in \Omega$. Como $T\left(z_{0}\right)$ é fechado, então $D\left(T\left(z_{0}\right)\right)$ é um espaço de Hilbert munido do produto interno $\langle u, v\rangle^{\prime} \doteq\langle u, v\rangle+\left\langle T\left(z_{0}\right) u, T\left(z_{0}\right) v\right\rangle$. Denotemos este espaço por $\mathrm{H}^{\prime}$ e definamos $U=I: \mathrm{H}^{\prime} \rightarrow \mathrm{H}$. Obviamente, $U$ é limitado. Definimos $V(z)=T(z): \mathrm{H}^{\prime} \rightarrow \mathrm{H}$; pelo teorema do gráfico fechado, $V(z)$ é limitado, para todo $z \in \Omega$. Como $V(z) u=T(z) u$ é holomorfa para cada $u \in \mathrm{H}$, segue, pelo princípio da limitação uniforme, que $V(z)$ é uma família holomorfa de operadores limitados, conforme [55], Theorem III-3.12. Pela proposição 1.2.72, temos que $T(z)$ é holomorfa, pois $T(z) U=V(z)$, para todo $z \in \Omega$.

Usando a mesma notação da prova do lema 1.2.79, definimos $T^{\prime}(z) u=\frac{d}{d z} T(z) u$. Vemos que $T^{\prime}(z) u=V^{\prime}(z) u$, onde $V^{\prime}(z)$ é interpretado no sentido usual. Fixado $z_{0} \in K \subset \Omega$ compacto, como $V(z)$ é holomorfa, segue que

$$
M=\sup _{\substack{z, w \in K \\ u \neq 0}} \frac{\|u\|+\|V(z) u\|}{\|u\|+\|V(w) u\|}<\infty .
$$

Como $V^{\prime}(z)$ é limitado, por 1.2 .36 existe $C>0$ tal que $\left\|V^{\prime}(z) u\right\| /(\|u\|+\|T(z) u\|) \leq C$, e portanto, existem $a, b \geq 0$ tais que

$$
\left\|T^{\prime}(z) u\right\| \leq a\|u\|+b\|T(z) u\|,
$$

para todo $z \in K$ e $u \in D$, i.e., $T^{\prime}$ é $T$-limitado. Assim, está provado o seguinte fato.

Proposição 1.2.80 Se $T(z)$ é uma família holomorfa de operadores de tipo $(A)$ e $K \subset \Omega$ é compacto, então existem $a, b \geq 0$ tais que 1.2.37 é válida para todo $z \in K e u \in \mathrm{D}$. Em outras palavras, $T^{\prime}(z)$ é uniformemente $T(z)$-limitado sobre cada compacto de $\Omega$.

Vamos agora estimar o crescimento dos autovalores de uma família holomorfa de operadores auto-adjuntos de tipo (A).

Proposição 1.2.81 Seja T(z) uma família holomorfa de operadores auto-adjuntos de tipo (A) e consideremos $z_{0} \in \Omega \cap \mathbb{R}$. Se $I \subset \mathbb{R}$ é um intervalo compacto contendo $z_{0}$ e $\lambda: I \subset \mathbb{R} \rightarrow \mathbb{R}$ representa um autovalor de $T(z), z \in \mathbb{R}$, obtido por perturbação do autovalor $\lambda\left(z_{0}\right)$ de $T\left(z_{0}\right)$ (cf. teorema 1.2.77), então

$$
\left|\lambda(z)-\lambda\left(z_{0}\right)\right| \leq \frac{a+b\left|\lambda\left(z_{0}\right)\right|}{b}\left(e^{b|z|}-1\right),
$$

onde $a, b$ são os mesmos obtidos na equação 1.2 .37 , com $K=I$. 
Prova. Usando os operadores $U(z)$ que antecedem a prova do teorema 1.2.77, podemos obter $u: I \rightarrow \mathrm{H}$, com $\|u(z)\|=1$, tal que

$$
(T(z)-\lambda(z)) u(z)=0,
$$

para todo $z \in I$. De fato, consideramos $\Sigma^{\prime}\left(z_{0}\right)=\left\{\lambda_{0}\right\}$ e definimos $u(z)=U(z) v$, onde $v \in$ $\operatorname{ker}\left(T\left(z_{0}\right)-\lambda\left(z_{0}\right)\right)$ é unitário.

Derivando a equação 1.2 .39 e tomando o produto interno com $u(z)$ temos

$$
\begin{aligned}
0 & =\left\langle\left(T^{\prime}(z)-\lambda^{\prime}(z)\right) u(z), u(z)\right\rangle+\left\langle(T(z)-\lambda(z)) u^{\prime}(z), u(z)\right\rangle \\
& =\left\langle T^{\prime}(z) u(z), u(z)\right\rangle-\lambda^{\prime}(z)+\left\langle u^{\prime}(z),(T(z)-\lambda(z)) u(z)\right\rangle \\
& =\left\langle T^{\prime}(z) u(z), u(z)\right\rangle-\lambda^{\prime}(z)
\end{aligned}
$$

portanto, por 1.2.37,

$$
\left|\lambda^{\prime}(z)\right| \leq\left\|T^{\prime}(z) u(z)\right\| \leq a+b\|T(z) u(z)\|=a+b|\lambda(z)| .
$$

A desigualdade 1.2.38 segue de 1.2 .40 e da desigualdade de Gronwall.

A proposição 1.2.81 é o passo crucial para concluirmos o teorema principal a respeito da continuação analítica, ao longo do eixo real, dos autovalores de uma família holomorfa de tipo (A) de operadores auto-adjuntos com resolvente compacto.

Definição 1.2.82 Um operador fechado $T$ tem resolvente compacto se existe $\zeta \in \mathrm{P}(T)$ tal que $\mathrm{R}(\zeta ; T)$ é compacto.

Segue da identidade do resolvente 1.1.1 que se $T$ tem resolvente compacto então $R(\zeta ; T)$ é compacto para todo $\zeta \in \mathrm{P}(T)$.

Teorema 1.2.83 Seja $T(z)$ uma família holomorfa de tipo $(A)$ de operadores auto-adjuntos definida para z em uma vizinhança de um intervalo $I \subset \mathbb{R}$ limitado. Suponhamos ainda que $T(z)$ tem resolvente compacto para algum e portanto, para todo $z$. Então, os autovalores de $T(z)$ podem ser representados por funções $\lambda_{n}(z)$ analíticas reais definidas em todo o intervalo $I$. Além disso, existem funções analíticas reais $u_{n}: I \rightarrow \mathrm{H}$ tais que $\left\|u_{n}(z)\right\|=1$, cada $u_{n}(z)$ é um autovetor correspondente ao autovalor $\lambda_{n}(z)$ e 0 conjunto $\left\{u_{n}(z)\right\}_{n=1}^{\infty}$ é uma base ortonormal de $\mathrm{H}$, para todo $z \in I$.

Prova. O passo crucial do teorema é verificar que os autovalores podem ser continuados analiticamente a todo o intervalo $I$. De fato, seja $\lambda(z)$ autovalor de $T(z)$ definido em um intervalo $(a, b) \subset I$, e suponhamos que $\lambda$ não pode ser continuada analiticamente a nenhum intervalo contendo propriamente $(a, b)$. Vamos supor que $(a, b) \neq I$ e chegar a uma contradição. Se $a$ é interior a $I$, vemos, pela proposição 1.2 .81 que $\lambda(z)$ é limitada, digamos $|\lambda(z)|<M$ para $z \downarrow a$. Como $T(z)$ tem resolvente compacto, segue que a parte do espectro de $T(a)$ contida em $(-M, M)$ é formada por um conjunto finito de autovalores $\mu_{1}, \ldots, \mu_{n}$ de $T(a)$. O teorema 1.2.51 e a observação 1.2.71 nos permitem concluir que, para $z$ suficientemente próximo de $a$, a parte do espectro de $T(z)$ contida em $(-M, M)$ pertence à uma pequena vizinhança dos pontos $\mu_{1}, \ldots, \mu_{n}$. Isso implica que $\lambda(z)$ converge para um autovalor de $T(a)$ quando $z \rightarrow a$ contrariando a maximalidade de $(a, b)$, pelo teorema 1.2.77.

A construção dos $u_{n}(z)$ é feita como segue. Consideramos $z_{0} \in I$ e uma base ortonormal $\left\{u_{n}\left(z_{0}\right)\right\}_{n=1}^{\infty}$ de $\mathrm{H}$ formada por autovetores correspondentes aos autovalores $\lambda_{n}\left(z_{0}\right)$. (Isto é 
possível porque $T\left(z_{0}\right)$ tem resolvente compacto). Para cada $u_{n}\left(z_{0}\right)$, consideramos $u_{n}(z)=$ $U(z) u_{n}\left(z_{0}\right)$, onde $U(z)$ é definida como nos comentários que antecedem a prova do teorema 1.2.77. Como os $U(z)$ são unitários, segue que $\left\{u_{n}(z)\right\}_{n=1}^{\infty}$ é de fato uma base ortonormal de $\mathrm{H}$ formada por autovetores correspondentes aos autovalores $\lambda_{n}(z)$.

ObSERVAção 1.2.84 Todos os resultados desta subseção valem para espaços reais, desde que nos restrinjamos a operadores auto-adjuntos.

\subsection{Teoria de Fredholm}

Nesta seção, trataremos de propriedades gerais de pares Fredholm de subespaços de $\mathrm{H}$ e também de operadores de Fredholm. Nosso objetivo principal é provar a estabilidade de pares Fredholm de subespaços e de operadores de Fredholm por pequenas perturbações. Discutiremos primeiramente o caso de operadores Fredholm limitados, passaremos aos pares Fredholm de subespaços e usaremos os resultados obtidos para estudar o caso de operadores Fredholm ilimitados. A seção encerra como uma breve discussão sobre a teoria de pares comensuráveis e perturbações compactas de subespaços.

\subsubsection{Operadores Fredholm}

Inicialmente, trataremos de propriedades gerais de operadores Fredholm, não necessariamente limitados. Quando tratarmos de operadores Fredholm ilimitados, veremos que a maioria das afirmações admite extensão à esta situação.

O próximo lema reflete uma propriedade interessante possuída pela imagem de um operador fechado com deficiência finita.

Lema 1.3.1 Se $T: \mathrm{D}(T) \subset \mathrm{H} \rightarrow \mathrm{H}^{\prime}$ é um operador fechado com $\operatorname{def} T<\infty$, então $\operatorname{Im} T$ é fechada.

Prova. Seja $M$ de dimensão finita $n$ tal que $\operatorname{Im} T \oplus M=H$. Consideremos o operador

$$
\begin{aligned}
S: \mathrm{D}(S) \subset(\mathrm{H} / \operatorname{ker} T) \oplus \mathbb{R}^{n} & \rightarrow \operatorname{Im} T \oplus \mathrm{M}=\mathrm{H}^{\prime} \\
(u+\operatorname{ker} T, x) & \mapsto \mathrm{T} u+A x,
\end{aligned}
$$

onde $A: \mathbb{R}^{n} \rightarrow \mathrm{M}$ é qualquer isomorfismo, $(\mathrm{H} / \operatorname{ker} T) \oplus \mathbb{R}^{n}$ é munido da norma da soma e $\mathrm{D}(S)=\mathrm{D}(\tilde{T}) \oplus \mathbb{R}^{n}$. Temos que $S^{-1}$ é um operador fechado definido em $\mathrm{H}^{\prime}$, portanto, pelo teorema do gráfico fechado, $S^{-1}$ é limitado. Logo, existe $C>0$ tal que

$$
\|u+\operatorname{ker} T\|+|x| \leq C(\|T u\|+\|A x\|)
$$

para todo $u \in \mathrm{D}(T)$ e $x \in \mathbb{R}^{n}$. Em particular, $\|u+\operatorname{ker} T\| \leq C\|T u\|$, para todo $u \in \mathrm{H}$, e portanto, $\gamma(T) \geq C^{-1}>0$. Pela proposição 1.2.14, segue que $\operatorname{Im} T$ é fechada. ${ }^{16}$

Os próximos resultados são úteis para entender a estrutura de um operador (semi) Fredholm.

\footnotetext{
${ }^{16}$ Deste argumento decorre um fato curioso: nem todo subespaço de codimensão finita é imagem de algum operador fechado definido em um espaço de Banach.
} 
Proposição 1.3.2 Seja $T \in \mathscr{C}\left(\mathrm{H}, \mathrm{H}^{\prime}\right)$ tal que $\operatorname{Im} T$ é fechada. Dados subespaços fechados $\mathrm{M} \subset \mathrm{H}$, $\mathrm{N} \subset \mathrm{H}^{\prime}$ tais que $\mathrm{M} \oplus \operatorname{ker} T=\mathrm{H} e \mathrm{~N} \oplus \operatorname{Im} T=\mathrm{H}^{\prime}$, existe $S \in \mathscr{L}\left(\mathrm{H}^{\prime}, \mathrm{H}\right)$ tal que $\operatorname{Im} S \subset \mathrm{D}(T)$, $S T \subset I-P_{\mathrm{M}, \operatorname{ker} T}$ e TS $=I-P_{\operatorname{Im} T, \mathrm{~N}}$.

Prova. Consideremos $\hat{T}$ a restrição de $T$ a $D(T) \cap M$. Vemos que $\hat{T}$ é um operador fechado bijetor com imagem fechada $(\operatorname{Im} \hat{T}=\operatorname{Im} T)$, portanto, pelo teorema do gráfico fechado segue que $\hat{T}^{-1}: \operatorname{Im} T \rightarrow \mathrm{H}$ é limitado.

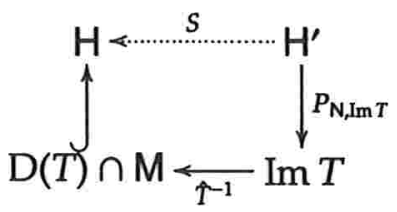

Definindo $S=\hat{T}^{-1} \circ P_{\mathrm{N}, \operatorname{Im} T} \in \mathscr{L}\left(\mathrm{H}^{\prime}, \mathrm{H}\right)$, temos que $\operatorname{Im} S=\mathrm{D}(T) \cap \mathrm{M} \subset \mathrm{D}(T), S T \subset I-P_{\mathrm{M}, \operatorname{ker} T}$ e $T S=I-P_{\operatorname{Im} T, N}$, provando o desejado.

Proposição 1.3.3 Se $T \in \mathscr{C}\left(\mathrm{H}, \mathrm{H}^{\prime}\right)$ é Fredholm então existem operadores $K \in \mathscr{L}(\mathrm{H}), L \in \mathscr{L}\left(\mathrm{H}^{\prime}\right)$ e $S \in \mathscr{L}\left(\mathrm{H}^{\prime}, \mathrm{H}\right)$ tais que

1. K, L têm posto finito;

2. $\operatorname{Im} S \subset \mathrm{D}(T)$;

3. $T S=I+L$ e $S T \subset I+K$.

Reciprocamente, se $\mathrm{T}: \mathrm{D}(T) \subset \mathrm{H} \rightarrow \mathrm{H}^{\prime}$ é um operador linear e existem $K, L, S$ como acima, então $T \in \mathscr{C}\left(\mathrm{H}, \mathrm{H}^{\prime}\right)$ é Fredholm.

Prova. Se $T$ é Fredholm, a existência de $K, L, S$ é garantida pela proposição 1.3.2. Reciprocamente, se existem $K, L, S$ satisfazendo os ítens $1,2,3$ acima, então $\operatorname{ker} T \subset \operatorname{ker}(I+K)$ e $\operatorname{Im} T \supset$ $\operatorname{Im}(I+L)$. Pelo o lema 1.3.4 a seguir, temos que nul $T<\infty$ e $\operatorname{Im} T$ é fechada de codimensão finita. Para provar que $T$ é fechado, basta observar que, pondo $\mathrm{M} \doteq \mathrm{D}(T) \cap(\operatorname{ker} K \cap S(\operatorname{ker} L))$ e $\mathrm{N} \doteq \operatorname{ker} L \cap T(\operatorname{ker} K \cap \mathrm{D}(T))$, temos $T(\mathrm{M}) \subset \mathrm{N}, S(\mathrm{~N}) \subset \mathrm{M}, S T=I$ em $\mathrm{M}$ e $T S=I$ em N. Como $S$ é limitado, temos que $N$ é fechado e $\left.T\right|_{M}: M \rightarrow N$ é fechado. Como $\operatorname{codim} M, \operatorname{codim} N<\infty$, segue que $T$ é fechado.

Lema 1.3.4 (Alternativa de Fredholm) Se $K \in \mathscr{L}(\mathrm{H})$ é compacto, então $K-\lambda$ é Fredholm $e$ ind $(K-\lambda)=0$, para todo $\lambda \neq 0$.

Prova. Pondo $\mathrm{N}=\operatorname{ker}(K-\lambda)$, temos que $\lambda=\left.K\right|_{\mathrm{N}}: \mathrm{N} \rightarrow \mathrm{N}$ é um operador compacto, portanto, $\operatorname{dim} N<\infty$. Provemos que $K-\lambda$ é um operador de Fredholm com índice zero. Isso é trivial se $\lambda \in \mathrm{P}(K)$, portanto, podemos supor que $\lambda \in \Sigma(K)$. Como $\lambda \neq 0$ e $K$ é compacto, então $\lambda$ é um autovalor isolado de $K$. Denotando por $P$ a projeção sobre $M^{\prime}=\operatorname{Im} P$ correspondente ao autovalor $\lambda$ e tendo em vista os resultados da subseção 1.2.3, em especial a proposição 1.2.48 e os comentários que a sucedem, temos que $\mathrm{M}^{\prime}$ é invariante por $K$, e portanto, $\operatorname{dim} \mathrm{M}^{\prime}<\infty$. Pondo $\mathrm{M}^{\prime \prime}=\operatorname{ker}(I-P), K^{\prime}=\left.K\right|_{M^{\prime}}$ e $K^{\prime \prime}=\left.K\right|_{M^{\prime \prime}}$, temos que $\lambda \notin \Sigma\left(K^{\prime \prime}\right)$, (pela proposição 1.2.48), portanto, $K^{\prime \prime}-\lambda$ é um isomorfismo de $\mathrm{M}^{\prime \prime}$. Disso,

$$
\operatorname{Im}(K-\lambda)=\operatorname{Im}\left(K^{\prime}-\lambda\right)+\operatorname{Im}\left(K^{\prime \prime}-\lambda\right)=\operatorname{Im}\left(K^{\prime}-\lambda\right)+M^{\prime \prime} .
$$


Em particular, $\operatorname{Im}(K-\lambda)$ é fechada, pois $\operatorname{dim} \operatorname{Im}\left(K^{\prime}-\lambda\right) \leq \operatorname{dim} \mathrm{M}^{\prime}<\infty$. Além disso, as decomposições 1.3.2 e $\mathrm{H}=\mathrm{M}^{\prime}+\mathrm{M}^{\prime \prime}$ provam que $\mathrm{H} / \operatorname{Im}(K-\lambda) \approx \mathrm{M}^{\prime} / \operatorname{Im}\left(K^{\prime}-\lambda\right)$. Portanto, como $\operatorname{dim} \mathrm{M}^{\prime}<\infty$, temos que

$$
\operatorname{def}(K-\lambda)=\operatorname{dim} M^{\prime}-\operatorname{dim} \operatorname{Im}\left(K^{\prime}-\lambda\right)=\operatorname{nul}\left(K^{\prime}-\lambda\right)=\operatorname{nul}(K-\lambda),
$$

de onde concluímos que ind $(K-\lambda)=0$.

ObSERvaÇão 1.3.5 Dado um operador de Fredholm $T \in \mathscr{C}\left(\mathrm{H}, \mathrm{H}^{\prime}\right)$, construindo $S$ como no diagrama 1.3.1, $\operatorname{com} \mathrm{M}=(\operatorname{ker} T)^{\perp} \mathrm{eN}=(\operatorname{Im} T)^{\perp}$, vemos que $\|S\|=\gamma(T)^{-1}$. De fato, obviamente, $\|S\| \leq\left\|\hat{T}^{-1}\right\|=\gamma(T)^{-1}$. Tomando $v=T u$ com $u \in(\operatorname{ker} T)^{\perp}$, temos $\|S v\|=\left\|\hat{T}^{-1} v\right\| ;$ isso implica que $\|S\|=\gamma(T)^{-1}$, pela observação 1.2.13.

OBSERVAÇão 1.3.6 Observando a prova da proposição 1.3.3, vemos que dado $T \in \mathscr{C}\left(\mathrm{H}, \mathrm{H}^{\prime}\right)$ (semi) Fredholm, o operador $S \in \mathscr{L}\left(\mathrm{H}^{\prime}, \mathrm{H}\right)$ construído é (semi) Fredholm e ind $S=-$ ind $T$.

OBSERVAÇÃo 1.3.7 $\mathrm{Na}$ ítem 1 da proposição 1.3.3, podemos trocar a expressão têm posto finito por são compactos e a referida proposição continua válida. De fato, se $T S=I+K$, com $K$ compacto, podemos obter $L$ de posto finito tal que $\|K-L\|<1$. Portanto, existe $(I+(K-L))^{-1}$ (usando a série de Neumann 1.2.10), e temos $T\left(S(I+(K-L))^{-1}\right)=I+L(I+(K-L))^{-1}$ com $L(I+(K-L))^{-1}$ de posto finito. O mesmo é válido para o produto $S T$.

Uma propriedade importante dos operadores Fredholm é a aditividade do índice por composição. Esta propriedade é verificada para operadores Fredholm ilimitados em geral, mas por enquanto, nos contentaremos com o caso limitado.

Teorema 1.3.8 Se $T_{1}: \mathrm{H}_{1} \rightarrow \mathrm{H}_{2}$ e $T_{2}: \mathrm{H}_{2} \rightarrow \mathrm{H}_{3}$ são operadores Fredholm limitados, então $T_{2} T_{1}$ é Fredholme

$$
\text { ind }\left(T_{2} T_{1}\right)=\operatorname{ind} T_{2}+\operatorname{ind} T_{1} \text {. }
$$

Prova. Suponhamos primeiramente que $T_{1}$ seja sobrejetor. Isso implica que

$$
\begin{aligned}
\operatorname{ind}\left(T_{2} T_{1}\right) & =\operatorname{nul}\left(T_{2} T_{1}\right)-\operatorname{def}\left(T_{2} T_{1}\right) \\
& =\operatorname{dim} T_{1}^{-1}\left(\operatorname{ker} T_{2}\right)-\operatorname{def} T_{2} \\
& =\operatorname{nul} T_{1}+\operatorname{nul} T_{2}-\operatorname{def} T_{2}=\operatorname{ind} T_{2}+\operatorname{ind} T_{1} .
\end{aligned}
$$

Caso $T_{1}$ não seja sobrejetor, consideremos $P_{\operatorname{Im} T_{1}}: \mathrm{H}_{2} \rightarrow \operatorname{Im} T_{1}, T_{1}^{\prime}=P_{\operatorname{Im} T_{1}} T_{1}: \mathrm{H}_{1} \rightarrow \operatorname{Im} T_{1}$ e $T_{2}^{\prime}=T_{2} \operatorname{Im} T_{1}$.

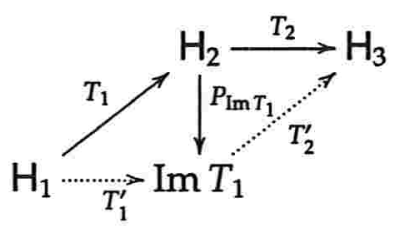

A seqüência

$$
0 \longrightarrow \operatorname{ker} T_{2} \cap \operatorname{Im} T_{1} \longrightarrow \operatorname{ker} T_{2} \stackrel{\pi}{\longrightarrow} \mathrm{H}_{2} / \operatorname{Im} T_{1} \stackrel{T_{2}}{\longrightarrow} \mathrm{H}_{3} / T_{2}\left(\operatorname{Im} T_{1}\right) \stackrel{p}{\longrightarrow} \mathrm{H}_{3} / \operatorname{Im} T_{2} \longrightarrow 0 ，
$$

é exata, onde $\pi$ denota a restrição a ker $T_{2}$ da projeção canônica $H_{2} \rightarrow H_{2} / \operatorname{Im} T_{1}, \bar{T}_{2}$ : $\mathrm{H}_{2} / \operatorname{Im} T_{1} \rightarrow \mathrm{H}_{3} / T\left(\operatorname{Im} T_{1}\right)$ é a aplicação induzida por $T$ e $p: \mathrm{H}_{3} / T\left(\operatorname{Im} T_{1}\right) \rightarrow \mathrm{H}_{3} / \operatorname{Im} T_{2}$ é a 
aplicação induzida pela inclusão $T_{2}\left(\operatorname{Im} T_{1}\right) \subset \operatorname{Im} T_{2}$. Em particular, $T_{1}^{\prime}, T_{2}^{\prime}$ são operadores Fredholm e vale a fórmula

$$
\operatorname{dim}\left(\operatorname{ker} T_{2} \cap \operatorname{Im} T_{1}\right)-\operatorname{nul} T_{2}+\operatorname{def} T_{1}-\operatorname{codim} T_{2}\left(\operatorname{Im} T_{1}\right)+\operatorname{def} T_{2}=0 .
$$

Como $T_{2} T_{1}=T_{2}^{\prime} T_{1}^{\prime}$ e $T_{1}^{\prime}$ é sobre,

$$
\begin{aligned}
\operatorname{ind}\left(T_{2} T_{1}\right)=\operatorname{ind}\left(T_{2}^{\prime} T_{1}^{\prime}\right) & =\operatorname{ind} T_{2}^{\prime}+\operatorname{ind} T_{1}^{\prime} \\
& =\left(\operatorname{dim}\left(\operatorname{ker} T_{2} \cap \operatorname{Im} T_{1}\right)-\operatorname{codim} T_{2}\left(\operatorname{Im} T_{1}\right)\right)+\left(\operatorname{ind} T_{1}+\operatorname{def} T_{1}\right) \\
& =\operatorname{nul} T_{2}-\operatorname{def} T_{2}+\operatorname{ind} T_{1}=\operatorname{ind} T_{2}+\operatorname{ind} T_{1} .
\end{aligned}
$$

Veremos agora que os operadores de Fredholm formam um conjunto aberto em $\mathscr{L}\left(\mathrm{H}, \mathrm{H}^{\prime}\right)$ e o índice é uma função localmente constante.

Teorema 1.3.9 Se $T \in \mathscr{C}\left(\mathrm{H}, \mathrm{H}^{\prime}\right)$ é Fredholm e $A \in \mathscr{L}\left(\mathrm{H}, \mathrm{H}^{\prime}\right)$ é tal que $\|A\|<\gamma(T)$, então $T+A$ é Fredholm $e$ ind $(T+A)=$ ind $T$.

Prova. Seja $T$ Fredholm e, de acordo com a proposição 1.3.3, tomemos $S \in \mathscr{L}\left(\mathrm{H}^{\prime} \mathrm{H}^{\prime}\right)$ tal que $S T=I+K$ e $T S=I+L$, com $K, L$ de posto finito. Portanto, $(T+A) S=(I+A S)+L$ e $S(T+A)=(I+S A)+K$. Se $\|A\|<\gamma(T)=\|S\|^{-1}$, (veja observação 1.3.5), usando a série de Neumann, concluímos que $(I+S A)^{-1}$ e $(I+A S)^{-1}$ são isomorfismos limitados de $\mathrm{H} \mathrm{e} \mathrm{H}^{\prime}$, respectivamente. Logo,

$$
\begin{aligned}
& (T+A)\left\{S(I+A S)^{-1}\right\}=I+L(I+A S)^{-1}, \\
& \left\{(I+S A)^{-1} S\right\}(T+A)=I+(I+S A)^{-1} K .
\end{aligned}
$$

Como $(I+S A)^{-1} S=S(I+A S)^{-1}$, pelas igualdades 1.3 .3 e 1.3 .4 e pela proposição 1.3 .3 temos que $T+A$ é Fredholm. Finalmente, pela observação 1.3.6, ind $T=-$ ind $S=-$ ind $S(I+A S)^{-1}=$ ind $(T+A)$, pois $(I+A S)^{-1}$ é um isomorfismo.

A proposição abaixo decorre diretamente do teorema 1.3.9.

Proposição 1.3.10 $\mathscr{F}\left(\mathrm{H}, \mathrm{H}^{\prime}\right)$ é um aberto de $\mathscr{L}\left(\mathrm{H}, \mathrm{H}^{\prime}\right) e$ ind : $\mathscr{F}\left(\mathrm{H}, \mathrm{H}^{\prime}\right) \rightarrow \mathbb{Z}$ é uma função localmente constante.

Vamos agora detalhar as informações sobre o comportamento da nulidade e da deficiência de um operador fechado quando sujeito a uma perturbação limitada.

Proposição 1.3.11 Se $T \in \mathscr{C}\left(\mathrm{H}, \mathrm{H}^{\prime}\right)$ é um operador (semi) Fredholm e $A \in \mathscr{L}\left(\mathrm{H}, \mathrm{H}^{\prime}\right)$ é tal que $\|A\|<\gamma(T)$, então $\operatorname{nul}(T+A) \leq \operatorname{nul} T e \operatorname{def}(T+A) \leq \operatorname{def} T$. Em particular, as funções nul, def : $\mathscr{F}\left(\mathrm{H}, \mathrm{H}^{\prime}\right) \rightarrow \mathbb{N}$ são semi-contínuas superiormente.

Prova. Seja $T$ semi-Fredholm e $S$ como na proposição 1.3 .3 , com $M=(\operatorname{ker} T)^{\perp}$ e $N=$ $(\operatorname{Im} T)^{\perp}$. Podemos supor que, de fato, $T$ é Fredholm, i.e., nul $T$ e $\operatorname{def} T$ são finitas. Temos que $S(T+A)=I+S A$ em $(\operatorname{ker} T)^{\perp}$. Como $\|A\|<\gamma(T)=\|S\|^{-1}$, então $I+S A$ é um isomorfismo, portanto, $\operatorname{ker}(T+A) \cap(\operatorname{ker} T)^{\perp}=0$. Isso implica que nul $(T+A) \leq \operatorname{nul} T$; basta observar que $P_{\operatorname{ker} T}^{\operatorname{ker} T+A)}: \operatorname{ker}(T+A) \rightarrow \operatorname{ker} T$ é um operador injetor. Provemos agora que $\operatorname{def}(T+A) \leq \operatorname{def} T$. De fato, temos que $(T+A) S=I+A S \operatorname{em} \operatorname{Im} T$. Como $\|A\|<\gamma(T)=\|S\|^{-1}$, então $I+A S$ é um isomorfismo. Isso implica que $\operatorname{Im}(T+A)$ contém um subespaço isomorfo a $\operatorname{Im} T$, e a conclusão segue. 
Proposição 1.3.12 Se $T \in \mathscr{C}\left(\mathrm{H}, \mathrm{H}^{\prime}\right)$ é (semi) Fredholm e $A \in \mathscr{L}\left(\mathrm{H}, \mathrm{H}^{\prime}\right)$ é tal que $\|A\|<\gamma(T)$, então Im $(T+A)$ é fechada. Em particular, $T+A$ é (semi) Fredholm.

Prova. Se def $T<\infty$, então pela proposição 1.3.11, temos $\operatorname{def}(T+A)<\infty$, e pelo lema 1.3.1 segue que $\operatorname{Im}(T+A)$ é fechada.

Suponhamos agora nul $T<\infty$ e $\operatorname{Im} T$ fechada. A proposição 1.3.11 e sua prova mostram que $\operatorname{nul}(T+A)<\infty$ e $\operatorname{ker}(T+A) \cap(\operatorname{ker} T)^{\perp}=0$. Já que $\operatorname{ker}(T+A) \oplus(\operatorname{ker} T)^{\perp}$ tem codimensão finita $(\leq \operatorname{nul} T)$, seja $M$ um subespaço de dimensão finita tal que $\operatorname{ker}(T+A) \oplus(\operatorname{ker} T)^{\perp} \oplus \mathrm{M}=\mathrm{H}$. Como

$$
\operatorname{Im}(T+A)=(T+A)\left((\operatorname{ker} T)^{\perp}\right)+(T+A)(\mathrm{M})
$$

e $M$ tem dimensão finita, para provar que $\operatorname{Im}(T+A)$ é fechada, basta provar que a restrição de $T+A$ a $(\operatorname{ker} T)^{\perp}$ tem imagem fechada. Como

$$
\|(T+A) u\| \geq\|T u\|-\|A u\| \geq(\gamma(T)-\|A\|)\|u\|,
$$

para todo $u \in(\operatorname{ker} T)^{\perp}$, o resultado decorre do lema 1.2.11.

ObSERVAÇão 1.3.13 As proposições 1.3.11 e 1.3.12 mostram que o conjunto dos operadores semi-Fredholm limitados é aberto em $\mathscr{L}\left(\mathrm{H}, \mathrm{H}^{\prime}\right)$ e nul, def são funções semi-contínuas superiormente com valores em $\mathbb{N} \cup\{ \pm \infty\}$.

O lema 1.3.12 contém uma afirmação que será útil no estudo de pares Fredholm de subespaços, a qual enunciamos a seguir.

Corolário 1.3.14 Se $T \in \mathscr{C}\left(\mathrm{H}, \mathrm{H}^{\prime}\right)$ e $A \in \mathscr{L}\left(\mathrm{H}, \mathrm{H}^{\prime}\right)$ são tais que nul $T=0$ e $\|A\|<\gamma(T)$, então $\gamma(T+A) \geq \gamma(T)-\|A\|$.

Prova. A proposição 1.3.11 implica que nul( $T+A) \leq \operatorname{nul} T=0, \operatorname{logo}, T+A$ é injetor. Em particular, a desigualdade 1.3 .5 vale para todo $u \in \mathrm{H}$, provando o que desejamos.

OBSERvaçÃo 1.3.15 Para T, $A$ como na proposição 1.3.12, em geral não é possível encontrar uma cota inferior para $\gamma(T+A)$ em termos de $\gamma(T)$ e $\|A\|$, como no corolário 1.3.14. Em outras palavras, $\gamma(\cdot)$ não é semi-contínua inferiormente. Ainda assim, em certos casos é possível contornar este defeito da função $\gamma$. T. Kato em [55], Pag.228, comenta sobre a impossibilidade, em geral, de encontrar limitações inferiores para $\gamma$.

\subsubsection{Pares Fredholm de subespaços}

Nesta subseção desenvolveremos a teoria de pares Fredholm de subespaços. Este tipo de par de subespaços será de fundamental importância quando estudarmos problemas de fronteira.

Definição 1.3.16 Dados subespaços fechados $\mathrm{M}, \mathrm{N}$ de $\mathrm{H}$, dizemos que $(\mathrm{M}, \mathrm{N})$ é um par Fredholm se $\operatorname{nul}(M, N)<\infty$ e $\operatorname{def}(M, N)<\infty$. O par $M, N$ é dito semi-Fredholm se $M+N$ é fechado e $\operatorname{nul}(\mathrm{M}, \mathrm{N})<\infty$ ou $\operatorname{def}(\mathrm{M}, \mathrm{N})<\infty$. O índice de Fredholm do par $(\mathrm{M}, \mathrm{N})$ é definido como

$$
\text { ind }(M, N)=\operatorname{nul}(M, N)-\operatorname{def}(M, N) \text {. }
$$


Em geral, exige-se na definição de par Fredholm que $\mathrm{M}+\mathrm{N}$ seja fechado. Veremos no lema 1.3.17 que esta condição é automaticamente satisfeita se $\operatorname{def}(M, N)<\infty$.

LEMA 1.3.17 São verdadeiras as seguintes afirmações:

1. Se $\mathrm{M}, \mathrm{N}$ são subespaços fechados de $\mathrm{H} e \operatorname{def}(\mathrm{M}, \mathrm{N})<\infty$, então $\mathrm{M}+\mathrm{N}$ é fechado.

2. Se $\mathrm{M} \supset \mathrm{N}^{\perp}$ são fechados então $\operatorname{codim} \mathrm{M}=\operatorname{codim}_{\mathrm{N}}(\mathrm{M} \cap \mathrm{N})$.

3. Se $\mathrm{M} \subset \mathrm{N} e$ codim $\mathrm{M}<\infty$, então $\mathrm{N}$ é fechado.

4. Se $\operatorname{dim} \mathrm{M}<\infty$ então $\operatorname{dim}\left(\mathrm{M}+\mathrm{N}^{\perp}\right) \cap \mathrm{N}<\infty$ para qualquer subespaço fechado $\mathrm{N}$.

Prova. O ítem 1 decorre diretamente do lema 1.3.1 considerando a aplicação

$$
\mathrm{M} \oplus \mathrm{N} \ni(u, v) \mapsto u+v \in \mathrm{H} .
$$

Para o ítem 2, observamos que a aplicação

$$
\begin{aligned}
& \mathrm{H} \rightarrow \mathrm{N} / \mathrm{M} \cap \mathrm{N} \\
& u \mapsto P_{\mathrm{N}} u+\mathrm{M} \cap \mathrm{N}
\end{aligned}
$$

é uma sobrejeção linear com núcleo $\mathrm{M}$, pois $P_{\mathrm{N}} u \in \mathrm{M} \cap \mathrm{N}$ implica que $u=\left(u-P_{\mathrm{N}} u\right)+P_{\mathrm{N}} u \in$ $\mathrm{N}^{\perp}+\mathrm{M} \subset \mathrm{M}$.

Para provar 3, consideremos a projeção $\pi: H \rightarrow \hat{H}=H / M$. Temos que $\pi(N)$ é fechado, pois $\hat{\mathrm{H}}$ tem dimensão finita, logo, $\mathrm{N}=\pi^{-1}(\pi(\mathrm{N}))$ é fechado.

Finalmente, como $\operatorname{dim} \operatorname{Im}\left(P_{\mathrm{N}}^{\mathrm{M}}\right) \leq \operatorname{dim} \mathrm{M}<\infty$ e $\left(P_{\mathrm{M}}^{\mathrm{N}}\right)^{*}=P_{\mathrm{N}^{\prime}}^{\mathrm{M}}$

$$
\left(M+N^{\perp}\right) \cap N=\left(M^{\perp} \cap N\right)^{\perp} \cap N=\operatorname{ker}\left(P_{M}^{N}\right)^{\perp} \cap N=\operatorname{Im}\left(P_{N}^{M}\right) \cap N,
$$

logo, $\operatorname{dim}\left(\mathrm{M}+\mathrm{N}^{\perp}\right) \cap \mathrm{N}<\infty$.

A próxima proposição fornece uma importante relação entre pares Fredholm de subespaços e operadores Fredholm.

Proposição 1.3.18 O par (M, N) é (semi) Fredholm se e só se $P_{\mathrm{N}^{\perp}}^{\mathrm{M}}: \mathrm{M} \rightarrow \mathrm{N}^{\perp}$ é um operador (semi) Fredholm. Além disso, $\operatorname{nul}(\mathrm{M}, \mathrm{N})=\operatorname{nul}\left(P_{\mathrm{N}^{+}}^{\mathrm{M}}\right), \operatorname{def}(\mathrm{M}, \mathrm{N})=\operatorname{def}\left(P_{\mathrm{N}^{+}}^{\mathrm{M}}\right) e \operatorname{ind}(\mathrm{M}, \mathrm{N})=\operatorname{ind}\left(P_{\mathrm{N}^{+}}^{\mathrm{M}}\right)$.

Prova. Como já observamos,

$$
\operatorname{ker}\left(P_{\mathrm{N}^{\perp}}^{\mathrm{M}}\right)=\mathrm{M} \cap \mathrm{N}
$$

Se $(M, N)$ é um par Fredholm, então, $\operatorname{ker}\left(P_{N^{+}}^{M}\right)$ tem dimensão finita. Como $M+N$ é fechado, pelo corolário 1.2.16, segue que $\operatorname{Im}\left(P_{\mathrm{N}^{1}}^{\mathrm{M}}\right)$ é fechada. Logo, pela proposição 1.2.19,

$$
\begin{aligned}
\operatorname{Im}\left(P_{N^{\perp}}^{M}\right) & =\left(\operatorname{ker} P_{M}^{N^{\perp}}\right)^{\perp} \cap N^{\perp} \\
& =\left(M^{\perp} \cap N^{\perp}\right)^{\perp} \cap N^{\perp} \\
& =(M+N) \cap N^{\perp} .
\end{aligned}
$$

Pelo ítem 2 do lema 1.3.17, segue que

$$
\operatorname{codim}_{N^{\perp}}\left(\operatorname{Im}\left(P_{N^{\perp}}^{M}\right)\right)=\operatorname{codim}_{N^{\perp}}\left((M+N) \cap N^{\perp}\right)=\operatorname{codim}(M+N) .
$$


Logo, $P_{\mathrm{N}^{\perp}}^{\mathrm{M}}$ é um operador de Fredholm. Reciprocamente, se $P_{\mathrm{N}^{\perp}}^{\mathrm{M}}$ é de Fredholm, então novamente pelo corolário 1.2.16, temos que $\mathrm{M}+\mathrm{N}$ é fechado. Logo, a igualdade 1.3.7 é verificada, e segue de 1.3.8 que $M+N$ tem codimensão finita.

A afirmação a respeito dos índices, nulidades e deficiências decorre imediatamente das igualdades 1.3 .6 e 1.3.8.

Agora veremos um critério de bastante importância teórica sobre pares de Fredholm.

Proposição 1.3.19 O par $(\mathrm{M}, \mathrm{N})$ é Fredholm se e só se $P_{\mathrm{M}}-P_{\mathrm{N}}$ é Fredholm. Além disso, $\gamma(\mathrm{M}, \mathrm{N})=$ $\gamma\left(P_{M}^{N^{\perp}}\right)=\gamma\left(P_{M}-P_{N}\right)$.

Prova. Como na prova do teorema 1.2.2, temos que

$$
P_{\mathrm{M}}-P_{\mathrm{N}}=P_{\mathrm{M}} P_{\mathrm{N}^{\perp}}-P_{\mathrm{M}^{\perp}} P_{\mathrm{N}}=P_{\mathrm{M}}^{\mathrm{N}^{\perp}} P_{\mathrm{N}^{\perp}}-P_{\mathrm{M}^{\perp}}^{\mathrm{N}} P_{\mathrm{N}} \text {. }
$$

Como $\operatorname{Im}\left(P_{\mathrm{M}}^{\mathrm{N}^{\perp}} P_{\mathrm{N}^{\perp}}\right) \subset \mathrm{M} \mathrm{e} \operatorname{Im}\left(P_{\mathrm{M}^{\perp}}^{\mathrm{N}} P_{\mathrm{N}}\right) \subset \mathrm{M}^{\perp}$, vemos imediatamente que

$$
\operatorname{ker}\left(P_{\mathrm{M}}-P_{\mathrm{N}}\right)=\mathrm{M} \cap \mathrm{N} \oplus(\mathrm{M}+\mathrm{N})^{\perp} \text { e } \operatorname{Im}\left(P_{\mathrm{M}}-P_{\mathrm{N}}\right)=\operatorname{Im}\left(P_{\mathrm{M}}^{\mathrm{N}^{\perp}}\right) \oplus \operatorname{Im}\left(P_{\mathrm{M}^{\perp}}^{\mathrm{N}}\right),
$$

sendo que ambas as somas diretas são ortogonais. Logo, se ( $M, N)$ é um par Fredholm então pela proposição 1.3.18, concluímos que $P_{\mathrm{M}}-P_{\mathrm{N}}$ é um operador Fredholm. Reciprocamente, se $P_{\mathrm{M}}-P_{\mathrm{N}}$ é Fredholm, então a primeira equação em 1.3.10 mostra que nul(M, $\left.\mathrm{N}\right)<\infty$; a segunda equação mostra que $\operatorname{Im}\left(P_{M^{\perp}}^{N}\right) \subset M^{\perp}$ é de codimensão finita igual a $\operatorname{def}(M, N)$, pela proposição 1.3.18.

Finalmente, a identidade 1.3 .9 e o fato que os operadores $P_{\mathrm{M}}^{\mathrm{N}^{1}} P_{\mathrm{N}^{\perp}}$ e $P_{\mathrm{M}^{\perp}}^{\mathrm{N}} P_{\mathrm{N}}$ têm imagem em subespaços ortogonais implicam que

$$
\begin{aligned}
\gamma\left(P_{\mathrm{M}}-P_{\mathrm{N}}\right)^{2} & =\inf _{\substack{u \in(\mathrm{M}+\mathrm{N}) \cap N^{\perp} \\
v \in(\mathrm{MnN})^{\perp} \cap \mathrm{N}}} \frac{\left\|P_{M}^{\mathrm{N}^{\perp}} u\right\|^{2}+\left\|P_{\mathrm{M}^{\perp}}^{\mathrm{N}} v\right\|^{2}}{\|u\|^{2}+\|v\|^{2}} \\
& =\inf _{\substack{u \in\left(\operatorname{ker}\left(P_{M}^{N^{\perp}}\right)\right)^{\perp} \cap N^{\perp} \\
v \in \operatorname{ker}\left(P_{M^{\perp}}^{\mathrm{N}}\right)^{\perp} \cap N}} \frac{\left\|P_{M}^{N^{\perp}} u\right\|^{2}+\left\|P_{\mathrm{M}^{\perp}}^{\mathrm{N}} v\right\|^{2}}{\|u\|^{2}+\|v\|^{2}} \\
& =\inf _{\substack{u \in\left(\operatorname{ker}\left(P_{M}^{N^{\perp}}\right)\right)^{\perp} \cap N^{\perp} \\
v \in \operatorname{ker}\left(P_{M^{\perp}}^{N}\right)^{\perp} \cap N}} \frac{\gamma\left(\mathrm{N}^{\perp}, \mathrm{M}^{\perp}\right)^{2}\|u\|^{2}+\gamma(\mathrm{N}, \mathrm{M})\|v\|^{2}}{\|u\|^{2}+\|v\|^{2}}=\gamma(\mathrm{M}, \mathrm{N})^{2}=\gamma\left(P_{\mathrm{M}}^{\mathrm{N}^{\perp}}\right)^{2},
\end{aligned}
$$

pelo corolário 1.2.18 e pelos lemas 1.2.8 e 1.2.15.

Vejamos que um par Fredholm é estável por pequenas perturbações, i.e., dado um par Fredholm $(M, N)$ então o par $\left(M^{\prime}, N\right)$ também é Fredholm, se $M, M^{\prime}$ são suficientemente próximos em termos da abertura máxima.

Teorema 1.3.20 Sejam $M, M^{\prime}, N$ subespaços fechados de $H$ tais que $\hat{\delta}\left(M^{\prime}, M\right)<\gamma(M, N)$. Então

$$
\operatorname{nul}\left(M^{\prime}, N\right) \leq \operatorname{nul}(M, N) \text { edef(}\left(M^{\prime}, N\right) \leq \operatorname{def}(M, N) .
$$

Além disso, se nul $(\mathrm{M}, \mathrm{N})<\infty$ e $\mathrm{M}+\mathrm{N}$ é fechado então $\mathrm{M}^{\prime}+\mathrm{N}$ é fechado. Em particular, se $(\mathrm{M}, \mathrm{N})$ é um par (semi) Fredholm então $\left(\mathrm{M}^{\prime}, \mathrm{N}\right)$ é um par (semi) Fredholm. 
Prova. Usando o segundo ítem da proposição 1.2.3, o lema 1.2.8, o lema 1.2.15 e considerando $P_{\mathrm{M}^{\prime \perp}}^{\mathrm{N}}, P_{\mathrm{M}^{\perp}}^{\mathrm{N}}: \mathrm{N} \rightarrow \mathrm{H}$, temos

$$
\left\|P_{\mathrm{M}^{\perp \perp}}^{\mathrm{N}}-P_{\mathrm{M}^{\perp}}^{\mathrm{N}}\right\| \leq\left\|P_{\mathrm{M}^{\prime \perp}}-P_{\mathrm{M}^{\perp}}\right\|=\hat{\delta}\left(\mathrm{M}^{\prime \perp}, \mathrm{M}^{\perp}\right)=\hat{\delta}\left(\mathrm{M}^{\prime}, \mathrm{M}\right)<\gamma(\mathrm{M}, \mathrm{N})=\gamma\left(P_{\mathrm{M}^{\perp}}^{\mathrm{N}}\right) .
$$

Logo, pelo teorema 1.3.9, temos $\operatorname{nul}\left(M^{\prime}, N\right)=\operatorname{nul}\left(P_{M^{\prime \perp}}^{N}\right) \leq \operatorname{nul}\left(P_{M^{\perp}}^{N}\right)=\operatorname{nul}(M, N)$. A afirmação sobre a deficiência é trivial se $\operatorname{def}(\mathrm{M}, \mathrm{N})=\infty$. Caso contrário, temos que o operador $P_{\mathrm{M}^{\perp}}^{\mathrm{N}}$ é semi-Fredholm. De fato, pelo ítem 1 da proposição 1.3.17, $\mathrm{M}+\mathrm{N}$ é fechado, portanto, pelo corolário 1.2.16, $\operatorname{Im}\left(P_{\mathrm{M}^{\perp}}^{\mathrm{N}}\right)$ é fechada. Isso nos permite concluir que a equação 1.3.7 é válida e portanto, codim $\mathrm{M}^{\perp}\left(\operatorname{Im}\left(P_{\mathrm{M}^{\perp}}^{\mathrm{N}}\right)\right)<\infty$, provando que $P_{\mathrm{M}^{\perp}}^{\mathrm{N}}$ é semi-Fredholm. Pela desigualdade 1.3.11 e pelas proposições 1.3.11, 1.3 .12 e 1.3 .18 concluímos que $P_{\mathrm{M}^{\prime 1}}^{\mathrm{N}}$ é semi-Fredholm e $\operatorname{def}\left(M^{\prime}, N\right)=\operatorname{def}\left(P_{M^{\prime 1}}^{N}\right) \leq \operatorname{def}\left(P_{M^{\perp}}^{N}\right)=\operatorname{def}(M, N)$, como queríamos.

OBservaçÃo 1.3.21 Pelo teorema 1.3.20, se $\hat{\delta}\left(M^{\prime}, M\right)<1=\gamma\left(M, M^{\perp}\right)$, então nul( $\left(M^{\prime}, M^{\perp}\right) \leq$ $\operatorname{nul}\left(M, M^{\perp}\right)=0$ e $\operatorname{def}\left(M^{\prime}, M^{\perp}\right) \leq \operatorname{def}\left(M, M^{\perp}\right)=0$. Isso significa que $M^{\prime} \cap M^{\perp}=0$ e $M^{\prime}+M^{\perp}=H$.

Vamos provar agora um resultado sobre a estabilidade dos pares (semi) Fredholm.

Teorema 1.3.22 Sejam $\mathrm{M}, \mathrm{M}^{\prime}, \mathrm{N}$ subespaços fechados de $\mathrm{H}$ e suponhamos que o par (M, N) é (semi) Fredholm. Se $\hat{\delta}\left(\mathrm{M}^{\prime}, \mathrm{M}\right)<\gamma(\mathrm{M}, \mathrm{N})$, então $\left(\mathrm{M}^{\prime}, \mathrm{N}\right)$ é (semi) Fredholm e ind $\left(\mathrm{M}^{\prime}, \mathrm{N}\right)=\operatorname{ind}(\mathrm{M}, \mathrm{N})$.

Prova. Pelo teorema 1.3.20, precisamos provar apenas a afirmação a respeito dos índices. A observação 1.3.21 implica que $\mathrm{M}^{\prime} \oplus \mathrm{M}^{\perp}=\mathrm{H}$.

Consideremos, como na proposição 1.3.19, os operadores $T=P_{\mathrm{M}}-P_{\mathrm{N}}=P_{\mathrm{M}^{\prime}}^{\mathrm{N}^{\perp}} P_{\mathrm{N}^{\perp}}-P_{\mathrm{M}^{\perp}}^{\mathrm{N}} P_{\mathrm{N}}$ e $T^{\prime}=P_{\mathrm{M}^{\prime}}-P_{\mathrm{N}}=P_{\mathrm{M}}^{\mathrm{N}^{\perp}} P_{\mathrm{N}^{\perp}}-P_{\mathrm{M}^{\perp}}^{\mathrm{N}} P_{\mathrm{N}}$. Vemos que $\operatorname{ker} T^{\prime}=\left(\mathrm{M}^{\prime}+\mathrm{N}\right)^{\perp} \oplus \mathrm{M} \cap \mathrm{N}$ e $\operatorname{Im} T^{\prime}=$ $M^{\prime} \cap\left(M^{\prime} \cap N\right)^{\perp} \oplus(M+N) \cap M^{\perp}$. Pelo ítem 2 do lema 1.3.17, temos que nul $T^{\prime}=\operatorname{def}\left(M^{\prime}, N\right)+\operatorname{nul}(M, N)$ e $\operatorname{def} T^{\prime}=\operatorname{nul}\left(M^{\prime}, N\right)+\operatorname{def}(M, N), \operatorname{logo}$, ind $T^{\prime}=\operatorname{ind}(M, N)-\operatorname{ind}\left(M^{\prime}, N\right)$. Além disso, pela proposição 1.3.19,

$$
\left\|T-T^{\prime}\right\|=\left\|P_{\mathrm{M}^{\prime}}-P_{\mathrm{M}}\right\|=\delta\left(\mathrm{M}, \mathrm{M}^{\prime}\right)<\gamma(\mathrm{M}, \mathrm{N})=\gamma(T),
$$

portanto, pelo teorema 1.3.9, temos que $T^{\prime}$ é Fredholm e $0=\operatorname{ind} T=\operatorname{ind} T^{\prime}$, i.e., ind $\left(M^{\prime}, N\right)=$ ind $(\mathrm{M}, \mathrm{N})$.

O teorema 1.3.22 é fundamental para estender alguns resultados de estabilidade sobre operadores de Fredholm limitados para operadores de Fredholm em geral. Isto será feito na próxima subseção.

Vamos agora provar alguns fatos relevantes para o estudo qualitativo de pares Fredholm de subespaços.

Proposição 1.3.23 Se $\mathrm{M}, \mathrm{N}$ são subespaços fechados tais que $\mathrm{M} \cap \mathrm{N}=0$ e $\mathrm{M}+\mathrm{N}$ é fechado, então $\operatorname{Im}\left(P_{\mathrm{M}}+P_{\mathrm{N}}\right)=\mathrm{M}+\mathrm{N}$.

Prova. Obviamente, $\operatorname{Im}\left(P_{\mathrm{M}}+P_{\mathrm{N}}\right) \subset \mathrm{M}+\mathrm{N}$. Como $\mathrm{M}+\mathrm{N}$ é fechado e $P_{\mathrm{M}} P_{\mathrm{M}+\mathrm{N}}=P_{\mathrm{M}}$, $P_{\mathrm{N}} P_{\mathrm{M}+\mathrm{N}}=P_{\mathrm{N}}$, podemos supor que $\mathrm{M}+\mathrm{N}=\mathrm{H}$. Vamos provar que $P_{\mathrm{M}}+P_{\mathrm{N}}$ é sobrejetora. Seja $S: \mathrm{M}^{\perp} \rightarrow \mathrm{M}$ tal que $\mathrm{G}(S)=\mathrm{N}$. Considerando ${ }^{17} S^{*}: \mathrm{M} \rightarrow \mathrm{M}^{\perp}$, temos que $\mathrm{G}\left(-S^{*}\right)=\mathrm{N}^{\perp}$. Assim, dado qualquer $w \in \mathrm{H}$, existem $u \in \mathrm{M}^{\perp}$ e $v \in \mathrm{M}$ tais que $w=(u+S u)+v$, pois $\mathrm{G}(S)+\mathrm{M}=\mathrm{H}$. Tomando $z=(u+S u)+\left(x-S^{*} x\right) \operatorname{com} x=v-S u$, temos que $P_{\mathrm{N}}(z)=u+S u$ e $P_{\mathrm{M}}(z)=x+S u=v$, portanto, $\left(P_{\mathrm{M}}+P_{\mathrm{N}}\right) z=u+S u+v=w$, portanto $P_{\mathrm{M}}+P_{\mathrm{N}}$ é sobrejetora.

\footnotetext{
${ }^{17}$ Veja os comentários após a observação 1.5 .19$.
} 
OBSERVAÇão 1.3.24 A prova da proposição 1.3.23 mostra que não só $P_{\mathrm{M}}+P_{\mathrm{N}}$ é sobrejetor, mas $\left(P_{\mathrm{M}}+P_{\mathrm{N}}\right)(\mathrm{M}+\mathrm{N})=\mathrm{M}+\mathrm{N}$.

OBSERvação 1.3.25 Temos que $\operatorname{ker}\left(P_{\mathrm{M}}+P_{\mathrm{N}}\right)=\dot{M}^{\perp} \cap \mathrm{N}^{\perp}=(\mathrm{M}+\mathrm{N})^{\perp}$. De fato, se $P_{\mathrm{M}} u+P_{\mathrm{N}} u=0$, então

$$
\left\|P_{\mathrm{M}} u\right\|^{2}=\left\langle P_{\mathrm{M}} u, u\right\rangle=-\left\langle P_{\mathrm{N}} u, u\right\rangle=-\left\|P_{\mathrm{N}} u\right\|^{2} .
$$

Em geral, para $\mathrm{M}, \mathrm{N}$ quaisquer,

$$
\overline{\mathrm{M}+\mathrm{N}}=\left(\operatorname{ker}\left(P_{\mathrm{M}}+P_{\mathrm{N}}\right)\right)^{\perp}=\overline{\operatorname{Im}\left(P_{\mathrm{M}}+P_{\mathrm{N}}\right)} .
$$

Estenderemos o resultado da proposição 1.3.23 para um par $(\mathrm{M}, \mathrm{N})$ com intersecção nãotrivial. Para tanto, observemos alguns fatos.

LemA 1.3.26 Se $\mathrm{M}, \mathrm{N}$ são subespaços fechados, $\operatorname{sejam} \mathrm{M}^{\prime}=\mathrm{M} \cap(\mathrm{M} \cap \mathrm{N})^{\perp}$ e $\mathrm{N}^{\prime}=\mathrm{N} \cap(\mathrm{M} \cap \mathrm{N})^{\perp}$. Então:

1. $M=(M \cap N) \oplus M^{\prime}$ e $=(M \cap N) \oplus N^{\prime}, \operatorname{com}(M \cap N)$ ortogonal a $M^{\prime}$ e $N^{\prime}$;

2. $M^{\prime}+N^{\prime}=M \cap(M \cap N)^{\perp}+N \cap(M \cap N)^{\perp}=(M+N) \cap(M \cap N)^{\perp}$;

3. $M \cap N+(M+N) \cap(M \cap N)^{\perp}=M+N$;

4. $\left.P_{\mathrm{M}^{\prime}}\right|_{(\mathrm{M} \cap N)^{\perp}}=\left.P_{\mathrm{M}}\right|_{(\mathrm{M} \cap \mathrm{N})^{\perp}}$ e $\left.P_{\mathrm{N}^{\prime}}\right|_{(\mathrm{M} \cap \mathrm{N})^{\perp}}=\left.P_{\mathrm{N}}\right|_{(\mathrm{M} \cap \mathrm{N})^{\perp}}$.

Prova. Provemos o primeiro ítem. Dado $u \in M$, seja $u=v+w \operatorname{com} v \in M \cap N$ e $w \in(M \cap N)^{\perp}$. Como $w=u-v \in \mathrm{M}$, temos que $w \in \mathrm{M}^{\prime}$, provando o desejado. A igualdade referente a $\mathrm{N}$ prova-se de maneira semelhante.

Observamos que, em geral, não é verdade que $\left(M+M^{\prime}\right) \cap N=M \cap N+M^{\prime} \cap N$; apenas a inclusão $\supset$ é verdadeira. No nosso caso, dado $u=u_{M}+u_{N} \in(M+N) \cap(M \cap N)^{\perp}, \operatorname{com} u_{M} \in M$ e $u_{\mathrm{N}} \in \mathrm{N}$, pelo primeiro ítem, existem $u_{\mathrm{M}^{\prime}}^{1} u_{\mathrm{N}}^{1} \in \mathrm{M} \cap \mathrm{N}, u_{\mathrm{M}}^{2} \in \mathrm{M}^{\prime}, u_{\mathrm{N}}^{2} \in \mathrm{N}^{\prime}$ tais que

$$
u=u_{\mathrm{M}}+u_{\mathrm{N}}=\left(u_{\mathrm{M}}^{1}+u_{\mathrm{M}}^{2}\right)+\left(u_{\mathrm{N}}^{1}+u_{\mathrm{N}}^{2}\right)=\left(u_{\mathrm{M}}^{1}+u_{\mathrm{N}}^{1}\right)+\left(u_{\mathrm{M}}^{2}+u_{\mathrm{N}}^{2}\right) .
$$

Como $u_{\mathrm{M}}^{1}+u_{\mathrm{N}}^{1} \in \mathrm{M} \cap \mathrm{N}, u_{\mathrm{M}}^{2}+u_{\mathrm{N}}^{2} \in \mathrm{M}^{\prime}+\mathrm{N}^{\prime} \subset(\mathrm{M} \cap \mathrm{N})^{\perp}$ e $u \in(\mathrm{M} \cap \mathrm{N})^{\perp}$, temos que $u_{\mathrm{M}}^{1}+u_{\mathrm{N}}^{1}=0$. Logo, $u=u_{M}^{2}+u_{N}^{2} \in M^{\prime}+N^{\prime}$, como queríamos. O terceiro ítem prova-se da mesma forma.

Para provar o quarto ítem, é suficiente provar que se $u \in(\mathrm{M} \cap \mathrm{N})^{\perp}$ então $P_{\mathrm{M}^{\prime}} u$ é o único vetor $v \in \mathrm{M}$ tal que $u-v \perp \mathrm{M}$. De fato, $P_{\mathrm{M}^{\prime}} u \in \mathrm{M}^{\prime} \subset \mathrm{M}$. Dado qualquer $v \in \mathrm{M}$, pelo primeiro ítem, $v=v_{1}+v_{2}$, com $v_{1} \in \mathrm{M} \cap \mathrm{N}$ e $v_{2} \in \mathrm{M}^{\prime}$. Como $u \in(\mathrm{M} \cap \mathrm{N})^{\perp}, P_{\mathrm{M}^{\prime}} u \in \mathrm{M}^{\prime} \subset(\mathrm{M} \cap \mathrm{N})^{\perp} \mathrm{e}$ $v_{1} \in \mathrm{M} \cap \mathrm{N}$ então $\left\langle u-P_{\mathrm{M}^{\prime}} u, v_{1}\right\rangle=0$. Por definição de $P_{\mathrm{M}^{\prime}}$, temos $\left\langle u-P_{\mathrm{M}^{\prime}} u, v_{2}\right\rangle=0$. Logo

$$
\left\langle u-P_{\mathbf{M}^{\prime}} u, v\right\rangle=\left\langle u-P_{\mathbf{M}^{\prime}} u, v_{1}\right\rangle+\left\langle u-P_{\mathbf{M}^{\prime}} u, v_{2}\right\rangle=0 .
$$

A afirmação correspondente a $\mathrm{N}$ e $\mathrm{N}^{\prime}$ prova-se de maneira semelhante.

OBSERVAÇÃo 1.3.27 Os resultados do lema 1.3.26 são verdadeiros substituindo $\mathrm{M} \cap \mathrm{N}$ por qualquer subespaço $S \subset M \cap N$ e pondo $M^{\prime}=M \cap S^{\perp}$ e $N^{\prime}=N \cap S^{\perp}$.

Proposição 1.3.28 Se M, Ne M + N são fechados, então $\operatorname{Im}\left(P_{\mathrm{M}}+P_{\mathrm{N}}\right)=\mathrm{M}+\mathrm{N}$. 
Prova. Definindo $\mathrm{M}^{\prime}, \mathrm{N}^{\prime}$ como no lema 1.3.26, temos pela proposição 1.3 .23 que $\operatorname{Im}\left(P_{\mathrm{M}^{\prime}}+\right.$ $\left.P_{\mathrm{N}^{\prime}}\right)=\mathrm{M}^{\prime}+\mathrm{N}^{\prime}$. Pelo último ítem do lema 1.3.26, concluímos que $\left(P_{\mathrm{M}}+P_{\mathrm{N}}\right)\left((\mathrm{M} \cap \mathrm{N})^{\perp}\right)=\mathrm{M}^{\prime}+\mathrm{N}^{\prime}$. Pelo segundo e pelo terceiro ítens do mesmo lema,

$$
\begin{aligned}
\operatorname{Im}\left(P_{\mathrm{M}}+P_{\mathrm{N}}\right) & =\left(P_{\mathrm{M}}+P_{\mathrm{N}}\right)(\mathrm{M} \cap \mathrm{N})+\left(P_{\mathrm{M}}+P_{\mathrm{N}}\right)\left((\mathrm{M} \cap \mathrm{N})^{\perp}\right) \\
& =\mathrm{M} \cap \mathrm{N}+\mathrm{M}^{\prime}+\mathrm{N}^{\prime}=\mathrm{M}+\mathrm{N} .
\end{aligned}
$$

OBSERVAÇÃo 1.3.29 A proposição 1.3.28 generaliza os resultados do apêndice de [21], pois não fazemos nenhuma hipótese sobre estruturas simpléticas ou lagrangianos, como é o caso no referido artigo.

Agora estamos prontos para obter um critério muito importante para determinar se um par de subespaços fechados é Fredholm.

Teorema 1.3.30 Sejam M, N subespaços fechados de $\mathrm{H}$.

1. Se $P_{\mathrm{M}}+P_{\mathrm{N}}$ é Fredholm e $\operatorname{nul}(\mathrm{M}, \mathrm{N})<\infty$ então $(\mathrm{M}, \mathrm{N})$ é um par Fredholm.

2. Se $\operatorname{def}(\mathrm{M}, \mathrm{N})<\infty$ então $P_{\mathrm{M}}+P_{\mathrm{N}}$ é Fredholm.

Prova. Se $P_{\mathrm{M}}+P_{\mathrm{N}}$ é Fredholm e nul(M, N) $<\infty$ então $\operatorname{Im}\left(P_{\mathrm{M}}+P_{\mathrm{N}}\right) \subset \mathrm{M}+\mathrm{N}$ é fechada de codimensão finita. Pelo ítem 3 do lema 1.3.17, segue que $M+N$ tem codimensão finita, e portanto, $(\mathrm{M}, \mathrm{N})$ é um par Fredholm. ${ }^{18}$

Provemos o ítem 2. Pelo ítem 1 do lema 1.3.17, segue que $\mathrm{M}+\mathrm{N}$ é fechado. Pela proposição 1.3.28, temos que $\operatorname{Im}\left(P_{\mathrm{M}}+P_{\mathrm{N}}\right)=\mathrm{M}+\mathrm{N}$ é fechada de codimensão finita. A conclusão segue da observação 1.3.25.

OBSERVAÇÃo 1.3.31 A prova das proposições 1.3 .23 e 1.3.28 pode ser adaptada para mostrar que se $\mathrm{M}+\mathrm{N}$ é fechado então $\operatorname{Im}\left(P_{\mathrm{M}}-P_{\mathrm{N}}\right)=\mathrm{M}+\mathrm{N}$. Isso melhora substancialmente o resultado da proposição 1.3.19.

\subsubsection{Operadores Fredholm ilimitados}

Nesta seção, vamos estender ao caso ilimitado os resultados de estabilidade obtidos para operadores Fredholm limitados. A ligação entre ambas as situações dá-se através da teoria de pares Fredholm de subespaços da subseção 1.3.2. Observamos inicialmente que o lema 1.3.1 e a proposição 1.3.3 não fazem nenhuma hipótese de limitação sobre os operadores envolvidos.

Iniciamos com um lema simples.

Lema 1.3.32 Sejam $\mathrm{M}, \mathrm{N}$ subespaços de $\mathrm{H}$ tais que $\mathrm{M}$ é denso e $\mathrm{N}$ é fechado de codimensão finita $n$. Então existe um subespaço $\mathrm{M}^{\prime} \subset \mathrm{M}$ de dimensão $n$ tal que $\mathrm{H}=\mathrm{M}^{\prime} \oplus \mathrm{N}$.

Prova. A imagem de M pela projeção $\pi: H \rightarrow H / N$ é um subespaço denso; $\operatorname{como} \operatorname{dim}(H / N)=$ $n$, então $\pi(\mathrm{M})=\mathrm{H} / \mathrm{N}$. Logo, existe $\left\{u_{1}, \ldots, u_{n}\right\} \subset \mathrm{M}$ tal que $\left\{u_{1}+\mathrm{N}, \ldots, u_{n}+\mathrm{N}\right\}$ é base de $\mathrm{H} / \mathrm{N}$. Tomando $\mathrm{M}^{\prime}$ o subespaço gerado por $\left\{u_{1}, \ldots, u_{n}\right\}$, segue o resultado.

\footnotetext{
${ }^{18}$ Em particular, $\operatorname{Im}\left(P_{\mathrm{M}}+P_{\mathrm{N}}\right)=\mathrm{M}+\mathrm{N}$, pela proposição 1.3.28.
} 
Corolário 1.3.33 Se Mé um subespaço denso e $\mathrm{N}$ tem codimensão finita, então $\mathrm{M} \cap \mathrm{N}$ é denso em $\mathrm{N}$.

Nosso próximo objetivo é estender o teorema 1.3.8 para uma classe substancialmente maior de operadores. Para isso, precisaremos da próxima proposição.

Proposição 1.3.34 Sejam $T_{1} \in \mathscr{C}\left(\mathrm{H}_{1}, \mathrm{H}_{2}\right)$ e $T_{2} \in \mathscr{C}\left(\mathrm{H}_{2}, \mathrm{H}_{3}\right)$ operadores tais que $\operatorname{Im} T_{2}$ é fechada $e$ $\operatorname{def} T_{1}<\infty$. Então existe um operador $S \in \mathscr{L}\left(\mathrm{H}_{3}, \mathrm{H}_{1}\right)$ tal que:

1. $\operatorname{Im} S \subset \mathrm{D}\left(T_{2} T_{1}\right)$;

2. $\left(T_{2} T_{1}\right) S=I-P_{\left(\operatorname{Im} T_{2}\right)^{\perp}}+K$, onde $K$ é um operador de posto finito;

3. Se nul $T_{2}<\infty$ então $S\left(T_{1} T_{2}\right) \subset I-P_{\operatorname{ker} T_{1}}+L$, onde $L$ é um operador de posto finito.

Em particular, se $T_{1} \in \mathscr{C} \mathscr{F}\left(\mathrm{H}_{1}, \mathrm{H}_{2}\right)$ e $T_{2} \in \mathscr{C} \mathscr{F}\left(\mathrm{H}_{2}, \mathrm{H}_{3}\right)$ então $T_{2} T_{1} \in \mathscr{C} \mathscr{F}\left(\mathrm{H}_{1}, \mathrm{H}_{3}\right){ }^{19}$

Prova. Pelo lema 1.3.32, existe um subespaço $M \subset D\left(T_{2}\right)$ tal que $M \oplus \operatorname{Im} T_{1}=H_{2}$. Pela proposição 1.3.2 existem $S_{1} \in \mathscr{L}\left(\mathrm{H}_{2}, \mathrm{H}_{1}\right), S_{2} \in \mathscr{L}\left(\mathrm{H}_{3}, \mathrm{H}_{2}\right)$ tais que $\operatorname{Im} S_{1} \subset \mathrm{D}\left(T_{1}\right), \operatorname{Im} S_{2} \subset \mathrm{D}\left(T_{2}\right)$ e são verificadas as relações:

$$
\begin{array}{cc}
T_{1} S_{1}=I-P_{\operatorname{Im} T_{1}, M} & T_{2} S_{2}=I-P_{\left(\operatorname{Im} T_{2}\right)^{\perp}} \\
S_{1} T_{1} \subset I-P_{\text {ker } T_{1}} & S_{2} T_{2} \subset I-P_{\text {ker } T_{2}}
\end{array} .
$$

Seja $S=S_{1} S_{2} \in \mathscr{L}\left(\mathrm{H}_{3}, \mathrm{H}_{1}\right)$. Claramente, $\operatorname{Im} S \subset \operatorname{Im} S_{1} \subset \mathrm{D}\left(T_{1}\right)$. Além disso, dado $u \in \mathrm{H}_{3}$, temos $T_{1}(S u)=S_{2} u-P_{\operatorname{Im} T_{1}, \mathrm{M}} S_{2} u \in \mathrm{D}\left(T_{2}\right)+\mathrm{M} \subset \mathrm{D}\left(T_{2}\right)$, portanto $\operatorname{Im} S \subset \mathrm{D}\left(T_{2} T_{1}\right)$, provando o ítem 1. As relações 1.3.12 implicam que

$$
\begin{aligned}
\left(T_{2} T_{1}\right) S & =T_{2}\left(T_{1} S_{1}\right) S_{2} \\
& =T_{2} S_{2}-T_{2} P_{\operatorname{Im} T_{1}, \mathrm{M}} S_{2} \\
& =I-P_{\left(\operatorname{Im} T_{2}\right)^{\perp}}-T_{2} P_{\operatorname{Im} T_{1}, \mathrm{M}} S_{2},
\end{aligned}
$$

provando o ítem 2. Além disso, as mesmas relações implicam que

$$
\begin{aligned}
S\left(T_{2} T_{1}\right) & \subset S_{1}\left(S_{2} T_{2}\right) T_{1} \\
& \subset S_{1} T_{1}-S_{1} P_{\operatorname{ker} T_{2}} T_{1} \\
& \subset I-P_{\operatorname{ker} T_{1}}-S_{1} P_{\operatorname{ker} T_{2}} T_{1} .
\end{aligned}
$$

Se nul $T_{2}<\infty$ então o operador $S_{1} P_{\text {ker } T_{2}} T_{1}$ é de posto finito (pois $P_{\text {ker } T_{2}}$ é de posto finito e $T_{1}^{*}$ é definido). Isso prova o ítem 3. Pela proposição 1.3.3, $T_{2} T_{1}$ é um operador Fredholm fechado.

Resta verificarmos que $T_{2} T_{1}$ é densamente definido. Pelo corolário 1.3.33, $\mathrm{D}\left(T_{1}\right) \cap\left(\operatorname{ker} T_{1}\right)^{\perp}$ é denso em $\left(\operatorname{ker} T_{1}\right)^{\perp}$. Vejamos que $\mathrm{D}\left(T_{2} T_{1}\right) \cap\left(\operatorname{ker} T_{1}\right)^{\perp}$ é denso em $\mathrm{D}\left(T_{1}\right) \cap\left(\operatorname{ker} T_{1}\right)^{\perp}$. De fato, novamente pelo corolário 1.3.33, dados $u \in \mathrm{D}\left(T_{1}\right) \cap\left(\operatorname{ker} T_{1}\right)^{\perp}$ e $\varepsilon>0$, existe $w \in \operatorname{Im} T_{1} \cap \mathrm{D}\left(T_{2}\right)$ tal que $\left\|T_{1} u-w\right\| \leq \varepsilon$. Se $v \in \mathrm{D}\left(T_{1}\right) \cap\left(\operatorname{ker} T_{1}\right)^{\perp}$ é tal que $T_{1} v=w$, então, pela definição 1.2.12, temos que

$$
\|u-v\| \leq \gamma\left(T_{1}\right)^{-1}\left\|T_{1} u-T_{1} v\right\| \leq \gamma\left(T_{1}\right)^{-1} \varepsilon
$$

e $v \in \mathrm{D}\left(T_{2} T_{1}\right)$, pois $T_{1} v \in \mathrm{D}\left(T_{2}\right)$. Como ker $T_{1} \subset \mathrm{D}\left(T_{2} T_{1}\right)$, segue que $T_{2} T_{1}$ é densamente definido.

\footnotetext{
${ }^{19} \mathrm{O}$ resultado vale também se $T_{1}, T_{2}$ são ambos semi-Fredholm à direita ou ambos semi-Fredholm à esquerda.
} 
Teórema 1.3.35 Se $T_{1} \in \mathscr{C} \mathscr{F}\left(\mathrm{H}_{1}, \mathrm{H}_{2}\right)$ e $T_{2} \in \mathscr{C} \mathscr{F}\left(\mathrm{H}_{2}, \mathrm{H}_{3}\right)$ então $T_{2} T_{1} \in \mathscr{C} \mathscr{F}\left(\mathrm{H}_{1}, \mathrm{H}_{3}\right)$ e

$$
\text { ind }\left(T_{2} T_{1}\right)=\text { ind } T_{2}+\text { ind } T_{1} \text {. }
$$

Prova. Pela proposição 1.3.34, $T_{2} T_{1} \in \mathscr{C} \mathscr{F}\left(\mathrm{H}_{1}, \mathrm{H}_{3}\right)$. Resta provarmos que a fórmula 1.3.13 é verificada.

Provemos a fórmula 1.3.13. Para tanto, observamos que se $T \in \mathscr{C} \mathscr{F}\left(\mathrm{H}, \mathrm{H}^{\prime}\right)$ e $S \in \mathscr{L}\left(\mathrm{H}^{\prime}, \mathrm{H}\right)$ é tal que $\operatorname{Im} S \subset \mathrm{D}(T)$ e $T S=I+L$, com $L$ de posto finito, então pelo lema 1.3.4, temos que ind $(T S)=0$. Considerando em $\mathrm{D}(T)$ a norma do gráfico $\|\cdot\|_{T}$, (cf. a observação 1.2.42), temos que $T$ é $\|\cdot\|_{T}$-limitado e

$$
\|S u\|_{T}^{2} \doteq\|u\|^{2}+\|T S u\|^{2}=\|u\|^{2}+\|u+L u\|^{2} \leq\left(3+2\|L\|^{2}\right)\|u\|^{2} .
$$

Portanto, $S$ é limitado com respeito à norma $\|\cdot\|_{T}$. Como o índice de Fredholm independe da norma utilizada, concluímos pelo teorema 1.3 .8 que $0=$ ind $(T S)=\operatorname{ind} T+$ ind $S$, i.e., ind $T=-$ ind $S$. No ${ }^{20}$ nosso caso, usando o operador $S=S_{1} S_{2}$ construído na proposição 1.3.34, temos, pelo teorema 1.3.8, que

$$
\text { ind }\left(T_{2} T_{1}\right)=- \text { ind }\left(S_{1} S_{2}\right)=- \text { ind } S_{1}-\text { ind } S_{2}=\text { ind } T_{2}+\text { ind } T_{1},
$$

como queríamos.

OBSERVAÇÃo 1.3.36 O teorema 1.3.35 continua válido, com a mesma prova, se substituirmos a palavra Fredholm por semi-Fredholm dे direita ou semi-Fredholm à esquerda.

Passaremos agora a estudar a estabilidade dos operadores (semi) Fredholm por pequenas perturbações na métrica $\hat{\delta}$. A idéia é fazer uma aplicação judiciosa dos resultados sobre estabilidade de pares (semi) Fredholm de subespaços obtidos na subseção 1.3.2.

Dado $T \in \mathscr{C}\left(\mathrm{H}, \mathrm{H}^{\prime}\right)$, temos que ker $T \oplus 0=\mathrm{G}(T) \cap \mathrm{He}(0 \oplus \operatorname{Im} T)+(\mathrm{H} \oplus 0)=\mathrm{G}(T)+(\mathrm{H} \oplus 0)$. Isto implica que nul $T=\operatorname{nul}(\mathrm{G}(T), \mathrm{H} \oplus 0)$ e $\operatorname{def} T=\operatorname{codim}(\mathrm{G}(T)+(\mathrm{H} \oplus 0))=\operatorname{def}(\mathrm{G}(T), \mathrm{H} \oplus 0)$. Decorre do fato de $T$ ser fechado que $\operatorname{Im} T$ é fechada se e só se $\mathrm{G}(T)+(H \oplus 0)$ é fechado. Isso nos permite concluir o seguinte fato.

Lema 1.3.37 Se $T \in \mathscr{C}\left(\mathrm{H}, \mathrm{H}^{\prime}\right)$ então Té (semi) Fredholm se e só se o par $(\mathrm{G}(T), \mathrm{H} \oplus 0)$ é (semi) Fredholm $e$ ind $T=$ ind $(\mathrm{G}(T), \mathrm{H} \oplus 0)$. Além disso,

$$
\gamma(\mathrm{G}(T), \mathrm{H} \oplus 0)=\frac{\gamma(T)}{\left(1+\gamma(T)^{2}\right)^{1 / 2}} .
$$

Prova. Temos que

$$
\begin{aligned}
\gamma(\mathrm{G}(T), \mathrm{H} \oplus 0) & =\inf _{u \notin \operatorname{ker} T} \frac{\operatorname{dist}((u, T u), \mathrm{H} \oplus 0)}{\operatorname{dist}((u, T u), \operatorname{ker} T \oplus 0)} \\
& =\inf _{u \notin \operatorname{ker} T} \frac{\|T u\|}{\left(\operatorname{dist}(u, \operatorname{ker} T)^{2}+\|T u\|^{2}\right)^{1 / 2}}=\frac{\gamma(T)}{\left(1+\gamma(T)^{2}\right)^{1 / 2}} .
\end{aligned}
$$

O próximo teorema decorre imediatamente dos teoremas 1.3.20, 1.3.22 e do lema 1.3.37.

\footnotetext{
${ }^{20}$ A mesma conclusão vale se $S T \subset I+K$, com $K$ de posto finito.
} 
TeOREma 1.3.38 Sejam $T, S \in \mathscr{C}\left(\mathrm{H}, \mathrm{H}^{\prime}\right)$ com $T$ (semi) Fredholm. Então $S$ é (semi) Fredholm, ind $S=$ ind $T$, nul $S \leq \operatorname{nul} T$ e def $S \leq \operatorname{def} T$ se $\hat{\delta}(S, T)<\gamma(T) /\left(1+\gamma(T)^{2}\right)^{1 / 2}$. Em particular, $\mathscr{C} \mathscr{F}\left(\mathrm{H}, \mathrm{H}^{\prime}\right) e^{\prime}$ aberto em $\mathscr{C}\left(\mathrm{H}, \mathrm{H}^{\prime}\right)$.

Segue do teorema 1.3.38 que o índice é constante em cada componente conexa dp espaço $\mathscr{C} \mathscr{F}\left(\mathrm{H}, \mathrm{H}^{\prime}\right)$. Nosso objetivo daqui por diante é provar que se dois operadores em $\mathscr{C} \mathscr{F}\left(\mathrm{H}, \mathrm{H}^{\prime}\right)$ têm mesmo índice, então ambos são conectados por uma curva contínua, ou seja, as componentes conexas (por caminhos) dos espaços $\mathscr{C} \mathscr{F}\left(\mathrm{H}, \mathrm{H}^{\prime}\right)$ e $\mathscr{F}\left(\mathrm{H}, \mathrm{H}^{\prime}\right)$ são os conjuntos

$$
\left\{T \in \mathscr{C} \mathscr{F}\left(\mathrm{H}, \mathrm{H}^{\prime}\right): \text { ind } T=k\right\} \text { e }\left\{T \in \mathscr{F}\left(\mathrm{H}, \mathrm{H}^{\prime}\right): \text { ind } T=k\right\}
$$

com $k \in \mathbb{Z}$, respectivamente.

LemA 1.3.39 Se $T \in \mathscr{C} \mathscr{F}\left(\mathrm{H}, \mathrm{H}^{\prime}\right) e$ ind $T \leq 0$ (ind $T \geq 0$ ) então existe uma curva contínua $T(t)$ em $\mathscr{C} \mathscr{F}\left(\mathrm{H}, \mathrm{H}^{\prime}\right)$ tal que $T(0)=T$ e nul $T(t)=0(\operatorname{def} T(t)=0)$ para todo $t \neq 0$. Se $T$ é limitado, podemos tomar $T(t)$ limitado.

Prova. Se ind $T \leq 0$ então nul $T \leq \operatorname{def} T$. Tomando $u_{1}, \ldots, u_{n}$ base ortonormal de $\operatorname{ker} T$ e $v_{1}, \ldots, v_{n} \in(\operatorname{Im} T)^{\perp}$ um conjunto ortonormal qualquer, seja $K u=\sum_{k=1}^{n}\left\langle u, u_{k}\right\rangle v_{k}$ e $T(t) \doteq T+t K$. Como $K$ é um operador de posto finito com imagem $(\operatorname{Im} T)^{\perp}$, segue que $\operatorname{ker} T(t)=0$ se $t \neq 0$. Além disso, pela desigualdade 1.2.9, segue que

$$
\hat{\delta}(T(t), T(s)) \leq|t-s| \mid K \|,
$$

e portanto $T(t)$ é contínua. $O$ caso ind $T \geq 0$ é análogo.

Observação 1.3.40 A prova do lema 1.3.39 mostra que se $T \in \mathscr{C} \mathscr{F}\left(\mathrm{H}, \mathrm{H}^{\prime}\right)$ tem índice $\leq 0(\geq 0)$ então existe um operador $K$ de posto finito tal que $T+K$ é injetor (sobrejetor).

ObSERVAÇão 1.3.41 Se $T \in \mathscr{F}\left(\mathrm{H}, \mathrm{H}^{\prime}\right)$ tem índice zero e $K$ é o operador construído na observação 1.3.40, então $\mathbb{R} \ni t \mapsto T(t)=T+t K \in \mathscr{F}\left(\mathrm{H}, \mathrm{H}^{\prime}\right)$ é uma curva em $\mathscr{L}(\mathrm{H})$ tal que $T(t)$ é inversível para $t \neq 0$. Em particular, GL(H) é denso no conjunto dos operadores Fredholm de índice zero. Um raciocínio semelhante prova que $\mathrm{GL}(H) \cap \mathscr{L}^{\text {sa }}(H)$ é denso em $\left\{T \in \mathscr{L}^{\text {sa }}(H): \gamma(T)>0\right\}$.

A próxima proposição mostra que existe uma decomposição de um operador fechado densamente definido análoga à decomposição polar $z=|z| e^{i \theta}$ de um número complexo. Antes, uma definição.

Definição 1.3.42 Uma isometria parcial é um operador $U$ tal que $\|U v\|=\|v\|$, para todo $v \in$ $(\operatorname{ker} U)^{\perp}$. Os subespaços $(\operatorname{ker} U)^{\perp}$ e $\operatorname{Im} U$ são chamados de subespaços inicial e final de $U$, respectivamente.

Proposição 1.3.43 (Decomposição Polar) Se $T \in \mathscr{C}\left(\mathrm{H}, \mathrm{H}^{\prime}\right)$, então existe um operador auto-adjunto positivo denotado por $|T|$ e uma isometria parcial $U$ tal que $T=U|T|$. Se T é limitado, então $|T|$ e $U$ também são limitados.

Prova. O operador $T^{*} T$ é auto-adjunto, pois $\left(I+T^{*} T\right)^{-1}$ é auto-adjunto limitado, conforme o lema 1.2.27 $27^{21}$. Além disso, $T^{*} T$ é positivo. Logo, existe a raiz quadrada $|T|=\left(T^{*} T\right)^{1 / 2}$. Temos

\footnotetext{
${ }^{21}$ Observamos que se $S$ é um operador auto-adjunto bijetor limitado em $\mathrm{H}$, então $S^{-1}$ é auto-adjunto (possivelmente ilimitado).
} 
que $|T|$ é auto-adjunto positivo e

$$
\||T| u\|^{2}=\langle|T| u,|T| u\rangle=\left\langle|T|^{2} u, u\right\rangle=\left\langle T^{*} T u, u\right\rangle=\langle T u, T u\rangle=\|T u\|^{2},
$$

para $u \in \mathrm{D}(T)$. Definimos $U$ em $\operatorname{Im}|T|$ como $U(|T| u)=T u$. Como $U$ é uma isometria (pela equação 1.3.15), $U$ se estende continuamente a $\overline{\operatorname{Im}|T|}$. Definimos $U$ como zero em $(\operatorname{Im}|T|)^{\perp}=\operatorname{ker}|T|=\operatorname{ker} T$. Logo, $U$ é uma isometria parcial tal que $T=U|T|$.

ObSERvação 1.3.44 A prova da proposição 1.3.43 mostra que $\operatorname{ker} U=\operatorname{ker} T$. Esta condição é suficiente para garantir a unicidade de $U$, i.e., existem únicos $U$ e $A \operatorname{com} T=U A, A$ positivo e $U$ isometria parcial com $\operatorname{ker} U=\operatorname{ker} T$. Toda vez que falarmos sobre $a$ decomposição polar de um operador fechado, subentenderemos estar se tratando desta decomposição única.

Observação 1.3.45 Se $U$ é uma isometria parcial, então $U^{*} U=P_{(\operatorname{ker} U)^{\perp}}$ e $U U^{*}=P_{\operatorname{Im} U}$. De fato, dados $u, v \in \mathrm{H}$, pondo $u=u_{0}+u_{0}^{\perp}$ e $v=v_{0}+v_{0}^{\perp}, \operatorname{com} u_{0}, v_{0} \in \operatorname{ker} U$ e $u_{0}^{\perp}, v_{0}^{\perp} \in(\operatorname{ker} U)^{\perp}$, temos que

$$
\left\langle U^{*} U u, v\right\rangle=\langle U u, U v\rangle=\left\langle U u_{0}^{\perp}, U v_{0}^{\perp}\right\rangle=\left\langle u_{0}^{\perp}, v_{0}^{\perp}\right\rangle=\left\langle P_{(\operatorname{ker} u)^{\perp}} u, v\right\rangle,
$$

sendo que a terceira igualdade decorre do fato que $U$ é isometria em $(\operatorname{ker} U)^{\perp}$. A igualdade referente à $U U^{*}$ prova-se de maneira análoga. Vale também uma recíproca deste fato: se $U$ é um operador limitado tal que $U^{*} U$ e $U U^{*}$ são projeções, então $U$ é uma isometria parcial.

Observação 1.3.46 Se $T \in \mathrm{GL}_{c}(\mathrm{H})$ e $T=U|T|$ é a decomposição polar de $T$ então $U \in U_{c}(\mathrm{H})$ e $|T| \in \mathrm{GL}_{c}(\mathrm{H})$ é positivo auto-adjunto. De fato, a raiz quadrada $|T|=\left(T^{*} T\right)^{1 / 2}$ pode ser obtida como limite da seqüência $\left\{T_{n}\right\}$ definida como $T_{0}=0$ e $T_{n+1}=\frac{1}{2}\left(T^{*} T+T_{n}^{2}\right)$ (veja [82], Capítulo VII). Vemos, por indução, que $|T| \in \mathrm{GL}_{c}(\mathrm{H})$ se $T \in \mathrm{GL}_{c}(\mathrm{H})$ e portanto, $U \in \mathrm{U}_{c}(\mathrm{H})$.

A proposição 1.3.43 é de grande importância para nossos propósitos, pois reduz o estudo de um operador fechado qualquer ao estudo de operadores positivos e isometrias parciais.

Proposição 1.3.47 Se $A \in \mathscr{C} \mathscr{F}$ sa $(\mathrm{H})$ é positivo, então existe uma curva contínua de operadores $\{A(t)\}_{0 \leq t \leq 1}$, em $\mathscr{C} \mathscr{F}^{\mathrm{sa}}(\mathrm{H})$ tal que $A(0)=A$ e $A(1)=I$. Além disso, $A(t)$ é limitado e bijetor 22 para todo $t \neq 0$.

Prova. Pelo lema 1.3.39, podemos supor que $\operatorname{ker} A=0$. Como $A$ é Fredholm auto-adjunto, temos que $\operatorname{Im} A$ é fechada, portanto, $A$ é bijetor. Logo, existe $\alpha>0$ tal que $\langle A u, u\rangle \geq \alpha\langle u, u\rangle$, para $u \in \mathrm{D}(A)$. Podemos supor $\alpha=1$.

Sejam $f_{t}$ a função com gráfico

\footnotetext{
${ }^{22}$ i.e., $A$ é injetor com imagem $\mathrm{H}$
} 


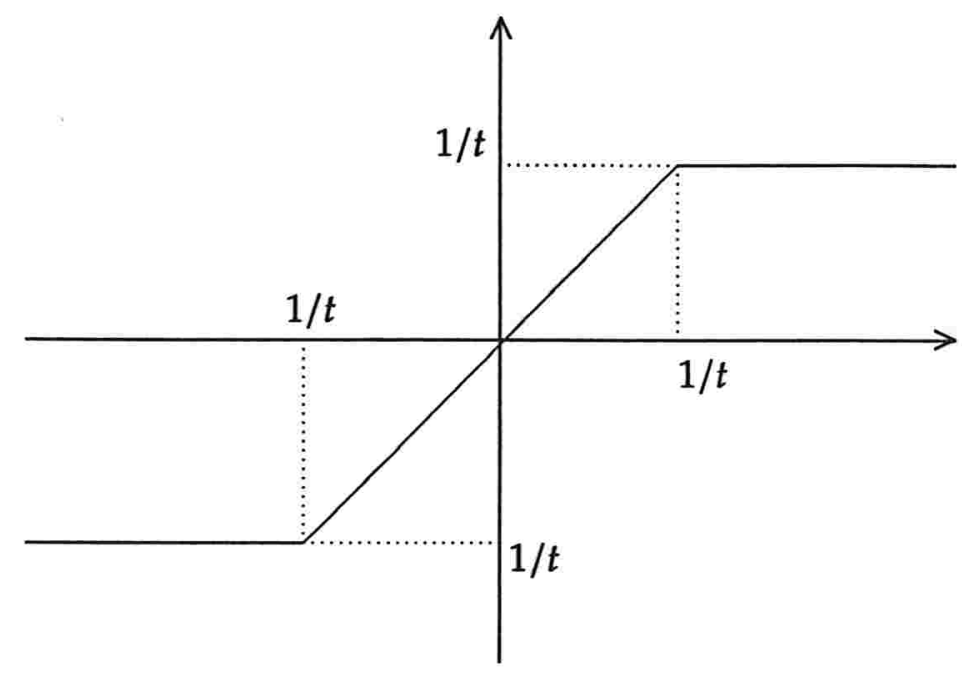

e

$$
A(t)=\int_{1}^{\infty} f_{t}(\lambda) d E_{\lambda},
$$

definido para $t \in(0,1]$, onde $\left\{E_{\lambda}\right\}_{\lambda \in \mathbb{R}}$ denota a família espectral de $A$. Definimos $A(0)=A$. Vemos que $A(1)=I$ e $A(t)$ é auto-adjunto (pois $f_{t}$ é real). Além disso, $\operatorname{ker} A(t)=0$ e $\operatorname{Im} A(t)$ é fechada, pois

$$
\|A(t) u\|^{2}=\int_{1}^{\infty}\left|f_{t}(\lambda)\right|^{2} d\left\|E_{\lambda}(u)\right\|^{2} \geq\|u\|^{2} .
$$

Como $A(t)$ é auto-adjunto, segue que $A(t)$ é bijetor para todo $t \neq 0$. Resta verificar que $A(t)$ é contínua em $\mathscr{C}(\mathrm{H})$; para isso, usaremos a caracterização da métrica $\hat{\delta}$ dada na proposição 1.2.29. Temos que

e

$$
R(A(t))=\int_{1}^{\infty} \frac{1}{1+f_{t}(\lambda)^{2}} d E_{\lambda}
$$

$$
A(t) R(A(t))=\int_{1}^{\infty} \frac{f_{t}(\lambda)}{1+f_{t}(\lambda)^{2}} d E_{\lambda} .
$$

Como $f_{t}(\lambda)$ é contínua em $t$ e $\lambda$ e limitada em cada conjunto da forma

$$
\{(t, \lambda): 0<a \leq t \leq b, \lambda \in \mathbb{R}\},
$$

segue pelo teorema da convergência dominada que $R(A(t))$ e $A(t) R(A(t))$ são contínuas em norma. Pela proposição 1.2.29, concluímos o desejado.

$\mathrm{O}$ argumento utilizado no final da prova da proposição 1.3 .47 pode ser repetido em situações bem mais gerais. Vamos destacar este fato no próximo lema.

Lema 1.3.48 Sejam A um operador auto-adjunto em $\mathrm{H},\left\{E_{\lambda}\right\}_{\lambda \in \mathbb{R}}$ a família espectral de A e $f:(x, \lambda) \ni$ $X \times \mathbb{R} \mapsto f(x, \lambda) \in \mathbb{C} \cup\{\infty\}$ contínua em $x \in I$ e mensurável em $\lambda$, com $X$ qualquer espaço topológico. Definindo ${ }^{23}$

$$
T(x)=\int_{\mathbb{R}} f(x, \lambda) d E_{\lambda},
$$

\footnotetext{
${ }^{23}$ Issio significa que para cada $x \in X$ fixo, $\lambda \mapsto f(x, \lambda)$ é mensurável e $x \mapsto f(x, \lambda)$ é contínua, para todo $\lambda \in \mathbb{R}$ fixado, com a convenção usual de que as vizinhanças abertas básicas de $\infty$ são conjuntos da forma $\{z \in \mathbb{C}:|z|>r\}$, com $r>0$.
} 
temos que $T(x)$ é um operador fechado para cada $x \in X$ e a aplicação $T: X \ni x \mapsto T(x) \in \mathscr{C}(\mathrm{H})$ é continua.

Prova. Basta observar que as funções $R(T(x))$ e $T(x) R(T(x))$ são contínuas em $x$, à valores em $\mathscr{L}(\mathrm{H})$. Isso decorre imediatamente das fórmulas 1.3.16, 1.3.17 (com $f(x, \lambda)$ em lugar de $f_{t}(\lambda)$ ), do teorema da convergência dominada e das estimativas óbvias

$$
\frac{1}{1+|f(x, \lambda)|^{2}} \leq 1 \quad \text { e } \quad \frac{|f(x, \lambda)|}{1+|f(x, \lambda)|^{2}}=\frac{1}{\left(1+|f(x, \lambda)|^{2}\right)^{1 / 2}} \frac{|f(x, \lambda)|}{\left(1+|f(x, \lambda)|^{2}\right)^{1 / 2}} \leq 1 \cdot 1=1 .
$$

A proposição a seguir mostra que um operador Fredholm injetor pode ser conectado por uma curva de operadores de Fredholm à uma isometria.

Proposição 1.3.49 Seja $T \in \mathscr{C} \mathscr{F}\left(\mathrm{H}, \mathrm{H}^{\prime}\right)$ um operador injetor. Então, existe uma curva contínua de operadores Fredholm ligando $T$ e a isometria U obtida na proposição 1.3.43.

Prova. Como $\operatorname{ker}|T|=\operatorname{ker} T=0$, então $|T|$ é um operador positivo injetor. Pela proposição 1.3.47, existe uma curva $A(t), 0 \leq t \leq 1$, de operadores Fredholm auto-adjuntos (de fato, bijeções de $\mathrm{H}$ ) tal que $A(0)=|T|$ e $A(1)=I$. Definindo $T(t)=U A(t)$, temos que $T(0)=T$ e $T(1)=U$. Vejamos que $T(t)$ é contínua. Temos que $R(T(t))=R(A(t))$, pois como ker $U=$ $\operatorname{ker} T=0$, pela observação 1.3.44, segue que $U^{*} U=I$, logo, $T(t)^{*} T(t)=A(t)^{*} A(t)$. Além disso, $T(t) R(T(t))=U A(t) R(A(t)) \mathrm{e}$

$$
I+T(t) T(t)^{*}=U\left(I+A(t) A(t)^{*}\right) U^{*}+P_{\operatorname{ker} U^{*}} .
$$

Assim, $\left.\left(I+T(t) T(t)^{*}\right)\right|_{\operatorname{ker} U^{*}}=I$ e $\left.\left(I+T(t) T(t)^{*}\right)\right|_{\operatorname{Im} U}=\left.U\left(I+A(t) A(t)^{*}\right) U^{*}\right|_{\operatorname{Im} U}$, portanto, $R\left(T(t)^{*}\right)=$

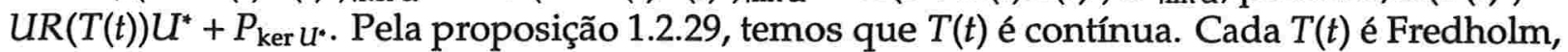
pois $A(t)$ é isomorfismo, $\operatorname{ker} U=\operatorname{ker} T$ e $\operatorname{Im} U=\operatorname{Im}|T|=\mathrm{H}$.

O próximo lema é o último passo para provar que o índice de Fredholm classifica as componentes conexas do espaço de operadores Fredholm.

Lema 1.3.50 Se $U_{0}, U_{1}: \mathrm{H} \rightarrow \mathrm{H}^{\prime}$ são isometrias tais que def $U_{0}=\operatorname{def} U_{1}$ então existe uma curva contínua $U(t), 0 \leq t \leq 1$, em $\mathscr{L}\left(\mathrm{H}, \mathrm{H}^{\prime}\right)$ tal que $U(0)=U_{0}$ e $U(1)=U_{1}$.

Prova. Seja $L:\left(\operatorname{Im} U_{0}\right)^{\perp} \rightarrow\left(\operatorname{Im} U_{1}\right)^{\perp}$ um isomorfismo unitário, o qual existe por hipótese. Definindo

$$
U=\left\{\begin{array}{ll}
U_{1} U_{0}^{-1} & \text { em } \operatorname{Im} U_{0} \\
L & \text { em }\left(\operatorname{Im} U_{0}\right)^{\perp}
\end{array},\right.
$$

temos que $U$ é um operador unitário. Pelo corolário 1.6 .7 da subseção $1.6 .1, \mathrm{U}(\mathrm{H})$ é conexo por caminhos, portanto existe uma curva contínua $U(t)$ em $U(H)$ tal que $U(0)=U$ e $U(1)=I$. Como $U_{1}=U U_{0}$, temos que $U(t) U_{0}$ é uma curva contínua conectando $U_{1}$ e $U_{0}$.

Teorema 1.3.51 Sejam $S, T \in \mathscr{C} \mathscr{F}\left(\mathrm{H}, \mathrm{H}^{\prime}\right)$. Existe uma curva contínua conectando $T$ e $S$ se e só se ind $T=$ ind $S$. Em particular, as componentes conexas (por caminhos) de $\mathscr{C} \mathscr{F}\left(\mathrm{H}, \mathrm{H}^{\prime}\right)$ e $\mathscr{F}\left(\mathrm{H}, \mathrm{H}^{\prime}\right)$ são os conjuntos 1.3.14, respectivamente. 
Prova. Obviamente, a existência de uma tal curva implica a igualdade dos índices, pelo teorema 1.3.38.

Reciprocamente, dado $T, S \in \mathscr{C} \mathscr{F}\left(\mathrm{H}, \mathrm{H}^{\prime}\right)$, podemos supor que ind $T=$ ind $S \leq 0$, senão trocamos $T, S$ por $T^{*}, S^{*}$. Pelo lema 1.3.39, podemos supor que $\operatorname{ker} T=\operatorname{ker} S=0$. Escrevendo as decomposições polares $T=U|T|$ e $S=V|S|$ de $S, T$, respectivamente, a proposição 1.3.49 mostra que existem curvas conectando $T$ e $U, S$ e $V$. Como def $U=-$ ind $T=-$ ind $S=\operatorname{def} V$, existem curvas em $\mathscr{C} \mathscr{F}\left(\mathrm{H}, \mathrm{H}^{\prime}\right)$ conectando $U$ e $V$, pela proposição 1.3.50, e isso encerra a prova do teorema.

Para encerrar esta seção, vamos provar que o índice de Fredholm é invariante também por perturbações relativas. Este tipo de perturbação não necessariamente é pequena com relação à métrica $\hat{\delta}$.

Proposição 1.3.52 Sejam $T \in \mathscr{C} \mathscr{F}\left(\mathrm{H}, \mathrm{H}^{\prime}\right)$ e A um operador qualquer entre $\mathrm{H} e \mathrm{H}^{\prime}$.

1. Se Té (semi) Fredholm e A é T-limitado com $2^{1 / 2} \max (a, b)<\gamma(T) /\left(1+\gamma(T)^{2}\right)^{1 / 2}$, então $T+A$ $e ́$ (semi) Fredholm, $\operatorname{nul}(T+A) \leq \operatorname{nul} T, \operatorname{def}(T+A) \leq \operatorname{def} T e$ ind $(T+A)=\operatorname{ind} T{ }^{24}$

2. Se Té (semi) Fredholm e Aé $T$-compacto então $T+A$ é (semi) Fredholm e ind $(T+A)=$ ind $T$.

Prova. Se $A$ satisfaz a hipótese do primeiro ítem, então

$$
\begin{aligned}
\|A u\| & \leq a\|u\|+b\|T u\| \\
& \leq \max (a, b)(\|u\|+\|T u\|) \\
& \leq 2^{1 / 2} \max (a, b)\|u\|_{T},
\end{aligned}
$$

usando a notação da observação 1.2.42. Por outro lado, pela definição de $\langle\cdot, \cdot\rangle_{T}$, temos que $\gamma(\hat{T})=\gamma(T) /\left(1+\gamma(T)^{2}\right)^{1 / 2}$, onde $\hat{T}$ denota o operador $T$ definido em $\mathrm{H}_{T}$. O resultado decorre do teorema 1.3.9.

Para o segundo ítem, basta observar que, usando a notação da observação 1.2.42, $\hat{A}$ é compacto, e portanto, a afirmação decorre da proposição 1.3.3 e da observação $1.3 .7 .{ }^{25}$

\subsubsection{Pares comensuráveis de subespaços}

Nesta subseção introduziremos uma propriedade de pares de subespaços que, em certo sentido, é mais forte do que a propriedade de ser Fredholm. Boas referências para o assunto são [1], [3].

Definição 1.3.53 Dados subespaços fechados $\mathrm{M}, \mathrm{N}$ de $\mathrm{H}$, dizemos que $\mathrm{M}, \mathrm{N}$ são comensuráveis se $P_{\mathrm{M}}-P_{\mathrm{N}}$ é compacto. Neste caso, $\mathrm{M}$ é dito uma perturbação compacta de $\mathrm{N}$ e este fato é denotado por $\mathrm{M} \sim \mathrm{N}$.

Observação 1.3.54 Como $P_{\mathrm{M}}-P_{\mathrm{N}}=P_{\mathrm{M}^{1}} P_{\mathrm{N}^{\perp}}-P_{\mathrm{M}^{\perp}} P_{\mathrm{N}}, P_{\mathrm{M}} P_{\mathrm{N}^{\perp}}=\left(P_{\mathrm{M}}-P_{\mathrm{N}}\right) P_{\mathrm{N}^{\perp}}$ e $P_{\mathrm{M}^{\perp}} P_{\mathrm{N}}=P_{\mathrm{M}^{\perp}}\left(P_{\mathrm{N}^{-}}-\right.$ $\left.P_{\mathrm{M}}\right)$, vemos que $\mathrm{M}, \mathrm{N}$ são comensuráveis se e só se $P_{\mathrm{M}} P_{\mathrm{N}^{\perp}}$ e $P_{\mathrm{M}^{\perp}} P_{\mathrm{N}}$ são compactos.

\footnotetext{
${ }^{24}$ Esta não é a melhor estimativa que se pode obter; em [55], Pág.236, Kato obtém o mesmo resultado com a estimativa mais fraca $a<(1-b) \gamma(T)$.

${ }^{25} \mathrm{E}$ interessante observar que as afirmações sobre índices, nulidades e deficiências decorrem do teorema 1.3.9 e do fato que tais números não dependem da topologia dos espaços $\mathrm{H}, \mathrm{H}^{\prime}$.
} 
Não é difícil verificar que é uma relação de equivalência entre subespaços fechados de $\mathrm{H}$ e $\mathrm{M} \sim \mathrm{N}$ se e só se $\mathrm{M}^{\perp} \sim \mathrm{N}^{\perp}$. A próxima definição segue a mesma linha da definição 1.3.53.

Definição 1.3.55 Dados $M, N$ subespaços fechados de $H$, definimos $M \approx N$ se e só se $\left(M, N^{\perp}\right)$ é um par Fredholm. $\mathrm{O}$ inteiro ind $\left(\mathrm{M}, \mathrm{N}^{\perp}\right)$ é chamado de dimensão relativa de $\mathrm{M}, \mathrm{N}$ e denotado por $\operatorname{dim}(\mathrm{M}, \mathrm{N})$.

ObSERVAÇÃo 1.3.56 Pela definição 1.3.55 e pela proposição 1.3 .18 , temos que $\operatorname{dim}(M, N)=$ ind $\left(P_{N}^{M}\right)$. Além disso, $\operatorname{dim}(M, N)=-\operatorname{dim}(N, M)$, pela proposição 1.2.19.

OBSERVAÇÃo 1.3.57 Se $\operatorname{dim} M, \operatorname{dim} N<\infty(\operatorname{codim} M, \operatorname{codim} N<\infty)$ então claramente $M \sim N$ e $\operatorname{dim}(M, N)=\operatorname{dim} M-\operatorname{dim} N(\operatorname{dim}(M, N)=\operatorname{codim} M-\operatorname{codim} N)$.

Ao contrário da relação , agora já não é tão evidente que é uma relação de equivalência. A próxima proposição prova este fato.

Proposição 1.3 .58 A relação $\approx$ é de equivalência.

Prova. A reflexividade é imediata. A simetria segue da proposição 1.2.19. Para verificar a transitividade, suponhamos que $L \approx M \approx N$. Temos $P_{L^{\perp}}^{N^{\perp}}-P_{L^{\perp}}^{M^{\perp}} P_{M^{\perp}}^{N^{\perp}}=P_{L^{\perp}} P_{M}^{N^{\perp}}$ e $\operatorname{ker}\left(P_{L^{\perp}} P_{M}^{N^{\perp}}\right)=$ $\left(L+M^{\perp}\right) \cap N^{\perp}$. Mas o último subespaço tem codimensão finita em $N^{\perp}$, pelo ítem 4 do lema 1.3.17, pois

$$
\left(\left(L+M^{\perp}\right) \cap N^{\perp}\right)^{\perp} \cap N^{\perp}=\left(\left(L^{\perp} \cap M\right)+N\right) \cap N^{\perp} .
$$

Em particular, $P_{L^{\perp}}^{N^{\perp}}$ é uma perturbação compacta do operador Fredholm $P_{L^{\perp}}^{\mathrm{M}^{\perp}} P_{\mathrm{M}^{\perp}}^{\mathrm{N}^{\perp}}$. Pelo ítem 2 da proposição 1.3.52 $P_{\mathrm{L}^{\perp}}^{\mathrm{N}^{\perp}}$ é um operador Fredholm com mesmo índice que $P_{\mathrm{L}^{\perp}}^{\mathrm{M}^{\perp}} P_{\mathrm{M}^{\perp}}^{\mathrm{N}^{\perp}}$, portanto, pela proposição 1.3.18 e pelo teorema 1.3.8, temos que $L \approx N$ e $\operatorname{dim}(L, N)=$ ind $\left(P_{L^{\perp}}^{N^{\perp}}\right)=$ ind $\left(P_{L^{\perp}}^{\mathrm{M}^{\perp}} P_{\mathrm{M}^{\perp}}^{\mathrm{N}^{\perp}}\right)=\operatorname{ind}\left(P_{\mathrm{L}^{\perp}}^{\mathrm{M}^{\perp}}\right)+\operatorname{ind}\left(P_{\mathrm{M}^{\perp}}^{\mathrm{N}^{\perp}}\right)=\operatorname{dim}(\mathrm{L}, \mathrm{M})+\operatorname{dim}(\mathrm{M}, \mathrm{N})$.

Corolário 1.3.59 Se $L \approx M \approx N$ então $\operatorname{dim}(L, N)=\operatorname{dim}(L, M)+\operatorname{dim}(M, N)$.

A próxima proposição mostra a relação entre pares comensuráveis e pares Fredholm de subespaços.

Proposição 1.3.60 Se $\mathrm{M} \sim \mathrm{N}$ então $\mathrm{M} \approx \mathrm{N}$, i.e., se $(\mathrm{M}, \mathrm{N})$ é um par comensurável, então $\left(\mathrm{M}, \mathrm{N}^{\perp}\right)$ é um par Fredholm.

Prova. Se $\mathrm{M}, \mathrm{N}$ são comensuráveis, então $P_{\mathrm{M}}-P_{\mathrm{N}}$ é compacto, logo, $P_{\mathrm{M}}+P_{\mathrm{N}^{1}}=I+\left(P_{\mathrm{M}}-P_{\mathrm{N}}\right)$ é uma perturbação compacta da identidade, em particular, $P_{M}+P_{N^{1}}$ é um operador Fredholm. Pela observação 1.3.25, $\mathrm{M} \cap \mathrm{N}^{\perp}=\operatorname{ker}\left(P_{\mathrm{M}}+P_{\mathrm{N}^{\perp}}\right)=\operatorname{ker}\left(I+\left(P_{\mathrm{M}}-P_{\mathrm{N}}\right)\right)$, $\operatorname{logo} \operatorname{dim}\left(\mathrm{M} \cap \mathrm{N}^{\perp}\right)<\infty$, pelo lema 1.3.4. Pelo ítem 1 do teorema 1.3.30, concluímos que $\left(\mathrm{M}, \mathrm{N}^{\perp}\right)$ é um par Fredholm.

Em geral, não é verdade que se $\left(\mathrm{M}, \mathrm{N}^{\perp}\right)$ é um par Fredholm então $\mathrm{M}, \mathrm{N}$ são comensuráveis, como veremos no próximo exemplo.

Exemplo 1.3.61 Consideremos no espaço de Hilbert $\mathrm{H} \oplus \mathrm{H}$ os subespaços $\mathrm{M}=\mathrm{H} \oplus 0$ e $\mathrm{N}=$ $\{(u, u): u \in H\}$ a diagonal. Temos que $M \cap N^{\perp}=0$ e $M+N^{\perp}=H$, logo $\left(M, N^{\perp}\right)$ é um par Fredholm. Mas $P_{\mathrm{M}} P_{\mathrm{N}}(u, v)=(u+v) / 2$, que não é um operador compacto. 
ObSERvação 1.3.62 O exemplo 1.3.61 mostra que o corolário 2.7 de [26] é falso. A versão correta para este corolário é o teorema 1.3.30. O referido corolário foi utilizado em [69] para provar que, no contexto simplético, o espaço $\mathscr{F}_{\mathrm{L}}(\Lambda)$ tem o tipo de homotopia de $\mathrm{U}(\infty) / \mathrm{O}(\infty)$, conforme notação da subseção 1.6.2. Uma versão corrigida deste resultado é obtida na referida seção.

Proposição 1.3.63 Se (L, N) é um par Fredholm de subespaços e M é uma perțurbação compacta de $L$ então $(M, N)$ é um par Fredholm e ind $(M, N)=\operatorname{ind}(L, N)+\operatorname{dim}(M, L)$.

Prova. Temos que

$$
P_{\mathrm{N}^{\perp}}^{\mathrm{M}}=P_{\mathrm{N}^{\perp}}^{\mathrm{L}} P_{\mathrm{L}}^{\mathrm{M}}+\left.P_{\mathrm{N}^{\perp}}\left(P_{\mathrm{M}}-P_{\mathrm{L}}\right)\right|_{\mathrm{M}} .
$$

Pelo teorema 1.3.8, a primeira parcela da soma acima é Fredholm; por hipótese, a segunda parcela é compacta, logo, pelo segundo ítem da proposição 1.3.52, $P_{\mathrm{N}^{\perp}}^{\mathrm{M}}$ é Fredholm e

$$
\operatorname{ind}(\mathrm{M}, \mathrm{N})=\operatorname{ind}\left(P_{\mathrm{N}^{1}}^{\mathrm{M}}\right)=\operatorname{ind}\left(P_{\mathrm{N}^{1}}^{\mathrm{L}} P_{\mathrm{L}}^{\mathrm{M}}\right)=\operatorname{ind}\left(P_{\mathrm{N}^{1}}^{\mathrm{L}}\right)+\operatorname{ind}\left(P_{\mathrm{L}}^{\mathrm{M}}\right)=\operatorname{ind}(\mathrm{L}, \mathrm{N})+\operatorname{dim}(\mathrm{M}, \mathrm{L}) \text {. }
$$

Vamos agora provar que um par Fredholm é, a menos de uma perturbação compacta, um par de subespaços complementares. Este resultado simples é muito importante no estudo da topologia de certas variedades grassmannianas, como veremos na subseção 1.6.3.

Proposição 1.3.64 Seja (M, N) um par Fredholm. Então existe $\mathrm{N}^{\prime}$ comensurável com $\mathrm{N}$ tal que $\left(\mathrm{M}, \mathrm{N}^{\prime}\right)$ é um par de subespaços complementares.

Prova. Sejam $S=(M+N)^{\perp}$ e $N_{0}=N+S$. Temos que nul $(M, N)=\operatorname{nul}\left(M, N_{0}\right)$ e $\operatorname{def}\left(M, N_{0}\right)=0$. Além disso, como dim $S<\infty$, temos que $\operatorname{Im}\left(P_{\mathrm{N}}+P_{\mathrm{S}}\right)=\mathrm{N}+\mathrm{S}=\mathrm{N}_{0}$, pela proposição 1.3.23, $\left(P_{\mathrm{N}}+P_{\mathrm{S}}\right)^{*}=P_{\mathrm{N}}+P_{\mathrm{S}}$ e $\left(P_{\mathrm{N}}+P_{\mathrm{S}}\right)^{2}=P_{\mathrm{N}}+P_{\mathrm{S}}, \log$, $P_{\mathrm{N}_{0}}=P_{\mathrm{N}}+P_{\mathrm{S}}$. Em particular, $\mathrm{N}_{0}$ é perturbação compacta de $\mathrm{N}$.

Podemos agora supor que $\operatorname{def}(M, N)=0$. Pondo $N^{\prime}=N \cap(M \cap N)^{\perp}$, temos que nul $\left(M, N^{\prime}\right)=0$ e $\operatorname{def}\left(\mathrm{M}, \mathrm{N}^{\prime}\right)=0$. De fato, a primeira igualdade é óbvia e a segunda decorre do ítem 1 do lema 1.3.26. Além disso, o ítem 4 do referido lema mostra que $\left.\left(P_{N^{\prime}}-P_{N}\right)\right|_{(M \cap N)^{\perp}}=0$, portanto, $P_{N^{\prime}}=P_{\mathrm{N}}+K, \operatorname{com} K$ de posto finito, logo $\mathrm{N}^{\prime}$ é perturbação compacta de $\mathrm{N}$.

Ainda não temos ferramentas adequadas para construir exemplos de pares comensuráveis de subespaços. Por exemplo, dado um subespaço fechado $\mathrm{M}$ e $\mathrm{K}: \mathrm{H} \rightarrow \mathrm{H}$ um operador compacto, não é inteiramente óbvio que $\mathrm{M} \sim(I+K)(\mathrm{M})$. O próximo teorema nos fornece uma maneira simples de construir pares comensuráveis de subespaços.

Teorema 1.3.65 Sejam $T_{1}, T_{2} \in \mathscr{L}\left(\mathrm{H}, \mathrm{H}^{\prime}\right)$ tais que $\operatorname{Im} T_{1}, \operatorname{Im} T_{2}$ são fechadas e $\mathrm{K} \doteq T_{1}-T_{2}$ é compacto. Então $\operatorname{ker} T_{1} \sim \operatorname{ker} T_{2}, \operatorname{Im} T_{1} \sim \operatorname{Im} T_{2} e$

$$
\operatorname{dim}\left(\operatorname{Im} T_{2}, \operatorname{Im} T_{1}\right)=-\operatorname{dim}\left(\operatorname{ker} T_{2}, \operatorname{ker} T_{1}\right) .
$$

Prova. Pela proposição 1.3.2, existem $S_{1}, S_{2} \in \mathscr{L}\left(\mathrm{H}^{\prime}, \mathrm{H}\right)$ tais que

$$
\begin{array}{cc}
T_{1} S_{1}=P_{\operatorname{Im} T_{1}} & T_{2} S_{2}=P_{\operatorname{Im} T_{2}} \\
S_{1} T_{1}=P_{\left(\operatorname{ker} T_{1}\right)^{\perp}} & S_{2} T_{2}=P_{\left(\operatorname{ker} T_{2}\right)^{\perp}}
\end{array}
$$


Logo,

$$
\begin{array}{r}
P_{\left(\operatorname{ker} T_{1}\right)^{\perp}} P_{\text {ker } T_{2}}=S_{1} T_{1} P_{\text {ker } T_{2}}=S_{1} T_{2} P_{\text {ker } T_{2}}+S_{1} K P_{\text {ker } T_{2}}=S_{1} K P_{\operatorname{ker} T_{2}} \text { e } \\
P_{\left(\operatorname{Im} T_{1}\right)^{\perp}} P_{\operatorname{Im} T_{2}}=P_{\left(\operatorname{Im} T_{1}\right)^{\perp}} T_{2} S_{2}=P_{\left(\operatorname{Im} T_{1}\right)^{\perp}} T_{1} S_{2}-P_{\left(\operatorname{Im} T_{1}\right)^{\perp}} K S_{2}=-P_{\left(\operatorname{Im} T_{1}\right)^{\perp} K S_{2}} .
\end{array}
$$

Fórmulas semelhantes valem para $P_{\text {ker } T_{1}} P_{\left(\operatorname{ker} T_{2}\right)^{\perp}}$ e $P_{\operatorname{Im} T_{1}} P_{\left(\operatorname{Im} T_{2}\right)^{\perp}}$, e portanto, pela observação 1.3.54, segue que $\operatorname{ker} T_{1} \sim \operatorname{ker} T_{2}$ e $\operatorname{Im} T_{1} \sim \operatorname{Im} T_{2}$. Temos que $\left(\operatorname{ker} T_{1}\right)^{\perp} \sim\left(\operatorname{ker} T_{2}\right)^{\perp} \mathrm{e}$ $\operatorname{dim}\left(\left(\operatorname{ker} T_{2}\right)^{\perp},\left(\operatorname{ker} T_{1}\right)^{\perp}\right)=-\operatorname{dim}\left(\operatorname{ker} T_{2}, \operatorname{ker} T_{1}\right)$. Como

$$
\left.T_{1}\right|_{\left(\operatorname{ker} T_{1}\right)^{\perp}}:\left(\operatorname{ker} T_{1}\right)^{\perp} \rightarrow \operatorname{Im} T_{1} \text { e }\left.T_{2}\right|_{\left(\operatorname{ker} T_{2}\right)^{\perp}}:\left(\operatorname{ker} T_{2}\right)^{\perp} \rightarrow \operatorname{Im} T_{2}
$$

são isomorfismos, concluímos que $T_{1} P_{\left(\operatorname{ker} T_{1}\right)^{\perp}}^{\left(\operatorname{ker} T_{2}\right.},\left.P_{\operatorname{Im} T_{1}}^{\operatorname{Im} T_{2}} T_{2}\right|_{\left(\operatorname{ker} T_{2}\right)^{\perp}}:\left(\operatorname{ker} T_{2}\right)^{\perp} \rightarrow \operatorname{Im} T_{1}$ são Fredholm de índices $\operatorname{dim}\left(\left(\operatorname{ker} T_{2}\right)^{\perp},\left(\operatorname{ker} T_{1}\right)^{\perp}\right)$ e $\operatorname{dim}\left(\operatorname{Im} T_{2}, \operatorname{Im} T_{1}\right)$, respectivamente. Além disso,

$$
\begin{aligned}
T_{1} P_{\left(\operatorname{ker} T_{1}\right)^{\perp}}^{\left(\operatorname{ker} T_{2}\right.}-P_{\operatorname{Im} T_{1}}^{\operatorname{Im} T_{2}} T_{2} & =T_{1}\left(I+\left(P_{\left(\operatorname{ker} T_{1}\right)^{\perp}}-P_{\left(\operatorname{ker} T_{2}\right)^{\perp}}\right)\right)-\left(I+\left(P_{\operatorname{Im} T_{1}}-P_{\operatorname{Im} T_{2}}\right)\right) T_{2} \\
& =T_{1}\left(P_{\left(\operatorname{ker} T_{1}\right)^{\perp}}-P_{\left(\operatorname{ker} T_{2}\right)^{\perp}}\right)+K+\left(P_{\operatorname{Im} T_{1}}-P_{\operatorname{Im} T_{2}}\right) T_{2} .
\end{aligned}
$$

Como o operador em 1.3.19 é compacto, o resultado segue.

Corolário 1.3.66 Se $\mathrm{M} \sim \mathrm{N} e U \in \mathrm{GL}(\mathrm{H})$, então $U(\mathrm{M}) \sim U(\mathrm{~N}) e \operatorname{dim}(U(\mathrm{M}), U(\mathrm{~N}))=\operatorname{dim}(\mathrm{M}, \mathrm{N})$. $O$ mesmo é verificado sob a hipótese que $\left.U\right|_{M},\left.U\right|_{N}$ são injetores e têm imagem fechada.

Prova. Como $U(\mathrm{M})=\operatorname{Im} U P_{\mathrm{M}}$ e $U(\mathrm{~N})=\operatorname{Im} U P_{\mathrm{N}}$ e $U P_{\mathrm{M}}-U P_{\mathrm{N}}=U\left(P_{\mathrm{M}}-P_{\mathrm{N}}\right)$, o teorema 1.3.65 implica que $U(\mathrm{M}) \sim U(\mathrm{~N})$ e

$$
\operatorname{dim}(U(\mathrm{M}), U(\mathrm{~N}))=\operatorname{dim}\left(\operatorname{Im} U P_{\mathrm{M}}, \operatorname{Im} U P_{\mathrm{N}}\right)=-\operatorname{dim}\left(\operatorname{ker} U P_{\mathrm{M}}, \operatorname{ker} U P_{\mathrm{N}}\right)=\operatorname{dim}(\mathrm{M}, \mathrm{N}) .
$$

Corolário 1.3.67 Dados um subespaço fechado $\mathrm{M}$ e $\mathrm{K}: \mathrm{H} \rightarrow \mathrm{H}$ um operador compacto, temos $(I+K)(\mathrm{M}) \sim \mathrm{M}$.

Prova. Basta observar que $(I+K)(\mathrm{M})$ é fechado, pelo ítem 4 da proposição 1.2.22 e pelo lema 1.3.4 e aplicar o teorema 1.3.65 aos operadores $\left.(I+K)\right|_{M},\left.I\right|_{M}: M \rightarrow H$.

Veremos na próxima proposição que toda perturbação compacta de um subespaço fechado com dimensão relativa zero pode ser obtida como no corolário 1.3.67.

Proposição 1.3.68 Sejam $\mathrm{M}, \mathrm{N}$ subespaços comensuráveis tais que $\operatorname{dim}(\mathrm{M}, \mathrm{N})=0$. Então existe $T \in \mathrm{GL}_{c}(\mathrm{H})$ tal que $T(\mathrm{M})=\mathrm{N}$.

Prova. Seja $T^{\prime}=I+\left(P_{\mathrm{N}}-P_{\mathrm{M}}\right)=P_{\mathrm{M}^{\perp}}+P_{\mathrm{N}} \in I+\mathscr{L}_{c}(\mathrm{H})$. Temos que $\left.T^{\prime}\right|_{\mathrm{M}}=P_{\mathrm{N}}^{\mathrm{M}}$ e por hipótese, ind $\left(P_{\mathrm{N}}^{\mathrm{M}}\right)=\operatorname{ind}(\mathrm{M}, \mathrm{N})=0$. Pela observação 1.3.40, existe $K: M \rightarrow N$ de posto finito, nulo no complementar de $\mathrm{M} \cap \mathrm{N}^{\perp}$, tal que $P_{\mathrm{N}}^{\mathrm{M}}+K$ é um isomorfismo entre $\mathrm{M}$ e $\mathrm{N}$. Estendendo $K=0 \mathrm{em} \mathrm{M}^{\perp}$, podemos considerar $K$ definido em todo $\mathrm{H}$. Como $\operatorname{ker} T^{\prime}=\mathrm{M} \cap \mathrm{N}^{\perp}$, temos que $T \doteq T^{\prime}+K \in \mathrm{GL}_{c}(\mathrm{H})$ e $T(\mathrm{M})=\mathrm{N}$. 


\subsection{O Fluxo Espectral}

Nesta seção, descreveremos o conceito de fluxo espectral de uma curva de operadores auto-adjuntos (não necessariamente limitados). Esta noção foi introduzida por M. Atiyah, V. Patodi e I. Singer em [14], [15]. Apesar disso, uma definição explícita do fluxo espectral para curvas de operadores limitados apareceu somente no artigo relativamente recente [75]. Vamos mostrar que a mesma idéia do referido artigo pode ser aplicada para definir o fluxo espectral de curvas de operadores Fredholm auto-adjuntos ilimitados contínuas na topologia gap, i.e., na topologia da métrica $\delta$.

\subsubsection{Decomposições do Espectro}

Vamos estudar algumas decomposições do espectro de um operador auto-adjunto em um espaço de Hilbert H. Referências básicas para esta subseção são [55], [80], [81], [88].

Inicialmente, recordamos um fato importante a respeito de medidas em $\mathbb{R}$, cuja prova pode ser encontrada em [41].

Teorema 1.4.1 (Decomposição de Lebesgue) Se $\mu$ é uma medida de Borel em $\mathbb{R}$, então existem medidas $\mu_{\mathrm{pp}}, \mu_{\text {cont }}, \mu_{\mathrm{ac}} e \mu_{\text {sing }}$ tais que:

1. $\mu_{\mathrm{pp}}$ é puramente pontual, i.e., $\mu_{\mathrm{pp}}$ tem suporte em um conjunto enumerável; ${ }^{26}$

2. $\mu_{\text {cont }}$ é contínua, i.e., $\mu_{\text {cont }}(\{x\})=0$ para todo $x \in \mathbb{R}$;

3. $\mu_{\mathrm{ac}} e$ absolutamente contínua com relação à medida de Lebesgue $\lambda$, i.e., se $\lambda(E)=0$ então $\mu_{\mathrm{ac}}(E)=0$, para qualquer $E \subset \mathbb{R}$ boreliano;

4. $\mu_{\text {sing }}$ é contínua e singular com relação à medida de Lebesgue $\lambda$, i.e., $\mu_{\text {sing }}$ é contínua $e$ existem $A, B \subset \mathbb{R}$ borelianos disjuntos tais que $A \cup B=\mathbb{R}$ e $\lambda(A)=\mu_{\text {sing }}(B)=0$;

5. $\mu=\mu_{\mathrm{pp}}+\mu_{\mathrm{cont}}=\mu_{\mathrm{pp}}+\mu_{\mathrm{ac}}+\mu_{\text {sing; }}$;

6. $\mu_{\text {cont }}=\mu_{\mathrm{ac}}+\mu_{\text {sing }}$.

Seja $T$ um operador auto-adjunto em $\mathrm{H} \mathrm{e}\left\{E_{\lambda}\right\}_{\lambda \in \mathbb{R}}$ a resolução espectral ${ }^{27}$ correspondente a T. A projeção espectral correspondente a $\lambda$ é definida como $P_{\lambda}=E_{\lambda}-E_{\lambda-0}$. Vemos ${ }^{28}$ que $\lambda$ é autovalor de $T$ se e só se $P_{\lambda} \neq 0$ e neste caso $P_{\lambda}$ é a projeção sobre o auto-espaço correspondente a $\lambda$. É bem conhecido que $\left\{E_{\lambda}\right\}_{\lambda \in \mathbb{R}}$ se estende à uma medida de Borel em $\mathbb{R}$ com valores no espaço de projeções ortogonais de $\mathrm{H}$. Vamos construir uma decomposição de $\mathrm{H}$ análoga à decomposição de Lebesgue de uma medida, como no teorema 1.4.1.

Fixado $u \in H$, a função real

$$
\lambda \mapsto\left\langle E_{\lambda} u, u\right\rangle
$$

é não-decrescente, portanto, define uma medida de Lebesgue-Stieltjes em $\mathbb{R}$, a qual denotaremos por $\mu_{u}$. Definimos os subespaços puramente pontual, absolutamente contínuo e singular

\footnotetext{
${ }^{26}$ Podemos expressar este fato dizendo que $\mu_{\mathrm{Pp}}=\sum_{n=1}^{\infty} a_{n} \delta_{x_{n}}$, onde $a_{n} \geq 0, x_{n} \in \mathbb{R}$ e $\delta_{x_{n}}$ é a medida de Dirac em $x_{n}$.

${ }^{27}$ Vamos supor que $\left\{E_{\lambda}\right\}_{\lambda \in R}$ é contínua d̀ direita, como de praxe.

${ }^{28} E_{\lambda-0}$ denota $\operatorname{slim}_{\mu \rightarrow \lambda^{-}} E_{\mu}$ e slim é o limite na topologia da convergência forte de operadores, i.e., convergência pontual.
} 
$\mathrm{H}_{\mathrm{pp}}, \mathrm{H}_{\mathrm{ac}}$ e $\mathrm{H}_{\text {sing }}$ como o conjunto dos $u \in \mathrm{H}$ tais que $\mu_{u}$ é puramente pontual, absolutamente contínua ou singular contínua com relação à medida de Lebesgue, respectivamente. $\mathrm{O}$ subespaço contínuo de $T$ é definido por $\mathrm{H}_{\text {cont }} \doteq \mathrm{H}_{\mathrm{pp}}^{\perp}$. Vemos que $\mathrm{H}_{\mathrm{pp}}=\bigoplus_{\lambda \in \mathbb{R}} \operatorname{Im} P(\lambda)$, em particular, $\mathrm{H}_{\mathrm{pp}}$ é invariante por $T$, e portanto, como $T$ é auto-adjunto, $\mathrm{H}_{\text {cont }}$ também é invariante por $T$. Não é difícil ver que $\mathrm{H}_{\mathrm{ac}}$ e $\mathrm{H}_{\text {sing }}$ também são invariantes e por $T$, e portanto, podemos dar a seguinte definição.

DeFINIÇÃo 1.4.2 Definimos o espectro pontual, o espectro contínuo, o espectro absolutamente contínuo e o espectro singular de $T$ como $\Sigma_{\mathrm{pp}}(T)=\Sigma\left(\left.T\right|_{\mathrm{H}_{\mathrm{pp}}}\right) \Sigma_{\text {cont }}(T)=\Sigma\left(\left.T\right|_{\mathrm{H}_{\text {cont }}}\right), \Sigma_{\mathrm{ac}}(T)=\Sigma\left(\left.T\right|_{\mathrm{H}_{\mathrm{ac}}}\right)$ e $\Sigma_{\text {sing }}(T)=\Sigma\left(\left.T\right|_{\mathrm{H}_{\text {sing }}}\right)$, respectivamente.

O próximo lema, que decorre do teorema 1.4.1, nos mostra que os subespaços que definimos fornecem uma decomposição ortogonal do espaço $\mathrm{H}$ em subespaços invariantes por $T$.

Lema 1.4.3 Temos que

$$
\mathrm{H}=\mathrm{H}_{\mathrm{pp}} \oplus \mathrm{H}_{\text {cont }}=\mathrm{H}_{\mathrm{pp}} \oplus \mathrm{H}_{\mathrm{ac}} \oplus \mathrm{H}_{\text {sing }},
$$

sendo que estes espaços são dois a dois ortogonais. Além disso, $\Sigma_{\text {cont }}(T)=\Sigma_{\mathrm{ac}}(T) \cup \Sigma_{\text {sing }}(T) e$ $\Sigma(T)=\overline{\Sigma_{\mathrm{pp}}(T)} \cup \Sigma_{\text {cont }}(T)$.

Podemos nos perguntar, independentemente do que foi feito acima, quais os pontos do espectro de um operador auto-adjunto $T$ podem ser removidos por meio de uma perturbação compacta de $T$, i.e., para quais pontos $\lambda \in \Sigma(T)$ existe $K \in \mathscr{L}_{c}(\mathrm{H})$ tal que $\lambda \notin \Sigma(T+K)$ ? A resposta para esta pergunta sugere a definição do espectro essencial de um operador autoadjunto. $^{29}$

DefinIÇÃo 1.4.4 O espectro essencial de um operador auto-adjunto $T$ é definido como

$$
\Sigma_{\mathrm{ess}}(T) \doteq \bigcap_{K \in \mathscr{L}_{\mathrm{c}}(\mathrm{H})} \Sigma(T+K)
$$

O espectro discreto de $T$ é definido como $\Sigma_{\text {disc }}(T)=\Sigma(T) \backslash \Sigma_{\text {ess }}(T)$.

Por definição, $\Sigma(T)$ é a reunião disjunta de $\Sigma_{\text {ess }}(T)$ e $\Sigma_{\text {disc }}(T)$. A próxima proposição fornece caracterizações importantes dos espectros essencial e discreto de um operador auto-adjunto.

Proposição 1.4.5 Se T é um operador auto-adjunto com resolução espectral $\left\{E_{\lambda}\right\}_{\lambda \in \mathbb{R}}$, então

$$
\begin{aligned}
\Sigma_{\text {ess }}(T) & =\left\{\lambda \in \Sigma(T): \operatorname{dim} \operatorname{Im}\left(E_{\lambda+\varepsilon}-E_{\lambda-\varepsilon}\right)=\infty \text { para todo } \varepsilon>0\right\} \\
& =\{\lambda \in \Sigma(T): T-\lambda \text { não é Fredholm }\} .
\end{aligned}
$$

Analogamente,

$$
\begin{aligned}
\Sigma_{\text {disc }}(T) & =\left\{\lambda \in \Sigma(T): 0<\operatorname{dim} \operatorname{Im}\left(E_{\lambda+\varepsilon}-E_{\lambda-\varepsilon}\right)<\infty \text { para algum } \varepsilon>0\right\} \\
& =\{\lambda \in \Sigma(T): \lambda \text { é autovalor isolado de multiplicidade finita }\}
\end{aligned}
$$

\footnotetext{
${ }^{29}$ É possivel definir o espectro essencial de qualquer operador fechado, conforme [55], [88], mas vamos nos ater ao caso auto-adjunto.
} 
Prova. Antes de mais nada, frisamos que a expressão $\lambda$ é autovalor isolado de multiplicidade finita usada na definição do conjunto em 1.4.5 significa que $\lambda$ é autovalor de multiplicidade finita que é ponto isolado de $\Sigma(T)$.

Se $\lambda \notin \Sigma_{\text {ess }}(T)$, então existe $K \in \mathscr{L}_{c}(H)$ tal que $\lambda \notin \Sigma(T+K)$, i.e., o operador $(T+K-\lambda)^{-1}$ existe e é limitado. Em particular, $T+K-\lambda$ é Fredholm de índice zero. Pelo ítem 2 da proposição 1.3.52, segue que $T-\lambda$ é Fredholm de índice zero. Reciprocamente, suponhamos que $T-\lambda$ é Fredholm. Se $\lambda \in \mathbb{C} \backslash \mathbb{R}$, então $\lambda \notin \Sigma_{\text {ess }}(T)$, pois $\Sigma_{\text {ess }}(T) \subset \Sigma(T) \subset \mathbb{R}$. Podemos, portanto, supor $\lambda \in \mathbb{R}$. Logo, $K=P_{\operatorname{Im}(T-\lambda)^{\perp}}$ é compacto auto-adjunto e $T+K-\lambda: \mathrm{D}(T) \rightarrow \mathrm{H}$ é bijetor. Pelo teorema do gráfico fechado, $(T+K-\lambda)^{-1} \in \mathscr{L}(\mathrm{H})$, portanto, $\lambda \notin \Sigma(T+K)$. Isso prova que $\Sigma_{\text {ess }}(T)$ coincide com o conjunto em 1.4.3. Um argumento análogo mostra que $\Sigma_{\text {disc }}(T)$ é igual ao conjunto definido em 1.4.5.

Para encerrar a demonstração, é suficiente provar que $\Sigma_{\text {disc }}(T)$ coincide com o conjunto em 1.4.4. Suponhamos que $\operatorname{dim} \operatorname{Im}\left(E_{\lambda+\varepsilon}-E_{\lambda-\varepsilon}\right)<\infty$ para algum $\varepsilon>0$. Temos que $P_{\lambda}=$ $E_{\lambda}-E_{\lambda-0} \leq E_{\lambda+\varepsilon}-E_{\lambda-\varepsilon}, \operatorname{logo}, \operatorname{dim} \operatorname{Im} P_{\lambda} \leq \operatorname{dim} \operatorname{Im}\left(E_{\lambda+\varepsilon}-E_{\lambda-\varepsilon}\right)<\infty$. Lembrando ${ }^{30}$ que $P_{\lambda}$ é a projeção ortogonal sobre $\operatorname{ker}(T-\lambda)$, segue que $\lambda$ é um autovalor de multiplicidade finita. $\lambda$ é ponto isolado de $\Sigma(T)$, pois os pontos de $\Sigma(T)$ são exatamente os pontos de não-constancia de $\left\{E_{\lambda}\right\}_{\lambda \in \mathbb{R}}, \mathrm{e}^{31}$ a existência de infinitos pontos de $\Sigma(T)$ em cada intervalo $(\lambda-\varepsilon, \lambda+\varepsilon)$ contradiria o fato que $\operatorname{dim} \operatorname{Im}\left(E_{\lambda-\varepsilon}-E_{\lambda+\varepsilon}\right)<\infty$. Reciprocamente, suponhamos que $\lambda \in \Sigma(T)$ é um autovalor isolado de multiplicidade finita. Logo, existe $\varepsilon>0$ tal que $[\lambda-\varepsilon, \lambda+\varepsilon] \cap \Sigma(T)=\{\lambda\}$. Em particular, $E_{\lambda+\varepsilon}-E_{\lambda-\varepsilon}=E_{\lambda}-E_{\lambda-0}=P_{\lambda}$. Como $P_{\lambda}$ é a projeção ortogonal sobre $\operatorname{ker}(T-\lambda)$, segue que $\operatorname{dim} \operatorname{Im}\left(E_{\lambda+\varepsilon}-E_{\lambda-\varepsilon}\right)=\operatorname{dim} \operatorname{Im} P_{\lambda}=\operatorname{mul}(\lambda)<\infty$.

CoROLÁrio 1.4.6 Se Ké um operador T-compacto, então $\Sigma_{\text {ess }}(T)=\Sigma_{\text {ess }}(T+K)$.

Prova. Basta usar a igualdade 1.4 .3 e a proposição 1.3.52.

ObSERVAÇão 1.4.7 A prova da proposição 1.4.5 mostra que

$$
\Sigma_{\text {ess }}(T)=\bigcap_{K \in \mathscr{L}_{c}(\mathrm{H})} \Sigma(T+K) .
$$

ObSERVAÇÃo 1.4.8 Podemos substituir na definição do conjunto 1.4.3 a palavra Fredholm pela expressão Fredholm de índice zero sem alterar a veracidade da proposição 1.4.5. De fato, se $\mathrm{H}$ é complexo, $\Omega=\{\lambda \in \mathbb{C}: T-\lambda$ é Fredholm $\}$ é aberto (pelo teorema 1.3.38) e contém os semi-planos superior e inferior. Logo, $\Omega$ tem no máximo duas componentes conexas, e portanto, como cada componente contém necessariamente um ponto $\lambda$ tal que $T-\lambda$ é isomorfismo, segue, pelo teorema 1.3.51, que ind $(T-\lambda)=0$ para todo $\lambda \in \Omega$. Caso $H$ seja real, basta considerar o operador $T^{\mathbb{C}}$ agindo no complexificado $\mathrm{H}^{\mathbb{C}}$ de $\mathrm{H}$ e observar que $\Sigma\left(T^{\mathbb{C}}\right)=\Sigma(T)+i \Sigma(T)$ e ind $T=$ ind $\left(T^{\mathbb{C}}\right)$.

Observação 1.4.9 Temos que $\Sigma_{\text {cont }}(T) \subset \Sigma_{\text {ess }}(T)$. De fato, pela proposição 1.4.5, se $\mu \in \Sigma_{\text {disc }}(T)$, então para qualquer $u \in \operatorname{ker}(T-\mu)$ não-nulo, a função 1.4 .1 é nula para $\lambda<\mu$ e igual a $\|u\|^{2}$ para $\lambda \geq \mu$. Em particular, tal função não é contínua em $\lambda=\mu$, e portanto, $u \notin \mathrm{H}_{\text {cont. }}$ Isso mostra

\footnotetext{
${ }^{30}$ Basta ver que se $P$ é uma projeção ortogonal, $A$ é auto-adjunto e $P \leq A$, então $\operatorname{ker} A \subset \operatorname{ker} P$, portanto, $\operatorname{Im} P \subset \overline{\operatorname{Im} A}$.

${ }^{31}$ Um ponto $\mu \in \mathbb{R}$ é dito ponto de constância de $\left\{E_{\lambda}\right\}_{\lambda \in \mathbb{R}}$ se existe $\varepsilon>0$ tal que $E_{\mu-\varepsilon}=E_{\mu+\varepsilon} ;$ o complementar do conjunto de tais $\mu$ é o conjunto dos pontos de não-constância de $\left\{E_{\lambda}\right\}_{\lambda \in \mathbf{R}}$.
} 
que $\left.(T-\mu)\right|_{H_{\text {cont }}}$ é inversível $\left(\operatorname{mul}(\mu)<\infty\right.$ implica que o operador auto-adjunto $\left.(T-\mu)\right|_{H_{\text {cont }}}$ é injetor e tem imagem fechada), portanto, $\lambda \notin \Sigma_{\text {cont }}(T)$.

A próxima proposição mostra que vale uma propriedade semelhante à proposição 1.2.38 para o espectro essencial.

Proposição 1.4.10 Se Ké um compacto tal que $K \cap \Sigma_{\text {ess }}(T)=\emptyset$, então existe $\delta>0$ tal que $\delta(S, T)<\delta$ implica $K \cap \Sigma_{\text {ess }}(S)=\emptyset$.

Prova. Se $K \cap \Sigma_{\text {ess }}(T)=\emptyset$ então $K \cap \Sigma_{\text {disc }}(T)$ é finito. Segue, portanto, que

$$
\alpha \doteq \inf _{\lambda \in K} \gamma(T-\lambda) /\left(1+\gamma(T-\lambda)^{2}\right)^{1 / 2}>0 .
$$

Pela proposição 1.2.35, $\hat{\delta}(S-\lambda, T-\lambda) \leq \max \left(2,\left(1+|\lambda|^{2}\right)\right) \delta(S, T)$; tomando

$$
\delta \doteq \alpha \sup _{\lambda \in K}\left\{\max \left(2,\left(1+|\lambda|^{2}\right)\right)^{-1}\right\} \text {, }
$$

o resultado segue pelo teorema 1.3.38 e pela igualdade 1.4.3 da proposição 1.4.5.

OBSERvação 1.4.11 Se $T$ tem resolvente compacto (cf. definição 1.2.82), então $\Sigma_{\text {ess }}(T)=\emptyset$. De fato, se $\mathrm{R}(\zeta ; T)$ é compacto, temos $T-\lambda=(T-\zeta)[I+(\zeta-\lambda) \mathrm{R}(\zeta ; T)]$, logo $T-\lambda$ é Fredholm de índice zero para todo $\lambda$, pelo lema 1.3.4 e pelo teorema 1.3.35. O resultado decorre da igualdade 1.4.3 na proposição 1.4.5.

ObSERVAÇão 1.4.12 Se $\zeta \in \mathrm{R}(\zeta ; T)$, então $\Sigma_{\text {ess }}(\mathrm{R}(\zeta ; T))=\left\{(\lambda-\zeta)^{-1}: \lambda \in \Sigma_{\text {ess }}(T)\right\}$, pois $\mathrm{R}(\zeta ; T)-$ $\lambda=-\lambda \mathrm{R}\left(\zeta+\lambda^{-1} ; T\right) \mathrm{R}(\zeta ; T)$, se $\lambda \neq 0$.

O próximo teorema mostra que o espectro essencial é estável por um tipo ainda mais geral de perturbação.

TEOREma 1.4.13 (WeYL) Se $S, T$ são operadores auto-adjuntos e existe $\zeta \in \mathrm{P}(S) \cap \mathrm{P}(T)$ tal que $\mathrm{R}(\zeta ; S)$ $\mathrm{R}(\zeta ; T)$ é compacto, então $\Sigma_{\text {ess }}(S)=\Sigma_{\text {ess }}(T)$.

Prova. O resultado segue do corolário 1.4.6 e da observação 1.4.12.

OвsERvaçÃo 1.4.14 O teorema 1.4.6 é verdadeiro em geral, para operadores não necessariamente auto-adjuntos, e a mesma prova se aplica, cf. [55], Pag.244.

Na próxima proposição vamos provar algumas relações entre os vários tipos de espectro definidos.

Proposição 1.4.15 Dado $\lambda \in \Sigma(T)$, temos que $\lambda \in \Sigma_{\text {ess }}(T)$ se e só se

1. $\lambda \in \Sigma_{\text {cont }}(T) o u$

2. $\lambda \in \overline{\Sigma_{\mathrm{pp}}(T)} \backslash \Sigma_{\mathrm{sing}}(T)$ ou

3. $\lambda$ é autovalor com $\operatorname{mul}(\lambda)=\infty$. 
Prova. Se $\lambda$ satisfaz o ítem (2) ou (3), então para qualquer $\varepsilon>0, \operatorname{dim} \operatorname{Im}\left(E_{\lambda+\varepsilon}-E_{\lambda-\varepsilon}\right)=\infty$, logo, pela proposição $1.4 .5, \lambda \in \Sigma_{\text {ess }}(T)$. Este fato e a observação 1.4 .9 mostram que se $\lambda$ satisfaz (1), (2) ou (3), então $\lambda \in \Sigma_{\text {ess }}(T)$. Reciprocạmente, se $\lambda \in \Sigma_{\text {ess }}(T)$ e $\lambda$ não é autovalor de multiplicidade infinita nem limite de autovalores de multiplicidade finita, segue que para todo $u \in \mathrm{H}$, a função monótona definida em 1.4.1 é contínua, logo o auto-espaço contínuo $\mathrm{H}_{\text {cont }}$ correspondente a $T$ coincide com $H$. Isso mostra que $\lambda \in \Sigma_{\text {cont }}(T)$.

Corolário 1.4.16 Se Té limitado, então $\Sigma_{\text {ess }}(T) \neq \emptyset$. Mais geralmente, se $\lambda$ é um ponto não-isolado de $\Sigma(T)$ que pertence à fronteira de $\mathrm{P}(T)$, então $\lambda \in \Sigma_{\mathrm{ess}}(T)$.

ObSERVAÇão 1.4.17 A despeito da não estabilidade dos outros tipos de espectro mediante perturbações, o espectro essencial além de ser invariante por perturbações relativamente compactas é também um conjunto fechado. Isso decorre da igualdade 1.4.3 no teorema 1.4.5 e do teorema 1.3.38.

ObSERvação 1.4.18 Como Hé separável, $\Sigma_{\mathrm{pp}}(T)$ é enumerável, pois se $\lambda \neq \mu$, então $\operatorname{ker}(T-\lambda) \perp$ $\operatorname{ker}(T-\mu)$. Em particular, $\Sigma_{\text {disc }}(T) \subset \Sigma_{\mathrm{pp}}(T)$ é enumerável.

Lema 1.4.19 Se T é auto-adjunto e $\Sigma_{\text {ess }}(T) \subset(\alpha, \infty)$ então existem $\beta>\alpha$ e $K \in \mathscr{L}_{c}(\mathrm{H})$ auto-adjunto tais que $T+K \geq \beta$; em particular, $\Sigma(T+K) \subset[\beta, \infty)$.

Prova. Se $\Sigma_{\text {ess }}(T) \subset(\alpha, \infty)$, então segue diretamente da definição de $\Sigma_{\text {ess }}(T)$ e da observação 1.4.7 que existem $\beta>\alpha$ e $K$ compacto auto-adjunto tais que $\Sigma(T+K) \subset[\beta, \infty)$. Portanto, se $\left\{E_{\lambda}\right\}_{\lambda \in \mathbb{R}}$ é a resolução espectral de $T+K$ então

$$
\langle(T+K) u, u\rangle=\int_{\beta}^{\infty} \lambda d\left\|E_{\lambda}(u)\right\|^{2} \geq \beta\|u\|^{2},
$$

provando o desejado.

Observação 1.4.20 Como é de praxe, podemos considerar a álgebra de Calkin $\mathrm{C}$, i.e., o quociente da álgebra $\mathscr{L}(\mathrm{H})$ pelo ideal de operadores compactos. Neste caso, o espectro essencial de um operador $T \in \mathscr{L}(\mathrm{H})$ é o espectro de $\pi(T)$ como elemento da álgebra de Banach $\mathcal{C}$, onde $\pi: \mathscr{L}(\mathrm{H}) \rightarrow \mathcal{C}$ é a aplicação quociente.

\subsubsection{Construção do Fluxo Espectral}

Nosso objetivo agora é definir o fluxo espectral de uma curva de operadores Fredholm auto-adjuntos (não necessariamente limitados). Isso foi feito pela primeira vez por B. BoossBavnbek, M. Lesch e J. Phillips em [23]. Nosso objetivo nesta subseção será dar uma definição rigorosa do fluxo espectral no caso ilimitado, tendo muito pontos em comum com o trabalho dos referidos autores. Vamos sempre ter em mente a idéia que o fluxo espectral de uma curva de operadores Fredholm auto-adjuntos mede intuitivamente a mudança de sinal dos autovalores a medida que o parâmetro varia.

Primeiramente, faremos uma construção abstrata (como em [44]) que será útil para a construção não só do fluxo espectral como também do índice de Maslov. Seja $X$ um espaço topológico e denotemos por $\pi(X)$ o grupóide fundamental de $X$, i.e., o conjunto de todas as 
classes de homotopia $[\mu]$ com extremos fixos de curvas $\mu:[0,1] \rightarrow X .^{32}$ Podemos definir a concatenação de duas curvas $\mu, \eta$ tais que $\mu(1)=\eta(0)$ de maneira usual

$$
\mu * \eta(t)=\left\{\begin{array}{rl}
\mu(2 t), & \text { se } 0 \leq t \leq 1 / 2 \\
\eta(2 t-1), & \text { se } 1 / 2 \leq t \leq 1
\end{array} .\right.
$$

Definimos $\mu^{-1}(t)=\mu(1-t)$; é fácil ver que $\mu * \mu^{-1}$ e $\mu^{-1} * \mu$ são homotópicas às curvas constantes $\mu(0)$ e $\mu(1)$, respectivamente. Se $G$ é um grupo, dizemos que $\varphi: \pi(X) \rightarrow G$ é um homomorfismo se $\varphi([\mu] *[\eta])=\varphi([\mu]) \varphi([\eta])$, para todas $[\mu],[\eta] \in \pi(X)$ tais que o produto $[\mu] *[\eta]$ esteja definido.

ExEMPLo 1.4.21 Se $f: X \rightarrow G$ é qualquer função, então a aplicação $\varphi: \pi(X) \rightarrow G$ dada por $\varphi([\mu])=f(\mu(0))^{-1} f(\mu(1))$ é um homomorfismo.

Veremos agora que se $\left\{U_{\alpha}\right\}_{\alpha \in A}$ é uma cobertura aberta de $X$ e $\varphi_{\alpha}: \pi\left(U_{\alpha}\right) \rightarrow G$ é uma família de homomorfismos satisfazendo uma certa relação de compatibilidade, então existe um único homomorfismo $\varphi: \pi(X) \rightarrow G$ cuja restrição à cada $\pi\left(U_{\alpha}\right)$ é $\varphi_{\alpha}$.

DefinIÇão 1.4.22 Se $\left\{U_{\alpha}\right\}_{\alpha \in A}$ é uma cobertura aberta de $X$ e $\varphi_{\alpha}: \pi\left(U_{\alpha}\right) \rightarrow G$ é uma família de homomorfismos, com $G$ um grupo qualquer, dizemos que $\left\{\varphi_{\alpha}\right\}_{\alpha \in A}$ é compatível se $\left.\varphi_{\alpha}\right|_{\pi\left(U_{\alpha} \cap U_{\beta}\right)}=$ $\left.\varphi_{\beta}\right|_{\pi\left(U_{\alpha} \cap U_{\beta}\right)}$, sempre que $U_{\alpha} \cap U_{\beta} \neq \emptyset$.

A próxima proposição mostra que a compatibilidade de uma família de homomorfismos é condição necessária e suficiente para que exista uma única extensão dos $\varphi_{\alpha}$.

Proposição 1.4.23 Seja $\left\{U_{\alpha}\right\}_{\alpha \in A}$ uma cobertura aberta de X e $\varphi_{\alpha}: \pi\left(U_{\alpha}\right) \rightarrow G$ uma família de homomorfismos. Então existe um único homomorfismo $\varphi: \pi(X) \rightarrow G$ cuja restrição d̀ cada $\pi\left(U_{\alpha}\right)$ é $\varphi_{\alpha}$ se e só se $\left\{\varphi_{\alpha}\right\}_{\alpha \in A}$ é compatível.

Prova. Obviamente, compatibilidade é uma condição necessária para que exista alguma extensão dos $\varphi_{a}$.

Suponhamos que $\left\{\varphi_{\alpha}\right\}_{\alpha \in A}$ é compatível. A idéia para construir a extensão é decompor uma curva $\mu$ qualquer em pequenas porções $\mu_{\alpha}$ contidas em algum $U_{a}$ e definir $\varphi([\mu])$ como o produto dos $\varphi_{\alpha}\left(\left[\mu_{\alpha}\right]\right)$.

Dada uma curva $\mu:[0,1] \rightarrow X$, por compacidade existem $\alpha_{1}, \ldots, \alpha_{n} \in A$ e uma partição $0=t_{0}<\ldots<t_{n}=1$ tais que $\mu\left(\left[t_{j-1}, t_{j}\right]\right) \subset U_{\alpha_{j}}$ para $j=1, \ldots, n$. Para facilitar a notação, vamos denotar $\varphi_{\alpha_{j}}, U_{\alpha_{j}}$ e $\left.\mu\right|_{\left[t_{j-1}, t_{j}\right]}$ por $\varphi_{j}, U_{j}$ e $\mu_{j}$, respectivamente. Definimos

$$
\varphi([\mu])=\varphi_{1}\left(\left[\mu_{1}\right]\right) \cdot \ldots \cdot \varphi_{n}\left(\left[\mu_{n}\right]\right) .
$$

Vamos mostrar que $\varphi$ é bem-definida, i.e., não depende nem da partição nem dos abertos utilizados. Se refinarmos a partição mantendo os abertos $U_{j}$ fixos, o fato que cada $\varphi_{j}$ é um homomorfismo em $\pi\left(U_{j}\right)$ mostra que $\varphi$ não se altera. Se mantivermos a partição fixa mas trocarmos os abertos $U_{j}$ por abertos $U_{j}^{\prime}$ tais que $\mu_{j}$ tem imagem contida em $U_{j}^{\prime}$, então $\varphi$ não altera o seu valor, pois, por hipótese, $\varphi_{j}$ e $\varphi_{j}^{\prime}$ coincidem quando restritas a $U_{j} \cap U_{j}^{\prime}$. Logo, $\varphi$ é bem-definida, i.e., independe da partição e dos abertos utilizados.

\footnotetext{
${ }^{32} \pi(X)$ é o quociente do conjunto de todas as curvas $[0,1] \rightarrow X$ pela relação de equivalência $\mu \equiv \eta$ se e só se $\mu(0)=\eta(0), \mu(1)=\eta(1)$ e existe uma homotopia entre $\mu$ e $\eta$ que mantém fixos $\mu(0)$ e $\mu(1)$.
} 
Vamos agora provar que $\varphi([\mu])$ depende somente da classe de homotopia com extremos fixos de $\mu$. Suponha que $\eta$ seja homotópica a $\mu$ nestas condições e seja $H:[0,1] \times[0,1] \rightarrow X$ uma homotopia tal que $H(t, 0)=\mu(t), H(t, 1)=\eta(t), H(0, s)=\mu(0)$ e $H(1, s)=\mu(1)$. Por compacidade, existem partições $0=t_{0}<\ldots<t_{m}=1,0=s_{0}<\ldots<s_{q}=1$ e abertos $U_{j, l}$ (notação como antes) tais que $H\left(\left[t_{j-1}, t_{j}\right] \times\left[s_{l-1}, s_{l}\right]\right) \subset U_{j, l}$. Podemos supor que $q=1$; o resultado para $q>1$ segue por indução. Como antes, denotemos $U_{j, l}, \varphi_{j, l},\left.\mu\right|_{\left[t_{j-1}, t_{j}\right]}$ e $\left.\eta\right|_{\left[t_{j-1}, t_{j}\right]}$ por $U_{j}$ e $\varphi_{j}, \mu_{j}, \eta_{j}$, respectivamente e para cada $j=0, \ldots, n$, seja $\gamma_{j}(s)=H\left(t_{j}, s\right)$. Como as curvas $\mu_{j}$ e $\gamma_{j-1} * \eta_{j} * \gamma_{j}^{-1}$ são homotópicas em $U_{j}$, temos que

$$
\varphi_{j}\left(\left[\mu_{j}\right]\right)=\varphi_{j}\left(\left[\gamma_{j-1} * \eta_{j} * \gamma_{j}^{-1}\right]\right)=\varphi_{j}\left(\left[\gamma_{j-1}\right]\right) \varphi_{j}\left(\left[\eta_{j}\right]\right) \varphi_{j}\left(\left[\gamma_{j}^{-1}\right]\right),
$$

para $j=1, \ldots, n$.

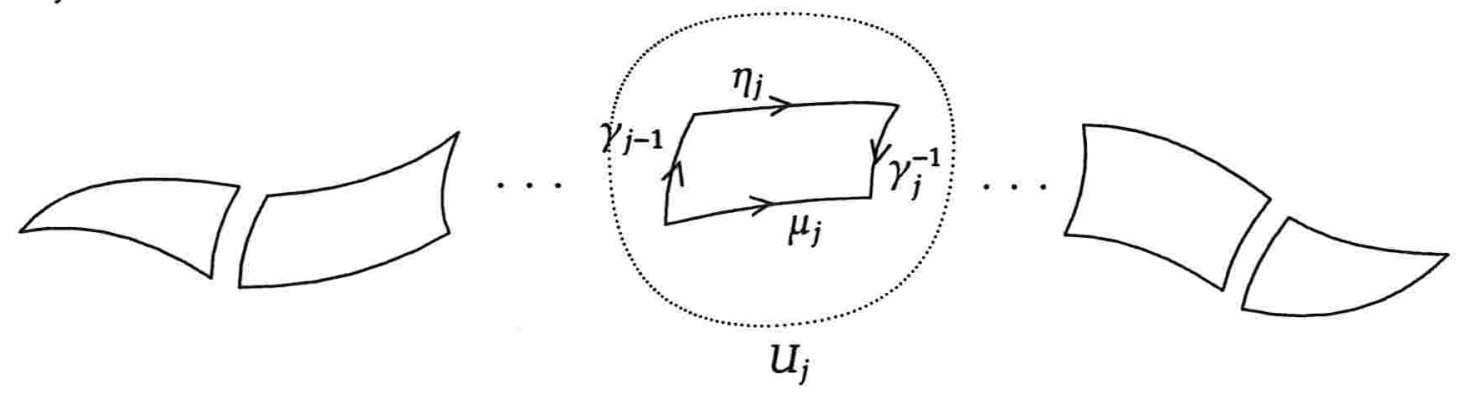

Portanto,

$$
\begin{aligned}
\varphi([\mu]) & =\varphi_{1}\left(\left[\mu_{1}\right]\right) \cdot \ldots \cdot \varphi_{j}\left(\left[\mu_{j}\right]\right) \cdot \ldots \cdot \varphi_{n}\left(\left[\mu_{n}\right]\right) \\
& =\varphi_{1}\left(\left[\gamma_{0} * \eta_{1} * \gamma_{1}^{-1}\right]\right) \cdot \ldots \cdot \varphi_{j}\left(\left[\gamma_{j-1} * \eta_{j} * \gamma_{j}^{-1}\right]\right) \cdot \ldots \cdot \varphi_{n}\left(\left[\gamma_{n-1} * \eta_{n} * \gamma_{n}^{-1}\right]\right) \\
& =\varphi_{1}\left(\left[\eta_{1}\right]\right) \cdot \ldots \cdot \varphi_{j}\left(\left[\eta_{j}\right]\right) \cdot \ldots \cdot \varphi_{n}\left(\left[\eta_{n}\right]\right)=\varphi([\eta]) .
\end{aligned}
$$

Isso mostra que $\varphi$ define um homomorfismo $\pi(X) \rightarrow G$, como queríamos.

Para darmos a definição do fluxo espectral, a próxima proposição é essencial, pois nos fornece uma maneira local de medir a mudança de sinal dos autovalores de uma família de operadores Fredholm auto-adjuntos.

Proposição 1.4.24 Dado $T \in \mathscr{C} \mathscr{F}$ sa então existem $\varepsilon, \delta>0$ tais que:

1. A projeção $\chi_{[-\delta, \delta]}(S)$ tem ${ }^{33}$ posto finito para todo $S \in \mathcal{U}_{T} \doteq\left\{S \in \mathscr{C}^{\mathrm{sa}}(\mathrm{H}): \delta(S, T)<\varepsilon\right\}$;

2. A aplicação $\mathcal{U}_{T} \ni S \mapsto \chi_{[-\delta, \delta]}(S) \in \mathscr{L}(\mathrm{H})$ é contínua.

Prova. A igualdade 1.4.3 da proposição 1.4.5 implica que $0 \notin \Sigma_{\text {ess }}(T)$. Como $\Sigma_{\text {ess }}(T)$ é fechado (observação 1.4.17), existe $\delta>0$ tal que $[-\delta, \delta] \cap \Sigma_{\text {ess }}(T)=\emptyset$. Pela proposição 1.4.15, $[-\delta, \delta] \cap \Sigma_{\text {sing }}(T)$ é finito, portanto, podemos supor, diminuindo $\delta$, que $[-\delta, \delta] \cap \Sigma(T)=\emptyset$ ou $[-\delta, \delta] \cap \Sigma(T)=\{0\}$. No primeiro caso, pela proposição 1.2 .38 , temos que existe $\varepsilon>0$ tal que todo elemento de $\mathcal{U}_{T}$ tem espectro disjunto de $[-\delta, \delta]$, em particular, o ítem (2) é satisfeito trivialmente. No segundo caso, a conclusão decorre do teorema 1.2.51.

\footnotetext{
${ }^{33} \chi_{A}$ denota a função característica do conjunto $A$.
} 
Para $S \in \mathcal{U}_{T}$, com $\delta>0$ como na proposição 1.4.24, definimos $\chi_{[-\delta, \delta]}^{+}(S)$ como a projeção espectral sobre o subespaço gerado pelos autovetores de $S$ correspondentes aos autovalores de $S$ em $[0, \delta]$. A proposição 1.4.24 mostra que para uma curva $\mu:[0,1] \rightarrow \mathcal{U}_{T}$, o inteiro

$$
\varphi_{T}(\mu)=\operatorname{dim} \operatorname{Im} \chi_{[-\delta, \delta]}^{+}(\mu(1))-\operatorname{dim} \operatorname{Im} \chi_{[-\delta, \delta]}^{+}(\mu(0))
$$

é bem-definido, aditivo por concatenação e invariante por uma homotopia em $\mathcal{U}_{T}$ que mantém fixos $\mu(0)$ e $\mu(1)$. Em outras palavras, $\varphi_{T}$ é um homomorfismo $\pi\left(\mathcal{U}_{T}\right) \rightarrow \mathbb{Z}$. Provemos agora que os homomorfismos $\varphi_{T}$ satisfazem a hipótese da proposição 1.4.23. De fato, seja $\mu:[0,1] \rightarrow \mathcal{U}_{T_{1}} \cap \mathcal{U}_{T_{2}}$ uma curva e $\delta_{i}>0$ correspondentes aos abertos $\mathcal{U}_{T_{i}}, i=1,2$, como na proposição 1.4.24. Podemos supor $\delta_{1} \leq \delta_{2}$. Logo, pela proposição 1.4.24, a aplicação

$$
[0,1] \ni t \mapsto \chi_{\left[-\delta_{2}, \delta_{2}\right]}^{+}(\mu(t))-\chi_{\left[-\delta_{1}, \delta_{1}\right]}^{+}(\mu(t))=\chi_{\left(\delta_{1}, \delta_{2}\right]}(\mu(t)) \in \mathscr{L}(\mathrm{H})
$$

é contínua a valores no espaço de projeções ortogonais de $\mathrm{H}$. Pelo ítem 3 da proposição 1.2.3, segue que $\operatorname{dim} \operatorname{Im} \chi_{\left(\delta_{1}, \delta_{2}\right]}(\mu(t))$ é constante, digamos igual a $n$. Logo,

$$
\begin{aligned}
\varphi_{T_{1}}(\mu) & =\operatorname{dim} \operatorname{Im} \chi_{\left[-\delta_{1}, \delta_{1}\right]}^{+}(\mu(1))-\operatorname{dim} \operatorname{Im} \chi_{\left[-\delta_{1}, \delta_{1}\right]}^{+}(\mu(0)) \\
& =\left(\operatorname{dim} \operatorname{Im} \chi_{\left[-\delta_{2}, \delta_{2}\right]}^{+}(\mu(1))-n\right)-\left(\operatorname{dim} \operatorname{Im} \chi_{\left[-\delta_{2}, \delta_{2}\right]}^{+}(\mu(0))-n\right) \\
& =\operatorname{dim} \operatorname{Im} \chi_{\left[-\delta_{2}, \delta_{2}\right]}^{+}(\mu(1))-\operatorname{dim} \operatorname{Im} \chi_{\left[-\delta_{2}, \delta_{2}\right]}^{+}(\mu(0))=\varphi_{T_{2}}(\mu) .
\end{aligned}
$$

A proposição 1.4.23 nos permite concluir o seguinte teorema.

Tвовема 1.4.25 Existe um único homomorfismo

$$
\text { sf }: \pi\left(\mathscr{C} \mathscr{F}^{\mathrm{sa}}\right) \rightarrow \mathbb{Z}
$$

chamado fluxo espectral, o qual coincide $\operatorname{com} \varphi_{T}$ em $\pi\left(\mathcal{U}_{T}\right)$, conforme notação da proposição 1.4.24.

O homomorfismo construído no teorema 1.4.25 restringe-se a um homomorfismo

$$
\text { sf }: \pi\left(\mathscr{F}^{\mathrm{sa}}\right) \rightarrow \mathbb{Z},
$$

de acordo com o corolário 1.2.32. Na próxima subseção, vamos estudar a topologia dos espaços de operadores auto-adjuntos limitados e ilimitados e suas relações com o fluxo espectral.

\subsubsection{A Topologia dos Espaços de Operadores Auto-adjuntos}

Iniciamos a seção definindo alguns espaços sobre os quais iremos trabalhar nesta seção.

DeFinição 1.4.26 Fixemos as seguintes notações:

$\cdot \mathscr{F}_{+}^{\text {sa }}:$ Espaço dos operadores $T \in \mathscr{F}^{\text {sa }}$ tais que $\Sigma_{\text {ess }}(T) \subset(0, \infty)$;

- $\mathscr{F}_{-}^{\text {sa }}$ : Espaço dos operadores $T \in \mathscr{F}^{\text {sa }}$ tais que $\Sigma_{\text {ess }}(T) \subset(-\infty, 0)$;

$\cdot \mathscr{F}_{*}^{\mathrm{sa}}=\mathscr{F}^{\mathrm{sa}} \backslash\left(\mathscr{F}_{+}^{\mathrm{sa}} \cup \mathscr{F}_{-}^{\mathrm{sa}}\right)$.

Os espaços $\mathscr{C} \mathscr{F}_{ \pm}^{\text {sa }}, \mathscr{C} \mathscr{F}_{*}^{\text {sa }}$ são os análogos de $\mathscr{F}_{ \pm}^{\text {sa }}, \mathscr{F}_{*}^{\text {sa }}$ no caso ilimitado. 
A topologia dos espaços $\mathscr{F}$ sa e suas variantes definidas em 1.4.26 foi estudada largamente por Atiyah e Singer em [13]. Eles mostraram que $\mathscr{F}_{ \pm}^{\text {sa }}$ são contráteis e $\mathscr{F}_{*}^{\text {sa }}$ é conexo por caminhos, tem grupo fundamental $\mathbb{Z}$ e sf : $\pi_{1}\left(\mathscr{F}_{*}^{\text {sa }}\right) \rightarrow \mathbb{Z}$ é um isomorfismo. Entre outros fatos, eles provaram que o espaço $\mathscr{F}_{*}^{\text {sa }}$ é um espaço classificante para o funtor $K^{-1}$ no caso complexo; veja [8] e [13].

Comecemos mostrando que, de fato,

Proposição 1.4.27 $\mathscr{F}_{+}^{\mathrm{sa}}, \mathscr{F}_{-}^{\mathrm{sa}} e \mathscr{F}_{*}^{\mathrm{sa}}$ são abertos.

Prova. Se $T \in \mathscr{F}_{+}^{\text {sa }}$, então pelo lema 1.4 .19 existem $K$ compacto auto-adjunto e $\beta=\beta(T)>0$ tais que $T+K \geq \beta$. Em particular, se $S$ é auto-adjunto limitado e $\|S-T\|<\beta / 2$, então $S+K=T+K+(S-T) \geq \beta / 2$. Logo, $\Sigma(S+K) \subset[\beta / 2, \infty)$, e por definição do espectro essencial, $\Sigma_{\text {ess }}(S) \subset(0, \infty)$ se $\|S-T\|<\beta / 2$. Isso prova que $\mathscr{F}_{+}^{\text {sa }}$ é aberto. Da mesma forma prova-se que $\mathscr{F}$ sa é aberto.

Vejamos agora que $\mathscr{F}_{*}^{\text {sa }}$ é aberto. Se $T \in \mathscr{F}_{*}^{\text {sa }}$, sejam $\mathrm{H}_{+}, \mathrm{H}_{-}$os subespaços correspondentes ao espectro positivo e negativo, respectivamente de $T$, i.e., $H_{-}=\operatorname{Im} \chi_{(-\infty, 0)}(T)$ e $H_{+}=$ $\operatorname{Im} \chi_{[0, \infty)}(T) . H_{ \pm}$são invariantes por $T,\left.T_{ \pm} \doteq T\right|_{H_{ \pm}}$são operadores Fredholm auto-adjuntos e $H$ se escreve como soma direta ortogonal $\mathrm{H}_{+} \oplus \mathrm{H}_{-}$. Podemos supor que $T_{+}$seja estritamente positivo e $T_{-}$seja estritamente negativo. Como $\Sigma_{\text {ess }}\left(T_{+}\right) \subset(0, \infty)$ e $\Sigma_{\text {ess }}\left(T_{-}\right) \subset(-\infty, 0)$, pelo parágrafo anterior existe $\delta>0$ tal que se $A \in \mathscr{L}^{\text {sa }}\left(\mathrm{H}_{+}\right), C \in \mathscr{L}^{\text {sa }}\left(\mathrm{H}_{-}\right)$e $\left\|A-T_{+}\right\|,\left\|C-T_{-}\right\|<\delta$, então $\Sigma_{\text {ess }}(A) \subset(0, \infty)$ e $\Sigma_{\text {ess }}(C) \subset(-\infty, 0)$. Como $\operatorname{dim} \mathrm{H}_{+}=\operatorname{dim} \mathrm{H}_{-}=\infty$, segue pelo corolário 1.4.16 que $\Sigma_{\text {ess }}(A), \Sigma_{\text {ess }}(C) \neq \emptyset$. Como para $B \in \mathscr{L}\left(\mathrm{H}_{+}, \mathrm{H}_{-}\right)$tal que $\|B\|<\min \left(\left\|A^{-1}\right\|^{-1},\left\|C^{-1}\right\|^{-1}\right) \doteq \varepsilon$ o operador auto-adjunto

$$
S=\left(\begin{array}{cc}
A & B^{*} \\
B & C
\end{array}\right)
$$

(com respeito a decomposição $\mathrm{H}=\mathrm{H}_{+} \oplus \mathrm{H}_{-}$) é inversível, segue pela limitação de $S$ que $S$ tem espectro essencial tanto em $(0, \infty)$ quanto em $(-\infty, 0)$, ou seja, $S \in \mathscr{F}_{*}^{\text {sa }}$.

Proposição 1.4.28 $\mathscr{F}_{+}^{\text {sa }} e \mathscr{F}_{-}^{\text {sa }}$ são contráteis.

Prova. Vamos construir uma homotopia entre a identidade e uma aplicação constante em $\mathscr{F}_{+}^{\text {sa }}$. Seja $H(T, t)=(1-t) T+t I, T \in \mathscr{F}_{+}^{\text {sa }}, 0 \leq t \leq 1$. Obviamente, $H$ é contínua. Como $\Sigma_{\text {ess }}(T) \subset(0, \infty)$, temos que $-\frac{t}{1-t} \notin \Sigma_{\text {ess }}(T)$, para $0 \leq t<1$. Pela proposição $1.4 .5, H(T, t)$ é Fredholm, para todos $T \in \mathscr{F}_{+}^{\text {sa }}, 0 \leq t \leq 1$. Claramente, $H(T, t)$ é auto-adjunto, limitado e $\Sigma_{\text {ess }}(H(T, t))=(1-t) \Sigma_{\text {ess }}(T)+t \subset(0, \infty)$.

\section{Teorema 1.4.29 $\mathscr{F}^{\text {sa }}$ é conexo por caminhos.}

Prova. Sejam $T \in \mathscr{F}_{*}^{\text {sa }}$ e $P$ a projeção ortogonal sobre $\operatorname{ker} T=(\operatorname{Im} T)^{\perp}$. A curva (contínua) $[0,1] \ni t \mapsto T+t P \in \mathscr{L}(\mathrm{H})$ conecta $T$ a um isomorfismo auto-adjunto de $\mathrm{H}(T+P$, por exemplo). Portanto, podemos supor que $T$ seja um isomorfismo auto-adjunto. Sejam $\alpha \doteq \inf \{|\lambda|: \lambda \in$ $\Sigma(T)\}$ e $f_{t}: \mathbb{R} \rightarrow \mathbb{R}, 0 \leq t \leq 1 / \alpha$, a função cujo gráfico aparece esboçado no teorema 1.3.47. Claramente a aplicação $T(t):[0,1 / \alpha] \ni t \mapsto f_{t}(T) \in \mathscr{L}(\mathrm{H})$ é contínua (pelo lema 1.3.48), $T(0)=T$ e $T(1 / \alpha)=(1 / \alpha)\left(P_{+}-P_{-}\right)=(1 / \alpha)\left(2 P_{+}-I\right)$, onde $P_{+}, P_{-}$são as projeções ortogonais sobre os auto-espaços positivo e negativo de $T$. Como $T$ tem espectro essencial tanto em $(-\infty, 0)$ como em $(0, \infty)$, segue que $P_{+}$é uma projeção sobre um subespaço de dimensão e 
codimensão infinita. Como o espaço de tais projeções é conexo por caminhos (conforme a proposição 1.6.33), temos que $\mathscr{F}_{*}^{\text {sa }}$ é conexo por caminhos.

O próximo corolário decorre diretamente das proposições 1.4.27, 1.4.28 e do teorema 1.4.29.

CoRolário 1.4.30 O espaço $\mathscr{F}^{\mathrm{sa}}$ possui três componentes conexas (por caminhos), a saber, $\mathscr{F}_{+}^{\mathrm{sa}}, \mathscr{F}_{-}^{\mathrm{sa}}$ $e \mathscr{F}_{*}^{\text {sa }}$, sendo que as duas primeiras são contráteis.

OBSERVAÇão 1.4.31 A mesma prova dada para o teorema 1.4 .29 mostra que $\mathscr{C} \mathscr{F}{ }_{*}^{\text {sa }}$ é conexo por caminhos. O único detalhe que precisamos observar é que a curva $T(t)$ construída é contínua, pela proposição 1.3.48. Em geral, dado $T \in \mathscr{C} \mathscr{F}$ sa , a prova do teorema 1.4 .29 mostra que sempre existe uma curva contínua em $\mathscr{C} \mathscr{F}$ sa ligando $T$ à uma conjugação, i.e., um operador da forma $2 P_{M}-I$, com $P_{M}$ projeção ortogonal sobre um subespaço fechado $M$. Mais adiante, exploraremos este aspecto da prova do teorema 1.4.29.

A próxima proposição fornece relações simples de densidade para os espaços de operadores Fredholm auto-adjuntos que estamos estudando.

Proposição 1.4.32 $\mathscr{L}^{\mathrm{sa}}, \mathscr{F}^{\mathrm{sa}}$ e $\mathscr{F}^{\mathrm{sa}}$ são abertos densos em $\mathscr{C}^{\mathrm{sa}}, \mathscr{C} \mathscr{F}^{\mathrm{sa}} e \mathscr{C}^{*}{ }^{\mathrm{sa}}$, respectivamente.

Prova. A abertura dos referidos espaços decorre do corolário 1.2.32 e da proposição 1.4.27. Para provar a densidade, seja $T \in \mathscr{C}^{\text {sa }}$ qualquer e $f_{t}$ a função definida no teorema 1.4.29. Como $f_{t}(T)$ é auto-adjunto limitado e $t \mapsto f_{t}(T)$ é contínua em $t=0$ (como no lema 1.3.48) temos que $\mathscr{L}^{\text {sa }}$ é denso em $\mathscr{C}^{\text {sa }}$. Se $T$ é Fredholm, então $f_{t}(T)$ também o é; isso prova a densidade de $\mathscr{F}$ sa em $\mathscr{C} \mathscr{F}^{\text {sa }}$. Se $T \in \mathscr{C}_{\mathscr{F}^{\text {sa }}}$, então, para $t$ suficientemente pequeno, $f_{t}(T)$ tem espectro essencial tanto em $(-\infty, 0)$ quanto em $(0, \infty)$, provando que $\mathscr{F}_{*}^{\text {sa }}$ é denso em $\mathscr{C} \mathscr{F}_{*}^{\text {sa }}$.

Vamos agora construir uma aplicação importante, a qual foi definida primeiramente em [75]. Para isso, definimos

$$
\hat{\mathrm{F}}_{*}^{\infty} \doteq\left\{S \in \mathscr{L}(\mathrm{H}):\|S\|=1, \Sigma_{\text {ess }}(S)=\{1,-1\} \text { e } \Sigma_{\text {disc }}(T) \text { é finito }\right\} \subset \mathscr{F}_{*}^{\text {sa }} .
$$

Proposição 1.4.33 Existe $F: \mathscr{C}_{*} \mathscr{F}^{\mathrm{sa}} \rightarrow \hat{\mathrm{F}}_{*}^{\infty}$ contínua cuja restrição a $\mathscr{F}_{*}^{\mathrm{sa}}$ é uma equivalência homotópica.

Prova. Para cada $T \in \mathscr{C} \mathscr{F}_{*}^{\text {sa }}$, existem uma vizinhança aberta $\mathcal{V}_{T}$ de $T$ em $\mathscr{C} \mathscr{F}$ sa $\delta>0$ tais que para cada $S \in \mathcal{V}_{T}, \Sigma(S) \cap[-\delta, \delta]$ é um sistema finito de autovalores de $S$ (cf. proposição 1.4.24). Seja $f_{T}: \mathbb{R} \rightarrow \mathbb{R}$ contínua tal que $f_{T}(\lambda)=-1$ se $\lambda<\delta, f_{T}(\lambda)=1$ se $\lambda>\delta$ e $f_{T}$ é linear em $[-\delta, \delta]$. Temos que

$$
\begin{aligned}
f_{T}: \mathcal{V}_{T} & \rightarrow \hat{\mathrm{F}}^{\infty} \\
S & \mapsto f_{T}(S)
\end{aligned}
$$

é contínua. Como $\mathscr{C} \mathscr{F}_{*}^{\text {sa }}$ é paracompacto (pois é um espaço métrico), é possível obter uma partição da unidade $\left\{\theta_{T}\right\}_{T \in \mathscr{C} \mathscr{F} \text { :a }}$ (localmente finita) subordinada à cobertura aberta $\left\{\mathcal{V}_{T}\right\}_{T \in \mathscr{C} \mathscr{F} \text { sa }}$. Definimos

$$
F(S)=\sum_{T \in \mathscr{C} F \text { sa }} \theta_{T}(S) f_{T}(S) .
$$

Vemos que $F$ é contínua e toma valores no espaço apropriado, pois qualquer combinação convexa de funções como as $f_{T}$ produz uma função do mesmo tipo. Para ver que a restrição 


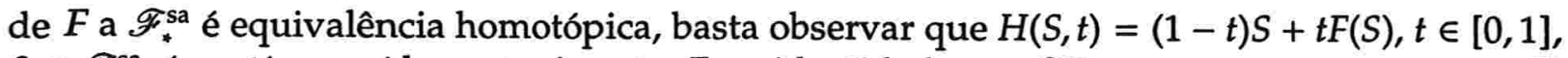
$S \in \mathscr{F}_{*}^{\text {sa }}$, é contínua e é homotopia entre $F$ e a identidade em $\mathscr{F}_{*}^{\text {sa }}$.

Em [13], Atiyah e Singer mostraram que, no caso complexo, a aplicação

$$
\Phi: \hat{\mathrm{F}}_{*}^{\infty} \ni S \mapsto \exp (i \pi(I+S)) \in \mathrm{U}(\infty)
$$

é uma equivalência homotópica. A prova deste resultado é um tanto delicada e não será dada aqui. A dificuldade reside no fato que $\Phi$ não é uma fibração. A idéia é verificar que os espaços envolvidos podem ser aproximados por espaços de dimensão finita, sobre os quais $\Phi$ é uma fibração e uma certa condição de compatibilidade entre estes espaços é verificada. Isto prova a seguinte proposição.

Proposição 1.4.34 $\mathscr{F}_{*}^{\text {sa }}$ tem o tipo de homotopia de $\mathrm{U}(\infty)$, se $\mathrm{H}$ é complexo.

O próximo argumento, devido a Phillips [75], mostra que o fluxo espectral é um isomorfismo $\pi_{1}\left(\mathscr{F}_{*}{ }^{\mathrm{s}}\right) \rightarrow \mathbb{Z}$, no caso complexo.

Teorema 1.4.35 O fluxo espectral sf: $\pi_{1}\left(\mathscr{F}_{*}^{\text {sa }}\right) \rightarrow \mathbb{Z}$ é um isomorfismo no caso complexo.

Prova. Pela proposição $1.4 .34, \pi_{1}\left(\mathscr{F}_{*}^{\text {sa }}\right)=\pi_{1}(\mathrm{U}(\infty))=\mathbb{Z}$, logo, basta mostrar que sf é sobre. De fato, se $\left\{e_{k}\right\}_{k \in \mathbb{Z}}$ é base ortonormal de $\mathrm{H}$, e $P_{k}$ denota a projeção ortogonal sobre o espaço gerado por $e_{k}$, então

$$
T(t)=\sum_{k \geq 1}\left(P_{k}-P_{-k}\right)+t P_{0}
$$

para $-1 \leq t \leq 1$ é uma curva contínua com fluxo espectral igual a 1.

O próximo teorema revela uma diferença crucial entre $\mathscr{C} \mathscr{F}^{\mathrm{sa}}$ e $\mathscr{F}^{\mathrm{sa}}$. A prova dada aqui não faz uso da transformada de Cayley, ao contrário de [23].

Teorema 1.4.36 $\mathscr{C} \mathscr{F}^{\text {sa }}$ é conexo por caminhos.

Prova. Vimos na observação 1.4.31 que, dado $T \in \mathscr{C} \mathscr{F}$ sa existe uma curva ligando $T$ à uma conjugação. Podemos portanto, supor que $T$ seja uma conjugação, i.e., $T=2 P_{M}-I=P_{M}-P_{M^{\perp}}$. Podemos pensar em $T$ como um operador que é igual a $I$ em $M$ e igual a $-I$ em $M^{\perp}$. Se codim $\mathrm{M}<\infty$, então $T(t)=P_{\mathrm{M}}-t P_{\mathrm{M}^{\perp}},-1 \leq t \leq 1$, é uma curva de operadores de Fredholm auto-adjuntos conectando $T$ a $I$.

Vamos agora à parte não-trivial do resultado, i.e., suporemos que $\operatorname{codim} M=\infty$. É suficiente provar que existe uma curva contínua em $\mathscr{C} \mathscr{F}$ sa $(\mathrm{M})$ conectando $I$ e $-I$. Podemos supor $\mathrm{M}=\mathrm{L}^{2}((0,1))$; neste caso o operador I pode ser entendido como o operador de multiplicação pela função identicamente 1 . Definindo para $t, \lambda \in[0,1]$

$$
f(t, \lambda)=(1-t)+t \operatorname{coth}(\lambda)
$$

temos ${ }^{34}$ que $f$ é contínua e real e $\left|f_{t}(\lambda)\right| \geq 1$. Portanto pelo lema 1.3 .48 ( $\operatorname{com} A=I$ ), segue que $T(t)$ definido como na equação 1.3.18 do referido lema é uma curva contínua de operadores bijetores $^{35}$ em $\mathscr{C} \mathscr{F}^{\text {sa }}(\mathrm{M})$. A vantagem que ganhamos aqui é que passamos do operador $I$, cujo

\footnotetext{
${ }^{34}$ coth denota a função cotangente hiperbólica, definida como $\operatorname{coth}(x) \doteq \cosh (x) / \sinh (x)=\left(e^{x}+e^{-x}\right) /\left(e^{x}-e^{-x}\right)$. Convencionamos que $\operatorname{coth}(0)=\infty$.

${ }^{35} T(t)$ é injetor com imagem $\mathrm{H}$.
} 
espectro é puramente pontual para o operador $T(1)$ que tem espectro $\Sigma(T(1))=\Sigma_{\text {ess }}(T(1))=$ $[\alpha, \infty), \operatorname{com} \alpha \doteq \operatorname{coth}(1)$. Para simplificar a notação, suponhamos $T=T(1)$. Considerando a apliçação

$$
g(t, \lambda)=\operatorname{coth}(\lambda-t),
$$

definida para $t, \lambda \in[0,1]$, temos que $g$ é contínua (inclusive em $t=\lambda$ ) e portanto, novamente pelo lema 1.3.48, $T(t)$ definida na equação 1.3.18, com $g$ em lugar de $f$, é uma curva contínua de operadores bijetores em $\mathscr{C} \mathscr{F}^{\text {sa }}(\mathrm{M})$.

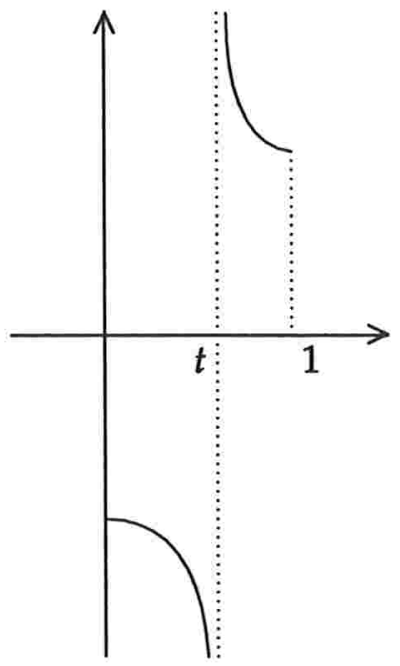

Vemos que $\Sigma(T(1))=\Sigma_{\text {ess }}(T(1))=(-\infty, \beta], \operatorname{com} \beta \doteq \operatorname{coth}(-1)$. Agora, basta usar um argumento análogo ao que fizemos com a função $f$ definida em 1.4.8 para construir uma curva de bijeções em $\mathscr{C}_{\mathscr{F}}{ }^{\mathrm{sa}}(\mathrm{M})$ conectando $T(1)$ e $-I$, como queríamos.

OBSERVAÇÃo 1.4.37 Um fato interessante demonstrado no teorema 1.4.36 é que existe uma curva contínua de bijeções ${ }^{36}$ em $\mathscr{C} \mathscr{F}$ sa conectando $I$ e $-I$. Em geral, se $T(t), 0 \leq t \leq 1$, é uma curva em $\mathscr{C}_{\mathscr{F}^{\text {sa }}}$ tal que $T(0)$ é bijetor (i.e., nul $\left.T(0)=\operatorname{def} T(0)=0\right)$, então, como ind $(T(t)$ ) é constante e as funções $t \mapsto \operatorname{nul}(T(t))$ e $t \mapsto \operatorname{def}(T(t))$ são semi-contínuas superiormente (pelo teorema 1.2.12), por conexidade concluímos que $\operatorname{nul}(T(t))=\operatorname{def}(T(t))=0$, para todo $t \in[0,1]$. Portanto, $T(t)$ é uma bijeção para todo $t \in[0,1]$.

ObSERVAÇÃo 1.4.38 A prova do teorema 1.4.36 funciona tanto no caso real quanto no caso complexo. Este fato não é inteiramente claro em [23]. Em loc.cit., Booss-Bavnbek, Lesch e Phillips usam a caracterização da imagem da transformada de Cayley dada no corolário 1.2.63 para provar que $\mathscr{C} \mathscr{F}$ sa é conexo por caminhos.

Um fato não muito explorado no estudo do espaço $\mathscr{C} \mathscr{F}^{\text {sa }}$ é que os conjuntos

$$
\mathscr{U}_{\alpha} \doteq\left\{T \in \mathscr{C} \mathscr{F}^{\text {sa }}: \inf _{\lambda \in \Sigma_{\text {ess }}(T)}|\lambda|>\alpha\right\},
$$

definidos para $\alpha>0$, são abertos (pela proposição 1.4.24) e $\mathscr{C} \mathscr{F}$ sa é a reunião dos $\mathscr{U}_{\alpha}$. Vamos obter algumas informações sobre os $\mathscr{U}_{\alpha}$.

\footnotetext{
${ }^{36}$ Como na nota de rodapé 35 .
} 
Proposição 1.4.39 A inclusão $i: \mathscr{U}_{\alpha} \subset \mathscr{U}_{\beta}, \alpha>\beta$ é uma equivalência homotópica.

Prova. Seja $\lambda(t)=\frac{(1-t) \beta+t \alpha}{\beta}, 0 \leq t \leq 1$. Pelo corolário 1.2.46, a aplicação $f_{t}(T)=\lambda(t) T$, $T \in \mathscr{C} \mathscr{F}^{\text {sa }}, 0 \leq t \leq 1$, é contínua. Além disso, como $f_{0}(T)=T$, para todo $T \in \mathscr{C} \mathscr{F}$ sa , $f_{1}\left(\mathscr{U}_{\beta}\right) \subset \mathscr{U}_{\alpha}, f_{t}\left(\mathscr{U}_{\beta}\right) \subset \mathscr{U}_{\beta}$ e $f_{t}\left(\mathscr{U}_{\alpha}\right) \subset \mathscr{U}_{\alpha}$, então $\left.f_{1}\right|_{\mathscr{U}_{\beta}}: \mathscr{U}_{\beta} \rightarrow \mathscr{U}_{\alpha}$ é uma inversa homotópica para $i$.

COROLÁrIo 1.4.40 $\mathscr{C} \mathscr{F}^{\text {sa }}$ tem o tipo de homotopia de $\mathscr{U}_{1}$.

Prova. Como os $\mathscr{U}_{\alpha}$ são abertos e $\mathscr{C} \mathscr{F}$ sa $(H)=\bigcup_{\alpha>0} \mathscr{U}_{\alpha}$, temos que $\mathscr{C} \mathscr{F}^{\text {sa }}$ tem o tipo de homotopia de $\lim _{\alpha} \mathscr{U}_{\alpha}$ (veja [93]). Pela proposição 1.4.39, segue que $\lim _{\alpha} \mathscr{U}_{\alpha}$ tem o tipo de homotopia de qualquer $\mathscr{U}_{\alpha}$.

\subsubsection{Assinaturas Parciais e Fluxo Espectral}

Nesta subseção, vamos desenvolver a teoria de assinaturas parciais e mostrar como a mesma pode ser utilizada para calcular o fluxo espectral de uma família analítica de operadores auto-adjuntos. Boa parte dos resultados aqui obtidos generaliza aqueles de [44] em dimensão finita.

Fixemos algumas notações. Se $T$ é um operador auto-adjunto definido em um espaço de dimensão finita, definimos o índice, a nulidade e o co-índice de $T$ como o número de autovalores de $T$, negativos, nulos e positivos, respectivamente, contados de acordo com a multiplicidade. Tais números são denotados por $n_{-}(T), n_{0}(T)$ e $n_{+}(T)$, respectivamente. A assinatura de $T$ é definida por $\sigma(T) \doteq n_{+}(T)-n_{-}(T)$. Podemos dar as mesmas definições para uma forma bilinear hermitiana, usando a identificação $T \mapsto\langle T \cdot, \cdot\rangle$ entre operadores auto-adjuntos e formas bilineares hermitianas.

Seja $T:\left[t_{0}-\varepsilon, t_{0}+\varepsilon\right] \rightarrow \mathscr{C} \mathscr{F}^{\mathrm{sa}}(\mathrm{H})$ uma família holomorfa de tipo (A) com domínio D (conforme a definição 1.2.78) e suponhamos que $t=t_{0}$ seja o único ponto de $\left[t_{0}-\varepsilon, t_{0}+\varepsilon\right]$ tal que $\operatorname{ker} T(t) \neq 0$. Queremos calcular a contribuição do ponto $t=t_{0}$ para o fluxo espectral de $T$. Se $\left.T^{\prime}\left(t_{0}\right)\right|_{\text {ker } T\left(t_{0}\right)}$ é injetor, então a mesma demonstração do teorema 4.1.32 de [76] mostra que

$$
\operatorname{sf}(T)=\sigma\left(\left.T^{\prime}\left(t_{0}\right)\right|_{\text {ker } T\left(t_{0}\right)}\right) .
$$

Porém, se $\left.T^{\prime}\left(t_{0}\right)\right|_{\text {ker } T\left(t_{0}\right)}$ é degenerada, as coisas se complicam. Considerando $H=\mathbb{R}^{2}, T(t)=$

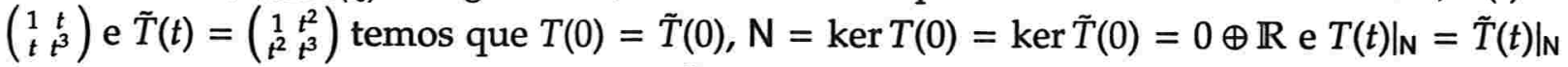
para todo $t$, mas $\operatorname{sf}\left(\left.T\right|_{[-\varepsilon, \varepsilon]}\right)=0 \neq 1=\operatorname{sf}\left(\left.\tilde{T}\right|_{[-\varepsilon, \varepsilon]}\right)$. Em particular, $T$ e $\tilde{T}$ têm a mesma expansão de Taylor restrita a $N$. Isso mostra que considerar simplesmente a restrição das derivadas $T^{(n)}\left(t_{0}\right)$ a $\operatorname{ker} T\left(t_{0}\right)$ não é suficiente para detectar a contribuição de $t_{0}$ para o fluxo espectral. A teoria de assinaturas parciais fornece as ferramentas adequadas para medir a contribuição de $t_{0}$ para o fluxo espectral da família $T$ em termos de restrições especiais.

Definição 1.4.41 O ponto $t_{0}$ é dito degenerescência da curva $T$ se $\operatorname{ker} T\left(t_{0}\right) \neq 0 . t_{0}$ é dito degenerescência isolada se $t_{0}$ for o único ponto de degenrescência em $\left[t_{0}-\varepsilon, t_{0}+\varepsilon\right]$. Uma função $u:\left[t_{0}-\varepsilon, t_{0}+\varepsilon\right] \rightarrow D$ de classe $C^{\infty}$ é dita função raiz para $T$ se $u\left(t_{0}\right) \in \operatorname{ker} T\left(t_{0}\right)$. A ordem de $u$ é a ordem do zero em $t=t_{0}$ da função $t \in\left[t_{0}-\varepsilon, t_{0}+\varepsilon\right] \mapsto T(t) u(t) \in \mathrm{H}$ e é denotada ${ }^{37}$ por ord $(u)$.

\footnotetext{
${ }^{37}$ Dado $k \in \mathbb{N}$, uma função $v:\left[t_{0}-\varepsilon, t_{0}+\varepsilon\right] \rightarrow \mathrm{H}$ de classe $\mathrm{C}^{\infty}$ tem um zero de ordem $k$ em $t=t_{0}$ se
} 
Vamos agora definir os objetos básicos para a teoria de assinaturas parciais. Dado $k \geq 1$, seja

$$
\mathrm{W}_{k} \doteq\left\{u_{0} \in \mathrm{W}_{0}: \text { Existe função raiz } u \text { para } T(t) \operatorname{com} u\left(t_{0}\right)=u_{0} \text { e ord }(u) \geq k\right\} \text {. }
$$

Para $u_{0}, v_{0} \in \mathrm{W}_{k}$, definimos

$$
B_{k}\left(u_{0}, v_{0}\right)=\frac{1}{k !}\left\langle\left.\frac{d^{k}}{d t^{k}}\right|_{t=t_{0}}[T(t) u(t)], v_{0}\right\rangle
$$

onde $u(t)$ é uma função-raiz para $T \operatorname{com} u\left(t_{0}\right)=u_{0}$ e ord $(u) \geq k$. Obviamente, cada $\mathrm{W}_{k}$ é um subespaço $W_{1} \supset W_{2} \supset \ldots W_{k} \supset \ldots$

PRoposição 1.4.42 $B_{k}$ é uma forma bilinear hermitiana (bem-definida) em $\mathrm{W}_{k}$, para todo $k \geq 1$. Além disso, $\mathrm{W}_{k+1} \subset \operatorname{ker} B_{k}$.

Prova. Dados $u_{0}, v_{0} \in \mathrm{W}_{k}$, se $u, v$ são funções-raiz para $T \operatorname{com} u\left(t_{0}\right)=u_{0}, v\left(t_{0}\right)=v_{0} \mathrm{e}$ $\operatorname{ord}(u), \operatorname{ord}(v) \geq k$, então

$$
\begin{aligned}
\left\langle\left.\frac{d^{k}}{d t^{k}}\right|_{t=t_{0}}[T(t) u(t)], v_{0}\right\rangle & =\left.\frac{d^{k}}{d t^{k}}\right|_{t=t_{0}}\langle T(t) u(t), v(t)\rangle \\
& =\left.\frac{d^{k}}{d t^{k}}\right|_{t=t_{0}}\langle u(t), T(t) v(t)\rangle \\
& =\left\langle u_{0},\left.\frac{d^{k}}{d t^{k}}\right|_{t=t_{0}}[T(t) v(t)]\right\rangle
\end{aligned}
$$

sendo que as igualdades 1.4 .11 e 1.4 .12 seguem do fato que $\left.\frac{d^{j}}{d t}\right|_{t=t_{0}} T(t) u(t)=\left.\frac{d^{j}}{d t}\right|_{t=t_{0}} T(t) v(t)=0$ para $j<k$.

OвSERVAÇão 1.4.43 É possível definir os subespaços $W_{k}$ e as formas $B_{k}$ de maneira algébrica, considerando a expansão de Taylor de $T$ em $t_{0}$. Veja [44], Pag.8.

Definição 1.4.44 Se $T:\left[t_{0}-\varepsilon, t_{0}+\varepsilon\right] \rightarrow \mathscr{C} \mathscr{F}$ sa $(\mathrm{H})$ é uma família holomorfa de tipo (A), $t=t_{0}$ é uma degenerescência isolada de $T$ e $k \geq 1$, definimos o $k$-ésimo índice parcial, o $k$-ésimo co-índice parcial e a $k$-ésima assinatura parcial em $t_{0}$ por

$$
n_{k}^{-}\left(T, t_{0}\right) \doteq n^{-}\left(B_{k}\right), n_{k}^{+}\left(T, t_{0}\right) \doteq n^{+}\left(B_{k}\right), \sigma_{k}\left(T, t_{0}\right) \doteq \sigma\left(B_{k}\right) .
$$

Tais inteiros são chamados genericamente de assinaturas parciais de $T$ em $t=t_{0}$.

Seja $T$ uma família como na definição 1.4.44. Denotemos por D o domínio de $T(t)$, o qual independe de $t$, por hipótese. Pelo teorema 1.2.77, se $\varepsilon>0$ é suficientemente pequeno, existe $\delta>0$ tal que a intersecção de $\Sigma(T(t)) \operatorname{com}(-\delta, \delta)$ consiste de uma quantidade finita de autovalores isolados, os quais podem ser representados por funções analíticas reais $\lambda_{1}(t), \ldots, \lambda_{N}(t)$ definidas em $\left[t_{0}-\varepsilon, t_{0}+\varepsilon\right]$. Além disso, é possível obter funções analíticas $u_{1}(t), \ldots, u_{N}(t)$

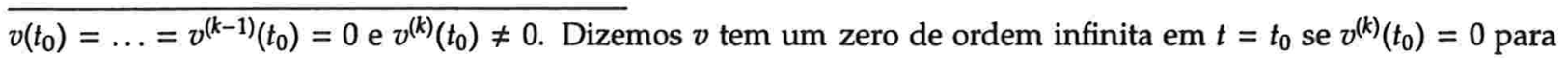
todo $k \in \mathbb{N}$. 
tais que $\left\{u_{j}(t)\right\}_{1 \leq j \leq N}$ é um conjunto ortonormal de $\mathrm{H}$ formado por autovetores de $T(t)$ correspondentes aos autovalores $\lambda_{1}(t), \ldots, \lambda_{N}(t)$, respectivamente. Vamos identificar as assinaturas parciais neste caso.

Primeiramente, provemos que

$$
\mathrm{W}_{k}=\operatorname{span}\left\{u_{j}\left(t_{0}\right): \lambda_{j}\left(t_{0}\right)=\lambda_{j}^{\prime}\left(t_{0}\right)=\ldots=\lambda_{j}^{(k-1)}\left(t_{0}\right)=0\right\},
$$

para qualquer $k \geq 1$. De fato, se $\lambda_{j}\left(t_{0}\right)=\lambda_{j}^{\prime}\left(t_{0}\right)=\ldots=\lambda_{j}^{(k-1)}\left(t_{0}\right)=0$, então $u_{j}(t)$ é uma função-raiz para $T$ com ordem $\geq k$, portanto, $u_{j}\left(t_{0}\right) \in \mathrm{W}_{k}$. Reciprocamente, se $u_{0} \in \mathrm{W}_{k}$, seja $u(t)$ uma função-raiz para $T$ tal que $u\left(t_{0}\right)=u_{0}$ e ord $(u) \geq k$. Pondo $\mu_{j}(t) \doteq\left\langle u(t), u_{j}(t)\right\rangle$, para $j \geq 1$, temos que $u(t)=\sum_{k=1}^{\infty} \mu_{j}(t) u_{j}(t)$. Logo, $T(t) u(t)=\sum_{k=1}^{\infty} \mu_{j}(t) \lambda_{j}(t) u_{j}(t)$, portanto, se $\mu_{j}\left(t_{0}\right) \neq 0$ então $\lambda_{j}\left(t_{0}\right)=\lambda_{j}^{\prime}\left(t_{0}\right)=\ldots=\lambda_{j}^{(k-1)}\left(t_{0}\right)=0$. Isso prova 1.4.14.

Se $u \in W_{k}$ é um autovetor correspondente ao autovalor $\lambda_{j}\left(t_{0}\right)$ e $\lambda_{j}\left(t_{0}\right)=\lambda_{j}^{\prime}\left(t_{0}\right)=\ldots=$ $\lambda_{j}^{(k-1)}\left(t_{0}\right)=0$, então, a definição de $B_{k}$ mostra que

$$
B_{k}(u, v)=\frac{1}{k !} \lambda_{j}^{(k)}\left(t_{0}\right)\langle u, v\rangle
$$

para todo $v \in \mathbf{W}_{k}$. Isso implica que

$$
\begin{aligned}
n_{k}^{+}\left(T, t_{0}\right) & =\#\left\{j: \lambda_{j}\left(t_{0}\right)=\lambda_{j}^{\prime}\left(t_{0}\right)=\ldots=\lambda_{j}^{(k-1)}\left(t_{0}\right)=0 \text { e } \lambda_{j}^{(k)}\left(t_{0}\right)>0\right\} \doteq n_{k}^{+}, \\
n_{k}^{-}\left(T, t_{0}\right) & =\#\left\{j: \lambda_{j}\left(t_{0}\right)=\lambda_{j}^{\prime}\left(t_{0}\right)=\ldots=\lambda_{j}^{(k-1)}\left(t_{0}\right)=0 \text { e } \lambda_{j}^{(k)}\left(t_{0}\right)<0\right\} \doteq n_{k}^{-}, \\
\sigma_{k}\left(T, t_{0}\right) & =n_{k}^{+}-n_{k}^{-} \doteq \sigma_{k} .
\end{aligned}
$$

Por outro lado, sabemos que um autovalor $\lambda_{j}(t)$ contribui na contagem do fluxo espectral se e só se $\lambda_{j}\left(t_{0}\right)=0$, e isso é verificado somente para um número finito de $j$ 's. Fixemos, portanto, um destes $j^{\prime}$ 's e seja $k$ o menor inteiro tal que $\lambda_{j}^{(k)}\left(t_{0}\right) \neq 0$. Temos quatro situações possíveis:

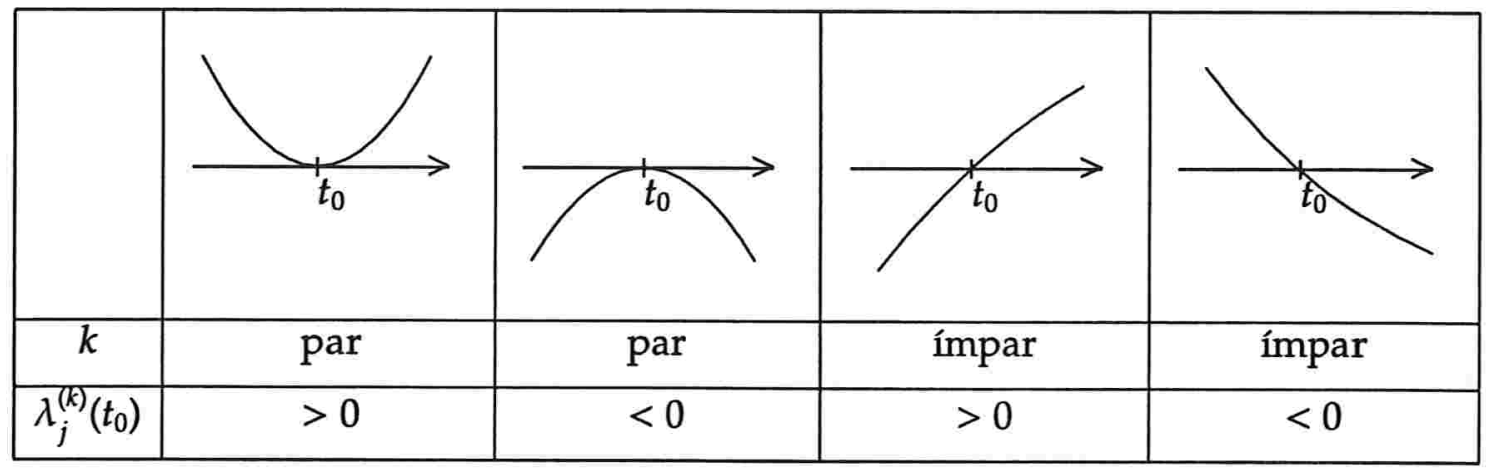

Estas observações e as equações 1.4.15, 1.4.16 e 1.4.17 nos permitem concluir o seguinte teorema. 
Trorema 1.4.45 Seja $T:\left[t_{0}-\varepsilon, t_{0}+\varepsilon\right] \rightarrow \mathscr{C} \mathscr{F}$ sa $(H)$ uma familia holomorfa de tipo $(A)$ e $t=t_{0}$ uma degenerescência isolada de T. Então

$$
\begin{aligned}
\operatorname{sf}\left(\left.T\right|_{\left[t_{0}-\varepsilon, t_{0}\right]}\right) & =\sum_{k \geq 1}\left\{n_{2 k}^{-}\left(T, t_{0}\right)+n_{2 k-1}^{+}\left(T, t_{0}\right)\right\}, \\
\operatorname{sf}\left(\left.T\right|_{\left[t_{0}, t_{0}+\varepsilon\right]}\right) & =-\sum_{k \geq 1} n_{k}^{-}\left(T, t_{0}\right) \\
\operatorname{sf}\left(\left.T\right|_{\left[t_{0}-\varepsilon, t_{0}+\varepsilon\right]}\right) & =\sum_{k \geq 1} \sigma_{2 k-1}\left(T, t_{0}\right) .
\end{aligned}
$$

A análise que antecede o teorema 1.4 .45 prova o seguinte resultado.

Proposição 1.4.46 Se $T:\left[t_{0}-\varepsilon, t_{0}+\varepsilon\right] \rightarrow \mathscr{C} \mathscr{F}$ sa $(\mathrm{H})$ é uma familia holomorfa de tipo $(A)$ e $t=t_{0} e ́$ a única degenerescência de $T(t)$ em $\left[t_{0}-\varepsilon, t_{0}+\varepsilon\right]$ então $\mathrm{W}_{k+1}=\operatorname{ker} B_{k}$ para todo $k \geq 1$.

Vamos agora estudar a invariância do fluxo espectral por certas ações com as quais nos depararemos posteriormente, em especial quando definirmos o índice de Maslov.

Consideremos a ação cogrediente de $\mathrm{GL}(\mathrm{H})$ em $\mathscr{C} \mathscr{F}$ sa $(\mathrm{H})$ dada pela fórmula

$$
\begin{aligned}
\mathrm{GL}(\mathrm{H}) \times \mathscr{C}^{\mathrm{sa}}(\mathrm{H}) & \rightarrow \mathscr{C F F}^{\mathrm{sa}}(\mathrm{H}) \\
(U, T) & \mapsto U^{*} T U
\end{aligned}
$$

A pergunta natural que surge é se o fluxo espectral de uma curva é invariante pela ação cogrediente. A próxima proposição nos dá uma sugestão de resposta para esta pergunta.

Proposição 1.4.47 Sejam $T:\left[t_{0}-\varepsilon, t_{0}+\varepsilon\right] \rightarrow \mathscr{C} \mathscr{F}$ sa $(H)$ uma família holomorfa de tipo $(A), t=t_{0}$ uma degenerescência isolada de $T$ e $U:\left[t_{0}-\varepsilon, t_{0}+\varepsilon\right] \rightarrow \mathrm{GL}(\mathrm{H})$ uma curva analítica real tal que $\mathrm{D} \doteq U(t)^{-1}(\mathrm{D})$ independe de $t \in[a, b]$. Então $\tilde{T}(t) \doteq U(t)^{*} T(t) U(t)$ é uma família holomorfa de tipo $(A), t=t_{0}$ é uma degenerescência isolada de $\tilde{T}(t) e$ as curvas $T e \tilde{T}$ têm as mesmas assinaturas parciais em $t=t_{0}$.

Prova. Obviamente, $\tilde{T}(t)$ é uma família holomorfa de tipo (A) e $t=t_{0}$ é uma degenerescência isolada de $\tilde{T}$. Sejam $W_{k}, \tilde{W}_{k}$ e $B_{k}, \tilde{B}_{k}$ os subespaços e formas bilineares definidos em 1.4 .9 e 1.4.10 correspondentes às curvas $T(t)$ e $\tilde{T}(t)$, respectivamente. Se $k \geq 1, u_{0}, v_{0} \in \mathrm{W}_{k}$ e $u(t), v(t)$ são funções-raiz para $T, \tilde{T}$, respectivamente, com ordem $\geq k$, então definindo $\tilde{u}(t)=U(t)^{-1} u(t)$ e $\tilde{v}(t)=U(t)^{-1} v(t)$, temos

$$
\tilde{T}(t) \tilde{u}(t)=U(t)^{*} T(t) u(t), \tilde{T}(t) \tilde{v}(t)=U(t)^{*} T(t) v(t)
$$

logo, $\tilde{u}$ e $\tilde{v}$ são funções-raiz para $T$ e $\tilde{T}$ de ordem $\geq k$ e $\tilde{W}_{k}=U\left(t_{0}\right)^{-1}\left(\mathrm{~W}_{k}\right)$, para todo $k \geq 0$. Além disso, pondo $\tilde{u}_{0} \doteq \tilde{u}\left(t_{0}\right)$ e $\tilde{v}_{0} \doteq \tilde{v}\left(t_{0}\right)$, temos

$$
\begin{aligned}
\tilde{B}_{k}\left(\tilde{u}_{0}, \tilde{v}_{0}\right) & =\frac{1}{k !}\left\langle\left.\frac{d^{k}}{d t^{k}}\right|_{t=t_{0}}[\tilde{T}(t) \tilde{u}(t)], \tilde{v}_{0}\right\rangle \\
& =\left.\frac{1}{k !} \frac{d^{k}}{d t^{k}}\right|_{t=t_{0}}\left\langle U(t)^{*} T(t) u(t), U(t)^{-1} v(t)\right\rangle \\
& =\left.\frac{1}{k !} \frac{d^{k}}{d t^{k}}\right|_{t=t_{0}}\langle T(t) u(t), v(t)\rangle \\
& =B_{k}\left(u_{0}, v_{0}\right),
\end{aligned}
$$


portanto, $B_{k}$ é o pull-back de $\tilde{B}_{k}$ por $U\left(t_{0}\right)^{-1}$; em particular, as assinaturas parciais de $T$ e $\tilde{T}$ em $t=t_{0}$ coincidem.

No próximo corolário, vamos utilizar a proposição 1.4.47 para mostrar que o fluxo espectral é invariante pela ação cogrediente, sob uma condição conveniente. Antes disso, precisamos de um fato técnico.

Lema 1.4.48 Se $T:[a, b] \rightarrow \mathscr{C} F^{\text {sa }}(\mathrm{H})$ é uma família holomorfa de tipo $(A)$, então existem $a=t_{0}<$ $\ldots<t_{r}=b$ tais que:

1. Para cada subintervalo $\left[t_{j-1}, t_{j}\right]$ existe $\delta_{j}>0$ tal que $\Sigma(T(t)) \cap(-\delta, \delta)$ consiste de uma quantidade finita (digamos $N_{j}$ ) de autovalores de multiplicidade finita, os quais podem ser representados por funções analíticas reais $\lambda_{1, j}(t), \ldots, \lambda_{N_{j}, j}(t)$ definidas em $\left[t_{j-1}, t_{j}\right]$, para $j=1, \ldots, r$;

2. O conjunto das degenerescências de $T$ em $\left[t_{j-1}, t_{j}\right]$ coincide com

$$
\left\{t \in\left[t_{j-1}, t_{j}\right]: \lambda_{i, j}(t)=0 \text { para alguma } \lambda_{i, j}\right\} .
$$

Em particular, em cada $\left[t_{j-1}, t_{j}\right]$ além dos autovalores identicamente nulos, $T$ possui somente degenrescências isoladas, e portanto, $\operatorname{sf}\left(\left.T\right|_{\left[t_{j-1}, t_{j}\right]}\right)$ pode ser calculado utilizando o teorema 1.4.45.

Prova. O primeiro ítem decorre imediatamente das observações que seguem a definição 1.4.44 e da compacidade de $[a, b]$. O segundo ítem decorre da definição das funções $\lambda_{i, j}$ e a conclusão segue da analiticidade das funções $\lambda_{i, j}$.

Decorre do lema 1.4.48 que uma família holomorfa de tipo (A) possui somente uma quantidade finita de degenerescências, além dos autovalores identicamente nulos, que não influenciam no cálculo do fluxo espectral. Em particular, podemos proceder como no caso em que as degenerescências são isoladas. Isso nos permite concluir o corolário abaixo.

Corolário 1.4.49 Sejam $T:[a, b] \rightarrow \mathscr{C} \mathscr{F}^{\mathrm{sa}}(\mathrm{H})$ uma família holomorfa de tipo $(A)$ e $U:[a, b] \rightarrow$ $\mathrm{GL}(\mathrm{H})$ analitica real tal que $\mathrm{D} \doteq U(t)^{-1}(\mathrm{D})$ independe de $t \in[a, b]$. Então $0^{38} \tilde{T}(t) \doteq U(t)^{*} T(t) U(t) e$ $T(t)$ têm mesmo fluxo espectral em $[a, b]$.

Prova. Basta usar o lema 1.4.48, o teorema 1.4.45, a proposição 1.4.47 e a aditividade do fluxo espectral por concatenação.

No caso limitado é possível obter uma versão bem mais precisa do corolário 1.4.49.

Corolário 1.4.50 Sejam $T:[a, b] \rightarrow \mathscr{F}^{\mathrm{sa}}(\mathrm{H}), U:[a, b] \rightarrow \mathrm{GL}(\mathrm{H})$ contínuas $e \tilde{T}(t)$ como no corolário 1.4.49. Então $\operatorname{sf}(T)=\operatorname{sf}(\tilde{T})$.

Prova. Como sf é invariante por homotopias com os extremos fixos, podemos supor que $T, U$ são analíticas reais. ${ }^{39}$ Como $T(t)$ é limitado com domínio $\mathrm{H}$, então $T$ é holomorfa de tipo (A) e $U(t)^{-1}(\mathrm{D})=\mathrm{H}$ independe de $t$. Logo, $\mathrm{sf}(T)=\operatorname{sf}(\tilde{T})$.

É importante ainda para nossos propósitos estudar mais um tipo especial de invariância do fluxo espectral.

\footnotetext{
${ }^{38} \mathrm{D} \doteq \mathrm{D}(T(t))$, conforme os comentários após a definição 1.4.44.

${ }^{39}$ Primeiramente, aproximamos $T$ e $U$ por curvas analíticas reais com os mesmos extremos (veja o final da seção 2.4). Como um aberto de um espaço de Banach é localmente conexo por caminhos e semi-localmente simplesmente conexo (pois as bolas são convexas), temos que, de fato, $T, U$ são homotópicas a curvas analíticas reais com os mesmos extremos. Veja [76], Pág.69.
} 
ProposiçÃo 1.4.51 Sejam $T:\left[t_{0}-\varepsilon, t_{0}+\varepsilon\right] \rightarrow \mathscr{C} \mathscr{F}$ sa $(H)$ uma família holomorfa de tipo $(A), t=t_{0}$ uma degenerescência isolada de $T$ e $U:\left[t_{0}-\varepsilon, t_{0}+\varepsilon\right] \rightarrow \mathrm{GL}(\mathrm{H})$ uma curva analítica real tais que

1. $\mathrm{D} \doteq U(t)^{-1}(\mathrm{D})$ independe de $t \in[a, b]$;

2. $U^{*}(t) T(t)=T(t) U(t)$, para todo $t \in[a, b]$;

3. $\left.U\left(t_{0}\right)\right|_{\operatorname{ker}\left(T\left(t_{0}\right)\right)}=I$;

4. $U(t)^{*} T(t)=T(t) U(t)$, para todo $t \in[a, b]$.

Então $\hat{T}(t) \doteq T(t) U(t)$ é uma família holomorfa de tipo $(A), t=t_{0}$ é degenerescência isolada de $\hat{T}$ e $T, \hat{T}$ têm as mesmas assinaturas parciais em $t=t_{0}$.

Prova. Claramente, os ítens (1), (2) e (3) da hipótese implicam que $\hat{T}$ é uma família holomorfa de tipo (A) de operadores auto-adjuntos com degenerescência isolada em $t=t_{0}$ e $\operatorname{ker} T\left(t_{0}\right)=$ $\operatorname{ker} \hat{T}\left(t_{0}\right)$. Consideremos $k \geq 1$, os espaços $W_{k}, \hat{W}_{k}$ e as formas bilineares $B_{k}, \hat{B}_{k}$ correspondentes a $T, \hat{T}$ em $t=t_{0}$, respectivamente. Se $u$ é função-raiz para $T \operatorname{com} u(t)=u_{0} \in \mathrm{W}_{k}$, então $\hat{u}(t) \doteq U(t)^{-1} u(t)$ é função-raiz para $\hat{T}$ com $\hat{u}\left(t_{0}\right)=u\left(t_{0}\right)=u_{0}$, pelo segundo ítem da hipótese. Como $T(t) u(t)=\hat{T}(t) \hat{u}(t)$, temos que ord $(u)=\operatorname{ord}(\hat{u})$ em $t=t_{0}, \operatorname{logo}, W_{k}=\hat{W}_{k}$, para todo $k \geq 1$. Além disso, dado qualquer $v_{0} \in \mathrm{W}_{k}$, temos

$$
\hat{B}_{k}\left(u_{0}, v_{0}\right)=\frac{1}{k !}\left\langle\left.\frac{d^{k}}{d t^{k}}\right|_{t=t_{0}}[\hat{T}(t) \mathfrak{u}(t)], v_{0}\right\rangle=\frac{1}{k !}\left\langle\left.\frac{d^{k}}{d t^{k}}\right|_{t=t_{0}}[T(t) u(t)], v_{0}\right\rangle=B_{k}\left(u_{0}, v_{0}\right) ，
$$

provando o desejado.

O próximo corolário mostra que o fluxo espectral é invariante por mais um tipo de ação.

Corolário 1.4.52 Sejam $T:[a, b] \rightarrow \mathscr{C} \mathscr{F}^{\mathrm{sa}}(\mathrm{H})$ uma família holomorfa de tipo $(A)$ e $U:[a, b] \rightarrow$ $\mathrm{GL}(\mathrm{H})$ uma curva analítica real tais que

1. $\mathrm{D} \doteq U(t)^{-1}(\mathrm{D})$ independe de $t \in[a, b]$;

2. $U^{*}(t) T(t)=T(t) U(t)$, para todo $t \in[a, b] ;$

3. $\left.U(t)\right|_{\operatorname{ker}(T(t))}=I$, para todo $t \in[a, b]$.

Definindo $\hat{T}$ como na proposição 1.4.51, temos que $\operatorname{sf}(\hat{T})=\operatorname{sf}(T)$.

Prova. Pelo lema 1.4.48, excetuando autovalores identicamente nulos, $T$ admite no máximo uma quantidade finita de degenerescências isoladas em $[a, b]$, portanto, podemos subdividir o intervalo $[a, b]$ em pequenos intervalos, cada qual contendo apenas um ponto de degenerescência. Usando o teorema 1.4 .45 e a aditividade do fluxo espectral por concatenação, o resultado segue pela proposição 1.4 .51 .

Corolário 1.4.53 Se $T:[a, b] \rightarrow \mathscr{F} \mathrm{sa}(\mathrm{H})$ é holomorfa no sentido usual e U é uma curva satisfazendo as hipóteses do corolário 1.4.52, então $\operatorname{sf}(T)=\operatorname{sf}(\hat{T})$. 


\subsection{Estruturas Simpléticas}

Nosso ambiente de trabalho nesta seção será um espaço simplético de dimensão infinita. A idéia é tentar preservar ao máximo a teoria já conhecida em dimensão finita (veja [6], [76], por exemplo). Os resultados aqui obtidos sobre subespaços lagrangianos serão muito importantes nas próximas subseções onde trabalharemos com certas variedades grassmannianas formadas por subespaços lagrangianos.

\subsubsection{Preliminares}

Seja $\mathrm{H}$ um espaço de Hilbert separável de dimensão infinita. Uma estrutura complexa em $\mathrm{H}$ é um operador $J \in \mathscr{L}(\mathrm{H})$ tal que $J^{2}=-I$ e $J^{*}=-J$.

Definição 1.5.1 Uma forma simplética em $\mathrm{H}$ é uma forma bilinear $\omega: \mathrm{H} \times \mathrm{H} \rightarrow \mathbb{R}$ dada por $\omega(u, v)=\langle J u, v\rangle, u, v \in \mathrm{H}$, onde $J$ é uma estrutura complexa. Um espaço de Hilbert munido de uma forma simplética é chamado de espaço simplético.

OвSERvação 1.5.2 Em [27], B. Booss-Bavnbek e C. Zhu desenvolvem a teoria no contexto de espaços de Banach, sem a hipótese que a forma $\omega$ seja representada por um operador $J$ como acima.

Se $T \in \mathscr{L}\left(\mathrm{H}, \mathrm{H}^{\prime}\right)$ e $\omega^{\prime}$ é uma forma simplética em $\mathrm{H}^{\prime}$, com estrutura complexa correspondente $J^{\prime}$, em geral, o pull-back $T^{*}\left(\omega^{\prime}\right)$ é uma forma bilinear anti-simétrica em $\mathrm{H}$. Faz sentido perguntar sob que condições $T^{*}\left(\omega^{\prime}\right)$ é uma forma simplética em $\mathrm{H}$. Para resolver esta questão, observamos que para $u, v \in \mathrm{H}$,

$$
\begin{aligned}
T^{*}\left(\omega^{\prime}\right)(u, v) & =\omega^{\prime}(T u, T v) \\
& =\left\langle J^{\prime} T u, T v\right\rangle=\left\langle T^{*} J^{\prime} T u, v\right\rangle,
\end{aligned}
$$

logo $T^{*}\left(\omega^{\prime}\right)$ é uma forma simplética se e só se $T^{*} J^{\prime} T$ é uma estrutura complexa. Como $\left(T^{*} J^{\prime} T\right)^{*}=$ $-T^{*} J^{\prime} T$, segue que $T^{*}\left(\omega^{\prime}\right)$ é forma simplética se e só se $\left(T^{*} J^{\prime} T\right)^{2}=-I$. Em particular, se $T^{*} T=I_{H}$ e $T T^{*}=I_{H^{\prime}}$, tal condição é satisfeita.

Definição 1.5.3 $\mathrm{Se}(\mathrm{H}, \omega, J),\left(\mathrm{H}^{\prime}, \omega^{\prime}, J^{\prime}\right)$ são espaços simpléticos, dizemos que $T \in \mathscr{L}\left(\mathrm{H}, \mathrm{H}^{\prime}\right)$ é um simplectomorfismo ou um isomorfismo simplético se $T$ é um isomorfismo (limitado) e $T^{*}\left(\omega^{\prime}\right)=\omega$, ou equivalentemente, $T^{*} J^{\prime} T=J$. Neste caso, $(H, \omega, J)$ é dito simplectomorfo a $\left(H, \omega^{\prime}, J^{\prime}\right)$.

Definimos o grupo simplético de $(\mathrm{H}, \omega, J)$ por

$$
\mathrm{Sp}(\mathrm{H})=\mathrm{Sp}(\mathrm{H}, \omega) \doteq\left\{T \in \mathrm{GL}(\mathrm{H}): T^{*}(\omega)=\omega\right\}=\left\{T \in \mathrm{GL}(\mathrm{H}): T^{*} J T=J\right\},
$$

i.e., $\mathrm{Sp}(\mathrm{H})$ é o conjunto dos simplectomorfismos de $H$. Sp $(H)$ é um subgrupo fechado de $G L(H)$.

Definimos o grupo unitário de $\mathrm{H}$ relativo a J por

$$
\mathrm{U}(\mathrm{H} ; J)=\{T \in \mathrm{O}(\mathrm{H}): T J-J T=0\} .
$$

Como antes, $\mathrm{U}_{c}\left(\mathrm{H}_{;} J\right)$ é o subgrupo formado pelos elementos que são perturbações compactas da identidade. O próximo lema fornece uma relação útil entre os grupos definidos anteriormente. 
Lema 1.5.4 Temos que $\mathrm{Sp}(\mathrm{H}) \cap \mathrm{O}(\mathrm{H})=\mathrm{U}(\mathrm{H} ; J)$. Em particular, $\mathrm{U}(\mathrm{H} ; J) \subset \mathrm{Sp}(\mathrm{H})$.

Prova. Se $T \in U(H ; J)$, então por definição $T \in \mathrm{O}(\mathrm{H})$ e $T^{*} J T=T^{*} T J=J$, $\log o T \in \mathrm{Sp}(\mathrm{H})$. Reciprocamente, se $T \in \mathrm{Sp}(\mathrm{H}) \cap \mathrm{O}(\mathrm{H})$, então $T J=T\left(T^{*} J T\right)=J T$, logo $T \in \mathrm{U}(\mathrm{H} ; J)$.

Voltaremos a tratar destes grupos oportunamente. Vamos agora dar um exemplo fundamental de espaço simplético de dimensão infinita.

Exemplo 1.5.5 Dado qualquer espaço de Hilbert, definimos em $\mathrm{H} \oplus \mathrm{H}$ a forma simplética

$$
\omega_{\text {can }}\left((u, v),\left(u^{\prime}, v^{\prime}\right)\right)=\left\langle u, v^{\prime}\right\rangle-\left\langle v, u^{\prime}\right\rangle .
$$

É imediato que $\mathrm{H} \oplus \mathrm{H}$ munido desta forma é um espaço simplético. A estrutura complexa correspondente a $\omega$ é $J(u, v)=(-v, u), u, v \in \mathrm{H}$. A forma simplética $\omega_{\text {can }}$ e a estrutura complexa $J$ correspondentes são chamadas de forma simplética e estrutura complexa canônicas de $\mathrm{H} \oplus \mathrm{H}$. Veremos adiante que qualquer espaço simplético é simplectomorfo a um espaço simplético deste tipo. Este fato mostra que boa parte da teoria pode ser desenvolvida como em dimensão finita, conforme [76], Cap.I.

\subsubsection{Subespaços Lagrangianos}

Dado um espaço simplético $(H, \omega, J)$ e $M \subset H$ subespaço fechado, podemos nos perguntar sob que condições a restrição da estrutura simplética de $\mathrm{H}$ a $\mathrm{M}$ torna-o um espaço simplético. Uma condição necessária óbvia é que $\mathrm{M}$ seja invariante por $J$. Um argumento simples mostra que, de fato, esta condição é suficiente. Neste caso, M é dito um subespaço simplético de $\mathrm{H}$. Uma situação diametralmente oposta a esta é aquela em que $J(M) \subset \mathrm{M}^{\perp}$. Este tipo de subespaço aparece mais freqüentemente na teoria do que os subespaços simpléticos; esta subseção é dedicada ao estudo dos mesmos.

Definição 1.5.6 Dado um espaço simplético $H$, um subespaço $L \subset H$ é dito isotrópico se $L \subset(J L)^{\perp}$. $L$ é dito lagrangiano se $L=(J L)^{\perp}$. Em particular, se $L$ é lagrangiano, então $L$ é fechado. O conjunto de todos os subespaços lagrangianos de $\mathrm{H}$ será denotado por $\Lambda \mathrm{e}$ chamado de grassmanniana de lagrangianos de $\mathrm{H}$.

Proposição 1.5.7 São verdadeiras as seguintes afirmações:

1. Se $\mathrm{W}$ é qualquer subespaço fechado, então $J(\mathrm{~W})^{\perp}=J\left(\mathrm{~W}^{\perp}\right)$.

2. Um subespaço fechado Lé lagrangiano se e só se $P_{\mathrm{L}} J+J P_{\mathrm{L}}=J$, ou equivalentemente, $J P_{\mathrm{L}}=P_{\mathrm{L}^{\perp}} J$.

Prova. A prova do ítem 1 segue diretamente da anti-simetria de $J$. Para provar o ítem 2 , observamos que se $L$ é lagrangiano, então a relação $P_{\mathrm{L}} J+J P_{\mathrm{L}}=J$ é verificada em $L$ e em $\mathrm{L}^{\perp}$, portanto em $\mathrm{H}$. Reciprocamente, se $P_{\mathrm{L}} J+J P_{\mathrm{L}}=J$, então dados $u, v \in \mathrm{L}$, temos $\omega(u, v)=\langle J u, v\rangle=\left\langle P_{L^{\perp}} J u, v\right\rangle=0$, portanto, $L$ é isotrópico. Se $v \in \mathrm{H}$ é tal que $\omega(u, v)=0$ para todo $u \in \mathrm{L}$, então $0=\left\langle J P_{\mathrm{L}} u, v\right\rangle=\left\langle P_{\mathrm{L}^{\perp}} J u, v\right\rangle$, portanto $v \in \mathrm{L}$, provando que $\mathrm{L}$ é lagrangiano.

Vamos agora estudar a estrutura de um espaço simplético via subespaços lagrangianos. Dado um lagrangiano $L$ de um espaço simplético $H$, como $H$ tem dimensão infinita, temos que 
$L$ também tem dimensão infinita. Dada $\left\{e_{j}\right\}_{j=1}^{\infty}$ uma base ortonormal de $L$, como J é estrutura complexa, temos que $\left\{-J e_{j}\right\}_{j=1}^{\infty}$ é uma base ortonormal de $L^{\perp}=J L$. Tem-se que

$$
\begin{gathered}
\omega\left(e_{j}, e_{k}\right)=\omega\left(-J e_{j},-J e_{k}\right)=0, \\
-\omega\left(e_{j},-J e_{k}\right)=\omega\left(-J e_{k}, e_{j}\right)=\delta_{j k},
\end{gathered}
$$

logo, com respeito à base ortonormal $\left\{e_{j}\right\}_{j=1}^{\infty} \cup\left\{-J e_{j}\right\}_{j=1}^{\infty}$ de $\mathrm{H}$ (ou, com respeito à decomposição $H=L \oplus L^{\perp}$ ), a forma $\omega$ (ou o operador $J$ ) tem a forma

$$
\left(\begin{array}{cc}
0 & -I \\
I & 0
\end{array}\right)
$$

É fácil construir um simplectomorfismo $T:(\mathrm{H}, \omega) \rightarrow\left(\mathrm{H} \oplus \mathrm{H}, \omega_{\text {can }}\right)$ : definimos $T e_{j}=\left(e_{j}, 0\right)$ e $T\left(J e_{j}\right)=\left(0,-e_{j}\right)$, para todo $j \geq 1$ e estendemos $T$ por linearidade para todo $H$. Para ver que $T$ é um simplectomorfismo, basta observar $T$ é um isomorfismo que comuta com as estruturas complexas correspondentes e usar o lema 1.5.4. Assim, a simples existência de um subespaço lagrangiano em um espaço simplético implica que é possível obter uma base de $\mathrm{H}$ na qual a matriz de $\omega$ é da forma 1.5.2 e também nos fornece um simplectomorfismo entre $(H, \omega)$ e o espaço $\left(H \oplus H, \omega_{\text {can }}\right)$.

Vamos agora estudar a existência de subespaços lagrangianos.

LEMA 1.5.8 L С Hé um subespaço lagrangiano se e só se Lé isotrópico maximal.

Prova. Se $L$ é lagrangiano e $L^{\prime} \supset L$ é isotrópico, então

$$
L^{\prime} \subset\left(J L^{\prime}\right)^{\perp} \subset(J L)^{\perp}=L,
$$

$\log 0 L^{\prime}=L$. Reciprocamente, se $L$ é isotrópico maximal, então dado qualquer $u \in(J L)^{\perp}, o$ subespaço gerado por $L$ e $u$ é isotrópico contendo $L$, portanto, $u \in L$. Logo, $L=(J L)^{\perp}$.

Lema 1.5.9 Se $\left\{\mathrm{L}_{\alpha}\right\}_{\alpha \in A}$ é uma cadeia de subespaços isotrópicos ${ }^{40}$ de $\mathrm{H}$, então $\mathrm{L}=\bigcup_{\alpha \in A} \mathrm{~L}_{\alpha}$ é um subespaço isotrópico de $\mathrm{H}$.

Prova. Seja $\mathrm{M}=\bigcap_{\alpha \in A}\left(J \mathrm{~L}_{\alpha}\right)^{\perp}$; como $\left\{\mathrm{L}_{\alpha}\right\}_{\alpha \in A}$ é uma cadeia, segue que $(J \mathrm{~L})^{\perp}=\mathrm{M}$. O lema estará provado se mostrarmos que $\mathrm{L} \subset \mathrm{M}$. Para isso, basta observar que, dado $\alpha \in A$, se $\beta \geq \alpha$, então $\mathrm{L}_{\alpha} \subset \mathrm{L}_{\beta} \subset\left(J \mathrm{~L}_{\beta}\right)^{\perp}$. Se $\beta<\alpha$ então $\mathrm{L}_{\alpha} \subset\left(J \mathrm{~L}_{\alpha}\right)^{\perp} \subset\left(J \mathrm{~L}_{\beta}\right)^{\perp}$. Assim, para qualquer $\beta \in A, \mathrm{~L}_{\alpha} \subset\left(J \mathrm{~L}_{\beta}\right)^{\perp}$, portanto, $\mathrm{L}_{\alpha} \subset \bigcap_{\beta \in A}\left(J \mathrm{~L}_{\beta}\right)^{\perp}=\mathrm{M}$. Como $\alpha \in A$ é arbitrário, temos que $\mathrm{L} \subset \mathrm{M}$.

Proposição 1.5.10 Em qualquer espaço simplético existe algum subespaço lagrangiano.

Prova. Seja $\Omega$ o conjunto de todos os subespaços isotrópicos. Como $0 \in \Omega$, então $\Omega \neq \emptyset$. Além disso, $\Omega$ é um conjunto parcialmente ordenado pela relação de inclusão. $O$ lema 1.5.9 mostra que $\Omega$ satisfaz as hipóteses do lema de Zorn. Portanto, existe um subespaço isotrópico $L \in \Omega$ maximal. Pelo lema 1.5.8, $L$ é um subespaço lagrangiano e a proposição está demonstrada.

A proposição 1.5 .10 mostra que toda a discussão que antecede o lema 1.5 .8 é válida em qualquer espaço simplético; vamos destacar este fato no teorema a seguir.

\footnotetext{
${ }^{40}$ Isso significa que $A$ é um conjunto totalmente ordenado e se $\alpha<\beta$ então $\mathrm{L}_{\alpha} \subset \mathrm{L}_{\beta}$.
} 
Teorema 1.5.11 Seja $(\mathrm{H}, \omega)$ um espaço simplético. Então existe uma base ortonormal de $\mathrm{H}$ da forma $\left\{e_{j}\right\}_{j=1}^{\infty} \cup\left\{-J e_{j}\right\}_{j=1}^{\infty}$ com respeito d̀ qual a matriz de $\omega$ (ou de J) é da forma 1.5.2. Existe também um simplectomorfismo de $(\mathrm{H}, \omega)$ em $\left(\mathrm{H} \oplus \mathrm{H}, \omega_{\text {can }}\right)$.

O teorema 1.5.11 permite-nos, portanto, fazer construções no espaço simplético canônico e transportá-las por meio de um simplectomorfismo a qualquer espaço simplético.

Vamos agora provar que a ação de $\mathrm{U}(\mathrm{H} ; J)($ em particular, de $\mathrm{Sp}(\mathrm{H}))$ no conjunto dos espaços lagrangianos é transitiva.

Proposição 1.5.12 Dados $\mathrm{L}_{0}$, $\mathrm{L}_{1}$ lagrangianos existe $T \in \mathrm{U}(\mathrm{H} ; J) \subset \mathrm{Sp}(\mathrm{H})$ tal que $T\left(\mathrm{~L}_{0}\right)=\mathrm{L}_{1}$.

Prova. Sejam $\left\{e_{j}^{0}\right\}_{j=1}^{\infty}$ e $\left\{e_{j}^{1}\right\}_{j=1}^{\infty}$ bases ortonormais de $L_{0}$ e $L_{1}$ respectivamente. Como já observamos, $\left\{J e_{j}^{0}\right\}_{j=1}^{\infty}$ e $\left\{J e_{j}^{1}\right\}_{j=1}^{\infty}$ são bases ortonormais de $L_{0}^{\perp}$ e $L_{1}^{\perp}$, respectivamente. Definimos $T: \mathrm{H} \rightarrow \mathrm{H}$ por $T e_{j}^{0}=e_{j}^{1}$ e $T\left(J e_{j}^{0}\right)=J e_{j}^{1}, j \geq 1$. Por definição, $T \in \mathrm{U}(\mathrm{H} ; J) \subset \mathrm{Sp}(\mathrm{H})$ e $T\left(\mathrm{~L}_{0}\right)=\mathrm{L}_{1}$.

É possível, usando a mesma idéia da proposição 1.5.12, estender um isomorfismo entre lagrangianos a um simplectomorfismo do espaço $\mathrm{H}$.

Proposıção 1.5.13 Sejam $L_{0}, L_{1}$ lagrangianos e $T: L_{0} \rightarrow L_{1}$ um isomorfismo limitado. Então existe $\tilde{T} \in \mathrm{U}(\mathrm{H} ; J) \subset \mathrm{Sp}(\mathrm{H})$ tal que $\left.\tilde{T}\right|_{L_{0}}=T$.

Prova. Como antes, seja $\left\{e_{j}\right\}_{j=1}^{\infty}$ uma base ortonormal de $L_{0}$. Definimos $\tilde{T}\left(J e_{j}\right)=J T e_{j}$ e $\tilde{T} e_{j}=T e_{j}$, se $j \geq 1$. Por definição, $\tilde{T} \in \mathrm{U}(\mathrm{H} ; J) \subset \mathrm{Sp}(\mathrm{H})$ e $\left.\tilde{T}\right|_{L_{0}}=T$.

OBSERvação 1.5.14 A prova da proposição 1.5.13 mostra que se $T \in U(H ; J)$ é tal que $T\left(L_{0}\right) \subset L_{0}$, para algum $L_{0}$ lagrangiano, então $T$ é determinado por sua restrição à $L_{0}$. Portanto, a aplicação $\left.T \mapsto T\right|_{L_{0}}$ é um isomorfismo contínuo entre os grupos $\left\{T \in \mathrm{U}(\mathrm{H} ; J): T\left(\mathrm{~L}_{0}\right)=\mathrm{L}_{0}\right\}$ e $\mathrm{O}\left(\mathrm{L}_{0}\right)$.

Temos um resultado análogo à proposição 1.3 .68 no caso de perturbações compactas (lagrangianas) de um lagrangiano.

Proposição 1.5.15 Se $\mathrm{L}_{0}, \mathrm{~L}_{1}$ são lagrangianos comensuráveis então existe $U \in \mathrm{U}_{c}\left(\mathrm{H}_{;} J\right)$ tal que $U\left(\mathrm{~L}_{0}\right)=\mathrm{L}_{1}$.

Prova. Seja $T_{1}=I+\left.\left(P_{\mathrm{L}_{1}}-P_{\mathrm{L}_{0}}\right)\right|_{\mathrm{L}_{0}}=P_{\mathrm{L}_{1}}^{\mathrm{L}_{0}}: \mathrm{L}_{0} \rightarrow \mathrm{L}_{1}$. Temos que ind $\left(T_{1}\right)=$ ind $\left(\mathrm{L}_{0}, \mathrm{~L}_{1}^{\perp}\right)=0$, portanto, pela observação 1.3.40, existe $K: L_{0} \rightarrow L_{1}$ de posto finito tal que $T_{2} \doteq T_{1}+K: L_{0} \rightarrow L_{1}$ é um isomorfismo. Pondo

$$
T=T_{2} P_{\mathrm{L}_{0}}-J T_{2} J P_{\mathrm{L}_{0}^{1}}=I+\left(\left(P_{\mathrm{L}_{1}}-P_{\mathrm{L}_{0}}\right)+K\right) P_{\mathrm{L}_{0}}-J\left(\left(P_{\mathrm{L}_{1}}-P_{\mathrm{L}_{0}}\right)+K\right) J P_{\mathrm{L}_{0}^{1}}
$$

temos que $T \in \mathrm{GL}_{c}(\mathrm{H}), T J=J T$ e $T\left(\mathrm{~L}_{0}\right)=\mathrm{L}_{1}$. Esta igualdade implica que $T^{*}\left(\mathrm{~L}^{\perp}\right)=\mathrm{L}_{0}^{\perp}, \operatorname{logo}$, como $T^{*} J=J T^{*}$, temos

$$
T^{*}(L)=T^{*} J\left(L^{\perp}\right)=J T^{*}\left(L^{\perp}\right)=J\left(L_{0}^{\perp}\right)=L_{0} .
$$

Em particular, $\left(T^{*} T\right)\left(L_{0}\right)=L_{0}$. Tomando $T=U|T|$ a decomposição polar de $T$, temos pela observação 1.3.46 que $U \in \mathrm{U}_{c}(\mathrm{H} ; J)$ e $|T|\left(\mathrm{L}_{0}\right)=\mathrm{L}_{0}$, portanto, $U\left(\mathrm{~L}_{0}\right)=\mathrm{L}$. 
OBSERVAÇão 1.5.16 Sejam $L_{0}$ um subespaço lagrangiano e $T \in \mathrm{Sp}(\mathrm{H})$. Com respeito à decomposição $H=L_{0} \oplus L_{0}^{\perp}$, o operador $J$ tem a forma

$$
\left(\begin{array}{cc}
0 & J L_{L_{0}^{+}} \\
J L_{L_{0}} & 0
\end{array}\right)
$$

Sejam $A: \mathrm{L}_{0} \rightarrow \mathrm{L}_{0}, B: \mathrm{L}_{0}^{\perp} \rightarrow \mathrm{L}_{0}, C: \mathrm{L}_{0} \rightarrow \mathrm{L}_{0}^{\perp}$ e $D: \mathrm{L}_{0}^{\perp} \rightarrow \mathrm{L}_{0}^{\perp}$ operadores limitados tais que, com respeito à decomposição $\mathrm{H}=\mathrm{L}_{0} \oplus \mathrm{L}_{0}^{\perp}$, o operador $T$ tem a forma $\left(\begin{array}{l}A \\ C\end{array}\right)$. Como $T \in \mathrm{Sp}(\mathrm{H})$, temos $T^{*} J T=J$, logo, $D^{*} J A+B^{*} J C=\left.J\right|_{L_{0}}, A^{*} J C \in \mathscr{L}^{\text {sa }}\left(\mathrm{L}_{0}\right)$ e $B^{*} J D \in \mathscr{L}^{\text {sa }}\left(\mathrm{L}_{0}^{\perp}\right)$. Às vezes, esta caracterização dos elementos de $\mathrm{Sp}(\mathrm{H})$ é útil para fazer cálculos explícitos.

Estudemos agora decomposições de um espaço simplético. Sejam $L_{0}, L_{1}$ subespaços complementares quaisquer de $\mathrm{H}$. $\mathrm{O}$ teorema do gráfico fechado implica que $P_{\mathrm{L}_{1}, L_{0}}, P_{\mathrm{L}_{0}, \mathrm{~L}_{1}}$ são limitados, e $\left\|P_{L_{0}, L_{1}}\right\|=\gamma\left(L_{0}, L_{1}\right)^{-1}$, pela observação 1.2.7, logo,

$$
\|u\|=\left\|P_{\mathrm{L}_{1}, \mathrm{~L}_{0}}(u)+P_{\mathrm{L}_{0}, \mathrm{~L}_{1}}(u)\right\| \leq\left\|P_{\mathrm{L}_{1}, \mathrm{~L}_{0}}(u)\right\|+\left\|P_{\mathrm{L}_{0}, \mathrm{~L}_{1}}(u)\right\| \leq 2 \gamma\left(\mathrm{L}_{0}, \mathrm{~L}_{1}\right)^{-1}\|u\|,
$$

para todo $u \in \mathrm{H}$, pelo lema 1.2.8. As desigualdades em 1.5.3 implicam que $\|u\|_{L_{0}, L_{1}} \doteq\left\|P_{L_{1}, L_{0}}(u)\right\|+$ $\left\|P_{L_{0}, L_{1}}(u)\right\|$ define uma norma equivalente em $\mathrm{H}$. Este fato será usado daqui por diante sem menção explícita.

Dado $L$ um subespaço fechado complementar a $L_{1}$, definimos $P_{L_{1}, L}^{L_{0}} \doteq P_{L_{1}, L} L_{L_{0}}: L_{0} \rightarrow L$.

Proposição 1.5.17 Se $\mathrm{L}_{0}, \mathrm{~L}_{1} e \mathrm{~L}, \mathrm{~L}_{1}$ são pares de subespaços complementares, então $P_{\mathrm{L}_{1}, \mathrm{~L}}^{\mathrm{L}_{0}}$ é um isomorfismo. ${ }^{41}$

Prova. Seja $\pi: H \rightarrow H / L_{1}$ a aplicação quociente. Temos que $\left.\pi\right|_{L_{0}}: L_{0} \rightarrow H / L_{1}$ é uma bijeção contínua, portanto, pelo teorema da aplicação aberta, $\left.\pi\right|_{L_{0}}$ tem inversa contínua. Como o diagrama

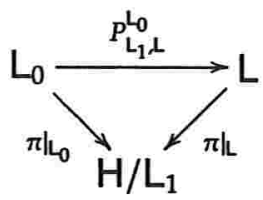

comuta, concluímos o desejado.

OBSERVAÇÃo 1.5.18 É imediato verificar que $\left(P_{L_{1}, L}^{L_{0}}\right)^{-1}=P_{L_{1}, L_{0}}^{L^{\prime}}$ se $L_{0}, L, L_{1}$ são como na proposição 1.5.17.

OBSERVAÇÃo 1.5.19 Se $L_{0}, L_{1}$ são subespaços complementares, usando a notação da proposição 1.5.17, temos que $\gamma\left(\left.\pi\right|_{L}\right)=\gamma\left(L, L_{1}\right)$, para qualquer subespaço $L$. De fato,

$$
\gamma\left(\left.\pi\right|_{\mathrm{L}}\right)=\inf _{\substack{u \in \mathrm{L} \\\|u\|=1}} \frac{\left\|u+\mathrm{L}_{1}\right\|}{\operatorname{dist}\left(u, \operatorname{ker}\left(\left.\pi\right|_{\mathrm{L}}\right)\right)}=\inf _{\substack{u \in \mathrm{L} \\\|u\|=1}} \frac{\operatorname{dist}\left(u, \mathrm{~L}_{1}\right)}{\operatorname{dist}\left(u, \mathrm{~L} \cap \mathrm{L}_{1}\right)}=\gamma\left(\mathrm{L}, \mathrm{L}_{1}\right) .
$$

Identificando $\left.\pi\right|_{L} \operatorname{com} P_{L_{1}}^{L}$, esta é simplesmente outra forma para o lema 1.2.15.

\footnotetext{
${ }^{41}$ i.e., $P_{L_{1}, L}^{L_{0}}$ é bijetor limitado com inverso limitado.
} 
Dado qualquer subespaço fechado $L$ complementar a $L_{1}$, existe um único operador linear $S: L_{0} \rightarrow L_{1}$ cujo gráfico é $L$. De fato,

$$
S=P_{\mathrm{L}_{0}, \mathrm{~L}_{1}}^{\mathrm{L}} \circ\left(P_{\mathrm{L}_{1}, L_{0}}^{\mathrm{L}}\right)^{-1}=P_{\mathrm{L}_{0}, \mathrm{~L}_{1}}^{\mathrm{L}} \circ P_{\mathrm{L}_{1}, \mathrm{~L}}^{\mathrm{L}_{0}} \text {. }
$$

A proposição 1.5.17 implica que $S$ é bem-definido e as observações anteriores provam que $S$ é limitado. Esta é a idéia fundamental utilizada para construir cartas locais em variedades grassmannianas, e será explorada mais adiante.

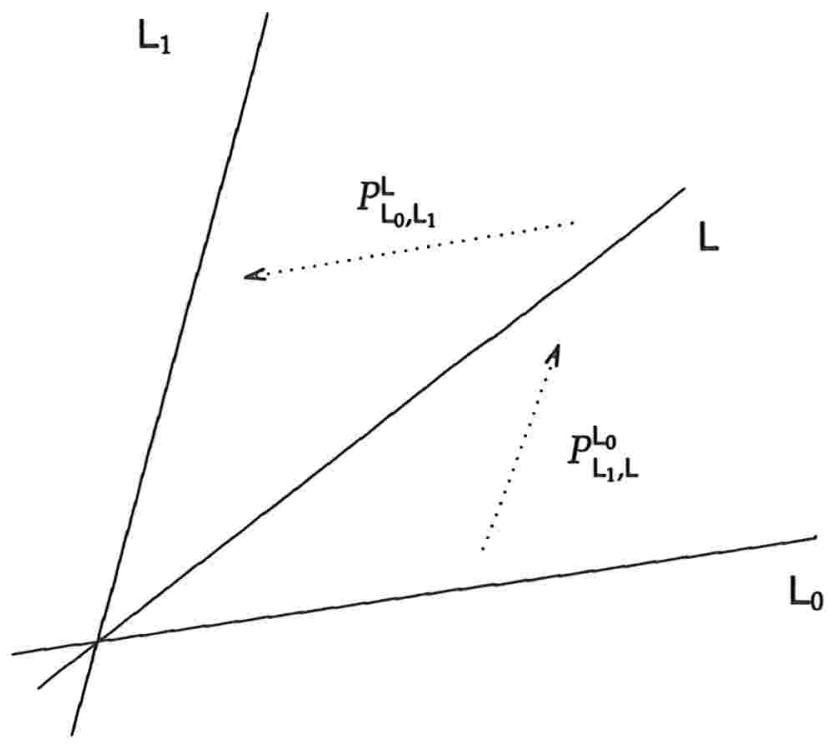

Por hora, retornemos ao caso de lagrangianos. Suponhamos daqui por diante que $L_{0}, L_{1}$ são lagrangianos complementares e que $L$ seja qualquer subespaço complementar a $L_{1}$. $O$ próximo lema fornece um critério importante para determinar se $L$ é lagrangiano.

LemA 1.5.20 Lé lagrangiano se e só se o operador $P_{\mathrm{L}_{0}} J S: \mathrm{L}_{0} \rightarrow \mathrm{L}_{0}$ é auto-adjunto.

Prova. Dados $u, v \in \mathrm{L}_{0}$, temos

$$
\begin{aligned}
\omega(u+S u, v+S v)=\omega(u, S v)+\omega(S u, v) & =\langle J u, S v\rangle+\langle J S u, v\rangle \\
& =-\left\langle u, P_{L_{0}} J S v\right\rangle+\left\langle P_{L_{0}} J S u, v\right\rangle .
\end{aligned}
$$

Logo, se $L=G(S)$ é lagrangiano, então $P_{L_{0}} J S$ é auto-adjunto. Reciprocamente, se $P_{\mathrm{L}_{0}} J S$ é auto-adjunto, então $L \subset(J L)^{\perp}$, pela igualdade 1.5.5. Dado $w \in(J L)^{\perp}$, pondo $w_{0}=P_{L_{1}, L_{0}} w$ e $w_{1}=P_{L_{0}, L_{1}} w$, e lembrando que $\mathrm{G}(S)=\mathrm{L}$, temos

$$
\begin{aligned}
0=\omega(w, u+S u) & =\omega\left(w_{0}, S u\right)+\omega\left(w_{1}, u\right) \\
& =-\left\langle w_{0}, J S u\right\rangle+\left\langle J w_{1}, u\right\rangle \\
& =-\left\langle w_{0}, P_{L_{0}} J S u\right\rangle+\left\langle P_{L_{0}} J w_{1}, u\right\rangle \\
& =-\left\langle P_{L_{0}} J S w_{0}, u\right\rangle+\left\langle P_{L_{0}} J w_{1}, u\right\rangle \\
& =\left\langle P_{\mathrm{L}_{0}} J\left(w_{1}-S w_{0}\right), u\right\rangle,
\end{aligned}
$$


para todo $u \in \mathrm{L}_{0}$. Logo, $P_{\mathrm{L}_{0}} J\left(w_{1}-S w_{0}\right) \in \mathrm{L}_{0}^{\perp} \cap \mathrm{L}_{0}=0$. Como $\left.P_{\mathrm{L}_{0}}\right|_{\mathrm{L}_{1}^{\perp}}=P_{\mathrm{L}_{0}^{\frac{1}{1}}, L_{0}}^{\mathrm{L}_{0}^{\perp}}$ é um isomorfismo (pela proposição 1.5.17), segue que $w_{1}=S w_{0}$ e portanto, $w=w_{0}+S w_{0} \in \mathrm{G}(S)=\mathrm{L}$.

OBSERVAÇão 1.5.21 No fim da prova da proposição 1.5.20 usamos o fato que $P_{L_{0}} L_{L_{1}^{+}}: L_{1}^{+} \rightarrow L_{0}$, (ou equivalentemente, $P_{L_{0}} J L_{L_{1}}: L_{1} \rightarrow L_{0}$ ) é um isomorfismo. De fato, $J L_{L_{1}}: L_{1} \rightarrow L_{1}^{\perp}$ é um isomorfismo, e $\left.P_{\mathrm{L}_{0}} J\right|_{\mathrm{L}_{1}}=\left.P_{\mathrm{L}_{0}^{+}, \mathrm{L}_{0}}^{\mathrm{L}_{0}^{+}} \circ J\right|_{\mathrm{L}_{1}}$.

Definição 1.5.22 Para $L_{0}, L_{1}$ lagrangianos complementares, definimos $\Psi_{L_{0}, L_{1}} \doteq\left(P_{L_{0}} J L_{L_{1}}\right)^{-1}$ : $\mathrm{L}_{0} \rightarrow \mathrm{L}_{1}$.

Temos que $\Psi_{L_{0}, L_{1}}$ é um isomorfismo e a correspondência

$$
T \mapsto S=\Psi_{L_{0}, L_{1}} \circ T
$$

define uma bijeção entre $\mathscr{L}^{\text {sa }}\left(L_{0}\right)$ e o conjunto dos operadores $L_{0} \rightarrow L_{1}$ cujo gráfico é um subespaço lagrangiano.

OBSERvação 1.5.23 Observamos que $\left(\Psi_{L_{0}, L_{1}}\right)^{*}=-\Psi_{L_{1}, L_{0}}$. De fato, para $v \in L_{1}$ e $u \in L_{0}$,

$$
\left\langle P_{\mathrm{L}_{0}} J v, u\right\rangle=\langle J v, u\rangle=-\langle v, J u\rangle=-\left\langle v, P_{\mathrm{L}_{1}} J u\right\rangle,
$$

logo, $\left(\left.P_{L_{0}} J\right|_{L_{1}}\right)^{*}=-\left.P_{L_{1}} J\right|_{L_{0}}$, e disso, segue a relação desejada.

Observação 1.5.24 Temos que $\left\|\Psi_{L_{0}, L_{1}}^{-1}\right\|=\delta\left(L_{0}, L_{1}\right)$ se $L_{0}, L_{1}$ são lagragianos complementares. De fato, $\left\|\Psi_{L_{0}, L_{1}}^{-1}\right\|=\left\|\left.\left(P_{L_{0}} J\right)\right|_{L_{1}}\right\|=\left\|P_{L_{0}}^{L_{1}^{-1}}\right\|=\delta\left(L_{1}^{\perp}, L_{0}^{+}\right)=\delta\left(L_{0}, L_{1}\right)$, pelo segundo ítem da proposição 1.2.3.

O lema 1.5.20 mostra que a seguinte definição faz sentido.

Definição 1.5.25 Sejam $L_{0}, L_{1}$ lagrangianos complementares de $H$. Definimos $\Lambda_{0}\left(L_{1}\right)$ como o conjunto de todos os lagrangianos complementares a $L_{1}$ e a aplicação

$$
\begin{aligned}
\varphi_{\mathrm{L}_{0}, \mathrm{~L}_{1}}: \Lambda_{0}\left(\mathrm{~L}_{1}\right) & \rightarrow \mathscr{L}^{\mathrm{sa}}\left(\mathrm{L}_{0}\right) \\
\mathrm{L} & \mapsto P_{\mathrm{L}_{0}} J S=\Psi_{\mathrm{L}_{0}, \mathrm{~L}_{1}}^{-1} \circ S,
\end{aligned}
$$

onde $S: L_{0} \rightarrow L_{1}$ é tal que $G(S)=L$.

Observação 1.5.26 Como já observamos, $S$ é determinado por $L$ e $\left.P_{L_{0}} J\right|_{L_{1}}: L_{1} \rightarrow L_{0}$ é um isomorfismo, logo, $\varphi_{L_{0}, L_{1}}$ é uma bijeção. Também é imediato que $\operatorname{ker} \varphi_{L_{0}, L_{1}}(L)=L \cap L_{0}$.

ObSERvaçÃo 1.5.27 Observamos que a aplicação $L \in \Lambda \mapsto P_{\mathrm{L}} \in \mathscr{L}(\mathrm{H})$ é uma bijeção entre $\Lambda$ e o conjunto dos operadores $P \in \mathscr{L}(\mathrm{H})$ tal que $P^{*}=P=P^{2}$ e $P J+J P=J$, conforme o ítem 2 da proposição 1.5.7. Em geral, dada uma projeção (não necessariamente ortogonal) $P \in \mathscr{L}(\mathrm{H})$, o operador $C \doteq 2 P-I$ é uma conjugação, i.e., $C^{2}=I$. Se $P^{*}=P$ (i.e., $P$ é ortogonal) então $C^{*}=C$ e se $P J+J P=J$, então $C J=-J C$. Portanto, a aplicação $L \mapsto C_{\mathrm{L}} \doteq 2 P_{\mathrm{L}}-I=P_{\mathrm{L}}-P_{\mathrm{L}^{+}}$é uma bijeção entre $\Lambda$ e o conjunto dos operadores $C \in \mathscr{L}(\mathrm{H})$ tais que $C^{2}=I, C J+J C=0$ e $C^{*}=C$. Em geral, dado $C \in \mathscr{L}(\mathrm{H})$ tal que $C^{2}=I$ e $C^{*}=C$, o subespaço $\operatorname{ker}(I-C)$ é lagrangiano se e só se $C J+J C=J$. Isso mostra que é possível utilizar as conjugações ao invés das projeções para estudar lagrangianos. 
As aplicações $\Psi_{L_{0}, L_{1}}$ podem ser utilizadas para construir simplectomorfismos entre subespaços lagrangianos.

Proposição 1.5.28 Dados pares $\left(L_{0}, L_{1}\right) e\left(L_{0}^{\prime}, L_{1}^{\prime}\right)$ de lagrangianos complementares de $H$ e $T: L_{0} \rightarrow L_{0}^{\prime}$ uma isometria, existe $\tilde{T} \in \mathrm{Sp}(\mathrm{H})$ tal que $\left.\tilde{T}\right|_{\mathrm{L}_{0}}=T$ e $\tilde{T}\left(\mathrm{~L}_{1}\right)=\mathrm{L}_{1}^{\prime}$.

Prova. Como $\Psi_{L_{0}, L_{1}}: L_{0} \rightarrow L_{1}$ é um isomorfismo, podemos definir $\tilde{T}$ como $\tilde{T}=T$ em $L_{0}$ e $\tilde{T}\left(\Psi_{L_{0}, L_{1}} u\right)=\Psi_{L_{0}^{\prime}, L_{1}^{\prime}} T u$, para $u \in L_{0}$. Obviamente, $\tilde{T}$ é um isomorfismo (limitado) de $H$. Resta provar que $T \in \mathrm{Sp}(\mathrm{H})$. Como todos os subespaços envolvidos são lagrangianos, basta provar que $\omega(\tilde{T} u, \tilde{T} v)=\omega(u, v)$, para quaisquer $u \in L_{0}, v \in L_{1}$. De fato,

$$
\begin{aligned}
\omega(\tilde{T} u, \tilde{T} v) & =\left\langle J T u, \Psi_{L_{0}^{\prime} L_{1}^{\prime}} T w\right\rangle, \operatorname{com} v=\Psi_{L_{0}, L_{1}} w \\
& =\left\langle\left(P_{L_{1}^{\prime}} J\right) T u, \Psi_{L_{0}^{\prime}, L_{1}^{\prime}} T w\right\rangle \\
& =-\left\langle\Psi_{L_{1}^{\prime}, L_{0}^{\prime}} \Psi_{L_{1}^{\prime}, L_{0}^{\prime}}^{-1} T u, T w\right\rangle, \text { pela observação 1.5.23 } \\
& =-\left\langle T u, T\left(P_{L_{0}} J v\right)\right\rangle \\
& =-\langle u, J v\rangle=\omega(u, v), \text { pois } T: L_{0} \rightarrow L_{0} \text { é isometria. }
\end{aligned}
$$

Vamos agora tratar de uma questão relativamente delicada a respeito de existência de subespaços lagrangianos. Em um espaço simplético de dimensão finita, dada uma família $\left\{L_{n}\right\}_{n \in \mathbb{N}}$ de lagrangianos, um argumento simples utilizando o teorema de Baire prova que existe um lagrangiano $L$ complementar a $L_{n}$ para todo $n \in \mathbb{N}$. Este argumento não se aplica em dimensão infinita. A seguir, vamos encontrar condições que garantam a existência de um lagrangiano complementar a um par de lagrangianos dados em um espaço simplético de dimensão infinita. O primeiro passo neste sentido é estender a proposição 1.5.28 para uma situação consideravelmente mais geral.

Proposição 1.5.29 Se $L_{0}, L_{1}$, $L_{1}^{\prime}$ são lagrangianos tais que $L_{0} \cap L_{1}=L_{0} \cap L_{1}^{\prime} e L_{0}+L_{1}, L_{0}+L_{1}^{\prime}$ são fechados, então existe $T \in \mathrm{Sp}(\mathrm{H})$ tal que $T\left(\mathrm{~L}_{0}\right)=\mathrm{L}_{0}$ e $T\left(\mathrm{~L}_{1}\right)=\mathrm{L}_{1}^{\prime}$.

Prova. Pondo $M \doteq L_{0} \cap\left(L_{0} \cap L_{1}\right)^{\perp}$ e $H_{0} \doteq M+J(M)$, temos que a restrição de $\omega$ e $J$ a $\mathrm{H}_{0}$ o tornam um espaço simplético. Além disso, os subespaços $L_{0} \cap H_{0}=M, L_{1} \cap H_{0}=L_{1} \cap\left(L_{0} \cap L_{1}\right)^{\perp}$ e $L_{1}^{\prime} \cap H_{0}=L_{1}^{\prime} \cap\left(L_{0} \cap L_{1}\right)^{\perp}$ são lagrangianos de $H_{0}$. Os mesmos argumentos utilizados na prova do lema 1.3.26 mostram que $\left(L_{0} \cap H_{0}\right) \cap\left(L_{1} \cap H_{0}\right)=\left(L_{0} \cap H_{0}\right) \cap\left(L_{1}^{\prime} \cap H_{0}\right)=0$ e $\left(L_{0} \cap H_{0}\right)+\left(L_{1} \cap H_{0}\right)=\left(L_{0} \cap H_{0}\right)+\left(L_{1}^{\prime} \cap H_{0}\right)=H_{0}$. Pela proposição 1.5.28, existe $T_{0} \in \mathrm{Sp}\left(\mathrm{H}_{0}\right)$ tal que $T_{0}\left(L_{0} \cap H_{0}\right)=L_{0} \cap H_{0}$ e $T_{0}\left(L_{1} \cap H_{0}\right)=L_{1}^{\prime} \cap H_{0}$. Como $J H_{0}=H_{0}$ e $J^{*}=-J$, então $J\left(H_{0}^{\perp}\right)=H_{0}^{\perp}$. Logo, definindo

$$
T=\left\{\begin{aligned}
T_{0} & , \mathrm{em} \mathrm{H}_{0} \\
I & , \mathrm{em} \mathrm{H} \mathrm{H}_{0}^{\perp}
\end{aligned}\right.
$$

temos que $T \in \mathrm{Sp}(\mathrm{H})$. Além disso, $T\left(\mathrm{~L}_{0}\right)=T\left(\mathrm{~L}_{0} \cap\left(\mathrm{L}_{0} \cap \mathrm{L}_{1}\right)^{\perp}+\left(\mathrm{L}_{0} \cap \mathrm{L}_{1}\right)\right)=T_{0}\left(\mathrm{~L}_{0} \cap \mathrm{H}_{0}\right)+\left(\mathrm{L}_{0} \cap \mathrm{L}_{1}\right)=$ $L_{0} \cap H_{0}+\left(L_{0} \cap L_{1}\right)=L_{0}$ e analogamente, $T\left(L_{1}\right)=L_{1}^{\prime}$, como queríamos.

Corolário 1.5.30 Se $L_{0}, L_{0}^{\prime}, L_{1}$, $L_{1}^{\prime}$ sãolagrangianos tais que $L_{0}+L_{1} e L_{0}^{\prime}+L_{1}^{\prime}$ são fechados, nul $\left(L_{0}, L_{1}\right)=$ $\operatorname{nul}\left(L_{0}^{\prime}, L_{1}^{\prime}\right) e \operatorname{codim}_{L_{0}}\left(L_{0} \cap\left(L_{0} \cap L_{1}\right)^{\perp}\right)=\operatorname{codim}_{L_{0}^{\prime}}\left(L_{0}^{\prime} \cap\left(L_{0}^{\prime} \cap L_{1}^{\prime}\right)^{\perp}\right)$ entäo existe $T \in S p(H)$ tal que $T\left(L_{0}\right)=L_{0}^{\prime}$ e $T\left(L_{1}\right)=L_{1}^{\prime}$. 
Prova. As hipóteses sobre a nulidade e a codimensão implicam a existência de um isomorfismo $S: L_{0} \rightarrow L_{0}^{\prime}$ tal que $S\left(L_{0} \cap L_{1}\right)=L_{0}^{\prime} \cap L_{1}^{\prime}$. Considerando a extensão unitária $\tilde{S}: H \rightarrow H$ dada pela proposição 1.5.13, e os lagrangianos $\tilde{S}\left(L_{0}\right)=L_{0}^{\prime}, \tilde{S}\left(L_{1}\right)$ e $\tilde{S}\left(L_{1}^{\prime}\right)$, a proposição 1.5.29 nos fornece $R \in \mathrm{Sp}(\mathrm{H})$ tal que $R\left(\mathrm{~L}_{0}^{\prime}\right)=\mathrm{L}_{0}^{\prime}$ e $R\left(\tilde{S}\left(\mathrm{~L}_{1}\right)\right)=\mathrm{L}_{1}^{\prime}$. Tomando $T \doteq R \tilde{S} \in \mathrm{Sp}(\mathrm{H})$, temos que $T$ satisfaz as propriedades requeridas.

Vamos agora utilizar a proposição 1.5 .29 para construir lagrangianos complementares.

Proposição 1.5.31 Se $L_{0}, L_{1}$ são lagrangianos com $L_{0}+L_{1}$ fechado, então existe $L_{\text {complementar } a} L_{0}$ $e L_{1}$.

Prova. Sejam $T \in \mathscr{L}^{\text {sa }}\left(\mathrm{L}_{0}\right)$ tal que $\operatorname{ker} T=\mathrm{L}_{0} \cap \mathrm{L}_{1}$ e $\gamma(T)>0 \mathrm{e}^{42} \mathrm{~L}_{1}^{\prime} \doteq \mathrm{G}\left(\Psi_{\mathrm{L}_{0}, \mathrm{~L}_{0}^{-1}} T\right)$. Pela segunda equação de 1.5.8, temos que $\gamma\left(L_{0}, L_{1}^{\prime}\right)>0$, e portanto, pelo lema 1.2.6, $L_{0}+L_{1}^{\prime}$ é fechado. Como $L_{1}^{\prime} \cap L_{0}=\operatorname{ker} T=L_{1} \cap L_{0}$, pela proposição 1.5.29, existe $T \in S p(H)$ tal que $T\left(L_{0}\right)=L_{0}$ e $T\left(L_{1}^{\prime}\right)=L_{1}$. Como $L_{0}^{+}$é complementar a $L_{0}$ e $L_{1}^{\prime}$, concluímos ${ }^{43}$ que $L=T\left(L_{0}^{+}\right)$é um lagrangiano complementar a $T\left(L_{0}\right)=L_{0}$ e $T\left(L_{1}^{\prime}\right)=L_{1}$, como queríamos.

A proposição 1.3.64 admite uma versão correspondente no caso de lagrangianos.

Proposição 1.5.32 Se $\mathrm{L}_{0}, \mathrm{~L}_{1}$ é um par Fredholm de lagrangianos, então existe um lagrangiano $\mathrm{L}_{1}^{\prime}$ comensurável com $\mathrm{L}_{1}$ tal que $\mathrm{L}_{0}$, $\mathrm{L}_{1}^{\prime}$ são subespaços complementares.

Prova. Seja $L_{2}$ um lagrangiano complementar a $L_{0}$ e $L_{1}$, conforme a proposição 1.5 .31 e $T=\varphi_{\mathrm{L}_{0}, \mathrm{~L}_{2}}\left(\mathrm{~L}_{1}\right)$. Temos que $T \in \mathscr{F}^{\mathrm{sa}}\left(\mathrm{L}_{0}\right), \operatorname{logo}$, pondo $T^{\prime}=T+\varepsilon P_{\mathrm{ker} T}, \varepsilon>0$, segue que $T^{\prime}$ é um isomorfismo e $L_{1}^{\prime}=\varphi_{L_{0}, L_{2}}^{-1}\left(T^{\prime}\right)$ é um lagrangiano complementar a $L_{0}$. Para verificar que $L_{1}^{\prime} \sim L_{1}$, vamos utilizar o lema 1.2.28: como $L_{1}=G(S)$, com $S=\Psi_{L_{0}, L_{2}} T$ e $L_{1}^{\prime}=G(S+K)$, com $K=\Psi_{\mathrm{L}_{0}, \mathrm{~L}_{2}} P_{\text {ker } T}$, basta verificar que $R(S+K)-R(S)$ é compacto. Isso decorre imediatamente da definição de $R(T)$ e da fórmula de Neumann, a qual é válida se $\varepsilon>0$ for suficientemente pequeno.

Vamos agora obter algumas informações sobre $\operatorname{Im} \varphi_{L_{0}, L_{1}}(L)$, para $L \in \Lambda_{0}\left(L_{1}\right)$.

Lema 1.5.33 Se $L_{0}, L_{1}$ são lagrangianos complementares de $H, L \in \Lambda_{0}\left(L_{1}\right)$ e $S: L_{0} \rightarrow L_{1}$ tem gráfico $\mathrm{L}$, então

$$
\|I+S\|^{-1} \gamma\left(P_{\mathrm{L}_{0}} J S\right) \leq \gamma\left(\mathrm{L}, \mathrm{L}_{0}\right) \leq \gamma\left(\mathrm{L}_{0}, \mathrm{~L}_{1}\right)^{-1} \gamma\left(P_{\mathrm{L}_{0}} J S\right) .
$$

Em particular, $P_{\mathrm{L}_{0}} J S$ tem imagem fechada se e só $\mathrm{L}+\mathrm{L}_{0}$ é fechado.

Prova. Para qualquer $u \in \mathrm{L}_{0}$, temos

$$
\gamma\left(\mathrm{L}_{0}, \mathrm{~L}_{1}\right) \operatorname{dist}\left(u, \mathrm{~L} \cap \mathrm{L}_{0}\right) \leq \operatorname{dist}\left(u+S u, \mathrm{~L} \cap \mathrm{L}_{0}\right) \leq\|I+S\| \operatorname{dist}\left(u, \mathrm{~L} \cap \mathrm{L}_{0}\right) .
$$

De fato, como $\operatorname{ker} S=L \cap L_{0}$

$$
\begin{aligned}
\operatorname{dist}\left(u+S u, L \cap L_{0}\right)=\inf _{v \in L_{n} L_{0}}\|(I+S) u+v\| & =\inf _{v \in \operatorname{Ln} L_{0}}\|(I+S)(u+v)\| \\
& \leq\|I+S\| \inf _{v \in L_{0} L_{0}}\|u+v\| \\
& =\|I+S\| \operatorname{dist}\left(u, L \cap L_{0}\right),
\end{aligned}
$$

\footnotetext{
${ }^{42}$ Podemos tomar $T=P_{\left(L_{0} \cap L_{1}\right)^{\perp} \cap L_{0^{\prime}}}^{L_{0}}$ por exemplo.

${ }^{43} \mathrm{Em}$ geral, se $L_{0}, L_{1}$ é qualquer par de lagrangianos complementares e $L=G\left(\Psi_{L_{0} L_{1}} T\right)$, com $T \in \mathscr{L}^{\text {sa }}\left(L_{0}\right)$ então $\gamma\left(L_{,} L_{1}\right) \geq \gamma\left(P_{L_{1}^{1}}^{L_{0}}\right)=\gamma\left(L_{0}, L_{1}\right)>0$.
} 
provando a desigualdade da direita em 1.5.7. Por outro lado,

$$
\begin{aligned}
\operatorname{dist}\left(u+S u, L \cap L_{0}\right) & =\inf _{v \in L_{n} L_{0}}\|u+S u+v\| \\
& \geq\left\|P_{\mathrm{L}_{1}, L_{0}}\right\|^{-1} \inf _{v \in \mathrm{L}_{0}}\|u+v\|, \text { pois } u+v \in \mathrm{L}_{0} \text { e } S u \in \mathrm{L}_{1}, \\
& =\gamma\left(\mathrm{L}_{0}, \mathrm{~L}_{1}\right) \operatorname{dist}\left(u, \mathrm{~L} \cap \mathrm{L}_{0}\right),
\end{aligned}
$$

pela observação 1.2.7. Isso completa a prova de 1.5.7. Portanto, como $\operatorname{dist}\left(u+S u, L_{0}\right)=$ $\left\|P_{L_{0}} J S u\right\|, 1.5 .7$ implica que

$$
\|I+S\|^{-1} \frac{\left\|P_{\mathrm{L}_{0}} J S u\right\|}{\operatorname{dist}\left(u, \mathrm{~L} \cap \mathrm{L}_{0}\right)} \leq \frac{\operatorname{dist}\left(u+S u, \mathrm{~L}_{0}\right)}{\operatorname{dist}\left(u+S u, \mathrm{~L} \cap \mathrm{L}_{0}\right)} \leq \gamma\left(\mathrm{L}_{0}, \mathrm{~L}_{1}\right)^{-1} \frac{\left\|P_{\mathrm{L}_{0}} J S u\right\|}{\operatorname{dist}\left(u, \mathrm{~L} \cap \mathrm{L}_{0}\right)},
$$

e 1.5 .6 segue.

OBSERVAÇÃo 1.5.34 Observamos que $\hat{\delta}\left(L_{0}, L_{1}\right)=\delta\left(L_{0}, L_{1}\right)$ para $L_{0}, L_{1}$ lagrangianos quaisquer. De fato, pelo primeiro e segundo ítens da proposição 1.2 .3 , temos que $\delta\left(L_{0}, L_{1}\right)=\delta\left(J L_{0}, J L_{1}\right)=$ $\delta\left(L_{0}^{\perp}, L_{1}^{\perp}\right)=\delta\left(L_{1}, L_{0}\right)$.

Tratemos agora de pares Fredholm de subespaços lagrangianos. Obviamente, toda a teoria desenvolvida na seção 1.3 continua válida, mas nesta situação mais específica, podemos obter resultados melhores.

Se $\left(L_{0}, L_{1}\right)$ é um par de subespaços lagrangianos, então

$$
\left(L_{0}+L_{1}\right)^{\perp}=L_{0}^{\perp} \cap L_{1}^{\perp}=J L_{0} \cap J L_{1}=J\left(L_{0} \cap L_{1}\right),
$$

portanto, $L_{0}, L_{1}$ é Fredholm se e só se nul $\left(L_{0}, L_{1}\right)<\infty$ e $L_{0}+L_{1}$ é fechado.

EXEMPLo 1.5.35 O exemplo 1.2.10 pode ser estendido para obtermos um par de subespaços lagrangianos cuja intersecção é trivial e cuja soma não é fechada. De fato, basta considerar $\mathrm{H} \oplus \mathrm{H}$ munido da forma simplética canônica (conforme o exemplo 1.5.5) e tomar o operador $T$ compacto auto-adjunto. Usando a relação 1.2.3, vemos que $\mathrm{G}(T)$ e $\mathrm{H} \oplus 0$ são lagrangianos de $\mathrm{H} \oplus \mathrm{H}$ com intersecção trivial e soma não fechada.

A próxima proposição fornece um critério para determinar se um par de lagrangianos é Fredholm.

Proposição 1.5.36 Sejam $L, L_{0}, L_{1}$ lagrangianos, com $L_{,} L_{0} \in \Lambda_{0}\left(L_{1}\right)$. São equivalentes as seguintes afirmações:

1. $\left(L, L_{0}\right)$ é um par Fredholm de lagrangianos.

2. $\varphi_{\mathrm{L}_{0}, \mathrm{~L}_{1}}(\mathrm{~L}) \in \mathscr{F}^{\mathrm{sa}}\left(\mathrm{L}_{0}\right)$.

3. $\operatorname{nul}\left(L, L_{0}\right)<\infty$ e $\gamma\left(L, L_{0}\right)>0$.

Prova. Temos que $\operatorname{ker}\left(\varphi_{L_{0}, L_{1}}(L)\right)=L \cap L_{0}$; pelos lemas 1.2.6, 1.5.33 e pela proposição 1.2.14, $\operatorname{Im}\left(\varphi_{L_{0}, L_{1}}(L)\right)$ é fechada se e só se $L+L_{0}$ o é. Estas afirmações implicam que o primeiro e o segundo ítens são equivalentes, pois $\varphi_{\mathrm{L}_{0}, \mathrm{~L}_{1}}(\mathrm{~L})$ é auto-adjunto, pelo lema 1.5.20.

O primeiro e o terceiro ítens também são equivalentes, pois $\left(L+L_{0}\right)^{\perp}=J\left(L \cap L_{0}\right)$ e $L+L_{0}$ é fechado se e só se $\gamma\left(L, L_{0}\right)>0$, pelo lema 1.2.6.

No caso de subespaços lagrangianos, o teorema 1.3.30 pode ser melhorado. 
TeOrema 1.5.37 Sejam $\mathrm{L}_{0}, \mathrm{~L}_{1}$ subespaços lagrangianos de $\mathrm{H}$. Então o par $\left(\mathrm{L}_{0}, \mathrm{~L}_{1}\right)$ é Fredholm se e só se $P_{L_{0}}+P_{L_{1}}$ é um operador Fredholm.

Prova. Basta observarmos que no caso de lagrangianos, pela observação 1.3.25,

$$
\operatorname{ker}\left(P_{\mathrm{L}_{0}}+P_{\mathrm{L}_{1}}\right)=\mathrm{L}_{0}^{\frac{1}{1}} \cap \mathrm{L}_{1}^{\perp}=J \mathrm{~L}_{0} \cap J \mathrm{~L}_{1}=J\left(\mathrm{~L}_{0} \cap \mathrm{L}_{1}\right),
$$

portantọ, se $P_{L_{0}}+P_{L_{1}}$ é Fredholm, então automaticamente nul $\left(L_{0}, L_{1}\right)<\infty$, e a conclusão segue do teorema 1.3.30.

Vamos agora provar um fato útil a respeito de lagrangianos complementares.

LEMA 1.5.38 Se $L_{0}, L_{1}$ são lagrangianos complementares e $\varepsilon>0$ então existe $L \in \Lambda_{0}\left(L_{0}\right) \cap \Lambda_{0}\left(L_{1}\right)$ tal que $\delta\left(\mathrm{L}_{1} \mathrm{~L}_{0}\right)<\varepsilon$.

Prova. Dado $T \in \mathscr{L}^{\text {sa }}\left(L_{0}\right)$, pondo $L=G\left(\Psi_{L_{0}, L_{1}} T\right) \in \Lambda_{0}\left(L_{1}\right)$, temos

$$
\delta\left(L, L_{0}\right)=\frac{\left\|\Psi_{L_{0}, L_{1}} T\right\|}{\left(1+\left\|\Psi_{L_{0}, L_{1}} T\right\|^{2}\right)^{1 / 2}} \quad \text { e } \quad \gamma\left(L, L_{0}\right)=\frac{\gamma\left(\Psi_{L_{0}, L_{1}} T\right)}{\left(1+\gamma\left(\Psi_{L_{0}, L_{1}} T\right)^{2}\right)^{1 / 2}} .
$$

Logo, tomando $T=t I$, com $t \neq 0$, obtemos subespaços lagrangianos em $\Lambda_{0}\left(L_{0}\right) \cap \Lambda_{0}\left(L_{1}\right)$ arbitrariamente próximos de $L_{0}$, como no enunciado.

Os cálculos do lema 1.5.38 podem ser utilizados para obter um exemplo patológico de subespaços lagrangianos.

EXEMPLo 1.5.39 Vamos provar que dado $\varepsilon>0$, existe $L \in \Lambda_{0}\left(L_{0}\right) \cap \Lambda_{0}\left(L_{1}\right)$ tal que $\delta\left(L, L_{1}\right)=1$, $\operatorname{mas} \delta\left(L, L_{0}\right)>1-\varepsilon, \gamma\left(L, L_{0}\right)=0$ e $L \cap L_{0}=0$. De fato, se $L=G\left(\Psi_{L_{0}, L_{1}} T\right)$, com $T \in \mathscr{L}^{\text {sa }}(H)$, então

$$
\delta\left(L_{,} L_{1}\right)=\frac{1}{\left(1+\gamma\left(\Psi_{L_{0}, L_{1}} T\right)^{2}\right)^{1 / 2}} .
$$

Tomando $T \in \mathscr{L}^{\text {sa }}\left(\mathrm{L}_{0}\right)$ compacto injetor e $t>0$, temos que $\gamma\left(\Psi_{\mathrm{L}_{0}, \mathrm{~L}_{1}} t T\right)=0$, logo $\delta\left(L_{,} \mathrm{L}_{1}\right)=$ 1. Como no exemplo 1.2.10, temos $\gamma\left(\mathrm{L}, \mathrm{L}_{0}\right)=0$ e $\mathrm{L} \cap \mathrm{L}_{0}=0$. Tomando $t>0$ tal que $t\left\|\Psi_{L_{0}, L_{1}} T\right\| /\left(1+t^{2}\left\|\Psi_{L_{0}, L_{1}} T\right\|^{2}\right)^{1 / 2}>1-\varepsilon$, segue que $L$ satisfaz as propriedades procuradas.

\subsection{Variedades Grassmannianas}

Nesta seção, vamos estudar um pouco mais detalhadamente algumas variedades grassmannianas em dimensão infinita. Também estudaremos alguns grupos de operadores em dimensão infinita e suas ações canônicas, especialmente em variedades grassmannianas. Construiremos explicitamente uma estrutura de variedade de Banach analítica real em algumas variedades grassmannianas. Finalmente, daremos uma descrição tão detalhada quanto possível da topologia de algumas variedades grassmannianas em dimensão infinita.

\subsubsection{Grupos de Operadores e Ações}

Vamos nesta subseção estudar os grupos $\mathrm{GL}(\mathrm{H}), \mathrm{U}(\mathrm{H}), \mathrm{O}(\mathrm{H})$ e $\mathrm{U}(\mathrm{H} ; J), \mathrm{Sp}(\mathrm{H})$ no caso simplético. Provaremos, entre outros resultados, o teorema de Kuiper. A referência original para o referido teorema é [58]. Também desenvolveremos uma teoria abstrata de espaços homôgeneos 
em dimensão infinita, a qual será utilizada na subseção 1.6.2. Em toda esta seção, $H$ denota um espaço de Hilbert real ou complexo separável de dimensão infinita.

Iniciemos com algumas relações canônicas entre os grupos $\mathrm{GL}(\mathrm{H})$ e $\mathrm{U}(\mathrm{H})$. A decomposição polar para operadores fechados dada na proposição 1.3.43 sugere que o estudo da topologia do grupo $\mathrm{GL}(\mathrm{H})$ seja reduzido ao estudo dos espaços de isometrias parciais e de operadores auto-adjuntos positivos. Aliás, já fizemos uso desta idéia para provar o teorema 1.3.51. A próxima proposição mostra que, de fato, a mesma idéia se aplica na situação em questão.

Proposição 1.6.1 GL(H) é homeomorfo a $\mathrm{U}(\mathrm{H}) \times \mathcal{P}(\mathrm{H})$, onde $\mathcal{P}(\mathrm{H})$ denota o conjunto dos isomorfismos positivos auto-adjuntos de $\mathrm{H}$.

Prova. Dado $T \in G L(H)$, pela proposição 1.2 .53 , temos que $|T|=\left(T^{*} T\right)^{1 / 2}$ é uma função contínua de $T$, logo,

$$
\begin{aligned}
\Phi: \mathrm{GL}(\mathrm{H}) & \rightarrow \mathrm{U}(\mathrm{H}) \times \mathcal{P}(\mathrm{H}) \\
T & \mapsto(U,|T|)=\left(T|T|^{-1},|T|\right)
\end{aligned}
$$

é um homeomorfismo entre $\mathrm{GL}(\mathrm{H})$ e $\mathrm{U}(\mathrm{H}) \times \mathcal{P}(\mathrm{H})$.

ObSERVAÇão 1.6.2 Mais adiante, veremos que $\mathrm{U}(\mathrm{H})$ admite estrutura de variedade de Banach. Também é verdade que a aplicação definida na proposição 1.6.1 é um difeomeorfismo, mas não precisaremos deste fato.

Lema 1.6.3 $\mathcal{P}(\mathrm{H})$ é contrátil.

Prova. Se $A \in \mathcal{P}(H)$, então a aplicação $H_{t}(A)=t I+(1-t) A$ é contínua, $H_{0}(A)=A, H_{1}(A)=I$ e $H_{t}(A) \in \mathcal{P}(\mathrm{H})$ para $0 \leq t \leq 1$.

\section{Corolário 1.6.4 $\mathrm{U}(\mathrm{H})$ é um retrato forte por deformação de $\mathrm{GL}(\mathrm{H})$.}

Prova. Basta observar que a aplicação $F_{t}(T) \doteq U H_{t}(|T|)$ é contínua (usando a notação da prova do lema 1.6.3 e da proposição 1.6.1), $F_{0}(T)=T, F_{1}(T)=U \in \mathrm{U}(\mathrm{H})$ e $F_{t}(T)=T$ para todo $T \in \mathrm{U}(\mathrm{H})$.

CorolárIo 1.6.5 GL(H) tem o mesmo tipo de homotopia que $\mathrm{U}(\mathrm{H})$.

Nosso próximo objetivo é provar o teorema de Kuiper, o qual mostra que GL(H) é contrátil. Este fato mostra quão diferentes são a topologia em dimensão finita e em dimensão infinita, uma vez que a topologia dos espaços $\mathrm{GL}(n), n \in \mathbb{N}$, influencia substancialmente a topologia das variedades de dimensão finita. O teorema de Kuiper mostra também que, ao contrário do caso de dimensão finita, em dimensão infinita não há distinção entre o caso real e o caso complexo. Uma excelente referência para este teorema é [18].

Teorema 1.6.6 (Kuiper) GL(H) é contrátil.

Prova. Faremos a prova em diversos passos. Como GL(H) é paracompacto (pois é um espaço métrico), pelo corolário ao teorema 15 de [73], basta provar que os grupos de homotopia $\pi_{k}(\mathrm{GL}(\mathrm{H}))$ são triviais para todo $k \geq 1$. 
Fixemos $k \geq 0$ e $f_{0}: S^{k} \rightarrow \mathrm{GL}(\mathrm{H})$ contínua. A idéia é construir uma sequência finita de homotopias que conecta $f_{0}$ à uma função constante.

Primeiro Passo. Vamos provar que $f_{0}$ é homotópica a uma função $f_{1}$ cuja imagem está contida em um complexo simplicial finito em $\mathrm{GL}(\mathrm{H})$. De fato, por compacidade, é possível cobrir $f_{0}\left(S^{k}\right)$ por um número finito de bolas abertas contidas em $\mathrm{GL}(\mathrm{H})$. Seja $\mathcal{T}$ uma triangulação de $S^{k}$ tal que a imagem de cada simplexo de $\mathcal{T}$ por $f_{0}$ está contida em alguma destas bolas. Definindo $f_{1}$ como a aplicação afim que coincide com $f_{0}$ nos vértices de $\mathcal{T}$, temos que a imagem de $f_{1}$ está contida em um complexo simplicial finito de $\mathrm{GL}(\mathrm{H})$. Como as bolas são convexas, a aplicação

$$
f_{t}=(1-t) f_{0}+t f_{1}
$$

define uma homotopia em $\mathrm{GL}(\mathrm{H})$ entre $f_{0}$ e $f_{1}$, como queríamos. Em particular, existe um subespaço $M$ de $\mathscr{L}(\mathrm{H})$ de dimensão $N<\infty$ tal que $M$ contém a imagem de $f_{1}$. Dados geradores $g_{1}, \ldots, g_{N}$ de $M$, segue que o subespaço gerado por $\{w(u): w \in M\}, u \in \mathrm{H}$, tem geradores $g_{1}(u), \ldots, g_{N}(u)$, em particular, $\left\{f_{1}(x) \cdot u: x \in S^{k}\right\}$ tem dimensão finita $\leq N$.

Segundo Passo. Agora, vamos provar que $f_{1}$ é homotópica a $f_{3}$ tal que existe $\mathrm{H}^{\prime}$ de dimensão infinita fechado com $\left.f_{3}(x)\right|_{H^{\prime}}=I$.

Esta é a parte mais delicada do teorema. Sejam $a_{1} \in \mathrm{H}$ unitário qualquer e $A_{1}$ um subespaço $N+2$-dimensional contendo $\left\{a_{1}^{0}, a_{1}, g_{1}\left(a_{1}\right), \ldots, g_{N}\left(a_{1}\right)\right\}$, onde $a_{1}^{0}$ é unitário ortogonal a $a_{1}, g_{1}\left(a_{1}\right), \ldots, g_{N}\left(a_{1}\right)$. Suponhamos obtidos $a_{i}, a_{i}^{0}, A_{i}$ para todo $i<k$ com estas propriedades. Como $\mathrm{H}$ tem dimensão infinita, existe um vetor unitário

$$
a_{k} \in \bigcap_{i<k}\left[A_{i}^{\perp} \cap\left(\bigcap_{j=1}^{N} g_{j}^{-1}\left(A_{i}^{\perp}\right)\right)\right] .
$$

Logo, $\left\{a_{k}, g_{1}\left(a_{k}\right), \ldots, g_{N}\left(a_{k}\right)\right\}$ é ortogonal a cada $A_{i}$ com $i<k$. Isso nos permite obter um subespaço $A_{k}$ de dimensão $N+2$ contendo $\left\{a_{k^{\prime}}^{0} a_{k}, g_{1}\left(a_{k}\right), \ldots, g_{N}\left(a_{k}\right)\right\}$, onde $a_{k}^{0}$ é unitário ortogonal a $a_{1}, g_{1}\left(a_{k}\right), \ldots, g_{N}\left(a_{k}\right)$. Observamos que, por construção, para $j, k$ distintos, $A_{j}$ e $A_{k}$ são ortogonais entre si. Seja $H^{\prime}$ o subespaço (fechado) gerado por $\left\{a_{i}: i \geq 1\right\}$.

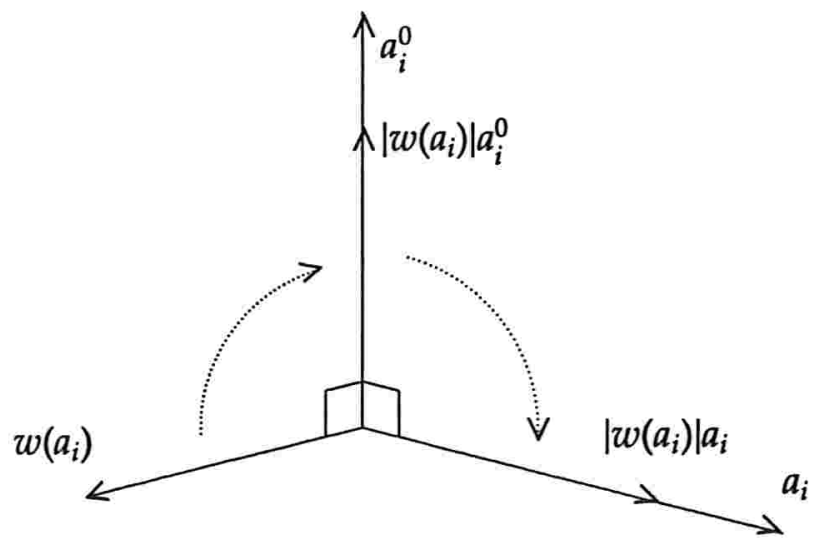

Vamos provar que é possível obter $f_{2}$ homotópica a $f_{1}$ tal que $f_{2}(x) a_{i}=\left|f_{1}(x)\right| a_{i}$, para todo $i \geq 1$. Como os $A_{i}$ são dois a dois ortogonais, basta construir a homotopia em cada $A_{i}$ e somar 
todas as homotopias obtidas. Para isso, seja $M_{C}=\left\{w \in M \cap G L(H):\|w\| \leq C,\|w\|^{-1} \leq C \|\right\}$. Temos que a imagem de $f_{1}$ está contida em $M_{C}$, para algum $C>0$. Para tais $w^{\prime}$ s, fixado $i \geq 1$, construímos uma homotopia no plano gerado por $w\left(a_{i}\right)$ e $a_{i}^{0}$ que rotaciona $w\left(a_{i}\right)$ até o vetor $\left|w\left(a_{i}\right)\right| a_{i}^{0}$, mantendo fixo o complemento ortogonal deste plano. Depois, construímos uma homotopia no plano gerado por $a_{i}^{0}$ que rotaciona $\left|w\left(a_{i}\right)\right| a_{i}^{0}$ até o vetor $\left|w\left(a_{i}\right)\right| a_{i}$. Podemos escrever explicitamente estas homotopias em termos das funções trigonométricas e observar que elas são contínuas em $w \in M_{C}$ e em $t$, uniformemente em $i$.

Tendo obtido $f_{2}$ como acima, é fácil verificar que $f_{2}$ é homotópica a $f_{3}$ tal que $f_{3}(x) a_{i}=a_{i}$, para todos $i \geq 1, x \in S^{k}$. De fato, basta tomar a homotopia $f_{t}(x)=I$ em $H^{\perp}$ e $f_{t}(x) a_{i}=$ $(1-t) f_{2}(x) a_{i}+t a_{i}$, para $i \geq 1$.

Terceiro Passo. Vejamos que $f_{3}$ é homotópica a $f_{4}$ tal que $\left.f_{4}(x)\right|_{\mathrm{H}^{\prime}}=I$ e $f_{4}(x)\left(\mathrm{H}^{\prime \perp}\right)=\mathrm{H}^{\perp \perp}$. Para provar este fato, observamos que, com respeito à decomposição $\mathrm{H}=\mathrm{H}^{\prime} \oplus \mathrm{H}^{\perp}$, o operador $f_{3}(x)$ tem a forma $\left(\begin{array}{ll}I & R \\ 0 & S\end{array}\right), \operatorname{com} R \in \mathscr{L}\left(\mathrm{H}^{\prime \perp}, \mathrm{H}^{\prime}\right)$ e $S \in \mathrm{GL}\left(\mathrm{H}^{\prime \perp}\right)$, logo

$$
\left(\begin{array}{cc}
I & t R \\
0 & S
\end{array}\right)
$$

para $0 \leq t \leq 1$ define uma homotopia em GL(H) entre $f_{3}$ e $f_{4}$ tal que $\left.f_{4}(x)\right|_{H^{\prime}}=I$ e $f_{4}(x)\left(H^{\prime \perp}\right)=$ $\mathrm{H}^{\prime \perp}$.

Quarto Passo. Finalmente, vamos mostrar que o conjunto

$$
\mathcal{S}=\left\{U \in \mathrm{GL}(\mathrm{H}):\left.U\right|_{\mathrm{H}^{\prime}}=I \text { e }\left.U\right|_{\mathrm{H}^{\prime \perp}} \in \mathrm{GL}\left(\mathrm{H}^{\prime \perp}\right)\right\}
$$

é contrátil. Isso encerra a demonstração, pois $f_{0}$ é homotópica a $f_{4}$ e a imagem de $f_{4}$ está contida em $\delta$.

Escrevamos $\mathrm{H}^{\prime}=\mathrm{H}_{1} \oplus \mathrm{H}_{2} \oplus \ldots \mathrm{H}_{n} \oplus \ldots$ com os espaços $\mathrm{H}_{n}$ fechados de dimensão infinita, dois a dois ortogonais. ${ }^{44} \mathrm{Um}$ operador $U \in \mathcal{S}$ tem a forma

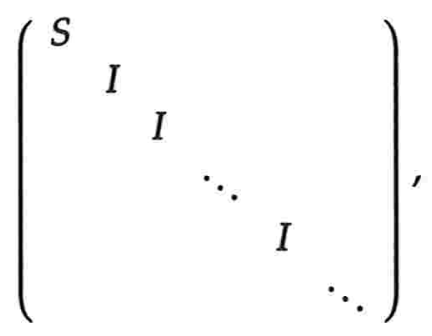

com respeito à decomposição ortogonal $\mathrm{H}=\mathrm{H}^{\perp} \oplus \mathrm{H}_{1} \oplus \ldots \oplus \mathrm{H}_{n} \oplus \ldots$, onde $S=\left.U\right|_{\mathrm{H}^{\prime}}$ e os termos que não aparecem são nulos. Como cada $\mathrm{H}_{n}$ é isomorfo $\mathrm{H}^{\perp \perp}$, podemos escrever os elementos de $\mathcal{S}$ na forma

$$
\left(\begin{array}{cccccc}
S & & & & & \\
& I & & & & \\
& S^{-1} S & & & \\
& & S & & \\
& & & S^{-1} S & \\
& & & & & \ddots
\end{array}\right) .
$$

\footnotetext{
${ }^{44}$ Basta tomar $\left\{e_{j}\right\}_{j=1}^{\infty}$ uma base ortonormal de $\mathrm{H}^{\prime}$ e conjuntos $N_{n} \subset \mathbb{N}$ infinitos dois a dois disjuntos tais que $\mathbb{N}=\bigcup_{n} N_{n}$ e definir $\mathrm{H}_{n}^{\prime}$ como o subespaço fechado gerado por $\left\{e_{j}: j \in N_{n}\right\}$.
} 
Agora, observamos que se $\mathrm{H}^{\prime}$ é um espaço de Hilbert e $R, T$ são funções definidas em um espaço qualquer $X$ com valores em $\mathscr{L}\left(\mathrm{H}^{\prime}\right)$, então as aplicações

$$
\left(\begin{array}{cc}
T R & 0 \\
0 & I
\end{array}\right) \text { e }\left(\begin{array}{ll}
R & 0 \\
0 & T
\end{array}\right)
$$

são homotópicas. De fato,

$$
\left(\begin{array}{cc}
(\cos t) I & -(\sin t) I \\
(\sin t) I & (\cos t) I
\end{array}\right)\left(\begin{array}{cc}
T & 0 \\
0 & I
\end{array}\right)\left(\begin{array}{cc}
(\cos t) I & (\sin t) I \\
-(\sin t) I & (\cos t) I
\end{array}\right)\left(\begin{array}{cc}
R & 0 \\
0 & I
\end{array}\right),
$$

com $0 \leq t \leq \pi / 2$ é uma homotopia entre tais aplicações. Logo, pela equação 1.6.2, a aplicação identidade em $S$ é homotópica à aplicação

$$
\left(\begin{array}{lllll}
S & & & & \\
& S^{-1} & & & \\
& & S & & \\
& & S^{-1} & \\
& & & & \ddots
\end{array}\right),
$$

a qual, pelo mesmo argumento, é homotópica à

$$
\left(\begin{array}{lllll}
I & & & & \\
& I & & & \\
& & I & & \\
& & & I & \\
& & & & \ddots
\end{array}\right),
$$

encerrando a demonstração do teorema.

O seguinte corolário decorre diretamente do teorema de Kuiper e do corolário 1.6.5.

COROLÁrIo 1.6.7 U(H) é contrátil.

Corolário 1.6.8 Se Hé um espaço simplético então $\mathrm{U}(\mathrm{H} ; J)$ é contrátil.

Prova. Seja $\mathrm{H}^{\mathbf{C}}$ a complexificação de $\mathrm{H}$ correspondente à estrutura complexa $J$, i.e., definimos a multiplicação por um escalar complexo como $(\alpha+i \beta) u=\alpha u+\beta J u$, para $\alpha, \beta \in \mathbb{R}$ e $u \in \mathrm{H}$. Não é difícil ver que $T \in \mathrm{U}\left(\mathrm{H}^{\mathbb{C}}\right)$ se e só se $T J=J T$ e $T \in \mathrm{O}(\mathrm{H}), \operatorname{logo} \mathrm{U}\left(\mathrm{H}^{\mathbb{C}}\right)=\mathrm{U}(\mathrm{H} ; J)$. Pelo teorema de Kuiper, $\mathrm{U}^{\left(\mathrm{H}^{\mathbb{C}}\right)}$, e portanto $\mathrm{U}(\mathrm{H} ; J)$, é contrátil.

Vamos agora introduzir uma estrutura diferenciável natural nos grupos $\mathrm{U}(\mathrm{H}), \mathrm{O}(\mathrm{H})$ e em $\mathrm{Sp}(\mathrm{H})$ e $\mathrm{U}(\mathrm{H} ; J)$, caso $\mathrm{H}$ seja um espaço simplético. A idéia é a mesma utilizada em dimensão finita, só precisamos tomar alguns cuidados. Obviamente, $\mathrm{GL}(\mathrm{H})$ admite uma estrutura diferenciável analítica real, pois é um aberto do espaço $\mathscr{L}(\mathrm{H})$. Esta estrutura torna analítica real a aplicação $\mathrm{GL}(\mathrm{H}) \times \mathrm{GL}(\mathrm{H}) \ni(S, T) \mapsto S T \in \mathrm{GL}(\mathrm{H})$, i.e., $\mathrm{GL}(\mathrm{H})$ é um grupo de Lie de dimensão infinita.

Proposição 1.6.9 Se $\mathrm{H}$ é um espaço de Hilbert (real ou complexo) então o grupo $\mathrm{U}(\mathrm{H})$ admite uma estrutura diferenciável analítica real que o torna subgrupo de Lie de $\mathrm{GL}(\mathrm{H})$. A álgebra de Lie $\mathrm{u}(\mathrm{H})$ de $\mathrm{U}(\mathrm{H})$ é o espaço $\mathscr{L}^{\operatorname{asim}}(\mathrm{H})$ dos operadores anti-simétricos em $\mathrm{H}$. 
Prova. Consideremos a aplicação analítica real

$$
\begin{aligned}
F: G L(H) & \rightarrow \mathscr{L}^{\text {sa }}(H) \\
T & \mapsto T^{*} T .
\end{aligned}
$$

Temos que $F^{\prime}(T) \cdot X=X^{*} T+T^{*} X$ para $T \in G L(H)$ e $X \in \mathscr{L}(H)$. Como $F^{-1}(I)=\mathrm{U}(\mathrm{H})$, pelo teorema da função implícita, a proposição fica demonstrada se mostrarmos que $I$ é um valor regular de $F$ e $\operatorname{ker} F^{\prime}(T)$ é um subespaço complementado ${ }^{45}$ de $\mathscr{L}(H)$. Se $F(T)=I$ então dado qualquer $Y \in \mathscr{L}^{\text {sa }}(\mathrm{H})$, temos que $F^{\prime}(T) \cdot(T Y / 2)=X$, logo, $I$ é valor regular de $F$. Temos que $\operatorname{ker} F^{\prime}(T)=\left\{X \in \mathscr{L}(\mathrm{H}): X^{*} T+T^{*} X=0\right\}$ e cada $X \in \mathscr{L}(\mathrm{H})$ se escreve como

$$
X=\left(\frac{X-T X^{*} T}{2}\right)+\left(\frac{X+T X^{*} T}{2}\right) .
$$

O primeiro fator da soma pertence a $\operatorname{ker} F^{\prime}(T)$ e o segundo pertence ao subespaço fechado $\left\{X \in \mathscr{L}(\mathrm{H}): X^{*} T-T^{*} X=0\right\}$. Como a intersecção destes subespaços é trivial, segue que $\operatorname{ker} F^{\prime}(T)$ é complementado, provando a proposição.

O mesmo argumento utilizado na prova da proposição 1.6 .9 pode ser utilizado no caso simplético.

Proposição 1.6.10 Se $\mathrm{H}$ é um espaço simplético então o grupo $\mathrm{Sp}(\mathrm{H})$ admite uma estrutura diferenciável analítica real que o torna um subgrupo de Lie de $\mathrm{GL}(\mathrm{H})$. A álgebra de Lie de $\mathrm{Sp}(\mathrm{H})$ é o espaço $\mathfrak{s p}(\mathrm{H})=\left\{X \in \mathscr{L}(\mathrm{H}): X^{*} J+J X=0\right\}$.

Prova. Consideramos $F(T)=T^{*} J T$ definida para $T \in \mathrm{GL}(\mathrm{H})$. Vemos que $F(T) \in \mathscr{L}^{\text {asim }}(\mathrm{H})$ e $F^{-1}(J)=\mathrm{Sp}(\mathrm{H})$; vamos provar que $J \in \mathscr{L}^{\text {asim }}(\mathrm{H})$ é um valor regular de $F$ e que $\operatorname{ker} F^{\prime}(T)$ é um subespaço complementado de $\mathscr{L}(\mathrm{H})$. Basta observar que $F^{\prime}(T) \cdot X=X^{*} J T+T^{*} J X, X \in \mathscr{L}(\mathrm{H})$ e se $T \in \mathrm{Sp}(\mathrm{H})$, então dado $Y \in \mathscr{L}^{\text {asim }}(\mathrm{H}), F^{\prime}(T) \cdot(-T J Y / 2)=Y$. Logo, $J$ é valor regular de $F$. Vejamos que $\operatorname{ker} F^{\prime}(T)$ é complementado: de fato, $\operatorname{ker} F^{\prime}(T)=L_{T^{*} J}^{-1}\left(\mathscr{L}^{\text {sa }}(H)\right)$, onde $L_{A}(X)=A X$, $\operatorname{logo} L_{T^{*} J}^{-1}\left(\mathscr{L}^{\operatorname{asim}}(\mathrm{H})\right)$ é um complementar para $\operatorname{ker} F^{\prime}(T)$.

OBSERVAÇÃo 1.6.11 Usando a notação da prova do corolário 1.6.8 e a proposição 1.6.9, vemos que $\mathrm{U}(\mathrm{H} ; J)$ admite uma estrutura de subgrupo de Lie de $\mathrm{GL}(\mathrm{H})$. A álgebra de Lie $\mathrm{u}(\mathrm{H} ; J)$ de $\mathrm{U}(\mathrm{H} ; J)$ é o espaço $\left\{\mathrm{X} \in \mathscr{L}(\mathrm{H}): X^{*}+X=J X-X J=0\right\}$.

OBSERVAÇão 1.6.12 Podemos dar uma caracterização dos elementos de $\mathfrak{s p}(\mathrm{H})$ semelhante àquela dada em 1.5.16 para os elementos de $\mathrm{Sp}(\mathrm{H})$. De fato, sejam $L_{0}$ um subespaço lagrangiano

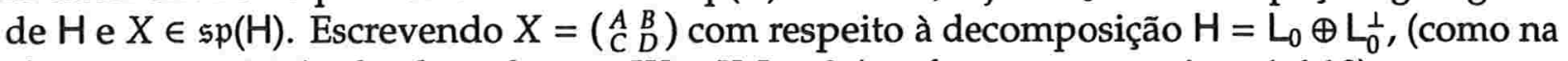
observação 1.5.16) e lembrando que $J X+X^{*} J=0$ (conforme a proposição 1.6.10), segue que $J B \in \mathscr{L}^{\text {sa }}\left(\mathrm{L}_{0}^{\frac{1}{}}\right), J C \in \mathscr{L}^{\text {sa }}\left(\mathrm{L}_{0}\right)$ e $D=J A^{*} J$, i.e., $X$ tem a forma

$$
\left(\begin{array}{cc}
A & B \\
C & J A^{*} J
\end{array}\right),
$$

$\operatorname{com} B, C$ como acima.

\footnotetext{
${ }^{45} \mathrm{Um}$ subespaço fechado $\mathrm{M}$ de um espaço de Banach $\mathrm{X}$ é dito complementado se existe um subespaço fechado $\mathrm{N}$ tal que $\mathrm{M} \cap \mathrm{N}=0$ e $\mathrm{M}+\mathrm{N}=\mathrm{X}$. Neste caso, $\mathrm{N}$ é dito um complementar de $\mathrm{M}$.
} 
Vamos agora fazer algumas considerações gerais sobre ações de grupos de Lie em dimensão infinita. Seja $G$ um grupo de Lie com álgebra de Lie g, $M$ uma variedade de Banach de classe $C^{k}(k=1, \ldots, \infty, \omega) \mathrm{e}$

$$
\begin{aligned}
G \times M & \rightarrow M \\
(g, p) & \mapsto g \cdot p
\end{aligned}
$$

uma ação à esquerda de $G$ sobre $M$. $G$ ou $M$ podem ter dimensão infinita. Isso ${ }^{46}$ significa que a aplicação 1.6.4 é de classe $C^{k}$, para cada $g \in G, \eta_{g}: p \mapsto g \cdot p$ é um difeomorfismo $C^{k}$ de $M$ e $g \cdot(h \cdot p)=(g h) \cdot p$, para todos $g, h \in G$ e $p \in M$. A ação é dita transitiva se dados quaisquer $p, q \in M$ existe $g \in G$ tal que $g \cdot p=q$. Para cada $p \in M$, o subgrupo fechado

$$
G_{p}=\{g \in G: g \cdot p=p\}
$$

é dito o grupo de isotropia de $p$. A teoria geral de ações de grupos de Lie (em dimensão finita) pode ser encontrada em [99] ou [102]. Boa parte dos resultados válidos em dimensão finita continua válida em dimensão infinita; vamos aqui descrever alguns deles. Uma excelente referência é o capítulo VI de [59].

Dado $g \in G$, denotaremos por $L_{g}$ o difeomorfismo $h \mapsto g h, h \in H$. O seguinte teorema é fundamental na teoria de ações de grupos de Lie.

Tвовемa 1.6.13 Suponhamos que $H \subset G$ seja um subgrupo de Lie fechado e que a álgebra de Lie h de $H$ seja um subespaço fechado complementado de $\mathrm{g}$, com complementar $\mathrm{M}$. Então o espaço $\mathrm{G} / \mathrm{H}$ de classes laterais de $\mathrm{H}$ em $\mathrm{G}$ admite uma estrutura de variedade diferenciável (de Banach) e a aplicação canônica $\pi: G \rightarrow G / H$ define um fibrado principal com grupo estrutural $H^{47}$

Prova. Munimos $G / H$ da topologia quociente, i.e., $U \subset G / H$ é aberto se e só se $\pi^{-1}(U) \subset G$ é aberto.. É imediato que a aplicação canônica é contínua e aberta. Vamos agora provar que é possível introduzir em $\mathrm{G} / \mathrm{H}$ uma estrutura diferenciável como no enunciado.

Consideremos a distribuição $C^{\infty}$ em $G$ definida por $D_{g}=\left(L_{g}\right)_{*}(\mathfrak{h}), g \in G$. Como h é subálgebra de Lie, segue que $D_{g}$ é uma distribuição integrável, e portanto, pelo teorema de Frobenius (veja [59], Pág.157), temos que existem $\varepsilon>0, V$ vizinhança aberta de $e \in G \mathrm{e}$

$$
\Phi: U \doteq\{u \in \mathfrak{h}:\|u\|<\varepsilon\} \times\{w \in M:\|w\|<\varepsilon\} \rightarrow V
$$

difeomorfismo tal que as variedades integrais conexas de $D$ em $V$ são exatamente os conjuntos da forma $\Phi(w+\{u \in \mathfrak{h}:\|u\|<\varepsilon\})$, com $w \in \mathrm{M}$. Diminuindo $V$ e $\varepsilon$ convenientemente, podemos supor que $V \cap H$ seja a variedade integral conexa de $D$ contida em $V$ passando por $e$. Vejamos como é possível diminuir $V$ de tal maneira que as variedades integrais de $D$ em $V$ sejam exatamente as classes laterais de $H$ em $V$. De fato, consideremos $V_{1}, V_{2} \subset V$ vizinhanças de $e$ tais que $V_{2} \cdot V_{2} \subset V$ e $V_{1}^{-1} \cdot V_{1} \subset V_{2}$. Logo, se $a H=b H \operatorname{com} a, b \in V_{1}$, então $b^{-1} a \in H \cap V_{2}$ e $H \cap V_{2}$ é subconjunto da variedade integral de $D$ passando por $e$. Logo, $a, b$ pertencem à mesma variedade integral de $D$, pois $a \in L_{b}\left(V_{2} \cap H\right)$ que é variedade integral de $D$ passando por $b$. Portanto, trocando $V$ por $V_{1}$, temos que as variedades integrais conexas de $D$ em $V_{2}$ coincidem com as classes laterais de $\mathrm{H}$ em $V$. Vamos manter $V$ fixo até o final da prova.

\footnotetext{
${ }^{46} \mathrm{Em}$ geral, um grupo de Lie de dimensão infinita é chamado de grupo de Banach-Lie, mas vamos abusar da notação e continuar a chamar um tal grupo simplesmente de grupo de Lie.

${ }^{47} \mathrm{Se} G$ tem estrutura diferenciável $C^{\infty}$ então $G / H$ também tem estrutura diferenciável $C^{\infty}$ e $\pi$ é $C^{\infty}$. $O$ mesmo é verificado para $C^{\omega}$ em lugar de $C^{\infty}$.
} 
Consideremos a subvariedade $S=\Phi(\mathrm{M}) \subset V$ e a aplicação $\left.\pi\right|_{S}: S \rightarrow G / H$. Como $S$ contém no máximo um elemento de cada classe lateral de $H$, segue que $\left.\pi\right|_{s}$ é injetora; como $\pi$ é aberta, temos que $\left.\pi\right|_{S}$ é um homeomorfismo de $S$ sobre o aberto $\pi(V)$. Deste fato, concluímos que $\left(\left.\pi\right|_{S}\right)^{-1}: \pi(V) \rightarrow S$ é uma seção de $\pi$ definida no aberto $\pi(V)$. Além disso, podemos considerar $\chi=\Phi^{-1} \circ\left(\left.\pi\right|_{S}\right)^{-1}: \pi(V) \rightarrow \mathrm{M}$ como um sistema de coordenadas em $\pi(V)$.
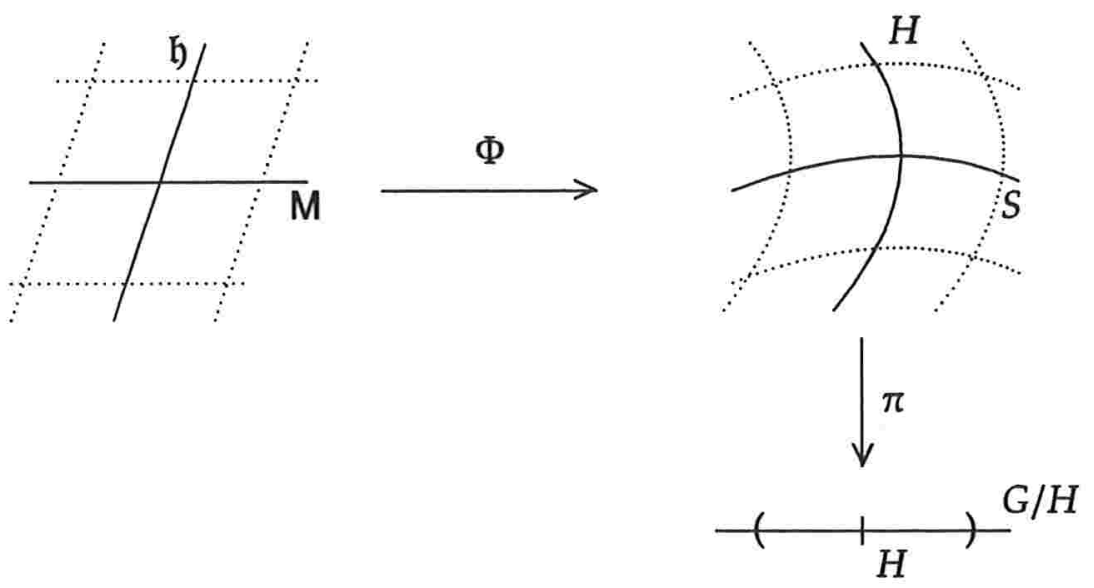

Para cada $g \in G$, consideremos $\chi_{g}: \pi(g \cdot V) \rightarrow \mathrm{M}, \chi_{g}=\chi \circ L_{g^{-1}}$. É fácil ver que $\left\{\chi_{g}\right\}_{g \in G}$ é um atlas diferenciável para $G / H$. Vejamos que $\pi: G \rightarrow G / H$ é $C^{\infty}$ (ou $C^{\omega}$, caso $G$ seja $C^{\omega}$ ). De fato, $\pi$ é $C^{\infty}$ se e só se para qualquer $g \in G$, a composta

$$
U \stackrel{\Phi}{\longrightarrow} V \stackrel{L_{g}}{\longrightarrow} g \cdot V \stackrel{\pi}{\longrightarrow} \pi(g \cdot V) \stackrel{x_{g}}{\longrightarrow} S \stackrel{\Phi^{-1}}{\longrightarrow} M
$$

o é. Mas a composta acima é a restrição a $U$ da projeção $P_{\mathfrak{h}, \mathrm{M}}$ sobre $\mathrm{M}$ paralelamente a $\mathfrak{h}$, que obviamente é $\mathrm{C}^{\infty}$.

Finalmente, vejamos que $\pi: G \rightarrow G / H$ define um fibrado principal com grupo estrutural $H$. Isso se faz usando a seção local $\chi: \pi(V) \rightarrow V$ definida no parágrafo anterior e o argumento clássico de [95], Sec.(7.4). Definimos uma trivialização de $V=\pi^{-1}(\pi(V))$ sobre $\pi(V)$ pondo

$$
\begin{aligned}
F: g \cdot V & \rightarrow \pi(g \cdot V) \times H \\
g x & \mapsto\left(\pi(g x),\left[L_{g} \circ \chi_{g}(g x H)\right]^{-1} g x\right) .
\end{aligned}
$$

É imediato que $F$ é um difeomorfismo. Além disso, pondo $\psi(g x)=\left[L_{g} \circ \chi_{g}(g x H)\right]^{-1} g x$, temos que $\psi(g x \cdot h)=\left[L_{g} \circ \chi_{g}(g x h H)\right]^{-1} g x h=\left[L_{g} \circ \chi_{g}(g x H)\right]^{-1} g x h=\psi(g x) \cdot h$, onde o último $\cdot$ denota a ação à direita de $H$ em $G$. Isso encerra a demonstração.

Suponhamos que a ação em 1.6.4 seja transitiva e fixemos $p \in M$. Neste caso, é possível definir uma bijeção

$$
\begin{aligned}
\varphi: G / G_{p} & \rightarrow M \\
g G_{p} & \mapsto g(p) .
\end{aligned}
$$

A próxima proposição mostra que $\varphi$ é, de fato, regular.

Proposição 1.6.14 Se a ação 1.6.4 é transitiva, então $\varphi$ é um difeomorfismo $C^{k}$. 
Prova. Como o seguinte diagrama

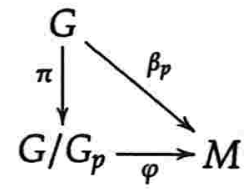

comuta, onde $\beta_{p}(g)=g \cdot p$, e pelo teorema 1.6.15 a aplicação $\pi$ admite seções locais $C^{\infty}$, segue que $\varphi$ é de classe $C^{k}$. Para provar que $\varphi$ é um difeomorfismo, basta verificar que $d \varphi$ é não-singular em cada ponto. Para isto, por homogeneidade, basta verificar que $\operatorname{ker}\left(d \beta_{p}\right)_{e} \subset \mathfrak{g}_{p}$. Dado $X \in \mathfrak{g}$ tal que $\left(d \beta_{p}\right)_{e} X=0$, para verificar que $X \in \mathfrak{g}_{p}$, basta verificar que $\exp (t X) \in G_{p}$ para todo $t \in \mathbb{R}$, pois $X=\left.\frac{d}{d t} \exp (t X)\right|_{t=0}$. Para $^{48}$ isso, basta provar que a curva $\sigma: t \mapsto \beta_{p}(\exp (t X))=\exp (t X) \cdot p$ é constante. De fato, temos

$$
\begin{aligned}
\sigma^{\prime}(t) & =\left(d \beta_{p}\right)\left(\left(L_{\exp (t X)}\right)_{e}(X)\right) \\
& =d\left(\eta_{\exp (t X)} \circ \beta_{p} \circ L_{\exp (-t X)}\right)\left(\left(L_{\left.\exp (t X))_{e}(X)\right)}\right.\right. \\
& =d \eta_{\exp (t X)} \circ d \beta_{p}(X)=0 .
\end{aligned}
$$

A proposição 1.6.14 e o teorema 1.6.13 implicam imediatamente o seguinte resultado.

Teorema 1.6.15 Consideremos a ação transitiva 1.6.4 e fixemos $p \in M$. Se a álgebra de Lie $\mathrm{g}_{p}$ de $G_{p}$ é um subespaço complementado de $\mathrm{g}$, então a aplicação $\beta_{p}: G \rightarrow M$ em 1.6.5 define um fibrado principal com grupo estrutural $G_{p}$.

Uma variedade $M$ sobre a qual age transitivamente um grupo de Lie $G$ é chamada de espaço homogêneo de $G$. O termo homogêneo é justificado pelo teorema 1.6.15, pois a estrutura do espaço $G / G_{p}$ é homogênea no sentido que é obtida a partir da estrutura local na classe trivial $G_{p}$. Na próxima subseção usaremos o teorema. 1.6.15 para obter algumas variedades grassmannianas como espaços homogêneos de certos grupos de Lie.

Para finalizar esta subseção, vamos introduzir um subgrupo importante de GL(H). O teorema de Kuiper mostra que, do ponto de vista topológico, o grupo GL(H) não é interessante. Para obter um subgrupo de GL(H) com topologia não-trivial, é necessário considerar o subgupo formado pelas perturbações compactas e de posto finito da identidade, o qual descreveremos agora.

Considerando as inclusões canônicas $\mathbb{R}^{n} \subset \mathbb{R}^{n+1}$ (ou $\mathbb{C}^{n} \subset \mathbb{C}^{n+1}$ ), obtemos inclusões $\mathrm{GL}(n) \subset$ $\mathrm{GL}(n+1)$ e portanto, podemos definir o grupo $\mathrm{GL}(\infty) \doteq \varliminf_{\longrightarrow} \mathrm{GL}(n)$. Este grupo tem topologia altamente não-trivial; de fato, $\pi_{i}(\mathrm{GL}(\infty))=\pi_{i}(\mathrm{GL}(n)), n>i$, e o último grupo é não trivial para uma infinidade de $i$ 's e pode ser calculado usando o teorema de periodicidade de Bott, conforme [64]. Para obter uma realização concreta de $G L(\infty)$, podemos pensar em seus elementos como "matrizes" infinitas que diferem da identidade (i.e., a matriz que possui somente 1's na diagonal e 0 's fora dela) por uma "matriz"que possui somente uma quantidade finita elementos não-nulos. Logo, podemos identificar $\mathrm{GL}(\infty)$ com o subgrupo de $\mathrm{GL}(\mathrm{H})$

\footnotetext{
${ }^{48} \mathrm{~A}$ aplicação exponencial é definida exatamente como no caso de dimensão finita. Para definí-la, é preciso apenas o teorema de existência e unicidade para equações ordinárias. Mais detalhes podem ser encontrados em [59].
} 
formado pelos operadores da forma $I+K$, onde $K$ é um operador de posto finito. $O$ mesmo vale para $\mathrm{U}(\infty)$.

Lembramos que $\mathrm{GL}_{c}(H)$ e $\mathrm{U}_{c}(\mathrm{H})$ denotam os subgrupos de $\mathrm{GL}(\mathrm{H})$ e $U(H)$, respectivamente, formados pelos operadores da forma $I+K$, com $K$ compacto. Caso $\mathrm{H}$ seja um espaço simplético, definimos $\mathrm{U}_{c}\left(\mathrm{H}_{\mathbb{R}}\right)$ de maneira análoga.

O seguinte resultado, (devido a Palais [72]) mostra que, topologicamente, os grupos GL( $\infty)$ e $\mathrm{GL}_{c}(\mathrm{H})$ são os mesmos.

Trorema 1.6.16 $\mathrm{GL}_{c}(\mathrm{H})$ tem o mesmo tipo de homotopia que $\mathrm{GL}(\infty)$. O mesmo vale para $\mathrm{U}$ em lugar de GL.

A proposição 1.6.1 continua válida se substituirmos $G L(H)$ por $G_{c}(H)$ e $U(H)$ por $U_{c}(H)$, como mostra a seguinte proposição.

Proposição 1.6.17 $\mathrm{GL}_{c}(\mathrm{H})$ é homeomorfo a $\mathrm{U}_{c}(\mathrm{H}) \times \mathcal{P}(\mathrm{H})$. Em particular, pelo lema 1.6.3, $\mathrm{U}_{c}(\mathrm{H})$ é retrato forte por deformação de $\mathrm{GL}_{c}(\mathrm{H})$.

Prova. Basta observar que, pela observação 1.3.46, $|T| \in \mathrm{U}_{c}(\mathrm{H})$ se $T \in \mathrm{GL}_{c}(\mathrm{H})$. Portanto, a aplicação $\mathrm{GL}_{c}(\mathrm{H}) \ni T \mapsto\left(T|T|^{-1},|T|\right) \in \mathrm{U}_{c}(\mathrm{H}) \times \mathcal{P}(\mathrm{H})$ é um homeomorfismo.

Observação 1.6.18 Obviamente, a aplicação $\mathrm{GL}_{c}(\mathrm{H}) \ni I+K \mapsto K \in \mathscr{L}_{c}(\mathrm{H})$ nos permite introduzir em $\mathrm{GL}_{c}(\mathrm{H})$ uma estrutura de variedade de Banach modelada no espaço $\mathscr{L}_{c}(\mathrm{H})$. Como o espaço $\mathscr{L}_{c}(\mathrm{H})$ não admite complementar fechado em $\mathscr{L}(\mathrm{H})$, segue que $\mathrm{GL}_{c}(\mathrm{H})$ não é uma subvariedade $C^{k}$ de $G L(H)$, para $k \geq 1$. Apesar disso, o teorema de Bartle-Graves [16] implica que $G_{c}(H)$ é uma subvariedade $C^{0}$ de $G L(H)$. $A^{49}$ mesma observação vale para $U_{c}(H)$ e $U(H)$.

\subsubsection{Estruturas Diferenciáveis}

Nesta subseção, vamos construir explicitamente estruturas de variedade de Banach analítica real em alguns conjuntos de subespaços e estudar propriedades destas estruturas. Uma referência interessante é [2].

Dado qualquer espaço de Hilbert $\mathrm{H}$, denotamos por $\mathcal{G}(\mathrm{H})$ o conjunto de todos os subespaços fechados de $H ; G(H)$ é chamado de grassmanniana total de $H$. Sejam $L_{0}, L_{1}$ subespaços complementares e consideremos a aplicação $\psi_{L_{0}, L_{1}}$ definida como

$$
\begin{aligned}
\psi_{\mathrm{L}_{0}, \mathrm{~L}_{1}}: \mathcal{G}_{0}\left(\mathrm{~L}_{1}\right) & \rightarrow \mathscr{L}\left(\mathrm{L}_{0}, \mathrm{~L}_{1}\right) \\
\mathrm{L} & \mapsto \psi_{\mathrm{L}_{0}, \mathrm{~L}_{1}}(\mathrm{~L}) \doteq P_{\mathrm{L}_{0}, \mathrm{~L}_{1}}^{\mathrm{L}} \circ P_{\mathrm{L}_{1}, \mathrm{~L}^{\mathrm{L}}}^{\mathrm{L}_{0}}
\end{aligned}
$$

onde $\mathcal{G}_{0}\left(L_{1}\right) \doteq\left\{L \in \mathcal{G}(H): L, L_{1} \in \mathcal{G}(H)\right.$ são complementares\}. Temos que $\psi_{L_{0}, L_{1}}$ é uma bijeção bem-definida, pelos comentários que antecedem a equação 1.5.4, e $\psi_{\mathrm{L}_{0}, \mathrm{~L}_{1}}(\mathrm{~L})$ é o único operador em $\mathscr{L}\left(L_{0}, L_{1}\right)$ cujo gráfico é $L$. Vamos provar que as aplicações $\psi_{L_{0}, L_{1}}$ formam um atlas analítico real para $\mathcal{G}(\mathrm{H})$, i.e., que as mudanças de coordenadas são analíticas reais.

\footnotetext{
${ }^{49}$ De fato, se $\mathrm{M} \subset \mathrm{X}$ é um subespaço fechado do espaço de Banach $\mathrm{X}$ e $s: \mathrm{X} / \mathrm{M} \rightarrow \mathrm{X}$ é uma seção contínua para a projeção canônica, então $S \doteq \operatorname{Im} s$ é um subconjunto fechado que contém exatamente um elemento de cada classe lateral $x+M, x \in \mathrm{X}$. Isso mostra que é possível construir em M uma estrutura de subvariedade $C^{0}$ de $X$.
} 
Fixemos, $L_{0}, L_{0}^{\prime}, L_{1} \in \mathcal{G}(H)$ tais que $\left(L_{0}, L_{1}\right)$ e $\left(L_{0}^{\prime}, L_{1}\right)$ são complementares. Se $L \in \mathcal{G}_{0}\left(L_{1}\right)$ e $L=G(S)$, com $S: L_{0} \rightarrow L_{1}$, então $\left(P_{L_{0}^{\prime}, L_{1}}^{L_{0}}+S\right) P_{L_{1}, L_{0}}^{L_{0}^{\prime}}: L_{0}^{\prime} \rightarrow L_{1}$, tem gráfico $L$. Logo, para qualquer $T \in \mathscr{L}\left(\mathrm{L}_{0}, \mathrm{~L}_{1}\right)$, temos que

$$
\psi_{\mathrm{L}_{0}^{\prime}, L_{1}} \circ \psi_{L_{0}, L_{1}}^{-1}(T)=\left(P_{L_{0}^{\prime}, L_{1}}^{L_{0}}+T\right) P_{L_{1}, L_{0}}^{L_{0}^{\prime}}
$$

Consideremos $L_{,} L_{0}, L_{1}, L_{1}^{\prime} \in \mathcal{G}(H)$ tais que os pares $\left(L_{0}, L_{1}\right)$ e $\left(L_{0}, L_{1}^{\prime}\right)$ são complementares, $L \in \mathcal{G}_{0}\left(L_{1}\right) \cap \mathcal{G}_{0}\left(L_{1}^{\prime}\right)$ e $T \in \mathscr{L}\left(L_{0}, L_{1}\right)$ tal que $G(T)=L$.

LEMA 1.6.19 Se $L_{,} L_{0}, L_{1}, L_{1}^{\prime}$ e $T$ são como acima, então $I+P_{L_{1}^{\prime}, L_{0}}^{L_{1}} T: L_{0} \rightarrow L_{0}$ é um isomorfismo.

Prova. Basta observar que dado $u \in L_{0}$,

$$
\left(I+P_{L_{1}^{\prime}, L_{0}}^{L_{1}} T\right) u=P_{L_{1}^{\prime}, L_{0}}(u+T u)=\left(P_{L_{1}^{\prime}, L_{0}}^{L_{0}} \circ(I+T)\right) u .
$$

Como $I+T: L_{0} \rightarrow L$ é um isomorfismo com inverso $P_{L_{1}, L_{0}}$, então, pela observação 1.5.18, temos que $I+P_{\mathrm{L}_{1}^{\prime}, L_{0}}^{\mathrm{L}_{1}} T$ é um isomorfismo com inverso $\left(I+P_{\mathrm{L}_{1}^{\prime}, L_{0}}^{\mathrm{L}_{1}} T\right)^{-1}=P_{\mathrm{L}_{1}, L_{0}}^{\mathrm{L}} P_{\mathrm{L}_{1}^{\prime}, L^{\prime}}^{\mathrm{L}_{0}}$

Como $u+T u=\left(I+P_{\mathrm{L}_{1}^{\prime}, L_{0}}^{\mathrm{L}_{1}} T\right) u+P_{\mathrm{L}_{0}, L_{1}^{\prime}}^{\mathrm{L}_{1}} T u, u \in \mathrm{L}_{0}$, o lema 1.6 .19 mostra que

$$
P_{\mathrm{L}_{0}, \mathrm{~L}_{1}^{\prime}}^{\mathrm{L}_{1}} T\left(I+P_{\mathrm{L}_{1}^{\prime}, L_{0}}^{\mathrm{L}_{1}} T\right)^{-1}: \mathrm{L}_{0} \rightarrow \mathrm{L}_{1}^{\prime}
$$

tem gráfico L, e portanto,

$$
\psi_{\mathrm{L}_{0}, \mathrm{~L}_{1}^{\prime}} \circ \psi_{\mathrm{L}_{0}, \mathrm{~L}_{1}}^{-1}(T)=P_{\mathrm{L}_{0}, \mathrm{~L}_{1}^{\prime}}^{\mathrm{L}_{1}} T\left(I+P_{\mathrm{L}_{1}^{\prime}, L_{0}}^{\mathrm{L}_{1}} T\right)^{-1},
$$

para todo $T \in \mathscr{L}\left(\mathrm{L}_{0}, \mathrm{~L}_{1}\right)$.

Para fixar a notação, vamos agora definir o que entendemos por uma função analítica real entre espaços de Banach.

DeFinição 1.6.20 Se X, Y são espaços de Banach (reais ou complexos) e $U \subset X$ é aberto, então $F: U \rightarrow$ Y é dita analítica real (ou de classe $C^{\omega}$ ) se para cada $x_{0} \in U$ existe $r>0$ tal que

$$
F(x)=\sum_{n=0}^{\infty} F^{(n)}\left(x_{0}\right) \cdot\left(x-x_{0}\right)^{n},
$$

se $\left\|x-x_{0}\right\|<r$, onde $F^{(n)}\left(x_{0}\right)$ denota a $n$-ésima derivada de $F$ em $x_{0}$ e $F^{(n)}\left(x_{0}\right) \cdot\left(x-x_{0}\right)^{n}$ denota a derivada $F^{(n)}\left(x_{0}\right)$ aplicada à $\left(x-x_{0}, \ldots, x-x_{0}\right)$. Dizemos que uma variedade de Banach é analítica real se possui um atlas tal que as mudanças de coordenadas são analíticas reais.

A próxima proposição fornece um exemplo importante de função analítica real.

Proposição 1.6.21 A função $F: G L(H) \ni T \mapsto T^{-1} \in G L(H)$ é analítica real.

Prova. Dado $T \in \mathrm{GL}(\mathrm{H})$, se $S \in \mathscr{L}(\mathrm{H})$ e $\|S-T\|<\left\|T^{-1}\right\|^{-1}$, então, usando a série de Neumann, (veja a observação 1.2.33) e a igualdade $S=T\left(I+T^{-1}(S-T)\right.$ ), segue que

$$
F(S)=S^{-1}=\left(I+T^{-1}(S-T)\right)^{-1} T^{-1}=\sum_{n=0}^{\infty}(-1)^{n}\left\{T^{-1}(S-T)\right\}^{n} T^{-1} \text {. }
$$


Logo $F$ é analítica real $\left(\operatorname{com} r=\left\|T^{-1}\right\|^{-1}\right) \mathrm{e}$

$$
F^{(n)}(T) \cdot\left(X_{1}, \ldots, X_{n}\right)=(-1)^{n} T^{-1} \cdot X_{1} \cdot \ldots \cdot T^{-1} \cdot X_{n} \cdot T^{-1} .
$$

TEOREMA 1.6.22 O conjunto

$$
\mathcal{A}=\left\{\psi_{\mathrm{L}_{0}, \mathrm{~L}_{1}}: \mathrm{L}_{0} \in \mathcal{G}_{0}\left(\mathrm{~L}_{1}\right)\right\}
$$

é um atlas analítico real de $\mathrm{G}(\mathrm{H})$.

Prova. Basta usar as equações 1.6.7, 1.6.8, a proposição 1.6.21 e a fórmula

$$
\psi_{\mathrm{L}_{0}^{\prime}, L_{1}^{\prime}} \circ \psi_{L_{0}, L_{1}}^{-1}=\left(\psi_{L_{0}^{\prime} L_{1}^{\prime}} \circ \psi_{L_{0}^{\prime}, L_{1}}^{-1}\right) \circ\left(\psi_{L_{0}^{\prime}, L_{1}} \circ \psi_{L_{0}, L_{1}}^{-1}\right),
$$

válida se $\mathcal{G}_{0}\left(L_{1}\right) \cap \mathcal{G}_{0}\left(L_{1}^{\prime}\right) \neq \emptyset$.

Podemos fazer uma construção semelhante no caso de lagrangianos. De fato, suponhamos que $\mathrm{H}$ seja um espaço de Hilbert simplético e consideremos a grassmanniana de lagrangianos de $\mathrm{H}$, i.e., o conjunto $\Lambda$ formado por todos os subespaços lagrangianos de $H$. Já construimos na definição 1.5.25 as aplicações $\varphi_{L_{0}, L_{1}}$ que servirão de cartas locais em $\Lambda$. Comparando a definição 1.5.25 e a definição da aplicação $\psi_{\mathrm{L}_{0}, \mathrm{~L}_{1}}$ em 1.6.6, vemos que $\varphi_{\mathrm{L}_{0}, \mathrm{~L}_{1}}=\Psi_{\mathrm{L}_{0}, \mathrm{~L}_{1}}^{-1} \circ \psi_{\mathrm{L}_{0}, \mathrm{~L}_{1}}$, com $\Psi_{L_{0}, L_{1}}$ definido em 1.5.22. Portanto, as fórmulas 1.6 .7 e 1.6 .8 se reduzem a

$$
\varphi_{\mathrm{L}_{0}^{\prime}, \mathrm{L}_{1}} \circ \varphi_{\mathrm{L}_{0}, \mathrm{~L}_{1}}^{-1}(T)=\Psi_{\mathrm{L}_{0}^{\prime}, \mathrm{L}_{1}}^{-1}\left(P_{\mathrm{L}_{0}^{\prime} \mathrm{L}_{1}}^{\mathrm{L}_{0}}+\Psi_{\mathrm{L}_{0}, \mathrm{~L}_{1}} T\right) P_{\mathrm{L}_{1}, \mathrm{~L}_{0}}^{\mathrm{L}_{0}^{\prime}} \text {. }
$$

e

$$
\varphi_{\mathrm{L}_{0}, \mathrm{~L}_{1}^{\prime}} \circ \varphi_{\mathrm{L}_{0}, \mathrm{~L}_{1}}^{-1}(T)=\left(\Psi_{\mathrm{L}_{0}, L_{1}^{\prime}}^{-1} P_{\mathrm{L}_{0}, L_{1}^{\prime}}^{\mathrm{L}_{1}} \Psi_{\mathrm{L}_{0}, \mathrm{~L}_{1}}\right) T\left(I+P_{\mathrm{L}_{1}^{\prime}, L_{0}}^{\mathrm{L}_{1}} \Psi_{\mathrm{L}_{0}, \mathrm{~L}_{1}} T\right)^{-1},
$$

respectivamente, onde $T \in \mathscr{L}^{\text {sa }}\left(\mathrm{L}_{0}\right)$. Fazendo $T=0$ em 1.6.10, temos que $\Psi_{\mathrm{L}_{0}^{\prime}, L_{1}}^{-1} P_{\mathrm{L}_{0}^{\prime}, L_{1}}^{\mathrm{L}_{0}} P_{\mathrm{L}_{1}, L_{0}}^{\mathrm{L}_{0}^{\prime}}=$ $\varphi_{L_{0}^{\prime}, L_{1}}\left(L_{0}\right)$. Como $\Psi_{L_{0}^{\prime}, L_{1}}^{-1} \Psi_{L_{0}, L_{1}}=\left(P_{L_{1}, L_{0}}^{L^{\prime}}\right)^{*}$, a equação 1.6 .10 reduz-se a

$$
\varphi_{L_{0}^{\prime}, L_{1}} \circ \varphi_{L_{0}, L_{1}}^{-1}(T)=\varphi_{L_{L_{0}^{\prime}}^{\prime} L_{1}}\left(L_{0}\right)+\left(P_{L_{1}, L_{0}}^{L_{0}^{\prime}}{ }^{*} T P_{L_{1}, L_{0}}^{L_{0}^{\prime}}\right. \text {. }
$$

Além disso, $\Psi_{\mathrm{L}_{0}, \mathrm{~L}_{1}^{\prime}}^{-1} P_{\mathrm{L}_{0}, \mathrm{~L}_{1}^{\prime}}^{\mathrm{L}_{1}} \Psi_{\mathrm{L}_{0}, \mathrm{~L}_{1}}=I: \mathrm{L}_{0} \rightarrow \mathrm{L}_{0}$, portanto,

$$
\varphi_{\mathrm{L}_{0}, \mathrm{~L}_{1}^{\prime}} \circ \varphi_{\mathrm{L}_{0}, \mathrm{~L}_{1}}^{-1}(T)=T\left(I+P_{\mathrm{L}_{1}^{\prime}, L_{0}}^{\mathrm{L}_{1}} \Psi_{\mathrm{L}_{0}, \mathrm{~L}_{1}} T\right)^{-1} \text {. }
$$

Usando as equações 1.6 .12 e 1.6.13, o mesmo argumento do teorema 1.6.22 prova o seguinte teorema.

Teorema 1.6.23 O conjunto

$$
\mathcal{B}=\left\{\varphi_{\mathrm{L}_{0}, \mathrm{~L}_{1}}: \mathrm{L}_{0} \in \Lambda_{0}\left(\mathrm{~L}_{1}\right)\right\}
$$

é um atlas analítico real de $\Lambda$.

Podemos nos perguntar qual é a relação entre a topologia obtida em $\mathcal{G}(\mathrm{H})$ e $\Lambda$ através da estrutura diferenciável e a topologia dada pela métrica $\hat{\delta}$. $\mathrm{A}^{50}$ próxima proposição nos diz que, de fato, ambas as topologias coincidem.

\footnotetext{
${ }^{50} \mathrm{~A}$ observação 1.5.34 implica que $\hat{\delta}=\delta \mathrm{em} \Lambda$.
} 
Proposição 1.6.24 Os espaços $\mathrm{G}(\mathrm{H})$ e $\Lambda$ são espaços completos munidos da métrica $\hat{\delta}$. Além disso, a topologia herdada pela estrutura diferenciável coincide com a topologia dada por $\hat{\delta}$. Em particular, $\mathcal{G}(\mathrm{H})$ e $\Lambda$ são variedades de Banach analíticas reais munidas da estrutura diferenciável fornecida pelos atlas 1.6 .9 e 1.6.14, respectivamente.

Prova. Vamos provar a proposição para o espaço $\Lambda$; o caso $\mathcal{G}(H)$ é essencialmente o mesmo. A proposição 1.2.2 e a observação 1.5.34 implicam que $\delta=\delta$ é uma métrica em $\Lambda$.

Dados quaisquer $L_{0}, L_{1} \in \Lambda$ complementares , $\beta>0$ e $0<\alpha \leq 1$, definimos

$$
\mathcal{U}\left(\beta, \mathrm{L}_{0} ; \mathrm{L}_{1}\right)=\varphi_{\mathrm{L}_{0}, \mathrm{~L}_{1}}^{-1}\left(\left\{T \in \mathscr{L}^{\text {sa }}\left(\mathrm{L}_{0}\right):\|T\|<\beta\right\}\right), \mathcal{V}\left(\alpha, \mathrm{L}_{0}\right)=\left\{\mathrm{L} \in \Lambda: \delta\left(\mathrm{L}_{,} \mathrm{L}_{0}\right)<\alpha\right\} .
$$

Para provar que ambas as topologias coincidem, vamos provar que para cada $U\left(\beta, L_{0} ; L_{1}\right)$ existe $V\left(\alpha, L_{0}\right) \subset U\left(\beta, L_{0} ; L_{1}\right)$ e que cada $V\left(\alpha, L_{0}\right)$ é de fato aberto na topologia induzida pela estrutura diferenciável. Para tanto, fixemos $L_{0}, L_{1} \in \Lambda$ complementares. Dado $T \in \mathscr{L}^{\text {sa }}\left(L_{0}\right)$, pondo $L \doteq \varphi_{L_{0}, L_{1}}^{-1}(T)=G\left(\Psi_{L_{0}, L_{1}} T\right)$, temos pela observação 1.2.24 que

$$
\delta\left(\mathrm{L}_{,} \mathrm{L}_{0}\right)=\delta\left(\mathrm{G}\left(\Psi_{\mathrm{L}_{0}, \mathrm{~L}_{1}} T\right), \mathrm{G}(0)\right)=\sup _{\substack{u \in \mathrm{L}_{0} \\ u \neq 0}} \frac{\left\|\Psi_{\mathrm{L}_{0}, \mathrm{~L}_{1}} T u\right\|}{\left(\|u\|^{2}+\left\|\Psi_{\mathrm{L}_{0}, \mathrm{~L}_{1}} T u\right\|^{2}\right)^{1 / 2}}=\frac{\left\|\Psi_{\mathrm{L}_{0} \mathrm{~L}_{1}} T\right\|}{\left(1+\left\|\Psi_{\mathrm{L}_{0}, L_{1}} T\right\|^{2}\right)^{1 / 2}} .
$$

Logo,

$$
v\left(\alpha, \mathrm{L}_{0}\right)=\varphi_{\mathrm{L}_{0}, \mathrm{~L}_{1}}^{-1}\left(\left\{T \in \mathscr{L}^{\mathrm{sa}}\left(\mathrm{L}_{0}\right):\left\|\Psi_{\mathrm{L}_{0} \mathrm{~L}_{1}} T\right\|<\alpha /\left(1-\alpha^{2}\right)^{1 / 2}\right\}\right) .
$$

Como $\left\{T \in \mathscr{L}^{\text {sa }}\left(L_{0}\right):\left\|\Psi_{L_{0}, L_{1}} T\right\|<\alpha /\left(1-\alpha^{2}\right)^{1 / 2}\right\}$ é aberto em $\mathscr{L}^{\text {sa }}\left(L_{0}\right)$, segue que $\mathcal{V}\left(\alpha, L_{0}\right)$ é aberto na topologia induzida pela estrutura diferenciável. ${ }^{51}$

Por outro lado, dado $\beta>0$, tomando $0<\alpha<\beta /\left(\delta\left(L_{0}, L_{1}\right)^{2}+\beta^{2}\right)^{1 / 2}$, se $T \in \mathscr{L}^{\text {sa }}\left(L_{0}\right)$ e $\left\|\Psi_{L_{0}, L_{1}} T\right\|<\alpha /\left(1-\alpha^{2}\right)^{1 / 2}$, então

$$
\|T\|=\left\|\Psi_{L_{0}, L_{1}}^{-1} \circ \Psi_{L_{0}, L_{1}} T\right\| \leq\left\|\Psi_{L_{0}, L_{1}}^{-1}\right\| \frac{\alpha}{\left(1-\alpha^{2}\right)^{1 / 2}}<\beta,
$$

pela observação 1.5.24. Logo, pela equação 1.6.15, segue que $U\left(\beta, L_{0} ; L_{1}\right) \supset \mathcal{V}\left(\alpha, L_{0}\right)$, provando o queremos. Em particular, $\mathcal{G}(\mathrm{H})$ e $\Lambda$ são Hausdorff, e portanto, são variedades de Banach analíticas reais.

Vamos estudar agora algumas ações dos grupos lineares usuais sobre as grassmannianas total e de lagrangianos. Primeiramente, provemos a regularidade da ação natural

$$
\begin{aligned}
\mathrm{GL}(\mathrm{H}) \times \mathrm{G}(\mathrm{H}) & \rightarrow \mathcal{G}(\mathrm{H}) \\
(A, \mathrm{~L}) & \mapsto A(\mathrm{~L}) .
\end{aligned}
$$

Fixemos $A \in \mathrm{GL}(\mathrm{H}), \mathrm{L}_{0} \in \mathcal{G}(\mathrm{H})$ e tomemos $\mathrm{L}_{1} \in \mathcal{G}(\mathrm{H})$ tal que $\mathrm{L}_{0}, A\left(\mathrm{~L}_{0}\right) \in \mathrm{G}_{0}\left(\mathrm{~L}_{1}\right)$. Dado qualquer $\mathrm{L} \in \mathcal{G}_{0}\left(\mathrm{~L}_{1}\right)$, existe $S: \mathrm{L}_{0} \rightarrow \mathrm{L}_{1}$ tal que $\mathrm{G}(S)=\mathrm{L}$. Dizer que $A\left(\mathrm{~L}_{0}\right)$ e $\mathrm{L}_{1}$ são complementares é o mesmo que dizer que $P_{\mathrm{L}_{1}, \mathrm{~L}_{0}} A: \mathrm{L}_{0} \rightarrow \mathrm{L}_{1}$ é um isomorfismo (veja a proposição 1.5.17). Como o conjunto dos isomorfismos $\mathrm{L}_{0} \rightarrow \mathrm{L}_{1}$ é aberto, segue que se $B \in \mathrm{GL}(\mathrm{H})$ é suficientemente próximo de $A$ e se $T \in \mathscr{L}\left(L_{0}, L_{1}\right)$ tem norma suficientemente pequena, então o operador

\footnotetext{
${ }^{51}$ Para $\alpha=1$, temos $\mathcal{V}\left(\alpha, L_{0}\right)=\Lambda_{0}\left(L_{1}\right)$
} 
$P_{\mathrm{L}_{1}, L_{0}} B(I+T): \mathrm{L}_{0} \rightarrow \mathrm{L}_{1}$ é um isomorfismo. Logo

$$
\begin{aligned}
B(\mathrm{~L}) & =\left\{B(u+T u): u \in \mathrm{L}_{0}\right\} \\
& =\left\{P_{\mathrm{L}_{1}, \mathrm{~L}_{0}} B(I+T) u+P_{\mathrm{L}_{0}, \mathrm{~L}_{1}} B(I+T) u: u \in \mathrm{L}_{0}\right\} \\
& =\left\{u+P_{\mathrm{L}_{0}, \mathrm{~L}_{1}} B(I+T)\left(P_{\mathrm{L}_{1}, \mathrm{~L}_{0}} B(I+T)\right)^{-1} u: u \in \mathrm{L}_{0}\right\} \\
& =\mathrm{G}\left(P_{\mathrm{L}_{0}, \mathrm{~L}_{1}} B(I+T)\left(P_{\mathrm{L}_{1}, \mathrm{~L}_{0}} B(I+T)\right)^{-1}\right) .
\end{aligned}
$$

Logo,

$$
\begin{aligned}
\psi_{\mathrm{L}_{0}, \mathrm{~L}_{1}}\left(B\left(\psi_{\mathrm{L}_{0}, \mathrm{~L}_{1}}^{-1}(T)\right)\right) & =\psi_{\mathrm{L}_{0}, \mathrm{~L}_{1}}(B(\mathrm{~L})) \\
& =P_{\mathrm{L}_{0}, \mathrm{~L}_{1}} B(I+T)\left(P_{\mathrm{L}_{1}, \mathrm{~L}_{0}} B(I+T)\right)^{-1} .
\end{aligned}
$$

A equação 1.6 .17 e a proposição 1.6 .21 mostram que se $A, B \in \mathrm{GL}(\mathrm{H})$ são suficientemente próximos e $T \in \mathscr{L}\left(L_{0}, L_{1}\right)$ tem norma suficientemente pequena, então a aplicação

$$
\begin{aligned}
\mathrm{GL}(\mathrm{H}) \times \mathscr{L}\left(\mathrm{L}_{0}, \mathrm{~L}_{1}\right) & \rightarrow \mathscr{L}\left(\mathrm{L}_{0}, \mathrm{~L}_{1}\right) \\
T & \mapsto \psi_{\mathrm{L}_{0}, \mathrm{~L}_{1}}\left(B\left(\varphi_{\mathrm{L}_{0}, \mathrm{~L}_{1}}^{-1}(T)\right)\right)
\end{aligned}
$$

é analítica real.

No caso simplético, podemos usar o mesmo raciocínio para provar que a ação natural

$$
\begin{aligned}
\mathrm{Sp}(\mathrm{H}) \times \Lambda & \rightarrow \Lambda \\
(A, \mathrm{~L}) & \mapsto A(\mathrm{~L}) .
\end{aligned}
$$

é analítica real. De fato, mantendo a mesma notação do caso anterior com as alterações óbvias, e tomando $L_{0}, L_{1}$ lagrangianos, temos que a aplicação correspondente

$$
\begin{aligned}
\varphi_{\mathrm{L}_{0}, \mathrm{~L}_{1}}\left(B\left(\varphi_{\mathrm{L}_{0}, \mathrm{~L}_{1}}^{-1}(T)\right)\right) & =\varphi_{\mathrm{L}_{0}, \mathrm{~L}_{1}}(B(\mathrm{~L})) \\
& =\Psi_{\mathrm{L}_{0}, \mathrm{~L}_{1}}^{-1} P_{\mathrm{L}_{0}, \mathrm{~L}_{1}} B\left(I+\Psi_{\mathrm{L}_{0}, \mathrm{~L}_{1}} T\right)\left(P_{\mathrm{L}_{1}, \mathrm{~L}_{0}} B\left(I+\Psi_{\mathrm{L}_{0}, \mathrm{~L}_{1}} T\right)\right)^{-1},
\end{aligned}
$$

definida para $T \in \mathscr{L}^{\text {sa }}\left(L_{0}\right)$ com norma suficientemente pequena, é analítica real. Em particular, a próxima proposição está provada.

Proposição 1.6.25 As ações 1.6.16 e 1.6 .18 são analíticas reais.

Consideremos agora a ação 1.6.18. Fixado $L_{0} \in \Lambda$, é fácil ver que a álgebra de Lie correspondente ao subgrupo de Lie fechado $\mathrm{Sp}\left(\mathrm{H}, \mathrm{L}_{0}\right) \doteq\left\{A \in \mathrm{Sp}(\mathrm{H}): A\left(\mathrm{~L}_{0}\right)=\mathrm{L}_{0}\right\}$ é a subálgebra

$$
\mathfrak{s p}\left(H, L_{0}\right)=\left\{X \in \mathfrak{p} p(H): X\left(L_{0}\right) \subset L_{0}\right\}
$$

de $\mathfrak{s p}(H)$. De acordo com a observação 1.6.12, vemos que $\mathfrak{s p}\left(H, L_{0}\right)$ é formada por elementos da forma 1.6.3 $\operatorname{com} C=0$. Portanto o subespaço dos operadores da forma $\left(\begin{array}{ll}0 & 0 \\ c & 0\end{array}\right), \operatorname{com} C \in \mathscr{L}\left(L_{0}, L_{0}^{\frac{1}{0}}\right)$ tal que $J C \in \mathscr{L}^{\text {sa }}\left(\mathrm{L}_{0}\right)$ é um complementar (fechado) para $\mathfrak{s p}\left(\mathrm{H}, \mathrm{L}_{0}\right)$. Mais precisamente, o subespaço $\left\{X \in \mathfrak{s p}(H): X\left(L_{0}\right) \subset L_{0}^{\perp}\right.$ e $\left.\left.X\right|_{L_{0}^{\perp}}=0\right\}$ é um complementar para $s p\left(H, L_{0}\right)$ em $\mathfrak{s p}(H)$. Este fato, juntamente com o teorema 1.6.15 implicam o seguinte.

Teorema 1.6.26 Para cada $L_{0} \in \Lambda$ fixo, a aplicação $\beta_{L_{0}}: S p(H) \rightarrow \Lambda$ definida no teorema 1.6 .15 com respeito d̀ ação 1.6 .18 torna $\mathrm{Sp}(\mathrm{H})$ um fibrado principal $\mathrm{C}^{\omega}$ sobre $\Lambda$ com grupo estrutural $\mathrm{Sp}\left(\mathrm{H}, \mathrm{L}_{0}\right)$. 
Vamos utilizar o teorema 1.6.26 para estudar a topologia de $\mathrm{Sp}(\mathrm{H})$. Dado $T \in \mathrm{GL}(\mathrm{H})$, escrevendo $T=\left(\begin{array}{l}A \\ C\end{array}\right)$ com respeito à decomposição $\mathrm{H}=\mathrm{L}_{0} \oplus \mathrm{L}_{0}^{\perp}$, vimos na observação 1.5.16 que $T \in \mathrm{Sp}(\mathrm{H})$ se e só se $D^{*} J A+B^{*} J C=J \mathrm{~L}_{0}, A^{*} J C \in \mathscr{L}^{\text {sa }}\left(\mathrm{L}_{0}\right)$ e $B^{*} J D \in \mathscr{L}^{\text {sa }}\left(\mathrm{L}_{0}^{\frac{1}{1}}\right)$. Se $T \in \mathrm{Sp}\left(\mathrm{H}, \mathrm{L}_{0}\right)$, então $C=0$, portanto, $A \in \mathrm{GL}\left(\mathrm{L}_{0}\right), D=-J\left(A^{-1}\right)^{*} J$ e $B=A J S$, com $S \in \mathscr{L}^{\text {sa }}\left(\mathrm{L}_{0}^{+}\right)$, logo, $T$ tem a forma

$$
\left(\begin{array}{cc}
A & A J S \\
0 & -J\left(A^{-1}\right)^{*} J
\end{array}\right)
$$

Isso nos permite definir

$$
\begin{aligned}
\Phi: \mathrm{Sp}\left(\mathrm{H}, \mathrm{L}_{0}\right) & \rightarrow \mathrm{GL}\left(\mathrm{L}_{0}\right) \times \mathscr{L}^{\mathrm{sa}}\left(\mathrm{L}_{0}^{\perp}\right) \\
T & \mapsto(A, S) .
\end{aligned}
$$

Como $S=-J A^{-1} B$, temos que $\Phi$ é analítica real, e isso prova a seguinte proposição.

Proposição 1.6.27 $\Phi$ é um difeomorfismo $C^{\omega}$ entre $\mathrm{Sp}\left(\mathrm{H}, \mathrm{L}_{0}\right)$ e $\mathrm{GL}\left(\mathrm{L}_{0}\right) \times \mathscr{L}^{\mathrm{sa}}\left(\mathrm{L}_{0}^{+}\right)$. Em particular, $\mathrm{Sp}\left(\mathrm{H}, \mathrm{L}_{0}\right)$ é contrátil.

Vamos agora estudar outras variedades grassmannianas importantes. Definimos $F p(H) \subset$ $\mathcal{G}(\mathrm{H}) \times \mathcal{G}(\mathrm{H})$, o espaço de pares Fredholm de $\mathrm{H}$, como o conjunto de todos os pares Fredholm de subespaços de $\mathrm{H}$. Dado $\mathrm{M} \in \mathcal{G}(\mathrm{H})$, definimos a grassmanniana Fredholm de $\mathrm{H}$ com respeito a $\mathrm{M}$ como o conjunto dos $N \in \mathcal{G}(H)$ tais que $(M, N) \in F p(H)$; denotamos tal conjunto por $\mathscr{F}_{M}(H)$. A próxima proposição decorre do teorema 1.3.22.

Proposição 1.6.28 $\mathrm{Fp}(\mathrm{H}) \subset \mathcal{G}(\mathrm{H}) \times \mathcal{G}(\mathrm{H}) e \mathscr{F}_{\mathrm{M}}(\mathrm{H}) \subset \mathcal{G}(\mathrm{H})$ são abertos.

Em particular, $F p(H)$ e $\mathscr{F}_{M}(H)$ admitem estrutura de variedade de Banach analítica real, pelo teorema 1.6.22. Temos também uma ação natural de $G L(H)$ sobre $F p(H)$, dada por

$$
\begin{aligned}
\mathrm{GL}(\mathrm{H}) \times \mathrm{Fp}(\mathrm{H}) & \rightarrow \mathrm{Fp}(\mathrm{H}) \\
T,(\mathrm{M}, \mathrm{N}) & \mapsto(T(\mathrm{M}), T(\mathrm{~N})) .
\end{aligned}
$$

Pela proposição 1.6.25, a aplicação 1.6.20 é uma ação $C^{\omega}$ do grupo $G L(H)$ sobre $F p(H)$.

Podemos também considerar ainda a grassmanniana essencial $\mathcal{G}_{e}(\mathrm{H})$, a qual é definida como o quociente de $\mathcal{G}(\mathrm{H})$ pela relação de equivalência definida em 1.3.53, munido da topologia quociente. Dado qualquer subespaço $L_{0} \in \mathcal{G}(\mathrm{H})$, definimos a grassmanniana de subespaços comensuráveis com $L_{0}$ como o conjunto $\mathcal{G}_{c}\left(L_{0} ; H\right)$ formado por todos os subespaços fechados de $H$ que são perturbações compactas de $L_{0}$. Consideremos a aplicação $\psi_{L_{0}, L_{0}^{+}}$definida em 1.6.6; como $P_{\mathrm{L}_{0}}^{\mathrm{L}}=\left.\left[I-\left(P_{\mathrm{L}}-P_{\mathrm{L}_{0}}\right)\left(P_{\mathrm{L}}-P_{\mathrm{L}^{\perp}}\right)\right]\right|_{\mathrm{L}}$, temos que

$$
\left.\eta_{\mathrm{L}_{0}, \mathrm{~L}_{0}^{\perp}} \doteq \psi_{\mathrm{L}_{0}, \mathrm{~L}_{0}^{1}}\right|_{W\left(\mathrm{~L}_{0}\right)}: W\left(\mathrm{~L}_{0}\right) \rightarrow P_{\mathrm{L}_{0}}+\mathscr{L}_{c}\left(\mathrm{~L}_{0}, \mathrm{~L}_{0}^{\perp}\right),
$$

onde $W\left(L_{0}\right) \doteq\left\{L \in \mathcal{G}_{c}\left(L_{0} ; H\right): \hat{\delta}\left(L_{,} L_{0}\right)<1\right\}$ é uma bijeção (bem-definida). Logo, podemos introduzir em $\mathcal{G}_{c}\left(\mathrm{~L}_{0} ; \mathrm{H}\right)$ uma estrutura de variedade de Banach analítica real modelada em $\mathscr{L}_{c}\left(\mathrm{~L}_{0}, \mathrm{~L}_{0}^{\frac{1}{0}}\right)$, de acordo com as equações 1.6 .7 e 1.6.8. Como já comentamos na observação 1.6.18, $\mathcal{G}_{c}\left(\mathrm{~L}_{0} ; \mathrm{H}\right)$ munido desta estrutura é apenas uma subvariedade $\mathrm{C}^{0}$ de $\mathcal{G}(\mathrm{H})$. Estas observações provam a seguinte proposição.

Proposição 1.6.29 O espaço $\mathcal{G}_{c}\left(\mathrm{~L}_{0} ; \mathrm{H}\right)$ admite estrutura de variedade de Banach analítica real modelada no espaço $\mathscr{L}_{c}\left(\mathrm{~L}_{0}, \mathrm{~L}_{0}^{+}\right)$. Munido desta estrutura, $\mathcal{G}_{c}\left(\mathrm{~L}_{0} ; \mathrm{H}\right)$ é uma subvariedade $\mathrm{C}^{0}$ de $\mathrm{G}(\mathrm{H})$. 
Podemos considerar a ação natural

$$
\begin{aligned}
\mathrm{GL}_{c}(\mathrm{H}) \times \mathrm{G}_{c}\left(\mathrm{~L}_{0} ; \mathrm{H}\right) & \rightarrow \mathcal{G}_{c}\left(\mathrm{~L}_{0} ; \mathrm{H}\right) \\
(A, \mathrm{~L}) & \mapsto A(\mathrm{~L}) .
\end{aligned}
$$

O corolário 1.3.67 implica que, de fato, $A(\mathrm{~L}) \in \mathcal{G}_{c}\left(\mathrm{~L}_{0} ; \mathrm{H}\right)$ se $A \in \mathrm{GL}_{c}(\mathrm{H})$ e $\mathrm{L} \in \mathcal{G}_{c}\left(\mathrm{~L}_{0} ; \mathrm{H}\right)$, portanto, a ação 1.6.21 é bem-definida. Argumentos inteiramente análogos àqueles da proposição 1.6.25 provam o seguinte resultado.

Proposição 1.6.30 A ação 1.6.21 é analítica real.

Passemos ao caso em que $H$ é um espaço simplético. Definimos $F p(\Lambda) \subset \Lambda \times \Lambda$ como o conjunto de todos os pares Fredholm de lagrangianos de $\mathrm{H}$; o espaço de pares comensuráveis de lagrangianos, Comp $(\Lambda)$, é definido de maneira análoga. Dado $L \in \Lambda$, definimos a grassmanniana de lagrangianos Fredholm com respeito a $L$ como o conjunto $\mathscr{F}_{L}(\Lambda) \subset \Lambda$ dos lagrangianos $L^{\prime}$ tais que $\left(L, L^{\prime}\right) \in F p(\Lambda)$. Define-se a grassmanniana de lagrangianos comensuráveis com $L$ como o conjunto de todos os lagrangianos comensuráveis com $L$ e denotamos tal conjunto por $\mathcal{G}_{c}(L ; \Lambda)$.

A proposição 1.6 .28 admite uma correspondente no caso simplético.

Proposição 1.6.31 $\mathrm{Fp}(\Lambda) \subset \Lambda \times \Lambda$ e $\mathscr{F}_{\mathrm{L}}(\Lambda) \subset \Lambda$ são abertos.

Temos também uma ação natural

$$
\begin{aligned}
\mathrm{U}_{c}(\mathrm{H} ; J) \times \mathcal{G}_{c}\left(\mathrm{~L}_{0} ; \Lambda\right) & \rightarrow \mathcal{S}_{c}\left(\mathrm{~L}_{0} ; \Lambda\right) \\
(A, \mathrm{~L}) & \mapsto A(\mathrm{~L}),
\end{aligned}
$$

a qual é analítica real, pelos mesmos motivos que a ação 1.6.21 o é, provando a proposição seguinte.

Proposição 1.6.32 A ação 1.6.22 é analítica real.

\subsubsection{Topologia}

Nesta subseção, vamos estudar o tipo de homotopia e outras propriedades topológicas de algumas das variedades grassmannianas definidas na subseção 1.6.2. Para algumas situações, será de fundamental importância a presença da estrutura diferenciável, por exemplo, na construção de fibrações utilizando o teorema 1.6.15.

Comecemos com a grassmanniana total $\mathcal{G}(\mathrm{H})$. Dados $n, k \in \mathbb{N} \cup\{\infty\} \operatorname{com} n+k=\infty$, definimos

$$
\mathcal{S}_{n, k}(\mathrm{H}) \doteq\{\mathrm{M} \in \mathcal{G}(\mathrm{H}): \operatorname{dim} \mathrm{M}=n, \operatorname{codim} \mathrm{M}=k\} .
$$

Usando o fato que GL(H) é conexo por caminhos (que decorre, em particular, do teorema de Kuiper), concluímos que os $\mathcal{G}_{n, k}(H)$ são, de fato, as componentes conexas de $\mathcal{G}(H)$. Obviamente, a aplicação $M \mapsto M^{\perp}$ é um difeomeorfismo que leva $\mathcal{G}_{n, k}(H)$ em $\mathcal{G}_{k, n}(H)$. Pelo teorema 1.6.25, a ação natural

$$
\begin{aligned}
\mathrm{GL}(\mathrm{H}) \times \mathcal{G}_{n, k}(\mathrm{H}) & \rightarrow \mathcal{G}_{n, k}(\mathrm{H}) \\
(A, \mathrm{~L}) & \mapsto A(\mathrm{~L}) .
\end{aligned}
$$


é analítica real e transitiva para quaisquer $n, k \in \mathbb{N} \cup\{\infty\}$. Pelo teorema 1.6.15, dado $M \in \mathcal{G}_{n, k}$, a aplicação $\beta_{M}: G L(H) \ni U \mapsto U(M) \in \mathcal{G}_{n, k}(H)$ define um fibrado principal com grupo estrutural $\left\{U \in G L(H): U(M)=M, U\left(M^{\perp}\right)=M^{\perp}\right\} \approx G L(M) \times G L\left(M^{\perp}\right)$. Usando o teorema de Kuiper e a seqüência exata da fibração, concluímos o seguinte resultado.

Proposição 1.6.33 As componentes conexas de $\mathcal{G}(\mathrm{H})$ são os conjuntos $\mathcal{G}_{n, k}(\mathrm{H})$ definidos em 1.6.23. Além disso, $\pi_{i}\left(\mathcal{G}_{n, \infty}(\mathrm{H})\right)=\pi_{i-1}(\mathrm{GL}(n))$ se $i \geq 1, n<\infty$ e $\pi_{i}\left(\mathcal{G}_{\infty, \infty}(\mathrm{H})\right)=0$. Em particular, $\mathcal{G}_{\infty, \infty}(\mathrm{H})$ é contrátil.

O caso da grassmanniana de lagrangianos é ainda mais simples. Fixado $L_{0} \in \Lambda$, pelas proposições 1.6.25, 1.5.12, a aplicação $\beta_{L_{0}}: U(H ; J) \ni T \mapsto T\left(L_{0}\right) \in \Lambda$ define um fibrado principal com grupo estrutural $\mathrm{O}\left(\mathrm{L}_{0}\right)$, pela observação 1.5.14. Pela seqüência exata da fibração e pelos corolários 1.6 .7 e 1.6.8, provamos, como antes, a proposição a seguir.

Proposição 1.6.34 $\Lambda$ é contrátil.

Corolário 1.6.35 $\mathrm{Sp}(\mathrm{H})$ é contrátil.

Prova. Basta usar o teorema de Kuiper, o teorema 1.6.26, as proposições 1.6 .27 e 1.6.34 e a seqüência exata da fibração.

OвSERVAÇÃo 1.6.36 O fibrado principal $\left.\beta_{\mathrm{L}_{0}}\right|_{\mathrm{U}(\mathrm{H} ;)}: \mathrm{U}(\mathrm{H} ; J) \rightarrow \Lambda$ com grupo estrutural $\mathrm{O}\left(\mathrm{L}_{0}\right)$ utilizado no corolário 1.6.35 permite-nos considerar $\Lambda$ como o espaço homogêneo $\mathrm{U}(\mathrm{H} ; J) / \mathrm{O}\left(\mathrm{L}_{0}\right)$. Nesse sentido, a grassmanniana de lagrangianos comporta-se como em dimensão finita, 52 conforme [76], Prop. (2.5.11).

O tipo de homotopia das grassmannianas de perturbações compactas e essencial pode ser facilmente calculado, mas não faremos os cálculos aqui, apenas enunciamos as seguintes proposições, cuja prova pode ser encontrada em [2].

Proposição 1.6.37 Se $\mathrm{M} \in \mathcal{G}_{\infty, \infty}(\mathrm{H})$, as componentes conexas de $\mathcal{G}_{c}(\mathrm{M} ; \mathrm{H})$ são os conjuntos da forma

$$
\mathcal{G}_{c}^{n}(\mathrm{M} ; \mathrm{H}) \doteq\left\{\mathrm{N} \in \mathcal{G}_{c}(\mathrm{M} ; \mathrm{H}) ; \operatorname{dim}(\mathrm{N}, \mathrm{M})=n\right\},
$$

com $n \in \mathbb{Z}$. Além disso, tais componentes são duas a duas homeomorfas e $\pi_{i}\left(\mathcal{G}_{c}^{n}(M ; H)\right)=\pi_{i-1}(G L(\infty))$, $i \geq 1$. . $^{53}$

Proposição 1.6.38 O espaço $\mathcal{G}_{e}(\mathrm{H})$ é formado por dois pontos isolados (os quais são imagem pela projeção canônica $\pi: \mathcal{G}(\mathrm{H}) \rightarrow \mathcal{S}_{e}(\mathrm{H})$ do conjunto dos subespaços de dimensão e codimensão finita, respectivamente) e por um conjunto conexo $\mathcal{S}_{e}^{*}(\mathrm{H})$, o qual é imagem de $\mathcal{G}_{\infty, \infty}(\mathrm{H})$ por $\pi$. Temos que $\mathcal{G}_{e}^{*}(\mathrm{H})$ tem grupo fundamental $\mathbb{Z} e \pi_{i}\left(\mathcal{G}_{e}^{*}(\mathrm{H})\right)=\pi_{i-2}(\mathrm{GL}(\infty)), i \geq 2$.

Passemos agora ao caso de pares Fredholm. Como ind : $\mathrm{Fp}(\mathrm{H}) \rightarrow \mathbb{Z}$ é contínua (pela proposição 1.3.22), temos que os conjuntos $\mathcal{G}_{m, \infty}(\mathrm{H}) \times \mathcal{G}_{\infty, n}(\mathrm{H}), \mathcal{G}_{\infty, m}(\mathrm{H}) \times \mathcal{G}_{n, \infty}(\mathrm{H}) \operatorname{com} m, n \in \mathbb{N}$ e $\mathrm{Fp}_{k}(\mathrm{H}) \doteq\left\{(\mathrm{M}, \mathrm{N}) \in \mathrm{Fp}(\mathrm{H}):\right.$ ind $(\mathrm{M}, \mathrm{N})=k$ e $\left.\mathrm{M}, \mathrm{N} \in \mathcal{G}_{\infty, \infty}(\mathrm{H})\right\}$, com $k \in \mathbb{N}$, são abertos e fechados em $F p(H)$. De fato, tais conjuntos são as componentes conexas de $F p(H)$. O tipo de homotopia das duas primeiras famílias pode ser facilmente calculado usando a proposição

\footnotetext{
${ }^{52} \mathrm{~A}$ grande diferença neste caso é que os grupos envolvidos são contráteis.

${ }^{53} \mathrm{Ou}$ seja, $\mathfrak{S}_{c}^{n}(\mathrm{M} ; \mathrm{H})$ tem o tipo de homotopia de BGL, o espaço classificante do grupo GL( $\left.\infty\right)$; veja [62].
} 
1.6.33. Cada $\mathrm{Fp}_{k}(\mathrm{H})$ tem o tipo de homotopia de $\mathcal{G}_{c}^{0}(\mathrm{M} ; \mathrm{H})$; este fato é provado em [2], usando as proposições 1.6 .37 e 1.6.38.

Estudemos o caso simplético. Seja $p: \mathrm{Fp}(\Lambda) \rightarrow \Lambda$ dada por $p\left(\mathrm{~L}, \mathrm{~L}^{\prime}\right)=\mathrm{L}$. Claramente, $p$ é analítica real.

Proposição 1.6 .39 p é uma fibração (no sentido de Serre). Em $m^{54}$ particular, vale a seqüência exata da fibração para $p$.

Prova. Basta observar que $[0,1]^{n}$ é compacto e cada $\Lambda_{0}(\mathrm{~L})$ é um aberto difeomorfo a um espaço de Banach, em particular, contrátil.

CoRolárIo 1.6.40 Dado $L \in \Lambda$, existe equivalencia homotópica entre $\mathscr{F}_{\mathrm{L}}(\Lambda)$ e $\mathrm{Fp}(\Lambda)$.

Prova. Usando a proposição 1.6.39, a seqüência exata da fibração e o teorema 1.6.34, obtemos uma equivalência homotópica fraca entre $\mathscr{F}_{L}(\Lambda)$ e $F p(\Lambda)$. Por [73], teorema 15 , obtemos a equivalência homotópica procurada.

No caso de pares comensuráveis de lagrangianos, as proposições 1.6 .32 e 1.5 .15 mostram que a ação

$$
\begin{aligned}
\mathrm{U}_{c}\left(\mathrm{H}_{;} J\right) \times \mathcal{G}_{c}\left(\mathrm{~L}_{0} ; \Lambda\right) & \rightarrow \mathcal{G}_{c}\left(\mathrm{~L}_{0} ; \Lambda\right) \\
(T, \mathrm{~L}) & \mapsto T(\mathrm{~L})
\end{aligned}
$$

é analítica real e transitiva. O grupo de isotropia em $L_{0}$ pode ser identificado, como na

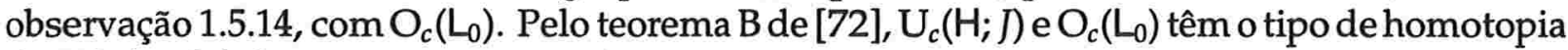
de $\mathrm{U}(\infty)$ e $\mathrm{O}(\infty)$, respectivamente. $\mathrm{O}$ teorema 1.6 .15 prova o seguinte.

Teorema 1.6.41 Existe um difeomorfismo entre $\mathcal{G}_{c}\left(\mathrm{~L}_{0}, \Lambda\right)$ e $\mathrm{U}(\infty) / \mathrm{O}(\infty)$.

OBSERVAÇÃo 1.6.42 O teorema 1.6.41 está enunciado incorretamente em [69], Pág. 489-492. Aí, não se faz restrição nenhuma à perturbações compactas. O mesmo erro repete-se em [22]. Apesar disso, o resultado é verdadeiro, e a prova será dada no teorema 1.6.46.

Observação 1.6.43 O teorema 1.6.41 foi originalmente demonstrado em [97], (I), Pág. 194, apesar de que no referido artigo, não se faz menção sobre a transitividade da ação do grupo unitário. Aliás, esta é a primeira vez em que é introduzido o espaço $\mathcal{G}_{c}\left(L_{0} ; \Lambda\right)$. Neste artigo, prova-se que $\mathcal{G}_{c}\left(L_{0} ; \Lambda\right)$ é conexo por caminhos (o que decorre, por sua vez do teorema 1.6.41), que $\pi_{1}\left(\mathcal{G}_{c}\left(L_{0} ; H\right)\right)=\mathbb{Z}$ (este fato decorre de $\pi_{1}\left(\mathcal{G}_{c}\left(L_{0} ; \Lambda\right)\right)=\pi_{1}\left(U_{\mathbb{R}}(n) / \mathrm{O}(n)\right)=\mathbb{Z}, n>1$; veja [76], Pág. 89) e $H_{1}\left(\mathcal{G}_{c}\left(L_{0} ; \Lambda\right) ; \mathbb{Z}\right)=H^{1}\left(\mathcal{G}_{c}\left(L_{0} ; \Lambda\right) ; \mathbb{Z}\right)=\mathbb{Z}$ (pelo teorema de Hurewicz; veja [93]). Isso nos permite definir o índice de Maslov para curvas em $\mathcal{G}_{c}\left(L_{0} ; \Lambda\right)$ exatamente como em dimensão finita, conforme [6] ou [76]; este é o ponto de vista adotado em [97].

Vamos agora mostrar que $\mathscr{F}_{L_{0}}(\Lambda)$ e $\mathcal{G}_{c}\left(L_{0} ; \Lambda\right)$ têm o mesmo tipo de homotopia. Para isso, será necessário introduzir a grassmanniana de lagrangianos essencial.

\footnotetext{
${ }^{54}$ Uma fibração de Serre é uma aplicação $p: E \rightarrow B$ tal que, dada qualquer homotopia $f_{t}:[0,1]^{n} \rightarrow B$ e $\tilde{f}_{0}:[0,1]^{n} \rightarrow E$ tal que $p \tilde{f}_{0}=f_{0}$, então existe uma homotopia $\tilde{f}_{t}:[0,1]^{n} \rightarrow E$ tal que $p \tilde{f}_{t}=f_{t}$ e $\tilde{f}_{t}$ coincide com $\tilde{f}_{0}$ em $t=0$, i.e., $p$ tem a propriedade de levantamento com respeito ao espaço $[0,1]^{n}$, para todo $n \in \mathbb{N}$. A seqüência exata da fibração é verificada mesmo para este tipo aparentemente mais fraco de fibrações. Veja [49], Pág. 373.
} 
Consideremos sobre a grassmanniana de lagrangianos $\Lambda$ a relação de equivalência $\sim$ dada por $L \sim L^{\prime}$ se e só se Lé perturbação compacta de L'. O quociente de $\Lambda$ por esta relação (munido da topologia quociente) é chamado de grassmanniana de lagrangianos essencial, e denotado por $\Lambda_{e}$.

Teorema 1.6.44 A aplicação natural $\pi: \Lambda \rightarrow \Lambda_{e}$ define um fibrado com fibra típica $\mathcal{S}_{c}\left(L_{0} ; \Lambda\right)$.

Prova. Fixado $L_{0} \in \Lambda$, podemos identificar $L_{0}$ com a projeção $P_{L_{0}}$. Consideremos a álgebra de Calkin $\mathrm{e} \doteq \mathscr{L}(\mathrm{H}) / \mathscr{L}_{c}(\mathrm{H})$ e a imagem $\left[P_{\mathrm{L}_{0}}\right]$ de $P_{\mathrm{L}_{0}}$ pela projeção canônica $p: \mathscr{L}(\mathrm{H}) \rightarrow \mathcal{C}$. Denotando por $\mathrm{U}(\mathcal{C})$ o conjunto dos elementos unitários de $\mathrm{C}$, $\mathrm{a}^{55}$ proposição 1.2 de [2] implica que a aplicação

$$
\begin{aligned}
\mathrm{U}(\mathrm{C}) & \rightarrow \mathrm{U}(\mathrm{C}) \cdot\left[P_{\mathrm{L}_{0}}\right] \\
u & \mapsto u\left[P_{L_{0}}\right] u^{-1}
\end{aligned}
$$

é um fibrado principal $C^{\omega}$ com grupo estrutural $\left\{u \in \mathrm{U}(\mathcal{C}): u\left[P_{L_{0}}\right] u^{-1}=\left[P_{L_{0}}\right]\right\}$. Restringindo o grupo estrutural ao subgrupo fechado

$$
p\left(\mathrm{O}\left(\mathrm{L}_{0}\right)\right) \doteq p\left(\left\{U \in \mathrm{U}(\mathrm{H} ; J): U J=J U \text { e } U\left(\mathrm{~L}_{0}\right)=\mathrm{L}_{0}\right\}\right)
$$

obtemos um fibrado principal

$$
\begin{aligned}
p(\mathrm{U}(\mathrm{H} ; J)) & \rightarrow p(\mathrm{U}(\mathrm{H} ; J)) \cdot\left[P_{\mathrm{L}_{0}}\right] \\
u & \mapsto u\left[P_{L_{0}}\right] u^{-1}
\end{aligned}
$$

com grupo estrutural $p\left(\mathrm{O}\left(\mathrm{L}_{0}\right)\right)$. Podemos reescrever a aplicação 1.6.25 como

$$
\begin{aligned}
p(\mathrm{U}(\mathrm{H} ; J)) & \rightarrow p(\mathrm{U}(\mathrm{H} ; J)) \cdot\left[P_{L_{0}}\right] \\
u & \mapsto\left[P_{u\left(L_{0}\right)}\right]
\end{aligned}
$$

onde $U \in \mathrm{U}(\mathrm{H} ; J)$ é tal que $p(U)=u$. Como o espaço $p(\mathrm{U}(\mathrm{H} ; J)) \cdot\left[P_{L_{0}}\right]$ identifica-se com $\Lambda_{e}$, vemos que existe uma vizinhança aberta $\mathcal{U}$ de $\left[L_{0}\right]$ em $\Lambda_{e}$ e uma aplicação contínua $s^{\prime}: U \rightarrow p(U(H ; J))$ tal que $s^{\prime}\left(\left[L_{0}\right]\right)=I$ e $\left[s^{\prime}([L])\left(L_{0}\right)\right]=[L]$, para $[L] \in \mathcal{U}$. Pelo ítem 1 da proposição 1.7 de $[2]$, a aplicação $\left.p\right|_{\mathrm{U}(\mathrm{H} ; J)}: \mathrm{U}(\mathrm{H} ; J) \rightarrow p(\mathrm{U}(\mathrm{H} ; J))$ admite uma seção local $s^{\prime \prime}$, portanto, compondo $s^{\prime}$ e $s^{\prime \prime}$, obtemos uma seção local $s: \mathcal{U} \rightarrow \mathrm{U}(\mathrm{H} ; J)$ tal que $\left[s([\mathrm{~L}])\left(\mathrm{L}_{0}\right)\right]=[\mathrm{L}]$, para $[\mathrm{L}] \in \mathcal{U}$. Logo,

$$
\begin{aligned}
\mathcal{U} \times \mathcal{G}_{c}\left(L_{0} ; \Lambda\right) & \rightarrow \pi^{-1}(\mathcal{U}) \\
\left([L], L^{\prime}\right) & \mapsto s([L])\left(L^{\prime}\right)
\end{aligned}
$$

é uma trivialização local de $\pi: \Lambda \rightarrow \Lambda_{e}$, como queríamos.

OBSERvação 1.6.45 Podemos usar a prova do teorema 1.6.44 (em especial, a aplicação 1.6.26) para construir uma estrutura de variedade de Banach analítica real em $\Lambda_{e}$.

Teorema 1.6.46 $\mathscr{F}_{L}(\Lambda)$ e $\mathcal{G}_{c}(L ; \Lambda)$ têm o mesmo tipo de homotopia. Em particular, $\mathscr{F}_{L}(\Lambda)$ tem o tipo de homotopia de $\mathrm{U}(\infty) / \mathrm{O}(\infty)$, pelo teorema 1.6.41.

\footnotetext{
${ }^{55} \mathrm{Ou}$ seja, $u \in \mathrm{U}(\mathcal{C})$ se e só se $u^{*} u=u u^{*}=1$.
} 
Prova. Consideremos a restrição $\left.\pi\right|_{\mathscr{F}_{L}(\Lambda)}: \mathscr{F}_{L}(\Lambda) \rightarrow \pi\left(\mathscr{F}_{L}(\Lambda)\right)$ do fibrado $\pi: \Lambda \rightarrow \Lambda_{e}$, a qual também define um fibrado com fibra típica $\mathcal{G}_{c}(L ; \Lambda)$, pela proposição 1.3 .63 e pelo teorema 1.6.44. A proposição 1.5 .32 mostra que $\pi\left(\mathscr{F}_{L}(\Lambda)\right)=\pi\left(\Lambda_{0}(L)\right)$. A aplicação

$$
[0,1] \times \pi\left(\Lambda_{0}(\mathrm{~L})\right) \ni\left(t, \pi\left(\varphi_{\mathrm{L}_{0}^{1}, L_{0}}^{-1}(T)\right)\right) \mapsto \pi\left(\varphi_{\mathrm{L}_{0}^{-}, L_{0}}^{-1}(t T)\right) \in \pi\left(\Lambda_{0}(\mathrm{~L})\right), T \in \mathscr{L}^{\text {sa }}\left(\mathrm{L}_{0}^{+}\right),
$$

é bem-definida (pois $\mathrm{G}(S) \sim \mathrm{G}(T)$ implica $\mathrm{G}(t S) \sim \mathrm{G}(t T)$ ) e é uma homotopia entre a identidade em $\pi\left(\Lambda_{0}(L)\right)$ e uma constante, portanto, $\pi\left(\Lambda_{0}(L)\right)$ é contrátil. O resultado segue usando a seqüência exata da fibração e o teorema 15 de [73].

Vamos agora estudar uma estratificação muito útil do espaço $\mathscr{F}_{L_{0}}(\Lambda)$ por subvariedades de codimensão finita. ${ }^{56}$ Sejam $k \geq 0 \mathrm{e}$

$$
\mathscr{F}_{L_{0}}^{k}(\Lambda) \doteq\left\{L \in \mathscr{F}_{L_{0}}(\Lambda): \operatorname{nul}\left(L, L_{0}\right)=k\right\}
$$

Obviamente, $\mathscr{F}_{L_{0}}(\Lambda)=\bigcup_{k \geq 0} \mathscr{F}_{L_{0}}^{k}(\Lambda)$. As próximas proposições mostram que esta estratificação é análoga ao caso de dimensão finita, conforme [6], [83].

Proposição 1.6.47 $\mathscr{F}_{\mathrm{L}_{0}}(\Lambda)$ admite uma cobertura formada por abertos da forma $\Lambda_{0}\left(\mathrm{~L}_{1}\right) \cap \mathscr{F}_{\mathrm{L}_{0}}(\Lambda)$, onde $L_{0}, L_{1}$ são complementares. Em particular, definindo

$$
\left.\zeta_{L_{0}, L_{1}} \doteq \varphi_{L_{0}, L_{1}}\right|_{\Lambda_{0}\left(L_{1}\right) \cap F_{L_{0}}(\Lambda)}: \Lambda_{0}\left(L_{1}\right) \cap \mathscr{F}_{L_{0}}(\Lambda) \rightarrow \mathscr{F}^{\text {sa }}\left(L_{0}\right),
$$

o conjunto $\left\{\zeta_{L_{0}, L_{1}}: L_{0} \in \Lambda_{0}\left(L_{1}\right)\right\}$ é um atlas de $\mathscr{F}_{L_{0}}(\Lambda)$.

Prova. Pela proposição 1.5 .36 e pelo teorema $1.6 .23, \zeta_{L_{0}, L_{1}}$ é um difeomorfismo $C^{\omega}$ com imagem $\mathscr{F}^{\text {sa }}\left(\mathrm{L}_{0}\right)$. A proposição 1.5.31 mostra que $\mathscr{F}_{L_{0}}(\Lambda)$ é a reunião dos $\Lambda_{0}\left(\mathrm{~L}_{1}\right) \cap \mathscr{F}_{L_{0}}(\Lambda)$, com $L_{1}$ complementar a $L_{0}$.

Proposição 1.6.48 Para cada $k \geq 0, \mathscr{F}_{L_{0}}^{k}(\Lambda)$ é uma subvariedade de Banach analítica real de $\mathscr{F}_{L_{0}}(\Lambda)$ de codimensão $k(k+1) / 2$.

Prova. Seja $L_{1} \in \Lambda$ complementar a $L_{0}$ e consideremos o difeomorfismo $\zeta_{L_{0}, L_{1}}$ definido em 1.6.27. Temos que $\zeta_{L_{0}, L_{1}}$ leva $\mathscr{F}_{L_{0}}^{k}(\Lambda) \cap \Lambda_{0}\left(L_{1}\right)$ em

$$
S_{k} \doteq\left\{T \in \mathscr{F}^{\mathrm{sa}}\left(\mathrm{L}_{0}\right): \operatorname{nul} T=k\right\} \text {. }
$$

Portanto, basta provar que $S_{k}$ é uma subvariedade de Banach analítica real de codimensão $k(k+1) / 2$ de $\mathscr{F}^{\text {sa }}\left(L_{0}\right)$. Para simplificar a notação, escreveremos $L_{0}=H$. Para cada subespaço $\mathrm{M} \subset \mathrm{H}$ com $\operatorname{dim} \mathrm{M}=k$, considerando a decomposição $\mathrm{H}=\mathrm{M} \oplus \mathrm{M}^{\perp}$, temos que $T \in \mathscr{L}^{\text {sa }}(\mathrm{H})$ se e só se $T$ tem a forma $\left({ }_{B^{*}}^{A}{ }_{C}^{B}\right), \operatorname{com} A \in \mathscr{L}^{\text {sa }}(\mathrm{M}), C \in \mathscr{L}^{\text {sa }}\left(\mathrm{M}^{\perp}\right)$ e $B \in \mathscr{L}\left(\mathrm{M}, \mathrm{M}^{\perp}\right)$. Com esta notação, seja

$$
\begin{aligned}
\Phi_{\mathrm{M}}: \mathscr{L}^{\mathrm{sa}}(\mathrm{M}) \times \mathscr{L}\left(\mathrm{M}, \mathrm{M}^{\perp}\right) \times\left(\mathrm{GL}\left(\mathrm{M}^{\perp}\right) \cap \mathscr{L}^{\mathrm{sa}}\left(\mathrm{M}^{\perp}\right)\right) & \rightarrow \mathcal{V}(\mathrm{M}) \\
(A, B, C) & \mapsto\left(\begin{array}{ll}
A & B \\
B^{*} & C
\end{array}\right),
\end{aligned}
$$

onde $\mathcal{V}(\mathrm{M}) \doteq\left\{T \in \mathscr{F}^{\mathrm{sa}}(\mathrm{H}): C \in \mathrm{GL}\left(\mathrm{M}^{\perp}\right)\right\}$. Temos que $\Phi_{\mathrm{M}}$ é um difeomorfismo $\mathrm{C}^{\omega} \mathrm{e} \Phi_{\mathrm{M}}(0,0, \cdot)=$ $S_{k} \cap \mathcal{V}(\mathrm{M})$. Variando $\mathrm{M} \subset \mathrm{H}$ (mantendo fixo $k$ ), os abertos $\mathcal{V}(\mathrm{M})$ cobrem $\delta_{k}$, portanto $\mathcal{S}_{k}$ é subvariedade $C^{\omega}$ de $\mathscr{F}^{\text {sa }}(\mathrm{H})$ de codimensão $k(k+1) / 2$.

\footnotetext{
${ }^{56}$ Dizemos que uma subvariedade $Y$ de uma variedade de Banach $X$ tem codimensão finita se o espaço tangente $T_{y} Y$ em cada ponto $y \in Y$ é um subespaço fechado de codimensão finita de $T_{y} X$.
} 
ObSERVAÇÃo 1.6.49 A subvariedade $S_{k}$ da proposição 1.6 .48 pode ser dada localmente como conjunto solução de $k(k+1) / 2$ equações algébricas, como em dimensão finita. De fato, tomando uma base $\left\{e_{1}, \ldots, e_{k}\right\}$ de M e $T \in \mathcal{V}(\mathrm{M})$, então $T \in S_{k}$ se e só se $\left\langle T e_{i}, e_{j}\right\rangle=0$ para todos $1 \leq i \leq j \leq k$. Isso mostra que $S_{k}$ é subvariedade algébrica de $\mathscr{F}^{\text {sa }}(\mathrm{H})$. O mesmo vale para $\mathscr{F}_{L_{0}}^{k}(\Lambda) \subset \mathscr{F}_{L_{0}}(\Lambda)$.

Para finalizar esta subseção, vamos provar alguns fatos gerais sobre a topologia de $\mathscr{F}_{L_{0}}(\Lambda)$.

Proposição 1.6.50 $\Lambda_{0}\left(L_{0}\right)$ é denso em $\left\{L \in \Lambda: L+L_{0}\right.$ é fechado $\}$. Em particular, $\Lambda_{0}\left(L_{0}\right)$ é denso em $\mathscr{F} L_{0}(\Lambda)$.

Prova. Fixemos $L \in \Lambda$ com $L+L_{0}$ fechado. Sejam $T \in \mathscr{L}^{\text {sa }}\left(L_{0}\right)$ tal que $\gamma(T)>0, \operatorname{ker} T=L_{\cap} \cap L_{0}$ e $L_{1}^{\prime} \doteq \mathrm{G}\left(\Psi_{L_{0}, L_{0}^{\prime}} T\right)$. Temos que $\operatorname{nul}\left(L_{0}, L_{1}\right)=\operatorname{nul}\left(L_{0}, L_{1}^{\prime}\right)$ e $L_{0}+L_{1}^{\prime}$ é fechado ${ }^{57}$, portanto, pela proposição 1.5.29, existe $U \in S p(H)$ tal que $U\left(L_{0}\right)=L_{0}$ e $U\left(L_{1}\right)=L$. Pela observação 1.3.41, temos que existem isomorfismos $T_{n} \in \mathscr{L}^{\text {sa }}\left(\mathrm{L}_{0}\right)$ tais que $\lim _{n} T_{n}=T$. Logo, $\mathrm{L}_{n}^{\prime} \doteq \mathrm{G}\left(\Psi_{\mathrm{L}_{0}, L_{0}^{\perp}} T_{n}\right)$ é um lagrangiano complementar a $L_{0}$ e $\left\{L_{n}^{\prime}\right\}$ converge para $L_{1}$. Pondo $L_{n}=U\left(L_{n}^{\prime}\right)$, temos que $L_{n}$ é complementar a $U\left(L_{0}\right)=L_{0}$ e $L_{n}$ converge para $U\left(L_{1}\right)=L$, pela proposição 1.6.25.

CoRolário 1.6.51 Se $\left\{\mathrm{L}_{n}\right\}_{n \in \mathbb{N}}$ é uma família de lagrangianos e existe algum lagrangiano $\mathrm{L}_{0}$ tal que $\mathrm{L}_{n}^{\perp} \in \mathscr{F}_{L_{0}}(\Lambda)$ para todo $n \in \mathbb{N}$, então existe $\mathrm{L} \in \mathscr{F}_{\mathrm{L}_{0}}(\Lambda)$ complementar a $\mathrm{L}_{n}$ para todo $n \in \mathbb{N}$.

Prova. Vejamos que $\mathscr{F}_{L_{n}}(\Lambda) \subset \mathscr{F}_{L_{0}}(\Lambda)$ para todo $n \in \mathbb{N}$. De fato, se $\left(L, L_{n}\right)$ é um par Fredholm, então pela proposição 1.2.19, temos $L^{\perp} \approx L_{n}$, usando a notação da definição 1.3.55. Como $L_{n} \approx L_{0}$, pelas proposições 1.3 .58 e 1.2.19, temos que $L^{\perp} \approx L_{0}$, logo, $\left(L_{,} L_{0}\right)$ é um par Fredholm, como queríamos. Da mesma forma, segue que $\mathscr{F}_{L_{0}}(\Lambda) \subset \mathscr{F}_{L_{n}}(\Lambda)$, para todo $n \in \mathbb{N}$. Em particular, $\Lambda_{0}\left(L_{n}\right) \subset \mathscr{F}_{L_{0}}(\Lambda)$ e $\mathscr{F}_{L_{0}}(\Lambda) \subset\left\{L \in \Lambda: L+L_{n}\right.$ é fechado\}. Pela proposição 1.6.50, temos que $\Lambda_{0}\left(L_{n}\right) \subset \mathscr{F}_{L_{0}}(\Lambda)$ é um aberto denso, para todo $n \in \mathbb{N}$. Como $\mathscr{F}_{L_{0}}(\Lambda)$ é um espaço de Baire ${ }^{58}$, o resultado segue do teorema de Baire.

\section{7 Índice de Maslov e Assinaturas Parciais}

Seja $\mathrm{H}$ um espaço de Hilbert simplético com forma simplética $\omega$ e estrutura complexa $J$. Nosso objetivo nesta seção é construir o índice de Maslov, para curvas de lagrangianos que formam um par Fredholm com um lagrangiano fixado e também para pares Fredholm de curvas de lagrangianos. Vamos utilizar na construção a técnica abstrata de [44] desenvolvida na subseção 1.4.2. Ao contrário de [69], não faremos nenhuma hipótese adicional sobre os pontos extremos das curvas envolvidas e obteremos um inteiro. Em [83], [84], J. Robbin e D. Salamon dão uma definição nestes moldes para o caso de dimensão finita, mas o resultado final é um semi-inteiro.

DefinIÇão 1.7.1 O ciclo de Maslov com vértice em $L_{0}$ é definido como

$$
\Sigma_{\mathrm{L}_{0}} \doteq \bigcup_{k \geq 1} \mathscr{F}_{\mathrm{L}_{0}}^{k}(\Lambda)
$$

\footnotetext{
${ }^{57}$ Pois $\gamma\left(L_{1}^{\prime}, L_{0}\right)>0$, pela segunda equação de 1.5 .8 .

${ }^{58} \mathrm{Um}$ espaço de Baire é um espaço topológico no qual a intersecção de uma família enumerável de abertos densos é densa. Todo espaço métrico completo é um espaço de Baire (teorema de Baire) e abertos de espaços de Baire são espaços de Baire. Como o espaço $\mathscr{F}_{L_{0}}(\Lambda)$ é um aberto do espaço métrico completo $\Lambda$, concluímos que $\mathscr{F}_{L_{0}}(\Lambda)$ é um espaço de Baire. Mais detalhes sobre espaços de Baire podem ser encontrados em [68].
} 
Quando $L_{0}$ estiver subentendido, chamaremos $\Sigma_{L_{0}}$ simplesmente de ciclo de Maslov.

A seguinte proposição será tecnicamente útil mais adiante.

PRoposição 1.7.2 Seja $\gamma:[0,1] \rightarrow \mathscr{F}_{L_{0}}(\Lambda)$ uma curva contínua qualquer. Então existe $k \in \mathbb{N}$ tal que a imagem de $\gamma$ não intersecta $\mathscr{F}_{L_{0}}^{j}(\Lambda)$, para qualquer $j>k$. Em particular, se $\gamma$ é analítica real, então a imagem de $\gamma$ ou está contida em algum $\mathscr{F}_{L_{0}}^{j}(\Lambda)$ ou intersecta $\Sigma_{L_{0}}$ somente uma quantidade finita de vezes.

Prova. Considerando a aplicação $f:[0,1] \ni t \mapsto \operatorname{nul}\left(\gamma(t), L_{0}\right) \in \mathbb{N}$, temos pelo teorema 1.3.20 que $f$ é semi-contínua superiormente. Em particular, existe $k \in \mathbb{N}$ tal que $\operatorname{nul}\left(\gamma(t), L_{0}\right) \leq k$, para todo $t \in[0,1]$, ou seja, a imagem de $\gamma$ não intersecta $\mathscr{F}_{L_{0}}^{j}(\Lambda)$, para qualquer $j>k$. A segunda afirmação decorre da proposição 1.6.48.

Dada uma curva $\gamma:[0,1] \rightarrow \mathscr{F}_{L_{0}}(\Lambda)$, os instantes $t$ nos quais $\gamma(t)$ intersecta não trivialmente $L_{0}$ são exatamente aqueles instantes em que a imagem de $\gamma$ intersecta o ciclo de Maslov $\Sigma_{\mathrm{L}_{0}}$. Para medir a estabilidade de tais instantes por perturbações de $\gamma$, poderíamos tentar utilizar a teoria de intersecção clássica (conforme [48] ou [66]) e obter um número inteiro que nos dá informação qualitativa sobre o tipo de intersecção de $\gamma \operatorname{com} \Sigma_{\mathrm{L}_{0}}$. A grosso modo, este seria o índice de Maslov, mas o problema que surge aqui é o fato de $\Sigma_{\mathrm{L}_{0}}$ não ser uma subvariedade de $\mathscr{F}_{L_{0}}(\Lambda)$, além do que, a teoria de intersecção utilizada pressupõe orientações nas subvariedades em questão. Mesmo assim, L. Nicolaescu em [69] constrói uma orientação transversa em $\overline{\mathscr{F}_{\mathrm{L}_{0} 1}(\Lambda)}$ e mostra que a teoria pode ser desenvolvida como em [6].

Vamos agora fazer as considerações necessárias para a definição do índice de Maslov. Sejam $L_{0}, L_{1}$ lagrangianos complementares de $H$. Definiremos um homomorfismo de grupóide $\nu_{L_{0}, L_{1}}: \pi\left(\Lambda_{0}\left(L_{1}\right) \cap \mathscr{F}_{L_{0}}(\Lambda)\right) \rightarrow \mathbb{Z}$ e verificaremos que a condição de compatibilidade da proposição 1.4.23 é satisfeita. Seja

$$
\begin{aligned}
v_{\mathrm{L}_{0}, L_{1}}: \pi\left(\Lambda_{0}\left(\mathrm{~L}_{1}\right) \cap \mathscr{F}_{L_{0}}(\Lambda)\right) & \rightarrow \mathbb{Z} \\
{[\gamma] } & \mapsto \operatorname{sf}\left(\varphi_{\mathrm{L}_{0}, L_{1}} \circ \gamma\right) .
\end{aligned}
$$

Temos que $v_{L_{0}, L_{1}}$ é um homomorfismo (bem-definido), pelo teorema 1.4.25. Seja $L_{1}^{\prime}$ outro lagrangiano complementar a $L_{0}$ e $\gamma$ uma curva definida em $[0,1]$ com imagem em $\Lambda_{0}\left(L_{1}\right) \cap$ $\Lambda_{0}\left(L_{1}^{\prime}\right) \cap \mathscr{F}_{L_{0}}(\Lambda)$. Vamos provar que $\nu_{L_{0}, L_{1}}(\gamma)=\nu_{L_{0}, L_{1}^{\prime}}(\gamma)$, i.e.,

$$
\operatorname{sf}\left(\varphi_{L_{0}, L_{1}} \circ \gamma\right)=\operatorname{sf}\left(\varphi_{L_{0}, L_{1}^{\prime}} \circ \gamma\right) \text {. }
$$

Como $\Lambda_{0}\left(L_{1}\right) \cap \Lambda_{0}\left(L_{1}^{\prime}\right) \cap \mathscr{F}_{L_{0}}(\Lambda)$ é um aberto de $\mathscr{F}_{L_{0}}(\Lambda)$, que é uma variedade de Banach analítica real, podemos aproximar $\gamma$ por uma curva analítica real por partes sem alterar nenhum dos inteiros em 1.7.2. Vamos supor daqui por diante que $\gamma$ seja analítica real. ${ }^{59}$ Pela fórmula 1.6 .13 , temos

$$
\varphi_{\mathrm{L}_{0}, \mathrm{~L}_{1}^{\prime}}(\gamma(t))=\varphi_{\mathrm{L}_{0}, \mathrm{~L}_{1}}(\gamma(t))\left(I+P_{\mathrm{L}_{1}^{\prime}, \mathrm{L}_{0}}^{\mathrm{L}_{1}} S(t)\right)^{-1},
$$

onde $S(t) \doteq \Psi_{L_{0}, L_{1}}\left(\varphi_{L_{0}, L_{1}}(\gamma(t))\right)$, i.e., $S:[0,1] \rightarrow \mathscr{L}\left(L_{0}, L_{1}\right)$ é analítica real e $G(S(t))=\gamma(t)$, para $t \in[0,1]$. Pelo segundo ítem da proposição 1.5.36, $\varphi_{\mathrm{L}_{0}, \mathrm{~L}_{1}}(\gamma(t)), \varphi_{\mathrm{L}_{0}, \mathrm{~L}_{1}^{\prime}}(\gamma(t)) \in \mathscr{F}^{\text {sa }}\left(\mathrm{L}_{0}\right)$, para todo

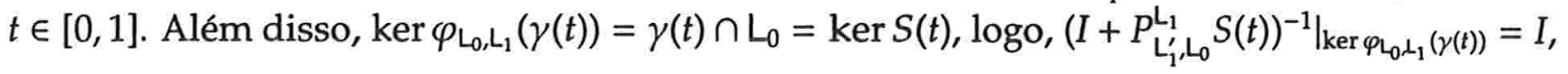

\footnotetext{
${ }^{59}$ Lembramos que uma curva $\eta$ definida em $[a, b]$ com valores em uma variedade analítica real é dita analítica real se $\eta$ é restrição a $[a, b]$ de uma curva analítica real definida em um intervalo aberto contendo $[a, b]$.
} 
portanto, pelo corolário 1.4.52 e pela observação 1.4.53, temos que $\operatorname{sf}\left(\varphi_{\mathrm{L}_{0}, \mathrm{~L}_{1}} \circ \gamma\right)=\operatorname{sf}\left(\varphi_{\mathrm{L}_{0}, \mathrm{~L}_{1}^{\prime}} \circ \gamma\right)$, como queríamos. Pelas proposições 1.4.23 e 1.6.47, o seguinte teorema está provado.

Teorema 1.7.3 Existe um único homomorfismo de grupóide $\mu_{\mathrm{L}_{0}}: \pi\left(\mathscr{F}_{L_{0}}(\Lambda)\right) \rightarrow \mathbb{Z}$, chamado índice de Maslov com respeito a $L_{0}$ tal que

$$
\mu_{\mathrm{L}_{0}}([\gamma])=\operatorname{sf}\left(\varphi_{\mathrm{L}_{0}, \mathrm{~L}_{1}} \circ \gamma\right)
$$

para qualquer $\gamma:[0,1] \rightarrow \Lambda_{0}\left(L_{1}\right) \cap \mathscr{F}_{L_{0}}(\Lambda)$ contínua.

Quando o lagrangiano $L_{0}$ estiver subentendido na definição de $\mu_{L_{0}}$, vamos chamá-lo simplesmente de índice de Maslov. Por vezes, também não faremos distinção entre uma curva $\gamma$ e sua classe de homotopia com extremos fixos $[\gamma]$. Dadas curvas $U:[a, b] \rightarrow \operatorname{Sp}(\mathrm{H})$ e $\gamma:[a, b] \rightarrow \Lambda$, denotamos por $U \cdot \gamma$ a curva $[a, b] \ni t \mapsto U(t)(\gamma(t))$. Vamos agora estudar algumas propriedades do índice de Maslov.

Proposição 1.7.4 O índice de Maslov $\mu_{\mathrm{L}_{0}}$ definido no teorema 1.7.3 tem as seguintes propriedades:

1. Se trocarmos a forma simplética $\omega$ por $-\omega$ então $\mu_{\mathrm{L}_{0}}$ também troca de sinal;

2. Se $\gamma:[0,1] \rightarrow \mathscr{F}_{L_{0}}^{k}(\Lambda)$ para algum $k \in \mathbb{N}$ então $\mu_{L_{0}}(\gamma)=0$;

3. Se $H:[0,1] \times[0,1] \rightarrow \mathscr{F}_{L_{0}}(\Lambda)$ é uma homotopia tal que $\operatorname{nul}\left(H(s, 0), L_{0}\right)$ e $\operatorname{nul}\left(H(s, 1), L_{0}\right)$ são constantes, então $\mu_{\mathrm{L}_{0}}\left(\gamma_{0}\right)=\mu_{\mathrm{L}_{0}}\left(\gamma_{1}\right)$, onde $\gamma_{i}(t)=H(i, t)$, para $i=0,1$;

4. Na notação do item 3 , se cada curva $\gamma_{s}(t) \doteq H(s, t)$ é fechada, para todo $s \in[0,1]$, então $\mu_{\mathrm{L}_{0}}\left(\gamma_{0}\right)=\mu_{\mathrm{L}_{0}}\left(\gamma_{1}\right)$;

5. Se $U \in \mathrm{U}(\mathrm{H} ; J)$ e $\gamma:[0,1] \rightarrow \mathscr{F}_{L_{0}}(\Lambda)$ então $\mu_{\mathrm{L}_{0}}(\gamma)=\mu_{U\left(\mathrm{~L}_{0}\right)}(U \cdot \gamma)$.

6. $\operatorname{Se} U \in \mathrm{Sp}(\mathrm{H}), U\left(\mathrm{~L}_{0}\right)=\mathrm{L}_{0}$ e $\gamma:[0,1] \rightarrow \mathscr{F}_{\mathrm{L}_{0}}(\Lambda)$ então $\mu_{\mathrm{L}_{0}}(U \cdot \gamma)=\mu_{\mathrm{L}_{0}}(\gamma)$;

7. Se $U:[0,1] \rightarrow \operatorname{Sp}(\mathrm{H}), U(t)\left(\mathrm{L}_{0}\right)=\mathrm{L}_{0}$ e $\gamma:[0,1] \rightarrow \mathscr{F}_{\mathrm{L}_{0}}(\Lambda)$ então $\mu_{\mathrm{L}_{0}}(U \cdot \gamma)=\mu_{\mathrm{L}_{0}}(\gamma)$;

8. $\operatorname{Se} U \in \mathrm{O}(\mathrm{H}), U J=-J U e U\left(\mathrm{~L}_{0}\right)=\mathrm{L}_{0}$, então $\mu_{\mathrm{L}_{0}}(U \cdot \gamma)=-\mu_{\mathrm{L}_{0}}(\gamma)$ para toda $\gamma:[0,1] \rightarrow \mathscr{F}_{\mathrm{L}_{0}}(\Lambda)$.

Prova. O ítem 1 é imediato. O ítem 2 segue da observação que uma família de operadores $T(t)$ tal que nul $T(t)$ é constante tem fluxo espectral nulo. Para provar o ítem 3, basta observar que pela invariância homotópica do índice de Maslov, temos

$$
\mu_{\mathrm{L}_{0}}(s \mapsto H(s, 0))+\mu_{\mathrm{L}_{0}}(t \mapsto H(1, t))=\mu_{\mathrm{L}_{0}}(t \mapsto H(0, t))+\mu_{\mathrm{L}_{0}}(s \mapsto H(s, 1))
$$

e utilizar o ítem 2. A fórmula 1.7.5 implica imediatamente o ítem 4.

Para provar o ítem 5, pelo corolário 1.6 .51 e pela aditividade do fluxo espectral por concatenação, podemos supor que $\gamma$ tem imagem em $\Lambda_{0}\left(L_{1}\right) \cap \mathscr{F}_{L_{0}}(\Lambda)$, com $L_{1} \in \Lambda$ complementar a $\mathrm{L}_{0}$. Temos que

$$
\begin{aligned}
\mu_{U\left(L_{0}\right)}(U \circ \gamma) & =\operatorname{sf}\left(\varphi_{U\left(L_{0}\right), U\left(\mathrm{~L}_{1}\right)} \circ(U \cdot \gamma)\right) \\
& \left.=\operatorname{sf}\left(P_{U\left(\mathrm{~L}_{0}\right)}\right) U S U^{-1}\right) \operatorname{com} S(t) \in \mathscr{L}\left(\mathrm{L}_{0}, \mathrm{~L}_{1}\right) \text { tal que } \mathrm{G}(S(t))=\gamma(t), \\
& =\operatorname{sf}\left(U P_{\mathrm{L}_{0}} U^{*} J U S U^{*}\right) \\
& =\operatorname{sf}\left(U P_{\mathrm{L}_{0}} J S U^{*}\right) \\
& =\operatorname{sf}\left(P_{\mathrm{L}_{0}} J S\right)=\mu_{\mathrm{L}_{0}}(\gamma),
\end{aligned}
$$


sendo que a penúltima igualdade decorre da invariância do fluxo espectral pela ação cogrediente, conforme o corolário 1.4.50.

Na prova do ítem 6, observamos que, como $U \in \operatorname{Sp}\left(H, L_{0}\right)$ e $\operatorname{Sp}\left(H, L_{0}\right)$ é conexo por caminhos (pela proposição 1.6.27), então existe uma curva $U(s) \in \mathrm{Sp}\left(\mathrm{H}, \mathrm{L}_{0}\right), 0 \leq s \leq 1$, tal que $U(0)=I$ e $U(1)=U$. Considerando $H(s, t) \doteq U(s) \cdot \gamma(t), 0 \leq s, t \leq 1$, temos que $H(0, t)=\gamma(t), H(1, t)=$ $U(\gamma(t))$ e $\operatorname{nul}\left(H(s, i), L_{0}\right)=\operatorname{nul}\left(U(s)(\gamma(i)), U(s)\left(L_{0}\right)\right)=\operatorname{nul}\left(\gamma(i), L_{0}\right)$, para $i=0,1$, portanto, $o$ resultado decorre do ítem 3 .

Para provar o ítem 7, basta observar que $H(s, t) \doteq U(s t) \cdot \gamma(t), 0 \leq s, t \leq 1$, é uma homotopia entre $t \mapsto U(t) \cdot \gamma(t)$ e $t \mapsto U(0) \cdot \gamma(t)$ enul $\left(U(s)(\gamma(j)), L_{0}\right)=\operatorname{nul}\left(U(s)(\gamma(j)), U(s)\left(L_{0}\right)\right)=\operatorname{nul}\left(\gamma(j), L_{0}\right)$, $j=0,1$, portanto o resultado segue dos ítens 3 e 6 .

Provemos 8. Antes de mais nada, um operador $U$ satisfazendo as hipóteses dadas é tal que $U(L)$ é lagrangiano se $L$ é lagrangiano. Além disso, os mesmos cálculos da prova do ítem 5 mostram que

$$
\begin{aligned}
\mu_{\mathrm{L}_{0}}(U \cdot \gamma) & =\operatorname{sf}\left(U P_{\mathrm{L}_{0}} U^{*} J U S U^{*}\right) \operatorname{com} S(t): \mathrm{L}_{0} \rightarrow \mathrm{L}_{1} \text { tal que } \mathrm{G}(S(t))=\gamma(t), \\
& =\operatorname{sf}\left(-U P_{\mathrm{L}_{0}} J S U^{*}\right) \\
& =-\operatorname{sf}\left(P_{\mathrm{L}_{0}} J S\right)=-\mu_{\mathrm{L}_{0}}(\gamma),
\end{aligned}
$$

pois o fluxo espectral é sensível à mudança de sinal.

Como $\mathscr{F}_{L_{0}}(\Lambda)$ é conexo por caminhos (pelo teorema 1.6.46), podemos considerar a restrição de $\mu_{L_{0}}$ a $\pi_{1}\left(\mathscr{F}_{L_{0}}(\Lambda)\right)$. Como no caso do fluxo espectral, temos o seguinte teorema.

Teorema 1.7.5 O indice de Maslov $\mu_{L_{0}}: \pi_{1}\left(\mathscr{F}_{L_{0}}(\Lambda)\right) \rightarrow \mathbb{Z}$ é um isomorfismo.

Prova. Pelo teorema 1.6.46, $\pi_{1}\left(\mathscr{F}_{L_{0}}(\Lambda)\right)=\mathbb{Z}$. Portanto, basta provar que $\mu_{L_{0}}: \pi_{1}\left(\mathscr{F}_{L_{0}}(\Lambda)\right) \rightarrow \mathbb{Z}$ é sobrejetor. Isso decorre da equação 1.6.24 e do teorema 1.4.35.

Agora que já conhecemos as principais propriedades do índice de Maslov, vamos usar a teoria de assinaturas parciais construída na subseção 1.4.4 para calcular o índice de Maslov de curvas analíticas reais em $\mathscr{F}_{L_{0}}(\Lambda)$.

Definição 1.7.6 Dada uma curva $\gamma:\left[t_{0}-\varepsilon, t_{0}+\varepsilon\right] \rightarrow \mathscr{F}_{L_{0}}(\Lambda)$, dizemos que $t=t_{0}$ é uma intersecção isolada de $\gamma \operatorname{com} \Sigma_{\mathrm{L}_{0}}$ se $\gamma(t) \in \Sigma_{\mathrm{L}_{0}}$ se e só se $t=t_{0}$.

Se $\gamma:\left[t_{0}-\varepsilon, t_{0}+\varepsilon\right] \rightarrow \mathscr{F}_{L_{0}}(\Lambda)$ é analítica real, $t=t_{0}$ é intersecção isolada de $\gamma$ com $\Sigma_{L_{0}}$ e $\varepsilon>0$ é suficientemente pequeno, então por continuidade existe $L_{1} \in \Lambda$ complementar a $L_{0}$ tal que $\gamma(t) \in \Lambda_{0}\left(L_{1}\right)$, para todo $t \in\left[t_{0}-\varepsilon, t_{0}+\varepsilon\right]$, pela proposição 1.5.31. Logo, a curva de operadores Fredholm auto-adjuntos limitados $\varphi_{\mathrm{L}_{0}, \mathrm{~L}_{1}} \circ \gamma$ tem uma degenerescência isolada em $t=t_{0}$, e portanto, podemos dar a seguinte definição.

DeFINIÇão 1.7.7 Se $\gamma$ é como na definição 1.7.6, definimos as assinaturas parciais de $\gamma$ em $t=t_{0}$ por

$$
\begin{gathered}
n_{k}^{+}\left(\gamma, t_{0} ; L_{0}\right) \doteq n_{k}^{+}\left(\varphi_{L_{0}, L_{1}} \circ \gamma, t_{0}\right), n_{k}^{-}\left(\gamma, t_{0} ; L_{0}\right) \doteq n_{k}^{-}\left(\varphi_{L_{0}, L_{1}} \circ \gamma, t_{0}\right) \\
\sigma_{k}\left(\gamma, t_{0} ; L_{0}\right) \doteq n_{k}^{+}\left(\gamma, t_{0} ; L_{0}\right)-n_{k}^{-}\left(\gamma, t_{0} ; L_{0}\right) .
\end{gathered}
$$


As mesmas observações feitas no início desta subseção para provar que os homomorfismos $v_{\mathrm{L}_{0}, \mathrm{~L}_{1}}$ satisfazem a condição de compatibilidade da proposição 1.4 .23 e a proposição 1.4 .51 mostram que as assinaturas parciais de $\gamma$ em $t=t_{0}$ são bem definidas, i.e., independem de $L_{1}$.

Vamos agora fazer algumas observações semelhantes àquelas que sucedem a definição. 1.4.44. Sejam $\gamma:\left[t_{0}-\varepsilon, t_{0}+\varepsilon\right] \rightarrow \mathscr{F}_{L_{0}}(\Lambda)$ uma curva analítica real que possui intersecção isolada com $L_{0}$ em $t=t_{0}$. Se $\varepsilon>0$ é suficientemente pequeno, podemos tomar $L_{1} \in \Lambda$ complementar a $L_{0}$ e a $\gamma(t)$, para todo $t \in\left[t_{0}-\varepsilon, t_{0}+\varepsilon\right]$; logo, $\gamma$ tem imagem em $\Lambda_{0}\left(L_{1}\right)$. Consideremos a curva analítica real de operadores Fredholm auto-adjuntos $S(t) \doteq \varphi_{L_{0}, L_{1}}(\gamma(t)): L_{0} \rightarrow L_{0}$ tais que $\mathrm{G}\left(\Psi_{\mathrm{L}_{0}, \mathrm{~L}_{1}} S(t)\right)=\gamma(t)$, conforme a definição 1.5.25. Temos que ker $S\left(t_{0}\right)=\gamma\left(t_{0}\right) \cap \mathrm{L}_{0} \neq 0$, logo, $0 \in \Sigma\left(S\left(t_{0}\right)\right)$. Se $\varepsilon>0$ é suficientemente pequeno, então pelo teorema 1.2.77 existe $\delta>0$ tal que $\Sigma(S(t)) \cap(-\delta, \delta)$ consiste de uma quantidade finita de autovalores isolados, os quais podem ser representados por funções analíticas reais $\lambda_{1}, \ldots, \lambda_{N}:\left[t_{0}-\varepsilon, t_{0}+\varepsilon\right] \rightarrow(-\delta, \delta)$. Também é possível obter funções analíticas reais $e_{1}, \ldots, e_{N}:\left[t_{0}-\varepsilon, t_{0}+\varepsilon\right] \rightarrow \mathrm{H}$ tais que $\left\{e_{j}(t)\right\}_{1 \leq j \leq N}$ é um conjunto ortonormal de autovetores correspondentes aos autovalores $\lambda_{1}(t), \ldots, \lambda_{N}(t)$. Logo, definindo $n_{k}^{ \pm}$e $\sigma_{k}$ como em 1.4.15, 1.4.16 e 1.4.17, concluímos o seguinte resultado análogo ao teorema 1.4.45.

TeORema 1.7.8 Se $\gamma:\left[t_{0}-\varepsilon, t_{0}+\varepsilon\right] \rightarrow \mathscr{F}_{L_{0}}(\Lambda)$ é uma curva analítica real que possui intersecção isolada com $\mathrm{L}_{0}$ em $t=t_{0}$, então,

$$
\begin{aligned}
\mu_{L_{0}}\left(\left.\gamma\right|_{\left[t_{0}-\varepsilon, t_{0}\right]}\right) & =\sum_{k \geq 1}\left\{n_{2 k}^{-}\left(\gamma, t_{0} ; L_{0}\right)+n_{2 k-1}^{+}\left(\gamma, t_{0} ; L_{0}\right)\right\}, \\
\mu_{L_{0}}\left(\left.\gamma\right|_{\left[t_{0}, t_{0}+\varepsilon\right]}\right) & =-\sum_{k \geq 1} n_{k}^{-}\left(\gamma, t_{0} ; L_{0}\right), \\
\mu_{L_{0}}\left(\left.\gamma\right|_{\left[t_{0}-\varepsilon, t_{0}+\varepsilon\right]}\right) & =\sum_{k \geq 1} \sigma_{2 k-1}\left(\gamma, t_{0} ; L_{0}\right),
\end{aligned}
$$

Como no caso do fluxo espectral, se $\gamma$ é analítica real temos uma fórmula bastante especial para o cálculo de $\mu_{L_{0}}(\gamma)$.

TEOREma 1.7.9 Seja $\gamma:[a, b] \rightarrow \mathscr{F}_{L_{0}}(\Lambda)$ uma curva analítica real cuja imagem não está contida em $\Sigma_{\mathrm{L}_{0}}$. Então

$$
\mu_{\mathrm{L}_{0}}(\gamma)=\sum_{\substack{\gamma(t) \in \Sigma_{L_{0}} \\ a<t<b}}\left[\sum_{k \geq 1} \sigma_{2 k-1}\left(\gamma, t_{0} ; \mathrm{L}_{0}\right)\right]+\sum_{k \geq 1}\left[n_{2 k}^{-}\left(\gamma, a ; \mathrm{L}_{0}\right)+n_{2 k-1}^{+}\left(\gamma, a ; \mathrm{L}_{0}\right)\right]-\sum_{k \geq 1} n_{k}^{-}\left(\gamma, b ; \mathrm{L}_{0}\right) .
$$

Prova. Pela proposição 1.7.2, vemos que a primeira soma tem somente uma quantidade finita de termos. Assim, tomando $\varepsilon>0$ suficientemente pequeno, os intervalos $(a, a+\varepsilon] \mathrm{e}$ $[b-\varepsilon, b)$ não contém nenhum instante de intersecção de $\gamma \operatorname{com} \Sigma_{\mathrm{L}_{0}}$. Um cálculo simples usando a definição das assinaturas parciais mostra que ambos os membros de 1.7 .9 são aditivos por concatenação. Por continuidade e pela proposição 1.5.31, podemos supor que existe $L_{1} \in \Lambda$ complementar a $L_{0}$ e a $\gamma(t)$, para todo $[a, b]$. Como $\mu_{L_{0}}$ é aditivo por concatenação, então

$$
\begin{aligned}
\mu_{L_{0}}(\gamma) & =\mu_{L_{0}}\left(\left.\gamma\right|_{[a+\varepsilon, b-\varepsilon]}\right)+\mu_{L_{0}}\left(\left.\gamma\right|_{[a, a+\varepsilon]}\right)+\mu_{L_{0}}\left(\left.\gamma\right|_{[b-\varepsilon, b]}\right) \\
& =\operatorname{sf}\left(\left.\varphi_{L_{0}, L_{1}} \circ \gamma\right|_{[a+\varepsilon, b-\varepsilon]}\right)+\operatorname{sf}\left(\left.\varphi_{L_{0}, L_{1}} \circ \gamma\right|_{[a, a+\varepsilon]}\right)+\operatorname{sf}\left(\left.\varphi_{L_{0}, L_{1}} \circ \gamma\right|_{[b-\varepsilon, b]}\right) .
\end{aligned}
$$


O teorema segue das fórmulas 1.4.18, 1.4.19 e 1.4.20.

Fixemos algumas notações necessárias para a definição do índice de Maslov para pares Fredholm de curvas de lagrangianos.

Definição 1.7.10 Dadas curvas $\gamma_{0}, \gamma_{1}:[a, b] \rightarrow \Lambda$, dizemos que $\left(\gamma_{0}, \gamma_{1}\right)$ é um par Fredholm de curvas de lagrangianos se para cada $t \in[a, b],\left(\gamma_{0}(t), \gamma_{1}(t)\right)$ é um par Fredholm, ou equivalentemente, a curva $\left(\gamma_{0}, \gamma_{1}\right)$ tem imagem $\operatorname{em} \operatorname{Fp}(\Lambda)$.

A idéia intuitiva do índice de Maslov para pares de curvas é a mesma utilizada para definir o índice de Maslov para uma única curva em $\mathscr{F}_{L_{0}}(\Lambda)$. Ao passo que naquela situação obtínhamos uma medida qualitativa dos instantes em que $\gamma(t)$ não era complementar a $L_{0}$, nesta situação queremos obter uma medida qualitativa dos instantes em que as duas curvas não são complementares. Vamos agora desenvolver as ferramentas que utilizaremos para formalizar esta idéia.

Consideremos o espaço simplético $\mathrm{H} \oplus \mathrm{H}$ munido da estrutura complexa $\hat{j}=(J,-J)$ e da forma simplética

$$
\hat{\omega}\left((u, v),\left(u^{\prime}, v^{\prime}\right)\right) \doteq \omega\left(u, u^{\prime}\right)-\omega\left(v, v^{\prime}\right) .
$$

Vemos que $\gamma_{0}(t) \cap \gamma_{1}(t) \neq 0$ se e só se $\left(\gamma_{0}(t) \oplus \gamma_{1}(t)\right) \cap \Delta \neq 0$, portanto, os instantes em que $\gamma_{0}(t)$ e $\gamma_{1}(t)$ não são complementares são exatamente os instantes nos quais $\gamma_{0}(t) \oplus \gamma_{1}(t)$ e $\Delta$ não são complementares. Obviamente, $\Delta$ é um lagrangiano de $\mathrm{H} \oplus \mathrm{H}$. O próximo lema mostra que o mesmo é verdade para $\gamma_{0}(t) \oplus \gamma_{1}(t)$.

Lema 1.7.11 Se $\mathrm{L}_{0}, \mathrm{~L}_{1}$ são lagrangianos de $\mathrm{H}$, então $\mathrm{L}_{0} \oplus \mathrm{L}_{1}$ é um lagrangiano de $(\mathrm{H} \oplus \mathrm{H}, \hat{\omega})$. Além disso, $\left(L_{0}, L_{1}\right)$ é um par Fredholm se e só se $\left(L_{0} \oplus L_{1}, \Delta\right)$ o ée ind $\left(L_{0}, L_{1}\right)=$ ind $\left(L_{0} \oplus L_{1}, \Delta\right)$.

Prova. Basta observar que $\left(L_{0} \oplus L_{1}\right)^{\perp}=L_{0}^{\perp} \oplus L_{1}^{\perp}=J L_{0} \oplus J L_{1}=j\left(L_{0} \oplus L_{1}\right)$, nul $\left(L_{0}, L_{1}\right)=$ $\operatorname{nul}\left(L_{0} \oplus L_{1}, \Delta\right)$ e def $\left(L_{0}, L_{1}\right)=\operatorname{def}\left(L_{0} \oplus L_{1}, \Delta\right)$.

Definição 1.7.12 Dado um par Fredholm de curvas $\gamma_{0}, \gamma_{1}:[a, b] \rightarrow \Lambda$, definimos o índice de Maslov do par $\left(\gamma_{0}, \gamma_{1}\right)$ por

$$
\mu\left(\gamma_{0}, \gamma_{1}\right)=\mu_{\Delta}\left(\gamma_{0} \oplus \gamma_{1}\right) \text {. }
$$

O índice de Maslov definido para pares Fredholm de curvas de lagrangianos tem propriedades análogas às propriedades do índice de Maslov enunciadas na proposição 1.7.4.

Proposição 1.7.13 O indice de Maslov para pares Fredholm de curvas $\gamma_{0}, \gamma_{1}:[a, b] \rightarrow \Lambda$ verifica as seguintes propriedades:

1. Trocar a forma simplética $\omega$ por $-\omega$ troca o sinal de $\mu\left(\gamma_{0}, \gamma_{1}\right)$;

2. Se $\operatorname{nul}\left(\gamma_{0}(t), \gamma_{1}(t)\right)$ é constante então $\mu\left(\gamma_{0}, \gamma_{1}\right)=0$;

3. Se $H, H^{\prime}:[0,1] \times[0,1] \rightarrow \Lambda$ são homotopias tais que $H(s, \cdot), H^{\prime}(s, \cdot)$ formam um par Fredholm de curvas em $\Lambda$ para todo $s \in[0,1]$ e $\operatorname{nul}\left(H(s, i), H^{\prime}(s, i)\right), i=0,1$, são constantes, então $\mu\left(\gamma_{0}, \gamma_{1}\right)=\mu\left(\gamma_{0}^{\prime}, \gamma_{1}^{\prime}\right)$, onde $\gamma_{i}(t) \doteq H(i, t)$ e $\gamma_{i}^{\prime}(t) \doteq H^{\prime}(i, t)$, para $i=0,1$;

4. Se $\gamma_{s}, \gamma_{s}^{\prime}, 0 \leq s \leq 1$, são homotopias entre $\gamma_{0}, \gamma_{1}$ e $\gamma_{0}^{\prime}, \gamma_{1}^{\prime}$, respectivamente, e $\gamma_{s}, \gamma_{s}^{\prime}$ são curvas fechadas, então $\mu\left(\gamma_{0}, \gamma_{1}\right)=\mu\left(\gamma_{0}^{\prime}, \gamma_{1}^{\prime}\right)$; 
5. Se $U:[0,1] \rightarrow \mathrm{Sp}(\mathrm{H})$ então $\mu\left(U \cdot \gamma_{0}, U \cdot \gamma_{1}\right)=\mu\left(\gamma_{0}, \gamma_{1}\right)$;

6. $\mu$ é anti-simétrico, i.e., $\mu\left(\gamma_{0}, \gamma_{1}\right)=-\mu\left(\gamma_{1}, \gamma_{0}\right)$.

Prova. Os ítens 1, 2, 3 e 4 decorrem imediatamente das afirmações correspondentes contidas na proposição 1.7.4 e da definição 1.7.12. Dado $U \in \mathrm{Sp}(\mathrm{H})$, o operador $\widehat{U}: \mathrm{H} \oplus \mathrm{H} \rightarrow \mathrm{H} \oplus \mathrm{H}$ definido por $\hat{U}\left(u, u^{\prime}\right)=\left(U u, U u^{\prime}\right)$ pertence a $\mathrm{Sp}(\mathrm{H} \oplus \mathrm{H})$ e $\hat{U}(\Delta)=\Delta$. Esta observação e o ítem 7 da proposição 1.7.4 provam o ítem 5.

Para provar o ítem 6, observamos que a aplicação $\Theta: \mathrm{H} \oplus \mathrm{H} \ni\left(u, u^{\prime}\right) \mapsto\left(u^{\prime}, u\right) \in \mathrm{H} \oplus \mathrm{H}$ pertence a $\mathrm{O}(H \oplus H)$, anti-comuta com $\hat{J}$ e fixa $\Delta$. Logo, pelo ítem 8 da proposição 1.7.4, segue que

$$
\mu\left(\gamma_{1}, \gamma_{0}\right)=\mu_{\Delta}\left(\gamma_{1} \oplus \gamma_{0}\right)=\mu_{\Delta}\left(\Theta \cdot\left(\gamma_{0} \oplus \gamma_{1}\right)\right)=-\mu_{\Delta}\left(\gamma_{0} \oplus \gamma_{1}\right)=-\mu\left(\gamma_{0}, \gamma_{1}\right)
$$

A próxima proposição mostra que a definição 1.7 .12 estende a definição de índice de Maslov dada no teorema 1.7.3.

Proposição 1.7.14 Sejam $L_{0}$ um lagrangiano e $\gamma:[a, b] \rightarrow \mathscr{F}_{L_{0}}(\Lambda)$ uma curva. Então

$$
\mu\left(\gamma, \mathrm{L}_{0}\right)=\mu_{\mathrm{L}_{0}}(\gamma) \text {. }
$$

Prova. Suponhamos inicialmente que $\gamma(t)$ é complementar a $L_{0}^{\perp}$ para todo $t \in[a, b]$. Dado qualquer $L_{1} \in \Lambda$ complementar a $L_{0}$ e $L_{0}^{\perp}$, segue pelo lema 1.7.11 que os pares de lagrangianos $\left(L_{0}^{\perp} \oplus L_{1}, \Delta\right)$ e $\left(L_{0}^{\perp} \oplus L_{1}, \gamma(t) \oplus L_{0}\right)$ são complementares em $H \oplus H$. Em outras palavras, a curva $\gamma(t) \oplus \mathrm{L}_{0}$ tem imagem em $\Lambda_{0}\left(\mathrm{~L}_{0}^{\perp} \oplus \mathrm{L}_{1}\right)$.

Sejam $U(t): L_{0} \rightarrow L_{0}^{\perp}$ operadores lineares tais que $G(U(t))=\gamma(t)$; portanto, $\varphi_{\mathrm{L}_{0}, L_{0}^{+}}(\gamma(t))=$ $J U(t)$. Definindo $\tilde{U}(t): H \rightarrow L_{0}^{+}$como $\tilde{U}(t)=U(t) P_{L_{0}}-P_{L_{0}^{1}}$, temos que $G(\tilde{U}(t))=\mathrm{G}(U(t))=\gamma(t)$. Além disso, a projeção $P_{L_{0}, L_{1}}: H \rightarrow L_{1}$ é tal que $\mathrm{G}\left(-P_{L_{0}, L_{1}}\right)=L_{0}$. Logo, definindo $T(t) \doteq$ $\left(\tilde{U}(t),-P_{L_{0}, L_{1}}\right): \Delta \rightarrow L_{0}^{\perp} \oplus L_{1}$, temos $G(T(t))=\gamma(t) \oplus L_{0}$, e portanto

$$
\begin{aligned}
\varphi_{\Delta, \mathrm{L}_{0}^{ \pm} \oplus \mathrm{L}_{1}}\left(\gamma(t) \oplus \mathrm{L}_{0}\right) & =P_{\Delta} \circ(J,-J) \circ T(t) \\
& =\frac{1}{2}\left(J U(t) P_{\mathrm{L}_{0}}-J\left(P_{\mathrm{L}_{0}^{\perp}}-P_{\mathrm{L}_{0}, \mathrm{~L}_{1}}\right), J U(t) P_{\mathrm{L}_{0}}-J\left(P_{\mathrm{L}_{0}^{\perp}}-P_{\mathrm{L}_{0}, L_{1}}\right)\right) \\
& =\frac{1}{2}(W(t), W(t)),
\end{aligned}
$$

onde $W(t)=J U(t) P_{\mathrm{L}_{0}}-J\left(P_{\mathrm{L}_{0}}^{\perp}-P_{\mathrm{L}_{0}, \mathrm{~L}_{1}}\right)$. Temos que $\left.W(t)\right|_{L_{0}}=J U(t)$ e $\left.W(t)\right|_{\mathrm{L}_{0}^{+}}=-J P_{\mathrm{L}_{1}, \mathrm{~L}_{0} \mathrm{~L}_{0}^{\perp}} ;$ em particular, $L_{0}, L_{0}^{\frac{1}{1}}$ são invariantes por $W(t)$. Logo, com respeito à decomposição $H=L_{0}+L_{0}^{\frac{1}{0}}$, $W(t)$ tem a forma

$$
W(t)=\left(\begin{array}{cc}
J U(t) & 0 \\
0 & -J P_{L_{0}, L_{1}} L_{L_{0}^{+}}
\end{array}\right),
$$

portanto, pela definição de fluxo espectral,

$$
\mu\left(\gamma, L_{0}\right)=\mu_{\Delta}\left(\gamma(t) \oplus L_{0}\right)=\operatorname{sf}(W(t))=\operatorname{sf}(J U(t))=\mu_{L_{0}}(\gamma),
$$

como queríamos. 
Vamos agora eliminar a hipótese que $\gamma(t)$ e $\mathrm{L}_{0}^{\perp}$ sejam complementares. Para isso, fixemos $\gamma$ qualquer. Como ambos os membros de 1.7 .11 são aditivos por concatenação, usando a proposição 1.5.31, podemos supor que existe $L_{2} \in \Lambda_{0}\left(L_{0}\right)$ tal que $\gamma(t)$ é complementar a $L_{2}$, para todo $t \in[a, b]$. Pela proposição 1.5.28, existe $U \in S p(H)$ tal que $U\left(L_{0}\right)=L_{0}$ e $U\left(L_{2}\right)=L_{0}^{+}$; em particular, $U(\gamma(t))$ é complementar a $\mathrm{L}_{0}^{\frac{1}{1}}$ para todo $t \in[a, b]$, portanto,

$$
\mu\left(\gamma, L_{0}\right)=\mu\left(U \cdot \gamma, L_{0}\right)=\mu_{L_{0}}(U \cdot \gamma)=\mu_{L_{0}}(\gamma),
$$

sendo que a primeira igualdade decorre do ítem 5 da proposição 1.7.13, a segunda igualdade decorre do que provamos no parágrafo anterior e a última igualdade decorre do ítem 6 da proposição 1.7.4.

Além da maneira apresentada, existe outra forma de reduzir o índice de Maslov de um par Fredholm de curvas ao índice de Maslov de uma única curva com relação a um lagrangiano fixado, utilizada por L. Nicolaescu em [69]. Dado um par Fredholm de curvas $\gamma_{0}, \gamma_{1}:[a, b] \rightarrow$ $\Lambda$ e $\mathrm{L}_{0} \in \Lambda$ qualquer, como a aplicação $\beta_{\mathrm{L}_{0}}: \mathrm{U}(\mathrm{H} ; J) \rightarrow \Lambda$ é uma fibração (pelo teorema 1.6.26 e pela observação 1.6.36), segue que existe uma curva $\eta:[a, b] \rightarrow U(H ; J)$ tal que $\eta(t)\left(L_{0}\right)=\gamma_{1}(t)$, para todo $t \in[a, b]$ (i.e., $\eta$ é um levantamento de $\gamma_{1}$ por $\beta_{L_{0}}$ ). A próxima proposição mostra que a curva $[a, b] \ni t \mapsto\left(\eta^{-1} \cdot \gamma_{0}\right)(t) \doteq \eta(t)^{-1}\left(\gamma_{0}(t)\right)$ pode ser utilizada para calcular o índice de Maslov do par $\left(\gamma_{0}, \gamma_{1}\right)$.

Proposição 1.7.15 Se $\gamma_{0}, \gamma_{1}, \eta$ são curvas como acima, então

$$
\mu\left(\gamma_{0}, \gamma_{1}\right)=\mu_{L_{0}}\left(\eta^{-1} \cdot \gamma_{0}\right) .
$$

Em particular, o inteiro $\mu_{\mathrm{L}_{0}}\left(\eta^{-1} \cdot \gamma_{0}\right)$ independe do levantamento $\eta$ utilizado.

Prova. Basta observar que

$$
\begin{aligned}
\mu\left(\gamma_{0}, \gamma_{1}\right) & =\mu\left(\eta^{-1} \cdot \gamma_{0}, \eta^{-1} \cdot \gamma_{1}\right), \text { pelo ítem } 5 \text { da proposição 1.7.13, } \\
& =\mu\left(\eta^{-1} \cdot \gamma_{0}, L_{0}\right) \\
& =\mu_{L_{0}}\left(\eta^{-1} \cdot \gamma_{0}\right), \text { pela proposição 1.7.14. }
\end{aligned}
$$

Como no caso de uma única curva, a teoria de assinaturas parciais pode ser utilizada para calcular o índice de Maslov no caso analítico real.

DeFinição 1.7.16 Dado um par Fredholm de curvas de lagrangianos $\gamma_{0}, \gamma_{1}:\left[t_{0}-\varepsilon, t_{0}+\varepsilon\right] \rightarrow \Lambda$, dizemos que $t=t_{0}$ é uma intersecção isolada de $\gamma_{0}, \gamma_{1}$ se $\gamma_{0}(t) \cap \gamma_{1}(t) \neq 0$ se e só se $t=t_{0}$.

Definição 1.7.17 Dado um par Fredholm $\gamma_{0}, \gamma_{1}:\left[t_{0}-\varepsilon, t_{0}+\varepsilon\right] \rightarrow \Lambda$ de curvas de lagrangianos com intersecção isolada em $t=t_{0}$, definimos as assinaturas parciais do par $\left(\gamma_{0}, \gamma_{1}\right)$ em $t=t_{0}$ por

$$
\begin{gathered}
n_{k}^{+}\left(\gamma_{0}, \gamma_{1}, t_{0}\right) \doteq n_{k}^{+}\left(\gamma_{0} \oplus \gamma_{1}, t_{0}\right), n_{k}^{-}\left(\gamma_{0}, \gamma_{1}, t_{0}\right) \doteq n_{k}^{-}\left(\gamma_{0} \oplus \gamma_{1}, t_{0}\right) \\
\sigma_{k}\left(\gamma_{0}, \gamma_{1}, t_{0}\right) \doteq n_{k}^{+}\left(\gamma_{0} \oplus \gamma_{1}, t_{0}\right)-n_{k}^{-}\left(\gamma_{0} \oplus \gamma_{1}, t_{0}\right) .
\end{gathered}
$$


Para finalizar esta seção, façamos alguns comentários úteis para o cálculo do índice de Maslov de um par Fredholm de curvas analíticas reais de lagrangianos. Seja $\gamma_{0}, \gamma_{1}:\left[t_{0}-\varepsilon, t_{0}+\right.$ $\varepsilon] \rightarrow \Lambda$ um tal par com intersecção isolada em $t=t_{0}$. Se $\varepsilon>0$ é suficientemente pequeno, a proposição 1.5.31 nos garante a existência de um lagrangiano $\mathrm{L}$ de $\mathrm{H} \oplus \mathrm{H}$ complementar a $\Delta \mathrm{e}$ $\gamma_{0}(t) \oplus \gamma_{1}(t)$, para todo $t \in\left[t_{0}-\varepsilon, t_{0}+\varepsilon\right]$. Consideremos a curva analítica real de operadores Fredholm auto-adjuntos $S(t) \doteq \varphi_{\Delta, L}(\gamma(t)): \Delta \rightarrow \Delta$ tais que $G\left(\Psi_{\Delta, L} S(t)\right)=\gamma_{0}(t) \oplus \gamma_{1}(t)$, para $t \in\left[t_{0}-\varepsilon, t_{0}+\varepsilon\right]$. Portanto, $\operatorname{ker} S\left(t_{0}\right)=\gamma\left(t_{0}\right) \cap \Delta$ e este último espaço é isomorfo a $\gamma_{0}\left(t_{0}\right) \cap \gamma_{1}\left(t_{0}\right) \neq$ 0 . Pelo teorema 1.2.77, se $\varepsilon>0$ é suficientemente pequeno, então existe $\delta>0$ tal que $\Sigma(S(t)) \cap(-\delta, \delta)$ consiste de uma quantidade finita de autovalores isolados, os quais podem ser representados por funções analíticas reais $\lambda_{1}, \ldots, \lambda_{N}:\left[t_{0}-\varepsilon, t_{0}+\varepsilon\right] \rightarrow(-\delta, \delta)$. Também é possível obter funções analíticas reais $e_{1}, \ldots, e_{N}:\left[t_{0}-\varepsilon, t_{0}+\varepsilon\right] \rightarrow \mathrm{H}$ tais que $\left\{e_{j}(t)\right\}_{1 \leq j \leq N}$ é um conjunto ortonormal de autovetores de $S(t)$ correspondentes aos autovalores $\lambda_{1}(t), \ldots, \lambda_{N}(t)$. Logo, definindo $n_{k}^{ \pm}$e $\sigma_{k}$ como em 1.4.15, 1.4.16 e 1.4.17, segue o resultado abaixo, análogo ao teorema 1.7.8.

Teorema 1.7.18 Se $\gamma_{0}, \gamma_{1}:\left[t_{0}-\varepsilon, t_{0}+\varepsilon\right] \rightarrow \Lambda$ é um par Fredholm de curvas analíticas reais que possui intersecção isolada em $t=t_{0}$, então são válidas as seguintes fórmulas:

$$
\begin{aligned}
\mu\left(\left.\gamma_{0}\right|_{[-\varepsilon, 0]},\left.\gamma_{1}\right|_{[-\varepsilon, 0]}\right) & =\sum_{k \geq 1}\left\{n_{2 k}^{-}\left(\gamma_{0}, \gamma_{1}, t_{0}\right)+n_{2 k-1}^{+}\left(\gamma_{0}, \gamma_{1}, t_{0}\right)\right\} \\
\mu\left(\left.\gamma_{0}\right|_{[0, \varepsilon]},\left.\gamma_{1}\right|_{[0, \varepsilon]}\right) & =\sum_{k \geq 1} n_{k}^{-}\left(\gamma_{0}, \gamma_{1}, t_{0}\right) \\
\mu\left(\left.\gamma_{0}\right|_{[-\varepsilon, \varepsilon]},\left.\gamma_{1}\right|_{[-\varepsilon, \varepsilon]}\right) & =\sum_{k \geq 1} \sigma_{2 k-1}\left(\gamma_{0}, \gamma_{1}, t_{0}\right) .
\end{aligned}
$$

No caso em que $\gamma_{0}, \gamma_{1}$ são curvas analíticas reais, temos o seguinte resultado, o qual decorre imediatamente da definição 1.7.17 e do teorema 1.7.9.

Trorema 1.7.19 Seja $\gamma_{0}, \gamma_{1}:[a, b] \rightarrow \Lambda$ um par de Fredholm de curvas analiticas reais, tal que a imagem de $\gamma_{0} \oplus \gamma_{1}$ não esteja contida em $\Delta$. Então,

$$
\mu\left(\gamma_{0}, \gamma_{1}\right)=\sum_{\substack{a<t<b \\ \gamma_{0}(t) \cap \gamma_{1}(t) \neq 0}}\left[\sum_{k \geq 1} \sigma_{2 k-1}\left(\gamma_{0}, \gamma_{1}, t_{0}\right)\right]+\sum_{k \geq 1}\left[n_{2 k}^{-}\left(\gamma_{0}, \gamma_{1}, a\right)+n_{2 k-1}^{+}\left(\gamma_{0}, \gamma_{1}, a\right)\right]-\sum_{k \geq 1} n_{k}^{-}\left(\gamma_{0}, \gamma_{1}, b\right) .
$$


. 


\section{Capítulo 2}

\section{Problemas de Fronteira e Operadores de Dirac}

\subsection{Preliminares}

Nesta seção, estabeleceremos os fatos básicos imprescindíveis para o estudo de problemas de fronteira em variedades com bordo. Consideraremos operadores pseudo-diferenciais e espaços de Sobolev em variedades, dando ênfase especial à classe dos operadores elípticos e suas principais propriedades. Também mostraremos que é válida a fórmula de GreenStokes, a qual é uma ferramenta indispensável para o nosso trabalho. A seção encerra com a construção do projetor de Calderón associado a um operador elíptico.

Fixemos algumas notações. Dada uma $n$-upla de inteiros não-negativos $\alpha=\left(\alpha_{1}, \ldots, \alpha_{n}\right)$, colocamos $|\alpha|=\alpha_{1}+\ldots+\alpha_{n}, \alpha !=\alpha_{1} ! \cdot \ldots \cdot \alpha_{n}$ ! e para $\xi \in \mathbb{R}^{n}$, definimos $\xi^{\alpha}=\xi_{1}^{\alpha_{1}} \cdot \ldots$. $\xi_{n}^{\alpha_{n}}$. Dadas coordenadas locais $x_{1}, \ldots, x_{n}$ em uma variedade $X$, definimos $i^{|\alpha|} D^{\alpha} \doteq \partial^{|\alpha|} / \partial x^{\alpha} \doteq$ $\partial^{|\alpha|} / \partial x_{1}^{\alpha_{1}} \ldots \partial x_{n}^{\alpha_{n}}$, para qualquer $\alpha \in \mathbb{Z}_{+}^{n}$. O produto interno de vetores $x, y \in \mathbb{R}^{n}$ será denotado por $x \cdot y$. Usaremos as letras $X, Y$ para denotar variedades riemannianas paracompactas com base enumerável. Os fibrados tangente e cotangente de uma variedade $X$ serão denotados por $T X$ e por $T^{*} X$, respectivamente. Toda vez que usarmos o termo variedade, subentenderemos tratar-se de uma variedade paracompacta com base enumerável. Dado um fibrado vetorial $E$ sobre uma variedade $X$, a dimensão de $E, \operatorname{dim} E$, é a dimensão de qualquer de suas fibras como espaço vetorial. Se $f: X^{\prime} \rightarrow X$ é uma aplicação contínua, denotamos por $f^{*}(E)$ o pull-back de $E$ por $f$, i.e., o fibrado cuja fibra em $x^{\prime} \in X^{\prime}$ é $E_{f\left(x^{\prime}\right)}$. Uma métrica riemanniana sobre $E$ é uma seção $C^{\infty}$ do fibrado $\operatorname{Met}(E)$ sobre $X$, cuja fibra em $x \in X$ é o cone positivo formado por todas os produtos internos definidos sobre $E_{x}$. Caso $E$ seja complexo, a fibra de $\operatorname{Met}(E)$ sobre $x \in X$ é o cone positivo formado por todos os produtos internos hermitianos sobre $E_{x}$. Em qualquer caso, um fibrado vetorial munido de uma métrica riemanniana será chamado de fibrado riemanniano.

Dado um fibrado riemanniano $E$ sobre uma variedade $X$, denotamos por $C^{k}(X ; E)$ o espaço de seções de $E$ de classe $C^{k}$. Quando o contexto estiver claro, indicaremos $C^{k}(X ; E)$ simplesmente por $C^{k}(E)$. O subescrito $c$ será usado para denotar seções de suporte compacto, assim, por exemplo, $C_{c}^{\infty}(E)$ denota o espaço de seções $C^{\infty}$ de $E$ com suporte compacto. $O$ espaço de seções $C^{\infty}$ de $E$ com suporte contido no compacto $K \subset X$ é denotado por $C_{c}^{\infty}(K ; E)$. Consideraremos também o espaço $\mathscr{D}^{\prime}(X ; E)$ de seções distribucionais de $E$, i.e., o espaço dos funcionais lineares $u: C_{c}^{\infty}(E) \rightarrow \mathbb{C}$ contínuos com respeito à topologia do limite indutivo em $C_{c}^{\infty}(E)$, 
conforme [80]. Equivalentemente, ${ }^{1}$ os elementos de $\mathscr{D}^{\prime}(X ; E)$ são tais que, em abertos coordenados restritos aos quais $E$ é trivial, fornecem distribuições a valores em $\mathbb{R}^{p}, \operatorname{com} p=\operatorname{dim} E$.

\subsubsection{Operadores Pseudo-Diferenciais}

Esta subseção apresenta a teoria básica de operadores pseudo-diferenciais em variedades e estabelece alguns resultados importantes para o estudo de equações diferenciais parciais. Todos os detalhes sobre as afirmações a seguir podem ser encontrados em [40], [46], [60] e [71]. Trabalharemos com fibrados complexos, mas, obviamente, todos os resultados e definições são válidos no caso real.

Definıção 2.1.1 Dados fibrados riemannianos $E, F$ de dimensões $p, q$, respectivamente, sobre uma variedade $X$, um operador diferencial de ordem $m$ em $X$ é qualquer operador linear $P$ : $C^{\infty}(E) \rightarrow C^{\infty}(F)$ com a seguinte propriedade: cada ponto de $X$ tem uma vizinhança aberta $U$ tal que $E, F$ restritos a $U$ são triviais e, em $U, P$ é representado por uma matriz

$$
P=\left(P_{j k}\right)_{1 \leq j \leq q, 1 \leq k \leq p},
$$

onde cada $P_{j k}$ é um operador diferencial escalar (i.e., que age em funções escalares) em $U$. Cada $P_{j k}$ tem ordem $\leq m$ e algum deles tem ordem exatamente $m$.

Se $X$ é um aberto de $\mathbb{R}^{n}, E=X \times \mathbb{C}^{p}, F=X \times \mathbb{C}^{q}$ e $P: C^{\infty}(E) \rightarrow C^{\infty}(F)$ é um operador diferencial então a aplicação

$$
\sigma(P): X \times \mathbb{R}^{n} \ni(x, \xi) \mapsto\left(\sigma\left(P_{j k}\right)\right)_{j, k} \in \mathscr{L}\left(\mathbb{C}^{p}, \mathbb{C}^{q}\right),
$$

onde $\sigma\left(P_{j k}\right)$ é o símbolo do operador $P_{j k}$, é chamada de símbolo de $P$ (ou símbolo total de $P$ ). O símbolo principal de $P, \sigma_{m}(P)$, é definido como na equação 2.1.1, trocando $\sigma\left(P_{j k}\right)$ por $\sigma_{m}\left(P_{j k}\right)$, o símbolo principal de ordem $m$. A parte principal de $P$ é o operador diferencial $P_{m}$ induzido pelo símbolo principal $\sigma_{m}(P)$.

No caso em que $X$ é uma variedade, a situação é mais delicada. Se $P=\sum_{|\alpha| \leq m} a_{\alpha}(x) D^{\alpha}$ é escalar e efetuamos uma mudança de coordenadas $\tilde{x}=\tilde{x}(x)$ em $X$, então, com relação as novas coordenadas o operador $P$ assume a forma

$$
P=\sum_{|\alpha| \leq m} \tilde{a}_{\alpha}(x) D_{\tilde{x}}^{\alpha}
$$

onde $\tilde{a}_{\alpha}(x)=\sum_{|\alpha|=m} S\left(\alpha_{1}, 1\right) \cdot \ldots \cdot S\left(\alpha_{n}, n\right) a_{\alpha}(x)$ e $S(k, l)=\sum_{i_{1}, \ldots, i_{k}=1}^{n} \frac{\partial \tilde{i}_{i_{1}}}{\partial x_{l}} \cdot \ldots \cdot \frac{\partial \tilde{x}_{i_{k}}}{\partial x_{l}}$ para $|\alpha|=m$. Levando em consideração as fórmulas de transição que a mudança de coordenadas induz no fibrado cotangente, vemos que $\sigma_{m}(P)$ é uma seção bem-definida do fibrado $\operatorname{Hom}\left(\pi^{*} E, \pi^{*} F\right)$. O mesmo vale para o caso não-escalar.

Dado um operador diferencial $P: C^{\infty}(E) \rightarrow C^{\infty}(F)$, com $X \subset \mathbb{R}^{n}$ aberto, usando o teorema de Stokes, vemos que existe um único operador diferencial $P^{*}: C^{\infty}(F) \rightarrow C^{\infty}(E)$ tal que

$$
\int_{X} P u \cdot v d x=\int_{X} u \cdot P^{*} v d x
$$

\footnotetext{
${ }^{1}$ Ou seja, dado $K \subset X$ compacto existem $N \in \mathbb{Z}_{+}$e $C_{K}>0$ tais que $|u(\varphi)| \leq C_{K} \sum_{|a| \leq N}$ sup $\left|\partial^{\alpha} \varphi\right|$ para toda $\varphi \in \mathrm{C}_{c}^{\infty}(K, E)$.
} 
para todas $u \in \mathrm{C}_{c}^{\infty}(E), v \in \mathrm{C}_{c}^{\infty}(F)$. Localmente, o operador $P^{*}$ tem a forma

$$
\left.P^{*}=\left(P_{k j}^{*}\right)_{k, j}\right),
$$

onde $P_{k j}^{*}$ denota o adjunto de $P_{k j}$. O operador $P^{*}$ é chamado adjunto de $P$.

$O$ conceito de operador pseudo-diferencial baseia-se na seguinte observação. Dado $P$ : $C^{\infty}(E) \rightarrow C^{\infty}(F)$ um operador diferencial definido no aberto $X \subset \mathbb{R}^{n}$ e $u \in C_{c}^{\infty}(E)$, pelo teorema de inversão de Fourier temos

$$
P u(x)=(2 \pi)^{-n} \int_{\mathbb{R}^{n}} e^{i x \cdot \xi} p(x, \xi) \mathfrak{u}(\xi) d \xi,
$$

onde $p(x, \xi)$ denota o símbolo de $P$. Um operador pseudo-diferencial em $X$ é um operador definido pela fórmula 2.1.2, onde permitimos que $p$ seja uma função arbitrária em $X \times \mathbb{R}^{n}$ que se comporte como uma função homogênea na variável $\xi$.

Definição 2.1.2 Se $X \subset \mathbb{R}^{n}$ é aberto, $E=X \times \mathbb{C}^{p}, F=X \times \mathbb{C}^{q}$ e $m \in \mathbb{R}$, dizemos que uma aplicação $p: X \times \mathbb{R}^{n} \rightarrow \mathscr{L}\left(\mathbb{C}^{p}, \mathbb{C}^{q}\right)$, de classe $\mathrm{C}^{\infty}$, com suporte compacto em $x$ para cada $\xi$ fixado, é um símbolo de ordem $m$ se dados $\alpha, \beta \in \mathbb{Z}_{+}^{n}$, existe $C_{\alpha, \beta}>0$ tal que

$$
\left|D_{x}^{\alpha} D_{\xi}^{\beta} p(x, \xi)\right| \leq C_{\alpha, \beta}(1+|\xi|)^{m-|\beta|},
$$

para todos $x \in X, \xi \in \mathbb{R}^{n}$. O conjunto dos símbolos de ordem $m$ é denotado por $S^{m}(E, F)$. Definimos $S^{-\infty}(E, F) \doteq \bigcap_{m \in \mathbb{R}} S^{m}(E, F)$ e $S^{\infty}(E, F) \doteq \bigcup_{m \in \mathbb{R}} S^{m}(E, F)$

OBSERVAÇÃo 2.1.3 A hipótese sobre o suporte de um símbolo na variável $x$ nãoé muito comum, mas evita alguns percalços, principalmente para definirmos a composição de operadores pseudo-diferenciais. Qualquer símbolo possui esta propriedade módulo $S^{-\infty}(E, F)$, conforme [40].

A próxima proposição mostra que, de fato, símbolos de ordem $m$ definem operadores lineares $\mathrm{C}_{c}^{\infty}(X, E) \rightarrow \mathrm{C}_{c}^{\infty}(X, F)$.

Proposição 2.1.4 Dado $p \in S^{m}(E, F)$ então a fórmula 2.1.2 define um operador linear $P: \mathrm{C}_{c}^{\infty}(E) \rightarrow$ $\mathrm{C}_{c}^{\infty}(F)$ que pode ser estendido continuamente a um operador $P: \mathscr{D}^{\prime}(X ; E) \rightarrow \mathscr{D}^{\prime}(X ; F)$.

Um operador pseudo-diferencial de ordem $m$ em um aberto $X \subset \mathbb{R}^{n}$ é um operador dado pela fórmula 2.1.2, com $p \in S^{m}(E, F)$. Dizemos que $P$ é suavizante se $p \in S^{-\infty}(E, F)$. Denotamos por $\Psi^{m}(E, F)$ o conjunto dos operadores pseudo-diferenciais de ordem $m$ em $X$ e por $\Psi^{\infty}(E, F)$ a reunião de todos os $\Psi^{m}(E, F)$ para $m \in \mathbb{R}$. Os espaços $S^{m}(E, F)$ são espaços de Frechét, munidos das seminormas $\varrho_{\alpha, \beta, m}(p)$, definidas como o ínfimo $\operatorname{dos} C_{\alpha, \beta}>0$ para os quais 2.1.3 é verificada.

Não é verdade em geral que existe uma correspondência biunívoca entre símbolos e operadores pseudo-diferenciais, ou seja, símbolos distintos podem produzir o mesmo operador pseudo-diferencial. Apesar disso, dado $P \in \Psi^{\infty}(E, F)$, o símbolo total $\sigma(P)$ de $P$ é bemdefinido módulo $S^{-\infty}(E, F)$. Uma maneira muito comum de construir símbolos para operadores pseudo-diferenciais é utilizar expansões assintóticas. O método consiste em mostrar que dados símbolos $p_{j} \in S^{m_{j}}(E, F)$ com $m_{j} \rightarrow-\infty$, existe $p \in S^{m_{0}}(E, F)$, único módulo $S^{-\infty}(E, F)$, tal que $p \sim \sum_{j=0}^{\infty} p_{j}$, i.e., $p-\sum_{j<k} p_{j} \in S^{m_{k}}(E, F)$, para todo $k \in \mathbb{N}$. Este método é especialmente útil na construção de uma parametriz para um operador elíptico, como veremos mais adiante. 
Os operadores pseudo-diferenciais são bem comportados com respeito a mudanças de coordenadas. De fato, se $E, E^{\prime}$ são fibrados hermitianos sobre abertos $X, X^{\prime} \subset \mathbb{R}^{n}, \Psi: E \rightarrow E^{\prime}$ é um difeomorfismo fibrado (i.e., $\Psi$ é difeomorfismo cuja restrição à cada fibra de $E$ é um isomorfismo linear) e $P \in \Psi^{m}\left(E^{\prime}, F\right)$ é um operador pseudo-diferencial em $X^{\prime}$, então definindo $\left(\Psi^{*} P\right)(\varphi)=P(\Psi \circ \varphi)$ para $\varphi \in C_{c}^{\infty}(E)$, temos que $\Psi^{*} P \in \Psi^{m}(E, F)$ é um operador pseudodiferencial em $X \operatorname{com} \sigma\left(\Psi^{*} P\right)=\sigma(P) \circ \Psi$.

Passemos agora ao caso de variedades. Sejam $X$ uma variedade $n$-dimensional e $E, F$ fibrados hermitianos sobre $X$ de dimensões $p, q$, respectivamente. Sejam $\mathscr{U}=\{U\}$ uma cobertura aberta de $X, \mathscr{A}_{E}=\left\{\Phi_{E}\right\}, \mathscr{A}_{F}=\left\{\Phi_{F}\right\}$ famílias de difeomorfismos fibrados $\Phi_{E}:$ $\pi_{E}^{-1}(U) \rightarrow \mathbb{R}^{n} \times \mathbb{C}^{p}, \Phi_{F}: \pi_{F}^{-1}(U) \rightarrow \mathbb{R}^{n} \times \mathbb{C}^{q}$ definidos para todo $U \in \mathscr{U}$, onde $\pi_{E}, \pi_{F}$ denotam as projeções de $E, F$, respectivamente, e tomemos uma partição da unidade $\left\{\theta_{F}\right\}$ subordinada à cobertura $\mathscr{U}$. $\operatorname{Para}^{2}$ cada $U \in \mathscr{U}$, podemos definir aplicações

$$
\begin{aligned}
\left(\Phi_{F}\right)_{*}: C_{c}^{\infty}(U, F) \ni \varphi & \mapsto \varphi \circ \Phi_{F}^{-1} \in C_{c}^{\infty}\left(\mathbb{R}^{n},\left(\Phi_{F}^{-1}\right)^{*}\left(\left.F\right|_{U}\right)\right), \\
\left(\Phi_{E}\right)^{*}: C_{c}^{\infty}\left(\mathbb{R}^{n},\left(\Phi_{E}^{-1}\right)^{*}\left(\left.E\right|_{U}\right)\right) \ni \varphi & \mapsto \varphi \circ \Phi_{E} \in C_{c}^{\infty}(U, E)
\end{aligned}
$$

Estas aplicações são úteis para definir operadores pseudo-diferenciais em variedades.

DeFINIÇÃo 2.1.5 Usando a notação do parágrafo anterior, dizemos que um operador linear $P: C_{c}^{\infty}(E) \rightarrow C_{c}^{\infty}(F)$ é um operador pseudo-diferencial de ordem $m$ se para cada par $\Phi_{E} \in \mathscr{A}_{E}$, $\Phi_{F} \in \mathscr{A}_{F}$, o operador

$$
\varphi \in C_{c}^{\infty}\left(\mathbb{R}^{n}, \mathbb{R}^{n} \times \mathbb{C}^{p}\right) \mapsto\left(\Phi_{F}\right)_{*} \theta_{F} P\left(\Phi_{E}\right)^{*}(\varphi) \in C_{c}^{\infty}\left(\mathbb{R}^{n}, \mathbb{R}^{n} \times \mathbb{C}^{q}\right)
$$

pertence a $\Psi^{m}\left(\mathbb{R}^{n} \times \mathbb{C}^{p}, \mathbb{R}^{n} \times \mathbb{C}^{q}\right)$.

Se $P \in \Psi^{m}(E, F)$, então o símbolo $\sigma(P)$ é bem-definido módulo $S^{m-1}(E, F)$ (como no caso euclidiano) e é uma seção do fibrado $\operatorname{Hom}\left(\pi^{*} E, \pi^{*} F\right)$, onde $\pi: T^{*} X \rightarrow X$ denota a projeção canônica. O símbolo principal de $P$ é a classe de equivalência de $\sigma(P)$ em $S^{m}(E, F) / S^{m-1}(E, F)$.

Se $P \in \Psi^{m}(E), Q \in \Psi^{l}(E)$ então a composição $P Q \in$ é bem-definida e o símbolo de $P Q$ admite a expansão assintótica

$$
\sigma(P Q) \sim \sum_{\alpha \in \mathbb{Z}_{+}^{n}} \frac{i^{|\alpha|}}{\alpha !} D_{\xi}^{\alpha} p D_{x}^{\alpha} q,
$$

onde $p, q$ denotam os símbolos totais de $P, Q$, respectivamente. Em particular, $\sigma(P Q)=\sigma(P) \sigma(Q)$ módulo $S^{m+l-1}(E)$. Como no caso de operadores diferenciais, podemos considerar o adjunto de um operador pseudo-diferencial. Este operador admite a expansão assintótica

$$
\sigma\left(P^{*}\right) \sim \sum_{\alpha \in \mathbb{Z}_{+}^{n}} \frac{i^{|\alpha|}}{\alpha !} D_{\xi}^{\alpha} D_{x}^{\alpha} p^{*},
$$

onde $p$ denota o símbolo de $P$. Dizemos que $P$ é (formalmente) auto-adjunto se $P^{*}=P$.

Ao contrário de um operador diferencial, em geral, um operador pseudo-diferencial não é local, no sentido que supp $P u \subset \operatorname{supp} u$, mas a próxima proposição mostra que uma propriedade de pseudo-localidade é verificada para operadores pseudo-diferenciais.

\footnotetext{
${ }^{2}$ Aqui e na definição $2.1 .5, \mathbb{R}^{n} \times \mathbb{C}^{p}$ e $\mathbb{R}^{n} \times \mathbb{C}^{q}$ são considerados fibrados triviais sobre $\mathbb{R}^{n}$.
} 
Proposição 2.1.6 Se $P \in \Psi^{\infty}(E, F)$, então $P$ é pseudo-local, i.e., sing supp $P u \subset$ sing supp $u$ para ${ }^{3}$ toda $u \in \mathscr{D}^{\prime}(X, E)$.

Vamos agora introduzir uma classe muito importante de operadores pseudo-diferenciais.

DefinıÇão 2.1.7 Dados fibrados triviais $E=X \times \mathbb{C}^{p}, F=X \times \mathbb{C}^{q}$ sobre o aberto $X \subset \mathbb{R}^{n}$, um operador $P \in \Psi^{m}(E, F)$ com símbolo $p \in S^{m}(E, F)$ é dito elíptico se $p(x, \xi): \mathbb{C}^{p} \rightarrow \mathbb{C}^{q}$ é inversível para todos $x \in X \mathrm{e} \xi \in \mathbb{R}^{n}$ e dado $K \subset X$ compacto existe $C_{K}>0$ tal que $\left|p(x, \xi)^{-1}\right| \leq C_{K}(1+|\xi|)^{-m}$ para $x \in K$ e $\xi \in \mathbb{R}^{n}$. No caso que $X$ é variedade, $P$ é elíptico se o operador 2.1.4 é elíptico para todo $U \in \mathscr{U}$. Um símbolo $p \in S^{m}(E, F)$ é dito elíptico se o operador pseudo-diferencial $P$ induzido por $p$ (conforme a proposição 2.1.4) é elíptico.

Dizemos que um operador $Q \in \Psi^{-m}(F, E)$ é uma parametriz para $P \in \Psi^{m}(E, F)$ se $P Q-I$ e $Q P-I$ são operadores suavizantes. Podemos pensar uma parametriz para um operador como um inverso módulo operadores suavizantes. O próximo teorema é fundamental na teoria de operadores elípticos.

Teorema 2.1.8 Se Xé uma variedade compacta sem bordo, então $P \in \Psi^{m}(E, F)$ é elíptico em $X$ se e só se $P$ admite uma parametriz $Q \in \Psi^{-m}(F, E)$.

\subsubsection{Espaços de Sobolev}

Nesta subseção, vamos introduzir os espaços de Sobolev em variedades compactas. Lembramos que, dado $s \in \mathbb{R}$, definimos

$$
\mathrm{H}^{s}\left(\mathbb{R}^{n}\right) \doteq\left\{u \in \mathscr{S}^{\prime}\left(\mathbb{R}^{n}\right):\|u\|_{s}^{2} \doteq \int\left(1+|\xi|^{2}\right)^{s / 2}|\hat{u}(\xi)|^{2} d \xi<\infty\right\},
$$

onde $\hat{u}$ denota a transformada de Fourier de $u \in \mathscr{S}^{\prime}\left(\mathbb{R}^{n}\right)$ e $\mathscr{S}^{\prime}\left(\mathbb{R}^{n}\right)$ é o espaço usual de distribuições temperadas em $\mathbb{R}^{n}$. Os espaços $\mathrm{H}^{s}(E)$ formam uma escala de espaços de Banach $\left\{\mathrm{H}^{s}\left(\mathbb{R}^{n}\right)\right\}_{s \in \mathbb{R}}$ tal que as inclusões $\mathrm{H}^{s}\left(\mathbb{R}^{n}\right) \subset \mathrm{H}^{t}\left(\mathbb{R}^{n}\right)$ são compactas para $s>t$, pelo lema de Rellich. Pelo teorema de Plancherel segue que $\mathrm{H}^{0}\left(\mathbb{R}^{n}\right)=\mathrm{L}^{2}\left(\mathbb{R}^{n}\right)$. É fácil ver que se $s=m \in \mathbb{Z}_{+}$ então a norma $m$ é equivalente à norma $\|u\|_{m}^{\prime 2}=\sum_{|\alpha| \leq m}\left\|D^{\alpha} u\right\|_{0}^{2}$.

No caso de um aberto $X \subset \mathbb{R}^{n}$ e $s=m \in \mathbb{Z}_{+}$, definimos

$$
\mathrm{H}^{m}(X) \doteq\left\{u \in \mathscr{D}^{\prime}(X): D^{\alpha} u \in \mathrm{L}^{2}(X) \text { para }|\alpha| \leq m \text { e }\|u\|_{m}^{2} \doteq \sum_{|\alpha| \leq m} \int_{X}\left|D^{\alpha} u\right|^{2} d x<\infty\right\} .
$$

Como antes, $\left\{\mathrm{H}^{m}(X)\right\}_{m \in \mathbf{Z}_{+}}$também define uma escala de espaços de Banach com as respectivas inclusões compactas. Decorre diretamente da definição de $\mathrm{H}^{m}(X)$ em 2.1.7 que $\mathrm{H}^{0}(X)=\mathrm{L}^{2}(X)$.

Se $E=X \times \mathbb{C}^{p}$ é um fibrado trivial sobre o aberto $X \subset \mathbb{R}^{n}$, podemos definir espaços de Sobolev de seções de $E$. Basta nas equações 2.1.6 e 2.1.7 considerar distribuições valoradas em $\mathbb{C}^{p}$ ao invés de distribuições escalares. Tais espaços serão denotados por $\mathrm{H}^{m}(X ; E)$ ou $\mathrm{H}^{m}(E)$ quando o papel de $X$ for implícito.

Podemos também definir espaços de Sobolev $\mathrm{H}^{s}(E)$ com $X$ uma variedade compacta sem bordo e $E$ um fibrado riemanniano sobre $X$. De fato, escolhamos uma família $\mathscr{A}_{E}$ como nos

\footnotetext{
${ }^{3} \mathrm{O}$ suporte singular de $u \in \mathscr{D}^{\prime}(X, E)$ é definido como o complemento do maior aberto restrito ao qual $u$ é de classe $\mathrm{C}^{\infty}$.
} 
comentários que antecedem a definição 2.1.5. Como $X$ é compacta, podemos supor que $\mathscr{A}_{E}=\left\{\Phi_{1}, \ldots, \Phi_{N}\right\}$. Seja $\left\{\chi_{j}\right\}_{j=1}^{N}$ uma partição da unidade subordinada à cobertura formada pelos abertos $U_{j}$ correspondentes. Dado $s \in \mathbb{R}$, definimos $\mathrm{H}^{s}(E)$ como o espaço das $u \in \mathscr{D}^{\prime}(X ; E)$ tais que $\Phi_{j} \circ\left(\chi_{j} \cdot \Phi_{j_{*}}(u)\right) \in \mathrm{H}^{s}\left(\mathbb{R}^{n} ;\left(\Phi_{j}^{-1}\right)^{*}\left(\left.E\right|_{U_{j}}\right)\right)=\mathrm{H}^{s}\left(\mathbb{R}^{n} ; \mathbb{R}^{n} \times \mathbb{C}^{p}\right)$, para $j=1, \ldots, N$, munido da norma

$$
\|u\|_{s}^{2}=\sum_{j=1}^{N}\left\|\Phi_{j} \circ\left(\chi_{j} \cdot \Phi_{j_{*}}(u)\right)\right\|_{s}^{2} .
$$

O espaços $\mathrm{H}^{s}(E)$ definidos desta forma têm propriedades inteiramente análogas aos correspondentes definidos em abertos de $\mathbb{R}^{n}$. Além disso, a estrutura de espaço de Banach de $\mathrm{H}^{s}(E)$ independe da cobertura e da partição da unidade utilizadas.

Podemos também considerar os espaços $C^{k}(E)$ de seções $C^{k}$ de $E$ munidos da norma

$$
\|u\|_{k}^{2}=\sum_{j=1}^{N}\left\|\Phi_{j} \circ\left(\chi_{j} \cdot \Phi_{j_{*}}(u)\right)\right\|_{\mathrm{C}^{k}}^{2},
$$

onde $\|v\|_{C^{k}}=\sup _{\substack{|\alpha| \leq k \\ x \in \mathbb{R}^{n}}}\left|D^{\alpha} v(x)\right|$, para $v$ seção de suporte compacto de um fibrado trivial sobre $\mathbb{R}^{n}$. A próxima proposição relaciona os espaços $\mathrm{H}^{s}(E)$ e $C^{k}(E)$.

Proposição 2.1.9 (Lema de Sobolev) Se $\operatorname{dim} X=n$ e $s>k+(n / 2)$ então a inclusão $\mathrm{H}^{s}(E) \subset C^{k}(E)$ é contínua.

De maneira análoga ao caso em que $X$ é um aberto de $\mathbb{R}^{n}$, também podemos definir os espaços $\mathrm{H}^{m}(E)$ se $X$ é uma variedade com bordo $Y$ e $E$ um fibrado vetorial hermitiano sobre $X$. Neste caso, temos um importante teorema, o qual nos dá informação sobre o valor de fronteira, ou traço de $u \in \mathrm{H}^{m}(E)$, cuja prova pode ser encontrada em [98], Cap.III-26.

\section{Teorema 2.1.10 A aplicação}

$$
\left.\mathrm{C}^{\infty}(E) \ni u \mapsto u\right|_{Y} \in \mathrm{C}^{\infty}\left(\left.E\right|_{Y}\right)
$$

estende-se continuamente a uma aplicação sobrejetora $\gamma_{0}: \mathrm{H}^{m}(E) \rightarrow \mathrm{H}^{m-1 / 2}\left(\left.E\right|_{Y}\right)$ para $m>0$.

No caso em que $X$ é uma variedade com bordo, os espaços $\mathrm{H}^{s}(E)$ foram definidos apenas para $s \in \mathbb{Z}_{+}$. Entretanto, é possível dar uma definição razoável para qualquer $s \in \mathbb{R}$. Seja $\tilde{X}$ o dobro de $X$, i.e., a variedade obtida a partir de duas cópias $X_{+}, X_{-}$de $X$ identificadas ponto-a-ponto pelo bordo $Y$ e $\tilde{E}$ a extensão óbvia de $E$ a $\tilde{X}$. Identificamos $X \operatorname{com} X_{+}$. Como $\tilde{X}$ é uma variedade compacta, podemos definir

$$
\mathrm{H}^{s}(E) \doteq\left\{\left.u\right|_{\text {int } X_{+}}: u \in \mathrm{H}^{s}(\tilde{E})\right\},
$$

onde int $X_{+}$denota o interior de $X_{+}$.

Considerando um colar de $Y$ em $X$, i.e., uma vizinhança aberta $U$ de $Y$ em $X$ difeomorfa ao produto $Y \times[0,1)$, (veja [100]) podemos definir coordenadas $(y, t) \in Y \times[0,1)$ em $U$ tais que $(y, 0)$ é identificado com $y \in Y$. Para $t \in(0,1)$, seja $\gamma_{t}:\left.C^{\infty}(E) \ni u \mapsto u_{t} \doteq u\right|_{\gamma_{X}\{t\}} \in C^{\infty}\left(\left.E\right|_{Y}\right)$ o operador de restrição. O próximo teorema, análogo ao teorema 2.1.10, mostra que para $s>1 / 2$, o operador $\gamma_{0} \doteq \lim _{t \downarrow 0} \gamma_{t}$ estende-se continuamente para $\mathrm{H}^{s}\left(\left.E\right|_{Y}\right)$. Sua prova pode ser encontrada em [25], Cap.11. 
TeоRema 2.1.11 O operador $\gamma_{0}$ admite extensão contínua a $\mathrm{H}^{s}(E)$ se $s>1 / 2$. Mais precisamente, dada $u \in \mathrm{H}^{s}(E)$, com $s>1 / 2$, existe $\gamma_{0}(u) \doteq \lim _{t \downarrow 0} u_{t} \in \mathrm{H}^{s-1 / 2}\left(\left.E\right|_{Y}\right)$ e a aplicação $\gamma_{0}: \mathrm{H}^{s}(E) \rightarrow \mathrm{H}^{s-1 / 2}\left(\left.E\right|_{Y}\right)$ é contínua. Além disso, $\gamma_{0}$ é sobrejetora.

Observação 2.1.12 Vemos que a definição de $\mathrm{H}^{s}(E)$ para $s>1 / 2$ qualquer coincide com a definição dada para $s \in \mathbb{Z}_{+}$. De fato, qualquer $u \in \mathrm{H}^{m}(E)$ se estende a uma seção de $\tilde{E}$ definida em $\tilde{X}$, conforme [98], Cap.III-26.

OBSERVAÇão 2.1.13 Às vezes, para estudar regularidade de funções (em especial, de soluções de equações diferenciais parciais), utiliza-se os espaços de potenciais de Bessel ao invés dos espaços de Sobolev, como, por exemplo, em [91]. Os teoremas usuais de extensão e traço são válidos também nesta situação e a teoria assim obtida é mais rica do ponto de vista de análise harmônica. Mais detalhes podem ser encontrados em [96].

OBSERVAÇão 2.1.14 A rigor, a aplicação $\gamma_{0}: \mathrm{H}^{s}(E) \rightarrow \mathrm{H}^{s-1 / 2}\left(\left.E\right|_{Y}\right)$ é bem-definida apenas para $s>1 / 2$, e portanto, o adjunto $\gamma_{0}^{*}: \mathrm{H}^{s}(E) \rightarrow \mathrm{H}^{s+1 / 2}\left(\left.E\right|_{Y}\right)$ é bem-definido para $s>0$. No entanto, usando a dualidade entre os espaços $\mathrm{H}^{s}$, (i.e., $\mathrm{H}^{-s}$ identifica-se canonicamente com o dual de $\mathrm{H}^{s}$ ) podemos definir $\gamma_{0}^{*}: \mathrm{H}^{s}\left(\left.E\right|_{Y}\right) \rightarrow \mathrm{H}^{s-1 / 2}(E)$ para todo $s \in \mathbb{R}$.

Também é possível definir um operador de extensão $\Xi: \mathrm{H}^{\mathrm{s}}\left(\left.E\right|_{Y}\right) \rightarrow \mathrm{H}^{s+1 / 2}(E)$, para $s>1 / 2$. De fato, usando as coordenadas $(y, t)$ em $U$, dadas $g \in C^{\infty}(Y)$ com suporte em um aberto coordenado de $Y, \chi \in C_{c}^{\infty}\left(\mathbb{R}^{n}\right) \operatorname{com} \int \chi=1$ e $\zeta \in C_{c}^{\infty}(\mathbb{R})$ tal que $\zeta(t)=0$ se $|t|>1$ e $\zeta(t)=1$ se $|t|<1 / 2$, definimos

$$
\Xi g(y, t)=\zeta(t) \int_{\mathbb{R}^{n}} \chi(\eta) g(y-t \eta) d \eta,
$$

e $\Xi g=0$ fora de $U$. Usando uma partição da unidade, podemos definir $\Xi g$ para $g \in C^{\infty}\left(\left.E\right|_{Y}\right)$ qualquer. $O$ operador assim obtido também estende-se continuamente a um operador $\Xi$ : $\mathrm{H}^{s}\left(\left.E\right|_{\gamma}\right) \rightarrow \mathrm{H}^{s+1 / 2}(E)$ tal que $\gamma_{0} \circ \Xi=I$, para qualquer $s>0$. Podemos também definir um operador de extensão $\mathrm{H}^{s}\left(\left.E\right|_{X_{+}}\right) \rightarrow \mathrm{H}^{s}(E)$ e um operador de restrição $r: \mathrm{H}^{s}(E) \rightarrow \mathrm{H}^{s}\left(\left.E\right|_{X_{+}}\right)$para todo $s>0$. Usando a dualidade entre os espaços de Sobolev, estes operadores podem ser definidos para todo $s \in \mathbb{R}$. Mais detalhes sobre operadores de extensão e restrição encontramse em [98].

Passemos agora a estudar a regularidade de soluções de equações elípticas utilizando os espaços de Sobolev. A próxima proposição é um primeiro passo nesta direção.

Proposição 2.1.15 Se P é um operador pseudo-diferencial de ordem m sobre X então P é contínuo $\mathrm{H}^{s}(E) \rightarrow \mathrm{H}^{s-m}(E)$.

Caso $P$ seja elíptico, temos o seguinte teorema fundamental.

Teorema 2.1.16 Se $X$ é uma variedade compacta, $E, F$ são fibrados riemannianos sobre $X$ e $P \in$ $\Psi^{m}(E, F)$ é elíptico então:

1. Pé hipoelíptico, i.e., se $u \in \mathscr{D}^{\prime}(X, E)$ e Pué $C^{\infty}$ em $U \subset X$ aberto, então ué $C^{\infty}$ em $U$;

2. $P: \mathrm{H}^{s}(E) \rightarrow \mathrm{H}^{s-m}(E)$ é Fredholm, para todo $s \in \mathbb{R}$. Em particular, pelo item 1, ind $P$ independe da extensão de $P$ utilizada e $\operatorname{ker} P \subset C^{\infty}(E)$ tem dimensão finita; 
3. (Desigualdade de Gårding) Dado $s \in \mathbb{R}$, existe $C_{s}>0$ tal que

$$
\|u\|_{s} \leq C_{s}\left(\|P u\|_{s-m}+\|u\|_{s-m}\right)
$$

para toda $u \in \mathrm{H}^{s}(E)$.

Prova. Seja $Q \in \Psi^{-m}(F, E)$ uma parametriz de $P$, i.e., $Q P=I+R$ e $P Q=I+S$, onde $R$ é um operador suavizante, de acordo com o teorema 2.1.8. Para provar o primeiro ítem, basta observar que se $P u$ é de classe $C^{\infty}$ em $U$, tomando $\varphi \in C_{c}^{\infty}(X)$ identicamente 1 sobre um aberto $U^{\prime} \subset U$ com suporte em uma $\varepsilon$-vizinhança $U_{\varepsilon}^{\prime}$ de $U^{\prime}$, temos que $\varphi u=R \varphi u-Q P \varphi u$ também é $\mathrm{C}^{\infty}$ se $\varepsilon$ é suficientemente pequeno; $\mathrm{em}^{4}$ particular, $u$ é $C^{\infty} \mathrm{em} U^{\prime}$. Como $U^{\prime} \subset U$ é arbitrário, $u$ é $C^{\infty}$ em $U$, provando o ítem 1. Provemos o ítem 2. Como $R$ é suavizante, dado $s \in \mathbb{R}$, temos que $R: \mathrm{H}^{s}(E) \rightarrow \mathrm{H}^{s+1}(E)$ é contínuo, portanto, como a inclusão $\mathrm{H}^{s+1}(E) \subset \mathrm{H}^{s}(E)$ é compacta, considerando $P: \mathrm{H}^{s}(E) \rightarrow \mathrm{H}^{s-m}(E), Q: \mathrm{H}^{s-m}(E) \rightarrow \mathrm{H}^{s}(E)$, temos que $P Q=I+K$ e $Q P=I+L$, onde $K, L: \mathrm{H}^{s}(E) \rightarrow \mathrm{H}^{s}(E)$ são compactos. Pela proposição 1.3.3, temos que $P$ é Fredholm.

Para finalizar, observamos que pela proposição 2.1.15, dado $s \in \mathbb{R}$ existe $C_{s}>0$ tal que $\|Q v\|_{s} \leq C_{s}\|v\|_{s-m}$ e $\|R u\|_{s} \leq C_{s}\|u\|_{s-m}$, para $u, v \in \mathrm{H}^{s-m}(E)$. Logo,

$$
\|u\|_{s}=\|Q P u-R u\|_{s} \leq C_{s}\left(\|P u\|_{s-m}+\|u\|_{s-m}\right),
$$

para toda $u \in \mathrm{H}^{s}(E)$, provando o ítem 3.

OBservação 2.1.17 Em geral, se $X$ é compacta e $P \in \Psi^{s}(E, F)$ é um operador elíptico bijetor, podemos definir o espaço de Sobolev $\mathrm{H}^{s}(E)$ como o espaço das $u \in \mathscr{D}^{\prime}(X ; E)$ tais que $P u \in \mathrm{L}^{2}(E)$ (conforme [28]) munido da norma $\|u\|_{s}^{2} \doteq \int_{X}|P u|^{2} d x$. A desigualdade de Gårding (ítem 3 do teorema 2.1.16) mostra que, de fato, esta definição e a definição que demos no início desta subseção coincidem.

Finalizamos esta subseção com um resultado crucial a respeito da teoria espectral de um operador pseudo-diferencial elíptico auto-adjunto.

Teorema 2.1.18 Seja $P \in \Psi^{m}(E, F), m>0$, um operador elíptico auto-adjunto definido em uma variedade compacta $n$-dimensional sem bordo $X$, onde $E, F$ são fibrados riemannianos sobre $X$. As seguintes afirmações são verdadeiras:

1. Existe um operador compacto $G: \mathrm{H}^{0}(E) \rightarrow \mathrm{H}^{0}(E)$ tal que

$$
G P=I-R,
$$

onde $R$ denota a projeção ortogonal $\mathrm{H}^{0}(E) \rightarrow \operatorname{ker} P$. G é chamado de operador de Green;

2. O espectro de $P$ como operador ilimitado em $\mathrm{H}^{0}(E)$ consiste de uma seqüuencia de autovalores reais $\left\{\lambda_{k}\right\}_{k \in \mathbb{N}}$ de multiplicidade finita, $\operatorname{com} 0 \leq\left|\lambda_{1}\right| \leq \ldots \leq\left|\lambda_{k}\right| \leq \ldots$;

3. Cada autoespaço de $P$ é formado por seções de classe $\mathrm{C}^{\infty}$ e $\mathrm{H}^{0}(E)$ é a soma direta (como espaço de Hilbert) dos autoespaços de P;

\footnotetext{
${ }^{4}$ Dado $\varepsilon>0$, um operador pseudo-diferencial $P$ é equivalente módulo $S^{-\infty}(E, F)$ a um operador $\varepsilon$-local $P^{\prime}$, no sentido que supp $P^{\prime} u$ esta contido em uma $\varepsilon$-vizinhança de supp $u$, conforme [60].
} 
4. Existem $C, \delta>0$ (dependendo somentede $P, X e E$ ) tais que $\left|\lambda_{k}\right| \geq C k^{\delta}$ para todok suficientemente grande;

5. Existe $C^{\prime}>0$ (dependendo somente de $P, X$ e E) tal que

$$
\sum_{|\lambda| \leq t} \operatorname{mul}(\lambda) \leq C^{\prime} t^{n(n+2 m+2) / 2 m}
$$

para todo $t>0$, usando a notação definida nos comentários que antecedem a definição 1.2.49.

Prova. Já observamos no ítem 2 do teorema 2.1.16 que $P: \mathrm{H}^{m}(E) \rightarrow \mathrm{H}^{0}(E)$ é Fredholm, em particular, $\operatorname{Im} P$ é fechada em $\mathrm{H}^{0}(E)$ e portanto, pelos comentários que antecedem a definição 1.2.12 e pela observação 1.2.13, o operador $G \doteq i P^{-1} P_{\operatorname{Im} T}$, onde $i: \mathrm{H}^{m}(E) \rightarrow \mathrm{H}^{0}(E)$ é a inclusão, é um operador bem-definido e compacto que verifica a igualdade 2.1.8. Isso prova o ítem 1. O ítem 2 decorre da igualdade 2.1 .8 e do teorema espectral para operadores compactos auto-adjuntos. $\mathrm{O}$ ítem 3 decorre diretamente da hipoelipticidade de $P$, conforme o teorema 2.1.16. Para a prova dos ítens 4 e 5, consulte [46] e [60].

OBSERVAÇão 2.1.19 O exemplo mais clássico de aplicação do teorema 2.1.18 é o famoso Teorema de Hodge. Este teorema afirma que os ítens 1,2,3 do teorema 2.1 .18 são verdadeiros para o caso do laplaciano $\Delta$ agindo em formas diferenciais. Isso será provado mais adiante, na subseção 2.3.

\subsubsection{A Fórmula de Green-Stokes}

Nesta breve subseção, provaremos a validade da fórmula de Green-Stokes para operadores diferenciais em variedades com bordo. Como no cálculo em várias variáveis, esta fórmula serve para relacionar o comportamento de uma função no interior da variedade com seu comportamento sobre o bordo da mesma. Utilizaremos um argumento semelhante àquele usado por Palais e Seeley em [71], Cap.XVII, para provar a fórmula de Green-Stokes para um operador de ordem $m$ possivelmente maior que 1.

Consideremos uma variedade riemanniana ${ }^{5}$ compacta orientada $X$ com bordo $Y, E, F$ fibrados riemannianos sobre $X$ de dimensões $p, q$ respectivamente e $P: C^{\infty}(E) \rightarrow C^{\infty}(F)$ um operador pseudo-diferencial de ordem $m \geq 0$ em $X$. Seja $U$ um colar de $Y$ em $X$, i.e., uma vizinhança aberta $U$ de $Y$ em $X$ na qual é possível definir um sistema de coordenadas $(y, t) \in Y \times[0,1)$ de maneira que os pontos da forma $(y, 0)$ pertencem a $Y$. Suponhamos que, em $U$, o operador $P$ coincida com um operador diferencial. Sejam $u \in C_{c}^{\infty}(V, E), v \in C_{c}^{\infty}(V, F)$, onde $V$ é uma pequena vizinhança coordenada de um ponto de $Y$. As estruturas métricas de $\left.E\right|_{V}$ e $\left.F\right|_{V}$ podem ser dadas em termos de aplicações $A: V \rightarrow \mathrm{GL}(p), B: V \rightarrow \mathrm{GL}(q)$ tomando valores no conjunto dos operadores positivos. Denotaremos por $g d y d t$ a restrição do elemento de volume de $X$ a $V$.

Podemos escrever em $V$

$$
P=\sum_{j=0}^{m} P_{j}(t) D_{t}^{m-j},
$$

\footnotetext{
${ }^{5} \mathrm{O}$ resultado vale se supusermos apenas a existência de um elemento de volume em $X$.
} 
onde $P_{j}(t)$ é um operador diferencial em $Y \cap V$ de ordem $\leq j$ para $j=0, \ldots, m$. Lembramos que, dadas $f, g:[0,1] \rightarrow \mathbb{C}^{r} \operatorname{com} g(1)=\ldots=g^{(k-1)}(1)=0$, integrando por partes temos

$$
\int_{0}^{1} f^{(k)}(t) \cdot g(t) d t=(-1)^{k} \int_{0}^{1} f(t) \cdot g^{(k)}(t) d t+\sum_{l=0}^{k-1}(-1)^{k-l} f^{(l)}(0) \cdot g^{(k-l-1)}(0),
$$

portanto,

$$
\begin{aligned}
\int_{X} P u \cdot v d x & =\int_{0}^{1} \int_{\mathbb{R}^{n-1}} \sum_{j=0}^{m} B P_{j} D_{t}^{m-j} u \cdot v g d y d t \\
& =\int_{0}^{1} \int_{\mathbb{R}^{n-1}} \sum_{j=0}^{m} D_{t}^{m-j} u \cdot P_{j}^{*} B v g d y d t \\
& =\int_{0}^{1} \int_{\mathbb{R}^{n-1}} u \cdot \sum_{j=0}^{m} D_{t}^{m-j} P_{j}^{*} B v g d y d t- \\
& \quad-\left.\int_{\mathbb{R}^{n-1}} \sum_{j=0}^{m-1} \sum_{l=0}^{m-j-1}\left\{D_{t}^{l} u \cdot D_{t}^{m-l-1}\left(P_{j}^{*} B v\right) g\right\}\right|_{t=0} d y \\
& \int_{X} u \cdot P^{*} v d x-\int_{Y} H R_{0}(u) \cdot R_{0}(v) d y,
\end{aligned}
$$

onde $R_{0}(u)=\left(\gamma_{0}(u), \ldots, \gamma_{m-1}(u)\right)$ e $\left.\gamma_{j}(u) \doteq \frac{\partial^{j} u}{\partial v^{j}}\right|_{t=0}$, com $v$ a normal unitária interior a $X$, é o j-ésimo traço de $u$ em $Y$ (conforme a subseção 2.1.2), $H$ é uma matriz triangular de operadores diferenciais em $Y$ cuja diagonal é formada por termos da forma $A^{-1} P_{0}(0)^{*} B$ e $H R_{0}(u) \cdot R_{0}(v)$ denota simplesmente o produto interno euclidiano entre $H R_{0}(u)$ e $R_{0}(v)$. Neste ponto, seria mais adequado utilizarmos a linguagem de jatos, mas, preferimos manter a notação até agora utilizada.

OBSERVAÇÃo 2.1.20 O caso $m=1$ é de particular interesse. Neste caso, o operador $P$ assume a forma

$$
P=G \frac{\partial}{\partial t}+\sum_{j=1}^{n-1} C_{j} \frac{\partial}{\partial y_{j}}+\text { termos de ordem zero }
$$

em $V$, onde $y_{1}, \ldots, y_{n-1}$ são coordenadas em $Y \cap V, i G(y, t)=\sigma(P)(y, t ; 0, d t)$ e $i C_{j}(y, t)=$ $\sigma(P)\left(y, t ; d y_{j}, 0\right), j=1, \ldots, n-1$. Observando a igualdade 2.1 .9 nesta situação específica, concluímos que

$$
\int_{X} P u \cdot v d x-\int_{X} u \cdot P^{*} v d x=-\int_{Y} G u \cdot v d y,
$$

para $u \in C_{c}^{\infty}(E)$ e $v \in C_{c}^{\infty}(F)$.

O próximo teorema segue diretamente das observações anteriores e da existência de trivializações para os fibrados $E, F$ como nos comentários que antecedem a definição 2.1.5.

Teorema 2.1.21 Seja $X$ uma variedade riemanniana compacta orientada com bordo $Y, E, F$ fibrados vetoriais hermitianos sobre $X$ de dimensões $p, q$ respectivamente e $P: C^{\infty}(E) \rightarrow C^{\infty}(F)$ um operador 
pseudo-diferencial de ordem $m \geq 0$ em $X$ que coincide com um operador diferencial em uma vizinhança de $Y$. Então existe uma matriz triangular de operadores diferenciais $H$ definida em $Y$, cuja diagonal depende somente da parte principal de $P$ tal que

$$
\int_{X} P u \cdot v d x-\int_{X} u \cdot P^{*} v d x=-\int_{Y} H R_{0}(u) \cdot R_{0}(v) d y,
$$

para quaisquer $u \in C^{\infty}(E), v \in C^{\infty}(F)$.

\subsubsection{Operadores Elípticos Bijetores}

Nesta subseção, vamos desenvolver a teoria necessária para estudarmos em grande generalidade os espaços de dados iniciais de problemas de fronteira para operadores elípticos.

Sejam $\tilde{E}, \tilde{F}$ fibrados riemannianos sobre uma variedade $\tilde{X}$ compacta de dimensão $n+1$ sem bordo que se escreve como reunião de variedades $X_{+}, X_{-} \subset \tilde{X}$ com bordo $Y$ tais que $X_{+} \cap X_{-}=Y$ e $P: C^{\infty}(\tilde{E}) \rightarrow C^{\infty}(\tilde{F})$ um operador pseudo-diferencial elíptico de primeira ordem que coincide com um operador diferencial numa vizinhança aberta $U$ de $Y$. Fixando uma estrutura de colar em $U$, i.e., um difeomorfismo $Y \times(-1,1) \rightarrow U$, podemos escrever os pontos de $U$ na forma $(y, t)$, com $y \in Y$ e $t \in(-1,1)$. Definimos $i G(y, t) \doteq \sigma_{1}(P)(y, t ; 0, d t)$ em $U$. Vamos ${ }^{6}$ supor que a função $t$ esteja definida em todo $\tilde{X}, \operatorname{com} t \geq 0$ em $X_{+}$e $t \leq 0$ em $X_{-}$, embora $t$ só funcione como coordenada em $U$.

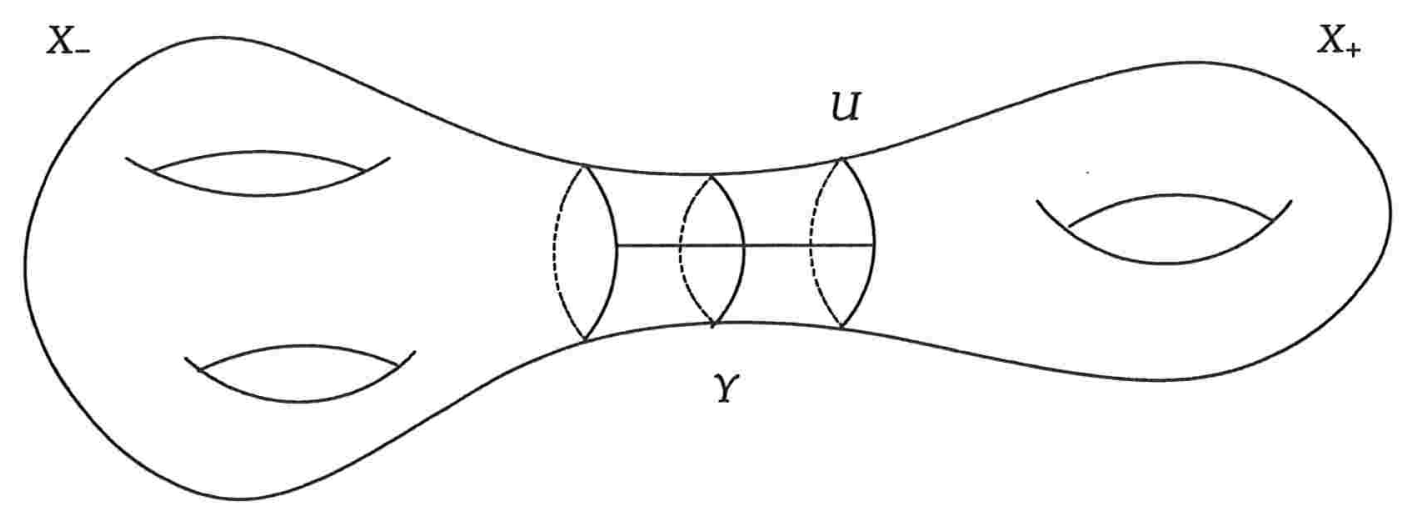

Definimos

$$
H_{ \pm}(P)=\left\{\left.u\right|_{Y}: u \in C^{\infty}\left(\left.\tilde{E}\right|_{X_{ \pm}}\right) \text {e } P u=0 \text { em int } X_{ \pm}\right\} .
$$

Veremos no teorema 2.1.22 que, sob uma hipótese especial, os espaços $H_{+}(P)$ e $H_{-}(P)$ fornecem informações geométricas interessantes sobre o problema de Cauchy para $P$ com dados iniciais em $Y$.

Teоrema 2.1.22 Suponhamos que P seja bijetor. Então as seguintes afirmações são verdadeiras:

1. $H_{+}(P) \cap H_{-}(P)=0$;

2. $P^{-1} \in \Psi^{-1}(\tilde{F}, \tilde{E})$ e tem símbolo principal $\sigma_{1}(P)^{-1}$;

\footnotetext{
${ }^{6}$ iG é o símbolo de $P$ na variável transversal.
} 
3. O operador $\tilde{S} \doteq r P^{-1} \gamma_{0}^{*} G: \mathrm{H}^{s-1 / 2}\left(\left.\tilde{E}\right|_{\gamma}\right) \rightarrow \mathrm{H}^{s}(\tilde{E})$ é limitado, onde $r$ é o operador de restrição dे $X_{+}$(conforme os comentários antecedentes à proposição 2.1.15) e $\gamma_{0}^{*}$ é o adjunto de $\gamma_{0}: \mathrm{H}^{s}(\tilde{E}) \rightarrow$ $\mathrm{H}^{s-1 / 2}\left(\left.\tilde{E}\right|_{\gamma}\right)$ (conforme a observação 2.1.14). Além disso, $P S g=0$ em int $X_{+} ;$

4. Se $g \in C^{\infty}\left(\left.\tilde{E}\right|_{Y}\right)$ então existem

$$
\begin{aligned}
& \tilde{\Pi}_{+} g=\lim _{\varepsilon \downarrow 0} \gamma_{\varepsilon} P^{-1} \gamma_{0}^{*} G g \\
& \tilde{\Pi}_{-} g=-\lim _{\varepsilon \uparrow 0} \gamma_{\varepsilon} P^{-1} \gamma_{0}^{*} G g .
\end{aligned}
$$

e são operadores pseudo-diferenciais de ordem zero em $Y$. O símbolo principal de $\tilde{\Pi}_{+} e m(y, \xi) \in$ $T^{*} Y$ é a projeção espectral sobre a soma dos autoespaços generalizados de $\tilde{E}_{y}$ correspondentes aos autovalores de $-G(y, 0) \sigma_{1}(P)(y, 0 ; \xi, 0)$ com parte imaginária $>0$;

5. $\tilde{\Pi}_{+}+\tilde{\Pi}_{-}=I$.

Prova. Se $u_{ \pm} \in C^{\infty}\left(\left.\tilde{E}\right|_{X_{ \pm}}\right)$são tais que $P u_{ \pm}=0 \mathrm{em} \operatorname{int} X_{ \pm}$e $\left.u_{+}\right|_{Y}=\left.u_{-}\right|_{Y}$, então definindo

$$
u=\left\{\begin{array}{ll}
u_{+} & \text {em } X_{+} \\
u_{-} & \text {em } X_{-}
\end{array},\right.
$$

temos que $u$ é contínua e para qualquer $\varphi \in \mathrm{C}^{\infty}(\tilde{E})$,

$$
\begin{aligned}
\int_{\tilde{X}} P u \cdot \varphi d x & =\int_{\tilde{X}} u \cdot P^{*} \varphi d x \\
& =\int_{X_{+}} u_{+} \cdot P^{*} \varphi d x+\int_{X_{-}} u_{-} \cdot P^{*} \varphi d x \\
& =\int_{X_{+}} P u_{+} \cdot \varphi d x-\int_{Y} G\left(\left.u_{+}\right|_{Y}\right) \cdot \varphi d y+\int_{X_{-}} P u_{-} \cdot \varphi d x+\int_{Y} G\left(\left.u_{-}\right|_{Y}\right) \cdot \varphi d y \\
& =0,
\end{aligned}
$$

pela fórmula de Green-Stokes. Logo, $P u=0$, e como $P$ é bijetor, $u=0$. O ítem 1 está provado.

Para provar o ítem 2, seja $Q \in \Psi^{-1}(\tilde{F}, \tilde{E})$ uma parametriz de $P$, dada pelo teorema 2.1.8. Logo, $Q P=I+R$, onde $R$ é um operador suavizante, e portanto, $P^{-1}=Q-R P^{-1} \in \Psi^{-1}(\tilde{F}, \tilde{E})$.

Provemos o ítem 5, supondo já provado o ítem 4. Dada $g \in C^{\infty}\left(\left.\tilde{E}\right|_{Y}\right)$, pondo $u \doteq P^{-1} \gamma_{0}^{*} G g$, temos que, para qualquer $\varphi \in C^{\infty}(\tilde{E})$,

$$
\int_{Y} G g \cdot \gamma_{0}(\varphi) d y=\int_{\bar{X}} \gamma_{0}^{*} G g \cdot \varphi d x=\int_{\bar{X}} P u \cdot \varphi d x=\int_{\bar{X}} u \cdot P^{*} \varphi d x=\lim _{\varepsilon \downarrow 0} \int_{|t|>\varepsilon} u \cdot P^{*} \varphi d x .
$$

Como $u$ é de classe $C^{\infty}$ em int $X_{+}$e int $X_{-}$(pois, pelo ítem $2, P^{-1}$ é um operador pseudodiferencial de ordem -1 , e portanto, $P^{-1}$ é pseudo-local, conforme a proposição 2.1.6), pela fórmula de Green-Stokes, temos

$$
\lim _{\varepsilon \downarrow 0} \int_{|t|>\varepsilon} u \cdot P^{*} \varphi d x=\lim _{\varepsilon \downarrow 0} \int_{Y} G \gamma_{\varepsilon}(u) \cdot \gamma_{\varepsilon}(\varphi) d y-\int_{Y} G \gamma_{-\varepsilon}(u) \cdot \gamma_{-\varepsilon}(\varphi) d y=\int_{Y} G\left(\tilde{\Pi}_{+}+\tilde{\Pi}_{-}\right) g \cdot \gamma_{0}(\varphi) d y,
$$

portanto,

$$
\int_{Y} G g \cdot \gamma_{0}(\varphi) d y=\int_{Y} G\left(\tilde{\Pi}_{+}+\tilde{\Pi}_{-}\right) g \cdot \gamma_{0}(\varphi) d y
$$


para toda $\varphi \in C^{\infty}(\tilde{E})$. Como $\gamma_{0}$ é sobre $C^{\infty}\left(\left.\tilde{E}\right|_{\gamma}\right)$, temos que $\Pi_{+}+\Pi_{-}=I$ em $C^{\infty}(\tilde{E})$.

Ainda assumindo provado o ítem 4, provemos o ítem 3. Dada $g \in C^{\infty}\left(\left.\tilde{E}\right|_{\gamma}\right)$ e $g_{0} \doteq \Pi_{+} g$, temos que existe $C_{1}$ (independente de $g$ e $g_{0}$ ) tal que $\left\|g_{0}\right\|_{s-1 / 2} \leq C_{1}\|g\|_{s-1 / 2}$. Se $E: H^{s}\left(\left.\tilde{E}\right|_{Y}\right) \rightarrow$ $\mathrm{H}^{s+1 / 2}(\tilde{E})$ é um operador de extensão, definimos $v \doteq E g_{0} \in \mathrm{H}^{s+1 / 2}(\tilde{E}), u \doteq P^{-1} \gamma_{0}^{*} G g$ e

$$
w=\left\{\begin{array}{l}
u \text { em } X_{+} \\
v \text { em } X_{-}
\end{array} .\right.
$$

Provemos que $w \in \mathrm{H}^{s}(\tilde{E})$. De fato, dada $\varphi \in \mathrm{C}^{\infty}(\tilde{E})$, temos

$$
\begin{aligned}
\int_{\bar{X}} P w \cdot \varphi d x= & \lim _{\varepsilon \downarrow 0} \int_{|t|>\varepsilon} w \cdot P^{*} \varphi d x \\
= & \lim _{\varepsilon \downarrow 0} \int_{|t|>\varepsilon} P w \cdot \varphi d x-\int_{Y} G(y, \varepsilon) \gamma_{\varepsilon}(w) \cdot \gamma_{\varepsilon}(\varphi) d y+ \\
& \quad+\int_{Y} G(y,-\varepsilon) \gamma_{-\varepsilon}(w) \cdot \gamma_{-\varepsilon}(\varphi) d y \\
= & \int_{X_{-}} P w \cdot \varphi d x,
\end{aligned}
$$

pelo ítem 4 e pelo fato que $\gamma_{0}(u)=\gamma_{0}(v)$. Logo, supp $P w \subset X$. Em particular, $\|P w\|_{s-1}=\|P v\|_{s-1}$ e portanto, pela proposição 2.1.15, existem $C_{2}, C_{3}>0$ tais que

$$
\|w\|_{s}=\left\|P^{-1} P w\right\|_{s} \leq C_{2}\|P w\|_{s-1}=C_{2}\|P v\|_{s-1} \leq C_{3}\|v\|_{s} .
$$

Em particular, $w \in \mathrm{H}^{\mathrm{s}}(\tilde{E})$ e existe $C_{5}>0$ tal que

$$
\left\|P^{-1} \gamma_{0}^{*} G g\right\|_{s}=\|u\|_{s} \leq\|w\|_{s} \leq C_{3}\|v\|_{s} \leq C_{3}\|E \mid\| g_{0}\left\|_{s-1 / 2} \leq C_{5}\right\| g \|_{s-1 / 2} ，
$$

como queríamos.

Vamos agora à prova do ítem 4 que é a parte mais delicada do teorema. Daremos apenas a idéia da prova, os detalhes podem ser consultados em [28], [71], Cap.XVII e [91].

É suficiente provar o resultado para o operador $M_{\varphi_{1}} P^{-1} M_{\varphi_{2}} \gamma_{0}^{*} G$, onde $\varphi_{1}, \varphi_{2} \in C^{\infty}(\tilde{E})$ têm suporte em alguma vizinhança aberta coordenada $V$ de $Y$, restrita à qual $\tilde{E}$ é trivial e $M_{\varphi}$ denota o operador de multiplicação por $\varphi$. Se $\operatorname{supp} \varphi_{1} \cap \operatorname{supp} \varphi_{2}=\emptyset$, então $M_{\varphi_{1}} P^{-1} M_{\varphi_{2}} \gamma_{0}^{*} G$ é um operador suavizante ${ }^{8}$, e portanto, trivialmente existe $\lim _{\varepsilon \rightarrow 0^{ \pm}} \gamma_{\varepsilon} M_{\varphi_{1}} P^{-1} M_{\varphi_{2}} \gamma_{0}^{*} G g \stackrel{{ }^{\prime}}{\doteq} \pm \Pi_{ \pm} g$ em $C^{\infty}\left(\left.E\right|_{Y}\right)$ e $\Pi_{ \pm}$são operadores suavizantes. Caso $V \cap Y=\emptyset$, então $M_{\varphi_{1}} P^{-1} M_{\varphi_{2}} \gamma_{0}^{*} G=$ 0 , logo $0=\lim _{\varepsilon \rightarrow 0^{ \pm}} \gamma_{\varepsilon} M_{\varphi_{1}} P^{-1} M_{\varphi_{2}} \gamma_{0}^{*} G g \doteq \pm \Pi_{ \pm} g$. Resta apenas o caso em que $V \cap Y \neq \emptyset$ e $\operatorname{supp} \varphi_{1} \cap \operatorname{supp} \varphi_{2} \neq \emptyset$. Tomemos $\varphi \in C_{c}^{\infty}(V, E)$ tal que $\varphi=1$ em uma vizinhança de supp $\left(\left|\varphi_{1}\right|+\left|\varphi_{2}\right|\right)$. Pelo ítem $2, B=M_{\varphi} P^{-1} M_{\varphi}$ é um operador pseudo-diferencial em $V$ de ordem -1. Se $\sigma(P)=a_{0}+a_{1}$, com $a_{j} \in S^{j}(E, F)$ e $\sigma(B) \sim \sum_{l<-1} b_{l}$, com $b_{l} \in S^{l}(E, F)$ e $B_{l}$ é o operador definido pela fórmula 2.1.2 com $p=b_{l}$, então $M_{\varphi_{1}} P^{-1} M_{\varphi_{2}}-\sum_{l>l_{0}} B_{l}$ tem ordem $\leq l_{0}$. Portanto,

$$
\left(M_{\varphi_{1}} P^{-1} M_{\varphi_{2}}-\sum_{l>l_{0}} B_{l}\right) \gamma_{0}^{*} G: \mathrm{H}^{-1 / 2}\left(\left.\tilde{E}\right|_{\gamma}\right) \rightarrow \mathrm{H}^{-1-l_{0}}(\tilde{E})
$$

\footnotetext{
${ }^{7}$ Conforme os comentários que antecedem a proposição 2.1.15.

${ }^{8}$ Isso porque operadores pseudo-diferenciais possuem núcleo $\mathrm{C}^{\infty}$ fora da diagonal.
} 
é limitado. Pela proposição 2.1.9, se $l_{0}<-1-\frac{n+1}{2}$, a inclusão $\mathrm{H}^{-1-l_{0}}(\tilde{E}) \rightarrow \mathrm{C}^{0}(\tilde{E})$ é contínua. Em particular, existe uniformemente $\lim _{\varepsilon \rightarrow 0^{ \pm}} \gamma_{\varepsilon}\left(M_{\varphi_{1}} P^{-1} M_{\varphi_{2}}-\sum_{l>l_{0}} B_{l}\right) \gamma_{0}^{*} G g$ para toda $g \in \mathrm{H}^{s}\left(\left.\tilde{E}\right|_{\gamma}\right)$. Se $D$ é qualquer operador diferencial e $l_{0}<-1-k-(n+1) / 2$, então o mesmo verifica-se com $\lim _{\varepsilon \rightarrow 0^{ \pm}} \dot{\gamma}_{\varepsilon} D\left(M_{\varphi_{1}} P^{-1} M_{\varphi_{2}}-\sum_{l>l_{0}} B_{l}\right) \gamma_{0}^{*} G g$, para $g \in \mathrm{H}^{s}\left(\left.\tilde{E}\right|_{Y}\right)$.

Portanto, basta provar que

$$
\lim _{\varepsilon \rightarrow 0^{+}} \gamma_{\varepsilon} B_{l} \gamma_{0}^{*} G g
$$

existe uniformemente para $g \in \mathrm{H}^{s}\left(\left.\tilde{E}\right|_{\gamma}\right)$. Para isso, A. Calderón e R. Seeley mostram que o símbolo de $B_{l} \gamma_{0}^{*}$ é dado em $V \cap U$ por

$$
\sigma\left(B_{l} \gamma_{0}^{*}\right)(y, t ; \xi, \tau)=\frac{1}{2 \pi} \int_{\Gamma(\xi)} e^{i t z} b_{l}(y, t ; \xi, z) d z,
$$

onde consideramos a extensão natural de $a_{1}(y, t ; \xi, \tau)$ para $\tau=z \in \mathbb{C}$ (pois $a_{1}$ é linear em $\tau$ ) e $\Gamma(\eta)$ é uma curva em $\{\operatorname{Im} z>0\}$ contendo o conjunto (compacto) dos $z \in \mathbb{C}$ tais que $a_{1}(y, t ; \xi, z)$ não é inversível com $|\xi|=1$ e $(y, t) \in V$, i.e., os pólos de $a_{1}(y, t ; \xi, \cdot)^{-1}$. Neste ponto, é usada a hipótese de elipticidade de $P$ ! No caso de $\tilde{\Pi}_{-}$, tomamos $\Gamma(\xi)$ de maneira análoga, porém no semiplano $\{\operatorname{Im} z<0\}$.

Em particular, existem uniformemente para $g \in \mathrm{H}^{s}\left(\left.\tilde{E}\right|_{Y}\right)$ os limites em 2.1.12. Argumentando como antes, vemos que também existem uniformemente os limites 2.1.12 com $\gamma_{\varepsilon} D$ em lugar de $\gamma_{\varepsilon}$. Portanto, são bem definidos os operadores $\tilde{\Pi}_{+}, \tilde{\Pi}_{-}$em 2.1.10 e 2.1.11. Vejamos que $\tilde{\Pi}_{+}, \tilde{\Pi}_{-}$são operadores pseudo-diferenciais de ordem zero. De fato, dada $g \in C^{\infty}\left(\left.E\right|_{Y}\right)$ suportada em $V \cap Y$, temos que

$$
\lim _{\varepsilon \downarrow 0} \gamma_{\varepsilon} B_{l} \gamma_{0}^{*} G g(y)=(2 \pi)^{-n} \int_{\mathbb{R}^{n}} e^{i y \cdot \xi} \hat{g}(\xi)\left\{(2 \pi)^{-1} \int_{\Gamma(\xi)} a_{1}(y, 0 ; \xi, z) G g(y) d z\right\} d \xi .
$$

Como $a_{1}(y, 0 ; \xi, z)=i z G(y, 0)+i \sigma_{1}(P)(y, 0 ; \xi, 0)$, concluímos que o símbolo principal de $\tilde{\Pi}_{+}$em $(y, 0 ; \xi, 0)$ é dado por

$$
\frac{1}{2 \pi i} \int_{\Gamma(\xi)}\left(\tau G(y, 0)+\sigma_{1}(P)(y, 0 ; \xi, 0)\right)^{-1} G(y, 0) d z=\frac{1}{2 \pi i} \int_{\Gamma(\xi)}\left(\tau+G(y, 0)^{-1} \sigma_{1}(P)(y, 0 ; \xi, z)\right)^{-1} d z .
$$

Como $\Gamma(\xi)$ contém os autovalores de $G(y, 0)^{-1} \sigma_{1}(P)(y, 0 ; \xi, z)$ com parte imaginária positiva, o ítem 4 segue dos comentários da subseção 1.2.3.

OBservaçÃo 2.1.23 Temos que $\sigma_{0}\left(\tilde{\Pi}_{+}\right)+\sigma_{0}\left(\tilde{\Pi}_{-}\right)=I$, usando os argumentos do final da prova do ítem 4.

Veremos agora que toda solução $u \in \mathrm{H}^{s}\left(\left.\tilde{E}\right|_{X_{+}}\right)$da equação $P u=0$ em int $X_{+}$possui restrição a $Y$ (traço) em $\mathrm{H}^{s-1 / 2}\left(\left.\tilde{E}\right|_{Y}\right.$ ) dependendo continuamente de $u$. Antes, provemos uma propriedade análoga no caso euclidiano. Denotemos os pontos de $\mathbb{R}^{n+1}$ na forma $(y, t)=\left(y_{1}, \ldots, y_{n}, t\right)$, com $y_{1}, \ldots, y_{n}, t \in \mathbb{R}$.

Proposição 2.1.24 Se $s \in \mathbb{R}, u \in \mathrm{H}^{s}\left(\mathbb{R}^{n+1}\right)$, supp $u \subset\{t \leq 0\}$ e $Q$ é um operador pseudo-diferencial de ordem -1 em $\mathbb{R}^{n}$, então

$$
\gamma_{0}(Q u) \doteq \lim _{\varepsilon \downarrow 0} \gamma_{\varepsilon} Q u
$$

existe em $\mathrm{H}^{s+1 / 2}\left(\mathbb{R}^{n}\right)$ e existe $C_{s}>0$ tal que $\left\|\gamma_{0}(Q u)\right\|_{s-1 / 2} \leq C_{s}\|u\|_{s}$, para toda $u$ nestas condições. Aqui, $\gamma_{\varepsilon}$ denota $a$ restrição de $u a\{t=\varepsilon\}$. 
Prova. Vamos provar esta proposição sob a hipótese $s>-1 / 2$. De fato, nosso interesse principal nesta proposição é o caso $s=1 / 2$, portanto, nossa hipótese não é muito restritiva. $\mathrm{A}$ prova para $s$ arbitrário pode ser encontrada em [91]].

Definimos $\Lambda_{+}^{s} \doteq(-\partial / \partial t+\sqrt{I+\Delta})^{s}$, ou seja

$$
\left(\Lambda_{+}^{s} u\right)(y, t) \doteq(2 \pi)^{-n-1} \int_{\mathbb{R}} \int_{\mathbb{R}^{n}} e^{i(\xi \cdot y+\tau t)}\left(-\tau+\sqrt{1+|\xi|^{2}}\right)^{s} \hat{u}(\xi, \tau) d \xi d \tau,
$$

para $u \in C_{c}^{\infty}\left(\mathbb{R}^{n+1}\right)$ e $(y, t) \in \mathbb{R}^{n} \times \mathbb{R} . \Lambda_{+}^{s}$ não é um operador pseudo-diferencial (pois o "símbolo" de $\Lambda_{+}^{s}$ não satisfaz as hipóteses de regularidade que deve possuir o símbolo de um operador pseudo-diferencial), mas possui as seguintes importantes propriedades:

1. $\Lambda_{+}^{s}: \mathrm{H}^{s}\left(\mathbb{R}^{n+1}\right) \rightarrow \mathrm{H}^{s-1}\left(\mathbb{R}^{n+1}\right)$ é um isomorfismo, para todo $s \in \mathbb{R}$;

2. Se $u$ se anula em $\{t>0\}$ então $\Lambda_{+}^{s} u$ também se anula em $\{t>0\}$;

3. Se $C_{k}\left(R, \Lambda_{+}\right)$denota o $k$-ésimo comutador de $R$ e $\Lambda_{+} \doteq \Lambda_{+}^{1}$, então a ordem de $C_{k}\left(R, \Lambda_{+}\right)$ não excede a ordem de $R$.

Suponhamos $s>-1 / 2$. Temos

$$
Q u=\left[Q, \Lambda_{+}\right] \Lambda_{+}^{-1} u+\Lambda_{+} Q \Lambda_{+}^{-1} u .
$$

A primeira parcela da direita em 2.1 .13 pertence a $\mathrm{H}^{s+2}\left(\mathbb{R}^{n+1}\right)$. Como $s+2>1 / 2$, existe o traço $\gamma_{0}\left(\left[Q, \Lambda_{+}\right] \Lambda_{+}^{-1} u\right) \in \mathrm{H}^{s+3 / 2}\left(\mathbb{R}^{n}\right)$. A segunda parcela em 2.1.13 pertence a $\mathrm{H}^{s+1}\left(\mathbb{R}^{n+1}\right)$; como $s+1>1 / 2$, então existe o traço $\gamma_{0}\left(\Lambda_{+} Q \Lambda_{+}^{-1} u\right) \in \mathrm{H}^{s+1 / 2}\left(\mathbb{R}^{n}\right)$. Além disso, existem constantes $C_{s}, C_{s}^{\prime}, C_{s}^{\prime \prime}>0$ tais que

$$
\begin{aligned}
\left\|\gamma_{0}(Q u)\right\|_{s+1 / 2} & \leq\left\|\gamma_{0}\left(\left[Q, \Lambda_{+}\right] \Lambda_{+}^{-1} u\right)\right\|_{s+1 / 2}+\left\|\gamma_{0}\left(\Lambda_{+} Q \Lambda_{+}^{-1} u\right)\right\|_{s+1 / 2} \\
& \leq C_{s}^{\prime}\left\|\left[Q, \Lambda_{+}\right] \Lambda_{+}^{-1} u\right\|_{s+1}+C_{s}^{\prime \prime}\left\|\Lambda_{+} Q \Lambda_{+}^{-1} u\right\|_{s+1} \\
& \leq C_{s}\|u\|_{s} .
\end{aligned}
$$

Teorema 2.1.25 Sob as mesmas hipóteses do teorema 2.1.22, dados $s \in \mathbb{R} e u \in \mathrm{H}^{s}\left(\left.\tilde{E}\right|_{\mathrm{X}_{+}}\right)$tais que $P u=0 e m$ int $X_{+}$, existem

$$
\gamma_{0}(u) \doteq \lim _{\varepsilon \downarrow 0} \gamma_{\varepsilon}(u)
$$

$e C_{s}>0$ tais que $\left\|\gamma_{0}(u)\right\|_{s-1 / 2} \leq C_{s}\|u\|_{s}$, para toda $u$ nestas condições.

Prova. Sejam $u$ como no enunciado e $\tilde{u} \in \mathrm{H}^{s}(\tilde{E})$ tal que $\left.\tilde{u}\right|_{\text {int } X_{+}}=u$. Logo, $w=P u \in \mathrm{H}^{s-1}(\tilde{E})$ e $w=0$ em int $X_{+}$. Portanto, é suficiente provar que se $w \in \mathrm{H}^{s}(\tilde{E})$ e $w=0$ em int $X_{+}$, então existem $\gamma_{0}\left(P^{-1} w\right) \doteq \lim _{\varepsilon \downarrow 0} \gamma_{\varepsilon} P^{-1} w$ e $C_{s}>0$ (independente de $w$ ) tais que $\left\|\gamma_{0}\left(P^{-1} w\right)\right\|_{s-1 / 2} \leq$ $C_{s}\|w\|_{s-1}$. Localizando o problema, vemos que é suficiente provar que se $\varphi, \psi \in C^{\infty}(\tilde{X})$ têm suporte em alguma vizinhança coordenada de um ponto de $Y$ e $B \doteq M_{\psi} P^{-1} M_{\varphi}$, então existem $\gamma_{0}(B w) \doteq \lim _{\varepsilon \downarrow 0} \gamma_{\varepsilon} B w$ e $C_{s}>0$ (independente de $w$ ) tais que $\left\|\gamma_{0}(B w)\right\|_{s-1 / 2} \leq C_{s}\|w\|_{s-1}$. Este fato decorre diretamente da proposição 2.1.24.

Nas proposições anteriores, provamos que soluções da equação $P u=0$ em int $X_{+}$têm um traço bem definido em $Y$. Vamos agora verificar como se comporta tal traço com respeito aos operadores $\tilde{S}, \tilde{\Pi}_{+}$definidos no teorema 2.1.22. 
Proposição 2.1.26 Se $u \in \mathrm{H}^{s}\left(\left.\tilde{E}\right|_{X_{+}}\right)$é tal que $P u=0$ em int $X_{+} e g \doteq \gamma_{0}(u)$, então $u=\tilde{S} g e \tilde{\Pi}_{+} g=g$. Em particular, $\tilde{\Pi}_{+}^{2}=\tilde{\Pi}_{+}$.

Prova. Se $s \in \mathbb{Z}_{+}$, então claramente $g=\tilde{\Pi}_{+} g$. Pondo $v=\tilde{S} g-u$ em $X_{+}$, temos pela fórmula de Green-Stokes que $P v=0, \log 0, v=0$.

Passemos ao caso $s \in \mathbb{R}$. Dado $x \in X_{+} \backslash Y$, seja $\varepsilon>0$ tal que $x \in X_{2 \varepsilon} \doteq\{t \geq 2 \varepsilon\}$. Como $u$ é $C^{\infty}$ em $\tilde{X} \backslash Y$ (pois $P$ é elíptico), o parágrafo anterior aplica-se e concluímos que $u=P^{-1} \gamma_{\varepsilon} G \gamma_{\varepsilon} u$ em $X_{\varepsilon}$. Tomando $\varphi, \psi \in C^{\infty}(\tilde{X})$ tais que $\varphi \psi=0$ e $\psi=1$ em $\{|t|<2 \varepsilon\}$, temos que $u(x)=M_{\varphi} P^{-1} M_{\psi} \gamma_{\varepsilon}^{*} G \gamma_{\varepsilon} u(x)$. Como $M_{\varphi} P^{-1} M_{\psi}$ é um operador pseudo-diferencial suavizante e $\lim _{\varepsilon \rightarrow 0^{+}} \gamma_{\varepsilon}^{*} G \gamma_{\varepsilon} u=\gamma_{0}^{*} G \gamma_{0} u$ em $H^{t}(\tilde{E}), t=\max (s, 0)$, segue pela proposição 2.1 .9 que

$$
u(x)=\lim _{\varepsilon \rightarrow 0^{+}} M_{\varphi} P^{-1} M_{\psi} \gamma_{\varepsilon}^{*} G \gamma_{\varepsilon} u(x)=M_{\varphi} P^{-1} M_{\psi} \gamma_{0}^{*} G \gamma_{0} u(x)=\tilde{S} g(x) .
$$

Como $x$ é arbitrário em $X \backslash Y$, temos que $\tilde{\Pi}_{+} g \doteq \lim _{\varepsilon \rightarrow 0^{+}} \gamma_{\varepsilon} \tilde{S} g=\lim _{\varepsilon \rightarrow 0^{+}} \gamma_{\varepsilon} u=\gamma_{0}(u)=g$, como queríamos.

\subsubsection{O Projetor de Calderón}

Toda a teoria desenvolvida na subseção 2.1.4 é muito interessante e revela propriedades importantes possuídas por soluções da equação $P u=0$ em int $X_{+}$, onde $P$ um operador elíptico bijetor em uma variedade compacta $\tilde{X}=X_{+} \cup X_{-}$particionada por uma hipersuperfície $Y$. No entanto, a hipótese de bijetividade do operador $P$ parece bastante artificial, uma vez que boa parte da teoria de equações elípticas ocupa-se justamente de estudar propriedades das soluções da equação $P u=0$; a teoria de funções holomorfas é um bom exemplo disso. Outra situação de interesse não contemplada na teoria da subseção 2.1.4 é o caso de problemas de fronteira em variedades com bordo. Nesta seção, descreveremos como reduzir o estudo de um operador elíptico em uma variedade com bordo à teoria já desenvolvida. O método é devido a A. Calderón e R. Seeley, conforme [91].

Sejam $X=X_{+}$uma variedade riemanniana compacta orientada com bordo $Y, E, F$ fibrados riemannianos sobre $X$ e $P: C^{\infty}(E) \rightarrow C^{\infty}(F)$ um operador pseudo-diferencial elíptico em $X$, que coincide com um operador diferencial em um colar $U$ de $Y$ em $X$. Consideremos uma estrutura de colar em $U$, i.e., um difeomorfismo $Y \times[0,1) \rightarrow U$; portanto, podemos escrever os pontos de $U$ na forma $(y, t)$, com $y \in Y$ e $t \in[0,1)$, de tal forma que $y \in Y$ é identificado com $(y, 0)$. Como antes, definimos $i G(y, t) \doteq \sigma_{1}(P)(y, t ; 0, d t)$ em $U$.

Sejam $\tilde{X}$ o dobro de $X$ (conforme os comentários após o teorema 2.1.10), $\tilde{E} \doteq E \oplus F, \tilde{F}=F \oplus E$ fibrados hermitianos sobre $\tilde{X}$ e o operador elíptico

$$
\tilde{P}=\left(\begin{array}{cc}
P & 0 \\
0 & P^{*}
\end{array}\right)
$$

definido em $C^{\infty}(\tilde{E})$ com imagem em $C^{\infty}(\tilde{F})$. 


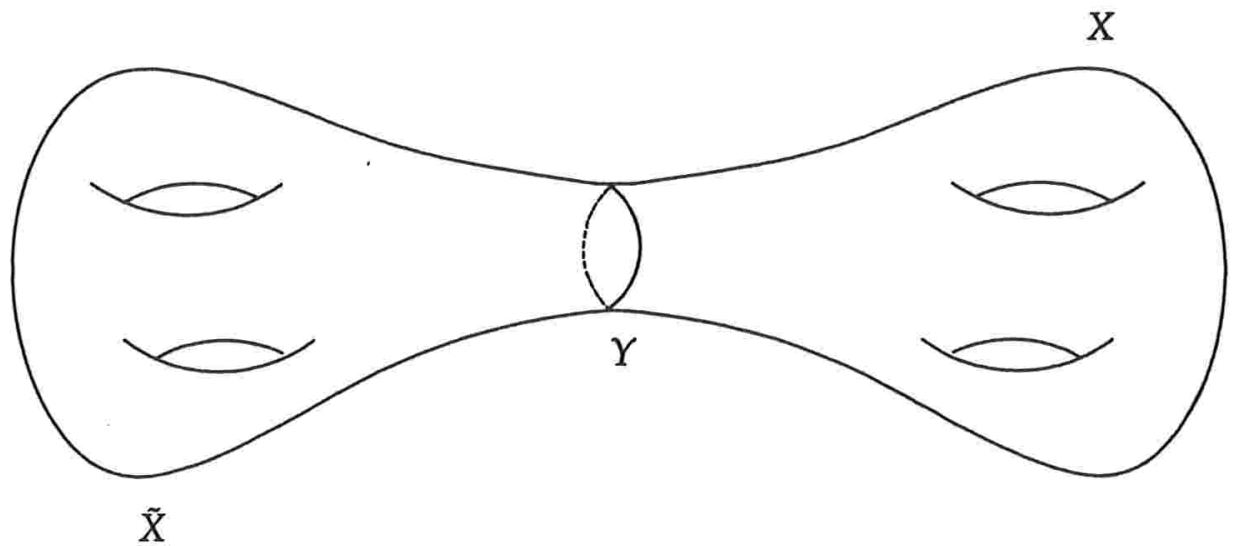

Usando uma homotopia simples, é possível construir um símbolo elíptico $q$ de classe $C^{\infty}$ tal que $\sigma(\tilde{P})=q$ para $t \geq-1 / 2$ e $q$ é um múltiplo de $I$ para $t \leq-1$. Seja ${ }^{9} Q$ um operador pseudo-diferencial com símbolo $q$ e $\varphi, \psi \in C^{\infty}(\tilde{X})$ tais que $\varphi^{2}+\psi^{2}=1$ em $\{t \leq-1 / 2\}$ e $\psi=1$ em $\{t \leq-1\}$. Então $B \doteq M_{\varphi} \tilde{P} M_{\varphi}+M_{\psi} Q M_{\psi}$ é elíptico e coincide com $\tilde{P}$ em $X$. Seja

$$
\mathscr{N}_{0}(P) \doteq\left\{u \in \mathscr{D}^{\prime}(X ; E): P u=0 \text { e } \gamma_{0}(u)=0\right\},
$$

onde $\gamma_{0}$ denota o traço em $Y$. Se $u \in \mathscr{N}_{0}(P)$ então definindo $\bar{u}$ coincidindo com $u$ em $X_{+} \mathrm{e}$ valendo zero em $X_{-,}$, segue da fórmula de Green-Stokes que $P \vec{u}=0$ no sentido de distribuições. Pela elipticidade de $P$ (veja o ítem 1 do teorema 2.1.16), temos que $\bar{u}$ é de classe $C^{\infty}$ em $\tilde{X}$. Como a aplicação $\mathscr{N}_{0}(P) \ni u \mapsto \vec{u} \oplus 0 \in \operatorname{ker} B$ é injetora, pelo teorema 2.1 .18 , segue que $\operatorname{dim} \mathscr{N}_{0}(P)<\infty$ e $\mathscr{N}_{0}(P) \subset C^{\infty}(E)$.

Se $N$ denota a projeção ortogonal de $\mathrm{H}^{0}(E \oplus F)$ sobre $\mathscr{N}_{0}(P) \oplus \mathscr{N}_{0}\left(P^{*}\right)$, então $N$ é um operador suavizante. Sejam $\theta \in C^{\infty}(\tilde{X})$ uma função que se anula exatamente sobre $X_{+}$e $A \doteq B+N+i M_{\theta}$. Temos por construção que $A$ é elíptico inversível, e portanto, podemos aplicar os resultados da subseção 2.1.4. Considerando os operadores $\tilde{S}=\tilde{S}(A)$ e $\tilde{\Pi}_{+}=\tilde{\Pi}_{+}(A)$ dados pelo teorema 2.1.22, definimos

$$
\begin{aligned}
S: \mathrm{H}^{s}\left(\left.E\right|_{Y}\right) & \rightarrow \mathrm{H}^{s+1 / 2}(E) \\
g & \mapsto \pi_{1} r(\tilde{S}(g \oplus 0))
\end{aligned}
$$

e

$$
\begin{aligned}
\Pi_{+}: \mathrm{H}^{s}\left(\left.E\right|_{Y}\right) & \rightarrow \mathrm{H}^{s}\left(\left.E\right|_{Y}\right) \\
g & \mapsto \pi_{1}\left(\tilde{\Pi}_{+}(A)(g \oplus 0)\right)
\end{aligned}
$$

onde $\pi_{1}: E \oplus F \rightarrow E$ é a projeção canônica e $r: \mathrm{H}^{s+1 / 2}(\tilde{E}) \rightarrow \mathrm{H}^{s+1 / 2}\left(\left.\tilde{E}\right|_{X_{+}}\right)=\mathrm{H}^{s+1 / 2}(E)$ é o operador de restrição comentado no parágrafo anterior à proposição 2.1.15. Os operadores definidos em 2.1.15 e 2.1.16 são chamados de operador de Poisson e projetor de Calderón correspondentes ao operador original $P$, respectivamente.

Antes de explorar as propriedades de $S$ e $\Pi_{+}$vamos definir um espaço importante. Seja

$$
\operatorname{ker}(P, s) \doteq\left\{u \in \mathrm{H}^{s}(E): P u=0 \text { em int } X_{+}\right\},
$$

para $s \in \mathbb{R}$. O teorema 2.1.25 mostra que todo elemento de $\operatorname{ker}(P, s)$ possui um traço bemdefinido sobre $Y$ em $\mathrm{H}^{s-1 / 2}\left(\left.E\right|_{Y}\right)$, portanto, podemos definir o espaço de dados de Cauchy de $P$ por

$$
\mathrm{H}(P, s) \doteq \gamma_{0}(\operatorname{ker}(P, s)) \subset \mathrm{H}^{s-1 / 2}\left(\left.E\right|_{Y}\right) .
$$

\footnotetext{
${ }^{9}$ Como antes, podemos supor que a função $t$ esteja definida em toda $\tilde{X} \operatorname{com} t \geq 0$ em $X_{+}$e $t \leq 0$ em $X$.
} 
Agora temos todos os ingredientes necessários para provar o principal teorema desta seção.

TEOREMa 2.1.27 Seja $X$ uma variedade riemanniana compacta orientada com bordo $Y, E, F$ fibrados riemannianos sobre $\mathrm{X}$ e $P: \mathrm{C}^{\infty}(E) \rightarrow \mathrm{C}^{\infty}(F)$ um operador pseudo-diferencial elíptico em $\mathrm{X}$, que coincide com um operador diferencial em um colar $U$ de $Y$ em $X$. São verdadeiras as seguintes afirmações:

1. Os operadores $S, \Pi_{+}$definidos em 2.1 .15 e 2.1.16 são limitados;

2. Para qualquer $u \in \operatorname{ker}(P, s)$, existe o limite $\gamma_{0}(u) \doteq \lim _{\varepsilon \downarrow 0} \gamma_{\varepsilon} u$;

3. O operador $S$ tem imagem em $\operatorname{ker}(P, s), \operatorname{Im} S \cap \mathscr{N}_{0}(P)=0 e \operatorname{Im} S+\mathscr{N}_{0}(P)=\operatorname{ker}(P, s)$;

4. $\left.S\right|_{\mathrm{H}(P, s)}: \mathrm{H}(P, s) \rightarrow \operatorname{Im} S$ é um isomorfismo linear (limitado);

5. $\Pi_{+}$é uma projeção de $\mathrm{H}^{s}\left(\left.E\right|_{Y}\right)$ sobre $\mathrm{H}(P, s)$ tal que $\gamma_{0} \circ S=\Pi_{+}$, para todo $s \in \mathbb{R}$;

6. $\Pi_{+}$é um operador pseudo-diferencial de ordem zero, cujo símbolo principal em $(y, \xi) \in T^{*} Y$ é a projeção espectral sobre a soma dos autoespaços generalizados de $E_{y}$ correspondentes aos autovalores de $-G(y, 0) \sigma_{1}(P)(y, 0 ; \xi, 0)$ com parte imaginária $>0$;

7. $\mathscr{N}_{0}(P) \subset C^{\infty}(E) e \operatorname{dim} \mathscr{N}_{0}(P)<\infty$.

Prova. As afirmações 1, 2 e 7 já foram provadas. $O$ ítem 5 decorre da definição de $\Pi_{+}$e da proposição 2.1.26.

Provemos o ítem 3. O fato que $S$ tem imagem em $\operatorname{ker}(P, s)$ decorre diretamente do ítem 3 do teorema 2.1.22. Dada qualquer $u \in \operatorname{ker}(P, s)$, definimos $g \doteq \gamma_{0}(u) \in \mathrm{H}^{s-1 / 2}\left(\left.E\right|_{Y}\right)$ e $v \doteq S g \in$ $\operatorname{Im} S \subset \operatorname{ker}(P, s)$. Temos que $w \doteq u-v \in \mathscr{N}_{0}(P)$ e $u=v+w$, portanto, $\operatorname{Im} S+\mathscr{N}_{0}(P)=\operatorname{ker}(P, s)$. Além disso, se $u \in \operatorname{Im} S \cap \mathscr{N}_{0}(P)$, então existe $g=\gamma_{0}(u) \in \mathrm{H}^{s-1 / 2}\left(\left.E\right|_{\gamma}\right)$ e $u=S g$ em int $X$. Como $u \in \mathscr{N}_{0}(P)$, temos $g=0$, portanto, $u=0$ e $\operatorname{Im} S \cap \mathscr{N}_{0}(P)=0$.

Para provar o ítem 4, basta observar que $\mathrm{H}(P, s)=\gamma_{0}(\operatorname{ker}(P, s))=\gamma_{0}(\operatorname{Im} S)$ e que $\gamma_{0} \lim s:$ $\operatorname{Im} S \rightarrow \mathrm{H}(P, s)$ é uma inversa para $\left.S\right|_{\mathrm{H}(P, s)}: \mathrm{H}(P, s) \rightarrow \operatorname{Im} S$.

Faremos agora alguns comentários sobre os espaços de dados de Cauchy. Inspirados nos espaços de Hardy da teoria de funções analíticas, definimos $\mathrm{H}_{\mathrm{Har}}(P, s)$ como o fecho do subespaço $\gamma_{0}\left(\left\{u \in C^{\infty}(E): P u=0\right.\right.$ em int $\left.\left.X\right\}\right)$ no espaço $H^{s-1 / 2}\left(\left.E\right|_{Y}\right)$. Definimos também $\mathrm{H}_{\text {Cald }}(P, s)$ como a imagem de $\mathrm{H}^{s-1 / 2}\left(\left.E\right|_{Y}\right)$ pelo projetor de Calderón $\Pi_{+}$. Assim como o espaço de dados de Cauchy $\mathrm{H}(P, s)$ definido em 2.1.18, os espaços $\mathrm{H}_{\mathrm{Har}}(P, s)$ e $\mathrm{H}_{\mathrm{Cal}}(P, s)$ também fornecem definições naturais (sob algum ponto de vista) para o que seria um bom espaço de dados iniciais em $Y$ para o problema de Cauchy para o operador $P$. A próxima proposição mostra que, de fato, tais espaços coincidem.

Proposição 2.1.28 Dado qualquer $s \in \mathbb{R}, \mathrm{H}(P, s)=\mathrm{H}_{\mathrm{Har}}(P, s)=\mathrm{H}_{\mathrm{Cal}}(P, s)$. Em particular, $\mathrm{H}(P, s) e ́$ fechado em $\mathrm{H}^{s-1 / 2}\left(\left.E\right|_{Y}\right)$.

Prova. Como a imagem de uma projeção é um subespaço fechado, $C^{\infty}(E)$ é denso em $\mathrm{H}^{s}(E)$ e $\operatorname{Im} \Pi_{+}=\gamma_{0}\left(\left\{u \in C^{\infty}(E): P u=0\right.\right.$ em int $\left.X\right\}$, pelo teorema 2.1.27, concluímos que $\mathrm{H}_{\mathrm{Har}}(P, s)=\mathrm{H}_{\mathrm{Cal}}(P, s)$. Este mesmo teorema também implica que $\mathrm{H}(P, s)=\mathrm{H}_{\mathrm{Cal}}(P, s)$. 


\subsection{Estruturas de Clifford}

Nesta seção será introduzida a técnica de álgebras de Clifford no estudo de operadores elípticos. Este procedimento foi sistematizado por M. Atiyah, R. Bott e A. Shapiro em [9]. O interesse no uso de álgebras de Clifford é justificado pelo fato que tais estruturas fornecem uma linguagem adequada para o tratamento genérico de uma quantidade razoável de operadores diferenciais invariantemente definidos em variedades riemannianas. $O$ caso mais freqüente é o dos operadores de Dirac, sobre os quais falaremos na seção 2.3. Também introduziremos o conceito de estrutura spin para fibrados e variedades. Este conceito é, sem dúvida, uma das grandes realizações da teoria desenvolvida por M. Atiyah e I. Singer. A grosso modo, um fibrado vetorial orientado é spin se for possível simplificar o seu grupo estrutural passando de $\mathrm{SO}(n)$ para o recobrimento de duas folhas $\mathrm{Spin}(n) \rightarrow \mathrm{SO}(n)$. Uma variedade orientada é spin se tiver fibrado tangente spin. A existência ou não deste tipo de simplificação revela propriedades topológicas profundas que serão discutidas oportunamente. Neste ponto, a teoria do índice para operadores elípticos (especialmente o teorema de Atiyah-Singer) pode ser utilizada para obter-se resultados (inacessíveis por outros métodos) sobre topologia das variedades e vice-versa: o conhecimento de propriedades topológicas pode traduzir-se em propriedades analíticas de operadores elípticos.

Também discutiremos alguns aspectos importantes sobre a construção de fibrados spinor em variedades spin. Este tipo de fibrado tem sua importância justificada pelo fato que suas fibras são módulos sobre a álgebra de Clifford, e portanto, o espaço de seções de um fibrado spinor é naturalmente um módulo sobre o espaço de seções do fibrado de Clifford. A seção encerra com um breve tratamento de conexões e estruturas métricas em fibrados spinor e suas relações com a estrutura algébrica em questão.

\subsection{1 Álgebras de Clifford e suas Representações}

Consideremos o espaço $\mathbb{R}^{n}$ munido do produto interno canônico, a álgebra

$$
\mathscr{T}\left(\mathbb{R}^{n}\right) \doteq \sum_{r=0}^{\infty} \otimes^{r} \mathbb{R}^{n}
$$

cujos elementos são tensores mistos sobre $\mathbb{R}^{n}$ e $\mathscr{I}$ o ideal de $\mathscr{T}\left(\mathbb{R}^{n}\right)$ gerado pelos elementos da forma $v \otimes v+|v|^{2}, \operatorname{com} v \in \mathbb{R}^{n}$. Definimos a álgebra de Clifford $\mathrm{Cl}(n)$ como a álgebra quociente $\mathscr{T}\left(\mathbb{R}^{n}\right) / \mathscr{I}$. Temos uma inclusão natural $\mathbb{R}^{n} \subset \mathrm{Cl}(n)$ obtida a partir da composição $\mathbb{R}^{n} \approx$ $\otimes 1 \mathbb{R}^{n} \rightarrow \mathrm{Cl}(n)$, onde a segunda aplicação é a restrição da projeção canônica $\mathscr{T}\left(\mathbb{R}^{n}\right) \rightarrow \mathrm{Cl}(n)$ a $\otimes 1 \mathbb{R}^{n}$. O produto definido para elementos de $\mathrm{Cl}(n)$ é chamado de produto de Clifford e denotado simplesmente por $\cdot$. Nesta seção, voltaremos a denotar o produto interno canônico em $\mathbb{R}^{n}$ por $\langle\cdot, \cdot\rangle$.

Dado qualquer $u \in \mathbb{R}^{n}$, por definição, temos que $u \cdot u=-|u|^{2}$. Em particular, para quaisquer $v, w \in \mathbb{R}^{n}$,

$$
-|v|^{2}-|w|^{2}+v \cdot w+w \cdot v=(v+w) \cdot(v+w)=-|v+w|^{2}=-|v|^{2}-|w|^{2}-2\langle v, w\rangle,
$$

logo,

$$
v \cdot w+w \cdot v=-2\langle v, w\rangle
$$


Dada uma base ortonormal $\left\{e_{j}\right\}_{j=1}^{n}$ de $\mathbb{R}^{n}$, segue que $e_{j} \cdot e_{j}=-1$ para $j=1, \ldots, n$ e $e_{j} \cdot e_{k}=-e_{k} \cdot e_{j}$, para $j \neq k$. A partir desta observação, vemos que os elementos de $\mathrm{Cl}(n)$ se escrevem como combinação linear de elementos da forma $e_{i_{1}} \cdot \ldots \cdot e_{i_{k}}$ com $1 \leq i_{1}<\ldots<i_{k} \leq n$ e $k=1, \ldots, n$. Isso prova a seguinte proposição.

Proposição 2.2.1 Como espaços vetoriais, $\mathrm{Cl}(n)$ e $\Lambda^{*}\left(\mathbb{R}^{n}\right)$ são ${ }^{10}$ isomorfos. Em particular, $\mathrm{Cl}(n)$ tem dimensão $2^{n}$.

OвSERVAÇão 2.2.2 Se $\mathscr{A}$ é qualquer álgebra associativa com unidade e $f: \mathbb{R}^{n} \rightarrow \mathscr{A}$ é uma aplicação linear tal que $f(v) \cdot f(v)=-|v|^{2}$ para todo $v \in \mathbb{R}^{n}$, então existe um único homomorfismo de álgebras $\tilde{f}: \mathrm{Cl}(n) \rightarrow \mathscr{A}$ estendendo $f$. Esta propriedade universal pode ser usada para caracterizar a álgebra de $\mathrm{Clifford} \mathrm{Cl}(n)$.

Vamos agora construir uma involução (i.e., um operador cujo quadrado é a identidade) muito importante de $\mathrm{Cl}(n)$. Considerando $\alpha: \mathbb{R}^{n} \rightarrow \mathbb{R}^{n} \subset \mathrm{Cl}(n), \alpha(v)=-v$, temos que $\alpha(v) \cdot \alpha(v)=-|v|^{2}, v \in \mathbb{R}^{n}$, logo, pela observação 2.2.2, existe uma única extensão de $\alpha$ a $\mathrm{Cl}(n)$, a qual continuaremos a denotar por $\alpha$. Como $\alpha \circ \alpha=I$, pondo $\mathrm{Cl}^{j}(n) \doteq \operatorname{ker}\left(\alpha-(-1)^{j}\right)$, para $j=0,1$, obtemos uma decomposição $\mathrm{Cl}(n)=\mathrm{Cl}^{0}(n) \oplus \mathrm{Cl}^{1}(n) \cdot \mathrm{Cl}^{0}(n)$ e $\mathrm{Cl}^{1}(n)$ são chamados de parte par e parte impar de $\mathrm{Cl}(n)$, respectivamente. Além disso, $\mathrm{Cl}^{i}(n) \cdot \mathrm{Cl}^{j}(n) \subset \mathrm{Cl}^{i+j}(n)$, com $i, j \in \mathbb{Z}_{2}$. A parte par (ímpar) de $\mathrm{Cl}(n)$ pode ser caracterizada como o subespaço gerado pelos elementos da forma $e_{i_{1}} \cdot \ldots \cdot e_{i_{k}} \operatorname{com} 1 \leq i_{1}<\ldots<i_{k} \leq n, k$ par (ímpar) e $\left\{e_{j}\right\}_{j=1}^{n}$ base ortonormal de $\mathbb{R}^{n}$.

Proposição 2.2.3 $\mathrm{Cl}^{0}(n)$ é uma subálgebra de $\mathrm{Cl}(n)$ de dimensão $2^{n-1}$ isomorfa a $\mathrm{Cl}(n-1)$.

Prova. Basta observar que a aplicação $\mathrm{Cl}(n-1) \ni \varphi=\varphi_{0}+\varphi_{1} \mapsto \varphi_{0}+\varphi_{1} \cdot e_{n} \in \mathrm{Cl}^{0}(n)$ é um isomorfismo, onde $\varphi=\varphi_{0}+\varphi_{1}$ é a decomposição de $\varphi$ em parte par e parte ímpar, respectivamente.

Vamos agora descrever outra involução de $\mathrm{Cl}(n)$. Dados $v_{1}, \ldots, v_{k} \in \mathbb{R}^{n}, k \in \mathbb{N}$, definimos $\left(v_{1} \otimes \ldots \otimes v_{k}\right)^{t}=v_{k} \otimes \ldots \otimes v_{1}$. Estendendo esta aplicação por linearidade a $\mathscr{T}\left(\mathbb{R}^{n}\right)$, obtemos uma involução $(\cdot)^{t}: \mathscr{T}\left(\mathbb{R}^{n}\right) \rightarrow \mathscr{T}\left(\mathbb{R}^{n}\right)$ que preserva o ideal $\mathscr{I}$. Portanto, $(\cdot)^{t}$ estende-se a uma involução $(\cdot)^{t}: \mathrm{Cl}(n) \rightarrow \mathrm{Cl}(n)$, a qual chamamos de transposição.

É possível dar uma caracterização bastante concreta da álgebra de $\mathrm{Clifford} \mathrm{Cl}(n)$ em termos da álgebra exterior $\Lambda^{*}\left(\mathbb{R}^{n}\right)$. Definimos o produto de Clifford de $\theta \in \Lambda^{1}\left(\mathbb{R}^{n}\right)$ e $\eta \in \Lambda^{p}\left(\mathbb{R}^{n}\right)$ por

$$
\begin{aligned}
\theta \cdot \eta & \doteq \theta \wedge \eta-\theta\llcorner\eta \\
\eta \cdot \theta & \doteq(-1)^{p}(\theta \wedge \eta+\theta\llcorner\eta)),
\end{aligned}
$$

onde $\theta\llcorner\eta$ denota a contração de $\theta$ e $\eta$ definida $\operatorname{como} \theta\llcorner\eta(\cdot, \ldots, \cdot)=\eta(Y, \cdot, \ldots, \cdot)$, $\operatorname{com} Y$ o único vetor de $\mathbb{R}^{n}$ que representa $\theta$ com respeito ao produto interno usual. Estendendo o produto de Clifford por linearidade e associatividade, obtemos um produto bem-definido em $\Lambda^{*}\left(\mathbb{R}^{n}\right)$ tal que $\theta \cdot \theta=-|\theta|^{2}$, para toda $\theta \in \Lambda^{1}\left(\mathbb{R}^{n}\right) \approx \mathbb{R}^{n}$. Isso mostra que $\mathrm{Cl}(n)$ é isomorfo a $\Lambda^{*}\left(\mathbb{R}^{n}\right)$ munido do produto de Clifford.

Consideremos o grupo multiplicativo $\mathrm{Cl}^{\times}(n)$ formado pelos inversíveis de $\mathrm{Cl}(n)$. Temos que $\mathrm{Cl}^{\times}(n)$ é um grupo de Lie de dimensão $2^{n}$. Além disso, como $v \cdot v=-|v|^{2}$ para $v \in \mathbb{R}^{n}$, então $v^{-1}=-v /|v|^{2}$, se $v \neq 0, \operatorname{logo} \mathbb{R}^{n} \backslash\{0\} \subset \mathrm{Cl}^{\times}(n)$.

\footnotetext{
${ }^{10} \Lambda^{*}\left(\mathbb{R}^{n}\right)$ denota a álgebra exterior de $\mathbb{R}^{n}$.
} 
DefinIÇão 2.2.4 Definimos Pin(n) como o subgrupo multiplicativo de $\mathrm{Cl}^{\times}(n)$ gerado pela esfera unitária $S^{n-1} \subset \mathbb{R}^{n}$. O grupo $\operatorname{Spin}(n)$ é definido como a parte par de $\operatorname{Pin}(n)$, i.e., $\operatorname{Spin}(n) \doteq \operatorname{Pin}(n) \cap \mathrm{Cl}^{0}(n)$.

Proposição 2.2.5 O grupo Spin(n) pode ser caracterizado como

$$
\begin{aligned}
\operatorname{Spin}(n) & =\left\{\varphi \in \operatorname{Pin}(n): \varphi \cdot \varphi^{t}=\varphi^{t} \cdot \varphi=1\right\} \\
& =\left\{v_{1} \cdot \ldots \cdot v_{k}: v_{1}, \ldots, v_{k} \in S^{n-1}, k \text { par }\right\}
\end{aligned}
$$

Prova. Basta ver que $\operatorname{se} \varphi=v_{1} \ldots v_{k} \in \operatorname{Pin}(n), \operatorname{com} v_{1}, \ldots, v_{k} \in S^{n-1}$, então $\varphi^{t} \cdot \varphi=\varphi \cdot \varphi^{t}=(-1)^{k}$. I

Consideremos a representação adjunta $\operatorname{Ad}: \mathrm{Cl}^{\times}(n) \ni \varphi \mapsto \operatorname{Ad}_{\varphi} \in \operatorname{Aut}(\mathrm{Cl}(n))$, dada por $\operatorname{Ad}_{\varphi}(x) \doteq \varphi \cdot x \cdot \varphi^{-1}, x \in \mathrm{Cl}(n)$. Vamos calcular uma restrição especial de Ad.

PROPosição 2.2.6 Se $v \in \mathbb{R}^{n} \backslash\{0\}$ então $-\operatorname{Ad}_{v}(w)=w-\frac{2\langle v, w\rangle}{|v|^{2}} v$, para todo $w \in \mathbb{R}^{n}$.

Prova. Pelos comentários que antecedem a proposição 2.2.1 e a definição 2.2.4, dado $v \in \mathbb{R}^{n} \backslash\{0\}$,

$$
\operatorname{Ad}_{v}(w)=v \cdot w \cdot v^{-1}=-\frac{v \cdot w \cdot v}{|v|^{2}}=-\frac{1}{|v|^{2}}(-w \cdot v-2\langle v, w\rangle) \cdot v=-w+\frac{2\langle v, w\rangle}{|v|^{2}} v,
$$

para qualquer $w \in \mathbb{R}^{n}$, como queríamos.

A proposição 2.2.6 mostra que se $v \in \mathbb{R}^{n} \backslash\{0\}$ então $-\mathrm{Ad}_{v}$ é a reflexão em torno do hiperplano ortogonal a $v$. Para livrar-se do sinal negativo inconveniente, introduzimos a representação $\tilde{\mathrm{Ad}}: \mathrm{Cl}^{\times}(n) \rightarrow \operatorname{Aut}(\mathrm{Cl}(n))$ dada como $\tilde{\mathrm{Ad}}{ }_{\varphi}(x) \doteq \alpha(\varphi) \cdot x \cdot \varphi^{-1}, x \in \mathbb{R}^{n}$. Claramente, $\tilde{\mathrm{Ad}} \tilde{\varphi}_{\varphi_{1} \cdot \varphi_{2}}=$ $\tilde{\operatorname{Ad}} \varphi_{\varphi_{1}} \tilde{A d}_{\varphi_{2}}$ para $\varphi_{1}, \varphi_{2} \in \mathrm{Cl}(n)$ e $\tilde{A d} d_{\varphi}=\operatorname{Ad}_{\varphi}$ se $\varphi \in \mathrm{Cl}^{0}(n)$. Pela proposição 2.2.6, $\tilde{A d}$ é a reflexão em torno do hiperplano ortogonal a $v$, para $v \in \mathbb{R}^{n} \backslash\{0\}$.

Consideremos a restrição $\left.\vartheta \doteq \tilde{\operatorname{Add}}\right|_{\operatorname{Pin}(n)}: \operatorname{Pin}(n) \rightarrow \operatorname{Aut}(\mathrm{Cl}(n))$. Observamos que se $v \in S^{n-1}$, então $\vartheta(v)=\tilde{A d}_{v}$ (pois $v^{-1}=-v$ ), logo, $\vartheta(v)$ é a reflexão em torno do hiperplano perpendicular a $v$. Como todo elemento de $T \in \mathrm{O}(n)$ admite uma base ortonormal com respeito à qual tem a forma

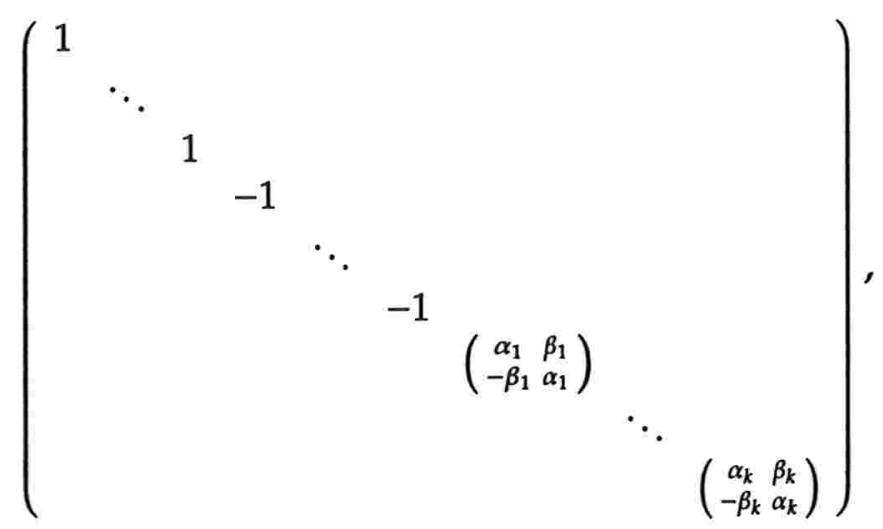

$\operatorname{com} \alpha_{j}, \beta_{j} \in \mathbb{R}, j=1, \ldots, k$ e cada rotação $\left(\begin{array}{cc}\alpha_{j} & \beta_{j} \\ -\beta_{j} & \alpha_{j}\end{array}\right)$ em $\mathbb{R}^{2}$ é produto de duas reflexões, concluímos que $T$ é produto de reflexões em torno de hiperplanos de $\mathbb{R}^{n}$. Em particular, como Pin $(n)$ é gerado por $S^{n-1}$, concluímos que $\operatorname{Im} \vartheta=\mathrm{O}(n)$. Como os elementos de $\operatorname{Spin}(n)$ são produto de uma quantidade par de elementos de $S^{n-1}$, concluímos que $\vartheta(\operatorname{Spin}(n))=S \mathrm{~S}(n)$ e a próxima proposição está provada. 
Proposição 2.2.7 O homomorfismo $\vartheta: \operatorname{Pin}(n) \rightarrow \mathrm{O}(n)$ é sobre e sua restrição $\left.\vartheta_{0} \doteq \vartheta\right|_{\text {Spin }(n)}$ tem imagem $\mathrm{SO}(n)$.

Lembramos que $\mathrm{SO}(n)$ é conexo para todo $n \in \mathbb{N}, \pi_{1}(\mathrm{SO}(n))=\mathbb{Z}_{2}$ para todo $n \geq 3 \mathrm{e}$ $\pi_{1}(\mathrm{SO}(2))=\mathbb{Z}$, conforme [49]. A próxima proposição revela uma propriedade fundamental do grupo $\operatorname{Spin}(n)$ para $n \geq 3$.

Proposição 2.2.8 Se $n \geq 3$ então $\vartheta_{0}: \operatorname{Spin}(n) \rightarrow \mathrm{SO}(n)$ é o recobrimento universal de $\mathrm{SO}(n)$. Em particular, $\operatorname{Spin}(n)$ é uma variedade compacta conexa e simplesmente conexa.

PRova. Vemos que ker $\vartheta_{0}=\{ \pm 1\}$, pois $\varphi \in \operatorname{ker} \vartheta_{0}$ se e só se $\varphi \cdot v=v \cdot \varphi$ para todo $v \in \mathbb{R}^{n}$. Logo, $\vartheta_{0}$ é um recobrimento de $\mathrm{SO}(n)$ com duas folhas. Para ver que $\vartheta_{0}$ é de fato o recobrimento universal, basta ver que $\operatorname{Spin}(n)$ é conexo. Isso equivale a mostrar que existe uma curva em $\operatorname{Spin}(n)$ conectando $1 \mathrm{e}-1$. De fato, $\alpha(t) \doteq\left(\cos t e_{1}+\sin t e_{2}\right) \cdot\left(-\cos t e_{1}+\sin t e_{2}\right)=$ $\cos (2 t)+\sin (2 t) e_{1} \cdot e_{2}$, com $0 \leq t \leq \pi / 2$ é uma tal curva, onde $e_{1}, e_{2}$ são vetores unitários ortogonais em $\mathbb{R}^{n}$.

OвSERVAção 2.2.9 O grupo Spin(2) é definido como o recobrimento de duas folhas de $\mathrm{SO}(2) \approx$ $S^{1}$.

Vamos agora introduzir um ingrediente essencial no estudo de álgebras de Clifford. $\mathrm{O}$ elemento de volume orientado em $\mathrm{Cl}(n)$ é definido por

$$
\omega \doteq e_{1} \cdot \ldots \cdot e_{n},
$$

onde $\left\{e_{j}\right\}_{j=1}^{n}$ denota, como antes, uma base ortonormal positiva de $\mathbb{R}^{n}$. É imediato ver que $\omega$ independe da base positiva utilizada, e portanto, é um elemento bem-definido de $\mathrm{Cl}(n)$. $\mathrm{Em}$ certos casos, $\omega$ pode ser utilizada para obtermos uma decomposição de $\mathrm{Cl}(n)$, como mostram a próxima proposição e seu corolário.

Proposição 2.2.10 Temos que $\omega^{2}=(-1)^{n(n+1) / 2} e \omega \cdot v=(-1)^{n-1} v \cdot \omega$ para todo $v \in \mathbb{R}^{n}$. Em particular,

$$
\omega^{2}=\left\{\begin{array}{r}
1 \text { se } n \equiv 0 \text { ou } 3 \bmod 4 \\
-1 \quad \text { se } n \equiv 1 \text { ou } 2 \bmod 4
\end{array} .\right.
$$

Se $n \equiv 0$ ou 3 mod 4 então, definindo $\pi_{ \pm} \doteq \frac{1}{2}(1 \pm \omega)$, temos que $\left(\pi_{ \pm}\right)^{2}=I, \pi_{+} \pi_{-}=\pi_{-} \pi_{+}=0$ e $\pi_{+}+\pi_{-}=I$. Em particular, $\left(\operatorname{Im} \pi_{+}\right) \oplus\left(\operatorname{Im} \pi_{-}\right)=\mathrm{Cl}(n)$.

Corolário 2.2.11 Se $V$ é um $\mathrm{Cl}(n)$-módulo à esquerda (i.e., $V$ é um espaço vetorial real e existe um homomorfismo $\rho: \mathrm{Cl}(n) \rightarrow \mathscr{L}(V))$, então pondo $V_{ \pm} \doteq\left(\operatorname{Im} \pi_{ \pm}\right) \cdot V$, temos que $V=V_{+} \oplus V_{-}$. Além disso, dado qualquer $v \in \mathbb{R}^{n} \backslash\{0\}$, a aplicação $V_{ \pm} \ni \varphi \mapsto v \cdot \varphi \in V_{\mp}$ é um isomorfismo.

Estudemos agora algumas representações de $\mathrm{Cl}(n)$. Uma representação de $\mathrm{Cl}(n)$ é simplesmente um homomorfismo $\rho: \mathrm{Cl}(n) \rightarrow \mathscr{L}(V)$, onde $V$ é um espaço vetorial (real ou complexo) de dimensão finita. Neste caso, $V$ pode ser munido de uma estrutura de $\mathrm{Cl}(n)$-módulo, definindo

$$
\varphi \cdot v \doteq \rho(\varphi)(v),
$$

para quaisquer $\varphi \in \mathrm{Cl}(n)$ e $v \in V$. A representação é dita real ou complexa conforme $V$ o seja. Dizemos que $\rho$ é redutivel se $V=V_{1} \oplus V_{2}$ com $V_{1}, V_{2}$ submódulos de $V$ não-triviais. Caso 
contrário, $V$ é dita irredutível. Como $V$ tem dimensão finita, vemos que toda representação de $\mathrm{Cl}(n)$ pode ser decomposta como soma direta de representações irredutíveis. Pondo $\mathbb{C l}(n) \doteq \mathrm{Cl}(n) \otimes_{\mathbb{R}} \mathbb{C}$, as mesmas definições anteriores podem ser dadas com $\mathbb{C l}(n)$ em lugar de $\mathrm{Cl}(n)$.

A teoria de representações de álgebras de Clifford é totalmente descrita no próximo teorema, cujo prova pode ser encontrada em [60].

TEOREMA 2.2.12 São verdadeiras as seguintes afirmações:

1. Se $n=2 m$ então, a menos de isomorfismo, existe um único $\mathbb{C l}(n)$-módulo irredutível;

2. Se $n=2 m+1$ então existem, a menos de isomorfismo, exatamente dois $\mathbb{C l}(n)$-módulos irredutíveis distintos.

3. Se $n=4 m$ então existe, a menos de isomorfismo, um único $\mathrm{Cl}(n)$-módulo irredutível;

4. Se $n=4 m+3$ então existem, a menos de isomorfismo, exatamente dois $\mathrm{Cl}(n)$-módulos irredutíveis distintos.

\subsubsection{Estruturas Spin}

Nesta subseção, discutiremos o conceito de estrutura spin para um fibrado vetorial real ou complexo sobre uma variedade. Este conceito é importante para o desenvolvimento da teoria de fibrados de Clifford e operadores de Dirac, conforme esclareceremos na subseção 2.2.3. Também trataremos de variedades spin e forneceremos alguns exemplos simples, conforme [60] e [65].

Seja $\pi: E \rightarrow X$ um fibrado riemanniano $n$-dimensional sobre uma variedade $X$. Podemos obter (usando uma métrica riemanniana em $X$ ) uma cobertura aberta $\left\{U_{\alpha}\right\}$ de $X$ tal que qualquer intersecção $U_{\alpha_{1}} \cap \ldots \cap U_{\alpha_{k}}$ é contrátil e $\left.E\right|_{U_{\alpha}}$ é trivial, para todo $\alpha$. Em particular, é possível definir um referencial ortonormal $E_{\alpha} \doteq\left(E_{\alpha, 1}, \ldots, E_{\alpha, n}\right)$ de $E$ em $U_{\alpha}$. Em cada intersecção não-vazia $U_{\alpha} \cap U_{\beta}$, obtemos funções de transição $\tau_{\alpha \beta}: U_{\alpha} \cap U_{\beta} \rightarrow O(n)$ definidas por $E_{\beta}=E_{\alpha} \cdot \tau_{\alpha \beta}$. Claramente, temos que $\tau_{\alpha \beta} \tau_{\beta \gamma}=\tau_{\alpha \gamma}$ quando $U_{\alpha} \cap U_{\beta}, U_{\alpha} \cap U_{\gamma}$ e $U_{\beta} \cap U_{\gamma}$ são não-vazios. Podemos reduzir as aplicações $\tau_{\alpha \beta}$ à det $\tau_{\alpha \beta}: U_{\alpha} \cap U_{\beta} \rightarrow \mathbb{Z}_{2}=\{ \pm 1\}$. Usando a linguagem de cohomologia de Čech, temos ${ }^{11}$ uma aplicação $f: I^{2} \rightarrow \mathbb{Z}_{2}$. Como $\left(\tau_{\alpha \beta}\right)^{-1}=\tau_{\beta \alpha}$,

\footnotetext{
${ }^{11} \mathrm{Em}$ linhas gerais, dada uma cobertura $\mathscr{U}=\left\{U_{a}\right\}$ de um espaço topológico $X$, podemos definir um complexo simplicial cujas faces de dimensão $k$ são as $(k+1)$-uplas $\left(\alpha_{0}, \ldots, \alpha_{k}\right)$ tais que $U_{\alpha_{0}} \cap \ldots \cap U_{\alpha_{k}} \neq \emptyset$. Sobre o grupo livre $I^{k}$ gerado por todas as faces de dimensão $k$ consideramos o subgrupo $J^{k}$ gerado pelos elementos da forma $\operatorname{sgn}(\sigma)\left(\alpha_{0}, \ldots, \alpha_{k}\right)-\left(\sigma\left(\alpha_{0}\right), \ldots, \sigma\left(\alpha_{k}\right)\right)$, com $\sigma$ permutação de $k+1$ elementos. Definimos o operador de bordo $\partial$ como $\partial\left(\alpha_{0}, \ldots, \alpha_{k}\right)=\sum_{j=0}^{k}(-1)^{j}\left(\alpha_{0}, \ldots, \hat{\alpha}_{j}, \ldots, \alpha_{k}\right)$. O operador $\partial$ deixa $J^{k}$ invariante, e portanto, define um operador em $I^{k} / J^{k}$. Obtemos assim um complexo simplicial abstrato $I=\left\{I^{k} / J^{k}, \partial\right\}_{k \in Z}$ associado à cobertura $\mathscr{U}$ cuja cohomologia com coeficientes em um grupo abeliano $G$ é denotada por $H_{\mathscr{U}}^{*}(X ; G)$. Observamos que os co-ciclos do complexo I são aplicações $f: I^{k} \rightarrow G$ alternadas, i.e., tais que $f \circ \sigma=\operatorname{sgn}(\sigma) f$ e $\delta f=0$, onde $\delta$ é o operador de co-bordo. Se $G$ não é abeliano, ainda assim podemos considerar $H_{\mathscr{U}}^{p}(X ; G)$ : basta observar que a definição de co-ciclos e co-bordos não usa a comutatividade de $G$ e definir, como em cohomologia ordinária, $H_{\mathscr{U}}^{p}(X ; G)$ como o quociente dos co-ciclos de grau $p$ pelos co-bordos de grau $p$. Neste caso, $H_{\mathscr{U}}^{p}(X ; G)$ é somente um conjunto com um elemento distingüido. A cohomologia de Čech com coeficientes em $G$ é definida como $\check{H}^{*}(X ; G) \doteq \varliminf_{\mathscr{U}} H_{\mathscr{U}}^{*}(X ; G)$, onde o limite indutivo é tomado sobre a família de todas as coberturas abertas $\mathscr{U}$ de $X$. Se $G$ é abeliano e $\mathscr{U}$ é uma cobertura aberta tal que $U_{\alpha_{0}} \cap \ldots \cap U_{a_{k}}$ é contrátil para todos $\alpha_{1}, \ldots, \alpha_{k}$, então
} 
$f$ é alternada. Um cálculo imediato usando o fato que

$$
\tau_{\alpha \beta} \tau_{\beta \gamma}=\tau_{\alpha \gamma}, \tau_{\alpha \alpha}=1,
$$

mostra que de fato $f$ é um co-ciclo, e portanto, representa um elemento $w_{1}(E) \in H^{1}\left(X ; \mathbb{Z}_{2}\right)$, o qual é chamado de $1^{a}$ classe de Stiefel-Whitney. As relações 2.2.2 são chamadas de condições de co-ciclo. Vemos que $E$ é orientável se e só se é possível escolher os referenciais $E_{\alpha}$ de tal maneira que $f$ seja constante igual a 1 ; mas isso é equivalente a termos $w_{1}(E)=0$. Logo, $E$ é orientável se e só se $w_{1}(E)=0$.

Assumindo que $E$ é orientável, podemos supor que os referenciais são escolhidos de maneira que $\tau_{\alpha \beta}: U_{\alpha} \cap U_{\beta} \rightarrow \mathrm{SO}(n)$. Lembrando do recobrimento $\vartheta_{0}: \operatorname{Spin}(n) \rightarrow \operatorname{SO}(n)$ definido na proposição 2.2.5, podemos nos perguntar se é possível obter levantamentos $\tilde{\tau}_{\alpha \beta}$ de $\tau_{\alpha \beta}$ a $\operatorname{Spin}(n)$ (por $\vartheta_{0}$ ) satisfazendo as condições de co-ciclo. Vemos que cada aplicação $\tau_{\alpha \beta}: U_{\alpha} \cap U_{\beta} \rightarrow \mathrm{SO}(n)$ admite dois levantamentos a $\operatorname{Spin}(n)$, pois $U_{\alpha} \cap U_{\beta}$ é simplesmente conexo e, se $\tilde{\tau}_{\alpha \beta}: U_{\alpha} \cap U_{\beta} \rightarrow \operatorname{Spin}(n)$ denota um destes levantamentos, como $\vartheta_{0}\left(\tilde{\tau}_{\alpha \beta} \tilde{\tau}_{\beta \gamma}\right)=$ $\vartheta_{0}\left(\tilde{\tau}_{\alpha \gamma}\right)$, então $\tilde{\tau}_{\alpha \beta} \tilde{\tau}_{\beta \gamma}\left(\tilde{\tau}_{\alpha \gamma}\right)^{-1} \in \operatorname{ker} \vartheta_{0}=\{ \pm 1\}=\mathbb{Z}_{2}$. Logo, a aplicação $f: I^{3} \rightarrow \mathbb{Z}_{2}$ dada por $f(\alpha, \beta, \gamma)=\tilde{\tau}_{\alpha \beta} \tilde{\tau}_{\beta \gamma}\left(\tilde{\tau}_{\alpha \gamma}\right)^{-1}$ é alternada. Um cálculo simples mostra que $f$ é um co-ciclo, $\mathrm{e}$ portanto, representa uma classe de cohomologia $w_{2}(E) \in H^{2}\left(X ; \mathbb{Z}_{2}\right)$ chamada de $2^{a}$ classe de Stiefel-Whitney. Como no caso da $1^{a}$ classe de Stiefel-Whitney, vemos que é possível escolher os levantamentos $\tilde{\tau}_{\alpha \beta}$ satisfazendo as propriedades requeridas se e só se $w_{2}(E)=0$.

Definição 2.2.13 O fibrado $E$ é dito spin se $w_{1}(E)=0$ e $w_{2}(E)=0$. Uma variedade $X$ é dita spin se TX for um fibrado spin.

ObSERVAÇÃo 2.2.14 Dado qualquer fibrado vetorial $n$-dimensional $E$ sobre uma variedade $X$, podemos definir as classes de Stiefel-Whitney $w_{k}(E) \in H^{k}\left(X ; \mathbb{Z}_{2}\right), k \in \mathbb{N}$. Tais classes podem ser definidas axiomaticamente, como em [67], mas para nossos propósitos, é mais útil uma apresentação em termos da teoria de obstrução. De fato, seja $V_{k}(E)$ o fibrado dos $k$-referenciais em $E$ (a fibra de $V_{k}(E)$ sobre $x \in X$ é o conjunto dos das $k$-uplas de vetores ortonormais em $E_{x}$ ). Cada fibra de $V_{k}(E)$ é homeomorfa ao espaço $V_{k}\left(\mathbb{R}^{n}\right)$ (das $k$-uplas de vetores ortonormais em $\left.\mathbb{R}^{n}\right)$, o qual é $(n-k)$-conexo, por ${ }^{12}$ um argumento simples usando a seqüência exata da fibração. Portanto, dada uma triangulação de $X$, é possível obter uma seção $s$ de $V_{k}(E)$ definida no esqueleto $X^{n-k}$. Usando o método de teoria da obstrução (conforme [53]), obtemos uma classe de cohomologia $o_{n-k+1}(E) \in H^{n-k+1}\left(X ;\left\{\pi_{n-k}\left(V_{k}\left(E_{x}\right)\right)\right\}_{x \in X}\right)$, a qual é nula se e só se a seção $s$ admite extensão ao esqueleto $X^{n-k+1}$. Aqui, usamos coeficientes locais no fibrado de coeficientes (feixe) $\left\{\pi_{n-k}\left(V_{k}\left(E_{x}\right)\right)\right\}_{x \in X}$. Em particular, se $E$ é trivial então $w_{k}(E)=0$ para todo $k=1, \ldots, n$. Como $\pi_{n-k}\left(V_{k}\left(E_{x}\right)\right)$ é $\mathbb{Z}$ ou $\mathbb{Z}_{2}$ (conforme [95], Cap.II-§25.6), podemos definir $w_{n-k}(E) \in H^{n-k}\left(X ; \mathbb{Z}_{2}\right)$ como a redução módulo 2 da classe $o_{n-k+1}(E)$. Esta foi a maneira original pela qual foram definidas as classes de Stiefel-Whitney, conforme [95]. Obviamente, esta definição e a definição de $w_{1}(E), w_{2}(E)$ dada anteriormente em termos de cohomologia de Čech coincidem, apesar de que nesta definição não é necessário que $w_{1}(E)=0$ para que $w_{2}(E)$ seja definida. Todos os detalhes podem ser encontrados em [67]. A classe de Stiefel-Whitney total é definida como $w(E)=w_{0}(E)+w_{1}(E)+\ldots+w_{n}(E) \in H^{*}\left(X ; \mathbb{Z}_{2}\right)$.

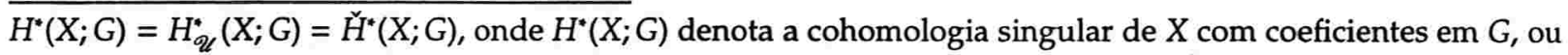
seja, não é necessário considerar o limite indutivo na definição de cohomologia de Čech, desde que utilizemos uma cobertura com a propriedade supra citada. Em particular, para variedades, a cohomologia de Čech coincide com a cohomologia singular. Para mais detalhes, veja [42], [68] e [93].

${ }^{12}$ Um espaço $Y$ é dito $r$-conexo, $r \geq 1$, se $\pi_{j}(Y)=0$, para todo $j \leq r$. 
Vamos agora olhar a situação sob outro ângulo. Dado um grupo topológico $G$ e uma cobertura aberta $\mathscr{U}=\left\{U_{\alpha}\right\}$ de $X$, consideremos $u$ um co-ciclo que representa uma classe de cohomologia em $H_{\mathscr{U}}^{1}(X ; G) ; u$ nada mais é que uma família de aplicações contínuas $u_{\alpha \beta}$ : $U_{\alpha} \cap U_{\beta} \rightarrow G$ definidas sempre que $U_{\alpha} \cap U_{\beta} \neq \emptyset$, satisfazendo a condição de co-ciclo. Vamos construir um fibrado $G$-principal associado a $u$. De fato, consideremos sobre a reunião disjunta $Y \doteq \bigcup_{\alpha} U_{\alpha} \times G$ a relação de equivalência dada por $\left(x_{\alpha}, g\right) \equiv\left(x_{\alpha}, g \cdot u_{\alpha \beta}\left(x_{\alpha}\right)\right)$, com $x_{\alpha} \in U_{\alpha} \mathrm{e}$ $g \in G$. Seja $E \doteq Y / \equiv$ munido da topologia quociente e $\pi: E \rightarrow X$ dada por $\pi\left(\left[\left(x_{\alpha}, g\right)\right]\right)=x_{\alpha}$, onde $\left[\left(x_{\alpha}, g\right)\right]$ denota a classe de equivalência de $\left(x_{\alpha}, g\right)$. Temos uma ação natural de $G$ sobre os conjuntos $\pi^{-1}(x)$ para cada $x \in X$ e trivializações locais compatíveis com esta ação. Portanto, $E$ é um fibrado $G$-principal associado à $u$.

Por outro lado, seja $\pi: E \rightarrow X$ um fibrado $G$-principal e uma cobertura aberta $\mathscr{U}=\left\{U_{a}\right\}$ de $X$ tal que a restrição de $E$ a cada $U_{\alpha}$ seja trivial. Dadas trivializações $h_{\alpha}: \pi^{-1}\left(U_{\alpha}\right) \rightarrow U_{\alpha} \times G$, se $U_{\alpha} \cap U_{\beta} \neq \emptyset$ definimos aplicações contínuas $u_{\alpha \beta}: U_{\alpha} \cap U_{\beta} \rightarrow G$ pela fórmula

$$
h_{\alpha} \circ h_{\beta}^{-1}\left(x_{\alpha}, g\right)=\left(x_{\alpha}, u_{\alpha \beta}(g)\right)
$$

$x_{\alpha} \in U_{\alpha}, g \in G$. É imediato verificar que a família $u \doteq\left\{u_{\alpha \beta}\right\}$ verifica a condição de co-ciclo 2.2.2, logo, $u$ representa um elemento de $H_{\mathscr{U}}^{1}(X ; G)$. Se $E^{\prime}$ é outro fibrado sobre $X$, não é difícil verificar que $E^{\prime}$ é equivalente a $E$ se e só se existe uma família de aplicações contínuas $v \doteq\left\{v_{\alpha}\right\}$, $v_{\alpha}: U_{\alpha} \rightarrow G$ tais que $u_{\alpha \beta}^{\prime}=v_{\alpha}^{-1} \cdot u_{\alpha \beta} \cdot v_{\beta}$ em $U_{\alpha} \cap U_{\beta}$, para todos $\alpha, \beta$. Esta última igualdade implica que $u$ e $u^{\prime}$ definem o mesmo elemento em $H_{\mathscr{U}}^{1}(X ; G)$, portanto, provamos o seguinte resultado.

Proposição 2.2.15 Existe uma correspondência biunívoca entre classes de equivalência de fibrados G-principais sobre X e $\check{H}^{1}(X ; G)$.

A correspondência enunciada na proposição 2.2.15 é muito útil para definirmos o que é uma estrutura spin. Consideremos $P_{\mathrm{O}}(E), P_{\mathrm{SO}}(E)$ os fibrados $\mathrm{O}(n)$-principal e $\mathrm{SO}(n)$-principal formados pelos referenciais ortonormais de $E$ e pelos referenciais ortonormais positivos de $E$, respectivamente e voltemos à construção que antecede a definição 2.2.13. Se $w_{1}(E)=0 \mathrm{e}$ $w_{2}(E)=0$, então existem os levantamentos $\tilde{\tau}_{\alpha \beta}: U_{\alpha} \cap U_{\beta} \rightarrow \operatorname{Spin}(n)$ satisfazendo a condição de co-ciclo 2.2.2. Fazendo agora a mesma construção que foi feita para provar a proposição 2.2.15, obtemos um fibrado Spin(n)-principal $P_{\text {Spin }}(E)$ e um recobrimento de duas folhas $\xi$ : $P_{\text {Spin }}(E) \rightarrow P_{\text {So }}(E)$ tal que $\xi(p \cdot g)=\xi(p) \vartheta_{0}(g)$ para todos $p \in P_{\text {Spin }}(E)$ e $g \in \operatorname{Spin}(n)$. Isso nos motiva a dar a seguinte definição.

Definição 2.2.16 Uma estrutura spin em um fibrado spin $E$ é um fibrado Spin(n)-principal $P_{\text {Spin }}(E)$ e um recobrimento de duas folhas $\xi: P_{\text {Spin }}(E) \rightarrow P_{\text {So }}(E)$ tal que $\xi(p \cdot g)=\xi(p) \cdot \vartheta_{0}(g)$ para todos $p \in P_{\text {Spin }}(E)$ e $g \in \operatorname{Spin}(n)$.

Observação 2.2.17 Considerando o quociente $\operatorname{Or}(E) \doteq P_{\mathrm{O}}(E) / \mathrm{SO}(n)$, obtemos um recobrimento de $E$ com duas folhas. $E$ é orientável se e só se $\operatorname{Or}(E)$ é trivial. Pela proposição 2.2.15, $\operatorname{Or}(E)$ define um elemento de $\breve{H}^{1}\left(X ; \mathbb{Z}_{2}\right) \approx H^{1}\left(X ; \mathbb{Z}_{2}\right)$, o qual claramente coincide com $w_{1}(E)$. Isto nos permite dar uma definição alternativa da $1^{a}$ classe de Stiefel-Whitney. Dizer que $E$ é orientável significa dizer que $w_{1}(E)=0$, ou equivalentemente, que é possível simplificar o grupo estrutural de $E$, passando do grupo desconexo $\mathrm{O}(n)$ para o grupo 0-conexo $\mathrm{SO}(n)$. A passagem de $\mathrm{SO}(n)$ a $\mathrm{Spin}(n)$ corresponde exatamente à segunda etapa deste processo, i.e., 
passar do grupo 0-conexo $\mathrm{SO}(n)$ ao grupo 1-conexo $\operatorname{Spin}(n)$, para $n \geq 3$, conforme a proposição 2.2.8.

Vamos agora relacionar estruturas spin em um fibrado $E$ com estruturas que dependem unicamente da estrutura topológica de $E$, conforme [65]. O referido artigo contém ainda outras definições interessantes de estruturas spin relacionadas com teoria de homotopia.

Proposição 2.2.18 Existe uma correspondência biunívoca entre estruturas spin em um fibrado orientado E e classes de cohomologia $u \in H^{1}\left(P_{\mathrm{SO}}(E), \mathbb{Z}_{2}\right)$ cuja restrição à cada fibra é não-trivial.

Prova. Basta observar que, pela proposição 2.2.15, existe uma correspondência biunívoca entre $H^{1}\left(P_{\mathrm{SO}}(E), \mathbb{Z}_{2}\right)$ e recobrimentos de duas folhas $\xi: E^{\prime} \rightarrow P_{\mathrm{SO}}(E)$. Estruturas spin em $E$ correspondem exatamente aos recobrimentos de duas folhas $\xi: E^{\prime} \rightarrow P_{\mathrm{SO}}(E)$ não-triviais. Mas um tal recobrimento é não-trivial em cada fibra $E_{x}^{\prime}$ de $E^{\prime}$ se e só se $E_{x}^{\prime}$ identifica-se com $\operatorname{Spin}(n)$ e a restrição de $\left.\xi\right|_{E_{x}^{\prime}}: E_{x}^{\prime} \rightarrow P_{\mathrm{SO}}(E)$ coincide com o único recobrimento de duas folhas não-trivial de $\mathrm{SO}(n)$, i.e., $\operatorname{com} \vartheta_{0}: \operatorname{Spin}(n) \rightarrow \mathrm{SO}(n)$. Portanto, $E^{\prime}$ admite estrutura de fibrado $\operatorname{Spin}(n)$-principal e $\xi: E^{\prime} \rightarrow P_{\mathrm{SO}}(E)$ é uma estrutura spin em $E$.

Proposição 2.2.19 Se $w_{2}(E)=0$ então existe uma correspondência biunívoca entre estruturas spin em E e elementos de $H^{1}\left(X, \mathbb{Z}_{2}\right)$.

Prova. Considerando a fibração $p: P_{\mathrm{SO}}(E) \rightarrow X$ e usando a seqüência espectral de Serre (conforme [93]), obtemos uma seqüência exata

$$
0 \longrightarrow H^{1}\left(X ; \mathbb{Z}_{2}\right) \stackrel{p^{*}}{\longrightarrow} H^{1}\left(P_{\mathrm{SO}}(E) ; \mathbb{Z}_{2}\right) \stackrel{i}{\longrightarrow} H^{1}\left(\mathrm{SO}(n) ; \mathbb{Z}_{2}\right) \stackrel{w_{E}}{\longrightarrow} H^{2}\left(X ; \mathbb{Z}_{2}\right),
$$

onde $i$ denota a inclusão natural da fibra $\mathrm{SO}(n)$ em $P_{\mathrm{SO}}(E)$. Como $w_{2}(E)$ é a imagem do gerador de $H^{1}\left(\mathrm{SO}(n) ; \mathbb{Z}_{2}\right) \approx \mathbb{Z}_{2}$ por $w_{E}$ (por naturalidade das classes características), temos que $w_{E}=0$, provando o desejado.

As próximas proposições nos fornecem condições geométricas interessantes para que um fibrado ou uma variedade sejam spin.

Proposição 2.2.20 Seja E um fibrado orientado sobre $X$ de dimensão $\geq 3$. Então $E$ é spin se e só se para qualquer superficie compacta $S$ e qualquer $f: S \rightarrow X$ contínua, o fibrado $f^{*}(E)$ é trivial. Se $X$ é simplesmente conexa e tem dimensão $>4$ então $E$ é spin se e só se a restrição de $E$ a qualquer esfera mergulhada em Xé trivial.

Prova. Se $E$ é spin, então $w_{2}(E)=0$, portanto, dada $f$ como no enunciado, temos que $w_{2}\left(f^{*}(E)\right)=f^{*}\left(w_{2}(E)\right)=0$. Isso implica que $f^{*}(E)$ é trivial. ${ }^{13}$ Reciprocamente, suponhamos que $f^{*}(E)$ é trivial para toda $f$ como no enunciado e provemos que $w_{2}(E)=0$. De fato, por dualidade de Poincaré, $H_{1}(X)$ é livre (conforme [49], Pag.236), logo, pelo teorema dos coeficientes universais, $H^{2}\left(X ; \mathbb{Z}_{2}\right) \approx \operatorname{Hom}\left(H_{2}(X), \mathbb{Z}_{2}\right)$. Como $H_{2}(X)$ é gerado por aplicações contínuas de superfícies compactas, a hipótese implica que $w_{2}(E)$ representa a homomorfismo nulo em $\operatorname{Hom}\left(H_{2}(X), \mathbb{Z}_{2}\right)$, portanto, $w_{2}(E)=0$.

Supondo $X$ simplesmente conexa de dimensão $>4$, temos pelo teorema de Hurewicz (veja [49], [93]) que $H_{1}(X)=0$ e $H_{2}(X) \approx \pi_{2}(X)$. Logo, $H_{2}(X)$ é gerado por aplicações contínuas

\footnotetext{
${ }^{13}$ Para concluir este fato, podemos utilizar a construção das classes características em termos de classes de obstrução dada na observação 2.2.14.
} 
definidas em esferas. A hipótese sobre $\operatorname{dim} X$ implica (pelos teoremas de aproximação e mergulho de Whitney, conforme [50]) que $\mathrm{H}_{2}(\mathrm{X})$ é gerado por mergulhos de esferas. Usando o mesmo argumento do parágrafo anterior, concluímos que $w_{2}(E)=0$.

Proposição 2.2.21 Seja X uma variedade riemanniana n-dimensional orientada e simplesmente conexa. Se $n \geq 5$ então X é spin se e só se qualquer superficie compacta mergulhada em X tem fibrado normal trivial. Se $n=4$ então $X$ é spin se e só se o fibrado normal de qualquer superficie compacta orientada mergulhada em $X$ tem classe de Euler par. ${ }^{14}$

Prova. Se $\operatorname{dim} X \geq 4$, temos que $H_{2}(X)$ é gerado por mergulhos de de superfícies compactas orientadas. ${ }^{15}$ Dado $i: S \rightarrow X$ um destes mergulhos, então $i^{*}\left(w_{2}(X)\right)=i^{*}\left(w_{2}(T X)\right)=$ $i^{*}\left(w_{2}(T S \oplus N S)\right)=w_{2}(N S)$, onde $N S$ denota o fibrado normal de $S$ em X. Calculando na classe fundamental de $S$, temos $\left(w_{2}(X), i_{*}([S])\right)=\left(i^{*}\left(w_{2}(X)\right),[S]\right)=\left(w_{2}(N S),[S]\right)$, logo, se $w_{2}(X)=0$ então $w_{2}(N S)=0$. Como $\mathrm{H}_{2}(\mathrm{X})$ é gerado por aplicações nestas condições, concluímos, como no primeiro parágrafo da proposição 2.2.20, que $w_{2}(X)=0$ se e só se $w_{2}(N S)=0$. A última afirmação decorre do fato que $e\left(E^{\prime}\right)=w_{n}\left(E^{\prime}\right) \bmod 2$, onde $e\left(E^{\prime}\right)$ denota a classe de Euler do fibrado $n$-dimensional $E^{\prime}$ sobre $X$.

Vejamos alguns exemplos de variedades spin.

EXEMPLO 2.2.22 As seguintes variedades são spin:

1. Se $X$ é 2-conexa então $X$ admite uma única estrutura spin, pela proposição 2.2.19 e pelo teorema de Hurewicz.

2. Se $E$ é estavelmente paralelizável ${ }^{16}$ então $E$ é spin. De fato, neste caso, $w_{k}(E)=0$ para todo $k \in \mathbb{N}$. Em particular, qualquer grupo de Lie e qualquer variedade dada como imagem inversa de um valor regular para $f: \mathbb{R}^{n+p} \rightarrow \mathbb{R}^{n}$ é spin. Se $X$ é uma variedade compacta tridimensional então X é paralelizável (Teorema de Stiefel, veja [67], Pag.148), portanto $X$ é spin. Se $X$ é bidimensional orientada, também é verdade que $X$ é spin, pois neste caso, $X$ é homeomorfa a um $n$-toro, o qual é estavelmente paralelizável.

3. Uma variedade complexa $X$ é spin se e só se $c_{1}(X) \equiv 0 \bmod 2$, onde $c_{k}(X)$ denota a $k$-ésima classe de Chern de $X$ (veja [67], §14). De fato, como toda variedade complexa $X$ é orientável, temos que $X$ é spin se e só se $c_{2}(X)=0$. A conclusão decorre do fato que $c_{k}(E) \equiv w_{k}(E) \bmod 2$, para todo $k \in \mathbb{N}$.

4. Dado $n \in \mathbb{N}$, temos que $w\left(\mathbb{R} \mathbb{P}^{n}\right)=(1+a)^{n+1}$, onde $a$ é um gerador de $H^{1}\left(\mathbb{R} \mathbb{P}^{n} ; \mathbb{Z}_{2}\right) \approx \mathbb{Z}_{2}$. Logo, $\mathbb{R P}^{n}$ é spin se e só se $n+1 \equiv 0 \bmod 2$ e $\left(\begin{array}{c}n+1 \\ 2\end{array}\right) \equiv 0 \bmod 2$, i.e., $n \equiv 3 \bmod 4$.

5. No caso complexo, um argumento semelhante ao ítem 4 mostra que $\mathbb{C P}^{n}$ é spin se e só se $n$ é ímpar.

\footnotetext{
${ }^{14} \mathrm{~A}$ classe de Euler e de um fibrado $n$-dimensional orientado $\pi: E \rightarrow X$ é definida como a única classe $e \in H^{n}(X ; \mathbb{Z})$, tal que $\pi^{*}(e)$ restrita às fibras $E_{x}$ produz a orientação escolhida de $E_{x}$, para todo $x \in X$.

${ }^{15}$ Novamente pelos teoremas de aproximação e mergulho de Whitney.

${ }^{16} \mathrm{Ou}$ seja, existe um fibrado trivial $E^{\prime}$ sobre $X$ tal que $E \oplus E^{\prime}$ é trivial
} 


\subsubsection{Fibrados de Clifford, fibrados spinor e conexões}

Nesta subseção, globalizaremos as construções de estruturas de Clifford da subsecão 2.2.1 e introduziremos os fibrados spinor. Vamos também estudar conexões definidas nestes fibrados e a sua correspondência com a estrutura algébrica.

Seja $\pi: P \rightarrow X$ um fibrado G-principal e seja Homeo $(F)$ o grupo de homeomorfismos de um espaço $F$ munido da topologia compacto-aberta usual. Dado um homomorfismo contínuo $\rho: G \rightarrow \operatorname{Homeo}(F)$, vamos construir o fibrado associado a $P$ por $\rho$. Consideremos a ação à esquerda de $G$ sobre $P \times F$ dada por

$$
g \cdot(p, f) \doteq\left(p \cdot g^{-1}, \rho(g) f\right),
$$

$\operatorname{com} p \in P, f \in F$ e $g \in G$. Definimos $P \times_{\rho} F$ como o espaço das órbitas desta ação. A composição $P \times F \rightarrow P \rightarrow X$, onde a primeira aplicação é a projeção na primeira coordenada e a segunda é $\pi$ passa ao quociente e define um fibrado $\pi_{\rho}: P \times_{\rho} F \rightarrow X$, chamado fibrado associado a $P$ por $\rho$. Caso trabalhemos na categoria diferenciável (i.e., $X, F$ são variedades $C^{\infty}, G$ é um grupo de Lie e $\rho$ toma valores no grupo de difeomorfismos de $F$ ), então $\pi_{\rho}: P \times_{\rho} F \rightarrow X$ é um fibrado $C^{\infty}$. Se $F=\mathbb{R}^{n}$ e $\rho: G \rightarrow \mathrm{GL}\left(\mathbb{R}^{n}\right)$ é um homomorfismo, então $P \times_{\rho} F$ é um fibrado vetorial sobre $X$.

Vejamos agora alguns exemplos importantes de fibrados associados.

Exemplo 2.2.23 Se $E$ é um fibrado vetorial $n$-dimensional, $P \doteq P_{\mathrm{GL}}(E)$ é o fibrado $\mathrm{GL}_{n}(\mathbb{R})$ principal ${ }^{17}$ dos referenciais em $E$ (a fibra de $P_{\mathrm{GL}}(E)$ sobre $x \in X$ é o conjunto de todos referenciais em $E_{x}$, i.e., o conjunto de todas as $n$-uplas $\left(v_{1}, \ldots, v_{n}\right)$, com $v_{j} \in E_{x}$, tal que $\left\{v_{1}, \ldots, v_{n}\right\}$ é uma base de $E_{x}$ ) e $\rho_{n}: \mathrm{GL}_{n}(\mathbb{R}) \rightarrow \mathrm{GL}(n)$ é a representação standard, então $P \times_{\rho_{n}} \mathbb{R}^{n}$ é equivalente a $E$. Utilizando a representação adjunta $\rho_{n}^{*}(g) \doteq \rho_{n}\left(g^{-1}\right)^{*}$, vemos que $P \times_{p_{n}^{*}} \mathbb{R}^{n} \approx E^{*}$, onde $E^{*}$ denota o fibrado dual de $E$. Considerando as representações induzidas $\Lambda^{k} \rho_{n}: \mathrm{GL}_{n}(\mathbb{R}) \rightarrow \operatorname{Aut}\left(\Lambda^{k} \mathbb{R}^{n}\right)$ e $\otimes^{k} \rho_{n}: \mathrm{GL}_{n}(\mathbb{R}) \rightarrow \operatorname{Aut}\left(\otimes^{k} \mathbb{R}^{n}\right)$, obtemos fibrados $P \times_{\Lambda^{k} \rho_{n}} \Lambda^{k} \mathbb{R}^{n} \approx \Lambda^{k}(E)$ e $P \times_{\otimes^{k} \rho_{n}} \otimes^{k} \mathbb{R}^{n} \approx \otimes^{k}(E)$, onde $\otimes^{k}(E)$ e $\Lambda^{k}(E)$ denotam os fibrados de tensores de grau $k$ e tensores de grau $k$ alternados sobre $E$, respectivamente.

Exemplo 2.2.24 Se E é um fibrado riemanniano, podemos utilizar no exemplo 2.2.23 o fibrado $P_{\mathrm{O}}(E)$ (definido após a proposição 2.2.15) ao invés de $P_{\mathrm{GL}}(E)$. Se além disso, $E$ é orientado, podemos ainda usar o fibrado $P_{\mathrm{SO}}(E)$.

Dado $T \in \mathrm{O}(n)$, definimos $T\left(v_{1} \otimes \ldots \otimes v_{k}\right)=T\left(v_{1}\right) \otimes \ldots \otimes T\left(v_{k}\right), k \in \mathbb{N}$, e estendemos $T$ por linearidade a $\mathscr{T}\left(\mathbb{R}^{n}\right)$, conforme notação no início da subseção 2.2.1. Como $T$ preserva o ideal $\mathscr{I}$, segue que $T$ define um automorfismo $\mathrm{Cl}(n) \rightarrow \mathrm{Cl}(n)$ tal que $T^{*} T=T T^{*}=I$. Obtemos desta maneira uma representação $\mathrm{cl}\left(\rho_{n}\right): \mathrm{SO}(n) \rightarrow \operatorname{Aut}(\mathrm{Cl}(n))$.

DEFINIÇão 2.2.25 O fibrado de Clifford associado ao fibrado orientado riemanniano $E$ é definido $\operatorname{como} \mathrm{Cl}(E) \doteq P_{\mathrm{SO}}(E) \times_{\mathrm{cl}\left(\rho_{n}\right)} \mathrm{Cl}(n)$.

Vemos que $\mathrm{Cl}(E)$ é um fibrado vetorial sobre $E$ cujas fibras admitem estrutura de $\mathrm{Cl}(n)$ módulo à esquerda. Todas as construções feitas para álgebras de Clifford podem ser feitas nesta situação mais geral. Por exemplo, temos uma decomposição $\mathrm{Cl}(E)=\mathrm{Cl}^{0}(E) \oplus \mathrm{Cl}^{1}(E)$,

\footnotetext{
${ }^{17}$ Neste exemplo, vamos fazer distinção entre o grupo $\mathrm{GL}_{n}(\mathbb{R})$ formado pelas matrizes reais $n \times n$ inversíveis e o grupo $G L(n)$ dos operadores inversíveis em $\mathbb{R}^{n}$.
} 
$\operatorname{com~} \mathrm{Cl}^{i}(E)$ sub-fibrados tais que $\mathrm{Cl}^{i}(n) \cdot \mathrm{Cl}^{j}(E) \subset \mathrm{Cl}^{i+j}(E)$, com $i, j \in \mathbb{Z}_{2}$. Além disso, obtemos também que $\mathrm{Cl}(E)$ e $\Lambda^{*}(E)$ são equivalentes como fibrados vetoriais.

Vamos agora definir uma classe muito importante de fibrados associados.

Definição 2.2.26 Se $\xi: P_{\text {Spin }}(E) \rightarrow P_{\mathrm{SO}}(E)$ é uma estrutura spin sobre o fibrado riemanniano $E$, então um fibrado spinor real é um fibrado da forma

$$
\mathrm{S}(E) \doteq P_{\mathrm{Spin}}(E) \times_{\mu} V,
$$

onde $V$ é um $\mathrm{Cl}(n)$-módulo à esquerda ${ }^{18}$ e $\mu: \operatorname{Spin}(n) \rightarrow \mathrm{SO}(V)$ é a representação dada por multiplicação à esquerda por elementos de $\operatorname{Spin}(n)$, i.e., $\mu(\varphi)$ é o automorfismo de multiplicação à esquerda por $\varphi \in \operatorname{Spin}(n) \subset \mathrm{Cl}(n)$. No caso complexo, um fibrado spinor complexo é um fibrado da forma

$$
\mathrm{S}_{\mathbf{C}}(E) \doteq P_{\mathrm{Spin}}(E) \times_{\mu} V_{\mathbf{C}},
$$

onde $V_{\mathbb{C}}$ é um $\mathbb{C l}(n)$-módulo à esquerda $M_{\mathbf{C}}$ e $\mu: \operatorname{Spin}(n) \rightarrow \mathrm{U}\left(V_{\mathbf{C}}\right)$ é como antes. Um spinor é simplesmente uma seção de um fibrado spinor.

Daremos agora um exemplo muito importante de fibrado spinor real.

EXEMPLo 2.2.27 Considerando $\mathrm{Cl}(n)$ como um $\mathrm{Cl}(n)$-módulo à esquerda, definimos

$$
\mathrm{Cl}_{\mathrm{Spin}}(E) \doteq P_{\mathrm{Spin}}(E) \times_{\ell} \mathrm{Cl}(n),
$$

onde $\ell: \operatorname{Spin}(n) \rightarrow \operatorname{Aut}(\mathrm{Cl}(n))$ é a representação por multiplicação à esquerda. Como existe uma inclusão natural $P_{\text {Spin }}(E) \subset \mathrm{Cl}_{\text {Spin }}(E)$, vemos que todo fibrado spinor real pode ser obtido a partir de $\mathrm{Cl}_{\text {Spin }}(E)$.

Vamos agora descrever a relação entre $\mathrm{Cl}(E)$ e $\mathrm{Cl}_{\text {Spin }}(E)$. Considerando a representação adjunta Ad : $\operatorname{Spin}(n) \rightarrow \operatorname{Aut}(\mathrm{Cl}(n))$, observamos que $\mathrm{Ad}( \pm 1)=I$, logo, Ad define uma representação $\mathrm{Ad}^{\prime}: \mathrm{SO}(n) \rightarrow \operatorname{Aut}(\mathrm{Cl}(n))$, a qual coincide com a representação $\mathrm{cl}\left(\rho_{n}\right)$, portanto $\mathrm{Cl}(E)=P_{\text {Spin }}(E) \times_{\mathrm{Ad}^{\prime}} \mathrm{Cl}(n)$.

A próxima proposição mostra que além da estrutura usual de fibrado vetorial, os fibrados spinor admitem uma importante estrutura de produto.

Proposição 2.2.28 Se $\mathrm{S}(E)$ é um fibrado spinor real sobre $\mathrm{X}$, então $\mathrm{S}(E)$ é um $\mathrm{Cl}(E)$-módulo d̀ esquerda, i.e., cada fibra $\mathrm{S}(E)_{x}$ é um $\mathrm{Cl}(E)_{x}$-módulo à esquerda. Em particular, o espaço de seções de $\mathrm{S}(E)$ é um módulo sobre o espaço de seções de $\mathrm{Cl}(E)$. O mesmo é verdadeiro para um fibrado spinor complexo $\mathrm{S}_{\mathbf{C}}(E)$.

Como no caso de álgebras de Clifford, podemos também definir o conceito de irredutibilidade. Dizemos que um fibrado $F$ de $\mathrm{Cl}(E)$-módulos à esquerda é irredutível se para cada $x \in X, F_{x}$ é um $\mathrm{Cl}(E)_{x}$-módulo irredutível. Nesta situação mais geral, também é verdade que um fibrado $F$ de $\mathrm{Cl}(E)$-módulos também é soma direta de fibrados irredutíveis. Em particular, é válida uma proposição análoga à proposição 2.2.12. O mesmo verifica-se no caso complexo.

\footnotetext{
${ }^{18} \mathrm{Com}$ isto, queremos dizer que $M$ é um espaço vetorial orientado com produto interno que admite estrutura de $\mathrm{Cl}(n)$-módulo à esquerda.
} 
Analisemos alguns casos importantes de fibrados spinor. Suponhamos $n$ par e seja $\mathrm{S}_{\mathbb{C}}(E)$ o fibrado spinor complexo irredutível associado a $E$. Definimos o elemento de volume complexo por

$$
\omega_{\mathbb{C}}=i^{n / 2} e_{1} \cdot \ldots \cdot e_{n},
$$

onde $\left\{\dot{e}_{j}\right\}_{j=1}^{n}$ é um referencial ortonormal local de $E$. Vemos, como na proposição 2.2.10, que $\omega_{\mathbf{C}}$ é bem-definido, $\omega_{\mathbf{C}}^{2}=1$ e $\omega_{\mathbf{C}} \cdot v=-v \cdot \omega_{\mathbb{C}}$, para qualquer $v \in E$. Logo, podemos definir $\mathrm{S}_{\mathbf{C}}^{ \pm}(E) \doteq \operatorname{ker}\left(\omega_{\mathbb{C}} \mp 1\right)$, onde identificamos $\omega_{\mathbb{C}}$ com o operador de multiplicação por $\omega_{\mathbb{C}}$. Vemos que $\mathrm{S}_{\mathbf{C}}^{ \pm}(E)$ são fibrados de $\mathbb{C l}^{0}(E)$-módulos à esquerda de mesma dimensão, $\mathbb{C l}^{1}(E) \cdot \mathrm{S}_{\mathbf{C}}^{ \pm}(E) \subset$ $\mathrm{S}_{\mathbf{C}}^{\mp}(E) \mathrm{e} \cdot \mathrm{S}_{\mathbf{C}}^{+}(E) \oplus \mathrm{S}_{\mathbf{C}}^{-}(E)=\mathrm{S}_{\mathbf{C}}(E)$.

No caso real, se $n=4 m$ e $S(E)$ é o fibrado spinor real irredutível associado a $E$, definimos $\omega=e_{1} \cdot \ldots \cdot e_{n}$. Temos que $\omega^{2}=1$ e $\omega \cdot v=-v \cdot \omega$ para $v \in \mathrm{Cl}^{0}(E)$. Definindo $\mathrm{S}^{ \pm}(E)$ como no caso complexo, obtemos uma decomposição $\mathrm{S}(E)=\mathrm{S}^{+}(E) \oplus \mathrm{S}^{-}(E)$, com $\mathrm{S}^{+}(E), \mathrm{S}^{-}(E) \mathrm{Cl}^{0}(E)$-módulos à esquerda $\mathrm{e}^{1}(E) \cdot \mathrm{S}^{ \pm}(E) \subset \mathrm{S}^{\mp}(E)$.

Uma vez construídos os fibrados de Clifford e spinor, podemos seguir adiante e estudar conexões nestes fibrados. A questão principal é relacionar a derivada covariante com a estrutura de produto (fórmula de Leibniz). Iniciaremos estudando a teoria geral de conexões em fibrados principais, seguindo [56], Vol.I e [94], Vol.II, e depois passaremos aos casos Clifford e spinor.

Dado um fibrado $G$-principal $\pi: P \rightarrow X$, podemos associar a cada $Y \in \mathfrak{g}$ (g denota a álgebra de Lie de $G$ ) o campo de vetores $\sigma(Y)$ definido em $P$ por

$$
\sigma\left(\left.Y_{p} \doteq \frac{d}{d t}\right|_{t=0} R_{\exp t \gamma(p)},\right.
$$

onde $R_{g}$ denota a ação à direita de $g \in G$ em $P$. É fácil ver que a aplicação $Y \in \mathfrak{g} \mapsto \sigma(Y) \in \mathfrak{X}(P)$ é linear injetora, onde $\mathfrak{X}(P)$ denota o espaço dos campos $C^{\infty}$ em $P$. Um vetor tangente a $p \in P$ é dito vertical se for da forma $\sigma(Y)_{p}$ para algum $Y \in \mathrm{g}$. O conjunto dos vetores tangentes verticais em $p$ é um subespaço de $T_{p} P$. Uma conexão em $P$ é uma escolha invariante de complementares para a distribuição de subespaços verticais.

Definição 2.2.29 Uma conexão em $P$ é uma 1-forma definida em $P$ à valores em $\mathrm{g}$ tal que

1. $\omega(\sigma(Y))=Y$ para todo $Y \in \mathfrak{g}$;

2. $R_{g}^{*}(\omega)=\operatorname{Ad}_{g^{-1}} \omega$, para todo $g \in G$.

A forma de curvatura correspondente a $\omega$ é a 2-forma definida como $\Omega \doteq d \omega-\omega \wedge \omega$.

OBSERVAÇão 2.2.30 Dada uma conexão $\omega$ em $P$, podemos definir uma distribuição de $n$-planos $(n=\operatorname{dim} X)$ complementar à distribuição vertical pondo $\theta_{p} \doteq \operatorname{ker} \omega_{p}, p \in P$. Esta distribuição é invariante pela ação de $G$ e é chamada de distribuição horizontal definida por $\omega$. Reciprocamente, dada $\theta$ nestas condições, podemos definir uma conexão $\omega$ satisfazendo os ítens 1,2 da definição 2.2.29 pondo $\omega=0$ sobre a distribuição $\theta$ e $\omega$ igual à identidade no subespaço vertical.

Definição 2.2.31 Se $E$ é um fibrado riemanniano sobre $X$, uma derivada covariante em $E$ é um operador linear $\nabla: C^{\infty}(E) \rightarrow C^{\infty}(T X \otimes E)$ tal que

$$
\nabla(f \varphi)=d f \otimes \varphi+f \nabla \varphi
$$


para toda $f \in C^{\infty}(X)$ e $\varphi \in C^{\infty}(E)$. Definimos $\nabla_{Y} \varphi \doteq \nabla \varphi(Y, \cdot)$, para $Y \in \mathfrak{X}(X)$ e $\varphi \in C^{\infty}(E)$. $O$ operador de curvatura associado à derivada covariante $\nabla$ é definido como

$$
R(Y, Z) \doteq \nabla_{Y} \nabla_{Z}-\nabla_{Z} \nabla_{Y}-\nabla_{[Y, Z]} \cdot
$$

Uma derivada covariante é dita compatível com a métrica de $E$ se $Y\langle Z, W\rangle=\left\langle\nabla_{Y} Z, W\right\rangle+\left\langle Z, \nabla_{Y} W\right\rangle$ para todos $Y, Z, W \in \mathfrak{X}(X)$. Às vezes, diremos simplesmente que $\nabla$ é uma conexão em $E$.

Vamos agora descrever a relação entre conexões e derivadas covariantes. Seja $P$ um fibrado $G$-principal sobre $X, \omega$ uma conexão em $P$ e $\rho: G \rightarrow \mathrm{O}(V)$ uma representação, onde $V$ é um espaço vetorial munido de produto interno. Consideremos o fibrado vetorial $E=P \times_{\rho} V$ associado a $P$ por $\rho$. Obviamente, $E$ herda a estrutura métrica de $V$, e portanto, é um fibrado riemanniano. Observamos que cada seção de $E$ está univocamente associada a uma aplicação G-equivariante $f: P \rightarrow V$, i.e., $f(p \cdot g)=\rho\left(g^{-1}\right) \cdot f(p)$, para $p \in P, g \in G$. Dados $\alpha:[0,1] \rightarrow X$ uma curva $C^{\infty} \operatorname{com} \alpha(0)=x_{0}, \alpha^{\prime}(0)=Y$ e $W=[p, v] \in E_{x_{0}}$, temos ${ }^{19}$ que existe um único levantamento $\tilde{\alpha}:[0,1] \rightarrow P$ de $\alpha$ tal que $\tilde{\alpha}(0)=p$ e $\tilde{\alpha}$ é horizontal, i.e., $\omega\left(\tilde{\alpha}^{\prime}(t)\right)=0$ para todo $t \in[0,1]$. O transporte paralelo $\pi_{t}: T_{\alpha(t)} X \rightarrow T_{x_{0}} X$ de $W$ ao longo de $\alpha$ é definido como $\pi_{t}^{-1}(W)=[\tilde{\alpha}(t), v]$, para $t \in[0,1]$. Se $\varphi$ é uma seção de $E \operatorname{com} \varphi\left(x_{0}\right)=W$, definimos

$$
\left.\nabla_{Y} \varphi \doteq \frac{d}{d t}\right|_{t=0} \pi_{t}(\varphi(\alpha(t))) .
$$

Como $\pi_{t}(\varphi(\alpha(t)))=\pi_{t}([\tilde{\alpha}(t), f(\tilde{\alpha}(t))])=[p, f(\tilde{\alpha}(t))]$, temos que

$$
\nabla_{Y} \varphi=\left.\frac{d}{d t}\right|_{t=0}[p, f(\tilde{\alpha}(t))]=[p,(\tilde{Y} f)(p)],
$$

$\tilde{Y} \doteq \tilde{\alpha}^{\prime}(0)$. A fórmula 2.2.6 mostra que a definição em 2.2.5 independe da curva $\alpha$. Como $\pi_{t}$ é uma isometria, temos que a derivada covariante definida em 2.2.5 é compatível com a métrica. Isso mostra como obter uma derivada covariante em $E$ a partir de uma conexão em $P$.

Vejamos qual a relação entre o operador de curvatura $R$ definido em 2.2 .4 e a forma de curvatura em $P$. Sejam $x_{0} \in X, p \in P \operatorname{com} \pi(p)=x_{0}, Y, Z \in T_{x_{0}} X$ e $\tilde{Y}, \tilde{Z} \in T_{p} P$ seus levantamentos horizontais (i.e., $\tilde{Y}, \tilde{Z}$ são os únicos vetores horizontais tangentes a $p$ tais que $\pi_{*}(\tilde{Y})=Y$ e $\left.\pi_{*}(\tilde{Z})=Z\right)$. Dada $\varphi \in C^{\infty}(S(E))$, se $f: P \rightarrow M$ é a aplicação G-equivariante correspondente a $\varphi$, então pela fórmula 2.2.6,

$$
\begin{aligned}
R(Y, Z) \varphi & =\nabla_{Y} \nabla_{Z} \varphi-\nabla_{Z} \nabla_{Y} \varphi-\nabla_{[Y, Z]} \varphi \\
& =[p,[\tilde{Y}, \tilde{Z}] f(p)-[\tilde{Y, Z}] f(p)] .
\end{aligned}
$$

Como $[\tilde{Y}, \tilde{Z}]-[\tilde{Y, Z}]$ é um campo vertical, segue que existe um único $W \in \mathfrak{g}$ tal que $\sigma(W)=$ $[\tilde{Y}, \tilde{Z}]-[\tilde{Y, Z}]$. Logo,

$$
\begin{aligned}
{[\tilde{Y}, \tilde{Z}] f(p)-[\tilde{Y, Z}] f(p) } & =\sigma(W) f(p) \\
& =\left.\frac{d}{d t}\right|_{t=0} f(p \cdot \exp (t W)) \\
& =\left.\frac{d}{d t}\right|_{t=0} \rho(\exp (-t W)) \cdot f(p) \\
& =-\rho_{*}(W) \cdot f(p),
\end{aligned}
$$

\footnotetext{
${ }^{19}$ Usamos a notação $[p, v]$ para indicar a classe de equivalência de $(p, v) \in P \times V$ em $E$.
} 
onde $\rho_{*}: \mathfrak{g} \rightarrow \mathfrak{s o}(V)$ denota a derivada do homomorfismo $\rho$ na identidade. Portanto,

$$
R(Y, Z) \varphi\left(x_{0}\right)=\left[p,-\rho_{*}(W) \cdot f(p)\right] .
$$

Por outro lado, temos $\Omega(\tilde{Y}, \tilde{Z})=d \omega(\tilde{Y}, \tilde{Z})=\tilde{Y}(\omega(\tilde{Z}))-\tilde{Z}(\omega(\tilde{Y}))-\omega([\tilde{Y}, \tilde{Z}])=-\omega([\tilde{Y}, \tilde{Z}])$ e também $W=\omega([\tilde{Y}, \tilde{Z}]-[\tilde{Y}, Z])=-\omega([\tilde{Y}, \tilde{Z}])=\Omega(\tilde{Y}, \tilde{Z})$, logo, por 2.2.7

$$
R(Y, Z) \varphi\left(x_{0}\right)=\left[p, \rho_{*}(\Omega(\tilde{Y}, \tilde{Z})) \cdot f(p)\right]=\rho_{*}(\Omega(\tilde{Y}, \tilde{Z})) \varphi\left(x_{0}\right) .
$$

O teorema a seguir está provado.

Teorema 2.2.32 Se P é um fibrado G-principal sobre $X, \rho: G \rightarrow \mathrm{O}(V)$ é uma representação, onde $V$ é um espaço vetorial munido de produto interno e $E=P \times_{\rho} V$ é o fibrado riemanniano associado $a P$ por $\rho$, então, a cada conexão $\omega$ em $P$ corresponde uma derivada covariante $\nabla$ em $E$ tal que $R(Y, Z)=\rho_{*}(\Omega(\tilde{Y}, \tilde{Z}))$ para quaisquer $Y, Z \in \mathfrak{X}(X)$.

Analisemos mais de perto o caso de fibrados spinor. Seja $E$ um fibrado spin sobre $X$, $\xi: P_{\text {Spin }}(E) \rightarrow P_{\text {SO }}(E)$ uma estrutura spin e $\omega$ uma conexão em $P_{\text {So }}(E)$. Definindo $\tilde{\omega} \doteq \xi^{*}(\omega)$, vemos que $\tilde{\omega}$ é uma conexão em $P_{\text {Spin }}(E)$. Dado um fibrado spinor $\mathrm{S}(E)=P_{\text {Spin }}(E) \times_{\ell} V$, (conforme a definição 2.2.26), a construção feita no parágrafo anterior mostra que é possível definir uma derivada covariante $\nabla$ em $S(E)$. Dados $x_{0} \in U, \chi \in \mathrm{C}^{\infty}(\mathrm{Cl}(E))$ e $\varphi \in \mathrm{C}^{\infty}(\mathrm{S}(E))$, pelos comentários do parágrafo anterior, podemos obter $U \subset X$ aberto contendo $x_{0}, g: P_{\text {Spin }}(E) \rightarrow$ $\mathrm{Cl}(n)$ Ad-equivariante e $f: P_{\text {Spin }}(E) \rightarrow V$ Spin $(n)$-equivariante tais que $\chi(x)=[e(x), g(e(x))] \mathrm{e}$ $\varphi(x)=[e(x), f(e(x))], x \in U$, onde $e$ é uma seção do fibrado $\pi: P_{\text {Spin }}(E) \rightarrow X$ definida em $U$. Logo, considerando $\alpha:[0,1] \rightarrow X$ de classe $C^{\infty}$ tal que $\alpha(0)=x_{0}$ e $\alpha^{\prime}(0)=Y$, temos que para $x \in U$ e $t \in[0,1]$

$$
\begin{gathered}
(\chi \cdot \varphi)(x)=[e(x), g(e(x)) \cdot f(e(x))], \\
\pi_{t}((\chi \cdot \varphi)(\alpha(t)))=\pi_{t}([\tilde{\alpha}(t), g(\tilde{\alpha}(t)) \cdot f(\tilde{\alpha}(t))])=[e, g(\tilde{\alpha}(t)) \cdot f(\tilde{\alpha}(t))],
\end{gathered}
$$

onde $\tilde{\alpha}:[0,1] \rightarrow P$ é o único levantamento horizontal de $\alpha$ com $\tilde{\alpha}(0)=p \doteq e\left(x_{0}\right)$. Portanto,

$$
\begin{aligned}
\nabla_{Y}(\chi \cdot \varphi)\left(x_{0}\right) & =\left.\frac{d}{d t}\right|_{t=0}[e, g(\tilde{\alpha}(t)) \cdot f(\tilde{\alpha}(t))] \\
& =\left[e, Y(g)_{p} \cdot f(p)+g(p) \cdot Y(f)_{p}\right] \\
& =\left[e, Y(g)_{p} \cdot f(p)\right]+\left[e, g(p) \cdot Y(f)_{p}\right]=\left(\nabla_{Y} \chi\right)_{x_{0}} \cdot \varphi\left(x_{0}\right)+\chi\left(x_{0}\right) \cdot\left(\nabla_{Y} \varphi\right)_{x_{0}} .
\end{aligned}
$$

Isso prova o seguinte teorema. ${ }^{20}$

TEorema 2.2.33 $S e \mathrm{~S}(E)=P_{\text {Spin }}(E) \times_{\ell} V$ é um fibrado spinor real associado ao fibrado spin $E$, então dada uma conexão em $P_{\mathrm{SO}}(E)$ e uma estrutura spin $\xi: P_{\mathrm{Spin}}(E) \rightarrow P_{\mathrm{SO}}(E)$ a conexão $\tilde{\omega} \doteq \xi^{*}(\omega)$ induz uma derivada covariante $\nabla$ em $\mathrm{S}(E)$ tal que

$$
\nabla_{Y}(\chi \cdot \varphi)=\nabla_{Y} \chi \cdot \varphi+\chi \cdot\left(\nabla_{Y} \varphi\right)
$$

para $\chi \in \mathrm{C}^{\infty}(\mathrm{Cl}(E)), \varphi \in \mathrm{C}^{\infty}(\mathrm{S}(E))$.

\footnotetext{
${ }^{20} \mathrm{~A}$ derivada covariante $\nabla_{Y} \chi$ já foi definida no teorema 2.2.32.
} 


\subsection{Operadores de Dirac}

Nesta seção trataremos do assunto principal do nosso trabalho, os operadores de Dirac e o teorema de Yoshida-Nicolaescu. A grosso modo, um operador de Dirac é um operador diferencial de primeira ordem agindo em seções de um fibrado $S$ de $\mathrm{Cl}(X)$-módulos sobre uma variedade $X$, definido em termos de uma conexão qualquer sobre $S$. De maneira um tanto surpreendente, o estudo de certas propriedades analíticas deste tipo de operador revela propriedades topológicas de $X$ e de $S$. Os exemplos mais famosos desta afirmação são os teoremas de Bochner e de Gallot-Meyer, os quais fornecem restrições topológicas para a existência de métricas de curvatura escalar e curvatura de Ricci não-negativas. Um caso de especial interesse é o caso de uma variedade spin $X$; neste caso, mostra-se que se $X$ admite uma métrica de curvatura escalar não-negativa e estritamente positiva em algum ponto, então $\hat{\mathrm{A}}(X)=0$, onde $\hat{\mathrm{A}}(X)$ denota o $\hat{\mathrm{A}}$-gênero de $X$, o qual é um número de Pontryagin racional de $X$, e portanto depende exclusivamente da topologia de $X$.

Exploraremos também a $\mathbb{Z}_{2}$-graduação usual de operadores de Dirac introduzida por $M$. Atiyah e I. Singer e mostraremos como escrever alguns invariantes topológicos de variedades diferenciáveis (como a característica de Euler e a assinatura para variedades de dimensão $4 k$ ) como índices de operadores elípticos.

\subsubsection{Definição e Exemplos}

Antes de mais nada, vamos discutir brevemente alguns aspectos históricos a respeito de operadores de Dirac. Na década de 20, o físico P. Dirac foi confrontado com o problema de encontrar uma raiz quadrada para o operador $-\Delta$ agindo em $\mathbb{R}^{3}$. A solução encontrada por ele foi considerar $-\Delta$ agindo não em uma única função complexa, mas em $n$-uplas de funções complexas. O operador procurado deve ter a forma

$$
D=\sum_{j=1}^{3} c_{j} \frac{\partial}{\partial x_{j}},
$$

onde $c_{1}, \ldots, c_{3}$ são matrizes $n \times n$ e a exigência que

$$
D^{2}=\left(\begin{array}{ccc}
\Delta & & \\
& \ddots & \\
& & \Delta
\end{array}\right)
$$

implica que $c_{j} c_{k}+c_{k} c_{j}=-2 \delta_{j k}$, para $1 \leq j, k \leq m$. Esta condição mostra que a estrutura de álgebra de $\mathrm{Clifford} \operatorname{de~} \mathrm{Cl}(n)$ fornece o ambiente de trabalho adequado para resolver o problema, conforme a fórmula 2.2.1. Vamos mostrar como construir, usando a teoria de fibrados de Clifford, um certo operador diferencial de primeira ordem $D$ invariantemente definido sobre um fibrado de $\mathrm{Cl}(\mathrm{X})$-módulos, com propriedades semelhantes às do caso euclidiano.

Sejam $X$ uma variedade riemanniana (com ou sem bordo) de dimensão $n, \mathrm{Cl}(X) \doteq \mathrm{Cl}(T X)$ o fibrado de Clifford de $X$ e $S$ um fibrado de $\mathrm{Cl}(X)$-módulos à esquerda (i.e., $S$ é um fibrado riemanniano cuja fibra $S_{x}$ é um $\mathrm{Cl}(X)_{x}$-módulo à esquerda, para todo $\left.x \in X\right)$. Vamos assumir que o operador de multiplicação por vetores unitários em TX é uma isometria de $S$, i.e.,

$$
\left\langle e \cdot s_{1}, e \cdot s_{2}\right\rangle=\left\langle s_{1}, s_{2}\right\rangle
$$


para todos $s_{1}, s_{2} \in S_{x}$ e $e \in T_{x} X$ unitário, com $x \in X$. Em particular, $\left\langle e \cdot s_{1}, s_{2}\right\rangle+\left\langle s_{1}, e \cdot s_{2}\right\rangle=0$. Sempre que falarmos de um fibrado de $\mathrm{Cl}(X)$-módulos, subentenderemos que a equação 2.3.1 é verificạda.

Definição 2.3.1 Sob as hipóteses anteriores, dada qualquer conexão $\nabla$ em $S$, definimos o operador de Dirac associado por

$$
D \doteq \sum_{j=1}^{n} e_{j} \cdot \nabla_{e_{j}}
$$

onde $\left\{e_{j}\right\}_{j=1}^{n}$ é um referencial ortonormal local em $X$. Se ${ }^{21}$ a conexão $\nabla$ em $S$ é compatível com a estrutura riemanniana e verifica a fórmula

$$
\nabla(\chi \cdot \varphi)=(\nabla \chi) \cdot \varphi+\chi \cdot(\nabla \varphi)
$$

para todas $\chi \in \mathrm{C}^{\infty}(\mathrm{Cl}(X))$ e $\varphi \in \mathrm{C}^{\infty}(S)$, então o operador $D$ correspondente é dito um operador Dirac compatível.

A próxima proposição mostra que, de fato, um operador de Dirac é um operador diferencial elíptico de primeira ordem e calcula o seu símbolo.

Proposição 2.3.2 Se D é um operador de Dirac então $D, D^{2}: C^{\infty}(S) \rightarrow C^{\infty}(S)$ são operadores diferenciais de primeira e segunda ordem com simbolos principais $\sigma_{1}(D)(\xi)=i \xi$ e $\sigma_{2}\left(D^{2}\right)(\xi)=-|\xi|^{2}$, para $\xi \in T^{*} X \approx T X$, respectivamente. ${ }^{22}$ Em particular, ambos são operadores elípticos.

Prova. Sejam $x_{1}, \ldots, x_{n}$ coordenadas em uma vizinhança aberta $U$ de $x_{0} \in X$ tal que $\left.\nabla_{\frac{\partial}{\partial x_{j}}} \frac{\partial}{\partial x_{j}}\right|_{x_{0}}=0$ para $1 \leq i, j \leq n$. Em particular, pondo $e_{j}=\left.\frac{\partial}{\partial x_{j}}\right|_{x_{0}}, j=1, \ldots, n$, segue que $\left\{e_{j}\right\}_{j=1}^{n}$ é uma base ortonormal de $T_{x_{0}} X$. Além disso, $\nabla_{e_{j}}-\frac{\partial}{\partial x_{j}}$ é um operador de ordem zero em $U$ cujos coeficientes se anulam em $x_{0}$. Assim, dado $\xi=\sum_{j=1}^{n} \xi_{j} d x_{j} \in T_{x_{0}}^{*} X$, temos que $\sigma_{1}(D)(\xi)=i \sum_{j=1}^{n} \xi_{j} e_{j}=i \xi$. A fórmula para $\sigma_{2}\left(D^{2}\right)$ decorre do cálculo direto ou então dos comentários após a fórmula 2.1.5.

A próxima proposição revela uma propriedade analítica muito importante dos operadores de Dirac compatíveis.

Proposição 2.3.3 Um operador de Dirac compatível é formalmente auto-adjunto.

Prova. Dados $x_{0} \in \operatorname{int} X, x_{1}, \ldots, x_{n}$ coordenadas locais em uma vizinhança aberta $U \subset$ int $X$ de $x_{0}$ como na proposição 2.3.2 e $u, v \in C_{c}^{\infty}(U)$, temos que

$$
\langle D u, v\rangle_{x}=\operatorname{div}(V)_{x}+\langle u, D v\rangle_{x}
$$

\footnotetext{
${ }^{21}$ Obviamente, é necessário verificar que $D$ independe do referencial utilizado. De fato, se $e_{j}^{\prime}=\sum_{k=1}^{n} \alpha_{j k} e_{k}$, $j=1, \ldots, n$ é outro referencial ortonormal local, então

$$
\sum_{j=1}^{n} e_{j}^{\prime} \cdot \nabla_{e_{j}^{\prime}}=\sum_{j, k, l=1}^{n} \alpha_{j k} \alpha_{j l} e_{k} \cdot \nabla_{e_{l}}=\sum_{k=1}^{n} e_{k} \cdot \nabla_{e_{k}} .
$$

${ }^{22}$ Isso significa que $\sigma_{1}(D)(\xi)$ é o operador de multiplicação por $i \xi$. A mesma notação vale para $\sigma_{2}\left(D^{2}\right)$.
} 
para todo $x \in U$, onde $V$ é o campo de vetores definido por $\langle V, Z\rangle \doteq-\langle u, Z \cdot v\rangle$, para todo $Z$ tangente a $X$. Em particular, pelo teorema de Stokes, segue o resultado.

A próxima proposição segue imediatamente da observação 2.1.20 e da proposição 2.3.2.

Proposição 2.3.4 Se D é um operador de Dirac sobre S e X é uma variedade compacta com bordo $Y$, então

$$
\int_{X}\langle D u, v\rangle d x-\int_{X}\left\langle u, D^{*} v\right\rangle d x=-\int_{Y}\langle v \cdot u, v\rangle d y,
$$

para todas $u, v \in \mathrm{C}^{\infty}(S)$, onde $v$ denota a normal interior a $X$.

Vamos agora dar alguns exemplos importantes de operadores de Dirac.

EXEMPLo 2.3.5 Vamos retomar neste exemplo a idéia original usada para definir um operador de Dirac comentada no início desta subseção. Sejam $X=\mathbb{R}^{n}$ e $S=\mathbb{R}^{n} \times V$, onde $V$ um $\mathrm{Cl}(n)$-módulo à esquerda para $\mathrm{Cl}(n)$ irredutível de dimensão finita. O operador de Dirac correspondente agindo em seções de $S$ é $D=\sum_{k=1}^{n} c_{k} \partial / \partial x_{k}$, com $c_{k} \in \mathscr{L}(V, V)$ verificando $c_{j} c_{k}+c_{k} c_{j}=-2 \delta_{j k}$, para $1 \leq j, k \leq n$.

Caso $n=1$, temos $\mathrm{Cl}(1) \approx \mathbb{C} \approx V$ e $D=i \partial / \partial x_{1}$.

Caso $n=2$, temos $\mathrm{Cl}(2) \approx \mathbb{H} \approx V$, onde $\mathbb{H}$ denota a álgebra dos quatérnios, $\mathbb{H}=\mathbb{C} \oplus \mathbb{C}$. Esta decomposição corresponde à decomposição de $\mathrm{Cl}(2)$ em partes par e ímpar. Fazendo as identificações necessárias, $D$ tem a forma

$$
D=\left(\begin{array}{cc}
0 & -\frac{\partial}{\partial z} \\
\frac{\partial}{\partial z} & 0
\end{array}\right),
$$

com $\partial / \partial \bar{z} \doteq \partial / \partial x_{1}+i \partial / \partial x_{2}$. Considerado como operador entre spinors pares e ímpares, $o$ operador de Dirac corresponde exatamente ao operador de Cauchy-Riemann. Para os casos $n=3$ e $n=4$, veja $[60]$.

Para podermos dar mais um exemplo muito importante de operador de Dirac, precisamos fazer alguns cálculos. Dada uma variedade riemanniana orientada $X$, consideremos o fibrado $\Lambda^{*} X$, cuja fibra em $x \in X$ é a álgebra exterior $\Lambda^{*}\left(T_{x}(X)\right)$. Introduzimos em $\Lambda^{*} X$ uma estrutura riemanniana declarando que $\left\{e_{i_{1}} \wedge \ldots \wedge e_{i_{k}}: 1 \leq i_{1}<\ldots<i_{k} \leq n, 1 \leq k \leq n\right\}$ é um conjunto ortonormal em $\left(\Lambda^{*} X\right)_{x}$, com $\left\{e_{j}\right\}_{j=1}^{n}$ base ortonormal positiva de $T_{x}(X)$. Aqui, obviamente, estamos identificando $T X$ e $T^{*} X$ por meio da estrutura riemanniana de $X$. Vamos usar a notação standard $e_{I} \doteq e_{i_{1}} \wedge \ldots \wedge e_{i_{k}}$ para uma $k$-upla de índices $I=\left\{1 \leq i_{1}<\ldots<i_{k} \leq n\right\}$. Dizemos que o comprimento de $I$ é $k$ e denotamos tal fato escrevendo $|I|=k$. Podemos definir o operador $\star$ de Hodge como

$$
\star\left(e_{I}\right)=\operatorname{sgn}(\sigma) e_{J},
$$

onde $J \doteq\{1, \ldots, n\} \backslash I$ é ordenado de maneira crescente e $\sigma$ é a permutação $(I, J)$. Em particular, o produto interno definido anteriormente em cada fibra de $\Lambda^{*} X$ pode ser dado por

$$
\langle\omega, \eta\rangle \star 1=\omega \wedge(\star \eta)
$$

para $\omega, \eta$ de mesmo grau e $\langle\omega, \eta\rangle=0$ caso $\omega, \eta$ tenham graus diferentes.

As seções de $\Lambda^{*} X$ são formas diferenciais de grau misto, portanto, podemos considerar o operador de diferenciação exterior usual, o qual é um operador diferencial de primeira ordem 
$d: C^{\infty}\left(\Lambda^{*} X\right) \rightarrow C^{\infty}\left(\Lambda^{*} X\right)$. Dados $x \in X$ e um referencial ortonormal positivo $\left\{e_{j}\right\}_{j=1}^{n}$ definido em um aberto $U$ contendo $x$ tal que $\left.\nabla_{e_{i}} e_{j}\right|_{x}=0$ para $1 \leq i, j \leq n$, e $\omega=\sum_{|l|=k} \omega_{I} e_{I}$ uma $k$-forma suportada em $U$, temos

$$
\begin{aligned}
\left.d \omega\right|_{x} & =\left.\sum_{|I|=k} d \omega_{I} \wedge e_{I}\right|_{x} \\
& =\left.\sum_{|I|=k} \sum_{j=1}^{n} e_{j}\left(\omega_{I}\right) e_{j} \wedge e_{I}\right|_{x} \\
& =\left.\sum_{j=1}^{n} e_{j} \wedge\left(\sum_{|I|=k} e_{j}\left(\omega_{I}\right) e_{I}\right)\right|_{x} \\
& =\left.\sum_{j=1}^{n} e_{j} \wedge \nabla_{e_{j}} \omega\right|_{x} .
\end{aligned}
$$

Isso mostra que, em termos de um tal referencial, o operador $d$ tem a forma

$$
d=\sum_{j=1}^{n} e_{j} \wedge \nabla_{e_{j}}
$$

em $x$. Aqui estamos usando a única extensão bilinear da conexão $\nabla$ em $X$ para formas, i.e., se $\omega$ é uma $k$-forma e $Y, Z_{1}, \ldots, Z_{k}$ são vetores tangentes,

$$
\left(\nabla_{Y} \omega\right)\left(Z_{1}, \ldots, Z_{k}\right)=Y\left(\omega\left(Z_{1}, \ldots, Z_{k}\right)\right)-\sum_{j=1}^{k} \omega\left(Z_{1}, \ldots, \nabla_{Y} Z_{j}, \ldots, Z_{k}\right) .
$$

De posse da fórmula 2.3.2, vamos calcular o adjunto formal de $d$. Definimos para uma $k$-forma $\omega$ e vetores tangentes $Z_{1}, \ldots, Z_{k-1}$, a divergência de $\omega$ como a $(k-1)$-forma dada por

$$
\begin{aligned}
\left.(\operatorname{div} \omega)\right|_{x}\left(Z_{1}, \ldots, Z_{k-1}\right) & \doteq \sum_{j=1}^{k-1}(-1)^{j+1}\left(\nabla_{e_{j}} \omega\right)\left(Z_{1}, \ldots, Z_{j-1}, e_{j}, Z_{j+1}, Z_{k-1}\right) \\
& =\sum_{j=1}^{n} e_{j}\left\llcorner\nabla_{e_{j}} \omega\left(Z_{1}, \ldots, Z_{k-1}\right),\right.
\end{aligned}
$$

onde $\left\{e_{j}\right\}_{j=1}^{n}$ é um referencial como antes, e $v\left\llcorner\eta\right.$ denota a contração de $v$ e $\eta$. Podemos ${ }^{23}$ escrever a divergência de uma $k$-forma $\omega$ sem depender do referencial $\left\{e_{j}\right\}_{j=1}^{n}$, observando que $\left.(\operatorname{div} \omega)\right|_{x}\left(Z_{1}, \ldots, Z_{k-1}\right)$ é justamente o traço da aplicação

$$
(Y, Z) \mapsto \sum_{j=1}^{k-1}(-1)^{j+1}\left(\nabla_{Y} \omega\right)\left(Z_{1}, \ldots, Z_{j-1}, Z, Z_{j+1}, Z_{k-1}\right) .
$$

\footnotetext{
${ }^{23} \mathrm{~A}$ contração de um campo de vetores $Y$ e uma $k$-forma diferencial $\eta$ é definida por $Y\left\llcorner\eta\left(Y_{1}, \ldots, Y_{k-1}\right) \doteq\right.$ $\eta\left(Y, Y_{1}, \ldots, Y_{n-1}\right)$ para quaisquer $Y_{1}, \ldots, Y_{k-1} \in \mathfrak{X}(X)$.
} 
Nosso objetivo é provar que o adjunto formal de $d$ é - div. Para tanto, observamos que se $\omega, \eta$ são $k$-formas suportadas em $U$,

$$
\begin{aligned}
\int_{X}\langle d \omega, \eta\rangle d x & =\int_{X} d \omega \wedge \star \eta \\
& =\int_{U} d(\omega \wedge \star \eta)-(-1)^{k} \omega \wedge d(\star \eta) \\
& =(-1)^{k+1} \int_{U} \omega \wedge d(\star \eta) \\
& =\int_{X}\left\langle\omega,(-1)^{k n+1} \star d \star \eta\right\rangle,
\end{aligned}
$$

pelo teorema de Stokes e por termos $\star \star=(-1)^{k(n-k)}$ em $\Lambda^{k}\left(T_{x} X\right)$. Portanto, $d^{*}=(-1)^{k n+1} \star d \star$. Por outro lado, se $\omega=f e_{1} \wedge \ldots \wedge e_{k}$ é uma $k$-forma em $X$, então $\star \omega=f e_{k+1} \wedge \ldots \wedge e_{n}$ e $d(\star \omega)=\sum_{j=1}^{k} e_{j}(f) e_{j} \wedge e_{k+1} \wedge \ldots \wedge e_{n}$ em $x$. Finalmente,

$$
\star(d \star \omega)=(-1)^{n(k+1)+1} \sum_{j=1}^{k}(-1)^{j+1} e_{j}(f) e_{1} \wedge \ldots \wedge \hat{e}_{j} \wedge \ldots \wedge e_{k}=-\operatorname{div} \omega,
$$

provando que

$$
d^{*}=-\operatorname{div}=-\sum_{j=1}^{n} e_{j}\left\llcorner\nabla_{e_{j}},\right.
$$

em $x$, como queríamos. Agora estamos aptos para o próximo exemplo.

Exemplo 2.3.6 Considerando a multiplicação de Clifford para formas diferenciais definida nos comentários que antecedem a definição 2.2.5, vemos que o operador de Dirac $D$ correspondente ao fibrado de Clifford $\Lambda^{*} X$ identifica-se com o operador $d+d^{*}$.

Usando a teoria de operadores elípticos desenvolvida na subseção 2.1.2, concluímos o seguinte resultado devido originalmente a W. Hodge. Mais informações podem ser encontradas em [102].

Teorema 2.3.7 (Hodge) Se X é uma variedade compacta sem bordo e $\Delta_{q} \doteq D^{2}=d d^{*}+d^{*} d$ é o laplaciano definido para formas diferenciais de grau $q \geq 0$, então existe uma seqüência de números reais $\left\{\lambda_{k}\right\}_{k \in \mathbb{N}}$ com $0 \leq\left|\lambda_{1}\right| \leq \ldots \leq\left|\lambda_{k}\right| \leq \ldots$ e uma base ortonormal $\left\{\omega_{k}\right\}_{k \in \mathbb{N}}$ de $\mathrm{H}^{0}\left(\Lambda^{q} X\right)$ tais que:

1. $\Delta \omega_{k}=\lambda_{k} \omega_{k}$ para todo $k \in \mathbb{N}$;

2. $\left|\lambda_{k}\right| \rightarrow \infty$;

3. Se $u \in C^{\infty}\left(\Lambda^{q} X\right)$ é fechada então existe uma única $v \in C^{\infty}\left(\Lambda^{q} X\right)$ tal que $\Delta v=0$ e $u, v$ são cohomólogas. Em particular, $\mathrm{H}^{q}(X) \approx \operatorname{ker} \Delta_{q}$ para todo $q \geq 0$.

Prova. Os ítens 1 e 2 decorrem diretamente dos ítens 2 e 3 do teorema 2.1.18. Se $G$ é o operador dado pelo ítem 1 do referido teorema, então $G d^{*} d+G d d^{*}=I-R$, onde $R$ é a projeção ortogonal sobre ker $\Delta_{q}$. Em particular, se $d u=0$, então $u-R u=d\left(d^{*} u\right)$, provando o ítem 3 . 
Vamos agora falar um pouco sobre a técnica de Bochner. Dado um operador de Dirac compatível em um fibrado de $\mathrm{Cl}(X)$-módulos $S$ sobre $X$, podemos definir o laplaciano para seções de $S$ como $\Delta \doteq D^{2}$. Este é um operador de segunda ordem canonicamente associado à conexão $\nabla$ de $S$. Por outro lado, como $\nabla$ define um operador diferencial de primeira ordem em $S$, temos que o operador $\nabla^{*} \nabla$ também é um operador diferencial de segunda ordem em $S$, invariantemente definido, e podemos nos perguntar sobre a diferença $D^{2}-\nabla^{*} \nabla$. As próximas proposições, cujas provas podem ser encontradas em [60] e [85], calculam $D^{2}-\nabla^{*} \nabla$ e fornecem importantes conseqüências desta fórmula.

Proposição 2.3.8 Temos

$$
D^{2}=\nabla^{*} \nabla+\frac{1}{2} \sum_{i, j=1}^{n} e_{i} \cdot e_{j} \cdot R\left(e_{i}, e_{j}\right),
$$

onde $\left\{e_{j}\right\}_{j=1}^{n}$ é um referencial ortonormal local em Xe R denota o operador de curvatura correspondente à conexão de $S$.

No caso em que $S=\Lambda^{*} X$, como discutido no exemplo 2.3.6, temos o seguinte resultado clássico devido a Bochner.

ProposiçÃo 2.3.9 Se $S=\Lambda^{*} X$, para $X$ compacta sem bordo, então o operador $D^{2}$ assume a forma familiar $\Delta=d^{*} d+d d^{*}$. Para 1-formas, o termo $\frac{1}{2} \sum_{i, j=1}^{n} e_{i} \cdot e_{j} \cdot R\left(e_{i}, e_{j}\right)$ coincide com o operador de curvatura de Ricci Ric, definido por $\operatorname{Ric}(Y) \doteq-\sum_{j=1}^{n} R\left(e_{j}, Y\right) e_{j}$.

Teorema 2.3.10 (Gallot-MeYer) Seja X uma variedade riemanniana compacta sem bordo. Se o operador de curvatura de Xé não-negativo e estritamente positivo em algum ponto, então $H^{j}(X ; \mathbb{R})=0$ para $j=1, \ldots, n-1$.

Proposição 2.3.11 (Bochner) Se Ric $\geq 0 \mathrm{em} X$, então $\operatorname{dim} H^{1}(X ; \mathbb{R}) \leq n$. Se Ric $>0$ em algum ponto, então $H^{1}(X ; \mathbb{R})=0$.

Prova. Basta observar que, dada uma 1-forma harmônica $\omega$, temos pelas proposições 2.3.8 e 2.3.9 que

$$
0=\int_{X}\langle\Delta \omega, \omega\rangle d x=\int_{X}\langle\nabla \omega, \nabla \omega\rangle d x+\int_{X}\langle\operatorname{Ric} \omega, \omega\rangle d x,
$$

$\operatorname{logo}, \nabla \omega=0$. Como esta é uma equação de primeira ordem, segue que o espaço de tais $\omega$ tem dimensão $\leq n$. Se Ric $>0$ em algum ponto, então $\omega=0$. O resultado segue do teorema de Hodge. 2.3.5.

No caso spin, a próxima proposição fornece uma interessante interpretação para a fórmula

Proposição 2.3.12 Se X é uma variedade spin compacta sem bordo e $S$ é um fibrado spinor sobre X munido da conexão canônica, então o termo $2 \sum_{i, j=1}^{n} e_{i} \cdot e_{j} \cdot R\left(e_{i}, e_{j}\right)$ em 2.3 .5 coincide com a curvatura escalar de X, definida como $s \doteq-\sum_{i, j=1}^{n}\left\langle R\left(e_{i}, e_{j}\right) e_{i}, e_{j}\right\rangle=\operatorname{tr}$ Ric. Em particular, vale a fórmula

$$
D^{2}=\nabla^{*} \nabla+\frac{1}{4} s .
$$

Se $s \geq 0$, então o espaço de seções harmônicas de $S$ (i.e., seções $u$ tais que $D^{2} u=0$ ) coincide com o espaço de seções paralelas $(\nabla u=0)$ e ambos têm dimensão finita. 
Do ponto de vista de teoria do índice, um operador de Dirac compatível $D$ não é muito interessante, pois a proposição 2.3.3 implica que $D$ é formalmente auto-adjunto, e portanto tem índice de Fredholm zero. Apesar disso, as proposições 2.3.8-2.3.12 mostram que tais operadores podem ser usados para obtermos resultados geométricos e topológicos interessantes. O grande passo da teoria neste momento, devido a M. Atiyah e I. Singer, é a quebra de simetria dos operadores de Dirac em certos casos. Esta técnica permite-nos escrever números que dependem somente da topologia de uma variedade em termos de índices de operadores elípticos definidos sobre a mesma. Tal técnica consiste em utilizar as decomposições dos fibrados de Clifford em parte par e parte ímpar e considerar restrições convenientes de operadores de Dirac.

Sejam $S$ um fibrado riemanniano de $\mathrm{Cl}(X)$-módulos à esquerda (como na definição 2.3.1) e $D$ o operador de Dirac compatível correspondente. Suponhamos que $S$ seja $\mathbb{Z}_{2}$-graduado, i.e., existe uma decomposição paralela ${ }^{24}$

$$
S=S^{0} \oplus S^{1}
$$

em sub-fibrados $S^{0}, S^{1}$ tal que $\mathrm{Cl}^{i}(X) \cdot S^{j} \subset S^{i+j}$, para $i, j \in \mathbb{Z}_{2}$. Neste caso, segue da definição de $D$ que, com respeito à decomposição 2.3.6, o operador $D$ tem a forma $\left(\begin{array}{cc}0 & D^{1} \\ D^{0} & 0\end{array}\right), \operatorname{com} D^{0}: C^{\infty}\left(S^{0}\right) \rightarrow$ $C^{\infty}\left(S^{1}\right)$ e $D^{1}: C^{\infty}\left(S^{1}\right) \rightarrow C^{\infty}\left(S^{0}\right)$. Como $D$ é auto-adjunto, temos que $D^{0}$ e $D^{1}$ são adjuntos. Além disso, $D^{j}$ têm símbolo $\sigma_{1}\left(D^{j}\right)=i \xi: S^{j} \rightarrow S^{j+1}$, pela proposição 2.3.2, para $j \in \mathbb{Z}_{2}$; em particular, $D^{0}, D^{1}$ são elípticos, e portanto, pelo ítem 2 do teorema 2.1.16, ambos têm um índice de Fredholm bem-definido, independente da extensão utilizada e ind $D^{0}=\operatorname{nul} D^{0}-\operatorname{nul} D^{1}$.

Vejamos alguns exemplos em que a situação anterior ocorre.

EXemplo 2.3.13 Se $X$ é orientada de dimensão $n=2 m$, os comentários após a proposição 2.2.28 mostram que é possível introduzir uma $\mathbb{Z}_{2}$-graduação em um fibrado de $\mathrm{Cl}(\mathrm{X})$-módulos $S$ sobre $X$. De fato, o elemento de volume complexo $\omega_{\mathbf{C}}$ definido em 2.2.3 é paralelo, anticomuta com elementos de $T X$ e $\omega_{\mathrm{C}}^{2}=1$. Supondo que $S$ seja complexo se $m$ é ímpar e definindo $S^{0} \doteq \operatorname{ker}\left(1-\omega_{\mathbf{C}}\right)$ e $S^{1} \doteq \operatorname{ker}\left(1+\omega_{\mathbf{C}}\right)$, obtemos uma $\mathbb{Z}_{2}$-graduação como no parágrafo anterior.

EXEMPlo 2.3.14 Considerando $S=\mathrm{Cl}(X) \approx \Lambda^{*} X$, para $X$ compacta sem bordo, vemos que $\mathrm{Cl}^{0}(X) \approx \Lambda^{+} X$ e $\mathrm{Cl}^{1}(X) \approx \Lambda^{-} X$, onde $\Lambda^{+} X=\oplus_{k \text { par }} \Lambda^{k} X$ e $\Lambda^{-} X=\oplus_{k \text { impar }} \Lambda^{k} X$. O operador de Dirac correspondente é $D=d+d^{*}$ (conforme o exemplo 2.3.6) e o operador $D^{0}$ corresponde à restrição $d+d^{*}: C^{\infty}\left(\Lambda^{+} X\right) \rightarrow C^{\infty}\left(\Lambda^{-} X\right)$. Como $D u=0$ se e só se $d u=0$ e $d^{*} u=0$, temos que $\operatorname{ker} D=\operatorname{ker} \Delta$. Como o último espaço é canonicamente isomorfo a $H^{*}(X ; \mathbb{R})$, pelo teorema de Hodge, temos que

$$
\text { ind } D^{0}=\operatorname{nul} D^{0}-\operatorname{nul} D^{1}=\sum_{k \text { par }} \operatorname{dim} H^{k}(X ; \mathbb{R})-\sum_{k \text { impar }} \operatorname{dim} H^{k}(X ; \mathbb{R})=\chi(X),
$$

onde $\chi(X)$ denota a característica de Euler de $X$.

EXEMPLo 2.3.15 Seja $X$ uma variedade riemanniana orientada compacta de dimensão $4 k$ e consideremos o fibrado de Clifford $S \doteq \mathrm{Cl}(X)=\mathrm{Cl}^{+}(X) \oplus \mathrm{Cl}^{-}(X)$, onde a $\mathbb{Z}_{2}$-graduação agora é dada pelo elemento de volume complexo $\omega_{\mathbb{C}}=(-1)^{k} \omega$. Como $\omega_{\mathbb{C}}$ é paralela, temos que $\operatorname{ker} D^{ \pm}=\left(1 \pm \omega_{\mathbf{C}}\right) \operatorname{ker} D$. Denotemos por $\mathbf{H}^{j}$ o espaço das formas harmônicas de grau $j$

\footnotetext{
${ }^{24}$ Isso significa que se $u$ é seção de $S^{i}$, então $\nabla_{Y} u$ também o é, para todos $Y \in \mathfrak{X}(X)$ e $i \in \mathbb{Z}_{2}$.
} 
(o qual é canonicamente isomorfo ao espaço $H^{j}(X ; \mathbb{R})$ ). Vemos que a restrição de $\omega_{\mathbf{C}}$ a $\mathbf{H}^{j}$ coincide com o operador $(-1)^{k+j(j-1) / 2} \star: \mathbf{H}^{j} \rightarrow \mathbf{H}^{4 k-j}$, onde $\star$ denota o operador $\star$ de Hodge definido anteriormente. Em particular, $\omega_{\mathbf{C}}: \mathbf{H}^{j} \rightarrow \mathbf{H}^{4 k-j}$ é um isomorfismo. Logo, o espaço $\mathbf{H}(j) \doteq \mathbf{H}^{j} \oplus \mathbf{H}^{4 k-j}$ admite uma decomposição $\mathbf{H}(j)=\mathbf{H}^{+}(j) \oplus \mathbf{H}^{-}(j)$, onde $\mathbf{H}^{ \pm}(j) \doteq\left(1 \pm \omega_{\mathbb{C}}\right) \mathbf{H}(j)$, $0 \leq j<2 k$. Como $k e r D^{ \pm}=\mathbf{H}^{ \pm}(0) \oplus \ldots \oplus \mathbf{H}^{ \pm}(2 k-1) \oplus\left(\mathbf{H}^{2 k}\right)^{ \pm}$, onde $\left(\mathbf{H}^{2 k}\right)^{ \pm} \doteq\left(1 \pm \omega_{\mathbb{C}}\right) \mathbf{H}^{2 k}$, e $\operatorname{dim} \mathbf{H}^{+}(j)=\operatorname{dim} \mathbf{H}^{-}(j)$, para $0 \leq j<2 k$, segue pelo teorema de Hodge que

$$
\text { ind } D^{+}=\operatorname{dim}\left(\mathbf{H}^{2 k}\right)^{+}-\operatorname{dim}\left(\mathbf{H}^{2 k}\right)^{-} \text {. }
$$

Por outro lado, podemos definir uma forma bilinear simétrica $Q: H^{2 k}(X ; \mathbb{R}) \times H^{2 k}(X ; \mathbb{R}) \rightarrow \mathbb{R}$ pela fórmula

$$
(\eta, \zeta) \mapsto \int_{\mathbf{X}} \eta \wedge \zeta=\int_{\mathbf{X}} \eta \wedge \star(\star \zeta)=\int_{X} \eta \wedge \star\left(\omega_{\mathbf{C}} \zeta\right)=\left\langle\eta, \omega_{\mathbf{C}} \cdot \zeta\right\rangle,
$$

onde usamos o fato que $\star \star=1 \mathrm{e} \star=\omega_{\mathrm{C}}$ em dimensão $2 k$ e denotamos por $\langle\cdot, \cdot\rangle$ o produto interno usual $\mathrm{L}^{2}$ para formas diferenciais. Como $Q(\eta, \eta)=\|\eta\|^{2}, Q$ é não-degenerada e tem autovalores \pm 1 . Isso nos permite definir a assinatura como no início da subseção 1.4.4, i.e., a diferença entre a dimensões dos autoespaços correspondentes aos autovalores +1 e -1 , respectivamente. Observando a definição dos espaços $\left(\mathbf{H}^{2 k}\right)^{ \pm}$, vemos pela fórmula 2.2.3 que

$$
\text { ind } D^{+}=\operatorname{sig}(X) \text {, }
$$

onde $\operatorname{sig}(X)$ denota a assinatura da forma bilinear simétrica $Q$. O operador $D$ é chamado de operador assinatura. A importância deste operador justifica-se pelo fato de podermos definir a forma $Q$ sem depender da estrutura diferenciável de $X$ pela fórmula $Q(\eta, \zeta)=(\eta \cup \zeta)([X])$, onde $\eta, \zeta \in H^{2 k}(X ; \mathbb{R}), \cup$ denota o produto cup em cohomologia e $[X]$ denota a classe fundamental de $X$. Dos teoremas de Hodge e de Rham (conforme [102]), segue que ambas as definições coincidem; em particular, $\operatorname{sig}(X)$ depende somente da topologia de $X$.

ExEMPLo 2.3.16 Seja $X$ uma variedade riemanniana compacta spin de dimensão $4 k$ e consideremos o fibrado spinor $\mathrm{S}_{\mathbb{C}} \doteq \mathrm{S}_{\mathbb{C}}(T X)$ com operador de Dirac $D$. Consideremos a decomposição canônica $S_{\mathbb{C}}=S_{\mathbb{C}}^{+} \oplus S_{\mathbb{C}}^{-}$em termos do elemento de volume complexo e o operador de Dirac correspondente $D^{+}$. O Teorema de Atiyah-Singer prova que

$$
\text { ind } D^{+}=\hat{\mathrm{A}}(X) \text {, }
$$

onde $\hat{A}(X)$ é um número de Pontryagin racional de $X$, conforme [60], [67] e [92].

Os exemplos anteriores mostram como a introdução de uma $\mathbb{Z}_{2}$-graduação em um fibrado de $\mathrm{Cl}(\mathrm{X})$-módulos pode ser útil para expressar invariantes topológicos em termos de índices de operadores elípticos.

\subsubsection{Propriedades de Continuação Única}

Discutiremos nesta subseção alguns resultados importantes sobre continuação única para operadores de Dirac. A maioria destes resultados decorre de técnicas bastante gerais de equações diferenciais parciais, portanto, iremos apenas indicar, quando necessário, as mudanças necessárias para adequação ao nosso caso.

Seja $P: C^{\infty}(E) \rightarrow C^{\infty}(F)$ um operador pseudo-diferencial, com $E, F$ fibrados riemannianos sobre uma variedade $X$. 
Definição 2.3.17 Dizemos que $P$ tem a propriedade de continuação única fraca, abreviadamente PCU fraca, se as condições $P u=0$ em $X$ e $\left.u\right|_{U}=0$ para $U \subset X$ aberto implicam que $u$ se anula em toda componente conexa de $X$ que intersecta $U$.

Definição 2.3.18 Dizemos que $P$ tem a propriedade de continuação única forte, abreviadamente PCU forte, se as condições $P u=0$ em $X$ e $\partial^{\alpha} u\left(x_{0}\right)=0$, para algum $x_{0} \in X$ e todo $\alpha \in \mathbb{Z}_{+}^{n}$ implicam que $u$ se anula na componente conexa de $X$ contendo $x_{0}$.

Obviamente, se $P$ tem PCU forte então $P$ tem PCU fraca. Também é verdade que se $P$ é analítico-hipoelíptico então $P$ tem PCU forte, conforme [98].

Ao contrário do que parece, elipticidade não garante PCU forte nem PCU fraca. De fato, em [78], A. Pliś mostra que existem $f=f(t, x), u=u(t, x) \in C^{\infty}\left(\mathbb{R}^{2}\right)$, com $u \neq 0$, tais que o operador

$$
P \doteq\left(\partial_{t}-i \partial_{x}\right)^{6}+i t^{6} \partial_{x}^{5}-\partial_{x}^{4}-f(t, x)
$$

é elíptico em $\mathbb{R}^{2}$ e $P u=0$ mas $u$ se anula em $\{(x, t): t \leq 0\}$.

Podemos nos perguntar que hipótese adicional deve ser satisfazer um operador elíptico para que o mesmo verifique PCU forte ou fraca. Assumindo que $P$ tenha coeficientes $\mathrm{C}^{\omega}, \mathrm{F}$. John mostra em [54] que $P$ é analítico-hipoelíptico. Em particular, $P$ tem PCU forte. ${ }^{25}$ Ainda no caso elíptico, em [52], Theorem 8.9.1, L. Hörmander mostra que $P$ tem a PCU fraca se for satisfeita uma hipótese de pseudo-convexidade forte. Obviamente, esta hipótese não é verificada no exemplo de $\mathrm{A}$. Pliś dado no parágrafo anterior.

Com respeito à PCU fraca, uma das técnicas mais utilizadas é a das estimativas de Carleman, desenvolvida por T. Carleman em [30], [31]. Esta é uma técnica muito versátil que se aplica à várias outras situações e consiste em obter estimativas à priori para uma função $u$ qualquer em termos de Pu. Por exemplo, em [25], B. Booss-Bavnbek e K. Wojciechowski utilizam estimativas de Carleman para provar que PCU fraca é válida para operadores de Dirac. Em [17], [20] e [24], são utilizadas estimativas de Carleman para provar a validade de PCU fraca para operadores de Dirac sujeitos à uma perturbação de ordem zero possivelmente não-linear e não-local. Tais perturbações aparecem naturalmente na teoria de Seiberg-Witten, conforme [70]. A observação crucial é que, dados um bi-colar $U$ de uma hipersuperfície $\Sigma$ de uma variedade riemanniana orientada $X$ cujo $^{26}$ fibrado normal é orientável, um operador de Dirac $D$ (conforme a definição 2.3.1) assume a forma

$$
D=d t \cdot\left(\frac{\partial}{\partial t}+B_{t}+C_{t}\right)
$$

em $U$, onde $t$ denota a coordenada transversal a $\Sigma$, $\cdot$ denota multiplicação de Clifford, $B_{t}$ é uma família a um parâmetro de operadores elípticos auto-adjuntos de $1^{\mathrm{a}}$ ordem na hipersuperfície paralela $\Sigma \times\{t\}$ e $C_{t}$ é uma família a um parâmetro de operadores anti-simétricos de ordem zero (de fato, homomorfismos anti-simétricos do fibrado de Clifford).

Um dos resultados mais importantes e clássicos a respeito de PCU forte é o teorema de Aronszajn-Cordes [7],[32]. Este teorema garante que se $P$ elíptico de $2^{a}$ ordem com símbolo

\footnotetext{
${ }^{25}$ Convém observar que no caso em que $P$ tem coeficientes $C^{\omega}$, o teorema de Holmgren (conforme [98], Section 21) implica que se $\Sigma$ é uma hipersuperfície não-característica para $P$ em um aberto $U$ e $u$ é uma distribuição satisfazendo a equação $P u=0 \mathrm{em} U$ e se anulando de um lado de $\Sigma$, então $u$ é nula em $U$.

${ }^{26} \mathrm{Ou}$ seja, uma vizinhança de $\Sigma$ difeomorfa a $\Sigma \times(-1,1)$.
} 
principal real positivo definido então $P$ tem PCU forte. Uma prova interessante pode ser encontrada em [79].

No caso de operadores de Dirac, como já comentamos, é possível obter uma prova da validade de PCU fraca usando estimativas de Carleman. Também é possível aplicar o teorema de Aronszajn-Cordes a $D^{2}$ e a proposição 2.3.2 para concluir a validade de PCU forte, portanto, o próximo teorema está provado.

Teorema 2.3.19 Sejam X uma variedade riemanniana orientada, $S$ um fibrado de $\mathrm{Cl}(\mathrm{X})$-módulos munido de uma conexão $\nabla$ e do operador de Dirac $D$ correspondente, conforme a definição 2.3.1. Então $D$ verifica PCU forte.

\subsection{O Teorema de Yoshida-Nicolaescu}

Sejam $X$ uma variedade riemanniana compacta orientada sem bordo e $E$ um fibrado riemanniano sobre $X$. Consideremos $Y \subset X$ uma hipersuperfície fechada que divide $X$ em duas porções $X_{+}, X_{-}$, i.e., $X_{+}, X_{-}$são variedades com bordo $Y=X_{+} \cap X_{-}$e $X=X_{+} \cup X_{-}$. Dizemos, neste caso, que $Y$ particiona $X$. Seja ainda $P$ um operador pseudo-diferencial elíptico agindo em seções de $E$ tal que, em um bicolar $U$ de $Y$ com coordenadas $(y, t) \in Y \times(-1,1), P$ tenha forma cilíndrica

$$
P=G \cdot\left(\frac{\partial}{\partial t}+B\right),
$$

com $G$ um endomorfismo de $E$ tal que $G^{2}=-I$ e $G^{*}=-G$ e $B$ um operador elíptico de primeira ordem em $Y$. Vamos assumir que tanto $G$ quanto $B$ independem da coordenada transversal $t$ e que $B$ seja auto-adjunto, embora para algumas construções que faremos isso não seja estritamente necessário. Podemos assumir também que $t \leq 0 \mathrm{em} X_{-}$e $t \geq 0 \mathrm{em} X_{+}$. Consideremos os operadores $\left.P_{ \pm} \doteq P\right|_{X_{ \pm}}$e os respectivos espaços de dados de Cauchy

$$
\mathrm{H}_{ \pm}(P) \doteq \mathrm{H}\left(P_{ \pm}, 1 / 2\right) \subset \mathrm{L}^{2}\left(\left.S\right|_{Y}\right),
$$

definidos na equação 2.1.18.

O estudo dos espaços de dados de Cauchy e dos projetores de Calderón correspondentes a $P_{ \pm}$pode ser feito por meio dos resultados da subseção 2.1.5. Isso envolve a construção de operadores elípticos bijetores $\tilde{P}_{+}, \tilde{P_{-}}$e um estudo cuidadoso dos objetos correspondentes a tais operadores. Porém, como $P_{+}, P_{-}$admitem a forma cilíndrica 2.4.1, é possível construir de maneira mais explícita os operadores $\tilde{P_{+}}$e $\tilde{P_{-}}$e, conseqüentemente, o projetores de Calderón correspondentes. Vamos agora descrever este método.

Consideremos a variedade $X_{+}$com bordo $Y$ e o dobro $\tilde{X}$ de $X_{+}$, i.e. a variedade orientada obtida a partir de duas cópias de $X_{+}$(uma delas com a orientação invertida) por identificação do bordo ponto a ponto. Definimos sobre $\tilde{X}$ o fibrado $\tilde{E}_{G}$ obtido como quociente do fibrado $E \cup E$ sobre $\tilde{X}$ pela relação de equivalência $u \equiv G \cdot u$ em $\left.E\right|_{Y}$. Em particular, $\tilde{E}_{G}$ coincide com $E$ sobre $X \backslash Y$. Seções de $\tilde{E}_{G}$ são pares de seções $\left(u_{1}, u_{2}\right)$ de $E$ tais que $u_{2}=G \cdot u_{1}$ em $Y$.

Vamos estender o operador $P$ a um operador elíptico de primeira ordem $\tilde{P}: C^{\infty}\left(\tilde{E}_{G}\right) \rightarrow$ $C^{\infty}\left(\tilde{E}_{G^{-1}}\right)$ definido em $\tilde{X}$ de tal forma que $\tilde{P}=P \cup P^{*}$, i.e., $\tilde{P}$ coincide com $P$ em $X_{+}$e com $P^{*}$ em $\tilde{X} \backslash X_{+}$. Definimos para uma seção $\left(u_{1}, u_{2}\right)$ de $\tilde{E}_{G}$

$$
\tilde{P}\left(u_{1}, u_{2}\right) \doteq\left(P u_{1}, P^{*} u_{2}\right) \text {. }
$$


$\tilde{P}$ é um operador bem-definido, pois se $u_{2}=G \cdot u_{1}$ então

$$
P^{*} u_{2}=\left(\frac{\partial}{\partial t}+B\right) G^{*} u_{2}=G^{-1} \cdot G\left(\frac{\partial}{\partial t}+B\right) u_{1}=G^{-1}\left(P u_{1}\right)
$$

onde o sinal positivo de $\frac{\partial}{\partial t}$ é devido à orientação. Vejamos que sob uma hipótese adequada $\tilde{P}$ é bijetor.

Proposição 2.4.1 Seja X uma variedade riemanniana compacta orientada com bordo Y particionada por uma hipersuperficie $Y$ e $P_{+}$um operador elíptico de primeira ordem que admite a forma cilíndrica 2.4.1 próximo de Y. Se $\mathscr{N}_{0}\left(P_{+}\right)=\mathscr{N}_{0}\left(P_{+}^{*}\right)=0$ então ${ }^{27} \tilde{P}$ é um operador elíptico bijetor.

Prova. Seja $u=\left(u_{1}, u_{2}\right)$ uma seção de $\tilde{E}$ tal que $\tilde{P} u=0$. Então, $P_{+} u_{1}=P_{+}^{*} u_{2}=0$ e portanto, pela formula de Green-Stokes,

$$
0=\int_{X_{+}} P_{+} u_{1} \cdot u_{2} d x-\int_{X_{+}} u_{1} \cdot P_{+}^{*} u_{2} d x=-\int_{Y} G u_{1} \cdot u_{2} d y=-\int_{Y}\left|u_{2}\right|^{2} d y .
$$

Logo, $u_{1}=u_{2}=0$ em $Y$ e portanto, $u_{1} \in \mathscr{N}_{0}\left(P_{+}\right)=0$ e $u_{2} \in \mathscr{N}_{0}\left(P_{+}^{*}\right)=0$, provando que $\tilde{P}$ é injetor. Da mesma forma, provamos que $\tilde{P}^{*}$ é injetor, logo $\tilde{P}$ é bijetor.

OвSERvaÇão 2.4.2 Se $P$ verifica PCU fraca então $\mathscr{N}_{0}\left(P_{+}\right)=\mathscr{N}_{0}\left(P_{+}^{*}\right)=0$. De fato, se $u \in \mathscr{N}_{0}\left(P_{+}\right)$, definindo $\bar{u}$ como

$$
\bar{u}= \begin{cases}u & \text { em } X_{+} \\ 0 & \text { em } X_{-}\end{cases}
$$

temos pela fórmula de Green-Stokes e pela elipticidade de $P$ que $\bar{u} \in C^{\infty}(E)$. Como $\bar{u}$ se anula em int $X_{-}$e $P$ verifica PCU fraca, temos que $\bar{u}=0$ e portanto, $u=0$. O mesmo é verdadeiro para $\mathscr{N}_{0}\left(P_{+}^{*}\right)$. Em particular, pelo teorema 2.3.19, se $P$ é um operador de Dirac sobre uma variedade particionada, então as hipóteses da proposição 2.4 .1 são verificadas.

Vamos agora estudar algumas propriedades dos projetores de Calderón de $P_{+}$e $P_{+}^{*}$. Observando a forma como foi definido o operador $\tilde{P}$, vemos que

$$
\Pi_{+}\left(P_{+}\right)=\tilde{\Pi}_{+}(\tilde{P}) \text { e } \Pi_{+}\left(P_{+}^{*}\right)=\tilde{\Pi}_{-}(\tilde{P}),
$$

onde $\tilde{\Pi}_{ \pm}(\tilde{P})$ são definidos em 2.1 .10 e 2.1 .11 e $\Pi_{+}\left(P_{+}\right), \Pi_{+}\left(P_{+}^{*}\right)$ são definidos em 2.1.16. Esta ${ }^{28}$ observação é crucial para a prova do próximo teorema.

TeоRema 2.4.3 Se X é uma variedade riemanniana compacta orientada com bordo $Y$ particionada por uma hipersuperficie $Y$ e $P_{+}$é um operador elíptico de primeira ordem que admite a forma cilíndrica 2.4.1 próximo de $Y$ e verifica $P C U$ fraca, então

$$
\mathrm{H}_{ \pm}(P)^{\perp}=G\left(\mathrm{H}_{ \pm}\left(P^{*}\right)\right) \text {. }
$$

\footnotetext{
${ }^{27} \mathscr{N}_{0}\left(P_{+}\right)$é definido na fórmula 2.1.14.

${ }^{28} \mathrm{As}$ fórmulas em 2.4 .3 podem ser tomadas como a definição de $\Pi_{+}\left(P_{+}\right), \Pi_{+}\left(P_{+}^{*}\right)$. Isso nos fornece uma maneira de definir o projetor de Calderón utilizando somente os resultados da subseção 2.1.4.
} 
Prova. Vamos provar para o caso de $\mathrm{H}_{+}(P)$; o outro caso é análogo. As fórmulas em 2.4.3, a definiçắo dos espaços de dados de Cauchy e o ítem 5 do teorema 2.1.22 mostram que

$$
\mathrm{G}\left(\mathrm{H}_{+}\left(P^{*}\right)\right)+\mathrm{H}_{+}(P)=\mathrm{L}^{2}(E) .
$$

Pela $^{29}$ fórmula de Green-Stokes, estes subespaços são ortogonais. $\mathrm{Como}_{+}(P)$ e $\mathrm{H}_{+}\left(P^{*}\right)$ são fechados (pela proposição 2.1.28), segue a igualdade 2.4.4.

Vamos fazer alguns comentários importantes sobre outro projetor importante na teoria de problemas de fronteira. Vimos no ítem 5 do teorema 2.1.27, fixando $s=1 / 2$, que o projetor de Calderón $\Pi_{+}\left(P_{+}\right)$é uma projeção com imagem $H_{+}(P)$. Vamos mostrar que é possível obter uma projeção ortogonal explicitamente construída em termos de $P$ com mesmo símbolo principal que $\Pi_{+}\left(P_{+}\right)$. Tal projeção é a chamada de projetor de Atiyah-Patodi-Singer, introduzida por M. Atiyah,; V. Patodi e I. Singer em [14]-I,III. Procedamos agora aos detalhes a respeito destas afirmações.

Seja $P_{+}$um operador satisfazendo as hipóteses da proposição 2.4.1. Como $B$ é auto-adjunto, pelo teorema 2.1.18, obtemos uma base ortonormal $\left\{u_{j}\right\}_{j \in \mathbb{Z}}$ de $\mathrm{L}^{2}\left(\left.E\right|_{Y}\right)$ formada por autofunções de $B$ correspondentes à autovalores (reais) $\left\{\lambda_{j}\right\}_{j \in \mathbb{Z}}$ tais que $\left|\lambda_{j}\right| \rightarrow \infty$ se $|j| \rightarrow \infty$. Seja $\pi_{\geq}$ a projeção ortogonal em $\mathrm{L}^{2}\left(\left.E\right|_{Y}\right)$ sobre o subespaço fechado gerado pelos $u_{j}$ correspondentes aos $\lambda_{j} \geq 0$. Podemos substituir $B$ pelo operador limitado $B^{\prime} \doteq\left(I+B^{2}\right)^{-1 / 2} B$ (conforme o lema 1.2.27) sem alterar o sinal dos autovalores de $B$. O operador $B^{\prime}$ tem autovetores $\left\{u_{j}\right\}_{j \in \mathbb{Z}}$ correspondentes aos autovalores $\left\{\lambda_{j} /\left(1+\lambda_{j}\right)^{1 / 2}\right\}_{j \in \mathbb{Z}}$; em particular, pela teoria da subseção 1.2.3,

$$
\pi_{\geq}=\frac{1}{2 \pi i} \int_{\gamma} \mathrm{R}\left(\zeta ; B^{\prime}\right) d \zeta,
$$

onde $\gamma$ é uma circunferência centrada em $z=1$ contendo em seu interior exatamente os autovalores não-negativos de $B^{\prime}$. Podemos aproximar o resolvente $\mathrm{R}\left(\zeta ; B^{\prime}\right)$ pela parametriz de $B^{\prime}-\zeta$, i.e., se $\sum_{j \leq 0} p_{j}$ é o desenvolvimento assintótico da parametriz de $B^{\prime}-\zeta$, então o operador $\sum_{j \geq N} P_{j}-\mathrm{R}\left(\zeta ; B^{\prime}\right)$ tem norma arbitrariamente pequena nos espaços de Sobolev usuais, para $N \rightarrow-\infty$ (conforme [46], Lemma 1.7.2), onde $P_{j}$ denota um operador pseudo-diferencial com símbolo $p_{j}$. Denotando por $b^{\prime}$ o símbolo principal de $B^{\prime}$ e por $p_{+}\left(p_{-}\right)$a projeção ortogonal sobre o autoespaço positivo (negativo) de $b^{\prime}$ (que coincide com a projeção sobre o autoespaço positivo (negativo) de $b$ ), temos

$$
\begin{aligned}
p_{0}(y, \xi ; \zeta) & =\left(b^{\prime}(y, \xi)-\zeta\right)^{-1} \\
& =\frac{1}{1-\zeta^{2}}\left(p_{+}-p_{-}+\zeta\right) \\
& =\frac{1}{1-\zeta^{2}}\left((1+\zeta) p_{+}(y, \xi)-(1-\zeta) p_{-}(y, \xi)\right) \\
& =\frac{1}{1-\zeta} p_{+}(y, \xi)-\frac{1}{1+\zeta} p_{-}(y, \xi) .
\end{aligned}
$$

Logo, o símbolo principal de $\pi_{+}$é

$$
\sigma_{0}\left(\pi_{+}\right)=\frac{1}{2 \pi i} \int_{\gamma}\left\{\frac{1}{1-\zeta} p_{+}(y, \xi)-\frac{1}{1+\zeta} p_{-}(y, \xi)\right\} d \zeta=p_{+}(y, \xi)=\sigma_{0}\left(\Pi_{+}\left(P_{+}\right)\right) .
$$

A próxima proposição está provada.

\footnotetext{
${ }^{29}$ Neste ponto, usamos o fato que $\mathrm{H}_{-}(\tilde{P})=G^{-1}\left(\mathrm{H}_{+}\left(P^{*}\right)\right)$.
} 
Proposição 2.4.4 Se $P_{+}$é um operador satisfazendo as hipóteses da proposição 2.4.3, então o projetor de Calderón e o projetor de Atiyah-Patodi-Singer correspondentes a $P_{+}$têm o mesmo símbolo principal. Em particular, a diferença $\pi_{\geq}-\Pi_{+}\left(P_{+}\right)$é um operador compacto..$^{30}$

Oвservação 2.4.5 Em [25], Pag. 93, B. Booss-Bavnbek e K. Wojciechowski indicam uma maneira de substituir uma projeção $Q$ qualquer em um espaço de Hilbert por uma projeção ortogonal com mesma imagem que $Q$. De fato,

$$
Q^{\prime} \doteq Q Q^{*}\left(Q Q^{*}+(I-Q)\left(I-Q^{*}\right)\right)^{-1}
$$

é uma projeção ortogonal sobre a imagem de $Q$. Além disso, vemos que $Q^{\prime}$ depende analiticamente de $Q$. Isso implica, levando em conta a estrutura diferenciável das variedades grassmannianas introduzida na subseção 1.6.2, que se $[a, b] \ni s \mapsto Q(s) \in \mathscr{L}(\mathrm{H})$ é uma curva de classe $C^{k}$ de projeções arbitrárias em um espaço de Hilbert $\mathrm{H}$, então a curva $[a, b] \ni s \mapsto \operatorname{Im} Q(s) \in \mathcal{G}(\mathrm{H})$ é uma curva de classe $C^{k}$, para $k=0, \ldots, \infty, \omega$.

Vamos agora introduzir uma ferramenta indispensável para o estudo da geometria dos espaços de dados de Cauchy. Definimos

$$
(J u)(y) \doteq G \cdot u(y),
$$

para $u \in \mathrm{L}^{2}\left(\left.E\right|_{Y}\right)$ e $y \in Y$. Vemos que $J$ é uma estrutura complexa em $\mathrm{L}^{2}\left(\left.E\right|_{Y}\right)$. Considerando a forma simplética $\omega$ correspondente definida por $\omega(u, v) \doteq\langle J u, v\rangle, u, v \in \mathrm{L}^{2}\left(\left.E\right|_{Y}\right)$, temos que $\left(\mathrm{L}^{2}\left(\left.E\right|_{Y}\right), \omega\right)$ é um espaço simplético, conforme a definição 1.5.1. Manteremos fixa esta estrutura simplética em $\mathrm{L}^{2}\left(\left.E\right|_{Y}\right)$, a menos de menção contrária.

O próximo teorema decorre imediatamente do teorema 2.4 .3 e da definição de subespaço lagrangiano dada em 1.5.17.

Teorema 2.4.6 Se Pé formalmente auto-adjunto então os espaços de dados de Cauchy $\mathrm{H}_{ \pm}(P)$ definidos em 2.4 .2 são subespaços lagrangianos de $\mathrm{L}^{2}\left(\left.E\right|_{Y}\right)$.

As propriedades de Fredholm para operadores e pares de subespaços são bastante típicas no estudo de problemas de fronteira para operadores elípticos. Veremos agora que também no nosso caso, são verificadas tais propriedades.

Teorema 2.4.7 Se $X, Y, E$ e $P$ são como no primeiro paragrafo desta seção, então $\left(\mathrm{H}_{+}(P), \mathrm{H}_{-}(P)\right)$ é um par Fredholm de subespaços de $\mathrm{L}^{2}\left(\left.E\right|_{Y}\right)$.

Prova. Se $g \in \mathrm{H}_{+}(P) \cap \mathrm{H}_{-}(P)$ então existem $u_{ \pm} \in \mathrm{C}^{\infty}\left(\left.E\right|_{X_{ \pm}}\right)$tais que $P_{ \pm} u_{ \pm}=0$ e $\gamma_{0}\left(u_{ \pm}\right)=g$. Definindo

$$
u=\left\{\begin{array}{ll}
u_{+} & \text {em } X_{+} \\
u_{-} & \text {em } X_{-}
\end{array},\right.
$$

a fórmula de Green-Stokes mostra que $P u=0$ no sentido de distribuições, $\operatorname{logo}, u \in C^{\infty}(E)$, pelo teorema 2.1.16. Além disso, a aplicação

$$
\mathrm{H}_{+}(P) \cap \mathrm{H}_{-}(P) \ni g \mapsto u \in \operatorname{ker} P
$$

\footnotetext{
${ }^{30}$ Obviamente, o mesmo é verdadeiro para $P_{-}$em lugar de $P_{+}$.
} 
é um isomorfismo e segue pelo referido teorema que nul $\left(\mathrm{H}_{+}(P), \mathrm{H}_{-}(P)\right)<\infty$.

Consideremos o projetor $\pi_{\leq} \doteq I-\pi_{\geq}\left(P_{+}\right)$, onde $\pi_{\geq}\left(P_{+}\right)$é o projetor de Atiyah-Patodi-Singer correspondente ao operador $P_{+}$. Vamos identificar qual a relação deste operador com o nosso problema. Assumimos desde o início que $t \geq 0 \mathrm{em} X_{+}$, portanto, ao fazermos as mesmas considerações para $P_{-}$em $X_{-}$, devemos escrever $P_{-}$na forma cilíndrica adequada

$$
P_{-}=(-G)\left(\frac{\partial}{\partial t}+(-B)\right)
$$

Em particular, vemos que $\pi_{\geq}\left(P_{-}\right)=\pi_{\leq}+q_{0}$, onde $\pi_{\geq}\left(P_{-}\right)$é o projetor de Atiyah-Patodi-Singer correspondente ao operador $P_{-}$e $q_{0}$ denota a projeção ortogonal sobre ker $B$ (que coincide com a projeção ortogonal sobre $\operatorname{ker}(-B)$ ). Portanto, pelo teorema 2.4.4, temos que

$$
\Pi_{+}(P)+\Pi_{-}(P)=\pi_{\geq}+\pi_{\leq}+K=I+K,
$$

onde $K: \mathrm{L}^{2}\left(\left.E\right|_{Y}\right) \rightarrow \mathrm{L}^{2}\left(\left.E\right|_{Y}\right)$ é um operador compacto. O resultado segue do teorema 1.3.30.

Vamos $^{31}$ agora relacionar o operador $P$ e os espaços de dados de Cauchy; o primeiro resultado nesta direção é a conjectura de Bojarski, conforme [19], [25].

TeORema 2.4.8 (BojARski) Dados uma variedade compacta $X$ particionada por uma hipersuperfície fechada $Y$ e $P$ um operador elíptico de primeira ordem que admite a forma cilíndrica em um colar de $Y$ em X satisfaz PCU fraca, temos que

$$
\text { ind } P=\text { ind }\left(\mathrm{H}_{+}(P), \mathrm{H}_{-}(P)\right) \text {, }
$$

onde o índice da esquerda é o indice de Fredholm do operador $P$ e o índice da direita é o indice de Fredholm de um par Fredholm de subespaços, conforme definições na seção 1.3.

Prova. Vimos no teorema 2.4.7 que existe um isomorfismo $\mathrm{H}_{+}(P) \cap \mathrm{H}_{-}(P) \approx \operatorname{ker} P$. Como $P^{*}$ verifica as mesmas hipóteses que $P$, pelo ítem 1 da proposição 1.5 .7 e pelo teorema 2.4.3, temos

$$
\operatorname{ker} P^{*} \approx \mathrm{H}_{+}\left(P^{*}\right) \cap \mathrm{H}_{-}\left(P^{*}\right)=\left(G\left(\mathrm{H}_{+}(P)\right)\right)^{\perp} \cap\left(G\left(\mathrm{H}_{-}(P)\right)\right)^{\perp}=\mathrm{G}\left(\left(\mathrm{H}_{+}(P)+\mathrm{H}_{-}(P)\right)^{\perp}\right) .
$$

Portanto,

$$
\text { ind } P=\operatorname{nul} P-\operatorname{nul} P^{*}=\operatorname{nul}\left(\mathrm{H}_{+}(P), \mathrm{H}_{-}(P)\right)-\operatorname{def}\left(\mathrm{H}_{+}(P), \mathrm{H}_{-}(P)\right)=\operatorname{ind}\left(\mathrm{H}_{+}(P), \mathrm{H}_{-}(P)\right) \text {, }
$$

como queríamos.

No caso em que $X$ tem dimensão impar ou $P$ é formalmente auto-adjunto, a fórmula 2.4.5 se trivializa, pois o índice de operadores elípticos em variedades compactas de dimensão ímpar é nulo (conforme [60], Theorem 13.12) e um par Fredholm de subespaços lagrangianos tem índice de Fredholm nulo, conforme o teorema 2.4.6. A variante adequada da conjectura de Bojarski em dimensão ímpar é o teorema de Yoshida-Nicolaescu, o qual passamos a descrever. Neste caso, ao invés de um único operador $P$, considera-se uma família a um

\footnotetext{
${ }^{31}$ Neste ponto, chamamos a atenção para o fato que a prova do teorema 2.4 .7 dada na literatura em geral (veja [26], Theorem 3.4, ou [69]) é incompleta. A parte substancial do teorema consiste em provar que $\mathrm{H}_{+}(P)+\mathrm{H}_{-}(P)$ é fechado. Vimos no exemplo 1.5.35 que existem lagrangianos com intersecção trivial e soma não-fechada.
} 
parâmetro $\{P(s)\}_{s \in[0,1]}$ de operadores de Dirac auto-adjuntos sobre a variedade particionada $X$ e a fórmula 2.4.5 transforma-se em

$$
\operatorname{sf}(\{P(s)\})=\mu\left(\left\{\mathrm{H}_{+}(s)\right\},\left\{\mathrm{H}_{-}(\mathrm{s})\right\}\right),
$$

onde $\operatorname{sf}(\{P(s)\})$ denota o fluxo espectral da família $\{P(s)\}_{s \in[0,1]}$ e $\mu\left(\left\{\mathrm{H}_{+}(s)\right\},\left\{\mathrm{H}_{-}(s)\right\}\right)$ denota o índice de Maslov do par Fredholm de curvas de lagrangianos formado pelos espaços de dados de Cauchy correspondentes aos operadores $P(s)_{+}$e $P(s)_{-}$.

Nosso objetivo é provar a fórmula 2.4.6 utilizando a teoria de assinaturas parciais, numa situação consideravelmente mais geral (vamos supor apenas que os $P(s)$ têm forma cilíndrica em um bicolar de $Y$ e verificam PCU fraca). Para isso, precisamos de alguns lemas técnicos.

Lema 2.4.9 Seja $\{T(s)\}_{s \in[0,1]}$ uma família a um parâmetro de operadores fechados densamente definidos em $\mathrm{H}$ com domínio comum $\mathrm{D}$. Suponhamos que exista uma norma $\|\cdot\|_{\mathrm{D}}$ em $\mathrm{D}$ tal que os operadores $T(s)$ são $\|\cdot\|_{D}$-contínuos. Então, $\{T(s)\}_{s \in I}$ é holomorfa de tipo $(A)$ se e só se

$$
I \ni s \mapsto T(s) \in \mathscr{L}(\mathrm{D}, \mathrm{H})
$$

é holomorfa, onde $\mathscr{L}(\mathrm{D}, \mathrm{H})$ denota o espaço dos operadores limitados $\mathrm{D} \rightarrow \mathrm{H}$ e $\mathrm{D}$ é munido da norma $\|\cdot\|_{\mathrm{D}}$.

Prova. Se 2.4 .7 é holomorfa, claramente $\{T(s)\}$ holomorfa de tipo (A). Se $\{T(s)\}$ é holomorfa de tipo (A), então, por definição, a aplicação $I \ni s \mapsto T(s) u \in H$ é holomorfa para todo $u \in \mathrm{D}$. Pelo princípio da limitação uniforme, concluímos que 2.4 .7 é holomorfa.

Lema 2.4.10 Seja $\{P(s)\}_{s \in[0,1]}$ uma família holomorfa de tipo $(A)$ de operadores elípticos auto-adjuntos de primeira ordem que têm a forma cilíndrica em um bicolar de $Y$ e verificam PCU fraca. Então as curvas $[0,1] \ni s \mapsto \mathrm{H}_{+}(P(s)) \in \Lambda e[0,1] \ni s \mapsto \mathrm{H}_{-}(P(s)) \in \Lambda$ são curvas analíticas reais na grassmanniana de lagrangianos $\Lambda \doteq \Lambda\left(\mathrm{L}^{2}\left(\left.E\right|_{Y}\right)\right)$.

Prova. Basta observar que os projetores de Calderón $\Pi_{+}\left(P(s)_{+}\right)$e $\Pi_{-}\left(P(s)_{-}\right)$dependem analiticamente dos operadores $\left(P(\tilde{s})_{+}\right)^{-1} \mathrm{e}\left(P(\tilde{s})_{-}\right)^{-1}$ os quais foram construídos explicitamente nos comentários antecedentes à proposição 2.4.1. O teorema decorre da observação 2.4.5.

Vamos agora estabelecer alguns fatos importantes sobre o cálculo do fluxo espectral para curvas de operadores. Seja $\{P(s)\}_{s \in[0,1]}$ uma família holomorfa de tipo (A) de operadores pseudo-diferenciais elípticos auto-adjuntos agindo em seções de um fibrado riemanniano $E$ sobre uma variedade riemanniana compacta $X$. Pelo teorema 2.1.16, os $P(s)$ são operadores Fredholm densamente definidos em $\mathrm{L}^{2}(E)$, e portanto é bem definido o fluxo espectral $\mathrm{sf}(\{P(s)\})$ da família $\{P(s)\}$. Pelo lema 1.4.48, a família $\{P(s)\}$ tem, além dos autovalores identicamente nulos (os quais não influenciam no cálculo do fluxo espectral), somente degenerescências isoladas. Podemos supor, sem perda de generalidade, que $s_{0} \in[0,1]$ seja a única degenerescência de $\{P(s)\}$ em $[0,1]$. A teoria de assinaturas parciais pode ser utilizada para calcular explicitamente o fluxo espectral de $\{P(s)\}$, mas nesta situação específica, podemos melhorar os métodos de cálculo fornecidos por esta teoria.

Definição 2.4.11 Uma função $u:\left(s_{0}-\varepsilon, s_{0}+\varepsilon\right) \rightarrow \mathrm{L}^{2}(E)$ é dita função-raiz generalizada para $P(s)$ se $P\left(s_{0}\right) u\left(s_{0}\right)=0$. Dizemos ${ }^{32}$ que uma função-raiz generalizada $u(s)$ tem ordem $k$ em $s=s_{0}$ se

\footnotetext{
${ }^{32}$ A igualdade $P\left(s_{0}\right) u\left(s_{0}\right)=0$ deve ser interpretada no sentido de distribuições. Em particular, pela elipticidade de $P\left(s_{0}\right)$, segue que $u\left(s_{0}\right) \in C^{\infty}(E)$.
} 
a função

$$
\left(s_{0}-\varepsilon, s_{0}+\varepsilon\right) \ni s \mapsto P(s) u(s) \in \mathscr{D}^{\prime}(X ; E)
$$

tem um zero de ordem $k$ em $s=s_{0}$.

A diferença crucial entre uma função-raiz segundo a definição 1.4 .41 e uma função-raiz generalizada é que no segundo caso, não exigimos que a função tenha imagem no domínio do operador e interpretamos os objetos envolvidos como distribuições. A próxima proposição fornece uma condição algébrica para a existência de uma função-raiz generalizada de determinada ordem.

Proposição 2.4.12 Existe uma função-raiz generalizada $u(s)$ de ordem $\geq k$ para $P(s)$ com $u\left(s_{0}\right)=$ $u_{0} \in \operatorname{ker} P\left(s_{0}\right)$ se e só se existem $u_{0} \doteq u_{0}^{(0)}, \ldots, u_{0}^{(k-1)} \in \mathrm{H}^{1}(E)$ tais que

$$
\sum_{j=0}^{r}\left(\begin{array}{l}
r \\
j
\end{array}\right) P^{(j)}\left(s_{0}\right) u_{0}^{(r-j)}=0,
$$

para $r=0, \ldots, k-1$, onde $P^{(j)}(s)$ denota a j-ésima derivada da aplicação

$$
[0,1] \ni s \mapsto P(s) \in \mathscr{L}\left(\mathrm{H}^{1}(E), \mathrm{H}^{0}(E)\right)
$$

Prova. Suponhamos que $u(s)$ é uma função-raiz generalizada para $P(s)$ de ordem $\geq k$ tal que $u\left(s_{0}\right)=u_{0}$. Definindo $u_{0}^{(r)} \doteq u^{(r)}\left(s_{0}\right) \in \mathrm{L}^{2}(E)$ para $r=0, \ldots, k-1$, a fórmula 2.4 .8 segue da fórmula de Leibniz. Como $P\left(s_{0}\right)$ é elíptico, temos que $u_{0}=u_{0}^{(0)} \in C^{\infty}(E)$. Procedendo indutivamente e usando as fórmulas 2.4 .8 e a elipticidade de $P\left(s_{0}\right)$, concluímos pelo teorema 2.1.16 que $u_{0}^{(r)} \in \mathrm{H}^{1}(E)$, para $r=0, \ldots, k-1$.

Reciprocamente, se existem $u_{0} \doteq u_{0}^{(0)}, \ldots, u_{0}^{(k-1)} \in \mathrm{H}^{1}(E)$ verificando 2.4 .8 , basta definir $u(s) \doteq \sum_{j=0}^{k-1}\left(s-s_{0}\right)^{j} u_{0}^{(j)}$ e observar que $u(s)$ é uma função-raiz para $P(s)$ de ordem $\geq k$ com $u\left(s_{0}\right)=u_{0}$.

Podemos definir, neste caso, os espaços

$$
\begin{aligned}
& W_{k}^{\prime}\left(P, s_{0}\right) \doteq\left\{u_{0} \in \operatorname{ker} P\left(s_{0}\right): \text { Existe função-raiz generalizada } u(s)\right. \\
& \text { para } \left.P(s) \text { de ordem } \geq k \operatorname{com} u\left(s_{0}\right)=u_{0}\right\}
\end{aligned}
$$

para $k \geq 1$. Como antes, temos que $W_{1}^{\prime}\left(P, s_{0}\right) \supset W_{2}^{\prime}\left(P, s_{0}\right) \supset \ldots$ são subespaços de $\operatorname{ker} P\left(s_{0}\right)$.

OBSERVAÇão 2.4.13 Observando a prova da proposição 2.4.12, vemos que para cada $u_{0} \in$ $\mathrm{W}_{k}^{\prime}\left(P, s_{0}\right)$, existe uma função-raiz $u(s)$ para $P(s)$ com $u\left(s_{0}\right)=u_{0}$ e $u(s) \in \mathrm{H}^{1}(E)$.

Sejam $u_{0}, v_{0} \in \mathrm{W}_{k}^{\prime}\left(P, s_{0}\right), u(s), v(s)$ funções-raiz generalizadas para $P(s)$ com $u\left(s_{0}\right)=u_{0} \mathrm{e}$ $v\left(s_{0}\right)=v_{0}$ e $v(s) \in \mathrm{H}^{1}(E)$. Denotando por $\langle\cdot, \cdot\rangle$ a dualidade natural entre $\mathrm{H}^{1}(E)$ e $^{-1}(E)$ (a qual coincide com o produto $L^{2}$ para funções em $L^{2}(E)$ ), temos que

$$
\begin{aligned}
\left\langle\left.\frac{d^{k}}{d s^{k}}\right|_{s=s_{0}} P(s) u(s), v_{0}\right\rangle & =\left.\frac{d^{k}}{d s^{k}}\right|_{s=s_{0}}\langle P(s) u(s), v(s)\rangle \\
& =\left.\frac{d^{k}}{d s^{k}}\right|_{s=s_{0}}\langle u(s), P(s) v(s)\rangle \\
& =\left\langle u_{0},\left.\frac{d^{k}}{d s^{k}}\right|_{s=s_{0}} P(s) v(s)\right\rangle .
\end{aligned}
$$


Logo, pela observação 2.4.13, para $u_{0}, v_{0} \in \mathrm{W}_{k}^{\prime}\left(P, s_{0}\right)$,

$$
B_{k}^{\prime}\left(P, s_{0}\right)\left(u_{0}, v_{0}\right) \doteq \frac{1}{k !}\left\langle\left.\frac{d^{k}}{d s^{k}}\right|_{s=s_{0}} P(s) u(s), v_{0}\right\rangle
$$

é uma forma bilinear bem definida e simétrica (hermitiana no caso complexo).

Proposição 2.4.14 A forma bilinear $B_{k}^{\prime}\left(P, s_{0}\right)$ definida em $\mathrm{W}_{k}^{\prime}\left(P, s_{0}\right)$ pela fórmula 2.4.11 é bem definida e simétrica.

A próxima proposição mostra que os espaços $W_{k}^{\prime}\left(P, s_{0}\right)$ e as formas bilineares $B_{k}^{\prime}\left(P, s_{0}\right)$ coincidem com $W_{k}\left(P, s_{0}\right)$ e $B_{k}\left(P, s_{0}\right)$ definidos na subseção 1.4.4, e portanto, podemos utilizar funções-raiz generalizadas para calcular o fluxo espectral de uma família $\{P(s)\}_{s \in[0,1]}$ como antes.

Proposição 2.4.15 Os espaços $W_{k}^{\prime}\left(P, s_{0}\right)$ e as formas $B_{k}^{\prime}\left(P, s_{0}\right)$ definidos em 2.4 .9 e 2.4.11 coincidem com $\mathrm{W}_{k}\left(P, s_{0}\right)$ e $B_{k}\left(P, s_{0}\right)$ definidos em 1.4.9 e 1.4.10.

Prova. Basta observar que, pela proposição 2.4.12, se existe uma função-raiz generalizada

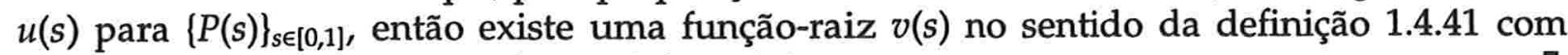
mesmas derivadas que $u(s)$ tal que $u\left(s_{0}\right)=v\left(s_{0}\right)$.

Lema 2.4.16 Sejam $S, T:\left[s_{0}-\varepsilon, s_{0}+\varepsilon\right] \rightarrow \mathscr{C} \mathscr{F}^{\mathrm{sa}}(\mathrm{H})$ familias holomorfas de tipo (A) que possuem uma única degenerescência em $s=s_{0}$. Suponhamos que exista um isomorfismo linear $\Phi: W_{1}\left(S, s_{0}\right) \rightarrow$ $\mathrm{W}_{1}\left(T, s_{0}\right)$ tal que

1. $\Phi\left(W_{k}\left(S, s_{0}\right)\right) \subset W_{k}\left(T, s_{0}\right)$ para todo $k \geq 2$;

2. $\Phi^{*}\left(B_{k}\left(T, s_{0}\right)\right)=B_{k}\left(S, s_{0}\right)$ para todo $k \geq 1$, onde $\Phi^{*}\left(B_{k}\left(T, s_{0}\right)\right)$ denota o pull-back da forma bilinear $B_{k}\left(T, s_{0}\right)$ por $\Phi$.

Então $\Phi\left(\mathrm{W}_{k}\left(S, s_{0}\right)\right)=\mathrm{W}_{k}\left(T, s_{0}\right)$ para todo $k \geq 1$. Em particular, $S$ e $T$ têm as mesmas assinaturas parciais em $s=s_{0}$ e portanto, o mesmo fluxo espectral em $\left[s_{0}-\varepsilon, s_{0}+\varepsilon\right]$.

Prova. Vamos provar que $\Phi\left(W_{k}\left(S, s_{0}\right)\right)=W_{k}\left(T, s_{0}\right)$ por indução em $k$. Isso é obviamente verdadeiro para $k=1$. Supondo que $\Phi\left(W_{k}\left(S, s_{0}\right)\right)=W_{k}\left(T, s_{0}\right)$, com $k \geq 1$, temos pela proposição 1.4.46 que

$$
\begin{aligned}
\Phi\left(\mathrm{W}_{k+1}\left(S, s_{0}\right)\right) & =\Phi\left(\operatorname{ker} B_{k}\left(S, s_{0}\right)\right) \\
& =\Phi\left(\Phi^{-1}\left(\operatorname{ker} B_{k}\left(T, s_{0}\right)\right)\right. \\
& =\operatorname{ker} B_{k}\left(T, s_{0}\right)=\mathrm{W}_{k+1}\left(T, s_{0}\right) .
\end{aligned}
$$

A conclusão segue do teorema 1.4.45.

Estamos prontos agora provar a fórmula 2.4 .6 no caso analítico real.

TEOREMA 2.4.17 Sejam X uma variedade riemanniana compacta orientada particionada por uma hipersuperficie fechada $Y, E$ um fibrado riemanniano sobre $X e\{P(s)\}_{s \in[0,1]}$ uma família holomorfa de 
tipo $(A)$ de operadores pseudo-diferenciais elípticos auto-adjuntos de primeira ordem em E que verificam PCU fraca e admitem a forma cilíndrica

$$
P(s)=G \cdot\left(\frac{\partial}{\partial t}+B(s)\right)
$$

em $u m$ bicolar de $Y$, com $G$ independente de te $s, G^{2}=-I, G^{*}=-G$ e $B(s)$ independente de $t$. Então a fórmula 2.4.6 é verificada.

Prova. Pelo lema 1.4.48, além dos autovalores identicamente nulos (os quais não influenciam no cálculo nem do fluxo espectral nem do índice de Maslov), $\{P(s)\}_{s \in[0,1]}$ possui somente degenerescências isoladas. Vimos no teorema 2.4.7 que existe um isomorfismo natural $\mathrm{H}_{+}(s) \cap$ $\mathrm{H}_{-}(s) \approx \operatorname{ker} P(s)$; em particular, o par de curvas $\left(\mathrm{H}_{+}(s), \mathrm{H}_{-}(s)\right)$ possui somente intersecções isoladas, conforme a definição 1.7.16. Tais intersecções ocorrem exatamente nos instantes de degenerescência da curva $\{P(s)\}_{s \in[0,1]}$. Pelos teoremas 1.4.45 e 1.7.18 e pelo lema 2.4.10, tanto o fluxo espectral quanto o índice de Maslov dependem unicamente das assinaturas parciais, portanto, é suficiente provar que as assinaturas parciais de $\{P(s)\}_{s \in[0,1]}$ e do par $\left(\mathrm{H}_{+}(P(s)), \mathrm{H}_{-}(P(s))\right)$ coincidem em cada degenerescência.

Podemos supor que exista um único ponto de degenerescência $s_{0} \in[0,1]$ para a curva $P(s)$. Consideremos um lagrangiano $L \subset \mathrm{L}^{2}\left(\left.E\right|_{Y}\right) \oplus \mathrm{L}^{2}\left(\left.E\right|_{Y}\right)$ complementar a $\Delta \mathrm{e} \mathrm{H}_{+}\left(s_{0}\right) \oplus \mathrm{H}_{-}\left(s_{0}\right)$ (o qual existe pela proposição 1.5.31 e pelo teorema 2.4.7). Pelo teorema 2.4.10, como $\Lambda_{0}(L)$ é aberto (conforme a notação e a estrutura diferenciável na grassmanniana de lagrangianos introduzida na subseção 1.6.2) podemos supor que a curva $[0,1] \ni s \mapsto H_{+}(s) \oplus H_{-}(s) \in \Lambda\left(L^{2}\left(\left.E\right|_{Y}\right) \oplus \mathrm{L}^{2}\left(\left.E\right|_{Y}\right)\right)$ tem imagem em $\Lambda_{0}(L)$. Consideremos uma curva de operadores $\left(U_{+}(s), U_{-}(s)\right): \Delta \rightarrow L$ tal que $\mathrm{G}\left(\left(U_{+}(s), U_{-}(s)\right)\right)=\mathrm{H}_{+}(s) \oplus \mathrm{H}_{-}(s), s \in[0,1]$. Usando a definição 2.3.2, concluímos que

$$
\begin{aligned}
\varphi_{\Delta, \mathrm{L}}\left(\mathrm{H}_{+}(s) \oplus \mathrm{H}_{-}(s)\right) & =P_{\Delta} \circ(G,-G) \circ\left(U_{+}(s), U_{-}(s)\right) \\
& =\left(\frac{1}{2} G\left(U_{+}(s)-U_{-}(s)\right), \frac{1}{2} G\left(U_{+}(s)-U_{-}(s)\right)\right),
\end{aligned}
$$

onde $P_{\Delta}(x, y)=\left(\frac{1}{2}(x+y), \frac{1}{2}(x+y)\right)$ é a projeção ortogonal sobre a diagonal $\Delta$ e $(G,-G)$ é a estrutura complexa correspondente ao espaço simplético $\mathrm{L}^{2}\left(\left.E\right|_{Y}\right) \oplus \mathrm{L}^{2}\left(\left.E\right|_{Y}\right)$, conforme os comentários após a definição 1.7.10. Pela definição 1.7.12 e pelo teorema 1.7.3, segue que

$$
\mu\left(\left\{\mathrm{H}_{+}(s)\right\},\left\{\mathrm{H}_{-}(s)\right\}\right)=\operatorname{sf}\left(\frac{1}{2} G\left(U_{+}(s)-U_{-}(s)\right), \frac{1}{2} G\left(U_{+}(s)-U_{-}(s)\right)\right) .
$$

Vamos utilizar a teoria desenvolvida na subseção 1.4.4 para calcular o fluxo espectral que aparece nas fórmulas 2.4 .6 e 2.4.14. Sejam $\Phi$ o isomorfismo dado por

$$
\begin{aligned}
\Phi: \Delta \cap\left(\mathrm{H}_{+}\left(s_{0}\right) \oplus \mathrm{H}_{-}\left(s_{0}\right)\right) & \rightarrow \operatorname{ker} P\left(s_{0}\right) \\
(g, g) & \mapsto u,
\end{aligned}
$$

onde $u$ é o único elemento de $\operatorname{ker} P\left(s_{0}\right)$ tal que $\left.u\right|_{Y}=g, \mathrm{~W}_{k}\left(P, s_{0}\right), B_{k}\left(P, s_{0}\right)$ os $^{33}$ objetos correspondentes à curva analítica de tipo (A) de operadores auto-adjuntos $\{P(s)\}$ e $W_{k}\left(\mathrm{H}, s_{0}\right), B_{k}\left(\mathrm{H}, s_{0}\right)$ os objetos correspondentes à curva analítica real de operadores auto-adjuntos 2.4.13. Pelo

\footnotetext{
${ }^{33}$ Aqui observamos que, segundo a teoria de regularidade para operadores elípticos desenvolvida na subseção 2.1.2, os elementos de $\operatorname{ker} P\left(s_{0}\right)$ são de classe $C^{\infty}$, portanto, $\left.u\right|_{Y}$ é bem definido.
} 
lema 2.4.16, como $\Phi: W_{1}\left(H, s_{0}\right) \rightarrow W_{1}\left(P, s_{0}\right)$ é um isomorfismo, é suficiente provar que $\Phi\left(\mathrm{W}_{k}\left(\mathrm{H}, s_{0}\right)\right) \subset \mathrm{W}_{k}\left(P, s_{0}\right)$ e $\Phi^{*}\left(B_{k}\left(P, s_{0}\right)\right)=B_{k}\left(\mathrm{H}, s_{0}\right)$, para todo $k \geq 1$.

Dados $\left(g_{0}, g_{0}\right),\left(h_{0,}, h_{0}\right) \in \mathrm{W}_{k}\left(\mathrm{H}, s_{0}\right)$ e $(g(s), g(s))$ uma função-raiz de ordem $\geq k$ para o operador 2.4.13 tal que $g\left(s_{0}\right)=g_{0}$, por definição de $U_{+}(s)$ e $U_{-}(s)$, temos que

$$
\begin{aligned}
& g(s)+U_{+}(s) g(s) \doteq f_{+}(s) \in \mathrm{H}_{+}(s) \\
& g(s)+U_{-}(s) g(s) \doteq f_{-}(s) \in \mathrm{H}_{-}(s)
\end{aligned}
$$

portanto, existem, pelo teorema 2.1.27, $F_{ \pm}(s) \in \mathrm{C}^{\infty}\left(\left.E\right|_{X_{ \pm}}\right) \cap \mathrm{H}^{1 / 2}\left(\left.E\right|_{X_{ \pm}}\right)$tais que

$$
\left\{\begin{array}{rl}
P(s) F_{ \pm}(s) & =0 \text { em int } X_{ \pm} \\
\left.F_{ \pm}(s)\right|_{Y} & =f_{ \pm}(s)
\end{array},\right.
$$

onde $\left.F_{ \pm}(s)\right|_{Y}$ é definido como o traço de $F_{ \pm}(s)$ sobre $Y$, conforme o teorema 2.1.27. Pelo referido teorema, $F_{ \pm}(s)$ também é analítica real com respeito ao parâmetro $s$. Pondo

$$
F(s)=\left\{\begin{array}{ll}
F_{+}(s) & \text { em } X_{+} \\
F_{-}(s) & \text { em } X_{-}
\end{array},\right.
$$

temos que $F(s) \in \mathrm{L}^{2}(E)$, pois $F_{ \pm}(s) \in \mathrm{H}^{1 / 2}\left(\left.E\right|_{X_{ \pm}}\right) \subset \mathrm{L}^{2}\left(\left.E\right|_{X_{ \pm}}\right)$. Vamos calcular $P(s) F(s)$ no sentido de distribuições. Por construção, $P(s) F(s)$ é uma distribuição suportada em $Y$ e para qualquer $w \in \mathrm{C}^{\infty}\left(\left.E\right|_{Y}\right)$, temos

$$
\begin{aligned}
\langle P(s) F(s), w\rangle & =\int_{X} F(s) \cdot P(s) \tilde{w} d y \text { onde } \tilde{w} \in C^{\infty}(E) \text { e }\left.\tilde{w}\right|_{Y}=w \\
& =\int_{X_{+}} F_{+}(s) \cdot P(s) \tilde{w} d x+\int_{X_{-}} F_{-}(s) \cdot P(s) \tilde{w} d x \\
& =\int_{Y} G\left(f_{+}(s)-f_{-}(s)\right) \cdot w d y,
\end{aligned}
$$

pela fórmula de Green-Stokes, onde $\langle\cdot, \cdot\rangle$ denota a dualidade usual entre distribuições e funções-teste e $\cdot$ denota o produto interno de $E$. Logo,

$$
P(s) F(s)=G\left(f_{+}(s)-f_{-}(s)\right)=G\left(U_{+}(s)-U_{-}(s)\right) g(s),
$$

no sentido de distribuições. Pondo $u_{0} \doteq \Phi\left(g_{0}, g_{0}\right)$, temos que $u_{0} \in \mathrm{W}_{k}\left(P, s_{0}\right)$, portanto $\Phi\left(\mathrm{W}_{k}\left(\mathrm{H}, s_{0}\right)\right) \subset \mathrm{W}_{k}\left(P, s_{0}\right)$, para todo $k \geq 1$. Além disso, de acordo com a definição de $B_{k}\left(\mathrm{H}, s_{0}\right)$ dada na fórmula 1.4 .10 e a proposição 1.4 .42 , se $v_{0}=\Phi\left(g_{0}, g_{0}\right)$ então

$$
\begin{aligned}
B_{k}\left(\mathrm{H}, s_{0}\right)\left(\left(g_{0}, g_{0}\right),\left(h_{0}, h_{0}\right)\right) & =\left.\frac{1}{k !} \int_{Y} \frac{d^{k}}{d s^{k}}\right|_{s=s_{0}} G\left(U_{+}(s)-U_{-}(s)\right) g(s) \cdot h_{0} d y \\
& =\frac{1}{k !}\left\langle\left.\frac{d^{k}}{d s^{k}}\right|_{s=s_{0}} P(s) F(s), h_{0}\right\rangle \\
& =B_{k}\left(P, s_{0}\right)\left(u_{0}, v_{0}\right) \\
& =\Phi^{*}\left(B_{k}\left(P, s_{0}\right)\right)\left(\left(g_{0}, g_{0}\right),\left(h_{0}, h_{0}\right)\right),
\end{aligned}
$$

encerrando a prova. 
Antes de estender o teorema para curvas arbitrárias, precisamos de um resultado simples de aproximação.

Seja $X$ um espaço de Banach e $\varphi: \mathbb{R} \rightarrow X$ uma curva. Dada $\chi: \mathbb{R} \rightarrow \mathbb{R}$ uma função $C^{\infty}$ com suporte compacto identicamente 1 em $[0,1]$, definimos

$$
\varphi_{\alpha}(s)=\sqrt{\frac{\alpha}{\pi}} \int_{\mathbb{R}} e^{-\alpha(s-t)^{2}} \chi(t) \varphi(t) d t,
$$

para $\alpha>0$. Claramente $\varphi_{\alpha}$ é uma função analítica real e dado $\varepsilon>0$, $\left\|\varphi_{\alpha}(s)-\varphi(s)\right\|<\varepsilon$ para $s \in[0,1]$ e $\alpha>0$ suficientemente pequeno. Definindo

$$
\psi_{\alpha}(s)=\varphi_{\alpha}(s)+(1-s)\left(\varphi(0)-\varphi_{\alpha}(0)\right)+s\left(\varphi(1)-\varphi_{\alpha}(1)\right),
$$

obtemos uma curva analítica real $\psi_{\alpha}$ tal que $\psi_{\alpha}(0)=\varphi(0)$ e $\psi_{\alpha}(1)=\varphi(1)$. Tomando $\alpha_{0}>0$ suficientemente pequeno tal que $\left\|\varphi_{\alpha_{0}}(s)-\varphi(s)\right\|<\varepsilon$ para todo $s \in[0,1]$, concluímos que $\left\|\psi_{\alpha_{0}}(s)-\varphi(s)\right\|<2 \varepsilon$ para todo $s \in[0,1]$. Logo, dada qualquer curva em um espaço de Banach, é possível obter uma aproximação (uniforme) analítica real com os mesmos pontos extremos. Este argumento é fundamental no próximo lema

Lema 2.4.18 Sejam $\varepsilon>0 e\left\{\nabla^{s}\right\}_{s \in[0,1]}$ uma família contínua de conexões em fibrado riemanniano $E$ sobre uma variedade compacta $X$, munido de uma estrutura de produto de Clifford. Então, existe uma família analítica real de conexões $\left\{\tilde{\nabla}^{s}\right\}_{s \in[0,1]}$ que coincide com $\left\{\nabla^{s}\right\}_{s \in[0,1]}$ nos extremos e tal que $\left\|\sigma^{s}-\tilde{\sigma}^{s}\right\|<\varepsilon, s \in[0,1]$, onde $\sigma^{s}$ e $\tilde{\sigma}^{s}$ denotam os símbolos dos operadores de Dirac correspondentes a $\nabla^{s} e \tilde{\nabla}^{s}$, respectivamente.

Prova. O espaço de conexões em $E$ é um espaço de Banach munido da norma

$$
\|\nabla\|=\sum_{\alpha=1}^{N} \sum_{j, k=1}^{n} \sup _{x \in U_{a}}\left|\nabla_{e_{j}^{\alpha}} e_{k}^{\alpha}(x)\right|,
$$

onde $\left\{e_{j}^{\alpha}\right\}_{j=1}^{n}, n=\operatorname{dim} E$, é um refeencial ortonormal definido sobre o aberto relativamente compacto $U_{\alpha}$ e $\left\{U_{\alpha}\right\}_{\alpha=1}^{N}$ é uma cobertura aberta de $X$. Como convergência na norma 2.4.19 implica convergência dos símbolos dos respectivos operadores de Dirac, o resultado segue dos comentários anteriores.

A versão clássica do teorema de Yoshida-Nicolaescu agora pode ser facilmente obtida.

TEOREMA 2.4.19 Se X é uma variedade riemanniana compacta orientada particionada por uma hipersuperfície fechada $Y, E$ é um fibrado de $\mathrm{Cl}(X)$-módulos à esquerda sobre $\mathrm{X}$ e $\{P(s)\}_{s \in[0,1]}$ é uma família contínua de operadores de Dirac auto-adjuntos em E que admitem a forma cilíndrica $2.4 .12 \mathrm{em}$ um bicolar de $Y$ então a fórmula 2.4.6 é verificada.

Prova. Seja $[0,1] \ni s \mapsto Q(s) \in \mathscr{L}\left(\mathrm{H}^{1}(E), \mathrm{H}^{0}(E)\right)$ uma família holomorfa de operadores de Dirac auto-adjuntos que aproxima $P(s)$ e tem a forma cilíndrica em um bicolar de $Y$, dada pelo lema 2.4.18. Como ambos os membros da equação 2.4 .6 são invariantes por pequenas perturbações, operadores de Dirac verificam PCU fraca (conforme o teorema 2.3.19) e o teorema é válido para a família $Q(s)$, o resultado segue. 


\section{Referências Bibliográficas}

[1] Abbondandolo, A., Morse Theory for Hamiltonian Systems, Pitman Research Notes in Mathematics, vol.425, Chapman \& Hall, 2001.

[2] Abbondandolo, A., Majer, P., Infinite Dimensional Grassmannians, arXiv:math.AT/0307192.

[3] Abbondandolo, A., Majer, P., Morse Homology in Hilbert Spaces, Comm. Pure Appl. Math. 54 (2001), 689-760.

[4] Agronovič, M., On the Problem of the Index of Elliptic Operators, Dokl. Akad. Nauk SSSR 142 (1962), 983-985.

[5] Agranovič, M., Dynin, A., General Boundary-value Problems for Elliptic Systems in Higherdimensional Regions, Dokl. Akad. Nauk SSSR 146 (1962), 511-514.

[6] Arnold, V., Characteristics Classes Entering in Quantization Conditions, Funct. Anal. Appl. 1 (1967), 1-13.

[7] Aronszajn, N., A Unique Continuation Theorem for Solutions of Elliptic Differential Equations or Inequalities of Second Order, J. Math. Pures Appl. 36 (1957), 235-249.

[8] АтіYAн, M., K-Theory, Benjamin, 1967.

[9] АтiYah, M., Botт, R., Shapiro, A., Clifford Modules, Topology 3 (1964) Suppl. 1, 3-38.

[10] AtiYAh, M., Singer, I., The Index of Elliptic Operators on Compact Manifolds, Bull. Amer. Math. Soc. 69 (1963), 422-433.

[11] Atryah, M., Singer, I., The Index of Elliptic Operators. I, Ann. of Math. (2) 87 (1968), 484-530.

[12] AтrYAh, M., Singer, I. The Index of Elliptic Operators. III., Ann. of Math. (2) 87 (1968), 546-604.

[13] АтIYAh, M., SingrR, I., Index Theory for Skew-adjoint Fredholm Operators, Publ. Math. IHES 37 (1969), 305-326.

[14] Atryah, M., V. Patodi, I. Singer, Spectral Asymmetry and Riemannian Geometry, Bull. London Math. Soc. 5 (1973), 229-234.

[15] Atryah, M., V. PAtodi, I. Singer, Spectral Asymmetry and Riemannian Geometry I, II, III; (I) Math. Proc. Camb. Phil. Soc. 77 (1975), 43-69; (II) 78 (1975), 405-432; (III) 79 (1976), 71-99.

[16] Bartle, R., Graves, L., Mappings Between Function Spaces, Trans. Amer. Math. Soc. 72 (1952), $400-413$

[17] Bleecker, D., Booss-Bavnbex, B., Spectral Invariants of Operators of Dirac Type on Partitioned Manifolds, Aspects of boundary problems in analysis and geometry, Oper. Theory Adv. Appl., 151,1-130. 
[18] Blezcker, D., Booss-BAvnbek, B., Topology and Analysis: The Atiyah-Singer Index Formula and Gauge-Theoretic Physics, Springer-Verlag, 1985.

[19] Bojarski, B., The Abstract Linear Conjugation Problem and Fredholm Pairs of Subspaces, in: "In Memorian I. N. Vekua", Tbilisi Univ. (1979), 45-60.

[20] Booss-BAvnBeK, B., The Unique Continuation Principle for Dirac Operators - Revisited, Geometry and Topology: Aarhus (1998), Contemporary Mathematics 258 (2000), 21-32.

[21] Booss-BAvnbeK, B., Furutani, K., Symplectic Functional Analysis and Spectral Invariants, Contemporary Mathematics 242, (1999), 53-83.

[22] Booss-Bavnbek, B., Furutani, K., The Maslov Index: a Functional Analytical Definition and the Spectral Flow Formula, Tokio J. Math. 21 (1998), 1-34.

[23] Booss-Bavnbek, B., Lesch, M., Phillips, J., Spectral flow of paths of self-adjoint Fredholm operators, Quantum gravity and spectral geometry (Napoli, 2001). Nuclear Phys. B Proc. Suppl. 104 (2002), 177-180. (arxiv-math.FA/0108014, 2001)

[24] Booss-Bavnbek, B., Marcolli, M., Wang, B., Weak UCP and Perturbed Monopole Equations, Internat. J. Math. 13 (2002), 987-1008.

[25] Booss-BAVnBek, B., Wojciechowski, K.P., Elliptic Boundary Value Problems for Dirac Operators, Birkhäuser, 1993.

[26] Booss-BavnвeK, B., Wojcizchowski, K.P., Desuspension of Splitting Elliptic symbols II, Ann. Global Anal. Geom. 4 (1986), 349-400.

[27] Booss-BavnbeK, B., ZHU, C., Weak Symplectic Functional Analysis and General Spectral Flow Formula, arXiv:math.DG/0406139, 2004.

[28] Calderón, A. Lectures on Pseudo-Differential Operators and Elliptic Boundary Value Problems, I, Instituto Argentino de Matematica, 1976.

[29] CAppell, S., Lee, R., Miller, E., Self-Adjoint Elliptic Operators and Manifold Decompositions Part III: Determinant Line Bundles and Lagrangian Intersection, Comm. Pure Appl. Math., 52 (1999), 543-611.

[30] Carleman, T., Sur les systèmes linéaires aux derivées partielles du premier ordre à deux variables, Comp. Rend. Acad. Sc. Paris 197 (1933), 471-474.

[31] Carleman, T., Sur un problème d'unicité pour les systemes d'équations aux dérivées partielles à deux variables indépendentes, Ark. Astr. Fys. 26 B 17 (1939), 1-9.

[32] CoRDes, H., Über die eindeutige Bestimmtheit der Lösungen elliptischer Differentialgleichungen durch Anfangsvorgaben, Nachr. Akad. Wiss. Göttingen Math.-Phys. Kl. IIa 11 (1956), 239-258.

[33] CoRdes, H., LABRousse, J.P., The Invariance of the Index in the Metric Space of Closed Operators, J. Math. Mech. 12 (1963), 693-720.

[34] Daniel, M., An Extension of a Theorem of Nicolaescu on Spectral Flow and Maslov Index, Proc. AMS 128 (1999), 611-619.

[35] Dynin, A., Singular Operators of Arbitrary Order on a Manifold, Dokl. Akad. Nauk SSSR 141 (1961), 21-23. 
[36] Dynin, A. S. n-dimensional Elliptic Boundary Value Problems with a Single Unknown Function, Dokl. Akad. Nauk SSSR 141 (1961), 285-287.

[37] Eidam, J. C. C., Piccione, P., Partial Signatures and the Yoshida-Nicolaescu Theorem, submetido à publicação; arXiv:math/DG.0502459.

[38] Eidam, J. C. C., Piccione, P., On the Homotopy Type of the Fredholm Lagrangian Grassmannian, submetido à publicação; arXiv:math/AT.0502480.

[39] FARBer, M., Levine, J., Jumps of the $\eta$-invariant, Math. Z., 223 (1996), 197-246.

[40] Folland, G., Introduction to Partial Differential Equations, Princeton University Press, 1995.

[41] Folland, G., Real analysis, Wiley Interscience Publishers, 1999.

[42] Fulton, W., Algebraic Topology: a First Course, Springer, 1995.

[43] GeL'rand, I., On Elliptic Equations, Russian Math. Surveys 15 (1960), no.3, 113.

[44] Giambó, R., Piccione, P., Portaluri, A., On the Maslov index of Lagrangians that are not Transversal to the Maslov cycle. Semi-Riemannian index theorems in the degenerate case, C. R. Math. Acad. Sci. Paris 338 (2004), no. 5, 397-402. / arxiv:math.DG/0306187.

[45] GiLKEx, P., Curvature and the Eigenvalues of the Laplace Operator for Elliptic Complexes, Adv. in Math. 10 (1973), 344-382.

[46] GILkEY, P., Invariance Theory, the Heat Equation, and the Atiyah-Singer Index Theorem, Publish or Perish, 1984.

[47] Goldberg, S., Unbounded Linear Operators, Dover, 1966.

[48] Guillemin, V., Pollack, A., Differential Topology, Prentice-Hall, 1974.

[49] Hatcher, A., Algebraic Topology, Cambridge University Press, 2002.

[50] Hirsch, M., Differential Topology, Springer, 1976.

[51] Hirzebruch, F., Arithmetic Genera and the Theorem of Riemann-Roch for Algebraic Varieties, Proc. Nat. Acad. Sci. USA. 40 (1954), 110-114.

[52] Hörmander, L., Linear Partial Differential Equations, Springer-Verlag, 1966.

[53] Hu, S., Homotopy Theory, Academic Press, 1959.

[54] JoHn, F., The Fundamental Solution of Linear Elliptic Differential Equations with Analytic Coefficients, Comm. Pure Appl. Math. 3 (1950), 273-304.

[55] Kato, T., Perturbation Theory for Linear Operators, Springer-Verlag, 1976.

[56] Ковауаsнi, S., Nomizu, K., Foundations of Differential Geometry, I,II, John Wiley \& Sons, Inc., 1963.

[57] KodaIrA, K., The Theorem of Riemann-Roch on Compact Analytic Surfaces, Amer. J. Math. 73, (1951) 813-875.

[58] KuIPER, N., The Homotopy Type of the Unitary Group of a Hilbert Space, Topology 3 (1964-65), 19-30. 
[59] LANG, S., Fundamentals of Differential Geometry, Springer, 1999.

[60] Lawson, B., Michelsohn, M-L., Spin Geometry, Princeton University Press, 1989.

:[61] Lufr, E., Maximal R-sets, Grassmann spaces and Stiefel spaces of a Hilbert space, Trans. Amer. Math. Soc. 126 (1967), 73-107

[62] May, J., A Concise Course in Algebraic Topology, Chicago University Press, 1999.

[63] McKean, H., Singer, I., Curvature and Eigenforms for the Laplacian, J. Diff. Geom. 1 (1967), 43-69.

[64] Milnor, J., Morse Theory, Annals of Mathematics Studies, Princeton University Press, 1963.

[65] Milnor, J., Spin Structures on Manifolds, L’Enseignement Math. 9 (1963), 198-203.

[66] MilnoR, J., Topology from the Differentiable Viewpoint, Princeton University Press, 1965.

[67] Milnor, J., Stasheff, J., Characteristic Classes, Princeton University Press, 1974.

[68] Munkres, J., Topology, Prentice-Hall, 2000.

[69] Nicolaescu, L., The Maslov Index, the Spectral Flow and Decompositions of Manifolds, Duke Math. Journal 80 (1995), 485-533.

[70] NicolaEscu, L., Notes on Seiberg-Witten theory, Graduate Studies in Mathematics, 28, American Mathematical Society, 2000.

[71] Palais, R., Seminar on the Atiyah-Singer Index Theorem, Princeton University Press, 1965.

[72] Palais, R., On the Homotopy Type of certain Groups of Operators, Topology 3 (1965), 271-279.

[73] Palais, R., Homotopy Theory in Infinite Dimensional Manifolds, Topology 5 (1966), 1-16.

[74] PAtodi, V., Curvature and the Eigenforms of the Laplace Operator, J. Diff. Geom. 5 (1971), 233-249. [75] PHILlips, J., Self-adjoint Fredholm Operators and Spectral Flow, Canad. Math. Bull. 39 (4) (1996),
460-467

[76] PICcione, P., TAusk, D. V, On the Geometry of Grassmannians and the Sympletic Group: the Maslov Index and its Applications, XI Escola de Geometria Diferencial, UFF-Niterói, 2000.

[77] PICCIONE, P., TAUSK, D., Complementary Lagrangians in Infinite Dimensional Symplectic Hilbert Spaces, arxiv:math.FA/0410031.

[78] Puiś, A., A Smooth Linear Elliptic Differential Equation without any Solution in a Sphere, Comm. Pure Appl. Math. 14 (1961), 599-617.

[79] Protrer, M., Unique Continuation for Elliptic Equations, Trans. Amer. Math. Soc. 95 (1960), 81-91.

[80] Reed, M., Simon, B., Methods of Modern Mathematical Physics, Vol.I - Functional analysis, Academic Press, 1980.

[81] REED, M., Simon, B., Methods of Modern Mathematical Physics, Vol.IV - Analysis of Operators, Academic Press, 1978. 
[82] Riesz, F., Sz.-NAGY, B., Functional analysis, Frederick Ungar Publishing Co., New York, 1955.

[83] Robbin, J., Salamon, D., The Maslov Index for Paths, Topology 32 (1993), 827-844.

[84] Robbin, J., Salamon, D., The Spectral Flow and the Maslov Index, Bull. London Math. Soc. 27 (1995), 1-33.

[85] Rosenberg, S., The Laplacian on a Riemannian Manifold, London Mt. Soc., 1997.

[86] Rudin, W., Real and Complex Analysis, McGraw-Hill, 1966.

[87] Rudin, W., Functional Analysis, McGraw-Hill, 1973.

[88] Schechter, M., Principles of Functional Analysis, Academic Press, 1971.

[89] SEeLey, R., Regularization of Singular Integral Operators on Compact Manifolds, Amer. J. Math. 83 (1961), 265-275.

[90] SeELey, R., The Index of Elliptic Systems of Singular Integral Operators, J. Math. Anal. Appl. 7 (1963), 289-309.

[91] SeELey, R., Singular Integrals and Boundary Value Problems, Amer. J. Math. 88 (1966), 781-809.

[92] Shanahan, P., The Atiyah-Singer Index Theorem, An Introduction, Lecture Notes in Mathematics, 638, Springer, 1978.

[93] Spanier, E., Algebraic Topology, Mc-Graw Hill, 1966.

[94] SPIVAK, M., A Comprehensive Introduction to Differential Geometry, Publish or Perish, 1975, 1979.

[95] Steenrod, N., The Topology of Fibre Bundles, Princeton University Press, 1951.

[96] Stein, E., Singular Integrals and Differentiability Properties of Functions, Princeton University Press,

[97] Swanson, R., Fredholm Intersection Theory and Elliptic Boundary Deformation Problems, I, J. Diff. Eq. 28 (1978), 189-201.

[98] Tréves, F., Basic Linear Partial Differential Equations, Academic Press, 1975.

[99] Varadarajan, V., Lie groups, Lie algebras and their Representations, Prentice-Hall, 1974.

[100] VICk, J. Homology Theory, Springer-Verlag, 1974.

[101] Vol'PERT, A., The Index of Systems of Two-dimensional Singular Integral Equations, Dokl. Akad. Nauk SSSR 142 (1962), 776-777.

[102] WARnER, F., Foundations of Differentiable Manifolds and Lie Groups, Springer-Verlag, 1983.

[103] YoshidA, T., Floer Homology and Splittings of Manifolds, Ann. of Math. 134 (1991), 227-323. 


\section{Índice Remissivo}

aplicação G-equivariante, 139

assimetria espectral, vii

assinatura de uma variedade $4 k$-dimensional, vii, 148

assinaturas parciais

de um par Fredholm de curvas, 105

de uma curva de lagrangianos, 101

autovalor(es)

expansão de Laurent em torno de um, 18

isolado, 18

multiplicidade algébrica de um, 18

nilpotente correspondente a um, 18

projetor correspondente a um, 18

sistema finito de, 19

cálculo funcional, 19

característica de Euler, 147

ciclo de Maslov, 98

classe

característica, 132

de Chern, 135

de Euler, 135

de obstrução, 132

co-ciclo, 131

condição de, 132

cohomologia

de Čech, 131

singular, 131

colar, 119

conexão, 138

conjectura de Bojarski, ix, 154

curvatura

de Ricci, 146

escalar, 146

forma de, 138

operador de, 139

deficiência

de um operador, $1,33,42$

de um par de subespaços, 3,34

derivada covariante, 138

desigualdade de Gårding, 116 divergência, 144

dobro de uma variedade, 114, 124

elemento de volume complexo, 138

espaço

de dados de Cauchy, ix, 125, 126, 150

de pares comensuráveis de lagrangianos, 93

de pares Fredholm, 92

de pares Fredholm de lagrangianos, 93

de seções

de classe $C^{k}, 109,114$

de suporte compacto, 109

distribucionais, 110

de Sobolev de seções, 113

homogêneo de um grupo de Lie, 86

simplético canônico, 69

simplético de dimensão infinita, 68

estavelmente paralelizável, 135

estimativas de Carleman, 149

estrutura complexa, 68, 153

expansão assintótica de um símbolo, 111

fórmula de Green-Stokes, 118

família holomorfa de operadores

auto-adjuntos, 27

de tipo (A), 28, 155

fechados, 25

fibrado

G-principal, 133, 136

associado, 136

cotangente de uma variedade, 109

de Clifford, 136

dos referenciais, 133, 136

irredutível, 137

riemanniano, 109

spinor, 137

tangente de uma variedade, 109

fluxo espectral, ix, 1, 50

forma cilíndrica, viii, 150

forma simplética, 68, 153

grassmanniana

lagrangianos comensuráveis, 93 
de lagrangianos, 89

de lagrangianos essencial, 96

de lagrangianos Fredholm, 93

de subespaços comensuráveis, 92

essencial, 92

Fredholm, 92

total, 87

grupo

ação de, 84

linear, 2, 78

ortogonal, 2,78

simplético, 78

unitário, 2, 78

espaço simplético, 78

unitário de um espaço simplético, 68

índice

de Fredholm

de um operador, vii, 2

de Maslov

de um par de curvas, 103

de uma curva de lagrangianos, 100

invariante de Casson, ix

isomorfismo

de Chern, vii

de Thom, vii

laplaciano, 146

métrica gap para operadores fechados, 9

$\eta$-invariante, viii

núcleo do calor, vii

norma do gráfico, 15

nulidade

de um operador, $1,33,42$

de um par de subespaços, 3,34

operador

adjunto, 111

analítico-hipoelíptico, 149

de Cauchy-Riemann, ix

de Dirac, ix, 141

compatível, 142

de Poisson, 125

diferencial, 110

$\star$ de Hodge, 143

fechado, 1 espectro de um, 2 módulo mínimo de um, 7 resolvente de um, 2

Fredholm, 2, 30, 39

hipoelíptico, 115

pseudo-diferencial, 111, 112

elíptico, 113

semi-Fredholm, 2

suavizante, 111

T-compacto, 14

T-limitado, 14

par Fredholm

de curvas de lagrangianos, 103

de lagrangianos, 77

parametriz, 113

problema de Cauchy, viii

projetor

de Atiyah-Patodi-Singer, 152, 153

de Calderón, 125, 152, 153

propriedade de continuação única

forte, 149

fraca, 149

pull-back de um fibrado, 109

quebra de simetria, 147

raio espectral, 2

símbolo(s), 112

classe de, 111

principal, 110, 112

total, 110

seção

harmônica, 146

paralela, 146

simplectomorfismo, 68

spin

estrutura, 131

fibrado, 132

variedade, 132

subespaço(s)

abertura máxima entre, 3

abertura mínima entre, 5

complementado, 83

complementares, 3

isotrópico, 69 
lagrangiano, 69, 153

par comensurável de, 46

par Fredholm de, 34, 153

simplético, 69

teorema

de Aronszajn-Cordes, 150

de Atiyah-Singer, vii, 148

de Bochner, 146

de Gallot-Meyer, 146

de Hellwig-Vekua, vii

de Hodge, 117, 145

de Kuiper, 79

de Riemann-Roch, vii

de Yoshida-Nicolaescu, x, 150, 155

traço de uma seção, 114, 115

transporte paralelo, 139

vertical

distribuição, 138

vetor, 138

$\mathbb{Z}_{2}$-graduação, 147 


\section{.}




\section{Índice de Símbolos e Notações}

$\doteq$ : Igual por definição, 3

$\langle\cdot, \cdot\rangle_{T}, 15$

L : Contração, 128

$\nabla$ : Derivada covariante, 138

$\star$ : Operador de Hodge, 143

Ad : Representação adjunta, 129

$B_{k}, 63$

C : Relação de extensão entre operadores lineares, inclusão de conjuntos, 2

$\mathscr{C}\left(\mathrm{H}, \mathrm{H}^{\prime}\right)$ : Espaço dos operadores lineares fechados entre $\mathrm{He} \mathrm{H}^{\prime}, 1$

$\mathscr{C}(\mathrm{H})$ : Espaço dos operadores lineares fechados em $\mathrm{H}, 1$

$\mathscr{C}^{\text {sa }}(\mathrm{H}), \mathscr{C}^{\text {sa }}$ : Espaço dos operadores auto-adjuntos em $\mathrm{H}$ (possivelmente ilimitados), 2

$\mathscr{C} \mathscr{F}\left(\mathrm{H}, \mathrm{H}^{\prime}\right)$ : Espaço dos operadores Fredholm (possivelmente ilimitados) entre $\mathrm{H}$ e $\mathrm{H}^{\prime}, 2$

$\mathscr{C} \mathscr{F}(\mathrm{H})$ : Espaço dos operadores Fredholm em $\mathrm{H}$ (possivelmente ilimitados), 2

$\mathscr{C} \mathscr{F}^{\text {sa }}(\mathrm{H}), \mathscr{C} \mathscr{F}^{\mathrm{sa}}$ : Espaço dos operadores Fredholm auto-adjuntos em $\mathrm{H}$ (possivelmente ilimitados), 2

$\operatorname{codim}_{H}(M)$ : Codimensão do subespaço $M$ no espaço $\mathrm{H}, 1$

$C^{k}(X ; E), C^{k}(E)$ : Espaço de seções de $E$ de classe $C^{k}, 110$

$C_{c}^{k}(E)$ : Espaço de seções de $E$ de classe $C^{k} \mathrm{e}$ suporte compacto, 110

$\mathrm{Cl}(E)$ : Fibrado de Clifford associado a $E$, 136

$\mathrm{Cl}(n), 127$

$\mathrm{Cl}(n), 131$

$\mathrm{Cl}^{j}(n), 128$

$\mathrm{Cl}^{\times}(n), 128$

$\mathrm{Cl}_{\text {Spin }}(E), 137$

$\operatorname{Comp}(\Lambda)$ : Espaço de pares comensuráveis de lagrangianos, 93

$C(\mathbb{R})$ : Espaço de Frechét das funções reais contínuas em $\mathbb{R}, 19$
$\mathrm{C}_{c}(Y)$ : Espaço de Banach das funções reais contínuas com suporte em $Y, 20$

$\mathrm{C}_{0}(\mathbb{R})$ : Espaço de Banach das funções reais contínuas em $\mathbb{R}$ que se anulam no infinito, 21

$D^{\alpha}, 109$

$\Delta$ : Diagonal do espaço $\mathrm{H} \oplus \mathrm{H}, 103$

$\operatorname{def} T$ : Deficiência do operador linear $T, 1$

$\operatorname{def}(M, N)$ : Deficiência do par de subespa$\operatorname{ços}(\mathrm{M}, \mathrm{N}), 3$

$\delta(M, N)$ : Abertura entre $M, N, 3$

$\delta(S, T)$ : Abertura entre $S$ e $T, 9$

$\tilde{\delta}(S, T)$ : Distância gap entre os operadores $S$ e $T, 9$

div : Divergência, 144

$\mathscr{D}^{\prime}(X ; E)$ : Espaço de seções distribucionais de $E, 110$

$\mathrm{D}(T)$ : Domínio do operador linear T, 1

$E$ : Fibrado vetorial, 109

$\mathscr{F}\left(\mathrm{H}, \mathrm{H}^{\prime}\right)$ : Espaço dos operadores Fredholm limitados entre $\mathrm{H} \mathrm{e} \mathrm{H}^{\prime}, 2$

$\mathscr{F}(\mathrm{H})$ : Espaço dos operadores Fredholm limitados em $\mathrm{H}, 2$

$\mathscr{F}_{L_{0}}^{k}(\Lambda), 97$

$\mathscr{F}_{\mathrm{L}}(\Lambda)$ : Grassmanniana de lagrangianos que formam par Fredholm com $L, 93$

$\mathscr{F}_{\mathrm{M}}(\mathrm{H}), 92$

$\mathrm{Fp}(\mathrm{H})$ : Espaço de pares Fredholm, 92

$\mathrm{Fp}(\Lambda)$ : Espaço de pares Fredholm de lagrangianos, 93

$\mathscr{F}^{\text {sa }}(\mathrm{H})$ : Espaço dos operadores Fredholm limitados auto-adjuntos em $\mathrm{H}, 2$

$\mathscr{F}_{*}^{\text {sa }}(\mathrm{H}): \mathscr{F}^{\text {sa }}(\mathrm{H}) \backslash\left(\mathscr{F}_{+}^{\text {sa }}(\mathrm{H}) \cup \mathscr{F}_{-}^{\text {sa }}(\mathrm{H})\right), 58$

$\mathscr{F}_{+}^{\text {sa }}(\mathrm{H}):$ Espaço dos operadores $T \in \mathscr{F}^{\text {sa }}(\mathrm{H})$ tais que $\Sigma_{\text {ess }}(T) \subset(0, \infty), 58$

$\mathscr{F}_{-}^{\text {sa }}(H):$ Espaço dos operadores $T \in \mathscr{F}^{\text {sa }}(H)$ tais que $\Sigma_{\text {ess }}(T) \subset(-\infty, 0), 58$

$\varphi_{\mathrm{L}_{0}, \mathrm{~L}_{1}, 74}$

$\gamma(\mathrm{M}, \mathrm{N})$ : Abertura mínima entre os subespaços $\mathrm{MeN}, 5$ 
$\gamma(T)$ : Módulo mínimo reduzido do operador linear $T, 7$

$\gamma_{0}:$ Traço, 114

GL(H) : Grupo linear de $\mathrm{H}, 2$

$\mathrm{GL}_{c}(\mathrm{H})$ : Grupo das perturbações compactas inversíveis da identidade de $\mathrm{H}$, 2

$\mathrm{GL}(\infty)$ : Grupo das perturbações inversíveis de posto finito da identidade, 87

$G_{p}$ : Grupo de isotropia em $p, 84$

$\mathrm{G}(T)$ : Gráfico do operador linear $T, 1$

$\mathcal{G}_{c}(L ; \Lambda)$ : Grassmanniana de lagrangianos comensuráveis com $L, 93$

$\mathcal{G}_{c}\left(\mathrm{~L}_{0} ; \mathrm{H}\right)$ : Grassmanniana de subespaços comensuráveis com $\mathrm{L}_{0}, 92$

$\mathcal{G}_{e}(\mathrm{H})$ : Grassmanniana essencial, 92

$\mathcal{G}(\mathrm{H})$ : Grassmanniana total de $\mathrm{H}, 87$

$\mathrm{H}$ : Espaço de Hilbert real ou complexo separável, 1

$\mathrm{H}_{\mathrm{ac}}$ : Espaço absolutamente contínuo de um operador auto-adjunto, 51

$\mathrm{H}_{\text {cont }}$ : Espaço contínuo de um operador auto-adjunto, 51

$\mathrm{H}_{\mathrm{pp}}$ : Espaço puramente pontual de um operador auto-adjunto, 51

$\mathrm{H}_{\text {sing }}$ : Espaço singular contínuo de um ope-

$\mathrm{H}_{\text {Cald }}(P, s), 126$ rador auto-adjunto, 51

$\mathrm{H}_{\mathrm{Har}}(P, s), 126$

$H_{ \pm}(P)$ : Espaço de dados de Cauchy, 119

$\mathrm{H}(P, s), 126$

$\mathrm{H}^{s}(E), 113,114$

$\breve{H}^{k}(X ; G)$ : Conjuntos de cohomologia de Čech, 131

Im $T$ : Imagem do operador linear $T, 1$

ind $T$ : Índice de Fredholm do operador linear $T, 2$

k: Transformada de Cayley, 22

$\operatorname{ker} T$ : Núcleo do operador linear $T, 1$

$\operatorname{ker}(P, s), 125$

$\Lambda$ : Grassmanniana de lagrangianos, 89

$L, L_{0}, L_{1}, \ldots$ : subespaços lagrangianos, 69

$\Lambda_{e}$ : Grassmanniana de lagrangianos essencial, 96
$\mathscr{L}\left(\mathrm{H}, \mathrm{H}^{\prime}\right)$ : Espaço dos operadores lineares limitados entre $\mathrm{He} \mathrm{H}^{\prime}, 2$

$\mathscr{L}(\mathrm{H})$ : Espaço dos operadores lineares limitados em $\mathrm{H}, 2$

$\mathscr{L}_{c}(\mathrm{H})$ : Espaço dos operadores lineares compactos em $\mathrm{H}, 2$

$\mathscr{L}_{\text {nor }}(\mathrm{H})$ : Espaço dos operadores lineares normais limitados, 20

$\mathscr{L}^{\text {sa }}(H)$ : Espaço dos operadores lineares limitados auto-adjuntos em $\mathrm{H}, 2$

$\mathscr{L}^{\operatorname{asim}}(\mathrm{H})$ : Espaço dos operadores lineares limitados anti-simétricos em $\mathrm{H}, 83$

$\Lambda_{0}\left(L_{1}\right)$ : Conjunto de todos os lagrangianos complementares a $\mathrm{L}_{1}, 74$

$\Lambda^{*}\left(\mathbb{R}^{n}\right)$ : Álgebra exterior de $\mathbb{R}^{n}, 128$

$\Lambda_{+}^{s}, 123$

$\mu_{\mathrm{L}_{0}}$ : Índice de Maslov com respeito a $\mathrm{L}_{0}$, 100

$\mu(\cdot, \cdot)$ : Índice de Maslov para pares Fredholm de curvas de lagrangianos, 103

$\mathrm{M}, \mathrm{N}$ : Subespaços fechados de ụm espaço de Hilbert, 3

$\mathrm{M} \oplus \mathrm{N}, 3$

$\operatorname{mul}(\lambda)$ : multiplicidade algébrica de $\lambda, 18$

$\mathscr{N}_{0}(P), 125$

$n_{k}^{+}\left(T, t_{0}\right): n$-ésimo co-índice parcial de $T$ em $t_{0}, 63$

$n_{k}^{+}\left(\gamma, t_{0} ; L_{0}\right): k$-ésimo co-índice de $\gamma$ em $t_{0}$, 102

$n_{k}^{+}\left(\gamma_{0}, \gamma_{1}, t_{0}\right): k$-ésimo co-índice de $\gamma_{0}, \gamma_{1}$ em $t_{0}, 106$

$n_{k}^{-}\left(T, t_{0}\right): n$-ésimo índice parcial de $T$ em $t_{0}$, 63

$n_{k}^{-}\left(\gamma, t_{0} ; L_{0}\right): k$-ésimo índice de $\gamma$ em $t_{0}, 102$

$n_{k}^{-}\left(\gamma_{0}, \gamma_{1}, t_{0}\right): k$-ésimo índice de $\gamma_{0}, \gamma_{1}$ em $t_{0}$, 106

nul $T$ : Nulidade do operador linear $T, 1$ nul $(M, N)$ : Nulidade do par de subespaços $(\mathrm{M}, \mathrm{N}), 3$

$\mathrm{O}(\mathrm{H})$ : Grupo ortogonal do espaço de Hilbert real $\mathrm{H}, 2$

$\mathrm{O}_{c}(\mathrm{H})$ : Grupo das perturbações compactas inversíveis ortogonais da identidade do espaço de Hilbert real $\mathrm{H}$, 
2

$\omega_{\text {can }}$ : Forma simplética canônica, 69

$\operatorname{ord}(u)$ : Ordem da função-raiz $u, 63$

$P(T)$ : Conjunto resolvente do operador linear $T, 2$

$P^{*}$ : Adjunto de $P, 111$

$P_{\mathrm{GL}}(E), 136$

$\mathcal{P}(H)$ : Conjunto dos isomorfismos positivos auto-adjuntos de $\mathrm{H}, 79$

$\pi_{\geq}, 152$

$\operatorname{Pin}(n), 129$

$\tilde{\Pi}_{ \pm}, 120$

$P_{M}$ : Projeção ortogonal sobre o subespaço fechado M, 3

$P_{m}$ : Parte principal de $P, 110$

$P_{\mathrm{M}}^{\mathrm{N}}$ : Restrição de $P_{\mathrm{M}}$ a N, 3

$P_{\mathrm{M}, \mathrm{N}}$ : Projeção sobre $\mathrm{N}$ paralelamente a $\mathrm{M}$, 3

$P_{\mathrm{L}_{1}, \mathrm{~L}}^{\mathrm{L}_{0}}:$ Restrição de $P_{\mathrm{L}_{1}, \mathrm{~L}}$ a $\mathrm{L}_{0}, 72$

$P_{\mathrm{O}}(E), 133$

$P \times_{\rho} F$ : Fibrado associado a $P$ por $\rho, 136$

$\Psi_{L_{0}, L_{1}}, 74$

$\psi_{\mathrm{L}_{0}, \mathrm{~L}_{1}}, 87$

$P_{\text {SO }}(E), 133$

$\mathrm{R}(\zeta ; T)$ : Resolvente do operador linear $T, 2$

$R(T)$ : Operador definido por $\left(I+T^{*} T\right)^{-1}, 10$ $R(Y, Z), 139$

$\Sigma_{\mathrm{L}_{0}}$ : Ciclo de Maslov com vértice em $\mathrm{L}_{0}, 98$

$\Sigma(T)$ : Espectro do operador linear $T, 2$

$\Sigma_{\mathrm{ac}}(T)$ : Espectro absolutamente contínuo do operador $T, 51$

$\Sigma_{\text {cont }}(T):$ Espectro contínuo do operador $T$, 51

$\Sigma_{\text {disc }}(T):$ Espectro discreto do operador $T$, 51

$\Sigma_{\text {ess }}(T)$ : Espectro essencial do operador $T$, 51

$\Sigma_{\mathrm{pp}}(T)$ : Espectro puramente pontual do operador $T, 51$

$\Sigma_{\text {sing }}(T)$ : Espectro singular contínuo do operador $T, 51$

sf : Fluxo espectral, 57

$\operatorname{sig}(X)$ : Assinatura da variedade $X, 148$

$\sigma(P):$ Símbolo de $P, 110$
$\sigma_{k}\left(T, t_{0}\right): n$-ésima assinatura parcial de $T$ em $t_{0}, 63$

$\sigma_{k}\left(\gamma, t_{0} ; L_{0}\right): k$-ésima assinatura parcial de $\gamma$ em $t_{0}, 102$

$\sigma_{k}\left(\gamma_{0}, \gamma_{1}, t_{0}\right): k$-ésima assinatura parcial de $\gamma_{0}, \gamma_{1}$ em $t_{0}, 106$

$S^{m}(E, F), 111$

$\mathrm{S}(E)$ : Fibrado spinor real, 137

$\mathrm{S}_{\mathbb{C}}(E)$ : Fibrado spinor complexo, 137

$\mathrm{Sp}(\mathrm{H})$ : Grupo simplético do espaço simplético $\mathrm{H}, 68$

$\mathrm{Sp}\left(\mathrm{H}, \mathrm{L}_{0}\right), 91$

$\mathfrak{s p}(\mathrm{H})$ : Álgebra de Lie de Sp(H), 83

$\mathfrak{s p}\left(\mathrm{H}, \mathrm{L}_{0}\right), 91$

$\operatorname{Spin}(n), 129$

$\sigma(Y), 138$

$\operatorname{spr}(T)$ : Raio espectral do operador $T, 2$

$\mathscr{T}\left(\mathbb{R}^{n}\right)$ : Álgebra tensorial de $\mathbb{R}^{n}, 127$

TX : Fibrado tangente de $X, 109$

$T^{*} X$ : Fibrado cotangente de $X, 109$

$\mathrm{U}(\mathrm{H})$ : Grupo unitário do espaço de Hilbert complexo $\mathrm{H}, 2$

$\mathfrak{u}(\mathrm{H})$ : Álgebra de Lie de $\mathrm{U}(\mathrm{H}), 83$

$\mathrm{U}_{c}(\mathrm{H})$ : Grupo das perturbações compactas inversíveis unitárias da identidade do espaço de Hilbert complexo $\mathrm{H}, 2$

$\mathrm{U}(\mathrm{H} ; J)$ : Grupo unitário do espaço simplético $\mathrm{H}, 68$

$\mathrm{U}(\infty)$ : Grupo das perturbações inversíveis de posto finito unitárias da identidade, 87

$\mathrm{U}_{\mathrm{inj}}(\mathrm{H})$ : Espaço dos operadores unitários $U$ tais que $\operatorname{ker}(I-U)=0,22$

$\mathrm{U}_{\text {inj }}^{\text {Fred }}(\mathrm{H})$ : Espaço dos $U \in U_{\mathrm{inj}}(\mathrm{H})$ tais que $U+I$ é Fredholm, 23

$w_{j}(E)$ : j-ésima classe de Stiefel-Whitney de E, 132

$W_{k}, 63$

$\mathfrak{X}(U)$ : Espaço dos campos vetoriais em $U$, 138 